\title{
Construction of Acyclic Quaternary Carbon Stereocenters by Catalytic Asymmetric Hydroalkynylation of Unactivated Alkenes
}

\author{
Zi-Xuan Wang and Bi-Jie Li* \\ Center of Basic Molecular Science (CBMS), Department of Chemistry, Tsinghua University
}

Table of Contents

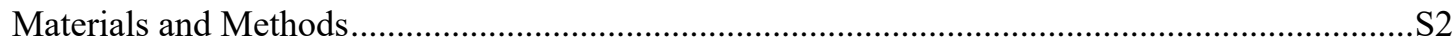

General Procedure for the Synthesis of $\beta, \gamma$-Unsaturated Amide....................................................S2

General Procedure for Ir-Catalyzed Hydroalkynylation .............................................................. 4

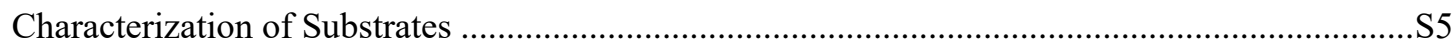

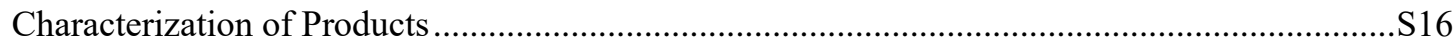

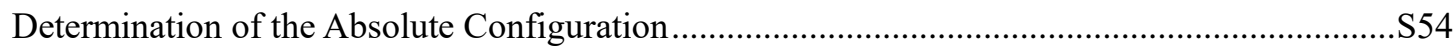

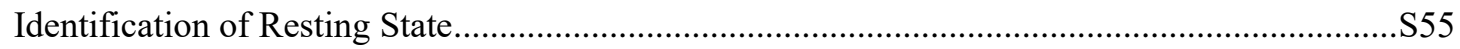

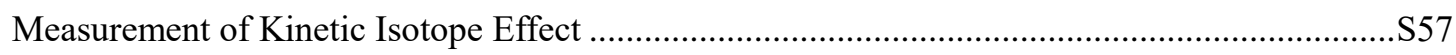

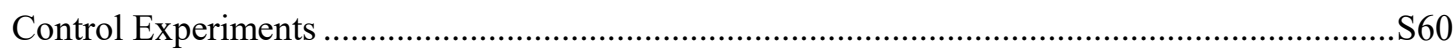

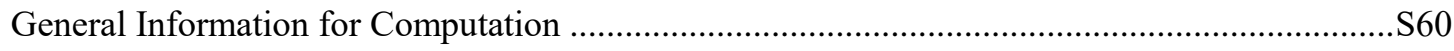

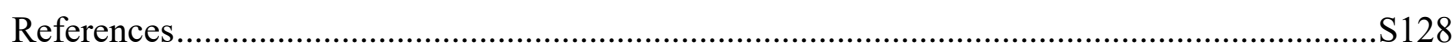

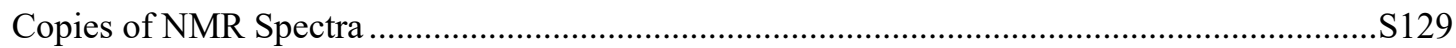




\section{Materials and Methods}

Unless otherwise noted, all reactions were assembled on a Schlenk vacuum line or in a glovebox using oven-dried glassware and were stirred with Teflon-coated magnetic stirring bars. All the ligands were purchased from Strem Chemicals and were used as received. $\left[\operatorname{Ir}(\operatorname{cod})_{2}\right] \mathrm{OTf}^{1}$ was prepared according to literature methods. Triisopropylsilylacetylene was purchased from Alfa Aesar and was used as received. Triisopropylsilylacetylene- $\mathrm{d} 1^{2}$ was prepared according to literature method. 1,2-difluorobenzene was degassed by purging with nitrogen and then distilled from $\mathrm{CaH}_{2}$. All other solvents and reagents were used as received. The amide substrates were prepared through condensation reaction of $\beta, \gamma$-unsaturated acid and amine ${ }^{3}$ according to literature methods. All work-up and purification procedures were carried out with reagent grade solvents in air. Reaction temperatures above $23{ }^{\circ} \mathrm{C}$ refer to temperatures of an aluminum heating block or a silicon oil bath, which were controlled by an electronic temperature modulator from IKA. NMR spectra were acquired on NMR spectrometer with $400 \mathrm{MHz}$ for ${ }^{1} \mathrm{H}$ NMR and $101 \mathrm{MHz}$ for ${ }^{13} \mathrm{C}$ NMR at the NMR facility at Center of Basic Molecular Science (CBMS). Chemical shifts ( $\delta$ ) are reported in ppm relative to the residual solvent signal $\left(\delta=7.26\right.$ for ${ }^{1} \mathrm{H}$ NMR and $\delta=77.0$ for ${ }^{13} \mathrm{C}$ NMR). Data for ${ }^{1} \mathrm{H}$ NMR spectra are reported as follows: chemical shift (multiplicity, coupling constants, number of hydrogens). Abbreviations are as follows: s (singlet), d (doublet), t (triplet), q (quartet), m (multiplet), br (broad). Infrared (IR) spectra were recorded on a Bruker FT-IR alpha (ATR mode) spectrophotometer. High-resolution mass spectral data was performed on a Thermo Scientific Q Exactive (positive mode) at the Mass Spectrometry Facility, CBMS. Enantiomeric ratio (er) values were determined by analytical liquid chromatography (HPLC) analysis on a Shimadzu chromatograph (Daicel chiral columns Chiralpak IA, IC, ID, IE, IF (4.6 x 250 mm)). Specific rotations were measured on a Jasco P-2000 Polarimeter.

\section{General Procedure for the Synthesis of $\beta, \gamma-$-Unsaturated Amide:}

To a solution of $\beta, \gamma$-unsaturated carboxylic acid (5.0 mmol, 1.0 equiv.) in dichloromethane (10.0 $\mathrm{mL}$ ) were added 1-ethyl-3-(3-(dimethylamino)propyl)-carbodiimide hydrochloride (EDCI) (7.5 mmol, 1.5 equiv.), $N, N$-dimethylaminopyridine $(0.50 \mathrm{mmol}, 10.0 \mathrm{~mol} \%)$ and then the amine $(5.5$ mmol, 1.1 equiv.). The reaction mixture was stirred at room temperature for $12 \mathrm{~h}$. Dichloromethane $(50 \mathrm{~mL})$ was added, and the mixture was washed with $1 \% \mathrm{HCl}(10 \mathrm{~mL})$, brine 
solution $(20 \mathrm{~mL})$ and dried over $\mathrm{Na}_{2} \mathrm{SO}_{4}$. After removal of the solvent, the residue was purified by column chromatography on silica gel with EtOAc/hexanes mixture as eluent.

\section{Synthesis of $(E)$ - $\beta, \gamma$-unsaturated carboxylic acid}

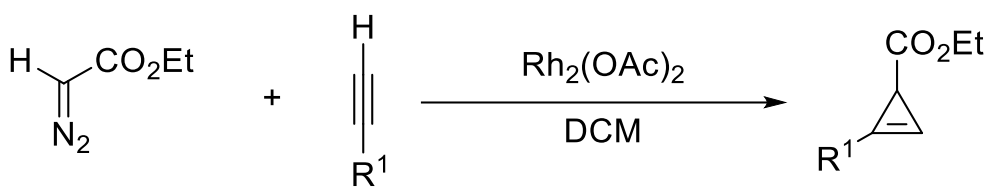

A solution of the diazoacetate $(10.0 \mathrm{mmol})$ in $20 \mathrm{~mL}$ DCM was slowly added to a stirred mixture of $\mathrm{Rh}_{2}(\mathrm{OAc})_{4}(0.10 \mathrm{mmol})$ and alkyne $(30 \mathrm{mmol})$ at room temperature. After the addition was complete, the mixture was allowed to stir for $2 \mathrm{~h}$ and then filtered through celite, concentrated, and chromatographed to give the corresponding cyclopropene derivative.<smiles>[R]C([R14])=CCC(=O)OCC</smiles>

Alkylmagnesium bromide (1.3 equiv.) was added dropwise to a suspension of $\mathrm{CuI}$ (19 mg, 0.10 mmol) in $\mathrm{Et}_{2} \mathrm{O}(10 \mathrm{~mL})$ and cyclopropene $(1.0 \mathrm{mmol})$ held at $-40{ }^{\circ} \mathrm{C}$. The resulting mixture was stirred at the same temperature for $1 \mathrm{~h}$, warmed to $0{ }^{\circ} \mathrm{C}$ and then stirred at $0{ }^{\circ} \mathrm{C}$ for $1 \mathrm{~h}$. The reaction was then quenched with $0.5 \mathrm{M} \mathrm{HCl}$. The aqueous layer was extracted twice with $\mathrm{Et}_{2} \mathrm{O}(20$ $\mathrm{mL}$ ). The combined organics were washed with brine, dried over $\mathrm{MgSO}_{4}$, filtered, and concentrated under reduced pressure. Crude mixtures were then purified by flash chromatography to give the corresponding $(E)-\beta, \gamma$-unsaturated esters.<smiles>[R]C([R])=CCC(=O)OCCO</smiles><smiles>[R]C([R])=CCC(=O)O</smiles>

A mixture of $(E)-\beta, \gamma$-unsaturated ester $(2.0 \mathrm{mmol})$ and $\mathrm{LiOH} \cdot \mathrm{H}_{2} \mathrm{O}(5$ equiv. $)$ in $\mathrm{EtOH}(10 \mathrm{~mL})$ and $\mathrm{H}_{2} \mathrm{O}(10 \mathrm{~mL})$ was vigorously stirred at room temperature for $8 \mathrm{~h}$. After the reaction was quenched with saturated aqueous $\mathrm{NH}_{4} \mathrm{Cl}$, the water layer was acidified with concentrated $\mathrm{HCl}(\mathrm{pH}=3)$ and extracted with DCM. The organic layer was dried with $\mathrm{Na}_{2} \mathrm{SO}_{4}$, concentrated in vacuo and chromatographically purified to yield (E)- $\beta, \gamma$-unsaturated carboxylic acid.

\section{Synthesis of (Z)- $\beta, \gamma$-unsaturated carboxylic acid}

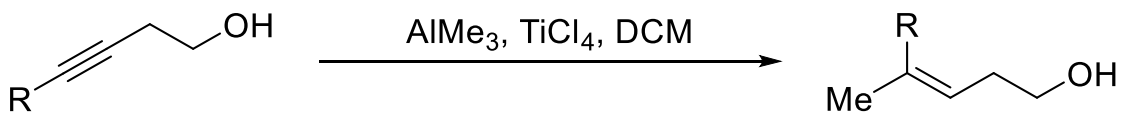


To a cooled $\left(0{ }^{\circ} \mathrm{C}\right)$ solution of trimethylaluminum $(16.0 \mathrm{~mL}, 2.0 \mathrm{M}$ solution in toluene, $32.0 \mathrm{mmol})$ in dichloromethane $(50 \mathrm{~mL})$ was added alkynol $(16.0 \mathrm{mmol})$ dropwise. The reaction was stirred for 20 minutes at the same temperature. The mixture was then cooled $\left(-45^{\circ} \mathrm{C}\right)$ followed by the addition of a cooled $\left(0{ }^{\circ} \mathrm{C}\right)$ solution of titanium(IV) chloride $(3.03 \mathrm{~g}, 16.0 \mathrm{mmol})$ in dichloromethane $(45 \mathrm{~mL})$ over the period of ca. $1 \mathrm{~min}$. via cannula. The resulting mixture was allowed to stir for 15 minutes and then quenched by the slow addition of pre-cooled $\left(0{ }^{\circ} \mathrm{C}\right)$ methanol $(8 \mathrm{~mL})$. An aqueous solution of $3 \mathrm{M} \mathrm{HCl}$ saturated with $\mathrm{NaCl}(30 \mathrm{~mL})$ was then added and the mixture was stirred for another 30 minutes. The organic layer was separated followed by extraction from the aqueous layer with diethyl ether $(3 \times 50 \mathrm{~mL})$. The combined organic extracts were dried (anhyd. $\mathrm{Na}_{2} \mathrm{SO}_{4}$ ) and concentrated under reduced pressure. Purification of the residue by flash chromatography affords the title compound.<smiles>[R]C(C)=CCCO</smiles>

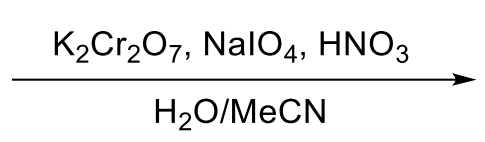<smiles>[R]C(C)=CCC(=O)O</smiles>

To a solution of $\mathrm{K}_{2} \mathrm{Cr}_{2} \mathrm{O}_{7}\left(6 \mathrm{mg}, 0.02 \mathrm{mmol}, 1 \mathrm{~mol} \%\right.$ ), $65 \%$ aq. $\mathrm{HNO}_{3}$ (39 mg, $0.20 \mathrm{mmol}, 10$ mol\%) and $\mathrm{NaIO}_{4}\left(941 \mathrm{mg}, 4.4 \mathrm{mmol}, 2.2\right.$ equiv.) in $\mathrm{H}_{2} \mathrm{O}(2.0 \mathrm{~mL})$ at $0{ }^{\circ} \mathrm{C}$ were added $4.0 \mathrm{~mL}$ of $\mathrm{MeCN}$ and (Z)-3-en-1-ol (2.0 mmol, 1 equiv.). The reaction mixture was stirred at $0{ }^{\circ} \mathrm{C}$ for $8 \mathrm{~h}$ and at $10{ }^{\circ} \mathrm{C}$ overnight. Inorganic salts were filtrated off and washed with $\mathrm{Et}_{2} \mathrm{O}$. The organic phase was separated and the aqueous phase was extracted with $\mathrm{Et}_{2} \mathrm{O}(3 \times 10 \mathrm{~mL})$. The combined organic phases were dried $\left(\mathrm{Na}_{2} \mathrm{SO}_{4}\right)$ and evaporated. The crude product was purified by flash chromatography to give (Z)- $\beta, \gamma$-unsaturated carboxylic acid

\section{General Procedure for Ir-Catalyzed Hydroalkynylation:}

In an $\mathrm{N}_{2}$-filled glovebox, $0.20 \mathrm{mmol}$ of the amide was weighed into a one-dram screw-capped vial. A stir bar was added, and the substrate was dissolved in 1,2-difluorobenzene $(0.30 \mathrm{~mL})$. The resulting solution was treated with $\left[\operatorname{Ir}(\operatorname{cod})_{2}\right]$ OTf $(20.0 \mu \mathrm{mol}, 10.0 \mathrm{~mol} \%)$, CTH-P-Phos $(24.0$ $\mu \mathrm{mol}, 12.0 \mathrm{~mol} \%)$ and then triisopropylsilylacetylene $(0.40 \mathrm{mmol}, 2.0$ equiv.). The vial was capped with a Teflon-lined screw cap, and the resulting solution was then removed from the glovebox, placed in a pre-set low temperature thermostatic stirring reaction bath at $20{ }^{\circ} \mathrm{C}$ for $84 \mathrm{~h}$. The reaction mixture was brought to room temperature and concentrated. The residue was directly 
purified by column chromatography on silica gel with EtOAc/hexanes mixture as eluent.

\section{Characterization of Substrates:}<smiles>CCN(CC)C(=O)C/C=C(\C)C(C)C</smiles>

Amide 1a: ${ }^{1} \mathrm{H}$ NMR $\left(400 \mathrm{MHz}, \mathrm{CDCl}_{3}\right) \delta 5.32(\mathrm{qt}, J=6.7,1.2 \mathrm{~Hz}, 1 \mathrm{H}), 3.36(\mathrm{q}, J=7.1 \mathrm{~Hz}, 2 \mathrm{H})$, $3.29(\mathrm{q}, J=7.1 \mathrm{~Hz}, 2 \mathrm{H}), 3.05(\mathrm{~d}, J=6.7 \mathrm{~Hz}, 2 \mathrm{H}), 2.00(\mathrm{t}, J=7.5 \mathrm{~Hz}, 2 \mathrm{H}), 1.62(\mathrm{~s}, 3 \mathrm{H}), 1.50-$ $1.34(\mathrm{~m}, 2 \mathrm{H}), 1.17(\mathrm{t}, J=7.1 \mathrm{~Hz}, 3 \mathrm{H}), 1.10(\mathrm{t}, J=7.1 \mathrm{~Hz}, 3 \mathrm{H}), 0.86(\mathrm{t}, J=7.3 \mathrm{~Hz}, 3 \mathrm{H}) .{ }^{13} \mathbf{C} \mathbf{N M R}$ $\left(101 \mathrm{MHz}, \mathrm{CDCl}_{3}\right) \delta 171.19,137.81,117.44,41.98,41.68,40.05,33.52,20.87,16.27,14.33,13.69$, 13.03. ESI-HR calcd for $\mathrm{C}_{12} \mathrm{H}_{24} \mathrm{NO}^{+}\left([\mathrm{M}+\mathrm{H}]^{+}\right)$198.1852, found 198.1847. IR $v\left(\mathrm{~cm}^{-1}\right) 2962,1604$, 1433,1139<smiles>CCCCN(CCCC)C(=O)C/C=C(\C)C(C)C</smiles>

Amide 1b: ${ }^{1} \mathbf{H}$ NMR (400 MHz, CDCl $) \delta 5.39-5.24(\mathrm{~m}, 1 \mathrm{H}), 3.30(\mathrm{t}, J=7.4 \mathrm{~Hz}, 2 \mathrm{H}), 3.20(\mathrm{t}, J=$ $7.4 \mathrm{~Hz}, 2 \mathrm{H}), 3.06(\mathrm{~d}, J=6.7 \mathrm{~Hz}, 2 \mathrm{H}), 2.00(\mathrm{t}, J=7.4 \mathrm{~Hz}, 2 \mathrm{H}), 1.63(\mathrm{~s}, 3 \mathrm{H}), 1.59-1.38(\mathrm{~m}, 6 \mathrm{H}), 1.37-$ $1.24(\mathrm{~m}, 4 \mathrm{H}), 0.98-0.89(\mathrm{~m}, 6 \mathrm{H}), 0.87(\mathrm{t}, J=7.3 \mathrm{~Hz}, 3 \mathrm{H}) .{ }^{13} \mathbf{C} \mathbf{N M R}\left(\mathbf{1 0 1} \mathbf{~ M H z}, \mathbf{C D C l}_{3}\right) \delta 171.53$, $137.70,117.56,47.79,45.60,41.67,33.57,31.27,29.86,20.86,20.23,20.16,16.30,13.86,13.82$, 13.68. ESI-HR calcd for $\mathrm{C}_{16} \mathrm{H}_{32} \mathrm{NO}^{+}\left([\mathrm{M}+\mathrm{H}]^{+}\right) 254.2478$, found 254.2470. IR $v\left(\mathrm{~cm}^{-1}\right) 2957,1640$, $1455,938$.<smiles>CCCCCCCN(CC)C(=O)C/C=C(\C)C(C)C</smiles>

Amide 1c: Two sets of peaks were observed in ${ }^{1} \mathrm{H}$ and ${ }^{13} \mathrm{C}$ NMR spectra due to amide rotamers, which are assigned to major and minor rotamers, respectively. ${ }^{1} \mathbf{H} \mathbf{~ N M R}\left(400 \mathrm{MHz}, \mathrm{CDCl}_{3}\right) \delta 5.38$ - $5.27(\mathrm{~m}, 1 \mathrm{H}$, overlapping), $3.36(\mathrm{q}, J=7.1 \mathrm{~Hz}, 2 \mathrm{H}$, major), $3.33-3.24(\mathrm{~m}, 4 \mathrm{H}$, overlapping), $3.18(\mathrm{t}$, $J=7.6 \mathrm{~Hz}, 2 \mathrm{H}$, major), $3.10-3.02(\mathrm{~m}, 2 \mathrm{H}$, overlapping), $2.00(\mathrm{t}, J=7.5 \mathrm{~Hz}, 2 \mathrm{H}$, overlapping), 1.62 (s, $3 \mathrm{H}$, overlapping), $1.59-1.48(\mathrm{~m}, 2 \mathrm{H}$, overlapping), $1.47-1.37$ (m, 2H, overlapping), $1.36-1.21(\mathrm{~m}$, 
$8 \mathrm{H}$, overlapping), $1.16(\mathrm{t}, J=7.1 \mathrm{~Hz}, 3 \mathrm{H}$, minor), $1.10(\mathrm{t}, J=7.1 \mathrm{~Hz}, 3 \mathrm{H}$, major) $0.92-0.82(\mathrm{~m}$, 6H,overlapping). ${ }^{13} \mathrm{C}$ NMR (101 MHz, $\left.\mathrm{CDCl}_{3}\right) \delta 171.38$ (major), 171.35 (minor), 137.79 (major), 137.72 (minor), 117.52 (overlapping), 47.72 (major), 45.37 (minor), 42.32 (minor), 41.69 (overlapping), 40.55 (major), 33.62 (major), 33.52 (minor), 31.79 (minor), 31.75 (major), 29.25 (major), 29.09 (minor), 29.05 (major), 27.84 (minor), 27.00 (minor), 26.94 (major), 22.57 (minor), 22.55 (major), 20.88 (overlapping), 16.31 (minor), 16.29 (major), 14.25 (minor), 14.03 (minor), 14.00 (major), 13.69 (overlapping), 12.94 (major). ESI-HR calcd for $\mathrm{C}_{17} \mathrm{H}_{34} \mathrm{NO}^{+}\left([\mathrm{M}+\mathrm{H}]^{+}\right)$268.2635, found 268.2628. IR $v\left(\mathrm{~cm}^{-1}\right) 2957,1641,1456,1133$.<smiles>CCN(CCc1ccccc1)C(=O)C/C=C(\C)C(C)C</smiles>

Amide 1d: Two sets of peaks were observed in ${ }^{1} \mathrm{H}$ and ${ }^{13} \mathrm{C}$ NMR spectra due to amide rotamers, which are assigned to major and minor rotamers, respectively. ${ }^{1} \mathbf{H} \mathbf{~ N M R}\left(400 \mathrm{MHz}, \mathrm{CDCl}_{3}\right) \delta 7.36$ -7.27 (m, 2H, overlapping), $7.25-7.12(\mathrm{~m}, 3 \mathrm{H}$, overlapping), $5.34(\mathrm{t}, J=6.7 \mathrm{~Hz}, 1 \mathrm{H}$, major), $5.27(\mathrm{t}$, $1 \mathrm{H}, J=6.7 \mathrm{~Hz}$, minor), $3.58-3.44(\mathrm{~m}, 2 \mathrm{H}$, overlapping), $3.40(\mathrm{q}, J=7.1 \mathrm{~Hz}, 2 \mathrm{H}$, minor $), 3.17(\mathrm{q}, J=$ $7.1 \mathrm{~Hz}, 2 \mathrm{H}$, major), $3.07(\mathrm{~d}, J=6.7 \mathrm{~Hz}, 2 \mathrm{H}$, major $), 2.91(\mathrm{~d}, J=6.7 \mathrm{~Hz}, 2 \mathrm{H}$, minor $), 2.88-2.78(\mathrm{~m}$, 2H, overlapping), $2.06-1.88(\mathrm{~m}, 2 \mathrm{H}$, overlapping), $1.64(\mathrm{~s}, 3 \mathrm{H}$, major $), 1.57$ (s, 3H, minor), $1.51-$ $1.36\left(\mathrm{~m}, 2 \mathrm{H}\right.$, overlapping), $1.19-1.07\left(\mathrm{~m}, 3 \mathrm{H}\right.$, overlapping), $0.94-0.81\left(\mathrm{~m}, 3 \mathrm{H}\right.$, overlapping). ${ }^{13} \mathrm{C}$ NMR (101 MHz, $\mathrm{CDCl}_{3}$ ) $\delta 171.54$ (overlapping), 139.51 (major), 138.36 (minor), 138.02 (major), 137.89 (minor), 128.87 (overlapping), 128.73 (overlapping), 128.67 (overlapping), 128.39 (overlapping), 126.69 (minor), 126.18 (major), 117.28 (minor), 117.25 (major), 49.15(minor) , 47.72 (major), 43.11 (major), 41.70 (major), 41.65 (minor), 40.56 (minor), 35.62 (minor), 34.18 (major), 33.51 (major), 33.41 (minor), 20.89 (major), 20.86 (minor), 16.31 (minor), 16.30 (major), 14.15 (major), 13.73 (major), 13.67 (minor), 12.87 (minor). ESI-HR calcd for $\mathrm{C}_{18} \mathrm{H}_{28} \mathrm{NO}^{+}\left([\mathrm{M}+\mathrm{H}]^{+}\right)$ 274.2165, found 274.2150. IR $v\left(\mathrm{~cm}^{-1}\right) 2930,1640,1454,748$.<smiles>CCN(CCc1cccs1)C(=O)C/C=C(\C)C(C)C</smiles> 
Amide 1e: Two sets of peaks were observed in ${ }^{1} \mathrm{H}$ and ${ }^{13} \mathrm{C}$ NMR spectra due to amide rotamers, which are assigned to major and minor rotamers, respectively. ${ }^{1} \mathrm{H} \mathrm{NMR}\left(400 \mathrm{MHz}, \mathrm{CDCl}_{3}\right) \delta 7.17$ (d, $J=5.0 \mathrm{~Hz}, 1 \mathrm{H}$, minor), $7.13(\mathrm{~d}, J=5.0 \mathrm{~Hz}, 1 \mathrm{H}$, major $), 6.98-6.89(\mathrm{~m}, 1 \mathrm{H}$, overlapping $), 6.85-$ $6.79(\mathrm{~m}, 1 \mathrm{H}$, overlapping), $5.34(\mathrm{t}, J=6.5 \mathrm{~Hz}, 1 \mathrm{H}$, major $), 5.28(\mathrm{t}, J=6.5 \mathrm{~Hz}, 1 \mathrm{H}$, minor $), 3.59-3.46$ (m, 2H, overlapping), $3.41(\mathrm{q}, J=7.1 \mathrm{~Hz}, 2 \mathrm{H}$, minor), $3.21(\mathrm{q}, J=7.1 \mathrm{~Hz}, 2 \mathrm{H}$, major $), 3.12-3.03(\mathrm{~m}$, 4H, overlapping), $2.94(\mathrm{~d}, J=6.5 \mathrm{~Hz}, 2 \mathrm{H}$, minor $), 2.06-1.92(\mathrm{~m}, 2 \mathrm{H}$, overlapping), $1.64(\mathrm{~s}, 3 \mathrm{H}$, major), 1.59 (s, 3H, minor), $1.50-1.35$ (m, 2H, overlapping), $1.19-1.07$ (m, 3H, overlapping), $0.93-$ $0.79\left(\mathrm{~m}, 3 \mathrm{H}\right.$, overlapping). ${ }^{13} \mathbf{C} \mathbf{N M R}\left(101 \mathrm{MHz}, \mathrm{CDCl}_{3}\right) \delta 171.66$ (overlapping), 141.74 (major), 140.25 (minor), 138.09 (overlapping), 127.13 (minor), 126.87 (major), 125.45 (minor), 125.19 (major), 124.08 (minor), 123.56 (major), 117.17 (overlapping), 49.20 (minor), 47.90 (major), 43.33 (major), 41.69 (major), 41.66 (minor), 40.57 (minor), 33.55 (minor), 33.50 (major), 29.55 (minor), 28.19 (major), 20.88 (overlapping), 16.34 (minor) ,16.31 (major), 14.15 (major), 13.73 (major), 13.68 (minor), 12.93 (minor). ESI-HR calcd for $\mathrm{C}_{16} \mathrm{H}_{26} \mathrm{NOS}^{+}\left([\mathrm{M}+\mathrm{H}]^{+}\right)$280.1730, found 280.1718. IR v $\left(\mathrm{cm}^{-1}\right) 2958,1639,1454,694$.<smiles>CCN(CCc1ccco1)C(=O)C/C=C(\C)c1ccccc1</smiles>

Amide 1f: Two sets of peaks were observed in ${ }^{1} \mathrm{H}$ and ${ }^{13} \mathrm{C}$ NMR spectra due to amide rotamers, which are assigned to major and minor rotamers, respectively. ${ }^{1} \mathbf{H}$ NMR $\left(400 \mathrm{MHz}, \mathbf{C D C l}_{3}\right) \delta$ $7.40-7.35$ (m, 1H, minor), $7.34-7.31(\mathrm{~m}, 1 \mathrm{H}$, major), $6.32(\mathrm{dd}, J=3.0,2.0 \mathrm{~Hz}, 1 \mathrm{H}$, minor $), 6.29(\mathrm{dd}$, $J=3.0,2.0 \mathrm{~Hz}, 1 \mathrm{H}$, major $), 6.07(\mathrm{~d}, J=3.0 \mathrm{~Hz}, 1 \mathrm{H}$, minor $), 6.05(\mathrm{~d}, J=3.0 \mathrm{~Hz}, 1 \mathrm{H}$, major $), 5.53-$ $5.16(\mathrm{~m}, 1 \mathrm{H}$, overlapping), $3.63-3.49(\mathrm{~m}, 2 \mathrm{H}$, overlapping), $3.38(\mathrm{q}, J=7.1 \mathrm{~Hz}, 2 \mathrm{H}$, minor) $3.17(\mathrm{q}, J$ $=7.1 \mathrm{~Hz}, 2 \mathrm{H}$, major $), 3.08(\mathrm{~d}, J=6.7 \mathrm{~Hz}, 2 \mathrm{H}$, major $), 2.97-2.82(\mathrm{~m}, 4 \mathrm{H}$, overlapping $), 2.09-1.94(\mathrm{~m}$, $2 \mathrm{H}$, overlapping), $1.65(\mathrm{~s}, 3 \mathrm{H}$, major $), 1.62(\mathrm{~s}, 3 \mathrm{H}$, minor $), 1.52-1.36(\mathrm{~m}, 2 \mathrm{H}$, overlapping $), 1.19-$ $1.07\left(\mathrm{~m}, 3 \mathrm{H}\right.$, overlapping), $0.94-0.81\left(\mathrm{~m}, 3 \mathrm{H}\right.$, overlapping) ${ }^{13} \mathbf{C} \mathbf{~ N M R}\left(\mathbf{1 0 1} \mathbf{M H z}, \mathbf{C D C l}_{3}\right) \delta 171.62$ (major), 171.58 (minor), 153.37 (major), 152.02 (minor), 141.57 (minor), 141.09 (major), 138.00 (major), 137.98 (minor), 117.22 (minor), 117.19 (major), 110.47 (minor), 110.28 (major), 106.75 (minor), 106.26 (major), 46.27 (minor), 44.78 (major), 43.04 (major), 41.67 (overlapping), 40.46 (minor), 33.48 (major), 33.33 (minor), 27.92 (minor), 26.49 (major), 20.85 (overlapping), 16.30 
(major), 16.26 (minor), 14.11 (major), 13.73 (major), 13.69 (minor), 12.90 (minor). ESI-HR calcd for $\mathrm{C}_{16} \mathrm{H}_{26} \mathrm{NO}_{2}{ }^{+}\left([\mathrm{M}+\mathrm{H}]^{+}\right)$264.1958, found 264.1950. IR $v\left(\mathrm{~cm}^{-1}\right)$ 2959, 1642, 1424, 1146.<smiles>CCN(CCOC)C(=O)C/C=C(\C)C(C)C</smiles>

Amide 1g: Two sets of peaks were observed in ${ }^{1} \mathrm{H}$ and ${ }^{13} \mathrm{C}$ NMR spectra due to amide rotamers, which are assigned to major and minor rotamers, respectively, ${ }^{1} \mathrm{H}$ NMR $\left(400 \mathrm{MHz}, \mathbf{C D C l}_{3}\right) \delta$ $5.42-5.20(\mathrm{~m}, 1 \mathrm{H}$, overlapping), $3.57-3.36(\mathrm{~m}, 6 \mathrm{H}$, overlapping), $3.35(\mathrm{~s}, 3 \mathrm{H}$, minor), $3.33(\mathrm{~s}, 3 \mathrm{H}$, major), $3.18-3.01(\mathrm{~m}, 2 \mathrm{H}$, overlapping), $2.01(\mathrm{t}, J=7.7 \mathrm{~Hz}, 2 \mathrm{H}$, overlapping), 1.63 (s, 3H, overlapping), $1.50-1.31(\mathrm{~m}, 2 \mathrm{H}$, overlapping), $1.17(\mathrm{t}, J=7.1 \mathrm{~Hz}, 3 \mathrm{H}$, major $), 1.11(\mathrm{t}, J=7.1 \mathrm{~Hz}, 3 \mathrm{H}$, minor), $0.87\left(\mathrm{t}, J=7.3 \mathrm{~Hz}, 3 \mathrm{H}\right.$, overlapping). ${ }^{13} \mathbf{C} \mathbf{N M R}\left(\mathbf{1 0 1} \mathbf{~ M H z}, \mathbf{C D C l}_{3}\right) \delta 171.91$ (minor), 171.82 (major), 137.95 (major), 137.85 (minor), 117.36 (minor), 117.23 (major), 71.11 (major), 71.03 (minor), 59.06 (minor), 58.75 (major), 47.16 (minor), 45.46 (major), 43.77 (major), 41.67 (overlapping), 41.12 (minor), 33.51 (minor), 33.24 (major), 20.85 (overlapping), 16.27 (overlapping), 14.06 (major), 13.67 (overlapping), 12.80 (minor). ESI-HR calcd for $\mathrm{C}_{13} \mathrm{H}_{26} \mathrm{NO}_{2}{ }^{+}\left([\mathrm{M}+\mathrm{H}]^{+}\right)$228.1958, found 228.1950. IR $v\left(\mathrm{~cm}^{-1}\right) 2959,1640,1455,1011$.<smiles>CCN(CCOC)C(=O)C/C=C(/C)c1ccccc1</smiles>

Amide 1g-Z: Two sets of peaks were observed in ${ }^{1} \mathrm{H}$ and ${ }^{13} \mathrm{C}$ NMR spectra due to amide rotamers, which are assigned to major and minor rotamers, respectively. ${ }^{1} \mathbf{H}$ NMR (400 $\left.\mathbf{M H z}, \mathbf{C D C l}_{3}\right) \delta 5.36$ (t, $J=6.4 \mathrm{~Hz}, 1 \mathrm{H}$, overlapping), $3.59-3.43(\mathrm{~m}, 4 \mathrm{H}$, overlapping), $3.40(\mathrm{q}, J=7.1 \mathrm{~Hz}, 2 \mathrm{H}$, overlapping), $3.35(\mathrm{~s}, 3 \mathrm{H}$, minor), $3.33(\mathrm{~s}, 3 \mathrm{H}$, major), $3.20-2.91(\mathrm{~m}, 2 \mathrm{H}$, overlapping), $2.04(\mathrm{t}, J=$ $7.8 \mathrm{~Hz}, 2 \mathrm{H}$, overlapping), $1.83-1.68(\mathrm{~m}, 3 \mathrm{H}$, overlapping), $1.51-1.35(\mathrm{~m}, 2 \mathrm{H}$, overlapping), 1.17 (t, $J=7.1 \mathrm{~Hz}, 3 \mathrm{H}$, major), $1.11(\mathrm{t}, J=7.1 \mathrm{~Hz}, 3 \mathrm{H}$, minor $), 0.90\left(\mathrm{t}, J=7.3 \mathrm{~Hz}, 3 \mathrm{H}\right.$, overlapping). ${ }^{13} \mathbf{C} \mathbf{N M R}$ (101 MHz, CDCl $\mathbf{3}$ ) $\delta 171.92$ (minor), 171.83 (major), 138.26 (major), 138.14 (minor), 117.96 (minor), 117.83 (major), 71.13 (major), 71.04 (minor), 59.09 (minor), 58.78 (major), 47.21, (minor) 45.54 (major), 43.83 (major), 41.16 (minor), 34.15 (overlapping), 33.09 (minor), 32.82 (major), 23.36 
(overlapping), 20.88 (overlapping), 14.10 (major), 14.01 (overlapping), 12.84 (minor). ESI-HR calcd for $\mathrm{C}_{13} \mathrm{H}_{26} \mathrm{NO}_{2}{ }^{+}\left([\mathrm{M}+\mathrm{H}]^{+}\right)$228.1958, found 228.1950. IR $v\left(\mathrm{~cm}^{-1}\right) 2961,1643,1455,1118$.<smiles>CCN(CCOC)C(=O)C/C=C(\C)C(C)C</smiles>

Amide 1h: Two sets of peaks were observed in ${ }^{1} \mathrm{H}$ and ${ }^{13} \mathrm{C}$ NMR spectra due to amide rotamers, which are assigned to major and minor rotamers, respectively. ${ }^{1} \mathbf{H}$ NMR $\left(400 \mathbf{~ M H z}, \mathbf{C D C l}_{3}\right) \delta 5.33$ $(\mathrm{t}, J=6.7 \mathrm{~Hz}, 1 \mathrm{H}$, overlapping $), 4.61(\mathrm{~s}, 1 \mathrm{H}$, minor $), 4.60(\mathrm{~s}, 1 \mathrm{H}$, major $), 3.60-3.71(\mathrm{~m}, 2 \mathrm{H}$, overlapping), 3.53 (t, $J=5.7 \mathrm{~Hz}, 2 \mathrm{H}$, major), $3.48(\mathrm{t}, J=5.7 \mathrm{~Hz}, 2 \mathrm{H}$, minor), $3.46-3.38$ (q, $J=7.1 \mathrm{~Hz}$, 2H, overlapping), 3.34 (s, 3H, overlapping), $3.15(\mathrm{~d}, J=6.7 \mathrm{~Hz}, 1 \mathrm{H}$, minor $), 3.09(\mathrm{~d}, J=6.7 \mathrm{~Hz}, 1 \mathrm{H}$, major), $2.00(\mathrm{t}, J=7.5 \mathrm{~Hz}, 2 \mathrm{H}$, overlapping), 1.63 (s, 3H, overlapping), $1.50-1.35(\mathrm{~m}, 2 \mathrm{H}$, overlapping), 1.19 (t, $J=7.1 \mathrm{~Hz}, 3 \mathrm{H}$, major), $1.12(\mathrm{t}, J=7.1 \mathrm{~Hz}, 3 \mathrm{H}$, minor), $0.87(\mathrm{t}, J=7.3 \mathrm{~Hz}, 3 \mathrm{H}$, overlapping). ${ }^{13} \mathbf{C}$ NMR (101 MHz, CDCl 3$) \delta 171.99$ (minor), 171.85 (major), 138.04 (major), 137.90 (minor), 117.37 (minor), 117.21 (major), 96.59 (minor), 96.56 (major), 66.09 (major), 65.82 (minor), 55.35 (minor), 55.21 (major), 47.12 (minor), 45.54 (major), 43.70 (major), 41.69 (overlapping), 40.98 (minor), 33.54 (minor), 33.35 (major), 20.88 (overlapping), 16.29 (major), 16.27 (minor), 14.14 (major), 13.70 (overlapping), 12.82 (minor). ESI-HR calcd for $\mathrm{C}_{14} \mathrm{H}_{28} \mathrm{NO}_{3}{ }^{+}\left([\mathrm{M}+\mathrm{H}]^{+}\right)$258.2064, found 258.2055. IR $v\left(\mathrm{~cm}^{-1}\right) 2957,1642,1455,919$.<smiles>COCCN(CCOC)C(=O)C/C=C(\C)C(C)C</smiles>

Amide 1i: ${ }^{1}$ H NMR (400 MHz, $\left.\mathbf{C D C l}_{3}\right) \delta 5.50-5.16(\mathrm{~m}, 1 \mathrm{H}), 3.61-3.45(\mathrm{~m}, 8 \mathrm{H}), 3.35(\mathrm{~s}, 3 \mathrm{H}), 3.32$ (s, 3H), $3.15(\mathrm{~d}, J=6.8 \mathrm{~Hz}, 2 \mathrm{H}), 2.02(\mathrm{t}, J=7.7 \mathrm{~Hz}, 2 \mathrm{H}), 1.62(\mathrm{~s}, 3 \mathrm{H}), 1.51-1.35(\mathrm{~m}, 2 \mathrm{H}), 0.87$ (t, $J=$ $7.3 \mathrm{~Hz}, 3 \mathrm{H}) .{ }^{13} \mathbf{C}$ NMR (101 MHz, $\left.\mathbf{C D C l}_{3}\right) \delta 172.58,137.94,117.27,71.18,70.93,59.05,58.76,48.88$, 46.43, 41.70, 33.29, 20.88, 16.29, 13.69. ESI-HR calcd for $\mathrm{C}_{14} \mathrm{H}_{28} \mathrm{NO}_{3}{ }^{+}\left([\mathrm{M}+\mathrm{H}]^{+}\right) 258.2064$, found 258.2055. IR $v\left(\mathrm{~cm}^{-1}\right) 2957,1642,1451,1191$. 
<smiles>CCN(CCC1=CCCCC1)C(=O)C/C=C(\C)c1ccccc1</smiles>

Amide 1j: 1H NMR (400 MHz, $\left.\mathbf{C D C l}_{3}\right) \delta 5.49-5.44(\mathrm{~m}, 1 \mathrm{H}$, major $), 5.43-5.39(\mathrm{~m}, 1 \mathrm{H}$, minor $)$, $5.32(\mathrm{t}, J=6.7 \mathrm{~Hz}, 1 \mathrm{H}$, overlapping), $3.44-3.33(\mathrm{~m}, 2 \mathrm{H}$, overlapping), $3.31-3.22(\mathrm{~m}, 2 \mathrm{H}$, overlapping), 3.06 (d, J = 6.7 Hz, 2H, overlapping), $2.21-2.08$ (m, 2H, overlapping), $2.06-1.88(\mathrm{~m}$, $6 \mathrm{H}$, overlapping), $1.65-1.50(\mathrm{~m}, 7 \mathrm{H}$, overlapping), $1.49-1.37(\mathrm{~m}, 2 \mathrm{H}$, overlapping), $1.16(\mathrm{t}, J=7.1$ $\mathrm{Hz}, 3 \mathrm{H}$, major $), 1.11(\mathrm{t}, J=7.1 \mathrm{~Hz}, 3 \mathrm{H}$, minor $), 0.87\left(\mathrm{t}, J=7.3 \mathrm{~Hz}, 3 \mathrm{H}\right.$, overlapping). ${ }^{13} \mathbf{C}$ NMR $(101$ MHz, CDCl3) $\delta 171.40$ (major), 171.31 (minor), 137.80 (overlapping), 135.27 (major), 134.34 (minor), 123.58 (major), 122.70 (minor), 117.48 (overlapping), 46.61 (major), 44.06 (minor), 42.39 (minor), 41.69 (overlapping), 40.55 (major), 37.51 (overlapping), 36.02 (overlapping), 33.53 (minor), 33.50 (major), 28.62 (major), 28.32 (minor), 25.28 (minor), 25.20 (major), 22.94 (minor), 22.85 (major), 22.36 (major), 22.21 (minor), 20.88 (overlapping), 16.35 (minor), 16.28 (major), 14.20 (major), 13.72 (minor), 13.69 (major), 12.93 (minor). ESI-HR calcd for $\mathrm{C}_{18} \mathrm{H}_{32} \mathrm{NO}^{+}\left([\mathrm{M}+\mathrm{H}]^{+}\right)$278.2478, found 278.2472. IR $v\left(\mathrm{~cm}^{-1}\right) 2928,1738,1643,1425,1376,797$.<smiles>CC(=CCC(=O)N1CCOCC1)C(C)C</smiles>

Amide 11: ${ }^{1} \mathbf{H}$ NMR (400 MHz, $\left.\mathbf{C D C l}_{3}\right) \delta 5.35-5.20(\mathrm{~m}, 1 \mathrm{H}), 3.73-3.56(\mathrm{~m}, 6 \mathrm{H}), 3.45(\mathrm{t}, J=4.7 \mathrm{~Hz}$, 2H), $3.08(\mathrm{~d}, J=6.8 \mathrm{~Hz}, 2 \mathrm{H}), 2.02(\mathrm{t}, J=7.6 \mathrm{~Hz}, 2 \mathrm{H}), 1.61(\mathrm{~s}, 3 \mathrm{H}), 1.50-1.33(\mathrm{~m}, 2 \mathrm{H}), 0.87(\mathrm{t}, J=7.3$ Hz, 3H). ${ }^{13}$ C NMR (101 MHz, CDCl 3$) \delta 170.74,138.70,116.41,66.92,66.68,46.18,42.01,41.63$, 33.59, 20.87, 16.31, 13.68. ESI-HR calcd for $\mathrm{C}_{12} \mathrm{H}_{22} \mathrm{NO}_{2}{ }^{+}\left([\mathrm{M}+\mathrm{H}]^{+}\right)$212.1645, found 212.1641. IR $v$ $\left(\mathrm{cm}^{-1}\right) 2959,1738,1646,1432,964$.<smiles>CCN(CCOC)C(=O)C/C=C(\C)c1ccccc1</smiles>

Amide 1m: Two sets of peaks were observed in ${ }^{1} \mathrm{H}$ and ${ }^{13} \mathrm{C}$ NMR spectra due to amide rotamers, which are assigned to major and minor rotamers, respectively. ${ }^{1} \mathbf{H}$ NMR $\left(400 \mathbf{M H z}, \mathbf{C D C l}_{3}\right) \delta 5.48$ $-5.18(\mathrm{~m}, 1 \mathrm{H}$, overlapping), $3.57-3.36(\mathrm{~m}, 6 \mathrm{H}$, overlapping), $3.35(\mathrm{~s}, 3 \mathrm{H}$, minor $), 3.33(\mathrm{~s}, 3 \mathrm{H}$, major $)$, 
$3.18-3.00(\mathrm{~m}, 2 \mathrm{H}$, overlapping), $2.02(\mathrm{t}, J=7.6 \mathrm{~Hz}, 2 \mathrm{H}$, overlapping), 1.63 (s, 3H, overlapping), 1.46 $-1.36(\mathrm{~m}, 2 \mathrm{H}$, overlapping), $1.34-1.20(\mathrm{~m}, 4 \mathrm{H}$, overlapping), $1.17(\mathrm{t}, J=7.1 \mathrm{~Hz}, 3 \mathrm{H}$, major $), 1.11(\mathrm{t}$, $J=7.1 \mathrm{~Hz}, 3 \mathrm{H}$, minor $), 0.88\left(\mathrm{t}, J=7.1 \mathrm{~Hz}, 3 \mathrm{H}\right.$, overlapping). ${ }^{13} \mathbf{C}$ NMR (101 MHz, CDCl $) \delta 171.90$ (minor), 171.82 (major), 138.23 (major), 138.13 (minor), 117.15 (minor), 117.02 (major), 71.12 (major), 71.06 (minor), 59.07 (minor), 58.76 (major), 47.18 (minor), 45.48 (major), 43.78 (major), 41.13 (minor), 39.55 (overlapping), 33.54 (minor), 33.25 (major), 31.51 (overlapping), 27.48 (overlapping), 22.52 (overlapping), 16.37 (overlapping), 14.08 (major), 14.02 (overlapping)), 12.81 (minor). ESI-HR calcd for $\mathrm{C}_{15} \mathrm{H}_{30} \mathrm{NO}_{2}{ }^{+}\left([\mathrm{M}+\mathrm{H}]^{+}\right)$256.2271, found 256.2260. IR $v\left(\mathrm{~cm}^{-1}\right)$ 2927, $1641,1455,1011$.<smiles>CCN(CCOC)C(=O)C/C=C(\C)[In]c1ccccc1</smiles>

Amide 1n: Two sets of peaks were observed in ${ }^{1} \mathrm{H}$ and ${ }^{13} \mathrm{C}$ NMR spectra due to amide rotamers, which are assigned to major and minor rotamers, respectively. ${ }^{1} \mathbf{H}$ NMR (400 $\left.\mathbf{M H z}, \mathbf{C D C l}_{3}\right) \delta 5.43$ - 5.14 (m, 1H, overlapping), $3.57-3.34$ (m, 6H, overlapping), 3.32 (s, 3H, minor), 3.31 (s, 3H, major), $3.12-3.00$ (m, 2H, overlapping), 1.99 (t, $J=7.6 \mathrm{~Hz}, 2 \mathrm{H}$, overlapping), 1.61 (s, 3H, overlapping), 1.46 $-1.32(\mathrm{~m}, 2 \mathrm{H}$, overlapping), $1.31-1.19(\mathrm{~m}, 10 \mathrm{H}$, overlapping), $1.15(\mathrm{t}, J=7.1 \mathrm{~Hz}, 3 \mathrm{H}$ major $), 1.08(\mathrm{t}$, $J=7.1 \mathrm{~Hz}, 3 \mathrm{H}$ minor $), 0.85\left(\mathrm{t}, J=6.9 \mathrm{~Hz}, 3 \mathrm{H}\right.$, overlapping). ${ }^{13} \mathbf{C}$ NMR $(\mathbf{1 0 1} \mathbf{M H z}, \mathbf{C D C l}) \delta 171.94$ (minor), 171.86 (major), 138.28 (major), 138.18 (minor), 117.13 (minor), 117.00 (major), 71.12 (major), 71.06 (minor), 59.07 (minor), 58.77 (major), 47.18 (minor), 45.49 (major), 43.78 (major), 41.14 (minor), 39.61 (overlapping), 33.55 (minor), 33.26 (major), 31.88 (overlapping), 29.49 (overlapping), 29.32 (overlapping), 29.26 (overlapping), 27.83 (overlapping), 22.65 (overlapping), 16.37 (major), 16.36 (minor), 14.08 (major), 14.07 (overlapping), 12.81 (minor). ESI-HR calcd for $\mathrm{C}_{18} \mathrm{H}_{36} \mathrm{NO}_{2}{ }^{+}\left([\mathrm{M}+\mathrm{H}]^{+}\right)$298.2741, found 298.2730. IR $v\left(\mathrm{~cm}^{-1}\right) 2924,1643,1455,1117$.<smiles>CCN(CCOC)C(=O)C/C=C(\C)CCC(C)C</smiles> 
Amide 10: Two sets of peaks were observed in ${ }^{1} \mathrm{H}$ and ${ }^{13} \mathrm{C}$ NMR spectra due to amide rotamers, which are assigned to major and minor rotamers, respectively. ${ }^{1} \mathbf{H}$ NMR $\left(400 \mathbf{~ M H z}, \mathbf{C D C l}_{3}\right) \delta 5.42$ $-5.25(\mathrm{~m}, 1 \mathrm{H}$, overlapping), $3.60-3.37(\mathrm{~m}, 6 \mathrm{H}$, overlapping), $3.34(\mathrm{~s}, 3 \mathrm{H}$, minor $), 3.33(\mathrm{~s}, 3 \mathrm{H}$, major $)$ $3.20-2.92(\mathrm{~m}, 2 \mathrm{H}$, overlapping), $2.02(\mathrm{t}, J=7.8 \mathrm{~Hz}, 2 \mathrm{H}$, overlapping), 1.64 (s, 3H, overlapping), 1.57 $-1.44(\mathrm{~m}, 1 \mathrm{H}$, overlapping), $1.36-1.25(\mathrm{~m}, 2 \mathrm{H}$, overlapping), $1.17(\mathrm{t}, J=7.1 \mathrm{~Hz}, 3 \mathrm{H}$, major $), 1.11(\mathrm{t}$, $J=7.1 \mathrm{~Hz}, 3 \mathrm{H}$, minor $), 0.88(\mathrm{~d}, J=6.6 \mathrm{~Hz}, 6 \mathrm{H}$, overlapping $) .{ }^{13} \mathbf{C} \mathbf{N M R}\left(\mathbf{1 0 1} \mathbf{M H z}, \mathbf{C D C l}_{3}\right) \delta 171.89$ (minor), 171.81 (major), 138.41 (major), 138.31 (minor), 117.00 (minor), 116.87 (major), 71.13 (major), 71.04 (minor), 59.08 (minor), 58.77 (major), 47.16 (minor), 45.48 (major), 43.78 (major), 41.13 (minor), 37.44 (overlapping), 37.10 (overlapping), 33.50 (minor), 33.22 (major), 27.72 (overlapping), 22.54 (overlapping), 16.46 (overlapping), 14.08 (major), 12.82 (minor). ESI-HR calcd for $\mathrm{C}_{15} \mathrm{H}_{30} \mathrm{NO}_{2}{ }^{+}\left([\mathrm{M}+\mathrm{H}]^{+}\right)$256.2271, found 256.2258. IR $v\left(\mathrm{~cm}^{-1}\right) 2954,1644,1455,1118$.<smiles>CCN(CCOC)C(=O)C/C=C(\C)CC(C)C</smiles>

Amide 1p: Two sets of peaks were observed in ${ }^{1} \mathrm{H}$ and ${ }^{13} \mathrm{C}$ NMR spectra due to amide rotamers, which are assigned to major and minor rotamers, respectively. ${ }^{1} \mathbf{H}$ NMR $\left(400 \mathbf{~ M H z}, \mathbf{C D C l}_{3}\right) \delta 5.41$ $-5.18(\mathrm{~m}, 1 \mathrm{H}$, overlapping), $3.57-3.37(\mathrm{~m}, 6 \mathrm{H}$, overlapping), $3.35(\mathrm{~s}, 3 \mathrm{H}$, minor $), 3.33(\mathrm{~s}, 3 \mathrm{H}$, major $)$, $3.18-3.01(\mathrm{~m}, 2 \mathrm{H}$, overlapping $), 1.90(\mathrm{~d}, J=7.3 \mathrm{~Hz}, 2 \mathrm{H}$, overlapping $), 1.81-1.69(\mathrm{~m}, 1 \mathrm{H}$, overlapping), 1.62 (s, 3H, overlapping), $1.18(\mathrm{t}, J=7.1 \mathrm{~Hz}, 3 \mathrm{H}$, major $), 1.10(\mathrm{t}, J=7.1 \mathrm{~Hz}, 3 \mathrm{H}$, minor), $0.85\left(\mathrm{~d}, J=6.6 \mathrm{~Hz}, 6 \mathrm{H}\right.$, overlapping). ${ }^{13} \mathbf{C}$ NMR $(\mathbf{1 0 1} \mathbf{~ M H z}, \mathbf{C D C l}) \delta 171.88$ (minor), 171.78 (major), 137.14 (major), 137.05 (minor), 118.56 (minor), 118.45 (major), 71.11 (major), 71.02 (minor), 59.05 (minor), 58.73 (major), 49.35 (overlapping), 47.15 (minor), 45.43 (major), 43.75 (major), 41.07 (minor), 33.57 (minor), 33.30 (major), 26.04 (overlapping), 22.36 (overlapping), 16.30 (overlapping), 14.06 (major), 12.76 (minor). ESI-HR calcd for $\mathrm{C}_{14} \mathrm{H}_{28} \mathrm{NO}_{2}{ }^{+}\left([\mathrm{M}+\mathrm{H}]^{+}\right)$242.2215, found 242.2108. IR $v\left(\mathrm{~cm}^{-1}\right) 2953,1641,1455,1117$.<smiles>CCN(CCOC)C(=O)C/C=C(\C)CCCCl</smiles> 
Amide 1r: Two sets of peaks were observed in ${ }^{1} \mathrm{H}$ and ${ }^{13} \mathrm{C}$ NMR spectra due to amide rotamers, which are assigned to major and minor rotamers, respectively. ${ }^{1} \mathbf{H}$ NMR $\left(400 \mathbf{M H z}, \mathbf{C D C l}_{3}\right) \delta 5.53$ $-5.25(\mathrm{~m}, 1 \mathrm{H}$, overlapping), $3.65-3.44(\mathrm{~m}, 6 \mathrm{H}$, overlapping), $3.40(\mathrm{q}, J=7.1 \mathrm{~Hz}, 2 \mathrm{H}), 3.36(\mathrm{~s}, 3 \mathrm{H}$, minor), 3.33 (s, 3H, major), 3.12 (d, $J=6.8 \mathrm{~Hz}, 2 \mathrm{H}$, minor), 3.09 (d, $J=6.8 \mathrm{~Hz}, 2 \mathrm{H}$, major), 2.18 (t, $J$ $=7.4 \mathrm{~Hz}, 2 \mathrm{H}$, overlapping $), 2.00-1.80(\mathrm{~m}, 2 \mathrm{H}$, overlapping $), 1.67-1.62(\mathrm{~m}, 3 \mathrm{H}$, overlapping $), 1.18(\mathrm{t}$, $J=7.1 \mathrm{~Hz}, 3 \mathrm{H}$, major), $1.11\left(\mathrm{t}, J=7.1 \mathrm{~Hz}, 3 \mathrm{H}\right.$, minor). ${ }^{13} \mathbf{C} \mathbf{N M R}\left(\mathbf{1 0 1} \mathbf{M H z}, \mathbf{C D C l}_{3}\right) \delta 171.58$ (minor), 171.44 (major), 136.22 (major), 136.08 (minor), 118.67 (minor), 118.51 (major), 71.11 (major), 70.95 (minor), 59.10 (minor), 58.78 (major), 47.20 (minor), 45.52 (major), 44.52 (minor), 44.49 (major), 43.79 (major), 41.10 (minor), 36.56 (overlapping), 33.25 (minor), 32.99 (major), 30.66 (overlapping), 16.37 (major), 16.34 (minor), 14.08 (major), 12.82 (minor). ESI-HR calcd for $\mathrm{C}_{13} \mathrm{H}_{25} \mathrm{ClNO}_{2}{ }^{+}$ $\left([\mathrm{M}+\mathrm{H}]^{+}\right)$262.1568, found 262.1559. IR $v\left(\mathrm{~cm}^{-1}\right) 2933,1639,1448,1117$.<smiles>CCN(CCOC)C(=O)C/C=C(\C)CCCOC(C)=O</smiles>

Amide 1s: Two sets of peaks were observed in ${ }^{1} \mathrm{H}$ and ${ }^{13} \mathrm{C}$ NMR spectra due to amide rotamers, which are assigned to major and minor rotamers, respectively. ${ }^{1} \mathbf{H}$ NMR $\left(400 \mathbf{~ M H z}, \mathbf{C D C l}_{3}\right) \delta 5.51$ $-5.22(\mathrm{~m}, 1 \mathrm{H}$, overlapping), $4.06(\mathrm{t}, J=6.7 \mathrm{~Hz}, 2 \mathrm{H}$, overlapping), $3.59-3.45(\mathrm{~m}, 4 \mathrm{H}$, overlapping), 3.42 (q, $J=7.1 \mathrm{~Hz}, 2 \mathrm{H}), 3.37$ (s, 3H, minor), 3.35 (s, 3H, major), $3.14(\mathrm{~d}, J=6.7 \mathrm{~Hz}, 2 \mathrm{H}$, minor), 3.10 (d, $J=6.7 \mathrm{~Hz}, 2 \mathrm{H}$, major), 2.12 (t, $J=7.6 \mathrm{~Hz}, 2 \mathrm{H}$, overlapping), 2.06 (s, 3H, overlapping), $1.82-1.74$ (m, 2H, overlapping), $1.70-1.64$ (m, 3H, overlapping), $1.19(\mathrm{t}, J=7.1 \mathrm{~Hz}, 3 \mathrm{H}$, major), $1.13(\mathrm{t}, J=7.1$ $\mathrm{Hz}, 3 \mathrm{H}$, minor). ${ }^{13} \mathbf{C} \mathbf{N M R}\left(\mathbf{1 0 1} \mathbf{M H z}, \mathbf{C D C l}_{3}\right) \delta 171.65$ (minor), 171.52 (major), 171.12 (overlapping), 136.65 (major), 136.50 (minor), 118.25 (minor), 118.09 (major), 71.09 (major), 70.96 (minor), 64.06 (overlapping), 59.08 (minor), 58.75 (major), 47.20 (minor), 45.53 (major), 43.79 (major), 41.13 (minor), 35.70 (overlapping), 33.26 (minor), 32.98 (major), 26.73 (overlapping), 20.94 (overlapping), 16.34 (major), 16.32 (minor), 14.08 (major), 12.81 (minor). ESI-HR calcd for $\mathrm{C}_{15} \mathrm{H}_{28} \mathrm{NO}_{4}{ }^{+}\left([\mathrm{M}+\mathrm{H}]^{+}\right)$ 286.2013, found 286.2003. IR $v\left(\mathrm{~cm}^{-1}\right) 2933,1736,1638,1448,1117$. 
<smiles>CCN(CCOC)C(=O)C/C=C(\C)CCCOc1ccccc1</smiles>

Amide 1t: Two sets of peaks were observed in ${ }^{1} \mathrm{H}$ and ${ }^{13} \mathrm{C}$ NMR spectra due to amide rotamers, which are assigned to major and minor rotamers, respectively. ${ }^{1} \mathbf{H}$ NMR $\left(400 \mathbf{M H z}, \mathbf{C D C l}_{3}\right) \delta 7.38$ $-7.33(\mathrm{~m}, 4 \mathrm{H}$, overlapping $), 7.33-7.26(\mathrm{~m}, 1 \mathrm{H}$, overlapping $), 5.48-5.25(\mathrm{~m}, 1 \mathrm{H}$, overlapping $), 4.52$ (s, 2H, overlapping), $3.58-3.38(\mathrm{~m}, 8 \mathrm{H}$, overlapping), $3.37(\mathrm{~s}, 3 \mathrm{H}$, minor), $3.35(\mathrm{~s}, 3 \mathrm{H}$, major), $3.13(\mathrm{~d}$ $J=6.8 \mathrm{~Hz}, 2 \mathrm{H}$, minor $), 3.10(\mathrm{~d}, J=6.8 \mathrm{~Hz}, 2 \mathrm{H}$, major $), 2.14(\mathrm{t}, J=7.7 \mathrm{~Hz}, 2 \mathrm{H}$, overlapping $), 1.81-$ 1.73 (m, 2H, overlapping), 1.67 (s, 3H, overlapping), 1.19 (t, $J=7.1 \mathrm{~Hz}, 3 \mathrm{H}$, major), 1.13 (t, $J=7.1$ Hz, 3H, minor). ${ }^{13} \mathbf{C}$ NMR (101 MHz, CDCl $\left.\mathbf{3}\right) \delta 171.80$ (minor), 171.69 (major), 138.66 (overlapping), 137.46 (major), 137.34 (minor), 128.32 (overlapping), 127.60 (overlapping), 127.45 (overlapping), 117.71 (minor), 117.57 (major), 72.87 (overlapping), 71.13 (major), 71.03 (minor), 70.03 (overlapping), 59.10 (minor), 58.79 (major), 47.21 (minor), 45.52 (major), 43.80 (major), 41.15 (minor), 36.07 (overlapping), 33.47 (minor), 33.20 (major), 27.96 (overlapping), 16.41 (overlapping), 14.11 (major), 12.85 (minor). ESI-HR calcd for $\mathrm{C}_{20} \mathrm{H}_{32} \mathrm{NO}_{3}{ }^{+}\left([\mathrm{M}+\mathrm{H}]^{+}\right)$334.2377, found 334.2367. IR $v\left(\mathrm{~cm}^{-1}\right) 2931,1639,1454,737$.<smiles>CCN(CCOC)C(=O)C/C=C(\C)CCCc1ccccc1</smiles>

Amide 1u: Two sets of peaks were observed in ${ }^{1} \mathrm{H}$ and ${ }^{13} \mathrm{C}$ NMR spectra due to amide rotamers, which are assigned to major and minor rotamers, respectively. ${ }^{1} \mathbf{H}$ NMR $\left(400 \mathbf{M H z}, \mathbf{C D C l}_{3}\right) \delta 7.32$ $-7.20(\mathrm{~m}, 2 \mathrm{H}$, overlapping), $7.20-7.11(\mathrm{~m}, 3 \mathrm{H}$, overlapping), $5.58-5.06(\mathrm{~m}, 1 \mathrm{H}$, overlapping), 3.56 - 3.43 (m, 4H, overlapping), 3.40 (q, J = 7.1 Hz, 2H, overlapping), 3.34 (s, $3 \mathrm{H}$, minor), 3.32 (s, $3 \mathrm{H}$, major), 3.13 (d, $J=6.7 \mathrm{~Hz}, 2 \mathrm{H}$, minor $), 3.09(\mathrm{~d}, J=6.7 \mathrm{~Hz}, 2 \mathrm{H}$, major $), 2.58(\mathrm{t}, J=7.8 \mathrm{~Hz}, 2 \mathrm{H}$, overlapping), $2.08(\mathrm{t}, J=7.6 \mathrm{~Hz}, 2 \mathrm{H}$, overlapping), $1.86-1.68(\mathrm{~m}, 2 \mathrm{H}$, overlapping), $1.64(\mathrm{~s}, 3 \mathrm{H}$, overlapping), $1.17(\mathrm{t}, J=7.1 \mathrm{~Hz}, 3 \mathrm{H}$, major $), 1.11(\mathrm{t}, J=7.1 \mathrm{~Hz}, 3 \mathrm{H}$, minor $) .{ }^{13} \mathbf{C} \mathbf{N M R}(\mathbf{1 0 1} \mathbf{M H z}$, $\mathbf{C D C l}_{3}$ ) $\delta 171.77$ (minor), 171.67 (major), 142.54 (minor), 142.52 (major), 137.61 (major), 137.50 (minor), 128.40 (overlapping), 128.21 (overlapping), 125.60 (overlapping), 117.74 (minor), 117.61 (major), 71.13 (major), 71.01 (minor), 59.08 (minor), 58.77 (major), 47.18 (minor), 45.50 (major), 
43.79 (major), 41.12 (minor), 39.15 (overlapping), 35.48 (overlapping), 33.43 (minor), 33.15 (major), 29.54 (overlapping), 16.38 (major), 16.36 (minor), 14.10 (major), 12.84 (minor). ESI-HR calcd for $\mathrm{C}_{19} \mathrm{H}_{30} \mathrm{NO}_{2}{ }^{+}\left([\mathrm{M}+\mathrm{H}]^{+}\right)$304.2271, found 304.2257. IR $v\left(\mathrm{~cm}^{-1}\right) 2932,1643,1454,1117,748$.<smiles>CCN(CCOC)C(=O)C/C=C(/C)CCCc1ccccc1</smiles>

Amide 1u-Z: Two sets of peaks were observed in ${ }^{1} \mathrm{H}$ and ${ }^{13} \mathrm{C}$ NMR spectra due to amide rotamers, which are assigned to major and minor rotamers, respectively. ${ }^{\mathbf{1}} \mathbf{H}$ NMR (400 $\left.\mathbf{M H z}, \mathbf{C D C l}_{3}\right) \delta 7.32$ $-7.23(\mathrm{~m}, 2 \mathrm{H}$, overlapping), $7.32-7.23(\mathrm{~m}, 3 \mathrm{H}$, overlapping), 5.37 (t, J = 6.7 Hz, 1H, overlapping), $3.65-3.33$ (m, 6H, overlapping), 3.33 (s, 3H, minor), 3.32 (s, 3H, major), 3.03 (d, J = 6.2 Hz, 2H, minor), 2.98 (d, $J=6.2 \mathrm{~Hz}, 2 \mathrm{H}$, major), $2.61(\mathrm{t}, J=7.6 \mathrm{~Hz}, 2 \mathrm{H}$, overlapping), 2.07 (t, $J=7.6 \mathrm{~Hz}, 2 \mathrm{H}$, overlapping), $1.81-1.67\left(\mathrm{~m}, 5 \mathrm{H}\right.$, overlapping), $1.19-0.97\left(\mathrm{~m}, 3 \mathrm{H}\right.$, overlapping). ${ }^{\mathbf{1 3}} \mathbf{C}$ NMR (101 MHz, $\mathbf{C D C l}_{3}$ ) $\delta 171.72$ (minor), 171.63 (major), 142.33 (minor), 142.23 (major), 137.78 (major), 137.67 (minor), 128.37 (overlapping), 128.26 (overlapping), 125.71 (major), 125.68 (minor), 118.33 (minor), 118.18 (major), 71.12 (major), 71.02 (minor), 59.06 (minor), 58.74 (major), 47.17 (minor), 45.54 (major), 43.74 (major), 41.17 (minor), 35.62 (minor), 35.57 (major), 32.97 (minor), 32.61 (major), 31.61 (minor), 31.53 (major), 29.22 (minor), 29.17 (major), 23.30 (overlapping), 14.07 (major), 12.83 (minor). ESI-HR calcd for $\mathrm{C}_{19} \mathrm{H}_{30} \mathrm{NO}_{2}{ }^{+}\left([\mathrm{M}+\mathrm{H}]^{+}\right)$304.2271, found 304.2262. IR v $\left(\mathrm{cm}^{-1}\right)$ 2931, 1643, 1454, 749.<smiles>CCN(CCOC)C(=O)CC=C(C)CCc1ccccc1</smiles>

Amide 1v: Two sets of peaks were observed in ${ }^{1} \mathrm{H}$ and ${ }^{13} \mathrm{C}$ NMR spectra due to amide rotamers, which are assigned to major and minor rotamers, respectively. ${ }^{1} \mathrm{H}$ NMR $\left(400 \mathrm{MHz}, \mathbf{C D C l}_{3}\right) \delta$ $7.31-7.23(\mathrm{~m}, 2 \mathrm{H}$, overlapping), $7.20-7.13(\mathrm{~m}, 3 \mathrm{H}$, overlapping), $5.49-5.27(\mathrm{~m}, 1 \mathrm{H}$, overlapping), $3.56-3.35$ (m, 6H, overlapping), 3.34 (s, 3H, minor), 3.33 (s, 3H, major), 3.11 (d, $J=6.8 \mathrm{~Hz}, 2 \mathrm{H}$, minor), $3.08(\mathrm{~d}, J=6.8 \mathrm{~Hz}, 2 \mathrm{H}$, major), $2.82-2.65(\mathrm{~m}, 2 \mathrm{H}$, overlapping), $2.44-2.24(\mathrm{~m}, 2 \mathrm{H}$, overlapping), $1.15(\mathrm{t}, J=7.1 \mathrm{~Hz}, 3 \mathrm{H}$, major $), 1.10(\mathrm{t}, J=7.1 \mathrm{~Hz}, 3 \mathrm{H}$, minor $) .{ }^{13} \mathbf{C} \mathbf{N M R}(\mathbf{1 0 1} \mathbf{M H z}$, 
$\mathbf{C D C l}_{3}$ ) $\delta 171.70$ (minor), 171.59 (major), 142.18 (minor), 142.15 (major), 137.31 (major), 137.15 (minor), 128.30 (overlapping), 128.22 (overlapping), 125.68 (major), 125.66 (minor), 117.98 (minor), 117.82 (major), 71.07 (major), 70.97 (minor), 59.07 (minor), 58.76 (major), 47.12 (minor), 45.49 (major), 43.75 (major), 41.41 (major), 41.38 (minor), 41.10 (minor), 34.48 (major), 34.45 (minor), 33.43 (minor), 33.11 (major), 16.62 (major), 16.58 (minor), 14.06 (major), 12.82 (minor). ESI-HR calcd for $\mathrm{C}_{18} \mathrm{H}_{28} \mathrm{NO}_{2}{ }^{+}\left([\mathrm{M}+\mathrm{H}]^{+}\right)$290.2115, found 290.2093. IR $v\left(\mathrm{~cm}^{-1}\right)$ 2930, 1641, 1454, 700 .<smiles>CC/C(=C\CC(=O)N(CC)CCOC)[PH2+]c1ccccc1</smiles>

Amide 1w: ${ }^{1}$ H NMR (400 MHz, CDCl 3 ) $\delta 5.35-5.25(\mathrm{~m}, 1 \mathrm{H}$, overlapping), $3.58-3.37$ (m, 6H, overlapping), 3.35 (s, 3H, minor), 3.33 (s, 3H, major), $3.16-2.99(\mathrm{~m}, 2 \mathrm{H}$, overlapping), $2.14-1.95$ (m, 4H, overlapping), $1.47-1.35(\mathrm{~m}, 2 \mathrm{H}$, overlapping), $1.33-1.21(\mathrm{~m}, 10 \mathrm{H}$, overlapping $), 1.17(\mathrm{t}, J=$ $7.1 \mathrm{~Hz}, 3 \mathrm{H}$, major), $1.11(\mathrm{t}, J=7.1 \mathrm{~Hz}, 3 \mathrm{H}$, minor $), 0.98(\mathrm{t}, J=7.5 \mathrm{~Hz}, 3 \mathrm{H}$, overlapping), $0.88(\mathrm{t}, J=$ $6.7 \mathrm{~Hz}, 3 \mathrm{H}$, overlapping). ${ }^{13} \mathbf{C}$ NMR (101 MHz, CDCl 3 ) $\delta 171.97$ (minor), 171.89 (major), 144.02 (major), 143.91 (minor), 116.55 (minor), 116.42 (major), 71.14 (major), 71.07 (minor), 59.08 (minor), 58.78 (major), 47.18 (minor), 45.51 (major), 43.80 (major), 41.13 (minor), 36.48 (overlapping), 33.01 (minor), 32.74 (major), 31.89 (overlapping), 29.52 (minor), 29.51 (major), 29.29 (overlapping), 28.07 (overlapping), 23.51 (overlapping), 22.66 (overlapping), 14.13 (major), 14.09 (overlapping), 12.83 (minor), 12.75 (overlapping). ESI-HR calcd for $\mathrm{C}_{19} \mathrm{H}_{38} \mathrm{NO}_{2}{ }^{+}\left([\mathrm{M}+\mathrm{H}]^{+}\right)$312.2897, found 312.2876. IR $v\left(\mathrm{~cm}^{-1}\right) 2925,1738,1644,1456,1117,790$.

\section{Characterization of Products:}<smiles>CCN(CC)C(=O)CC[C@](C)(C#C[Se-])C(C)C</smiles>

Amide 3a: Following the general procedure, amide 1a (39.4 mg, $0.200 \mathrm{mmol})$ was converted to the alkynyl amide. Purification by silica gel chromatography gave $49.4 \mathrm{mg}$ (65\% yield) of $\mathbf{3 a}$ as a colorless oil. ${ }^{1} \mathbf{H}$ NMR $\left(400 \mathrm{MHz}, \mathrm{CDCl}_{3}\right) \delta 3.41-3.27(\mathrm{~m}, 4 \mathrm{H}), 2.62-2.38(\mathrm{~m}, 2 \mathrm{H}), 1.87-1.75$ (m, 1H), $1.73-1.61(\mathrm{~m}, 1 \mathrm{H}), 1.55-1.30(\mathrm{~m}, 4 \mathrm{H}), 1.19-1.09(\mathrm{~m}, 9 \mathrm{H}), 1.08-0.98(\mathrm{~m}, 21 \mathrm{H})$, 
$0.95-0.88(\mathrm{~m}, 3 \mathrm{H}) .{ }^{13} \mathbf{C}$ NMR $\left(101 \mathrm{MHz}, \mathrm{CDCl}_{3}\right) \delta 172.32,114.89,80.73,44.33,41.88,40.02$, 36.97, 35.65, 29.41, 26.49, 18.64 (overlapping), 18.11, 14.59, 14.40, 13.06, 11.21 (overlapping). ESI-HR calcd for $\mathrm{C}_{23} \mathrm{H}_{46} \mathrm{NOSi}^{+}\left([\mathrm{M}+\mathrm{H}]^{+}\right) 380.3343$, found 380.3328. IR $v\left(\mathrm{~cm}^{-1}\right)$ 2956, 1646, $1461,883$.<smiles>CCN(CC)C(=O)CC[C@](C)(C#CPc1ccccc1)C(C)C</smiles>

Amide 3a-Ph: Amide 3a (37.9 mg, $0.100 \mathrm{mmol}), \mathrm{PdCl}_{2}\left(\mathrm{PPh}_{3}\right)_{2}(3.2 \mathrm{mg}, 4.5 \mathrm{~mol} \%)$, $\mathrm{CuI}(0.9 \mathrm{mg}$, $5 \mathrm{~mol} \%$ ) were placed in a $10 \mathrm{~mL}$ Schlenk tube. The tube was evacuated and refilled with nitrogen. THF (0.50 mL), NEt $3(0.10 \mathrm{~mL}), \mathrm{TBAF}(0.20 \mathrm{~mL}, 1 \mathrm{M}$ in THF) and $\mathrm{PhI}(24.5 \mathrm{mg}, 1.2$ equiv.) were successively added into the tube through syringes. The reaction was stirred at $45{ }^{\circ} \mathrm{C}$ for $4 \mathrm{~h}$. The volatiles were removed on a rotary evaporator. Further purification by silica gel chromatography gave $17.1 \mathrm{mg}$ (57\% yield) of $\mathbf{3 a}-\mathbf{P h}$ as a colorless oil. ${ }^{1} \mathbf{H} \mathbf{~ N M R}\left(400 \mathrm{MHz}, \mathrm{CDCl}_{3}\right.$ ) $\delta 7.42-7.36(\mathrm{~m}, 2 \mathrm{H}), 7.33-7.26(\mathrm{~m}, 3 \mathrm{H}), 3.50-3.28(\mathrm{~m}, 4 \mathrm{H}), 2.71-2.42(\mathrm{~m}, 2 \mathrm{H}), 1.99-1.87(\mathrm{~m}$, $1 \mathrm{H}), 1.86-1.76(\mathrm{~m}, 1 \mathrm{H}), 1.63-1.41(\mathrm{~m}, 4 \mathrm{H}), 1.28(\mathrm{~s}, 3 \mathrm{H}), 1.20(\mathrm{t}, J=7.1 \mathrm{~Hz}, 3 \mathrm{H}), 1.14(\mathrm{t}, J=7.1 \mathrm{~Hz}$ $3 \mathrm{H}), 0.98(\mathrm{t}, J=7.0 \mathrm{~Hz}, 3 \mathrm{H}),{ }^{13} \mathbf{C}$ NMR $\left(101 \mathrm{MHz}, \mathrm{CDCl}_{3}\right) \delta 172.37,131.48$ (overlapping), 128.15 (overlapping), 127.50, 123.91, 95.72, 82.04, 44.21, 42.04, 40.15, 37.25, 35.36, 29.40, 26.26, 18.16, 14.57, 14.41, 13.09. ESI-HR calcd for $\mathrm{C}_{20} \mathrm{H}_{30} \mathrm{NO}^{+}\left([\mathrm{M}+\mathrm{H}]^{+}\right) 300.2322$, found 300.2310 .

IR $v\left(\mathrm{~cm}^{-1}\right) 2963,1642,1460,757 .[\alpha]^{30} \mathrm{D}=-2.0\left(c=0.46, \mathrm{CHCl}_{3}\right)$ for a 95.0:5.0 er sample. The enantiomeric purity of this compound was determined by HPLC analysis in comparison with authentic racemic material (Chiralpak ID, 99:1 hexanes: $i-\mathrm{PrOH}, 1.0 \mathrm{~mL} / \mathrm{min}, 254 \mathrm{~nm}$ ): $t_{\text {major }}=$ $59.8 \mathrm{~min}, t_{\mathrm{minor}}=63.4 \mathrm{~min}$.

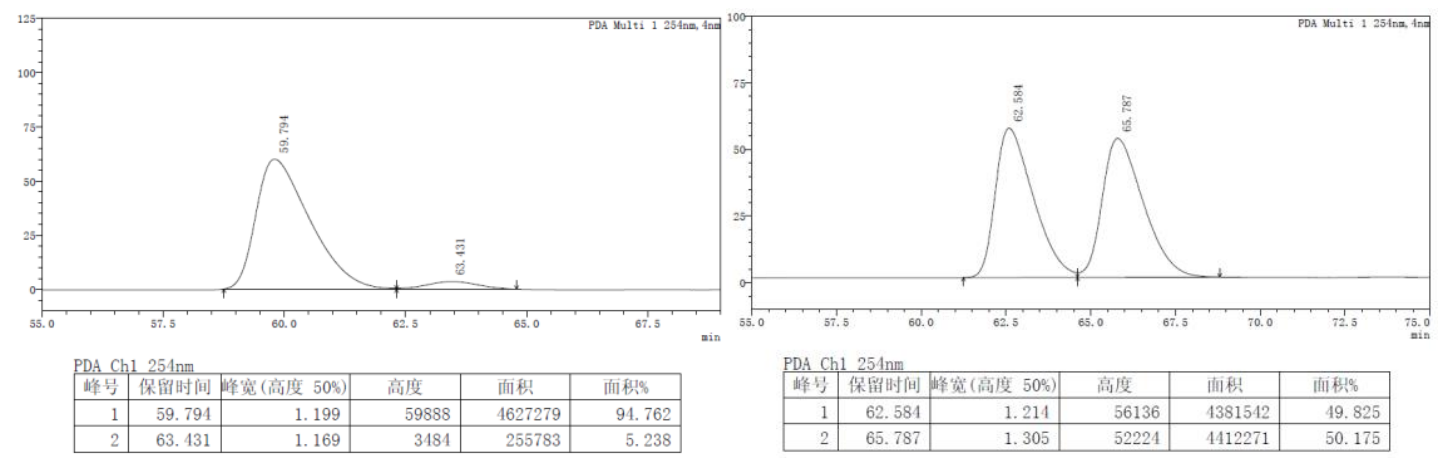




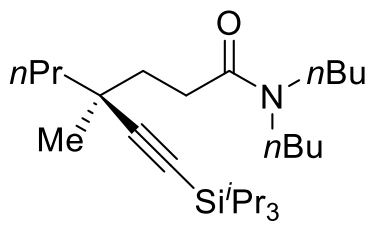

Amide 3b: Following the general procedure, amide $1 \mathbf{b}(50.6 \mathrm{mg}, 0.200 \mathrm{mmol})$ was converted to the alkynyl amide. Purification by silica gel chromatography gave $59.5 \mathrm{mg}$ (68\% yield) of $\mathbf{3 b}$ as a colorless oil. ${ }^{1} \mathbf{H}$ NMR $\left(400 \mathrm{MHz}, \mathrm{CDCl}_{3}\right) \delta 3.30(\mathrm{t}, J=7.5 \mathrm{~Hz}, 2 \mathrm{H}), 3.24(\mathrm{t}, \mathrm{J}=7.5 \mathrm{~Hz}, 2 \mathrm{H}), 2.65$ $-2.36(\mathrm{~m}, 2 \mathrm{H}), 1.87-1.76(\mathrm{~m}, 1 \mathrm{H}), 1.74-1.63(\mathrm{~m}, 1 \mathrm{H}), 1.56-1.40(\mathrm{~m}, 7 \mathrm{H}), 1.37-1.24(\mathrm{~m}$, 5H), $1.15(\mathrm{~s}, 3 \mathrm{H}), 1.08-0.97(\mathrm{~m}, 21 \mathrm{H}), 0.97-0.88(\mathrm{~m}, 9 \mathrm{H}) .{ }^{13} \mathbf{C} \mathbf{~ N M R}\left(101 \mathrm{MHz}, \mathrm{CDCl}_{3}\right) \delta$ $172.60,114.95,80.69,47.62,45.60,44.35,36.82,35.56,31.22,29.87,29.29,26.55,20.26,20.13$, 18.63 (overlapping), 18.11, 14.59, 13.88, 13.83, 11.23 (overlapping). ESI-HR calcd for $\mathrm{C}_{27} \mathrm{H}_{54} \mathrm{NOSi}^{+}\left([\mathrm{M}+\mathrm{H}]^{+}\right) 436.3969$, found 436.3956. IR $v\left(\mathrm{~cm}^{-1}\right)$ 2957, 1646, 1462, 883.<smiles>CCCCN(CCCC)C(=O)CC[C@](C)(C#Cc1ccccc1)C(C)C</smiles>

Amide 3b-Ph: Amide 3b (43.5 mg, $0.100 \mathrm{mmol}), \mathrm{PdCl}_{2}\left(\mathrm{PPh}_{3}\right)_{2}(3.2 \mathrm{mg}, 4.5 \mathrm{~mol} \%)$, $\mathrm{CuI}(0.9 \mathrm{mg}$, $5 \mathrm{~mol} \%$ ) were placed in a $10 \mathrm{~mL}$ Schlenk tube. The tube was evacuated and refilled with nitrogen. THF $(0.50 \mathrm{~mL}), \mathrm{NEt}_{3}(0.10 \mathrm{~mL})$, TBAF $(0.20 \mathrm{~mL}, 1 \mathrm{M}$ in THF) and $\mathrm{PhI}(24.5 \mathrm{mg}, 1.2$ equiv.) were successively added into the tube through syringes. The reaction was stirred at $45{ }^{\circ} \mathrm{C}$ for $4 \mathrm{~h}$. The volatiles were removed on a rotary evaporator. Further purification by silica gel chromatography gave $28.4 \mathrm{mg}$ ( $80 \%$ yield) of $\mathbf{3 b}-\mathbf{P h}$ as a colorless oil. ${ }^{\mathbf{1}} \mathbf{H} \mathbf{N M R}\left(400 \mathrm{MHz}, \mathrm{CDCl}_{3}\right)$ $\delta 7.40-7.32(\mathrm{~m}, 2 \mathrm{H}), 7.30-7.22(\mathrm{~m}, 3 \mathrm{H}), 3.35-3.28(\mathrm{~m}, 2 \mathrm{H}), 3.27-3.20(\mathrm{~m}, 2 \mathrm{H}), 2.64-2.43(\mathrm{~m}$, 2H), $1.97-1.84(\mathrm{~m}, 1 \mathrm{H}), 1.83-1.72(\mathrm{~m}, 1 \mathrm{H}), 1.63-1.39(\mathrm{~m}, 8 \mathrm{H}), 1.35-1.21(\mathrm{~m}, 7 \mathrm{H}), 1.00-0.81$ (m, 9H). ${ }^{13} \mathbf{C}$ NMR $\left(101 \mathrm{MHz}, \mathrm{CDCl}_{3}\right) \delta$ 172.72, 131.49 (overlapping), 128.11 (overlapping), 127.49, $123.92,95.74,82.07,47.82,45.71,44.24,37.27,35.34,31.35,29.95,29.47,26.31,20.27,20.10,18.16$, 14.57, 13.88, 13.77. ESI-HR calcd for $\mathrm{C}_{24} \mathrm{H}_{38} \mathrm{NO}^{+}\left([\mathrm{M}+\mathrm{H}]^{+}\right) 356.2948$, found 356.2932. IR $v\left(\mathrm{~cm}^{-1}\right)$ 2958, $1644,1465,756 .[\alpha]^{30} \mathrm{D}=-5.5\left(c=0.6, \mathrm{CHCl}_{3}\right)$ for a $92.5: 7.5$ er sample. The enantiomeric purity of this compound was determined by HPLC analysis in comparison with authentic racemic 
material (Chiralpak IC, 97:3 hexanes: $i-\mathrm{PrOH}, 1.0 \mathrm{~mL} / \mathrm{min}, 235 \mathrm{~nm}$ ): $t_{\text {major }}=11.5 \mathrm{~min}, t_{\text {minor }}=12.5$ $\min$.
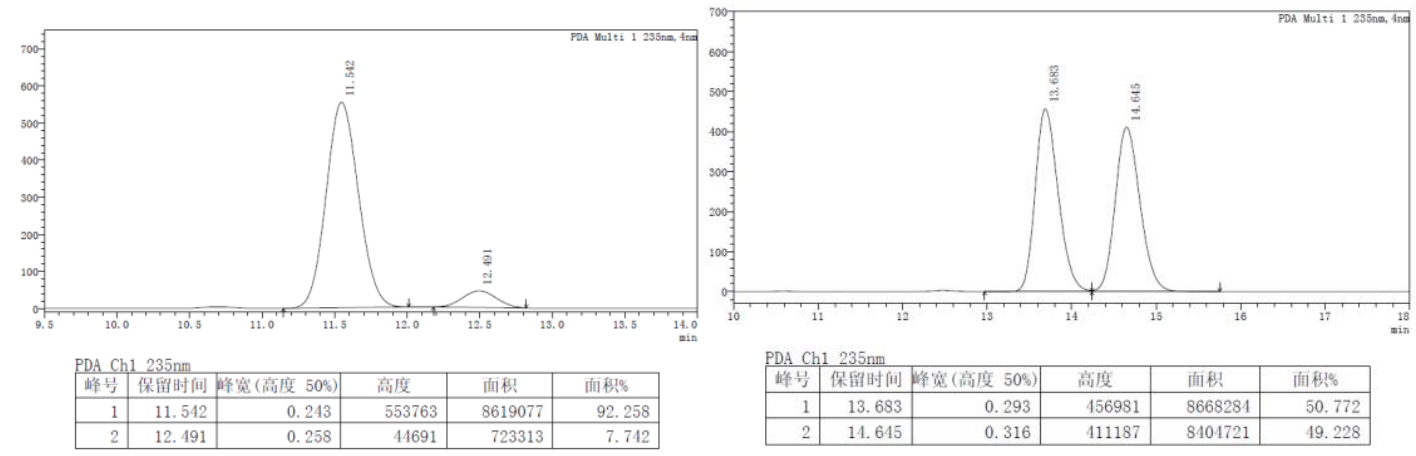<smiles>CCN(C[AsH2])C(=O)CC[C@](C)(C#C[AsH2])C(=O)c1ccccc1</smiles>

Amide 3c: Following the general procedure, amide 1c (53.4 mg, $0.200 \mathrm{mmol})$ was converted to the alkynyl amide. Purification by silica gel chromatography gave $56.3 \mathrm{mg}$ (63\% yield) of $\mathbf{3 c}$ as a colorless oil. Two sets of peaks were observed in ${ }^{13} \mathrm{C}$ NMR spectra due to amide rotamers, which are assigned to major and minor rotamers, respectively. ${ }^{1} \mathbf{H} \mathbf{~ N M R}\left(400 \mathrm{MHz}, \mathrm{CDCl}_{3}\right) \delta 3.44-$ $3.17(\mathrm{~m}, 4 \mathrm{H}), 2.67-2.34(\mathrm{~m}, 2 \mathrm{H}), 1.88-1.75(\mathrm{~m}, 1 \mathrm{H}), 1.74-1.62(\mathrm{~m}, 1 \mathrm{H}), 1.58-1.40(\mathrm{~m}, 5 \mathrm{H})$, $1.35-1.22(\mathrm{~m}, 9 \mathrm{H}), 1.20-1.08(\mathrm{~m}, 6 \mathrm{H}), 1.08-0.95(\mathrm{~m}, 21 \mathrm{H}), 0.94-0.84(\mathrm{~m}, 6 \mathrm{H}),{ }^{13} \mathbf{C}$ NMR (101 MHz, $\mathrm{CDCl}_{3}$ ) $\delta 172.52$ (major), 172.41 (minor), 114.92 (overlapping), 80.71 (overlapping), 47.51 (minor), 45.39 (major), 44.35 (minor), 44.33 (major), 42.26 (major), 40.50 (minor), 37.01 (major), 36.80 (minor), 35.65 (minor), 35.56 (major), 31.79 (overlapping), 29.39 (major), 29.33 (minor), 29.22 (minor), 29.13 (minor), 29.10 (major), 27.87 (major), 27.01 (major), 26.94 (minor), 26.56 (minor), 26.49 (major), 22.59 (overlapping), 18.65 (overlapping), 18.12 (overlapping), 14.60 (overlapping), 14.31 (major), 14.07 (major), 14.05 (minor), 12.94 (minor), 11.23, (overlapping) 11.22 (overlapping). ESI-HR calcd for $\mathrm{C}_{28} \mathrm{H}_{56} \mathrm{NOSi}^{+}\left([\mathrm{M}+\mathrm{H}]^{+}\right) 450.4126$, found 450.4114. IR $v\left(\mathrm{~cm}^{-1}\right) 2957,1648,1461,883$. 
<smiles>CCN(C[14CH3])C(=O)CC[C@](C)(C#Cc1ccccc1)C(=O)c1ccccc1</smiles>

Amide 3c-Ph: Amide 3c (44.9 mg, $0.100 \mathrm{mmol}), \mathrm{PdCl}_{2}\left(\mathrm{PPh}_{3}\right)_{2}(3.2 \mathrm{mg}, 4.5 \mathrm{~mol} \%)$, $\mathrm{CuI}(0.9 \mathrm{mg}$, $5 \mathrm{~mol} \%$ ) were placed in a $10 \mathrm{~mL}$ Schlenk tube. The tube was evacuated and refilled with nitrogen. THF (0.50 mL), NEt $3(0.10 \mathrm{~mL}), \mathrm{TBAF}(0.20 \mathrm{~mL}, 1 \mathrm{M}$ in THF) and $\mathrm{PhI}(24.5 \mathrm{mg}, 1.2$ equiv.) were successively added into the tube through syringes. The reaction was stirred at $45{ }^{\circ} \mathrm{C}$ for $4 \mathrm{~h}$. The volatiles were removed on a rotary evaporator. Further purification by silica gel chromatography gave $30.7 \mathrm{mg}$ ( $83 \%$ yield) of $\mathbf{3 c - P h}$ as a colorless oil. Two sets of peaks were observed in ${ }^{13} \mathrm{C}$ NMR spectra due to amide rotamers, which are assigned to major and minor rotamers, respectively. ${ }^{1} \mathbf{H}$ NMR $\left(400 \mathrm{MHz}, \mathrm{CDCl}_{3}\right) \delta 7.42-7.32(\mathrm{~m}, 2 \mathrm{H}), 7.31-7.22(\mathrm{~m}, 3 \mathrm{H}), 3.42$ $-3.32(\mathrm{~m}, 2 \mathrm{H}), 3.31-3.19(\mathrm{~m}, 2 \mathrm{H}), 2.64-2.40(\mathrm{~m}, 2 \mathrm{H}), 1.96-1.84(\mathrm{~m}, 1 \mathrm{H}), 1.82-1.72(\mathrm{~m}, 1 \mathrm{H})$, $1.61-1.40(\mathrm{~m}, 6 \mathrm{H}), 1.32-1.06(\mathrm{~m}, 14 \mathrm{H}), 0.95(\mathrm{t}, J=6.9 \mathrm{~Hz}, 3 \mathrm{H}), 0.90-0.78(\mathrm{~m}, 3 \mathrm{H}) .{ }^{13} \mathbf{C}$ NMR $\left(101 \mathrm{MHz}, \mathrm{CDCl}_{3}\right) \delta 172.55$ (minor), 172.53 (major), 131.48 (overlapping), 128.14 (overlapping), 128.11 (overlapping), 127.49 (overlapping), 123.92 (major), 123.90 (minor), 95.74 (minor), 95.69 (major), 82.07 (major), 82.03 (minor), 47.73 (major), 45.52 (minor), 44.23 (major), 44.22 (minor), 42.41 (minor), 40.61 (major), 37.28 (minor), 37.25 (major), 35.36 (minor), 35.34 (major), 31.78 (major), 31.69 (minor), 29.50 (major), 29.39 (minor), 29.35 (major), 29.09 (minor), 29.04 (major), 27.91 (minor), 27.02 (minor), 26.89 (major), 26.30 (major), 26.26 (minor), 22.58(minor), 22.51 (major), 18.16 (overlapping), 14.57 (overlapping), 14.32 (minor), 14.05 (minor), 14.01 (major), 12.98 (major). ESI-HR calcd for $\mathrm{C}_{25} \mathrm{H}_{40} \mathrm{NO}^{+}\left([\mathrm{M}+\mathrm{H}]^{+}\right)$370.3104, found 370.3090. IR $v\left(\mathrm{~cm}^{-1}\right)$ 2957, $1644,1424,756 .[\alpha]^{30} \mathrm{D}=-6.4\left(c=0.77, \mathrm{CHCl}_{3}\right)$ for a $93: 7$ er sample. The enantiomeric purity of this compound was determined by HPLC analysis in comparison with authentic racemic material (Chiralpak IA, 99:1 hexanes: $i-\operatorname{PrOH}, 1.0 \mathrm{~mL} / \mathrm{min}, 204 \mathrm{~nm}$ ): $t_{\text {major }}=27.2 \mathrm{~min}, t_{\mathrm{minor}}=30.5 \mathrm{~min}$. 

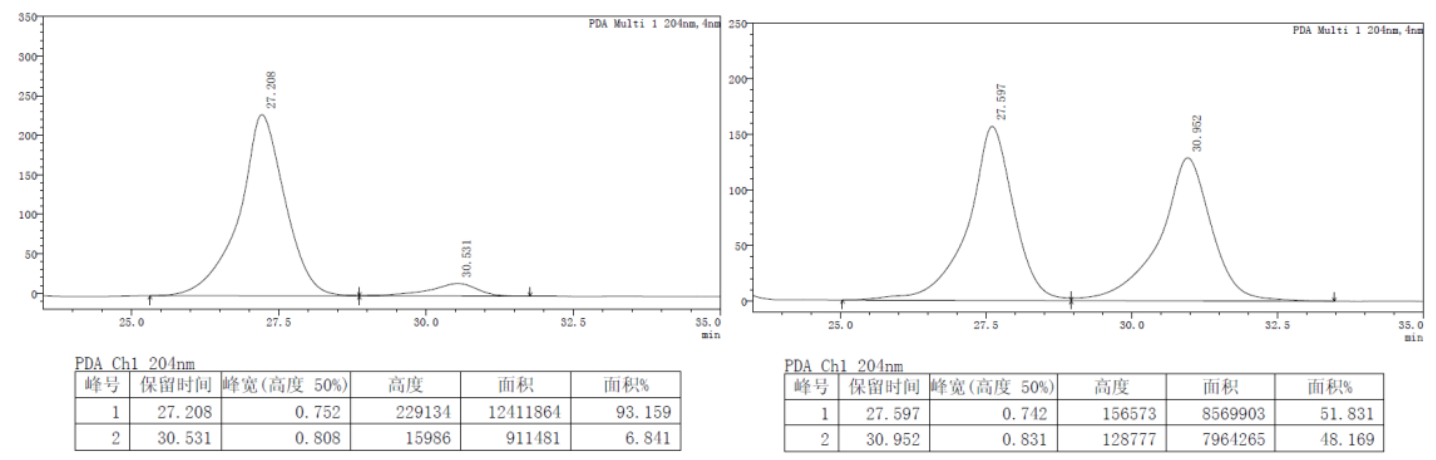<smiles>CCN(Cc1ccccc1)C(=O)CCC(C)(C#C[SeH])C(C)C</smiles>

Amide 3d: Following the general procedure for $96 \mathrm{~h}$, amide 1d (54.7 mg, $0.200 \mathrm{mmol})$ was converted to the alkynyl amide. Purification by silica gel chromatography gave $60.3 \mathrm{mg}(66 \%$ yield) of $\mathbf{3 a}$ as a colorless oil. Two sets of peaks were observed in ${ }^{1} \mathrm{H}$ and ${ }^{13} \mathrm{C}$ NMR spectra due to amide rotamers, which are assigned to major and minor rotamers, respectively. ${ }^{\mathbf{1}} \mathbf{H}$ NMR (400 $\left.\mathrm{MHz}, \mathrm{CDCl}_{3}\right) \delta 7.34-7.26(\mathrm{~m}, 2 \mathrm{H}$, overlapping), $7.25-7.09$ (m, 3H, overlapping), $3.59-3.46$ (m, 2H, overlapping), 3.37 (q, $J=7.1 \mathrm{~Hz}, 2 \mathrm{H}$, minor), 3.23 (q, $J=7.1 \mathrm{~Hz}, 2 \mathrm{H}$, major), $2.90-2.78$ (m, $2 \mathrm{H}$, overlapping), $2.61-2.42(\mathrm{~m}, 2 \mathrm{H}$, major $), 2.41-2.24(\mathrm{~m}, 2 \mathrm{H}$, minor $), 1.87-1.64(\mathrm{~m}, 2 \mathrm{H}$, overlapping), $1.56-1.27$ (m, 4H, overlapping), $1.21-1.09$ (m, 6H, overlapping), $1.09-0.96$ (m, 21H, overlapping), $0.96-0.87\left(\mathrm{~m}, 3 \mathrm{H}\right.$, overlapping). ${ }^{13} \mathbf{C ~ N M R}\left(101 \mathrm{MHz}, \mathrm{CDCl}_{3}\right) \delta 172.72$ (major), 172.58 (minor), 139.49 (major), 138.31 (minor), 128.84 (overlapping), 128.72 (overlapping), 128.69 (overlapping), 128.41 (overlapping), 126.66 (minor), 126.20 (major), 114.93 (minor), 114.85 (major), 80.82 (major), 80.73 (minor), 48.90 (minor), 47.73 (major), 44.33 (major), 44.24 (minor), 43.00 (major), 40.62 (minor), 36.95 (major), 36.62 (minor), 35.63 (major), 35.54 (minor), 35.50 (minor), 34.23 (major), 29.44 (major), 29.06 (minor), 26.55 (minor), 26.49 (major), 18.63 (overlapping), 18.12 (overlapping), 14.59 (overlapping), 14.23 (major), 12.85 (minor), 11.22 (overlapping). ESI-HR calcd for $\mathrm{C}_{29} \mathrm{H}_{50} \mathrm{NOSi}^{+}\left([\mathrm{M}+\mathrm{H}]^{+}\right) 456.3656$, found $456.3646 \mathbf{I R} v\left(\mathrm{~cm}^{-1}\right) 2957,1645,1422,676$. 
$[\alpha]^{29} \mathrm{D}=6.5\left(c=2.67, \mathrm{CHCl}_{3}\right)$ for a 93.0:7.0 er sample. The enantiomeric purity of this compound was determined by HPLC analysis in comparison with authentic racemic material (Chiralpak IC, 99:1 hexanes: $i-\mathrm{PrOH}, 1.0 \mathrm{~mL} / \mathrm{min}, 209 \mathrm{~nm}$ ): $t_{\text {major }}=37.3 \mathrm{~min}, t_{\mathrm{minor}}=44.9 \mathrm{~min}$.

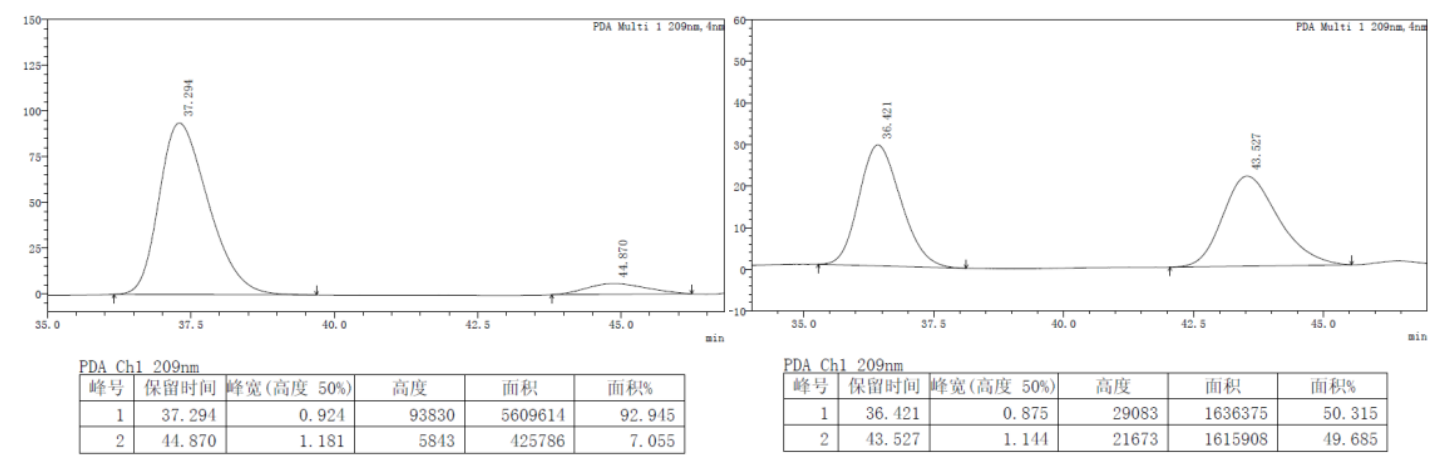<smiles>CCN(CCc1cccs1)C(=O)CC[C@](C)(C#C[SeH])C(C)C</smiles>

Amide 3e: Following the general procedure, amide 1e (55.9 mg, $0.200 \mathrm{mmol})$ was converted to the alkynyl amide. Purification by silica gel chromatography gave $60.1 \mathrm{mg}$ (65\% yield) of $\mathbf{3 e}$ as a colorless oil. Two sets of peaks were observed in ${ }^{1} \mathrm{H}$ and ${ }^{13} \mathrm{C}$ NMR spectra due to amide rotamers, which are assigned to major and minor rotamers, respectively. ${ }^{1} \mathbf{H}$ NMR $\left(400 \mathrm{MHz}, \mathrm{CDCl}_{3}\right) \delta 7.16$ (dd, $J=5.1,1.0 \mathrm{~Hz}, 1 \mathrm{H}$, minor), $7.13(\mathrm{dd}, J=5.1,1.0 \mathrm{~Hz}, 1 \mathrm{H}$, major), $6.96-6.89(\mathrm{~m}, 1 \mathrm{H}$, overlapping), $6.86-6.78(\mathrm{~m}, 1 \mathrm{H}$, overlapping), $3.59-3.50$ (m, 2H, overlapping), 3.39 (q, J = 7.1 Hz, 2H, minor), 3.24 (q, J = 7.1 Hz, 2H, major), $3.12-3.00$ (m, 2H, overlapping), $2.62-2.43$ (m, 2H, major), $2.42-2.26(\mathrm{~m}, 2 \mathrm{H}$, minor), $1.91-1.75(\mathrm{~m}, 1 \mathrm{H}$, overlapping), $1.74-1.64(\mathrm{~m}, 1 \mathrm{H}$, overlapping), $1.56-1.30(\mathrm{~m}, 4 \mathrm{H}$, overlapping), $1.18(\mathrm{~s}, 3 \mathrm{H}$, major $), 1.16-1.09(\mathrm{~m}, 6 \mathrm{H}$, overlapping), $1.08-0.98\left(\mathrm{~m}, 21 \mathrm{H}\right.$, overlapping), $0.95-0.87\left(\mathrm{~m}, 3 \mathrm{H}\right.$, overlapping). ${ }^{13} \mathbf{C}$ NMR (101 MHz, $\mathrm{CDCl}_{3}$ ) $\delta 172.82$ (major), 172.65 (minor), 141.74 (major), 140.22 (minor), 127.08 (minor), 126.87 (major), 125.46 (minor), 125.15 (major), 124.06 (minor), 123.58 (major), 114.87 (minor), 114.84 (major), 80.85 (major), 80.78 (minor), 48.87 (minor), 47.92 (major), 44.34 (major), 44.23 (minor), 43.24 (major), 40.60 (minor), 36.92 (major), 36.64 (minor), 35.65 (major), 35.50 (minor), 29.48 (minor), 29.45 (major), 29.11 (minor), 28.24 (major), 26.54 (minor), 26.50 (major), 18.65 (overlapping), 18.13 (overlapping), 14.59 (overlapping), 14.22 (major), 12.90 
(minor), 11.24 (overlapping). ESI-HR calcd for $\mathrm{C}_{27} \mathrm{H}_{48} \mathrm{NOSSi}^{+}\left([\mathrm{M}+\mathrm{H}]^{+}\right) 462.3220$, found 462.3207. IR $v\left(\mathrm{~cm}^{-1}\right) 2957,1646,1461,883 .[\alpha]^{30} \mathrm{D}=1.4\left(c=2.66, \mathrm{CHCl}_{3}\right)$ for a 94.0:6.0 er sample. The enantiomeric purity of this compound was determined by HPLC analysis in comparison with authentic racemic material (Chiralpak IF, 99:1 hexanes:i-PrOH, $1.0 \mathrm{~mL} / \mathrm{min}, 207$ $\mathrm{nm}): t_{\mathrm{major}}=34.4 \mathrm{~min}, t_{\mathrm{minor}}=37.7 \mathrm{~min}$.
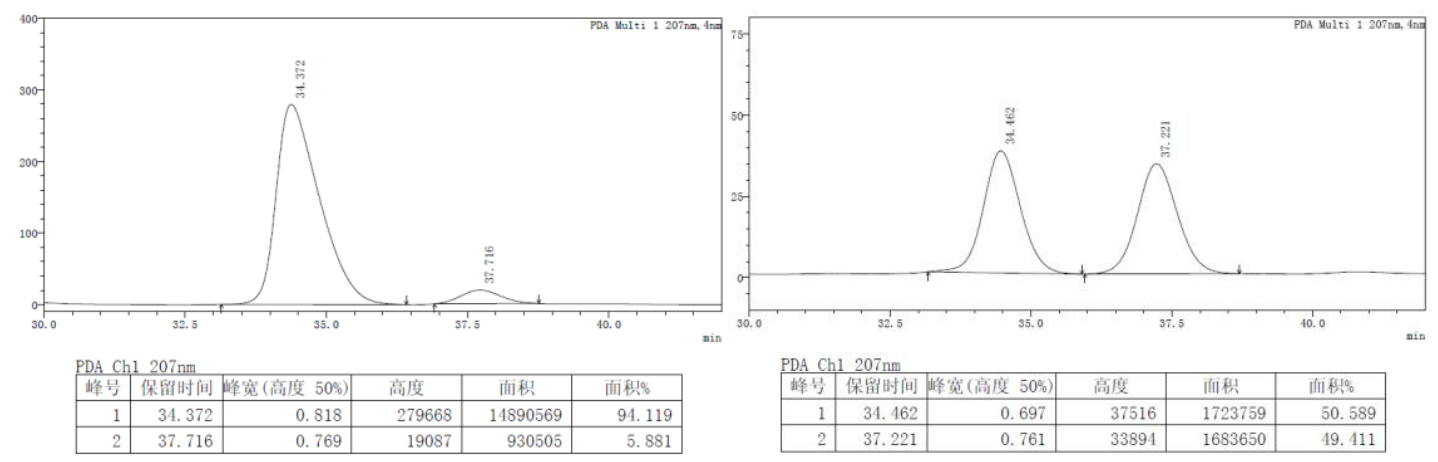<smiles>CCN(CCc1ccco1)C(=O)CC[C@](C)(C#C[Se-])C(C)C</smiles>

Amide 3f: Following the general procedure, amide $1 \mathbf{1}(52.6 \mathrm{mg}, 0.200 \mathrm{mmol})$ was converted to the alkynyl amide. Purification by silica gel chromatography gave $56.7 \mathrm{mg}$ (64\% yield) of $\mathbf{3 f}$ as a colorless oil. Two sets of peaks were observed in ${ }^{1} \mathrm{H}$ and ${ }^{13} \mathrm{C}$ NMR spectra due to amide rotamers, which are assigned to major and minor rotamers, respectively. ${ }^{1} \mathbf{H} \mathbf{~ N M R}\left(400 \mathrm{MHz}, \mathrm{CDCl}_{3}\right) \delta 7.34$ $-7.28(\mathrm{~m}, 1 \mathrm{H}$, overlapping), $6.39-6.19(\mathrm{~m}, 1 \mathrm{H}$, overlapping), $6.09-5.91(\mathrm{~m}, 1 \mathrm{H}$, overlapping $)$, $3.56(\mathrm{t}, J=7.2 \mathrm{~Hz}, 2 \mathrm{H}$, overlapping), $3.34(\mathrm{q}, J=7.1 \mathrm{~Hz}, 2 \mathrm{H}$, minor), 3.19 (q, $J=7.1 \mathrm{~Hz}, 2 \mathrm{H}$, major), $2.93-2.79$ (m, 2H, overlapping), $2.61-2.26(\mathrm{~m}, 2 \mathrm{H}$, overlapping), $1.86-1.74(\mathrm{~m}, 1 \mathrm{H}$, overlapping), $1.73-1.60(\mathrm{~m}, 1 \mathrm{H}$, overlapping), $1.54-1.31(\mathrm{~m}, 4 \mathrm{H}$, overlapping), 1.17 (s, 3H, major), $1.15(\mathrm{~s}, 3 \mathrm{H}$, minor), $1.10(\mathrm{t}, J=7.1 \mathrm{~Hz}, 3 \mathrm{H}$, overlapping), $1.08-0.97(\mathrm{~m}, 21 \mathrm{H}$, overlapping), $0.95-0.87$ (m, 3H, overlapping). ${ }^{13} \mathbf{C} \mathbf{~ N M R}\left(101 \mathrm{MHz}, \mathrm{CDCl}_{3}\right) \delta 172.79$ (major), 172.62 (minor), 153.42 (major), 151.99 (minor), 141.54 (minor), 141.11 (major), 114.90 (minor), 114.86 (major), 110.41 (minor), 110.29 (major), 106.74 (minor), 106.22 (major), 80.83 (major), 80.78 (minor), 46.03 (minor), 44.84 (major), 44.34 (major), 44.26 (minor), 42.97 (major), 40.52 (minor), 36.94 (major), 36.72 (minor), 35.67 (major), 35.57 (minor), 29.43 (major), 28.98 (minor), 
27.92 (minor), 26.58 (major), 26.53 (minor), 26.49 (major), 18.64 (overlapping), 18.13 (overlapping), 14.59 (overlapping), 14.20 (major), 12.87 (minor), 11.24 (overlapping). ESI-HR calcd for $\mathrm{C}_{27} \mathrm{H}_{48} \mathrm{NO}_{2} \mathrm{Si}^{+}\left([\mathrm{M}+\mathrm{H}]^{+}\right)$446.3449, found 446.3438. IR $v\left(\mathrm{~cm}^{-1}\right)$ 2958, 1647, 1423, 729. $[\alpha]^{30} \mathrm{D}=2.8\left(c=2.36, \mathrm{CHCl}_{3}\right)$ for a 94.5:5.5 er sample. The enantiomeric purity of this compound was determined by HPLC analysis in comparison with authentic racemic material (Chiralpak IE, 99:1 hexanes: $i-\mathrm{PrOH}, 1.0 \mathrm{~mL} / \mathrm{min}, 210 \mathrm{~nm}$ ): $t_{\text {major }}=49.6 \mathrm{~min}, t_{\mathrm{minor}}=52.5 \mathrm{~min}$.

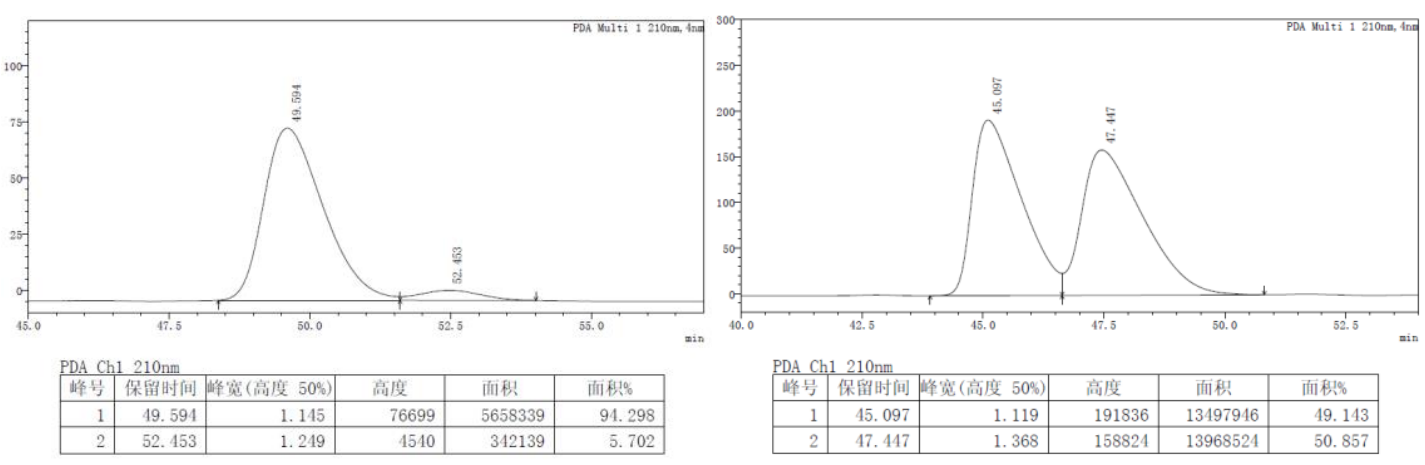<smiles>CCN(CCOC)C(=O)CCC(C)(C#C[Se-])C(C)C</smiles>

Amide 3g: Following the general procedure, amide $1 \mathrm{~g}(45.4 \mathrm{mg}, 0.200 \mathrm{mmol})$ was converted to the alkynyl amide. Purification by silica gel chromatography gave $65.8 \mathrm{mg}$ ( $80 \%$ yield) of $\mathbf{3 g}$ as a colorless oil. Two sets of peaks were observed in ${ }^{13} \mathrm{C}$ NMR spectra due to amide rotamers, which are assigned to major and minor rotamers, respectively. ${ }^{1} \mathbf{H} \mathbf{~ N M R}\left(400 \mathrm{MHz}, \mathrm{CDCl}_{3}\right) \delta 3.55-$ $3.45(\mathrm{~m}, 4 \mathrm{H}), 3.42(\mathrm{q}, J=7.2 \mathrm{~Hz}, 2 \mathrm{H}), 3.33(\mathrm{~s}, 3 \mathrm{H}), 2.62-2.38(\mathrm{~m}, 2 \mathrm{H}), 1.87-1.75(\mathrm{~m}, 1 \mathrm{H})$, $1.73-1.62(\mathrm{~m}, 1 \mathrm{H}), 1.56-1.30(\mathrm{~m}, 4 \mathrm{H}), 1.19-1.08(\mathrm{~m}, 6 \mathrm{H}), 1.08-0.96(\mathrm{~m}, 21 \mathrm{H}), 0.92(\mathrm{t}, J=$ $6.9 \mathrm{~Hz}, 3 \mathrm{H}) .{ }^{13} \mathbf{C} \mathbf{N M R}\left(101 \mathrm{MHz}, \mathrm{CDCl}_{3}\right) \delta 172.99$ (major), 172.93 (minor), 114.94 (minor), 114.81 (major), 80.80 (major), 80.65 (minor), 71.14 (major), 71.04 (minor), 59.00 (minor), 58.77 (major), 47.04 (minor), 45.43 (major), 44.32 (major), 44.28 (minor), 43.68 (major), 41.13 (minor), 36.87 (major), 36.77 (minor), 35.61 (major), 35.56 (minor), 29.40 (minor), 29.24 (major), 26.49 (overlapping), 18.63 (overlapping), 18.11 (overlapping), 14.59 (overlapping), 14.13 (major), 12.80 (minor), 11.20 (overlapping). ESI-HR calcd for $\mathrm{C}_{24} \mathrm{H}_{48} \mathrm{NO}_{2} \mathrm{Si}^{+}\left([\mathrm{M}+\mathrm{H}]^{+}\right) 410.3449$, found 410.3434. IR $v\left(\mathrm{~cm}^{-1}\right) 2938,1646,1461,675$. 
<smiles>C#CC#C[C@@](C)(CCC(=O)N(CC)CCOC)C(C)C</smiles>

Amide 3g-Ph: Amide 3g (40.9 mg, $0.100 \mathrm{mmol}), \mathrm{PdCl}_{2}\left(\mathrm{PPh}_{3}\right)_{2}$ (3.2 mg, $\left.4.5 \mathrm{~mol} \%\right)$, $\mathrm{CuI}(0.9 \mathrm{mg}$, $5 \mathrm{~mol} \%$ ) were placed in a $10 \mathrm{~mL}$ Schlenk tube. The tube was evacuated and refilled with nitrogen. THF (0.50 mL), NEt 3 (0.10 mL), TBAF ( $0.20 \mathrm{~mL}, 1 \mathrm{M}$ in THF) and PhI (24.5 mg, 1.2 equiv.) were successively added into the tube through syringes. The reaction was stirred at $45{ }^{\circ} \mathrm{C}$ for $4 \mathrm{~h}$. The volatiles were removed on a rotary evaporator. Further purification by silica gel chromatography gave $22.1 \mathrm{mg}$ (67\% yield) of $\mathbf{3 g}-\mathbf{P h}$ as a colorless oil. Two sets of peaks were observed in ${ }^{1} \mathrm{H}$ and ${ }^{13} \mathrm{C}$ NMR spectra due to amide rotamers, which are assigned to major and minor rotamers, respectively. ${ }^{1} \mathbf{H}$ NMR $\left(400 \mathrm{MHz}, \mathrm{CDCl}_{3}\right) \delta 7.41-7.33(\mathrm{~m}, 2 \mathrm{H}$, overlapping), $7.31-7.22(\mathrm{~m}, 3 \mathrm{H}$, overlapping), $3.56-3.37(\mathrm{~m}, 6 \mathrm{H}$, overlapping), $3.33(\mathrm{~s}, 3 \mathrm{H}$, major $), 3.28(\mathrm{~s}$, $3 \mathrm{H}$, minor $), 2.68-2.48(\mathrm{~m}, 2 \mathrm{H}$, overlapping), $1.98-1.85(\mathrm{~m}, 1 \mathrm{H}$, overlapping $), 1.84-1.72(\mathrm{~m}$, $1 \mathrm{H}$, overlapping), $1.60-1.37(\mathrm{~m}, 4 \mathrm{H}$, overlapping), $1.29-1.21(\mathrm{~m}, 3 \mathrm{H}$, overlapping $), 1.17(\mathrm{t}, J=$ $7.1 \mathrm{~Hz}, 3 \mathrm{H}$, major $), 1.10(\mathrm{t}, J=7.1 \mathrm{~Hz}, 3 \mathrm{H}$, minor $), 0.95\left(\mathrm{t}, J=7.0 \mathrm{~Hz}, 3 \mathrm{H}\right.$, overlapping). ${ }^{13} \mathrm{C}$ NMR (101 MHz, $\left.\mathrm{CDCl}_{3}\right) \delta 173.05$ (overlapping), 131.54 (overlapping), 131.50 (overlapping), 128.17 (overlapping), 128.12 (overlapping), 127.53 (major), 127.48 (minor), 123.97 (minor), 123.91 (major), 95.80 (minor), 95.66 (major), 82.11 (major), 82.04 (minor), 71.18 (major), 70.97 (minor), 59.00 (minor), 58.78 (major), 47.18 (minor), 45.56 (major), 44.24 (major), 44.21 (minor), 43.84 (major), 41.15 (minor), 37.16 (major), 37.10 (minor), 35.35 (major), 35.31 (minor), 29.47 (minor), 29.26 (major), 26.28 (overlapping), 18.18 (overlapping), 14.59 (overlapping), 14.15 (major), 12.86 (minor). ESI-HR calcd for $\mathrm{C}_{21} \mathrm{H}_{32} \mathrm{NO}_{2}{ }^{+}\left([\mathrm{M}+\mathrm{H}]^{+}\right) 330.2428$, found 330.2414. IR v $\left(\mathrm{cm}^{-1}\right) 2959,1643,1456,1117,757 .[\alpha]^{30} \mathrm{D}=-3.6\left(c=0.7, \mathrm{CHCl}_{3}\right)$ for a $96.5: 3.5$ er sample. The enantiomeric purity of this compound was determined by HPLC analysis in comparison with authentic racemic material (Chiralpak IC, 95:5 hexanes:i-PrOH, $1.0 \mathrm{~mL} / \mathrm{min}, 195 \mathrm{~nm}$ ): $t_{\text {major }}=$ $15.0 \mathrm{~min}, t_{\mathrm{minor}}=16.2 \mathrm{~min}$. 

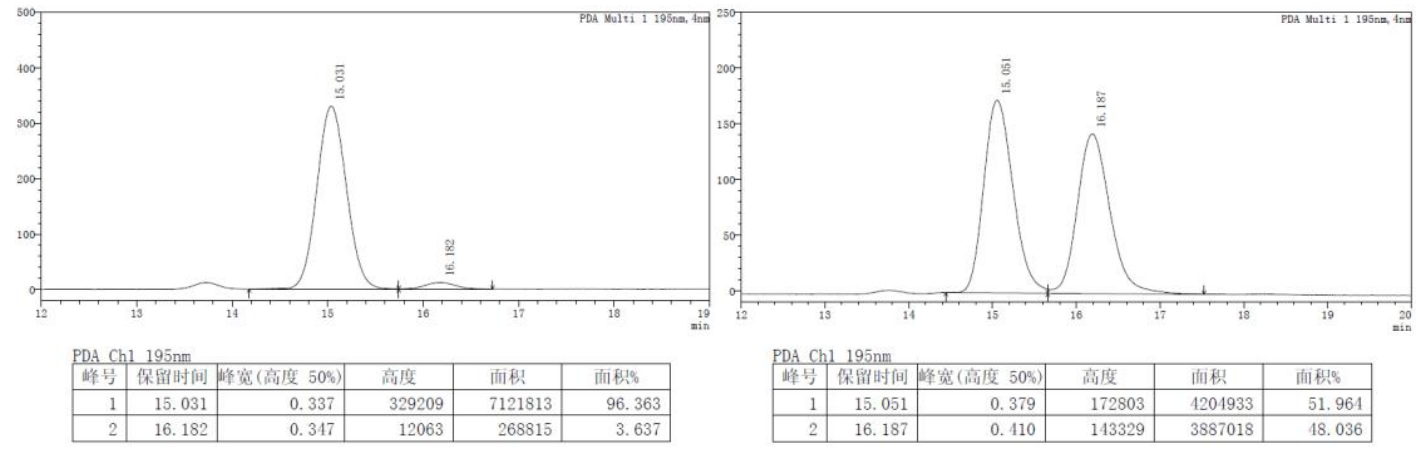<smiles>CCN(CCOCOC)C(=O)CC[C@](C)(C#C[SnH3])C(C)C</smiles>

Amide 3h: Following the general procedure, amide $1 \mathbf{h}(51.5 \mathrm{mg}, 0.200 \mathrm{mmol})$ was converted to the alkynyl amide. Purification by silica gel chromatography gave $63.8 \mathrm{mg}$ (73\% yield) of $\mathbf{3 h}$ as a colorless oil. Two sets of peaks were observed in ${ }^{13} \mathrm{C}$ NMR spectra due to amide rotamers, which are assigned to major and minor rotamers, respectively. ${ }^{1} \mathbf{H}$ NMR $\left(400 \mathrm{MHz}, \mathrm{CDCl}_{3}\right) \delta 4.69-$ $4.51(\mathrm{~m}, 2 \mathrm{H}), 3.72-3.60(\mathrm{~m}, 2 \mathrm{H}), 3.57-3.48(\mathrm{~m}, 2 \mathrm{H}), 3.44(\mathrm{q}, J=7.1 \mathrm{~Hz}, 2 \mathrm{H}), 3.38-3.24(\mathrm{~m}$, 3H), $2.66-2.41(\mathrm{~m}, 2 \mathrm{H}), 1.87-1.75(\mathrm{~m}, 1 \mathrm{H}), 1.75-1.61(\mathrm{~m}, 1 \mathrm{H}), 1.57-1.40(\mathrm{~m}, 3 \mathrm{H}), 1.39-$ $1.30(\mathrm{~m}, 1 \mathrm{H}), 1.23-1.09(\mathrm{~m}, 6 \mathrm{H}), 1.09-0.95(\mathrm{~m}, 21 \mathrm{H}), 0.95-0.89(\mathrm{~m}, 3 \mathrm{H}) .{ }^{13} \mathbf{C}$ NMR $(101$ $\left.\mathrm{MHz}, \mathrm{CDCl}_{3}\right) \delta 173.04$ (minor), 173.00 (major), 114.98 (minor), 114.85 (major), 96.55 (overlapping), 80.86 (major), 80.72 (minor), 66.10 (major), 65.83 (minor), 55.30 (minor), 55.21 (major), 47.04 (minor), 45.54 (major), 44.35 (major), 44.28 (minor), 43.61 (major), 40.95 (minor), 36.93 (major), 36.81 (minor), 35.65 (major), 35.60 (minor), 29.40 (minor), 29.32 (major), 26.52 (minor), 26.50 (major), 18.64 (overlapping), 18.12 (overlapping), 14.58 (overlapping), 14.20 (major), 12.81 (minor), 11.24 (overlapping). ESI-HR calcd for $\mathrm{C}_{25} \mathrm{H}_{50} \mathrm{NO}_{3} \mathrm{Si}^{+}\left([\mathrm{M}+\mathrm{H}]^{+}\right) 440.3554$, found 440.3543. IR $v\left(\mathrm{~cm}^{-1}\right) 2938,1647,1462,676$.<smiles>C#CC#C[C@@](C)(CCC(=O)N(CC)CCOCOC)C(C)C</smiles> 
Amide 3h-Ph: Amide 3h (43.9 mg, $0.100 \mathrm{mmol}), \mathrm{PdCl}_{2}\left(\mathrm{PPh}_{3}\right)_{2}$ (3.2 mg, $\left.4.5 \mathrm{~mol} \%\right)$, CuI (0.9 mg, $5 \mathrm{~mol} \%$ ) were placed in a $10 \mathrm{~mL}$ Schlenk tube. The tube was evacuated and refilled with nitrogen. THF (0.50 mL), NEt $3(0.10 \mathrm{~mL}), \mathrm{TBAF}(0.20 \mathrm{~mL}, 1 \mathrm{M}$ in THF) and $\mathrm{PhI}(24.5 \mathrm{mg}, 1.2$ equiv.) were successively added into the tube through syringes. The reaction was stirred at $45{ }^{\circ} \mathrm{C}$ for $4 \mathrm{~h}$. The volatiles were removed on a rotary evaporator. Further purification by silica gel chromatography gave $34.9 \mathrm{mg}$ (97\% yield) of $\mathbf{3 h}-\mathbf{P h}$ as a colorless oil. Two sets of peaks were observed in ${ }^{1} \mathrm{H}$ and ${ }^{13} \mathrm{C}$ NMR spectra due to amide rotamers, which are assigned to major and minor rotamers, respectively. ${ }^{1} \mathbf{H}$ NMR $\left(400 \mathrm{MHz}, \mathrm{CDCl}_{3}\right) \delta 7.47-7.33(\mathrm{~m}, 2 \mathrm{H}$, overlapping), $7.28-7.25$ (m, 3H, overlapping), $4.62(\mathrm{~s}, 2 \mathrm{H}$, major), $4.53(\mathrm{~s}, 2 \mathrm{H}$, minor), $3.72-3.59(\mathrm{~m}, 2 \mathrm{H}$, overlapping), 3.53 (t, $J=5.6 \mathrm{~Hz}, 2 \mathrm{H}$, overlapping), $3.50-3.38(\mathrm{~m}, 2 \mathrm{H}$, overlapping), $3.35(\mathrm{~s}, 3 \mathrm{H}$, major), 3.26 (s, 3H, minor), $2.68-2.47$ (m, 2H, overlapping), $1.97-1.84(\mathrm{~m}, 1 \mathrm{H}$, overlapping), $1.83-1.69(\mathrm{~m}, 1 \mathrm{H}$, overlapping), $1.57-1.38(\mathrm{~m}, 4 \mathrm{H}$, overlapping $), 1.25(\mathrm{~s}, 3 \mathrm{H}$, overlapping $)$, $1.19(\mathrm{t}, J=7.1 \mathrm{~Hz}, 3 \mathrm{H}$, major), $1.12(\mathrm{t}, J=7.1 \mathrm{~Hz}, 3 \mathrm{H}$, minor $), 0.95(\mathrm{t}, J=6.8 \mathrm{~Hz}, 2 \mathrm{H}$, overlapping). ${ }^{13} \mathbf{C}$ NMR (101 $\left.\mathrm{MHz}, \mathrm{CDCl}_{3}\right) \delta 173.14$ (minor), 173.06 (major), 131.54 (overlapping), 131.49, (overlapping) 128.17 (overlapping), 128.13 (overlapping), 127.54 (major), 127.50 (minor), 123.96 (minor), 123.89 (major), 96.56 (major), 96.51 (minor), 95.77 (major), 95.64 (minor), 82.10 (major), 82.00 (minor), 66.10 (major), 65.71 (minor), 55.25 (overlapping), 47.14 (minor), 45.63 (major), 44.22 (overlapping), 43.75 (major), 40.94 (minor), 37.18 (major), 37.09 (minor), 35.35 (major), 35.28 (minor), 29.45 (minor), 29.30 (major), 26.29 (minor), 26.26 (major), 18.16 (overlapping), 14.57 (overlapping), 14.20 (major), 12.85 (minor). ESI-HR calcd for $\mathrm{C}_{22} \mathrm{H}_{34} \mathrm{NO}_{3}{ }^{+}\left([\mathrm{M}+\mathrm{H}]^{+}\right)$360.2533, found 360.2522. IR $v\left(\mathrm{~cm}^{-1}\right)$ 2958, 1644, 1444, 1037, 757. $[\alpha]^{30}{ }_{\mathrm{D}}=-3.6\left(c=0.45, \mathrm{CHCl}_{3}\right)$ for a 95.5:4.5 er sample. The enantiomeric purity of this compound was determined by HPLC analysis in comparison with authentic racemic material (Chiralpak ID, 98:2 hexanes: $i-\mathrm{PrOH}, 1.0 \mathrm{~mL} / \mathrm{min}, 200 \mathrm{~nm}$ ): $t_{\text {major }}=36.5 \mathrm{~min}, t_{\mathrm{minor}}=39.5 \mathrm{~min}$.
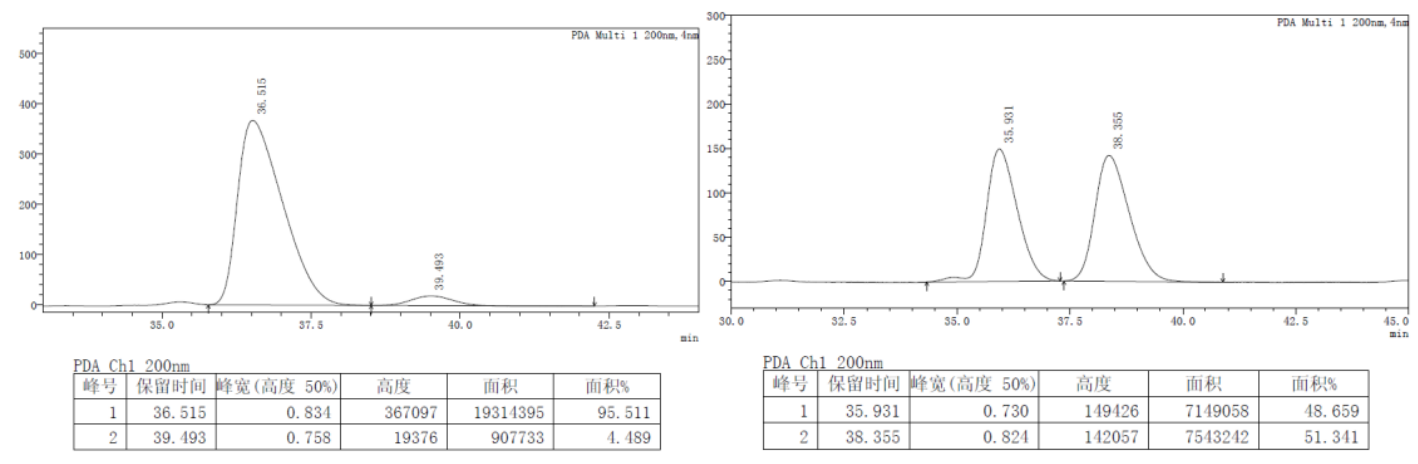


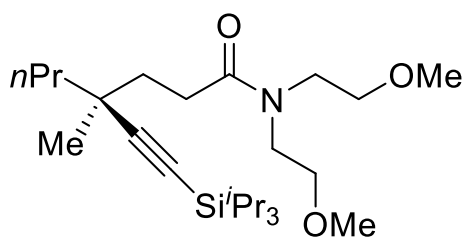

Amide 3i: Following the general procedure, amide 1i (51.4 mg, $0.200 \mathrm{mmol})$ was converted to the alkynyl amide. Purification by silica gel chromatography gave $45.5 \mathrm{mg}$ (52\% yield) of $\mathbf{3 i}$ as a colorless oil. ${ }^{1} \mathbf{H}$ NMR $\left(400 \mathrm{MHz}, \mathrm{CDCl}_{3}\right) \delta 3.70-3.42(\mathrm{~m}, 8 \mathrm{H}), 3.34(\mathrm{~s}, 6 \mathrm{H}), 2.69-2.40(\mathrm{~m}$, 2H), $1.87-1.75(\mathrm{~m}, 1 \mathrm{H}), 1.73-1.64(\mathrm{~m}, 1 \mathrm{H}), 1.53-1.30(\mathrm{~m}, 4 \mathrm{H}), 1.17(\mathrm{~s}, 3 \mathrm{H}), 1.12-0.96(\mathrm{~m}$, 21H), $0.95-0.85(\mathrm{~m}, 3 \mathrm{H}) .{ }^{13} \mathbf{C}$ NMR $\left(101 \mathrm{MHz}, \mathrm{CDCl}_{3}\right) \delta 173.62,114.94,80.72,71.15,70.95$, 58.96, 58.75, 48.70, 46.34, 44.28, 36.73, 35.57, 29.30, 26.51, 18.64 (overlapping), 18.12, 14.59, 11.24 (overlapping). ESI-HR calcd for $\mathrm{C}_{25} \mathrm{H}_{50} \mathrm{NO}_{3} \mathrm{Si}^{+}\left([\mathrm{M}+\mathrm{H}]^{+}\right) 440.3554$, found 440.3543 . IR $v$ $\left(\mathrm{cm}^{-1}\right) 2939,1647,1462,883$.<smiles>COCCN(CCOC)C(=O)CCC(C)(C#CPc1ccccc1)C(C)C</smiles>

Amide 3i-Ph: Amide 3i (43.9 mg, $0.100 \mathrm{mmol}), \mathrm{PdCl}_{2}\left(\mathrm{PPh}_{3}\right)_{2}$ (3.2 mg, $\left.4.5 \mathrm{~mol} \%\right)$, $\mathrm{CuI}(0.9 \mathrm{mg}, 5$ mol\%) were placed in a $10 \mathrm{~mL}$ Schlenk tube. The tube was evacuated and refilled with nitrogen. THF (0.50 mL), NEt 3 ( $0.10 \mathrm{~mL})$, TBAF $(0.20 \mathrm{~mL}, 1 \mathrm{M}$ in THF) and PhI (24.5 mg, 1.2 equiv.) were successively added into the tube through syringes. The reaction was stirred at $45{ }^{\circ} \mathrm{C}$ for $4 \mathrm{~h}$. The volatiles were removed on a rotary evaporator. Further purification by silica gel chromatography gave $28.0 \mathrm{mg}$ (78\% yield) of $\mathbf{3 i - P h}$ as a colorless oil. ${ }^{\mathbf{1}} \mathbf{H} \mathbf{~ N M R}\left(400 \mathrm{MHz}, \mathrm{CDCl}_{3}\right)$ $\delta 7.42-7.34(\mathrm{~m}, 2 \mathrm{H}), 7.30-7.22(\mathrm{~m}, 3 \mathrm{H}), 3.73-3.44(\mathrm{~m}, 8 \mathrm{H}), 3.34(\mathrm{~s}, 3 \mathrm{H}), 3.27$ (s, 3H), $2.75-$ $2.50(\mathrm{~m}, 2 \mathrm{H}), 1.98-1.84(\mathrm{~m}, 1 \mathrm{H}), 1.83-1.70(\mathrm{~m}, 1 \mathrm{H}), 1.61-1.38(\mathrm{~m}, 4 \mathrm{H}), 1.25(\mathrm{~s}, 3 \mathrm{H}), 0.95(\mathrm{t}$, $J=6.8 \mathrm{~Hz}, 3 \mathrm{H}) \cdot{ }^{13} \mathbf{C} \mathbf{N M R}\left(101 \mathrm{MHz}, \mathrm{CDCl}_{3}\right) \delta 173.72,131.53$ (overlapping), 128.11 (overlapping), 127.47, 123.95, 95.75, 82.04, 71.18, 70.83, 58.95, 58.76, 48.81, 46.35, 44.20, 36.98, 35.26, 29.33, 26.28, 18.15, 14.57. ESI-HR calcd for $\mathrm{C}_{22} \mathrm{H}_{34} \mathrm{NO}_{3}{ }^{+}\left([\mathrm{M}+\mathrm{H}]^{+}\right)$360.2533, found 360.2521. IR $v\left(\mathrm{~cm}^{-1}\right) 2958,1644,1464,1117,693 .[\alpha]^{30} \mathrm{D}=-5.2\left(c=0.48, \mathrm{CHCl}_{3}\right)$ for a $98.5: 1.5$ er sample. The enantiomeric purity of this compound was determined by HPLC analysis in 
comparison with authentic racemic material (Chiralpak ID, 98:2 hexanes:i-PrOH, $1.0 \mathrm{~mL} / \mathrm{min}$, $254 \mathrm{~nm}): t_{\text {major }}=32.9 \mathrm{~min}, t_{\text {minor }}=38.6 \mathrm{~min}$.
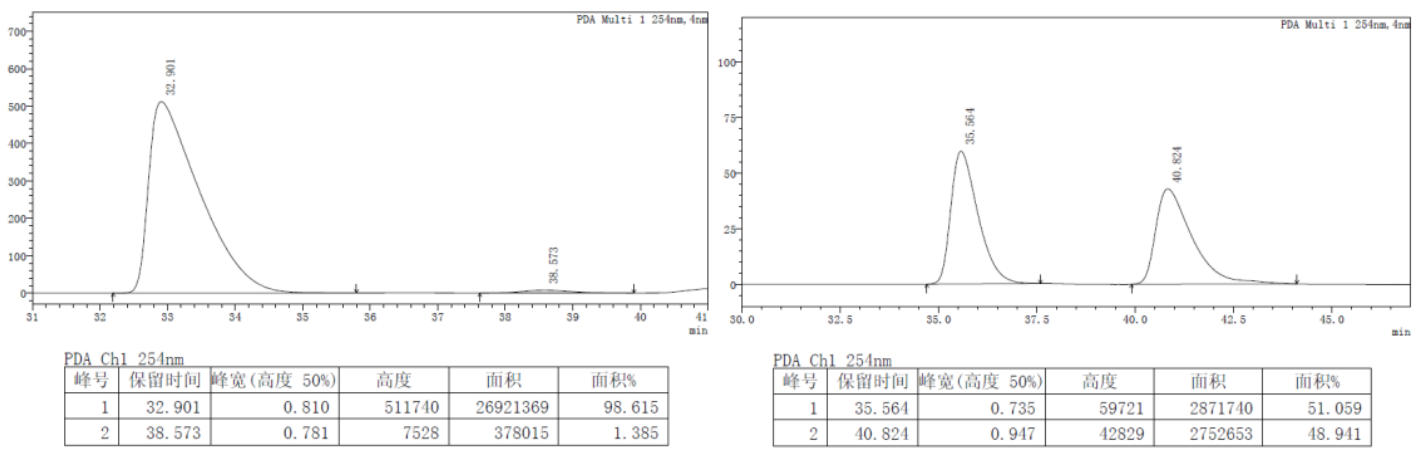<smiles>CCN(CCC1=CCCCC1)C(=O)CC[C@](C)(C#C[AsH2-])C(C)C</smiles>

Amide 3j: Following the general procedure, amide $(55.5 \mathrm{mg}, 0.200 \mathrm{mmol})$ was converted to the alkynyl amide. Purification by silica gel chromatography gave $47.0 \mathrm{mg}$ (51\% yield) of $\mathbf{3 j}$ as a colorless oil. ${ }^{1} \mathbf{H}$ NMR (400 MHz, $\left.\mathrm{CDCl}_{3}\right) \delta 5.49-5.33(\mathrm{~m}, 1 \mathrm{H}), 3.45-3.24(\mathrm{~m}, 4 \mathrm{H}), 2.62-2.30(\mathrm{~m}, 2 \mathrm{H}), 2.21$ $-2.09(\mathrm{~m}, 2 \mathrm{H}), 2.03-1.90(\mathrm{~m}, 4 \mathrm{H}), 1.88-1.74(\mathrm{~m}, 1 \mathrm{H}), 1.73-1.65(\mathrm{~m}, 1 \mathrm{H}), 1.63-1.57(\mathrm{~m}, 2 \mathrm{H})$, $1.57-1.50(\mathrm{~m}, 2 \mathrm{H}), 1.50-1.29(\mathrm{~m}, 4 \mathrm{H}), 1.19-1.08(\mathrm{~m}, 6 \mathrm{H}), 1.08-0.94(\mathrm{~m}, 21 \mathrm{H}), 0.94-0.87(\mathrm{~m}$, 3H). ${ }^{13} \mathrm{C}$ NMR (101 MHz, $\mathrm{CDCl}_{3}$ ) $\delta 172.49$ (major), 172.42 (minor), 135.29 (major), 134.24 (minor), 123.47 (minor), 122.70 (major), 114.93 (overlapping), 80.75 (overlapping), 46.34 (minor), 44.34 (overlapping), 44.08 (major), 42.32 (major), 40.61 (minor), 37.40 (minor), 37.09 (major), 36.76 (minor), 36.10 (major), 35.66 (major), 35.57 (minor), 29.45 (major), 29.19 (minor), 28.64 (minor), 28.31 (major), 26.61 (minor), 26.49 (major), 25.29 (major), 25.20 (minor), 22.96 (major), 22.87 (minor), 22.37 (major), 22.23 (minor), 18.65 (overlapping), 18.13 (overlapping), 14.60 (overlapping), 14.28 (major), 12.93 (minor), 11.24 (overlapping). ESI-HR calcd for $\mathrm{C}_{29} \mathrm{H}_{54} \mathrm{NOSi}^{+}\left([\mathrm{M}+\mathrm{H}]^{+}\right)$ 460.3969, found 460.3958. IR $v\left(\mathrm{~cm}^{-1}\right) 2929,1738,1650,1366,883$.<smiles>CCN(CCC1=CCCCC1)C(=O)CC[C@](C)(C#CC(C)C)c1ccccc1</smiles> 
Amide 3j-Ph: Amide 3j (45.9 mg, 0.100 mmol), $\mathrm{PdCl}_{2}\left(\mathrm{PPh}_{3}\right)_{2}$ (3.2 mg, $\left.4.5 \mathrm{~mol} \%\right), \mathrm{CuI}(0.9 \mathrm{mg}, 5$ mol\%) were placed in a $10 \mathrm{~mL}$ Schlenk tube. The tube was evacuated and refilled with nitrogen. THF $(0.50 \mathrm{~mL}), \mathrm{NEt}_{3}(0.10 \mathrm{~mL}), \mathrm{TBAF}(0.20 \mathrm{~mL}, 1 \mathrm{M}$ in THF) and $\mathrm{PhI}(24.5 \mathrm{mg}, 1.2$ equiv. $)$ were successively added into the tube through syringes. The reaction was stirred at $45{ }^{\circ} \mathrm{C}$ for $4 \mathrm{~h}$. The volatiles were removed on a rotary evaporator. Further purification by silica gel chromatography gave $34.1 \mathrm{mg}$ (90\% yield) of $\mathbf{3 j - P h}$ as a colorless oil. ${ }^{1} \mathbf{H} \mathbf{~ N M R}\left(400 \mathrm{MHz}, \mathrm{CDCl}_{3}\right)$ $\delta 7.38-7.33(\mathrm{~m}, 2 \mathrm{H}$, overlapping), $7.31-7.26(\mathrm{~m}, 3 \mathrm{H}$, overlapping), $5.46-5.34(\mathrm{~m}, 1 \mathrm{H}$, overlapping), $3.46-3.25(\mathrm{~m}, 4 \mathrm{H}$, overlapping), $2.69-2.40(\mathrm{~m}, 2 \mathrm{H}$, overlapping), $2.21-2.06(\mathrm{~m}, 2 \mathrm{H}$, overlapping), $2.02-1.94(\mathrm{~m}, 2 \mathrm{H}$, overlapping), $1.93-1.84$ (m, 3H, overlapping), $1.83-1.72(\mathrm{~m}, 1 \mathrm{H}$, overlapping), $1.59-1.36(\mathrm{~m}, 8 \mathrm{H}$, overlapping), $1.27-1.23(\mathrm{~m}, 3 \mathrm{H}$, overlapping), $1.17(\mathrm{t}, J=7.1 \mathrm{~Hz}, 3 \mathrm{H}$, minor $)$, $1.11(\mathrm{t}, J=7.1 \mathrm{~Hz}, 3 \mathrm{H}$, major $), 0.99-0.87\left(\mathrm{~m}, 3 \mathrm{H}\right.$, overlapping). ${ }^{13} \mathbf{C} \mathbf{~ N M R}\left(101 \mathrm{MHz}, \mathrm{CDCl}_{3}\right) \delta$ 172.54 (major), 172.52 (minor), 135.29 (minor), 134.18 (major), 131.54 (overlapping), 131.49 (overlapping), 128.16 (overlapping), 128.12 (overlapping), 127.51 (major), 127.47 (minor), 123.93 (overlapping), 123.68 (major), 122.69 (minor), 95.73 (minor), 95.70 (major), 82.09 (major), 82.04 (minor), 46.71 (minor), 44.31 (major), 44.22 (minor), 44.18 (major), 42.44 (minor), 40.62 (major), 37.65 (major), 37.35 (minor), 37.21 (major), 36.12 (minor), 35.37 (minor), 35.30 (major), 29.44 (minor), 29.40 (major), 28.53 (major), 28.33 (minor), 26.40 (major), 26.26 (minor), 25.29 (minor), 25.14 (major), 22.95 (minor), 22.78 (major), 22.37 (minor), 22.16 (major), 18.18 (overlapping), 14.58 (overlapping), 14.29 (major), 12.99 (minor). ESI-HR calcd for $\mathrm{C}_{26} \mathrm{H}_{38} \mathrm{NO}^{+}\left([\mathrm{M}+\mathrm{H}]^{+}\right)$380.2948, found 380.2938. IR $v\left(\mathrm{~cm}^{-1}\right) 2930,1643,1442,1366,756 .[\alpha]^{20} \mathrm{D}=-43.2\left(c=0.69, \mathrm{CHCl}_{3}\right)$ for a 95.5:5.5 er sample. The enantiomeric purity of this compound was determined by HPLC analysis in comparison with authentic racemic material (Chiralpak IC, 97:3 hexanes: $i-\mathrm{PrOH}, 1.0 \mathrm{~mL} / \mathrm{min}$, $210 \mathrm{~nm}): t_{\text {major }}=17.3 \mathrm{~min}, t_{\text {minor }}=19.5 \mathrm{~min}$.
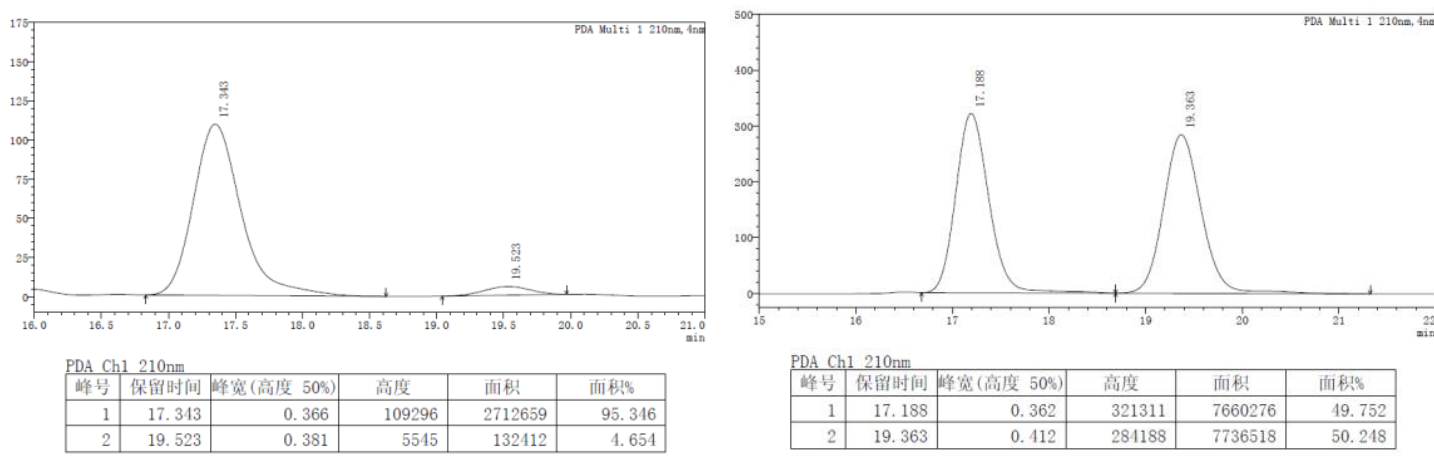
<smiles>CC(C)C[C@@](C)(C#Cc1ccccc1)CCC(=O)N1CCOCC1</smiles>

Amide 31-Ph: Amide 31 (39.3 mg, $0.100 \mathrm{mmol}), \mathrm{PdCl}_{2}\left(\mathrm{PPh}_{3}\right)_{2}$ (3.2 mg, $\left.4.5 \mathrm{~mol} \%\right), \mathrm{CuI}(0.9 \mathrm{mg}, 5$ mol\%) were placed in a $10 \mathrm{~mL}$ Schlenk tube. The tube was evacuated and refilled with nitrogen. THF (0.50 mL), NEt $3(0.10 \mathrm{~mL}), \mathrm{TBAF}(0.20 \mathrm{~mL}, 1 \mathrm{M}$ in THF) and $\mathrm{PhI}(24.5 \mathrm{mg}, 1.2$ equiv.) were successively added into the tube through syringes. The reaction was stirred at $45{ }^{\circ} \mathrm{C}$ for $4 \mathrm{~h}$. The volatiles were removed on a rotary evaporator. Further purification by silica gel chromatography gave $22.6 \mathrm{mg}$ (72\% yield) of $\mathbf{3 1 - P h}$ as a colorless oil. ${ }^{1} \mathbf{H} \mathbf{~ N M R}\left(400 \mathrm{MHz}, \mathrm{CDCl}_{3}\right)$ $\delta 7.42-7.32(\mathrm{~m}, 2 \mathrm{H}), 7.32-7.26(\mathrm{~m}, 3 \mathrm{H}), 3.80-3.57(\mathrm{~m}, 6 \mathrm{H}), 3.56-3.44(\mathrm{~m}, 2 \mathrm{H}), 2.69-2.43(\mathrm{~m}$, 2H), $1.95-1.83(\mathrm{~m}, 1 \mathrm{H}), 1.83-1.70(\mathrm{~m}, 1 \mathrm{H}), 1.55-1.36(\mathrm{~m}, 4 \mathrm{H}), 1.25(\mathrm{~s}, 3 \mathrm{H}), 0.96(\mathrm{t}, J=6.8 \mathrm{~Hz}$, 3H). ${ }^{13} \mathrm{C}$ NMR (101 MHz, $\left.\mathrm{CDCl}_{3}\right) \delta 171.89,131.48$ (overlapping), 128.22 (overlapping), 127.65, 123.76, 95.46, 82.19, 66.92, 66.67, 46.03, 44.19, 41.95, 36.94, 35.32, 29.39, 26.27, 18.17, 14.56 .

ESI-HR calcd for $\mathrm{C}_{20} \mathrm{H}_{28} \mathrm{NO}_{2}{ }^{+}\left([\mathrm{M}+\mathrm{H}]^{+}\right) 314.2115$, found 314.2107. IR $v\left(\mathrm{~cm}^{-1}\right)$ 2969, 1649, 1433, 883. $[\alpha]^{20} \mathrm{D}=18.5\left(c=0.19, \mathrm{CHCl}_{3}\right)$ for a $93.0: 7.0$ er sample. The enantiomeric purity of this compound was determined by HPLC analysis in comparison with authentic racemic material (Chiralpak IC, 95:5 hexanes: $i-\mathrm{PrOH}, 1.0 \mathrm{~mL} / \mathrm{min}, 254 \mathrm{~nm}$ ): $t_{\text {major }}=21.2 \mathrm{~min}, t_{\mathrm{minor}}=22.5 \mathrm{~min}$.
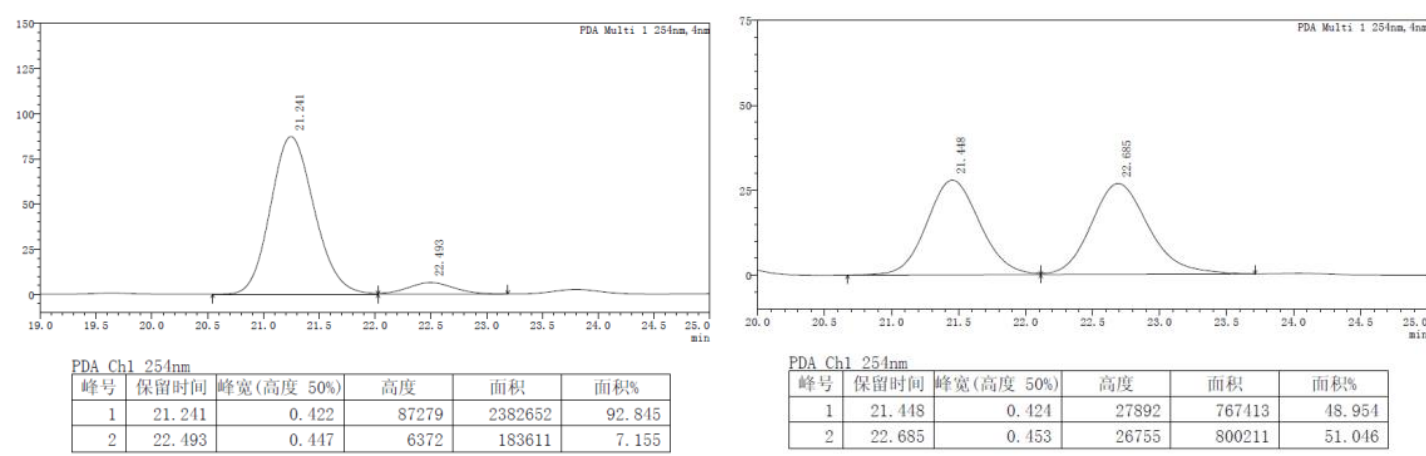<smiles>CCN(CCOC)C(=O)CCC(C)(C#C[SeH])COC</smiles>

Amide 3m: Following the general procedure, amide $\mathbf{1 j}$ (51.1 mg, $0.200 \mathrm{mmol}$ ) was converted to the alkynyl amide. Purification by silica gel chromatography gave $66.3 \mathrm{mg}$ (76\% yield) of $\mathbf{3} \mathbf{j}$ as a 
colorless oil. Two sets of peaks were observed in ${ }^{13} \mathrm{C}$ NMR spectra due to amide rotamers, which are assigned to major and minor rotamers, respectively. ${ }^{1} \mathbf{H}$ NMR $\left(400 \mathrm{MHz}, \mathrm{CDCl}_{3}\right) \delta 3.62-$ $3.38(\mathrm{~m}, 6 \mathrm{H}), 3.33$ (s, 3H), $2.65-2.37$ (m, 2H), $1.90-1.76(\mathrm{~m}, 1 \mathrm{H}), 1.74-1.61(\mathrm{~m}, 1 \mathrm{H}), 1.54-$ $1.40(\mathrm{~m}, 3 \mathrm{H}), 1.37-1.22(\mathrm{~m}, 5 \mathrm{H}), 1.20-1.10(\mathrm{~m}, 6 \mathrm{H}), 1.09-0.95(\mathrm{~m}, 21 \mathrm{H}), 0.88(\mathrm{t}, J=6.8 \mathrm{~Hz}$, 3H). ${ }^{13} \mathrm{C}$ NMR (101 MHz, $\mathrm{CDCl}_{3}$ ) $\delta 173.02$ (major), 172.95 (minor), 115.01 (minor), 114.88 (major), 80.88 (major), 80.73 (minor), 71.16 (major), 71.08 (minor), 59.00 (minor), 58.77 (major), 47.10 (minor), 45.48 (major), 43.71 (major), 42.02 (major), 41.97 (minor), 41.17 (minor), 36.89 (major), 36.79 (minor), 35.67 (major), 35.63 (minor), 32.30 (overlapping), 29.45 (minor), 29.29 (major), 26.50 (overlapping), 24.57 (overlapping), 22.61 (overlapping), 18.65 (overlapping), 14.15 (major), 14.00 (overlapping), 12.81 (minor), 11.25 (overlapping). ESI-HR calcd for $\mathrm{C}_{26} \mathrm{H}_{52} \mathrm{NO}_{2} \mathrm{Si}^{+}\left([\mathrm{M}+\mathrm{H}]^{+}\right)$438.3762, found 438.3749. IR $v\left(\mathrm{~cm}^{-1}\right) 2933,1649,1462,893$.<smiles>C=CC#C[C@@](C)(CCC(=O)N(CC)CCOC)c1ccccc1</smiles>

Amide 3m-Ph: Amide 3j (43.7 mg, 0.100 mmol), $\mathrm{PdCl}_{2}\left(\mathrm{PPh}_{3}\right)_{2}(3.2 \mathrm{mg}, 4.5 \mathrm{~mol} \%)$, $\mathrm{CuI}$ (0.9 mg, $5 \mathrm{~mol} \%$ ) were placed in a $10 \mathrm{~mL}$ Schlenk tube. The tube was evacuated and refilled with nitrogen. THF (0.50 mL), NEt 3 (0.10 mL), TBAF ( $0.20 \mathrm{~mL}, 1 \mathrm{M}$ in THF) and $\mathrm{PhI}(24.5 \mathrm{mg}, 1.2$ equiv.) were successively added into the tube through syringes. The reaction was stirred at $45{ }^{\circ} \mathrm{C}$ for $4 \mathrm{~h}$. The volatiles were removed on a rotary evaporator. Further purification by silica gel chromatography gave $18.2 \mathrm{mg}$ (51\% yield) of $\mathbf{3 j}$-Ph as a colorless oil. Two sets of peaks were observed in ${ }^{1} \mathrm{H}$ and ${ }^{13} \mathrm{C}$ NMR spectra due to amide rotamers, which are assigned to major and minor rotamers, respectively. ${ }^{1} \mathbf{H}$ NMR $\left(400 \mathrm{MHz}, \mathrm{CDCl}_{3}\right) \delta 7.42-7.33$ (m, 2H, overlapping), $7.31-7.23$ (m, 3H, overlapping), $3.56-3.37$ (m, 6H, overlapping), 3.33 (s, 3H, major), 3.28 (s, $3 \mathrm{H}$, minor), $2.69-2.42(\mathrm{~m}, 2 \mathrm{H}$, overlapping), $1.98-1.84(\mathrm{~m}, 1 \mathrm{H}$, overlapping), $1.83-1.72(\mathrm{~m}$, $1 \mathrm{H}$, overlapping), $1.62-1.40(\mathrm{~m}, 4 \mathrm{H}$, overlapping), $1.38-1.27$ (m, 4H, overlapping), $1.27-1.22$ (m, 3H, overlapping), 1.17 (t, $J=7.1 \mathrm{~Hz}, 3 \mathrm{H}$, major), 1.10 (t, $J=7.1 \mathrm{~Hz}, 3 \mathrm{H}$, minor), 0.90 (t, $J=$ $7.0 \mathrm{~Hz}, 3 \mathrm{H}$, overlapping). ${ }^{13} \mathbf{C}$ NMR (101 $\left.\mathrm{MHz}, \mathrm{CDCl}_{3}\right) \delta 173.04$ (overlapping), 131.52 (overlapping), 131.48 (overlapping), 128.15 (overlapping), 128.11 (overlapping), 127.51 (major), 
127.46 (minor), 123.98 (minor), 123.91 (major), 95.83 (minor), 95.71 (major), 82.07 (major), 82.01 (minor), 71.17 (major), 70.95 (minor), 58.98 (minor), 58.76 (major), 47.17 (minor), 45.55 (major), 43.82 (major), 41.86 (overlapping), 41.14 (minor), 37.09 (major), 37.03 (minor), 35.31 (major), 35.28 (minor), 32.30 (overlapping), 29.47 (minor), 29.26 (major), 26.26 (overlapping), 24.52 (overlapping), 22.60 (overlapping), 14.13 (major), 14.06 (overlapping), 12.84 (minor). ESI-HR calcd for $\mathrm{C}_{23} \mathrm{H}_{36} \mathrm{NO}_{2}{ }^{+}\left([\mathrm{M}+\mathrm{H}]^{+}\right)$358.2741, found 358.2731. IR $v\left(\mathrm{~cm}^{-1}\right)$ 2931, 1644, $1458,1117,692 .[\alpha]^{30} \mathrm{D}=-3.5\left(c=0.68, \mathrm{CHCl}_{3}\right)$ for a 97.0:3.0 er sample. The enantiomeric purity of this compound was determined by HPLC analysis in comparison with authentic racemic material (Chiralpak IC, 95:5 hexanes: $i-\mathrm{PrOH}, 1.0 \mathrm{~mL} / \mathrm{min}, 254 \mathrm{~nm}$ ): $t_{\text {major }}=15.6 \mathrm{~min}, t_{\text {minor }}=16.9$ $\min$.
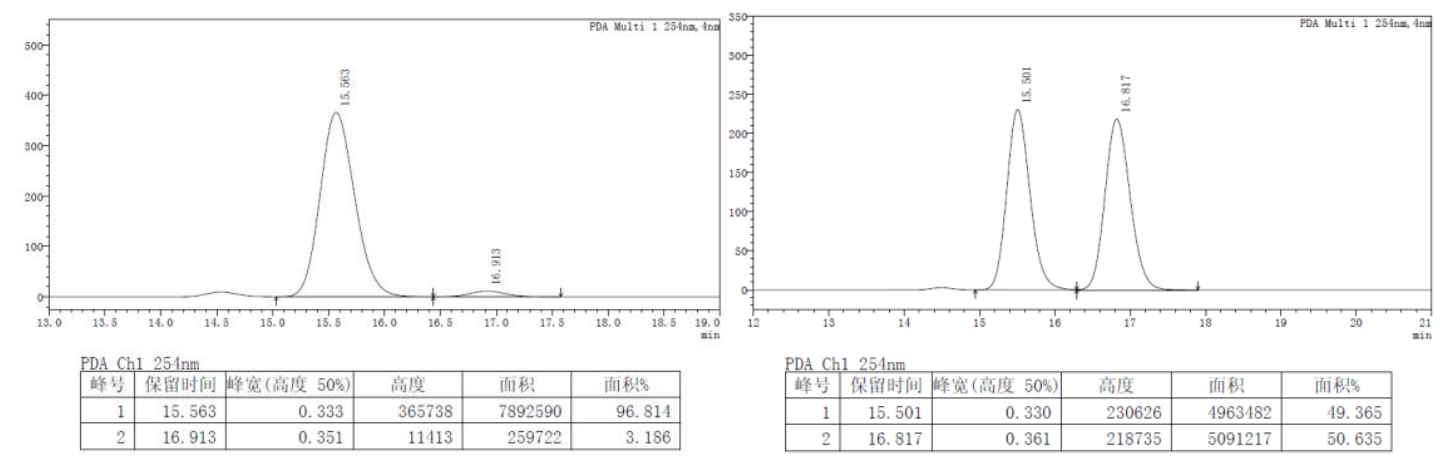<smiles>CCN(CCOC)C(=O)CC[C@](C)(C#C[Se-])C(=O)[O-]</smiles>

Amide 3n: Following the general procedure, amide 1k (59.5 mg, $0.200 \mathrm{mmol})$ was converted to the alkynyl amide. Purification by silica gel chromatography gave $71.5 \mathrm{mg}$ (75\% yield) of $\mathbf{3 k}$ as a colorless oil. Two sets of peaks were observed in ${ }^{13} \mathrm{C}$ NMR spectra due to amide rotamers, which are assigned to major and minor rotamers, respectively. ${ }^{1} \mathbf{H} \mathbf{~ N M R}\left(400 \mathrm{MHz}, \mathrm{CDCl}_{3}\right) \delta 3.55-3.46$ (m, 4H), $3.42(\mathrm{q}, J=7.2 \mathrm{~Hz}, 2 \mathrm{H}), 3.34(\mathrm{~s}, 3 \mathrm{H}), 2.65-2.41(\mathrm{~m}, 2 \mathrm{H}), 1.86-1.75(\mathrm{~m}, 1 \mathrm{H}), 1.73-$ $1.66(\mathrm{~m}, 1 \mathrm{H}), 1.54-1.32(\mathrm{~m}, 4 \mathrm{H}), 1.31-1.22(\mathrm{~m}, 10 \mathrm{H}), 1.19-1.09(\mathrm{~m}, 6 \mathrm{H}), 1.08-0.95(\mathrm{~m}$, 21H), 0.88 (t, $J=6.8 \mathrm{~Hz}, 3 \mathrm{H}) .{ }^{13} \mathbf{C}$ NMR (101 MHz, $\mathrm{CDCl}_{3}$ ) $\delta 173.03$ (major), 172.97 (minor), 115.00 (minor), 114.87 (major), 80.86 (major), 80.72 (minor), 71.15 (major), 71.07 (minor), 58.99 (minor), 58.77 (major), 47.09 (minor), 45.47 (major), 43.70 (major), 42.06 (major), 42.02 
(minor), 41.16 (minor), 36.89 (major), 36.79 (minor), 35.66 (major), 35.62 (minor), 31.89 (overlapping), 30.08 (overlapping), 29.56 (overlapping), 29.44 (minor), 29.28 (major), 29.23 (overlapping), 26.49 (overlapping), 24.89 (overlapping), 22.66 (overlapping), 18.65 (overlapping), 14.14 (major), 14.08 (overlapping), 12.80 (minor), 11.24 (overlapping). ESI-HR calcd for $\mathrm{C}_{29} \mathrm{H}_{58} \mathrm{NO}_{2} \mathrm{Si}^{+}\left([\mathrm{M}+\mathrm{H}]^{+}\right) 480.4231$, found 480.4216. IR $v\left(\mathrm{~cm}^{-1}\right) 2927,1649,1462,883$.

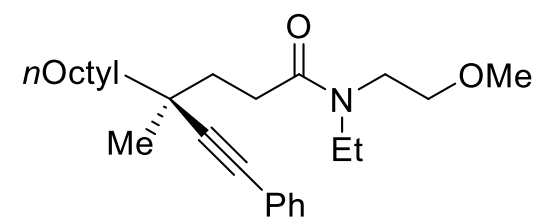

Amide 3n-Ph: Amide 3k (47.9 mg, $0.100 \mathrm{mmol}), \mathrm{PdCl}_{2}\left(\mathrm{PPh}_{3}\right)_{2}(3.2 \mathrm{mg}, 4.5 \mathrm{~mol} \%), \mathrm{CuI}(0.9 \mathrm{mg}$, $5 \mathrm{~mol} \%$ ) were placed in a $10 \mathrm{~mL}$ Schlenk tube. The tube was evacuated and refilled with nitrogen. THF (0.50 mL), NEt 3 (0.10 mL), TBAF ( $0.20 \mathrm{~mL}, 1 \mathrm{M}$ in THF) and PhI (24.5 mg, 1.2 equiv.) were successively added into the tube through syringes. The reaction was stirred at $45{ }^{\circ} \mathrm{C}$ for $4 \mathrm{~h}$. The volatiles were removed on a rotary evaporator. Further purification by silica gel chromatography gave $30.4 \mathrm{mg}$ (76\% yield) of $\mathbf{3 k - P h}$ as a colorless oil. Two sets of peaks were observed in ${ }^{1} \mathrm{H}$ and ${ }^{13} \mathrm{C}$ NMR spectra due to amide rotamers, which are assigned to major and minor rotamers, respectively. ${ }^{1} \mathbf{H}$ NMR $\left(400 \mathrm{MHz}, \mathrm{CDCl}_{3}\right) \delta 7.40-7.33(\mathrm{~m}, 2 \mathrm{H}$, overlapping), $7.31-7.24(\mathrm{~m}, 3 \mathrm{H}$, overlapping), $3.59-3.37$ (m, 6H, overlapping), 3.33 (s, 3H, major), 3.28 (s, $3 \mathrm{H}$, minor $), 2.80-2.35(\mathrm{~m}, 2 \mathrm{H}$, overlapping), $1.98-1.85(\mathrm{~m}, 1 \mathrm{H}$, overlapping), $1.84-1.72(\mathrm{~m}$, $1 \mathrm{H}$, overlapping), $1.62-1.40(\mathrm{~m}, 4 \mathrm{H}$, overlapping), $1.39-1.21(\mathrm{~m}, 13 \mathrm{H}$, overlapping), $1.18(\mathrm{t}, J$ $=7.1 \mathrm{~Hz}, 3 \mathrm{H}$, major $), 1.10(\mathrm{t}, J=7.1 \mathrm{~Hz}, 3 \mathrm{H}$, minor $), 0.88(\mathrm{t}, J=6.9 \mathrm{~Hz}, 3 \mathrm{H}$, overlapping $) .{ }^{13} \mathbf{C}$ NMR (101 MHz, $\left.\mathrm{CDCl}_{3}\right) \delta 173.07$ (overlapping), 131.52 (overlapping), 131.48 (overlapping), 128.14 (overlapping), 128.10 (overlapping), 127.50 (major), 127.45 (minor), 123.98 (minor), 123.91 (major), 95.84 (minor), 95.72 (major), 82.06 (major), 82.00 (minor), 71.18 (major), 70.97 (minor), 58.97 (minor), 58.76 (major), 47.17 (minor), 45.54 (major), 43.82 (major), 41.90 (overlapping), 41.14 (minor), 37.08 (major), 37.03 (minor), 35.30 (major), 35.27 (minor), 31.87 (overlapping), 30.11 (overlapping), 29.55 (overlapping), 29.29 (overlapping), 26.26 (overlapping), 24.85 (overlapping), 22.65 (overlapping), 14.14 (major), 14.08 (overlapping), 12.86 (minor). ESI-HR calcd for $\mathrm{C}_{26} \mathrm{H}_{42} \mathrm{NO}_{2}{ }^{+}\left([\mathrm{M}+\mathrm{H}]^{+}\right)$400.3210, found 400.3198. IR $v\left(\mathrm{~cm}^{-1}\right)$ 2927, 1644, 
$1459,1118,756 .[\alpha]^{30} \mathrm{D}=-1.9\left(c=1.06, \mathrm{CHCl}_{3}\right)$ for a 96.0:4.0 er sample. The enantiomeric purity of this compound was determined by HPLC analysis in comparison with authentic racemic material (Chiralpak IC, 95:5 hexanes: $i-\mathrm{PrOH}, 1.0 \mathrm{~mL} / \mathrm{min}, 240 \mathrm{~nm}$ ): $t_{\text {major }}=13.5 \mathrm{~min}, t_{\text {minor }}=14.7$ $\min$.
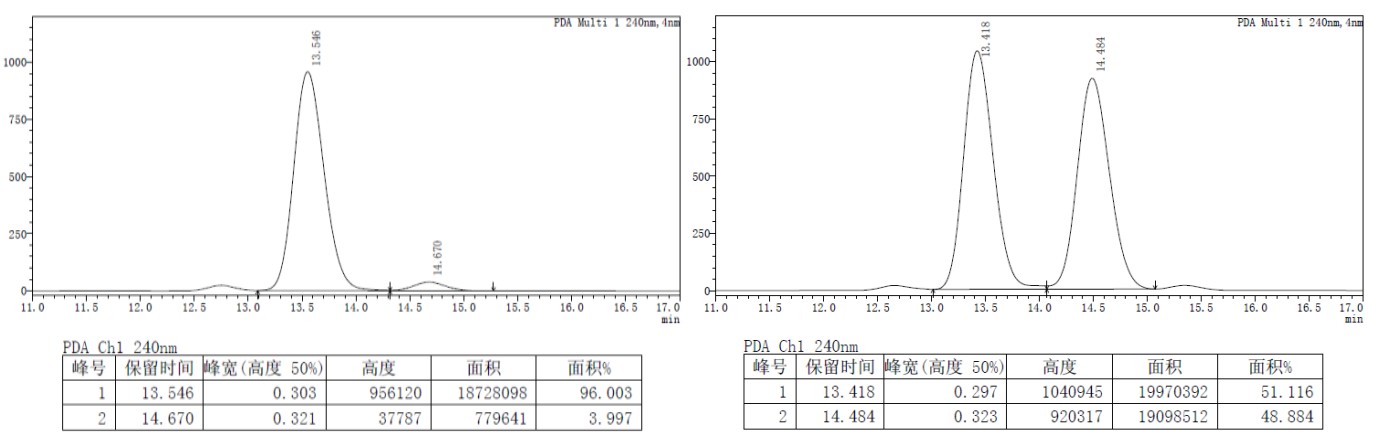<smiles>CCN(CCOC)C(=O)CC[C@](C)(C#C[SnH3])CCC(C)C</smiles>

Amide 3o: Following the general procedure, amide 11 (51.1 mg, $0.200 \mathrm{mmol})$ was converted to the alkynyl amide. Purification by silica gel chromatography gave $63.3 \mathrm{mg}$ (72\% yield) of $\mathbf{3 1}$ as a colorless oil. Two sets of peaks were observed in ${ }^{13} \mathrm{C}$ NMR spectra due to amide rotamers, which are assigned to major and minor rotamers, respectively. ${ }^{1} \mathbf{H} \mathbf{~ N M R}\left(400 \mathrm{MHz}, \mathrm{CDCl}_{3}\right) \delta 3.55-$ $3.46(\mathrm{~m}, 4 \mathrm{H}), 3.42(\mathrm{q}, J=7.2 \mathrm{~Hz}, 2 \mathrm{H}), 3.33(\mathrm{~s}, 3 \mathrm{H}), 2.65-2.39(\mathrm{~m}, 2 \mathrm{H}), 1.87-1.76(\mathrm{~m}, 1 \mathrm{H})$, $1.73-1.66(\mathrm{~m}, 1 \mathrm{H}), 1.54-1.28(\mathrm{~m}, 5 \mathrm{H}), 1.19-1.08(\mathrm{~m}, 6 \mathrm{H}), 1.08-1.00(\mathrm{~m}, 21 \mathrm{H}), 0.87(\mathrm{~d}, J=$ $6.5 \mathrm{~Hz}, 6 \mathrm{H}) .{ }^{13} \mathrm{C}$ NMR $\left(101 \mathrm{MHz}, \mathrm{CDCl}_{3}\right) \delta 173.02$ (major), 172.96 (minor), 114.95 (minor), 114.82 (major), 80.90 (major), 80.76 (minor), 71.15 (major), 71.06 (minor), 58.99 (minor), 58.77 (major), 47.10 (minor), 45.48 (major), 43.71 (major), 41.17 (minor), 39.85 (major), 39.82 (minor), 36.88 (major), 36.77 (minor), 35.66 (major), 35.61 (minor), 33.99 (overlapping), 29.44 (minor), 29.27 (major), 28.50 (overlapping), 26.47 (overlapping), 22.66 (overlapping), 22.61 (overlapping), 18.65 (overlapping), 14.15, 12.81, 11.25 (overlapping). ESI-HR calcd for $\mathrm{C}_{26} \mathrm{H}_{52} \mathrm{NO}_{2} \mathrm{Si}^{+}\left([\mathrm{M}+\mathrm{H}]^{+}\right)$ 438.3762, found 438.3744. IR $v\left(\mathrm{~cm}^{-1}\right) 2932,1647,1462,883$. 
<smiles>CCN(CCOC)C(=O)CC[C@](C)(C#Cc1ccccc1)CCC(C)C</smiles>

Amide 3o-Ph: Amide 31 (43.7 mg, $0.100 \mathrm{mmol}), \mathrm{PdCl}_{2}\left(\mathrm{PPh}_{3}\right)_{2}(3.2 \mathrm{mg}, 4.5 \mathrm{~mol} \%)$, $\mathrm{CuI}(0.9 \mathrm{mg}$, $5 \mathrm{~mol} \%$ ) were placed in a $10 \mathrm{~mL}$ Schlenk tube. The tube was evacuated and refilled with nitrogen. THF (0.50 mL), NEt $3(0.10 \mathrm{~mL}), \mathrm{TBAF}(0.20 \mathrm{~mL}, 1 \mathrm{M}$ in THF) and $\mathrm{PhI}(24.5 \mathrm{mg}, 1.2$ equiv.) were successively added into the tube through syringes. The reaction was stirred at $45{ }^{\circ} \mathrm{C}$ for $4 \mathrm{~h}$. The volatiles were removed on a rotary evaporator. Further purification by silica gel chromatography gave $25.4 \mathrm{mg}$ ( $71 \%$ yield) of 31-Ph as a colorless oil. Two sets of peaks were observed in ${ }^{1} \mathrm{H}$ and ${ }^{13} \mathrm{C}$ NMR spectra due to amide rotamers, which are assigned to major and minor rotamers, respectively. ${ }^{1} \mathbf{H}$ NMR $\left(400 \mathrm{MHz}, \mathrm{CDCl}_{3}\right) \delta 7.47-7.32(\mathrm{~m}, 2 \mathrm{H}$, overlapping), $7.31-7.22(\mathrm{~m}, 2 \mathrm{H}$, overlapping), $3.58-3.37(\mathrm{~m}, 6 \mathrm{H}$, overlapping), $3.33(\mathrm{~s}, 3 \mathrm{H}$, major $), 3.28(\mathrm{~s}$, $3 \mathrm{H}$, minor), $2.68-2.46(\mathrm{~m}, 2 \mathrm{H}$, overlapping), $1.98-1.85(\mathrm{~m}, 1 \mathrm{H}$, overlapping), $1.83-1.71(\mathrm{~m}$, $1 \mathrm{H}$, overlapping), $1.61-1.31$ (m, 5H, overlapping), $1.30-1.21(\mathrm{~m}, 3 \mathrm{H}$, overlapping), $1.18(\mathrm{t}, J=$ $7.1 \mathrm{~Hz}, 3 \mathrm{H}$, major), $1.11(\mathrm{t}, J=7.1 \mathrm{~Hz}, 3 \mathrm{H}$, minor), $0.92(\mathrm{~d}, J=1.2 \mathrm{~Hz}, 3 \mathrm{H}$, overlapping), 0.90 (d, $J=1.2 \mathrm{~Hz}, 3 \mathrm{H}$, overlapping). ${ }^{13} \mathbf{C}$ NMR $\left(101 \mathrm{MHz}, \mathrm{CDCl}_{3}\right) \delta 173.05$ (overlapping), 131.53 (overlapping), 131.48 (overlapping), 128.16 (overlapping), 128.11 (overlapping), 127.51 (major), 127.47 (minor), 123.98 (minor), 123.92 (major), 95.84 (minor), 95.72 (major), 82.07 (major), 82.01 (minor), 71.17 (major), 70.95 (minor), 58.98 (minor), 58.77 (major), 47.18 (minor), 45.56 (major), 43.83 (major), 41.15 (minor), 39.67 (overlapping), 37.02 (major), 36.97 (minor), 35.24 (major), 35.21 (minor), 33.85 (overlapping), 29.48 (minor), 29.25 (major), 28.48 (overlapping), 26.25 (overlapping), 22.70 (overlapping), 22.63 (overlapping), 14.14 (major), 12.85 (minor). ESI-HR calcd for $\mathrm{C}_{23} \mathrm{H}_{36} \mathrm{NO}_{2}{ }^{+}\left([\mathrm{M}+\mathrm{H}]^{+}\right)$358.2741, found 358.2726. IR $v\left(\mathrm{~cm}^{-1}\right)$ 2955, 1644, $1464,757 .[\alpha]^{30} \mathrm{D}=-0.8\left(c=0.59, \mathrm{CHCl}_{3}\right)$ for a $96.0: 4.0$ er sample. The enantiomeric purity of this compound was determined by HPLC analysis in comparison with authentic racemic material (Chiralpak IC, 95:5 hexanes: $i-\mathrm{PrOH}, 1.0 \mathrm{~mL} / \mathrm{min}, 218 \mathrm{~nm}$ ): $t_{\mathrm{major}}=14.2 \mathrm{~min}, t_{\mathrm{minor}}=15.5 \mathrm{~min}$. 

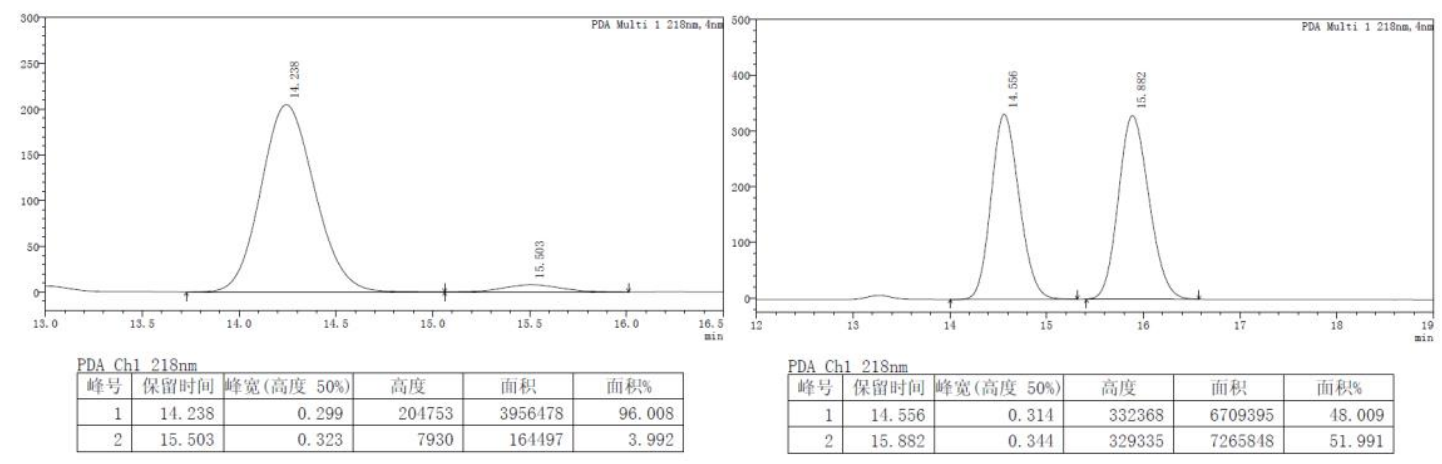<smiles>CC#CC[C@@](C)(CCC(=O)N(CC)CCOC)CC(C)C</smiles>

Amide 3p: Following the general procedure, amide 1m (48.3 mg, $0.200 \mathrm{mmol})$ was converted to the alkynyl amide. Purification by silica gel chromatography gave $41.4 \mathrm{mg}$ (49\% yield) of $\mathbf{3 m}$ as a colorless oil. Two sets of peaks were observed in ${ }^{13} \mathrm{C}$ NMR spectra due to amide rotamers, which are assigned to major and minor rotamers, respectively. ${ }^{1} \mathbf{H} \mathbf{~ N M R}\left(400 \mathrm{MHz}, \mathrm{CDCl}_{3}\right) \delta 3.59-3.48$ (m, 4H), $3.44(\mathrm{q}, J=7.2 \mathrm{~Hz}, 2 \mathrm{H}), 3.35(\mathrm{~s}, 3 \mathrm{H}), 2.67-2.43(\mathrm{~m}, 2 \mathrm{H}), 1.98-1.78(\mathrm{~m}, 2 \mathrm{H}), 1.76-$ $1.69(\mathrm{~m}, 1 \mathrm{H}), 1.48-1.38(\mathrm{~m}, 1 \mathrm{H}), 1.36-1.26(\mathrm{~m}, 1 \mathrm{H}), 1.23-1.13(\mathrm{~m}, 6 \mathrm{H}), 1.13-1.04(\mathrm{~m}, 21 \mathrm{H})$, $1.02(\mathrm{~d}, J=6.7 \mathrm{~Hz}, 3 \mathrm{H}), 1.00(\mathrm{~d}, J=6.7 \mathrm{~Hz}, 3 \mathrm{H}) .{ }^{13} \mathbf{C ~ N M R}\left(101 \mathrm{MHz}, \mathrm{CDCl}_{3}\right) \delta 172.99$ (major), 172.94 (minor), 115.14 (minor), 115.01 (major), 81.25 (major), 81.11 (minor), 71.14 (major), 71.05 (minor), 58.99 (minor), 58.77 (major), 50.46 (major), 50.40 (minor), 47.08 (minor), 45.47 (major), 43.68 (major), 41.15 (minor), 37.86 (major), 37.77 (minor), 35.32 (major), 35.27 (minor), 29.35 (minor), 29.18 (major), 27.23 (overlapping), 25.28 (overlapping), 24.84 (overlapping), 24.66 (overlapping), 18.64 (overlapping), 14.15 (major), 12.80 (minor), 11.27 (overlapping). ESI-HR calcd for $\mathrm{C}_{25} \mathrm{H}_{50} \mathrm{NO}_{2} \mathrm{Si}^{+}\left([\mathrm{M}+\mathrm{H}]^{+}\right) 424.3605$, found 424.3597. IR $v\left(\mathrm{~cm}^{-1}\right)$ 2941, 1648, $1462,883$.<smiles>CCN(CCOC)C(=O)CC[C@](C)(CC#Cc1ccccc1)CC(C)C</smiles> 
Amide 3p-Ph: Amide 3m (42.3 mg, $0.100 \mathrm{mmol}), \mathrm{PdCl}_{2}\left(\mathrm{PPh}_{3}\right)_{2}$ (3.2 mg, $\left.4.5 \mathrm{~mol} \%\right)$, $\mathrm{CuI}(0.9 \mathrm{mg}$, $5 \mathrm{~mol} \%$ ) were placed in a $10 \mathrm{~mL}$ Schlenk tube. The tube was evacuated and refilled with nitrogen. THF (0.50 mL), NEt $3(0.10 \mathrm{~mL}), \mathrm{TBAF}(0.20 \mathrm{~mL}, 1 \mathrm{M}$ in THF) and $\mathrm{PhI}(24.5 \mathrm{mg}, 1.2$ equiv.) were successively added into the tube through syringes. The reaction was stirred at $45{ }^{\circ} \mathrm{C}$ for $4 \mathrm{~h}$. The volatiles were removed on a rotary evaporator. Further purification by silica gel chromatography gave $23.0 \mathrm{mg}$ (67\% yield) of $\mathbf{3 m}-\mathbf{P h}$ as a colorless oil. Two sets of peaks were observed in ${ }^{1} \mathrm{H}$ and ${ }^{13} \mathrm{C}$ NMR spectra due to amide rotamers, which are assigned to major and minor rotamers, respectively. ${ }^{1} \mathbf{H}$ NMR $\left(400 \mathrm{MHz}, \mathrm{CDCl}_{3}\right) \delta 7.40-7.31(\mathrm{~m}, 2 \mathrm{H}$, overlapping), $7.31-7.22(\mathrm{~m}, 3 \mathrm{H}$, overlapping), $3.63-3.36(\mathrm{~m}, 6 \mathrm{H}$, overlapping), $3.33(\mathrm{~s}, 3 \mathrm{H}$, major $), 3.28(\mathrm{~s}$, $3 \mathrm{H}$, minor), $2.71-2.45(\mathrm{~m}, 2 \mathrm{H}$, overlapping), $2.00-1.85(\mathrm{~m}, 2 \mathrm{H}$, overlapping), $1.84-1.70(\mathrm{~m}$, $1 \mathrm{H}$, overlapping), $1.52(\mathrm{~d}, J=6.3 \mathrm{~Hz}, 1 \mathrm{H}$, minor $), 1.49(\mathrm{~d}, J=6.3 \mathrm{~Hz}, 1 \mathrm{H}$, major $), 1.43-1.38(\mathrm{~m}$, $1 \mathrm{H}$, major $), 1.38-1.34(\mathrm{~m}, 1 \mathrm{H}$, minor $), 1.32-1.23(\mathrm{~m}, 3 \mathrm{H}$, overlapping $), 1.17(\mathrm{t}, J=7.1 \mathrm{~Hz}, 3 \mathrm{H}$, major), 1.10 (t, $J=7.1 \mathrm{~Hz}, 3 \mathrm{H}$, minor), $1.05(\mathrm{~d}, J=6.7 \mathrm{~Hz}, 3 \mathrm{H}$, overlapping), 1.01 (d, $J=6.7 \mathrm{~Hz}$, 3H, overlapping). ${ }^{13} \mathrm{C}$ NMR (101 MHz, $\left.\mathrm{CDCl}_{3}\right) \delta 173.04$ (overlapping), 131.39 (overlapping), 131.34 (overlapping), 128.18 (overlapping), 128.14 (overlapping), 127.50 (major), 127.45 (minor), 124.08 (minor), 124.01 (major), 96.18 (minor), 96.05 (major), 82.26 (major), 82.20 (minor), 71.18 (major), 70.98 (minor), 58.99 (minor), 58.78 (major), 50.52 (overlapping), 47.18 (minor), 45.57 (major), 43.84 (major), 41.15 (minor), 38.13 (major), 38.06 (minor), 34.91 (major), 34.87 (minor), 29.40 (minor), 29.18 (major), 26.82 (overlapping), 25.26 (overlapping), 24.83 (overlapping), 24.45 (overlapping), 14.16 (major), 12.85 (minor). ESI-HR calcd for $\mathrm{C}_{22} \mathrm{H}_{34} \mathrm{NO}_{2}{ }^{+}$ $\left([\mathrm{M}+\mathrm{H}]^{+}\right) 344.2584$, found 344.2575. IR $v\left(\mathrm{~cm}^{-1}\right) 2956,1635,1461,908 .[\alpha]^{30} \mathrm{D}=-3.9(c=0.28$ $\mathrm{CHCl}_{3}$ ) for a 98.5:1.5 er sample. The enantiomeric purity of this compound was determined by HPLC analysis in comparison with authentic racemic material (Chiralpak ID, 98:2 hexanes: $i-\mathrm{PrOH}, 1.0 \mathrm{~mL} / \mathrm{min}, 242 \mathrm{~nm}$ ): $t_{\text {major }}=23.4 \mathrm{~min}, t_{\mathrm{minor}}=24.9 \mathrm{~min}$.
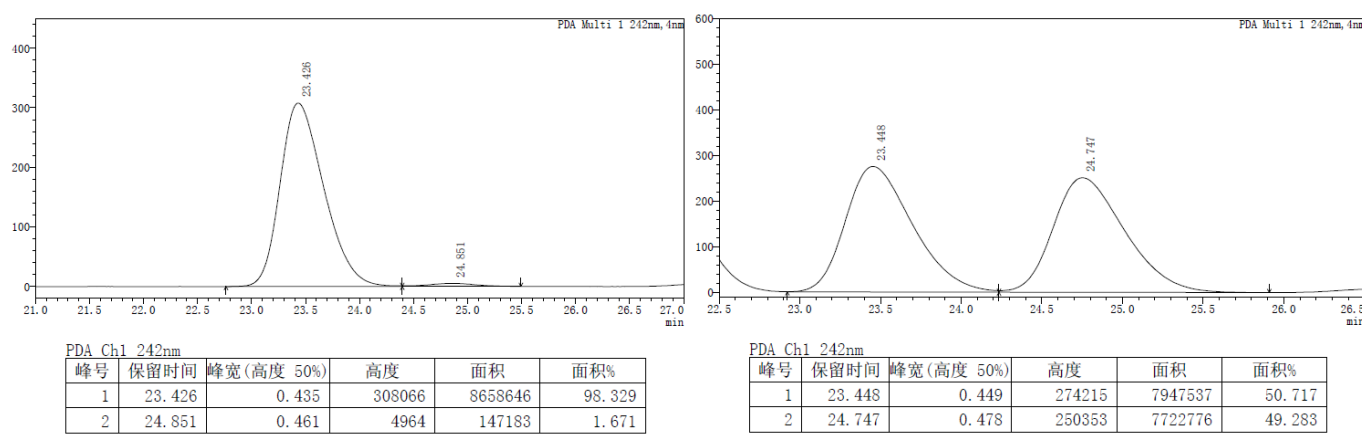
<smiles>CC[AsH2-]C#C[C@@](C)(CCCCl)CCC(=O)N(CC)CCOC</smiles>

Amide 3r: Following the general procedure, amide $10(52.4 \mathrm{mg}, 0.200 \mathrm{mmol})$ was converted to the alkynyl amide. Purification by silica gel chromatography gave $59.6 \mathrm{mg}$ (67\% yield) of $\mathbf{3 0}$ as a colorless oil. Two sets of peaks were observed in ${ }^{13} \mathrm{C}$ NMR spectra due to amide rotamers, which are assigned to major and minor rotamers, respectively. ${ }^{1} \mathbf{H} \mathbf{N M R}\left(400 \mathrm{MHz}, \mathrm{CDCl}_{3}\right) \delta 3.58(\mathrm{t}, J=$ $6.5 \mathrm{~Hz}, 2 \mathrm{H}), 3.55-3.47(\mathrm{~m}, 4 \mathrm{H}), 3.44(\mathrm{q}, J=7.1 \mathrm{~Hz}, 2 \mathrm{H}), 3.38-3.29(\mathrm{~m}, 3 \mathrm{H}), 2.72-2.41(\mathrm{~m}$, 2H), $2.14-1.90(\mathrm{~m}, 2 \mathrm{H}), 1.89-1.80(\mathrm{~m}, 1 \mathrm{H}), 1.79-1.71(\mathrm{~m}, 1 \mathrm{H}), 1.68-1.59(\mathrm{~m}, 1 \mathrm{H}), 1.58-$ $1.47(\mathrm{~m}, 1 \mathrm{H}), 1.23-1.11(\mathrm{~m}, 6 \mathrm{H}), 1.11-0.95(\mathrm{~m}, 21 \mathrm{H}) .{ }^{13} \mathbf{C} \mathbf{N M R}\left(101 \mathrm{MHz}, \mathrm{CDCl}_{3}\right) \delta 172.66$ (overlapping), 113.95 (minor), 113.81 (major), 81.80 (major), 81.63 (minor), 71.12 (major), 70.98 (minor), 59.00 (minor), 58.76 (major), 47.10 (minor), 45.50 (major), 45.47 (minor), 45.44 (major), 43.68 (major), 41.14 (minor), 39.19 (major), 39.12 (minor), 36.81 (major), 36.71 (minor), 35.32 (major), 35.27 (minor), 29.27 (minor), 29.11 (major), 28.46 (minor), 28.45 (major), 26.53 (minor), 26.51 (major), 18.63 (overlapping), 14.12 (major), 12.79 (minor), 11.19 (overlapping). ESI-HR calcd for $\mathrm{C}_{24} \mathrm{H}_{47} \mathrm{ClNO}_{2} \mathrm{Si}^{+}\left([\mathrm{M}+\mathrm{H}]^{+}\right)$444.3059, found 444.3045. IR $v\left(\mathrm{~cm}^{-1}\right)$ 2940, 1644, 1461, 658.<smiles>CCN(CCOC)C(=O)CC[C@](C)(C#Cc1ccccc1)CCCCl</smiles>

Amide 3r-Ph: Amide 30 (44.4 mg, $0.100 \mathrm{mmol}), \mathrm{PdCl}_{2}\left(\mathrm{PPh}_{3}\right)_{2}$ (3.2 mg, $4.5 \mathrm{~mol} \%$ ), $\mathrm{CuI}$ (0.9 mg, $5 \mathrm{~mol} \%$ ) were placed in a $10 \mathrm{~mL}$ Schlenk tube. The tube was evacuated and refilled with nitrogen. THF (0.50 mL), NEt $3(0.10 \mathrm{~mL})$, TBAF ( $0.20 \mathrm{~mL}, 1 \mathrm{M}$ in THF) and $\mathrm{PhI}(24.5 \mathrm{mg}, 1.2$ equiv.) were successively added into the tube through syringes. The reaction was stirred at $45{ }^{\circ} \mathrm{C}$ for $4 \mathrm{~h}$. The volatiles were removed on a rotary evaporator. Further purification by silica gel chromatography gave $26.6 \mathrm{mg}$ (73\% yield) of 3o-Ph as a colorless oil. Two sets of peaks were observed in ${ }^{1} \mathrm{H}$ and ${ }^{13} \mathrm{C}$ NMR spectra due to amide rotamers, which are assigned to major and 
minor rotamers, respectively. ${ }^{1} \mathbf{H}$ NMR $\left(400 \mathrm{MHz}, \mathrm{CDCl}_{3}\right) \delta 7.43-7.33(\mathrm{~m}, 2 \mathrm{H}$, overlapping), $7.32-7.23(\mathrm{~m}, 3 \mathrm{H}$, overlapping), 3.59 (t, $J=6.6 \mathrm{~Hz}, 2 \mathrm{H}$, overlapping), $3.54-3.36(\mathrm{~m}, 6 \mathrm{H}$, overlapping), 3.33 (s, 3H, major), 3.28 (s, 3H, minor), $2.71-2.44(\mathrm{~m}, 2 \mathrm{H}$, overlapping), 2.12 $1.88(\mathrm{~m}, 3 \mathrm{H}$, overlapping), $1.87-1.77(\mathrm{~m}, 1 \mathrm{H}$, overlapping $), 1.75-1.68(\mathrm{~m}, 1 \mathrm{H}$, overlapping $)$, $1.65-1.53(\mathrm{~m}, 1 \mathrm{H}$, overlapping), $1.34-1.23(\mathrm{~m}, 3 \mathrm{H}$, overlapping), $1.18(\mathrm{t}, J=7.1 \mathrm{~Hz}, 3 \mathrm{H}$, major), 1.11 (t, $J=7.1 \mathrm{~Hz}, 3 \mathrm{H}$, minor). ${ }^{13} \mathbf{C}$ NMR (101 MHz, $\left.\mathrm{CDCl}_{3}\right) \delta 172.76$ (minor), 172.70 (major), 131.53 (overlapping), 131.49 (overlapping), 128.20 (overlapping), 128.16 (overlapping), 127.74 (major), 127.68 (minor), 123.61 (minor), 123.53 (major), 94.76 (minor), 94.62 (major), 82.66 (major), 82.58 (minor), 71.14 (major), 70.89 (minor), 59.00 (minor), 58.77 (major), 47.18 (minor), 45.57 (major), 45.46 (minor), 45.44 (major), 43.81 (major), 41.13 (minor), 39.13 (major), 39.10 (minor), 37.02 (major), 36.96 (minor), 34.99 (major), 34.96 (minor), 29.29 (minor), 29.08 (major), 28.44 (overlapping), 26.28 (overlapping), 14.12 (major), 12.84 (minor). ESI-HR calcd for $\mathrm{C}_{21} \mathrm{H}_{31} \mathrm{ClNO}_{2}{ }^{+}\left([\mathrm{M}+\mathrm{H}]^{+}\right) 364.2038$, found 364.2030.

IR $v\left(\mathrm{~cm}^{-1}\right) 2970,1639,1444,758 .[\alpha]^{30} \mathrm{D}=-4.3\left(c=0.65, \mathrm{CHCl}_{3}\right)$ for a 95.5:4.5 er sample. The enantiomeric purity of this compound was determined by HPLC analysis in comparison with authentic racemic material (Chiralpak ID, 98:2 hexanes:i-PrOH, $1.0 \mathrm{~mL} / \mathrm{min}, 240 \mathrm{~nm}$ ): $t_{\text {major }}=$ $43.4 \mathrm{~min}, t_{\mathrm{minor}}=46.8 \mathrm{~min}$.
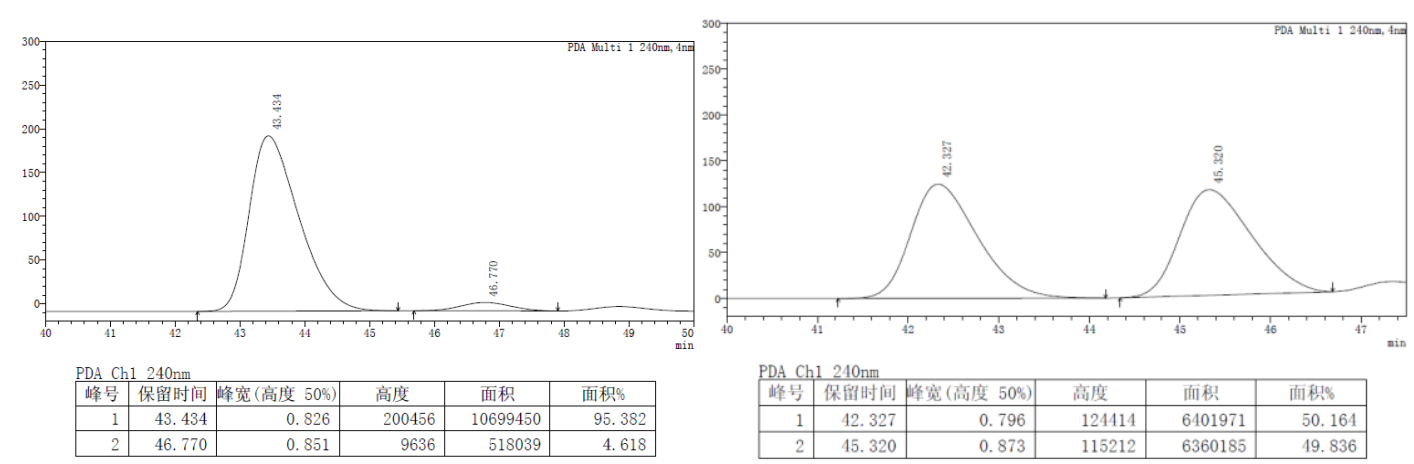<smiles>CCN(CC[AsH2-])C(=O)CC[C@@](C)(CCCOC)CCCOC(C)=O</smiles>

Amide 3s: Following the general procedure, amide 1p $(57.1 \mathrm{mg}, 0.200 \mathrm{mmol})$ was converted to the alkynyl amide. Purification by silica gel chromatography gave $68.1 \mathrm{mg}$ (73\% yield) of $\mathbf{3 p}$ as a 
colorless oil. Two sets of peaks were observed in ${ }^{13} \mathrm{C}$ NMR spectra due to amide rotamers, which are assigned to major and minor rotamers, respectively. ${ }^{1} \mathbf{H}$ NMR $\left(400 \mathrm{MHz}, \mathrm{CDCl}_{3}\right) \delta 4.13-$ $4.03(\mathrm{~m}, 2 \mathrm{H}), 3.58-3.45(\mathrm{~m}, 4 \mathrm{H}), 3.42(\mathrm{q}, J=7.2 \mathrm{~Hz}, 2 \mathrm{H}), 3.33(\mathrm{~s}, 3 \mathrm{H}), 2.65-2.43(\mathrm{~m}, 2 \mathrm{H})$, $2.04(\mathrm{~s}, 3 \mathrm{H}), 1.89-1.77(\mathrm{~m}, 3 \mathrm{H}), 1.76-1.70(\mathrm{~m}, 1 \mathrm{H}), 1.60-1.48(\mathrm{~m}, 1 \mathrm{H}), 1.48-1.39(\mathrm{~m}, 1 \mathrm{H})$, $1.22-1.09(\mathrm{~m}, 6 \mathrm{H}), 1.08-0.94(\mathrm{~m}, 21 \mathrm{H}) .{ }^{13} \mathbf{C}$ NMR (101 MHz, CDCl 3 ) $\delta 172.73$ (overlapping), 171.14 (overlapping), 114.05 (minor), 113.91 (major), 81.64 (major), 81.48 (minor), 71.12 (major), 70.98 (minor), 64.78 (minor), 64.75 (major), 59.00 (minor), 58.77 (major), 47.10 (minor), 45.49 (major), 43.69 (major), 41.15 (minor), 38.32 (major), 38.27 (minor), 36.82 (major), 36.72 (minor), 35.40 (major), 35.36 (minor), 29.33 (minor), 29.17 (major), 26.39 (overlapping), 24.47 (overlapping), 20.93 (overlapping), 18.63 (overlapping), 14.13 (major), 12.79 (minor), 11.19 (overlapping). ESI-HR calcd for $\mathrm{C}_{26} \mathrm{H}_{50} \mathrm{NO}_{4} \mathrm{Si}^{+}\left([\mathrm{M}+\mathrm{H}]^{+}\right) 468.3504$, found 468.3494. IR $v\left(\mathrm{~cm}^{-1}\right)$ $2941,1741,1646,1462,883$.<smiles>CCN(CCOC)C(=O)CCC(C)(C#CCOC(C)=O)CCCOC</smiles>

Amide 3s-Ph: Amide 3p (46.7 mg, $0.100 \mathrm{mmol}), \mathrm{PdCl}_{2}\left(\mathrm{PPh}_{3}\right)_{2}$ (3.2 mg, $4.5 \mathrm{~mol} \%$ ), $\mathrm{CuI}$ (0.9 mg, 5 mol\%) were placed in a $10 \mathrm{~mL}$ Schlenk tube. The tube was evacuated and refilled with nitrogen. THF (0.50 mL), NEt 3 (0.10 mL), TBAF ( $0.20 \mathrm{~mL}, 1 \mathrm{M}$ in THF) and $\mathrm{PhI}(24.5 \mathrm{mg}, 1.2$ equiv.) were successively added into the tube through syringes. The reaction was stirred at $45^{\circ} \mathrm{C}$ for $4 \mathrm{~h}$. The volatiles were removed on a rotary evaporator. Further purification by silica gel chromatography gave $32.2 \mathrm{mg}$ ( $83 \%$ yield) of $\mathbf{3 p}$-Ph as a colorless oil. Two sets of peaks were observed in ${ }^{1} \mathrm{H}$ and ${ }^{13} \mathrm{C}$ NMR spectra due to amide rotamers, which are assigned to major and minor rotamers, respectively. ${ }^{1} \mathbf{H}$ NMR $\left(400 \mathrm{MHz}, \mathrm{CDCl}_{3}\right) \delta 7.41-7.34(\mathrm{~m}, 2 \mathrm{H}$, overlapping), $7.32-7.24(\mathrm{~m}, 3 \mathrm{H}$, overlapping), $4.11(\mathrm{t}, J=6.6 \mathrm{~Hz}, 2 \mathrm{H}$, overlapping), $3.55-3.37(\mathrm{~m}, 6 \mathrm{H}$, overlapping), 3.34 (s, 3H, major), 3.28 (s, 3H, minor), $2.69-2.47$ (m, 2H, overlapping), 2.05 (s, 3H, overlapping), $1.98-1.74$ (m, 4H, overlapping), $1.67-1.58$ (m, 1H, overlapping), $1.56-1.46$ (m, 1H, overlapping), $1.32-1.22(\mathrm{~m}, 3 \mathrm{H}$, overlapping), $1.18(\mathrm{t}, J=7.1 \mathrm{~Hz}, 3 \mathrm{H}$, major $), 1.11(\mathrm{t}, J$ $=7.1 \mathrm{~Hz}, 3 \mathrm{H}$, minor). ${ }^{13} \mathbf{C}$ NMR $\left(101 \mathrm{MHz}, \mathrm{CDCl}_{3}\right) \delta 172.79$ (minor), 172.73 (major), 171.12 
(overlapping), 131.53 (overlapping), 131.49 (overlapping), 128.19 (overlapping), 128.15 (overlapping), 127.70 (major), 127.65 (minor), 123.66 (minor), 123.59 (major), 94.84 (minor), 94.70 (major), 82.60 (major), 82.52 (minor), 71.15 (major), 70.90 (minor), 64.77 (minor), 64.74 (major), 58.99 (minor), 58.76 (major), 47.18 (minor), 45.57 (major), 43.81 (major), 41.12 (minor), 38.15 (major), 38.11 (minor), 37.04 (major), 36.98 (minor), 35.07 (major), 35.03 (minor), 29.35 (minor), 29.14 (major), 26.20 (overlapping), 24.44 (overlapping), 20.97 (overlapping), 14.12 (major), 12.84 (minor). ESI-HR calcd for $\mathrm{C}_{23} \mathrm{H}_{34} \mathrm{NO}_{4}{ }^{+}\left([\mathrm{M}+\mathrm{H}]^{+}\right)$388.2482, found 388.2469. IR $v$ $\left(\mathrm{cm}^{-1}\right) 2974,1731,1628,1460,1243,724$.

$[\alpha]^{30} \mathrm{D}=-5.3\left(c=0.7, \mathrm{CHCl}_{3}\right)$ for a 95.0:5.0 er sample. The enantiomeric purity of this compound was determined by HPLC analysis in comparison with authentic racemic material (Chiralpak ID, 94:6 hexanes: $i-\mathrm{PrOH}, 1.0 \mathrm{~mL} / \mathrm{min}, 240 \mathrm{~nm}): t_{\text {major }}=29.2 \mathrm{~min}, t_{\text {minor }}=31.8 \mathrm{~min}$.
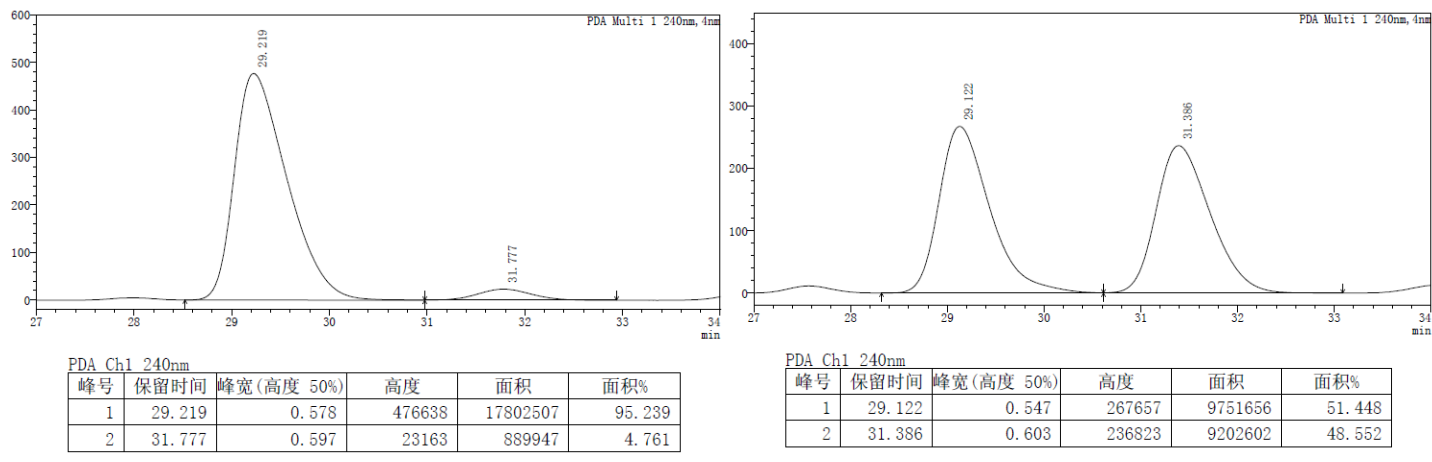<smiles>CCN(CCOC)C(=O)CC[C@](C)(C#C[Se-])CCCOc1ccccc1</smiles>

Amide 3t: Following the general procedure, amide 1q $(66.7 \mathrm{mg}, 0.200 \mathrm{mmol})$ was converted to the alkynyl amide. Purification by silica gel chromatography gave $73.9 \mathrm{mg}$ ( $72 \%$ yield) of $\mathbf{3 q}$ as a colorless oil. Two sets of peaks were observed in ${ }^{13} \mathrm{C}$ NMR spectra due to amide rotamers, which are assigned to major and minor rotamers, respectively. ${ }^{1} \mathbf{H} \mathbf{~ N M R}\left(400 \mathrm{MHz}, \mathrm{CDCl}_{3}\right) \delta 7.37-$ $7.30(\mathrm{~m}, 4 \mathrm{H}), 7.30-7.25(\mathrm{~m}, 1 \mathrm{H}), 4.50(\mathrm{~s}, 2 \mathrm{H}), 3.57-3.45(\mathrm{~m}, 6 \mathrm{H}), 3.41(\mathrm{q}, J=7.1 \mathrm{~Hz}, 2 \mathrm{H})$, $3.36-3.30(\mathrm{~m}, 3 \mathrm{H}), 2.63-2.43(\mathrm{~m}, 2 \mathrm{H}), 1.91-1.68(\mathrm{~m}, 4 \mathrm{H}), 1.61-1.51(\mathrm{~m}, 1 \mathrm{H}), 1.51-1.40(\mathrm{~m}$, 1H), $1.21-1.08(\mathrm{~m}, 6 \mathrm{H}), 1.08-0.94(\mathrm{~m}, 21 \mathrm{H}) .{ }^{13} \mathbf{C} \mathbf{~ N M R}\left(101 \mathrm{MHz}, \mathrm{CDCl}_{3}\right) \delta 172.88$ (major), 172.84 (minor), 138.69 (minor), 138.68 (major), 128.31 (overlapping), 127.52 (overlapping), 
127.43 (overlapping), 114.51 (minor), 114.38 (major), 81.28 (major), 81.14 (minor), 72.64 (overlapping), 71.13 (major), 71.03 (minor), 70.71 (minor), 70.68 (major), 58.99 (minor), 58.77 (major), 47.08 (minor), 45.46 (major), 43.68 (major), 41.15 (minor), 38.45 (major), 38.40 (minor), 36.92 (major), 36.83 (minor), 35.45 (major), 35.41 (minor), 29.39 (minor), 29.23 (major), 26.44 (overlapping), 25.34 (overlapping), 18.65 (overlapping), 14.13 (major), 12.79 (minor), 11.22 (overlapping). ESI-HR calcd for $\mathrm{C}_{31} \mathrm{H}_{54} \mathrm{NO}_{3} \mathrm{Si}^{+}\left([\mathrm{M}+\mathrm{H}]^{+}\right)$516.3867, found 516.3844. IR $v\left(\mathrm{~cm}^{-1}\right)$ $2940,1645,1455,734 .[\alpha]^{30} \mathrm{D}=-2.3\left(c=3.20, \mathrm{CHCl}_{3}\right)$ for a $96.5: 3.5$ er sample. The enantiomeric purity of this compound was determined by HPLC analysis in comparison with authentic racemic material (Chiralpak IC, 95:5 hexanes: $i-\mathrm{PrOH}, 1.0 \mathrm{~mL} / \mathrm{min}, 190 \mathrm{~nm}$ ): $t_{\text {major }}=14.1 \mathrm{~min}, t_{\text {minor }}=14.9$ $\min$.
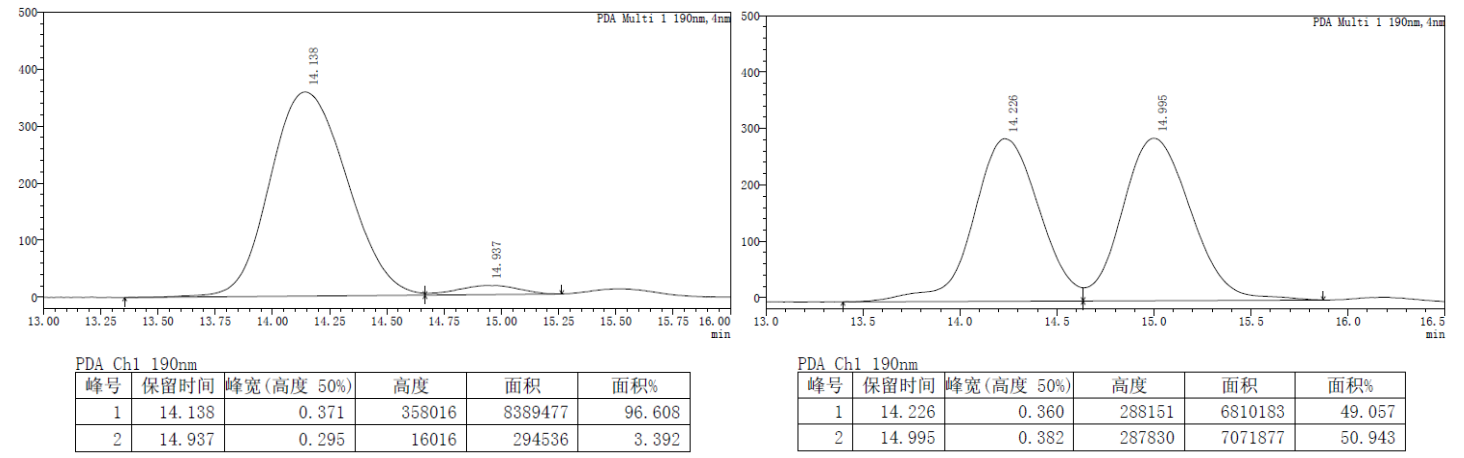<smiles>CC[AsH2-]C#C[C@@](C)(CCCc1ccccc1)CCC(=O)N(CC)CCOC</smiles>

Amide 3u: Following the general procedure, amide 1r $(60.7 \mathrm{mg}, 0.200 \mathrm{mmol})$ was converted to the alkynyl amide. Purification by silica gel chromatography gave $80.8 \mathrm{mg}$ ( $83 \%$ yield) of $\mathbf{3 r}$ as a colorless oil. Two sets of peaks were observed in ${ }^{13} \mathrm{C}$ NMR spectra due to amide rotamers, which are assigned to major and minor rotamers, respectively. ${ }^{1} \mathbf{H} \mathbf{~ N M R}\left(400 \mathrm{MHz}, \mathrm{CDCl}_{3}\right) \delta 7.29-$ $7.23(\mathrm{~m}, 2 \mathrm{H}), 7.20-7.12(\mathrm{~m}, 3 \mathrm{H}), 3.57-3.43(\mathrm{~m}, 4 \mathrm{H}), 3.43-3.35(\mathrm{~m}, 2 \mathrm{H}), 3.34-3.28(\mathrm{~m}, 3 \mathrm{H})$, $2.68-2.58(\mathrm{~m}, 2 \mathrm{H}), 2.56-2.40(\mathrm{~m}, 2 \mathrm{H}), 1.93-1.75(\mathrm{~m}, 3 \mathrm{H}), 1.73-1.65(\mathrm{~m}, 1 \mathrm{H}), 1.58-1.47(\mathrm{~m}$, 1H), $1.47-1.36(\mathrm{~m}, 1 \mathrm{H}), 1.18-1.08(\mathrm{~m}, 6 \mathrm{H}), 1.08-0.96(\mathrm{~m}, 21 \mathrm{H}) .{ }^{13} \mathbf{C ~ N M R}\left(101 \mathrm{MHz}, \mathrm{CDCl}_{3}\right)$ $\delta 172.88$ (major), 172.83 (minor), 142.44 (minor), 142.41 (major), 128.35 (overlapping), 128.22 (overlapping), 125.62 (overlapping), 114.68 (minor), 114.55 (major), 81.15 (major), 81.01 
(minor), 71.14 (major), 71.03 (minor), 58.98 (minor), 58.76 (major), 47.08 (minor), 45.48 (major), 43.69 (major), 41.60 (overlapping), 41.15 (minor), 36.89 (major), 36.80 (minor), 36.26 (overlapping), 35.61 (major), 35.57 (minor), 29.37 (minor), 29.20 (major), 26.81 (overlapping), 26.44 (overlapping), 18.66 (overlapping), 14.13 (major), 12.80 (minor), 11.23 (overlapping). ESI-HR calcd for $\mathrm{C}_{30} \mathrm{H}_{52} \mathrm{NO}_{2} \mathrm{Si}^{+}\left([\mathrm{M}+\mathrm{H}]^{+}\right) 486.3762$, found 486.3751. IR $v\left(\mathrm{~cm}^{-1}\right)$ 2940, 1646, 1461, 749. $[\alpha]^{30} \mathrm{D}=0.9\left(c=3.73, \mathrm{CHCl}_{3}\right)$ for a 95.0:5.0 er sample. The enantiomeric purity of this compound was determined by HPLC analysis in comparison with authentic racemic material (Chiralpak IF, 98:2 hexanes: $i-\mathrm{PrOH}, 1.0 \mathrm{~mL} / \mathrm{min}, 207 \mathrm{~nm}$ ): $t_{\text {major }}=16.9 \mathrm{~min}, t_{\mathrm{minor}}=18.1 \mathrm{~min}$.
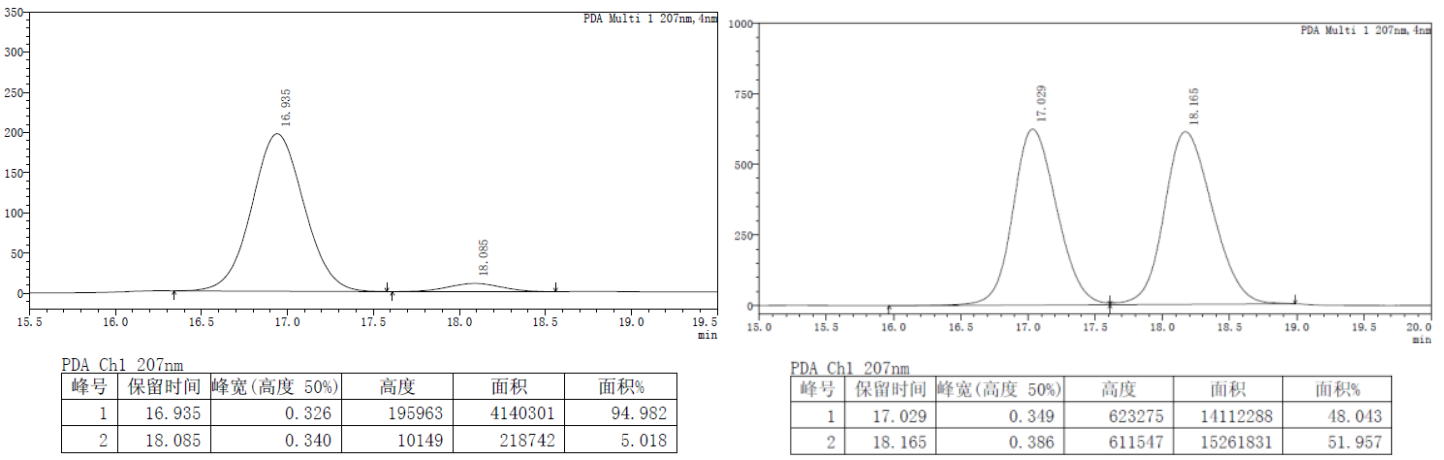<smiles>CCN(CCOC)C(=O)CC[C@](C)(C#C[AsH2-])CCc1ccccc1</smiles>

Amide 3v: Following the general procedure, amide 1s $(289.3 \mathrm{mg}, 1.000 \mathrm{mmol})$ was converted to the alkynyl amide. Purification by silica gel chromatography gave $377.2 \mathrm{mg}$ (80\% yield) of $3 \mathrm{~s}$ as a colorless oil. Two sets of peaks were observed in ${ }^{13} \mathrm{C}$ NMR spectra due to amide rotamers, which are assigned to major and minor rotamers, respectively. ${ }^{1} \mathbf{H} \mathbf{~ N M R}\left(400 \mathrm{MHz}, \mathrm{CDCl}_{3}\right) \delta 7.32-7.24$ (m, 2H), $7.22-7.13(\mathrm{~m}, 3 \mathrm{H}), 3.60-3.38(\mathrm{~m}, 6 \mathrm{H}), 3.33(\mathrm{~s}, 3 \mathrm{H}), 2.92-2.71(\mathrm{~m}, 2 \mathrm{H}), 2.68-2.45$ (m, 2H), $1.98-1.84(\mathrm{~m}, 1 \mathrm{H}), 1.83-1.61(\mathrm{~m}, 3 \mathrm{H}), 1.32-1.22(\mathrm{~m}, 3 \mathrm{H}), 1.19-1.01(\mathrm{~m}, 24 \mathrm{H}) .{ }^{13} \mathrm{C}$ NMR (101 MHz, $\mathrm{CDCl}_{3}$ ) $\delta 172.74$ (major), 172.73 (minor), 142.75 (minor), 142.69 (major), 128.38 (overlapping), 128.36 (overlapping), 125.69 (major), 125.67 (minor), 114.24 (minor), 114.10 (major), 81.68 (major), 81.51 (minor), 71.12 (major), 70.99 (minor), 59.00 (minor), 58.77 (major), 47.07 (minor), 45.48 (major), 44.37 (major), 44.31 (minor), 43.68 (major), 41.13 (minor), 36.77 (major), 36.66 (minor), 35.83 (major), 35.78 (minor), 31.69 (overlapping), 29.35 (minor), 
29.19 (major), 26.53 (minor), 26.51 (major), 18.69 (overlapping), 14.14 (major), 12.79 (minor), 11.23 (overlapping). ESI-HR calcd for $\mathrm{C}_{29} \mathrm{H}_{50} \mathrm{NO}_{2} \mathrm{Si}^{+}\left([\mathrm{M}+\mathrm{H}]^{+}\right) 472.3605$, found 472.3599. IR $v$ $\left(\mathrm{cm}^{-1}\right)$ 2940, 1646, 1458, 883.<smiles>CCN(CCOC)C(=O)CCC(C#CPc1ccccc1)(C(=O)O[O-])[N+](=O)[O-]</smiles>

Amide 3w-Ph: Amide 3w (49.3 mg, $0.100 \mathrm{mmol}), \mathrm{PdCl}_{2}\left(\mathrm{PPh}_{3}\right)_{2}$ (3.2 mg, $\left.4.5 \mathrm{~mol} \%\right)$, CuI (0.9 mg, 5 mol\%) were placed in a $10 \mathrm{~mL}$ Schlenk tube. The tube was evacuated and refilled with nitrogen. THF $(0.50 \mathrm{~mL}), \mathrm{NEt}_{3}(0.10 \mathrm{~mL})$, TBAF $(0.20 \mathrm{~mL}, 1 \mathrm{M}$ in THF) and $\mathrm{PhI}(24.5 \mathrm{mg}, 1.2$ equiv. $)$ were successively added into the tube through syringes. The reaction was stirred at $45{ }^{\circ} \mathrm{C}$ for $4 \mathrm{~h}$. The volatiles were removed on a rotary evaporator. Further purification by silica gel chromatography gave $23.1 \mathrm{mg}\left(56 \%\right.$ yield) of $\mathbf{3 w}-\mathbf{P h}$ as a colorless oil. ${ }^{1} \mathbf{H} \mathbf{~ N M R}\left(400 \mathrm{MHz}, \mathrm{CDCl}_{3}\right) \delta 7.45-7.35(\mathrm{~m}, 2 \mathrm{H}$, overlapping), $7.34-7.28$ (m, 3H, overlapping), $3.64-3.39$ (m, 6H, overlapping), 3.35 (s, 3H, major), $3.30(\mathrm{~s}, 3 \mathrm{H}$, minor $), 2.66-2.44(\mathrm{~m}, 2 \mathrm{H}$, overlapping), $1.94-1.77(\mathrm{~m}, 2 \mathrm{H}$, overlapping), $1.70-1.56(\mathrm{~m}$, $4 \mathrm{H}$, overlapping), $1.55-1.49$ (m, 2H, overlapping), $1.48-1.39$ (m, 2H, overlapping), $1.38-1.23$ (m, $10 \mathrm{H}$, overlapping), $1.19(\mathrm{t}, J=7.1 \mathrm{~Hz}, 3 \mathrm{H}$, major $), 1.13(\mathrm{t}, J=7.1 \mathrm{~Hz}, 3 \mathrm{H}$, minor $), 1.01(\mathrm{t}, J=7.3 \mathrm{~Hz}$, 3H, overlapping), $0.90\left(\mathrm{t}, J=6.7 \mathrm{~Hz}, 3 \mathrm{H}\right.$, overlapping). ${ }^{13} \mathbf{C}$ NMR $\left(101 \mathrm{MHz}, \mathrm{CDCl}_{3}\right) \delta 173.11$ (overlapping), 131.55 (overlapping), 131.51 (overlapping), 128.16 (overlapping), 128.12 (overlapping), 127.47 (major), 127.42 (minor), 124.04 (overlapping), 95.46 (minor), 95.33 (major), 82.93 (major), 82.86 (minor), 71.19 (major), 70.98 (minor), 58.99 (minor), 58.78 (major), 47.18 (minor), 45.56 (major), 43.82 (major), 41.14 (minor), 39.08 (overlapping), 37.74 (overlapping), 33.67 (major), 33.59 (minor), 31.89 (overlapping), 30.83 (overlapping), 30.20 (overlapping), 29.57 (overlapping), 29.33 (overlapping), 29.15 (minor), 28.96 (major), 24.26 (overlapping), 22.67 (overlapping), 14.15 (major), 14.09 (overlapping), 12.87 (minor), 8.81 (overlapping). ESI-HR calcd for $\mathrm{C}_{27} \mathrm{H}_{44} \mathrm{NO}_{2}{ }^{+}\left([\mathrm{M}+\mathrm{H}]^{+}\right)$ 414.3367, found 414.3359. IR $v\left(\mathrm{~cm}^{-1}\right) 2928,1646,1118,756 .[\alpha]^{20} \mathrm{D}=-14.6\left(c=0.28, \mathrm{CHCl}_{3}\right)$ for a 67.0:33.0 er sample. The enantiomeric purity of this compound was determined by HPLC analysis in comparison with authentic racemic material (Chiralpak IC, 99:1 hexanes:i-PrOH, $0.4 \mathrm{~mL} / \mathrm{min}, 205 \mathrm{~nm}$ ): $t_{\text {major }}=178.6 \mathrm{~min}, t_{\text {minor }}=184.6 \mathrm{~min}$. 

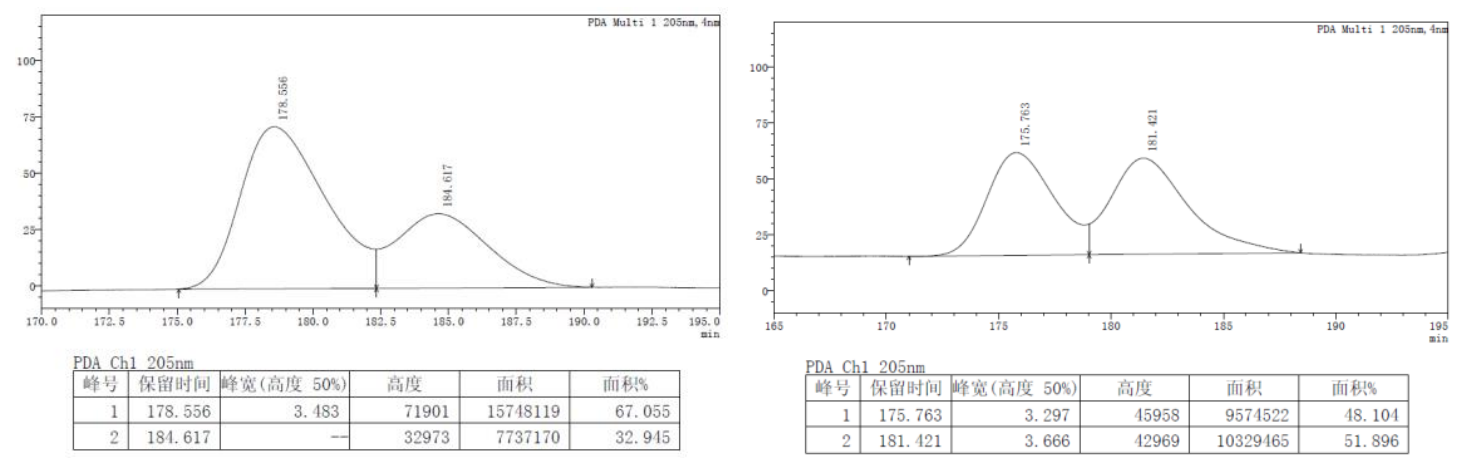<smiles>CCN(CC)C(=O)CC[C@](C)(CC)CCc1ccccc1</smiles>

Amide 4a: To a solution of $\mathbf{3 r}$ ( $350 \mathrm{mg}, 0.721 \mathrm{mmol})$ in $3.0 \mathrm{~mL}$ of THF was added TBAF (1.4 mmol, $1.0 \mathrm{M}$ in THF). The solution was stirred at room temperature for $2 \mathrm{~h}$. The mixture was diluted with $20 \mathrm{~mL}$ of EtOAc and was washed by $\mathrm{HCl}$ solution $(15 \mathrm{~mL} \times 2,1 \mathrm{M})$ and water $(15$ $\mathrm{mL}$ ). The organic layer was dried over $\mathrm{Na}_{2} \mathrm{SO}_{4}$ and concentrated. Purification by silica gel chromatography gave $214 \mathrm{mg}$ (90\% yield) of $\mathbf{4}$ as a colorless oil. Two sets of peaks were observed in ${ }^{1} \mathrm{H}$ and ${ }^{13} \mathrm{C}$ NMR spectra due to amide rotamers, which are assigned to major and minor rotamers, respectively. ${ }^{1} \mathbf{H}$ NMR $\left(400 \mathrm{MHz}, \mathrm{CDCl}_{3}\right) \delta 7.30-7.24(\mathrm{~m}, 2 \mathrm{H}$, overlapping), $7.21-$ $7.14(\mathrm{~m}, 3 \mathrm{H}$, overlapping), $3.54-3.45(\mathrm{~m}, 4 \mathrm{H}$, overlapping $), 3.45-3.36(\mathrm{~m}, 2 \mathrm{H}$, overlapping), $3.34(\mathrm{~s}, 3 \mathrm{H}$, minor), 3.33 (s, 3H, major), 2.62 (t, $J=7.6 \mathrm{~Hz}, 2 \mathrm{H}$, overlapping), $2.55-2.38(\mathrm{~m}, 2 \mathrm{H}$, overlapping), 2.11 (s, 1H, major), $2.10(\mathrm{~s}, 1 \mathrm{H}$, minor), $1.91-1.67$ (m, 4H, overlapping), 1.59 $1.49(\mathrm{~m}, 1 \mathrm{H}$, overlapping), $1.48-1.37(\mathrm{~m}, 1 \mathrm{H}$, overlapping $), 1.20-1.14(\mathrm{~m}, 6 \mathrm{H}$, overlapping), $1.10\left(\mathrm{t}, J=7.0 \mathrm{~Hz}, 3 \mathrm{H}\right.$, minor). ${ }^{13} \mathbf{C}$ NMR $\left(101 \mathrm{MHz}, \mathrm{CDCl}_{3}\right) \delta 172.75$ (overlapping), 142.32 (minor), 142.29 (major), 128.37 (overlapping), 128.25 (overlapping), 125.70 (overlapping), 90.04 (minor), 89.92 (major), 71.14 (major), 70.97 (minor), 69.64 (major), 69.55 (minor), 59.05 (minor), 58.76 (major), 47.09 (minor), 45.55 (major), 43.75 (major), 41.21 (overlapping), 36.64 (overlapping), 36.20 (overlapping), 34.51 (overlapping), 29.09 (minor), 28.88 (major), 26.53 (overlapping), 26.14 (overlapping), 14.09 (major), 12.83 (minor). ESI-HR calcd for $\mathrm{C}_{21} \mathrm{H}_{32} \mathrm{NO}_{2}{ }^{+}$ $\left([\mathrm{M}+\mathrm{H}]^{+}\right) 330.2428$, found 330.2414. IR $v\left(\mathrm{~cm}^{-1}\right) 2972,1639,1454,750 .[\alpha]^{20} \mathrm{D}=0.1(c=3.7$, $\mathrm{CHCl}_{3}$ ) for a 95.5:4.5 er sample. The enantiomeric purity of this compound was determined by 
HPLC analysis in comparison with authentic racemic material (Chiralpak IA, 98:2 hexanes: $i$-PrOH, $1.0 \mathrm{~mL} / \mathrm{min}, 209 \mathrm{~nm}$ ): $t_{\text {major }}=18.5 \mathrm{~min}, t_{\mathrm{minor}}=20.0 \mathrm{~min}$.
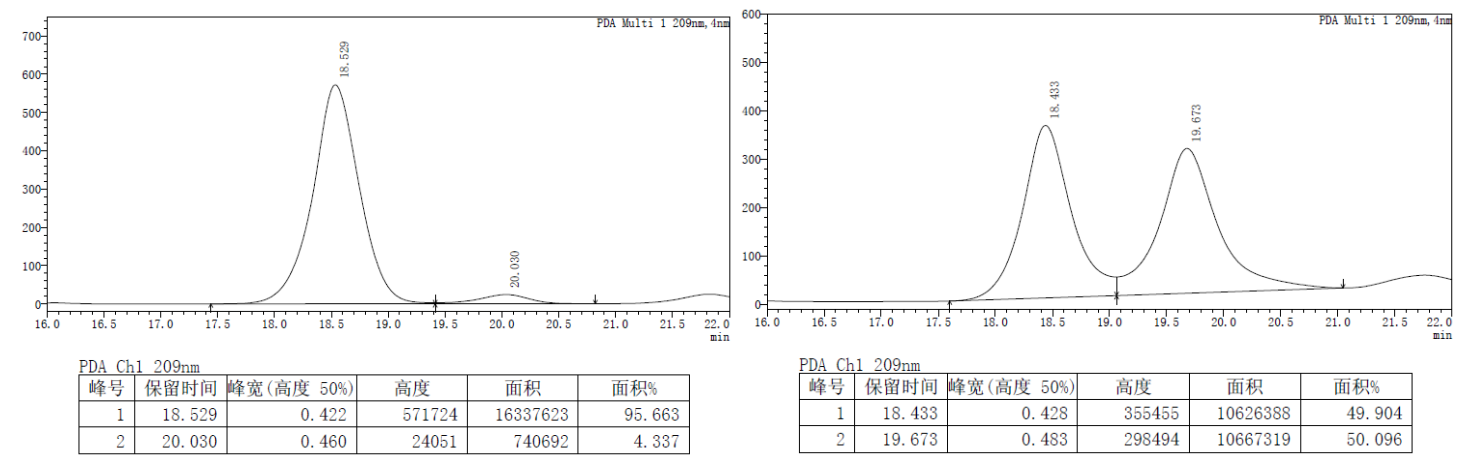<smiles>C=C[C@](C)(CCBr)CCC(=O)N(CC)CCOC</smiles>

Amide 5a: Amide 4 (16.5 mg, $0.050 \mathrm{mmol}$ ) and Lindlar's catalyst (palladium, 5\% on calcium carbonate, lead poisoned, $10.6 \mathrm{mg}$ ) were placed in a $10 \mathrm{~mL}$ Schlenk tube. The tube was evacuated and refilled with hydrogen through a hydrogen balloon. After addition of $1.0 \mathrm{~mL}$ of EtOAc, the mixture was stirred at room temperature for $24 \mathrm{~h}$ under balloon pressure of hydrogen. The reaction mixture was filtered through celite and washed with EtOAc. After removal of solvent, the residue was purified by silica gel chromatography to give $15.2 \mathrm{mg}$ (97\% yield) of amide $\mathbf{5 a}$ as a colorless oil. Two sets of peaks were observed in ${ }^{13} \mathrm{C}$ NMR spectra due to amide rotamers, which are assigned to major and minor rotamers, respectively. ${ }^{1} \mathbf{H} \mathbf{~ N M R}\left(400 \mathrm{MHz}, \mathrm{CDCl}_{3}\right) \delta 7.29-7.23(\mathrm{~m}$, 2H), $7.21-7.07$ (m, 3H), $5.65(\mathrm{dd}, J=17.6,10.8 \mathrm{~Hz}, 1 \mathrm{H}), 5.06-4.95(\mathrm{~m}, 1 \mathrm{H}), 4.89$ (d, $J=17.6$ $\mathrm{Hz}, 1 \mathrm{H}), 3.62-3.33(\mathrm{~m}, 6 \mathrm{H}), 3.33-3.30(\mathrm{~m}, 3 \mathrm{H}), 2.56(\mathrm{t}, J=7.6 \mathrm{~Hz}, 2 \mathrm{H}), 2.25-2.08(\mathrm{~m}, 2 \mathrm{H})$, $1.71-1.61(\mathrm{~m}, 2 \mathrm{H}), 1.61-1.47(\mathrm{~m}, 2 \mathrm{H}), 1.41-1.30(\mathrm{~m}, 2 \mathrm{H}), 1.18-1.04(\mathrm{~m}, 3 \mathrm{H}), 1.00-0.87(\mathrm{~m}$, 3H). ${ }^{13}$ C NMR (101 MHz, $\mathbf{C D C l}_{3}$ ) $\delta 173.23$ (minor), 173.21 (major), 146.47 (minor), 146.36 (major), 142.60 (minor), 142.56 (major), 128.36 (overlapping), 128.21 (overlapping), 125.62 (overlapping), 112.38 (major), 112.27 (minor), 71.16 (major), 70.95 (minor), 59.04 (minor), 58.75 (major), 47.06 (minor), 45.50 (major), 43.71 (major), 41.09 (minor), 40.77 (overlapping), 39.23 (overlapping), 36.57 (overlapping), 35.89 (major), 35.84 (minor), 28.16 (minor), 27.95 (major), 25.95 (overlapping), 21.98 (minor), 21.94 (major), 14.09 (major), 12.83 (minor). ESI-HR calcd for $\mathrm{C}_{21} \mathrm{H}_{34} \mathrm{NO}_{2}{ }^{+}\left([\mathrm{M}+\mathrm{H}]^{+}\right) 332.2584$, found 332.2570. IR $v\left(\mathrm{~cm}^{-1}\right) 2935,1644,1455,750 .[\alpha]^{20}{ }_{\mathrm{D}}=$ 
$-1.3\left(c=0.65, \mathrm{CHCl}_{3}\right)$ for a $95.5: 4.5$ er sample. The enantiomeric purity of this compound was determined by HPLC analysis in comparison with authentic racemic material (Chiralpak ID, 98:2 hexanes: $i-\mathrm{PrOH}, 0.5 \mathrm{~mL} / \mathrm{min}, 190 \mathrm{~nm}$ ): $t_{\text {major }}=58.9 \mathrm{~min}, t_{\mathrm{minor}}=62.7 \mathrm{~min}$.
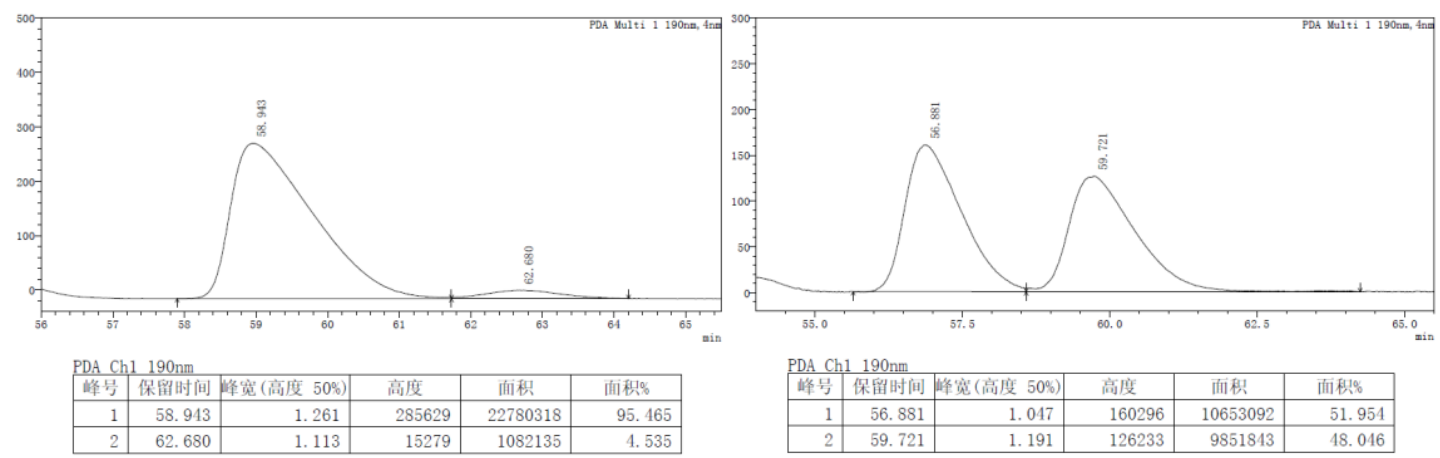<smiles>CCN(CCOC)C(=O)CC[C@](C)(CC)CCc1ccccc1</smiles>

Amide 5b: Amide $4(16.5 \mathrm{mg}, 0.050 \mathrm{mmol})$ and palladium, $10 \%$ on carbon $(2.1 \mathrm{mg})$ were placed in a $10 \mathrm{~mL}$ Schlenk tube. The tube was evacuated and refilled with hydrogen through a hydrogen balloon. After addition of $0.5 \mathrm{~mL}$ of methanol, the mixture was stirred at room temperature for 24 $\mathrm{h}$ under balloon pressure of hydrogen. The reaction mixture was filtered through celite and washed with EtOAc. After removal of solvent, the residue was purified by silica gel chromatography to give $15.1 \mathrm{mg}$ (91\% yield) of amide $\mathbf{5 b}$ as a colorless oil Two sets of peaks were observed in ${ }^{13} \mathrm{C}$ NMR spectra due to amide rotamers, which are assigned to major and minor rotamers, respectively. ${ }^{1} \mathbf{H}$ NMR $\left(400 \mathrm{MHz}, \mathrm{CDCl}_{3}\right) \delta 7.30-7.24(\mathrm{~m}, 2 \mathrm{H}), 7.21-7.08(\mathrm{~m}, 3 \mathrm{H}), 3.64-3.34$ (m, 6H), $3.33(\mathrm{~s}, 3 \mathrm{H}), 2.57(\mathrm{t}, J=7.6 \mathrm{~Hz}, 2 \mathrm{H}), 2.30-2.07(\mathrm{~m}, 2 \mathrm{H}), 1.65-1.45(\mathrm{~m}, 4 \mathrm{H}), 1.27-$ $1.19(\mathrm{~m}, 4 \mathrm{H}), 1.17-1.06(\mathrm{~m}, 3 \mathrm{H}), 0.85-0.79(\mathrm{~m}, 3 \mathrm{H}), 0.79-0.71(\mathrm{~m}, 3 \mathrm{H}) .{ }^{13} \mathbf{C} \mathbf{N M R}(101 \mathrm{MHz}$, $\left.\mathrm{CDCl}_{3}\right) \delta 173.50$ (minor), 173.45 (major), 142.73 (minor), 142.67 (major), 128.37 (overlapping), 128.21 (overlapping), 125.62 (overlapping), 71.19 (major), 70.99 (minor), 59.07 (minor), 58.77 (major), 47.16 (minor), 45.51 (major), 43.79 (major), 41.04 (minor), 38.35 (minor), 38.27 (major), 36.76 (overlapping), 34.72 (overlapping), 34.20 (major), 34.09 (minor), 31.33 (major), 29.68 (minor), 27.65 (minor), 27.45 (major), 25.47 (overlapping), 24.21 (overlapping), 14.17 (major), 12.87 (minor), 7.92 (overlapping). ESI-HR calcd for $\mathrm{C}_{21} \mathrm{H}_{36} \mathrm{NO}_{2}{ }^{+}\left([\mathrm{M}+\mathrm{H}]^{+}\right)$334.2741, found 334.2725. IR $v\left(\mathrm{~cm}^{-1}\right) 2932,1644,1456,751 .[\alpha]^{20} \mathrm{D}=-1.0\left(c=0.735, \mathrm{CHCl}_{3}\right)$ for a 96.0:4.0 er 
sample. The enantiomeric purity of this compound was determined by HPLC analysis in comparison with authentic racemic material (Chiralpak AD, 97:3 hexanes:i-PrOH, $0.5 \mathrm{~mL} / \mathrm{min}$, $225 \mathrm{~nm}): t_{\text {major }}=40.2 \mathrm{~min}, t_{\mathrm{minor}}=42.8 \mathrm{~min}$.

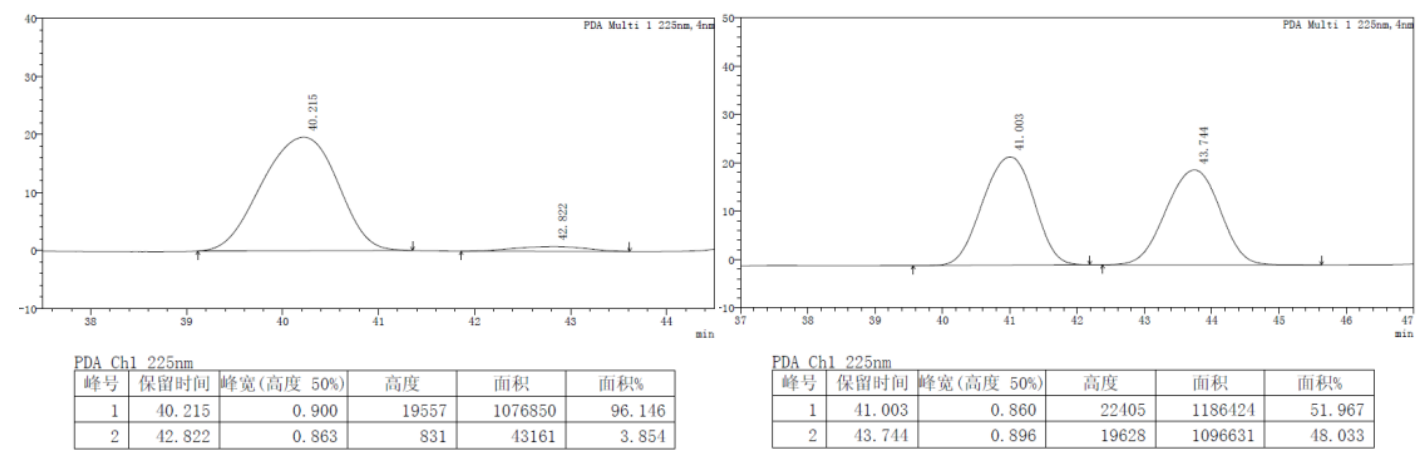<smiles>CCN(CCOC)C(=O)CC[C@](C)(CC#Cc1ccccc1)CCc1ccccc1</smiles>

Amide 5c: Amide 4 (16.5 mg, $0.050 \mathrm{mmol}), \mathrm{PdCl}_{2}\left(\mathrm{PPh}_{3}\right)_{2}(1.5 \mathrm{mg}, 4.5 \mathrm{~mol} \%)$, $\mathrm{CuI}(0.5 \mathrm{mg}, 4.5$ mol\%) were placed in a $10 \mathrm{~mL}$ Schlenk tube. The tube was evacuated and refilled with nitrogen. THF (0.50 mL), NEt $3(0.05 \mathrm{~mL})$ and $\mathrm{PhI}(12.3 \mathrm{mg}, 1.2$ equiv.) were successively added into the tube through syringes. The reaction was stirred at $45^{\circ} \mathrm{C}$ for $4 \mathrm{~h}$. The volatiles were removed on a rotary evaporator. Further purification by silica gel chromatography gave $17.4 \mathrm{mg}$ (91\% yield) of 5c as a colorless oil. Two sets of peaks were observed in ${ }^{1} \mathrm{H}$ and ${ }^{13} \mathrm{C}$ NMR spectra due to amide rotamers, which are assigned to major and minor rotamers, respectively. ${ }^{\mathbf{1}} \mathbf{H}$ NMR (400 MHz, $\left.\mathrm{CDCl}_{3}\right) \delta 7.39-7.32(\mathrm{~m}, 2 \mathrm{H}$, overlapping), $7.31-7.23(\mathrm{~m}, 5 \mathrm{H}$, overlapping $), 7.23-7.13(\mathrm{~m}, 3 \mathrm{H}$, overlapping), $3.59-3.36(\mathrm{~m}, 6 \mathrm{H}$, overlapping), 3.33 (s, 3H, major), 3.26 (s, 3H, minor), $2.65(\mathrm{t}, J$ $=7.5 \mathrm{~Hz}, 2 \mathrm{H}$, overlapping $), 2.60-2.43(\mathrm{~m}, 2 \mathrm{H}$, overlapping $), 1.97-1.73(\mathrm{~m}, 4 \mathrm{H}$, overlapping $)$, $1.68-1.57(\mathrm{~m}, 1 \mathrm{H}$, overlapping), $1.56-1.45(\mathrm{~m}, 1 \mathrm{H}$, overlapping $), 1.31-1.20(\mathrm{~m}, 3 \mathrm{H}$, overlapping), $1.16(\mathrm{t}, J=7.1 \mathrm{~Hz}, 3 \mathrm{H}$, major $), 1.10(\mathrm{t}, J=7.1 \mathrm{~Hz}, 3 \mathrm{H}$, minor $) .{ }^{13} \mathbf{C}$ NMR $(101 \mathrm{MHz}$, $\left.\mathrm{CDCl}_{3}\right) \delta 172.92$ (minor), 172.90 (major), 142.40 (minor), 142.36 (major), 131.54 (overlapping), 131.49 (overlapping), 128.40 (overlapping), 128.27 (overlapping), 128.17 (overlapping), 128.12 (overlapping), 127.57 (major), 127.52 (minor), 125.70 (major), 125.68 (minor), 123.87 (minor), 123.80 (major), 95.50 (minor), 95.38 (major), 82.25 (major), 82.19 (minor), 71.16 (major), 70.93 (minor), 58.98 (minor), 58.76 (major), 47.16 (minor), 45.55 (major), 43.80 (major), 41.44 
(overlapping), 41.13 (minor), 37.06 (major), 37.02 (minor), 36.24 (minor), 36.23 (major), 35.24 (major), 35.22 (minor), 29.40 (minor), 29.17 (major), 26.70 (overlapping), 26.24 (overlapping), 14.12 (major), 12.85 (minor). ESI-HR calcd for $\mathrm{C}_{27} \mathrm{H}_{36} \mathrm{NO}_{2}{ }^{+}\left([\mathrm{M}+\mathrm{H}]^{+}\right)$406.2741, found 406.2726. IR $v\left(\mathrm{~cm}^{-1}\right) 2933,1644,1454,757 .[\alpha]^{20}{ }_{\mathrm{D}}=0.9\left(c=0.84, \mathrm{CHCl}_{3}\right)$ for a 96.0:4.0 er sample. The enantiomeric purity of this compound was determined by HPLC analysis in comparison with authentic racemic material (Chiralpak IF, 98:2 hexanes: $i-\mathrm{PrOH}, 1.0 \mathrm{~mL} / \mathrm{min}, 205 \mathrm{~nm}$ ): $t_{\text {major }}=34.3$ $\min , t_{\operatorname{minor}}=36.4 \mathrm{~min}$.

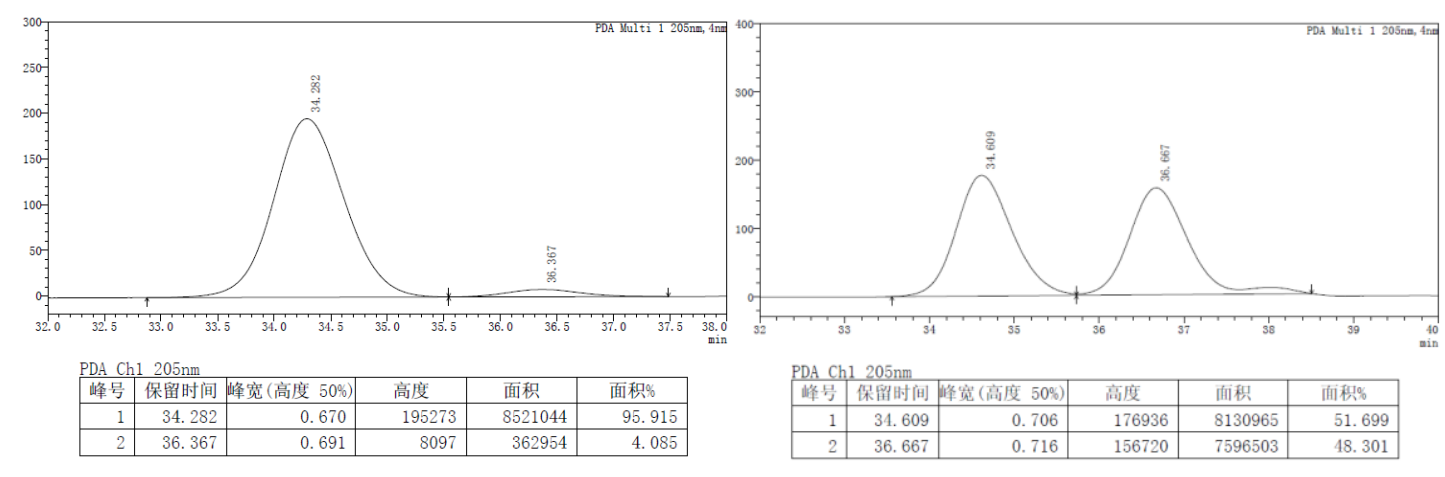<smiles>CCN(CCOC)C(=O)CC[C@](C)(CCc1ccccc1)c1cn(C)nn1</smiles>

Amide 5d: Amide 4 (16.5 mg, $0.050 \mathrm{mmol})$ and copper thiophene-2-carboxylate $(0.010 \mathrm{mmol}$, $1.9 \mathrm{mg}$ ) were added in toluene $(0.30 \mathrm{~mL})$. The reaction mixture was cooled in an ice-water bath. Subsequently, sulfonyl azide $(0.100 \mathrm{mmol}, 19.7 \mathrm{mg})$ was added slowly, and the reaction mixture was allowed to warm to room temperature and stirred until completion as judged by TLC. The mixture was diluted with saturated aq $\mathrm{NH}_{4} \mathrm{Cl}(10 \mathrm{~mL})$, extracted with EtOAc $(2 \times 10 \mathrm{~mL})$, dried over $\mathrm{Na}_{2} \mathrm{SO}_{4}$ and concentrated. Further purification by silica gel chromatography gave $22.7 \mathrm{mg}$ (86\% yield) of amide $\mathbf{5 d}$ as a colorless oil. Two sets of peaks were observed in ${ }^{13} \mathrm{C}$ NMR spectra due to amide rotamers, which are assigned to major and minor rotamers, respectively. ${ }^{1} \mathbf{H}$ NMR $\left(400 \mathrm{MHz}, \mathrm{CDCl}_{3}\right) \delta 7.97(\mathrm{~d}, J=8.3 \mathrm{~Hz}, 2 \mathrm{H}), 7.85-7.75(\mathrm{~m}, 1 \mathrm{H}), 7.36$ (d, $\left.J=8.3 \mathrm{~Hz}, 2 \mathrm{H}\right), 7.26$ $-7.20(\mathrm{~m}, 2 \mathrm{H}), 7.19-7.12(\mathrm{~m}, 1 \mathrm{H}), 7.12-7.03(\mathrm{~m}, 2 \mathrm{H}), 3.52-3.40(\mathrm{~m}, 2 \mathrm{H}), 3.39-3.13(\mathrm{~m}$, 7H), $2.56-2.47(\mathrm{~m}, 2 \mathrm{H}), 2.44(\mathrm{~s}, 3 \mathrm{H}), 2.26-1.86(\mathrm{~m}, 4 \mathrm{H}), 1.81-1.62(\mathrm{~m}, 2 \mathrm{H}), 1.57-1.42(\mathrm{~m}$, 1H), $1.40-1.30(\mathrm{~m}, 1 \mathrm{H}), 1.30-1.23(\mathrm{~m}, 3 \mathrm{H}), 1.09-0.93(\mathrm{~m}, 3 \mathrm{H}) .{ }^{13} \mathbf{C} \mathbf{~ N M R}\left(101 \mathrm{MHz}, \mathrm{CDCl}_{3}\right)$ 
$\delta 172.57$ (minor), 172.45 (major), 154.59 (minor), 154.51 (major), 147.05 (major), 147.01 (minor), 142.12 (minor), 142.06 (major), 133.30 (minor), 133.27 (major), 130.35 (overlapping), 130.34 (overlapping), 128.54 (overlapping), 128.29 (overlapping), 128.26 (overlapping), 128.24 (overlapping), 125.76 (major), 125.73 (minor), 119.93 (overlapping), 71.03 (major), 70.81 (minor), 58.96 (minor), 58.74 (major), 47.05 (minor), 45.46 (major), 43.60 (major), 41.02 (minor), 40.32 (minor), 40.26 (major), 36.86 (overlapping), 36.23 (major), 36.12 (minor), 36.10 (major), 36.02 (minor), 28.08 (minor), 27.88 (major), 25.83 (major), 25.82 (minor), 23.95 (minor), 23.91 (major), 21.78 (overlapping), 13.90 (major), 12.76 (minor). ESI-HR calcd for $\mathrm{C}_{28} \mathrm{H}_{39} \mathrm{~N}_{4} \mathrm{O}_{4} \mathrm{~S}^{+}$ $\left([\mathrm{M}+\mathrm{H}]^{+}\right)$527.2687, found 527.2675. IR $v\left(\mathrm{~cm}^{-1}\right) 2934,1637,1454,1391,674 .[\alpha]^{20} \mathrm{D}=1.8(c=$ 1.03, $\mathrm{CHCl}_{3}$ ) for a 96.0:4.0 er sample. The enantiomeric purity of this compound was determined by HPLC analysis in comparison with authentic racemic material (Chiralpak IF, 90:10 hexanes: $i-\mathrm{PrOH}, 1.0 \mathrm{~mL} / \mathrm{min}, 205 \mathrm{~nm}$ ): $t_{\text {major }}=37.8 \mathrm{~min}, t_{\mathrm{minor}}=42.6 \mathrm{~min}$.
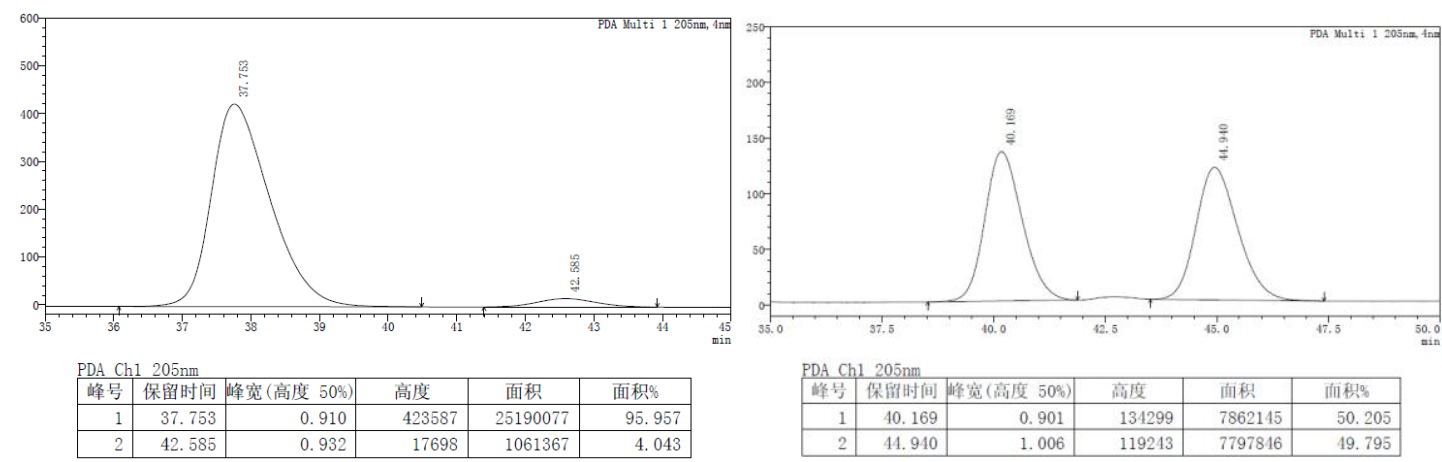<smiles>[3H]n1c([C@@](C)(CCC(=O)N(CC)CCOC)CCc2ccccc2)cc2ccccc21</smiles>

Amide 5e: Amide 4 (16.5 mg, $0.050 \mathrm{mmol}), \mathrm{PdCl}_{2}\left(\mathrm{PPh}_{3}\right)_{2}(1.8 \mathrm{mg}, 5 \mathrm{~mol} \%), \mathrm{CuI}(0.5 \mathrm{mg}, 5$ mol\%) N-tosyl-2-iodobenzene (18.7 mg, 1 equiv.) were placed in a $10 \mathrm{~mL}$ Schlenk tube. The tube was evacuated and refilled with nitrogen. DMF $(0.20 \mathrm{~mL})$ and TMG $(0.02 \mathrm{~mL}, 3$ equiv.) were successively added into the tube through syringes. The reaction was stirred at $40^{\circ} \mathrm{C}$ for $4 \mathrm{~h}$. The volatiles were removed on a rotary evaporator. Further purification by silica gel chromatography gave $22.6 \mathrm{mg}$ (79\% yield) of $\mathbf{5 e}$ as a colorless oil. Two sets of peaks were observed in ${ }^{1} \mathrm{H}$ and ${ }^{13} \mathrm{C}$ NMR spectra due to amide rotamers, which are assigned to major and minor rotamers, 
respectively. ${ }^{1} \mathbf{H}$ NMR $\left(400 \mathrm{MHz}, \mathrm{CDCl}_{3}\right) \delta 7.68-7.60(\mathrm{~m}, 2 \mathrm{H}$, overlapping), $7.58-7.51(\mathrm{~m}, 1 \mathrm{H}$, overlapping), $7.44-7.36$ (m, 1H, overlapping), $7.31-7.25$ (m, 2H, overlapping), $7.24-7.17$ (m, 5H, overlapping), $7.15-7.11(\mathrm{~m}, 2 \mathrm{H}$, overlapping), $7.00-6.93(\mathrm{~m}, 1 \mathrm{H}$, overlapping), $3.54-3.45$ (m, 4H, overlapping), $3.44-3.34(\mathrm{~m}, 2 \mathrm{H}$, overlapping), $3.30(\mathrm{~s}, 3 \mathrm{H}$, major $), 3.24(\mathrm{~s}, 3 \mathrm{H}$, minor), $2.72-2.61(\mathrm{~m}, 2 \mathrm{H}$, overlapping), $2.53(\mathrm{t}, J=8.0 \mathrm{~Hz}, 2 \mathrm{H}$, minor $), 2.43(\mathrm{t}, J=8.0 \mathrm{~Hz}, 2 \mathrm{H}$, major $)$, $2.32(\mathrm{~s}, 3 \mathrm{H}$, overlapping), $1.99-1.87(\mathrm{~m}, 1 \mathrm{H}$, overlapping), $1.86-1.72(\mathrm{~m}, 3 \mathrm{H}$, overlapping), $1.64-1.56(\mathrm{~m}, 1 \mathrm{H}$, overlapping $), 1.55-1.44(\mathrm{~m}, 1 \mathrm{H}$, overlapping $), 1.25(\mathrm{~s}, 3 \mathrm{H}$, major $), 1.23(\mathrm{~s}$, 3H, minor), $1.17-1.04$ (m, 3H, overlapping). ${ }^{13} \mathbf{C}$ NMR (101 MHz, $\left.\mathrm{CDCl}_{3}\right) \delta 172.53$ (minor), 172.34 (major), 143.80 (major), 143.78 (minor), 142.06 (minor), 141.98 (major), 137.75 (major), 137.62 (minor), 136.41 (overlapping), 131.95 (minor), 131.80 (major), 129.54 (overlapping), 128.93 (major), 128.84 (minor), 128.41 (overlapping), 128.36 (overlapping), 128.35 (overlapping), 127.19 (overlapping), 125.84 (major), 125.81 (minor), 124.11 (minor), 123.99 (major), 119.61 (minor), 119.20 (major), 114.90 (minor), 114.43 (major), 103.02 (minor), 102.95 (major), 71.11 (major), 70.87 (minor), 59.00 (minor), 58.73 (major), 47.24 (minor), 45.59 (major), 43.76 (major), 41.28 (minor), 41.19 (major), 41.06 (minor), 36.51 (major), 36.44 (minor), 36.18 (minor), 36.14 (major), 35.60 (minor), 35.55 (major), 29.13 (minor), 28.81 (major), 26.71 (overlapping), 26.33 (major), 26.24 (minor), 21.47 (overlapping), 14.08 (major), 12.82 (minor).ESI-HR calcd for $\mathrm{C}_{34} \mathrm{H}_{43} \mathrm{~N}_{2} \mathrm{O}_{4} \mathrm{~S}^{+}\left([\mathrm{M}+\mathrm{H}]^{+}\right)$575.2938, found 575.2921. IR $v\left(\mathrm{~cm}^{-1}\right)$ 2931, $1631,1490,1453,730 .[\alpha]^{20} \mathrm{D}=6.7\left(c=0.98, \mathrm{CHCl}_{3}\right)$ for a $96.5: 4.5$ er sample. The enantiomeric purity of this compound was determined by HPLC analysis in comparison with authentic racemic material (Chiralpak IA, 94:6 hexanes: $i-\mathrm{PrOH}, 0.5 \mathrm{~mL} / \mathrm{min}, 190 \mathrm{~nm}$ ): $t_{\text {major }}=41.3 \mathrm{~min}, t_{\text {minor }}=47.0$ $\min$.
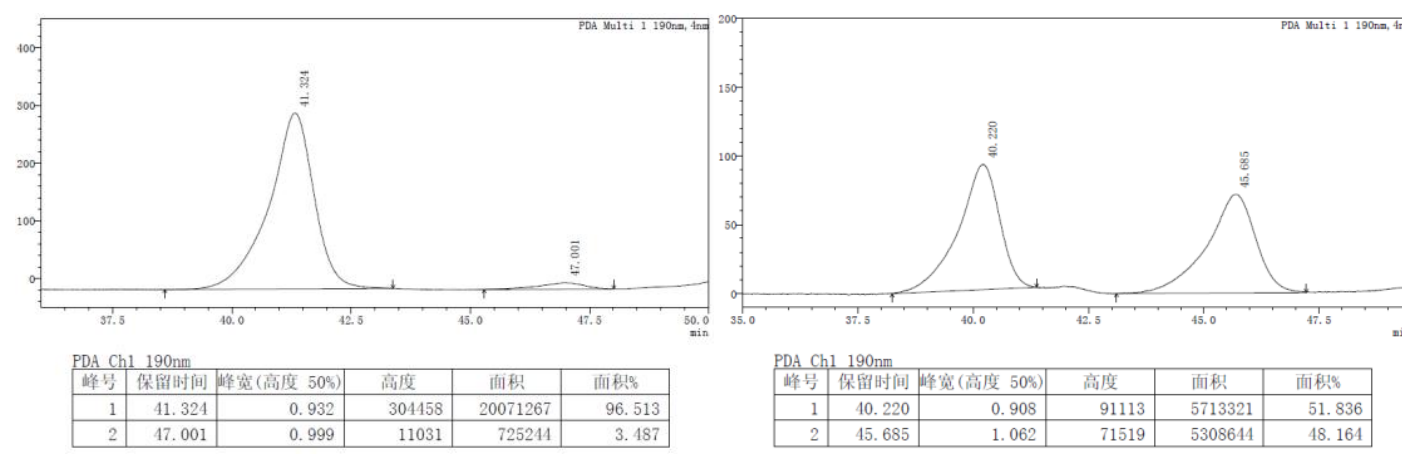
<smiles>CCC[C@](C)(CCC(=O)N(CC)CC)C(=O)O</smiles>

Amide 5f: Amide 4 (184.4 mg, $0.825 \mathrm{mmol}$ ), $\mathrm{NaIO}_{4}(706.2 \mathrm{mg}, 4$ equiv.) were placed in a $25 \mathrm{~mL}$ flask. $\mathrm{CCl}_{4}(1.60 \mathrm{~mL}) \mathrm{MeCN}(1.60 \mathrm{~mL}) \mathrm{H}_{2} \mathrm{O}(2.50 \mathrm{~mL})$ and $\mathrm{RuCl}_{3}(8.6 \mathrm{mg}, 5 \mathrm{~mol} \%)$, were successively added into the flask. The reaction was stirred at $\mathrm{rt}$ for $2 \mathrm{~h}$. After the completion of the reaction, the reaction mixture was extracted with DCM. The combined organic layers were dried over anhydrous $\mathrm{Na}_{2} \mathrm{SO}_{4}$. After removal of the solvent, the residue was purified by silica gel chromatography gave $113.5 \mathrm{mg}$ ( $57 \%$ yield) of $\mathbf{5 f}$ as a colorless oil. ${ }^{1} \mathbf{H}$ NMR (400 MHz, $\left.\mathrm{CDCl}_{3}\right)$ $\delta 3.49-3.22(\mathrm{~m}, 4 \mathrm{H}), 2.40-2.21(\mathrm{~m}, 2 \mathrm{H}), 2.10-1.95(\mathrm{~m}, 1 \mathrm{H}), 1.88-1.73(\mathrm{~m}, 1 \mathrm{H}), 1.71-1.56$ $(\mathrm{m}, 1 \mathrm{H}), 1.51-1.40(\mathrm{~m}, 1 \mathrm{H}), 1.40-1.22(\mathrm{~m}, 3 \mathrm{H}), 1.22-1.14(\mathrm{~m}, 6 \mathrm{H}), 1.10(\mathrm{t}, J=7.1 \mathrm{~Hz}, 3 \mathrm{H})$, $0.91(\mathrm{t}, J=7.2 \mathrm{~Hz}, 3 \mathrm{H}) .{ }^{13} \mathrm{C}$ NMR $\left(101 \mathrm{MHz}, \mathrm{CDCl}_{3}\right) \delta 181.93,172.26,45.33,42.07,41.70$, 40.29, 34.58, 28.64, 21.02, 17.70, 14.51, 14.31, 13.00. ESI-HR calcd for $\mathrm{C}_{13} \mathrm{H}_{26} \mathrm{NO}_{3}{ }^{+}\left([\mathrm{M}+\mathrm{H}]^{+}\right)$ 244.1907, found 244.1901. IR $v\left(\mathrm{~cm}^{-1}\right)$ 2962, 1721, 1601, 1466, 1222.<smiles>CCN(CCC[C@](C)(C#C[SnH3])C(C)C)CCOC</smiles>

Amine 5g: To a solution of $\mathrm{LiAlH}_{4}(0.500 \mathrm{mmol}, 19.0 \mathrm{mg})$ in THF $(0.50 \mathrm{~mL})$ was slowly added amide $3 \mathrm{~g}$ (20.5 $\mathrm{mg}, 0.050 \mathrm{mmol})$ at room temperature under a nitrogen atmosphere. After the addition, the solution was heated to reflux and stirred for $12 \mathrm{~h}$, followed by cooling to room temperature. The reaction mixture was quenched with water and filtered through celite. The filtrate was concentrated under reduced pressure and the residue was purified by column chromatography to give $19.7 \mathrm{mg}$ (99\% yield) of $\mathbf{5 g}$ as a colorless oil.

${ }^{1}$ H NMR $\delta 3.68-3.53(\mathrm{~m}, 2 \mathrm{H}), 3.35(\mathrm{~s}, 3 \mathrm{H}), 2.98-2.77(\mathrm{~m}, 4 \mathrm{H}), 2.76-2.60(\mathrm{~m}, 2 \mathrm{H}), 1.85-$ $1.65(\mathrm{~m}, 2 \mathrm{H}), 1.53-1.37(\mathrm{~m}, 4 \mathrm{H}), 1.35-1.28(\mathrm{~m}, 2 \mathrm{H}), 1.22-1.10(\mathrm{~m}, 6 \mathrm{H}), 1.09-0.95(\mathrm{~m}, 21 \mathrm{H})$, $0.94-0.87(\mathrm{~m}, 3 \mathrm{H}) .{ }^{13} \mathbf{C}$ NMR $\left(101 \mathrm{MHz}, \mathrm{CDCl}_{3}\right) \delta 115.26,80.37,69.67,58.87,53.52,52.43$, 47.89, 44.17, 39.18, 35.82, 26.54, 20.87, 18.64 (overlapping), 18.12, 14.58, 11.22 (overlapping), 10.51. ESI-HR calcd for $\mathrm{C}_{24} \mathrm{H}_{50} \mathrm{NOSi}^{+}\left([\mathrm{M}+\mathrm{H}]^{+}\right) 396.3656$, found 396.3673. IR $v\left(\mathrm{~cm}^{-1}\right) 2957$, $1463,1112,676$. 


\section{Determination of the Absolute Configuration:}
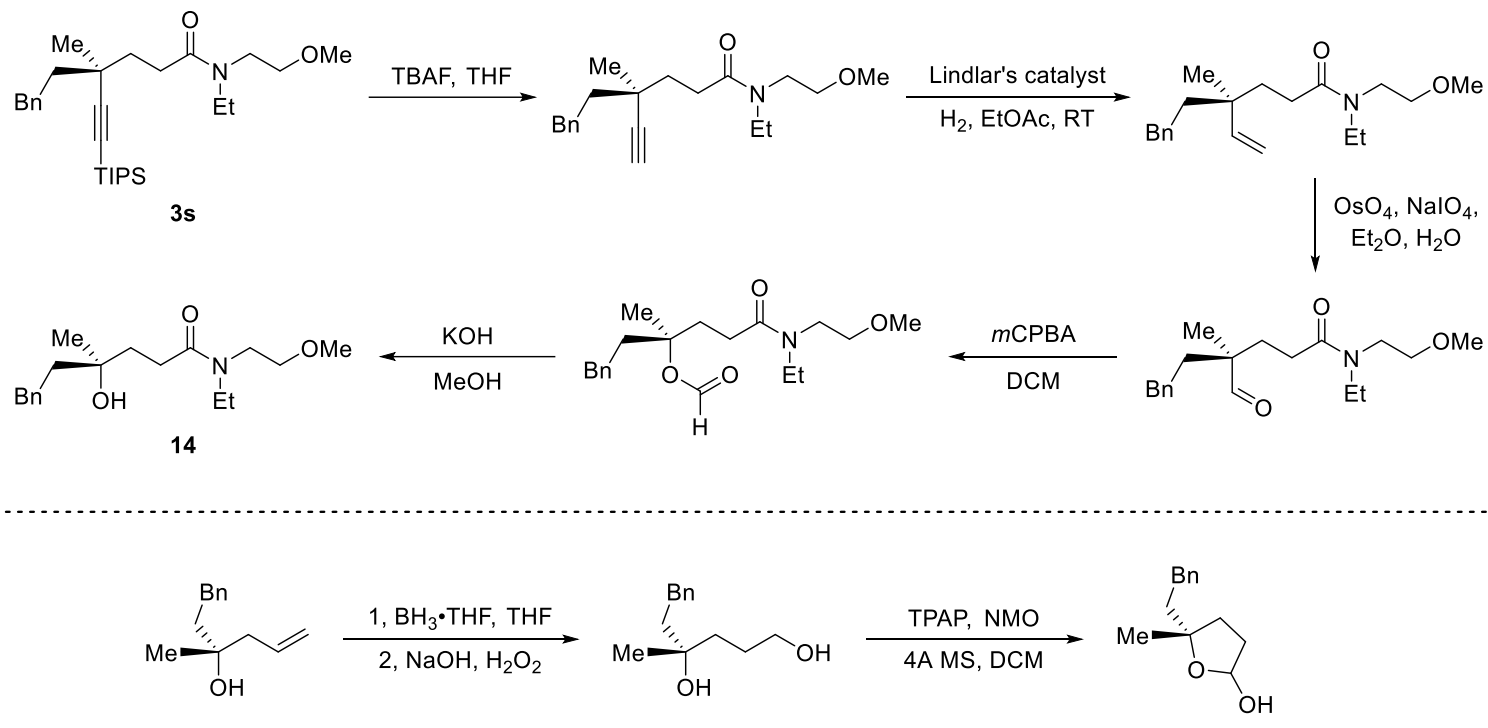

(3R)-3-methyl-1-phenylhex-5-en-3-ol

$\mathrm{OH}$

$\mathrm{Me}_{2} \mathrm{C}=\mathrm{CMe}_{2}, \mathrm{NaH}_{2} \mathrm{PO}_{4}$ $\mathrm{NaClO}_{2}, \mathrm{tBuOH}, \mathrm{H}_{2} \mathrm{O}$<smiles>CCNCCOCCN(CC)C(=O)CC[C@@](C)(O)Cc1ccccc1</smiles>

(R)-14

Amide 14: Two sets of peaks were observed in ${ }^{1} \mathrm{H}$ and ${ }^{13} \mathrm{C}$ NMR spectra due to amide rotamers, which are assigned to major and minor rotamers, respectively. ${ }^{1} \mathbf{H}$ NMR $\left(400 \mathrm{MHz}, \mathrm{CDCl}_{3}\right) \delta 7.32$ - 7.26 (m, 2H, overlapping), $7.24-7.12$ (m, 3H, overlapping), $3.57-3.37$ (m, 6H, overlapping), $3.36-3.26(\mathrm{~m}, 3 \mathrm{H}$, overlapping $), 2.82-2.62(\mathrm{~m}, 2 \mathrm{H}$, overlapping $), 2.60-2.45(\mathrm{~m}, 2 \mathrm{H}$, overlapping), $2.07-1.91(\mathrm{~m}, 1 \mathrm{H}$, overlapping), $1.88-1.66(\mathrm{~m}, 3 \mathrm{H}$, overlapping), $1.30-1.22(\mathrm{~m}$, 3H, overlapping), $1.19(\mathrm{t}, J=7.1 \mathrm{~Hz}, 3 \mathrm{H}$, major $), 1.11(\mathrm{t}, J=7.1 \mathrm{~Hz}, 3 \mathrm{H}$, minor $) .{ }^{13} \mathbf{C}$ NMR (101 $\mathrm{MHz}, \mathrm{CDCl}_{3}$ ) $\delta 174.17$ (minor), 173.96 (major), 143.05 (minor), 142.94 (major), 128.52 (overlapping), 128.49 (overlapping), 125.82 (major), 125.77 (minor), 71.37 (major), 71.36 (minor), 71.22 (major), 70.93 (minor), 59.29 (minor), 58.98 (major), 47.60 (minor), 46.06 (major), 44.98 (major), 44.92 (minor), 44.13 (major), 41.56 (minor), 36.07 (overlapping), 30.57 (overlapping), 27.89 (minor), 27.58 (major), 26.86 (overlapping), 14.10 (major), 12.94 (minor).

ESI-HR calcd for $\mathrm{C}_{18} \mathrm{H}_{30} \mathrm{NO}_{3}{ }^{+}\left([\mathrm{M}+\mathrm{H}]^{+}\right)$308.2220, found 308.2210. IR $v\left(\mathrm{~cm}^{-1}\right)$ 3403, 2918, 1622, 1456, 1118, 732. $[\alpha]^{20}{ }_{\mathrm{D}}=21.8\left(c=0.23, \mathrm{CHCl}_{3}\right)$ for a 95.0:5.0 er sample derived from 3s. The enantiomeric purity of this compound was determined by HPLC analysis in comparison with 
authentic racemic material (Chiralpak IC, 90:10 hexanes: $i-\mathrm{PrOH}, 1.0 \mathrm{~mL} / \mathrm{min}, 210 \mathrm{~nm}$ ): $t_{\text {major }}=$ $40.8 \mathrm{~min}, t_{\mathrm{minor}}=47.8 \mathrm{~min}$.
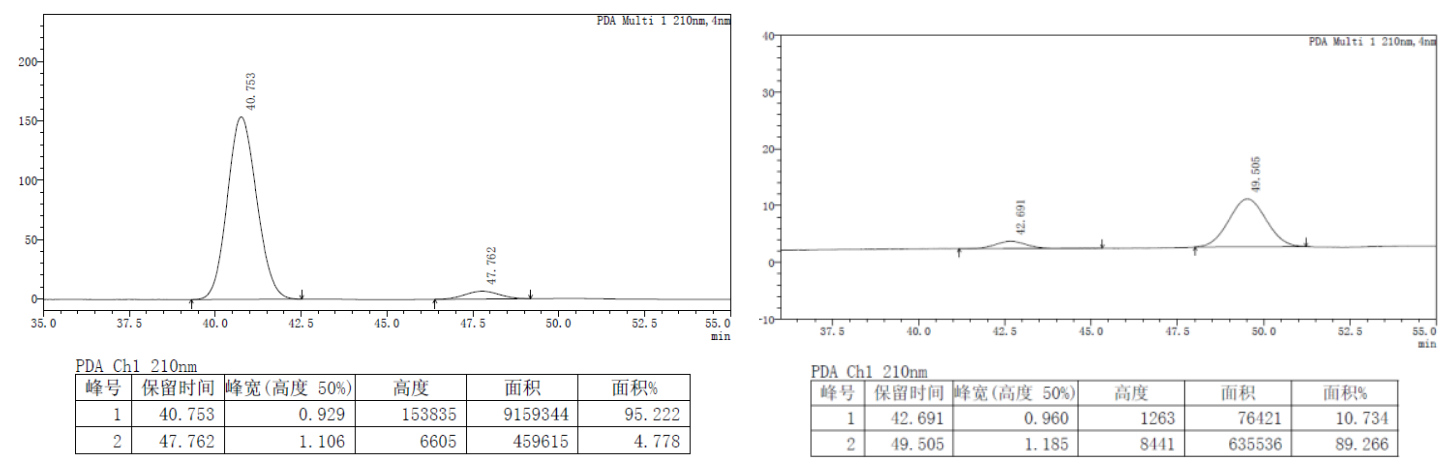

14 derived from $3 \mathrm{~s}$

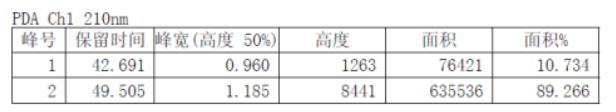

(R)-14
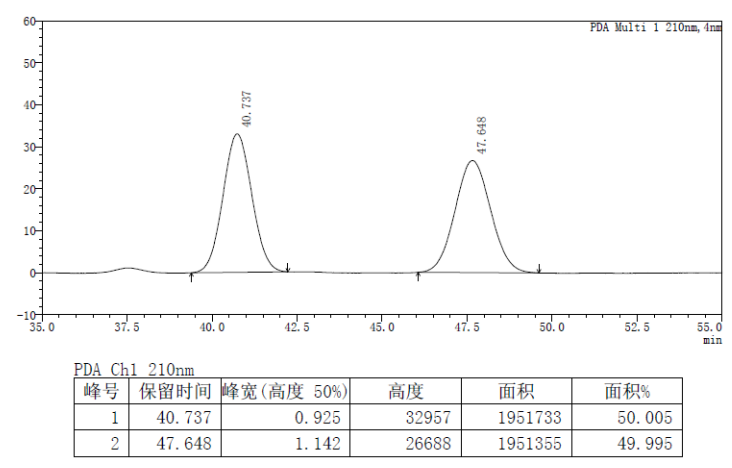

The HPLC data of $\mathbf{1 4}$ derived from $\mathbf{3 s}$, was compared to the HPLC data of $(\boldsymbol{R})-\mathbf{1 4}$ derived from reported (3R)-3-methyl-1-phenylhex-5-en-3-ol ${ }^{4,5}$ to conclude that the absolute configuration of the product, 3 s formed by the hydroalkynylation reaction is $(S)$. The absolute configuration of other amide products was assigned by analogy.

\section{Identification of Resting State:}

\section{Synthesis and characterization of complex 6}

In an $\mathrm{N}_{2}$-filled glovebox, $\operatorname{Ir}(\mathrm{COD})_{2} \mathrm{OTf}(0.150 \mathrm{~mol}, 1.0$ equiv), CTH-(R)-P-Phos (0.150 mol, 1.0 equiv) was weighed into a schlenk tube. THF $(3.0 \mathrm{~mL})$ was added via syringe. After 1 hours, the reaction mixture was concentrated in vacuo. The solid was dissolved in $0.5 \mathrm{~mL}$ of THF. After addition of $3.0 \mathrm{~mL}$ of $\mathrm{Et}_{2} \mathrm{O}$, solid of $\mathbf{6}$ precipitated out. The solid was filtered and dried under vacuum. ${ }^{31} \mathbf{P}$ NMR $\left(162 \mathrm{MHz}, \mathrm{CDCl}_{3}\right) \delta$ 12.51. ${ }^{1} \mathbf{H}$ NMR $\left(400 \mathrm{MHz}, \mathrm{CDCl}_{3}\right) \delta 7.68-7.27(\mathrm{~m}, 20 \mathrm{H})$, $6.74-6.59(\mathrm{~m}, 2 \mathrm{H}), 4.60-4.33(\mathrm{~m}, 2 \mathrm{H}), 4.30-4.02(\mathrm{~m}, 2 \mathrm{H}), 3.76(\mathrm{~s}, 6 \mathrm{H}), 3.49(\mathrm{~s}, 6 \mathrm{H}), 2.70-2.28$ (m, 4H), $2.17-1.92(\mathrm{~m}, 2 \mathrm{H}), 1.81-1.53(\mathrm{~m}, 2 \mathrm{H}) .{ }^{19} \mathbf{F}$ NMR $\left(376 \mathrm{MHz}, \mathrm{CDCl}_{3}\right) \delta$-77.92. ESI-HR calcd for $\mathrm{C}_{46} \mathrm{H}_{46} \mathrm{~N}_{2} \mathrm{O}_{4} \mathrm{P}_{2} \mathrm{Ir}^{+}\left([\mathrm{M}]^{+}\right)$945.2557, found 945.2730. 


\section{Kinetic profile}

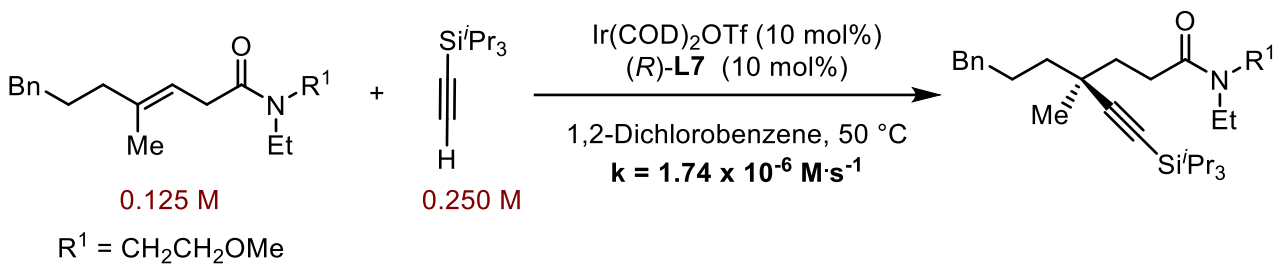

Reaction conditions: $\beta, \gamma$-unsaturated amide $=0.125 \mathrm{M}$, alkyne $=0.250 \mathrm{M}, \operatorname{Ir}(\mathrm{COD})_{2} \mathrm{OTf}=12.5$ $\mathrm{mM},(R)-\mathbf{L} 7=12.5 \mathrm{mM}, 50.0{ }^{\circ} \mathrm{C}$.

The initial rate was obtained by linear regression of the concentration-time data monitored by GC.

The figure for linear regression to obtain the initial rate is shown in Fig S1

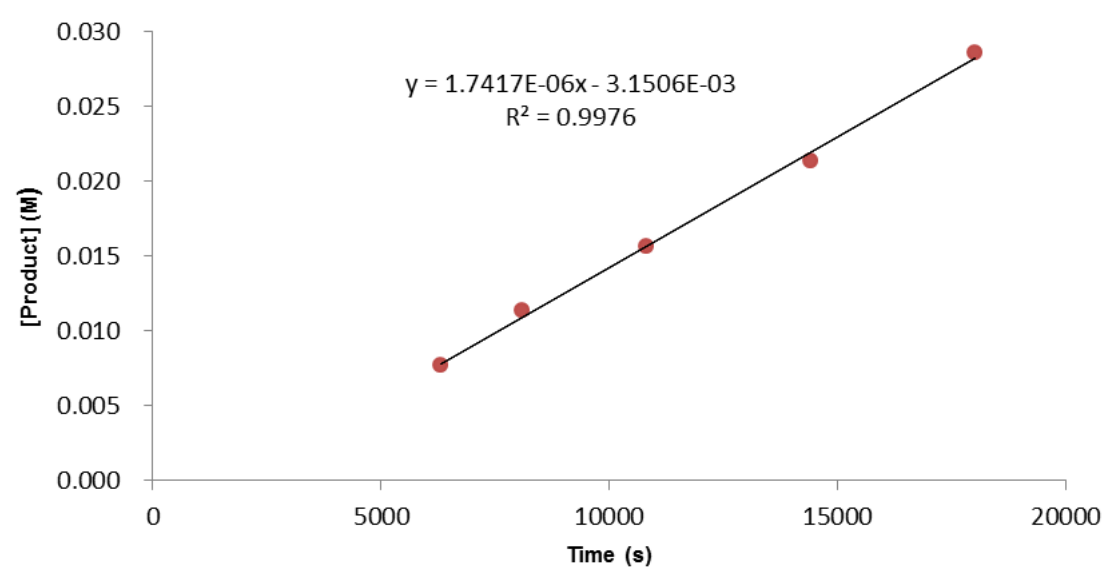

Fig S1. Initial rate of hydroalkynylation of $\beta, \gamma$-unsaturated amide

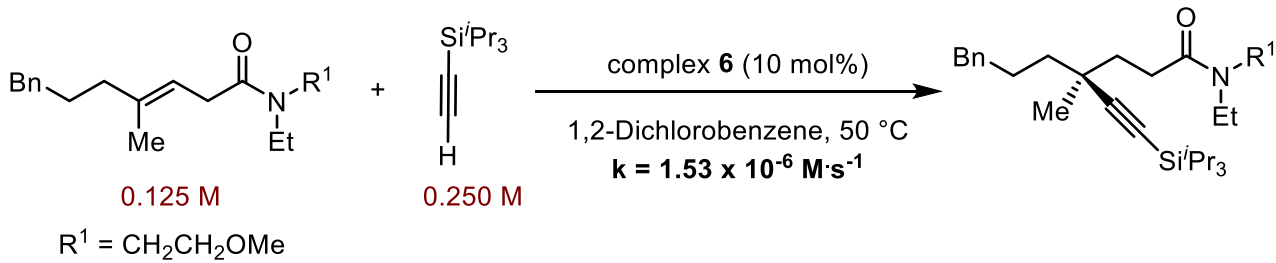

Reaction conditions: $\beta, \gamma$-unsaturated amide $=0.125 \mathrm{M}$, alkyne $=0.250 \mathrm{M}$, complex $\mathbf{6}=12.5 \mathrm{mM}$, $50.0^{\circ} \mathrm{C}$.

The initial rate was obtained by linear regression of the concentration-time data monitored by GC.

The figure for linear regression to obtain the initial rate is shown in Fig S2 


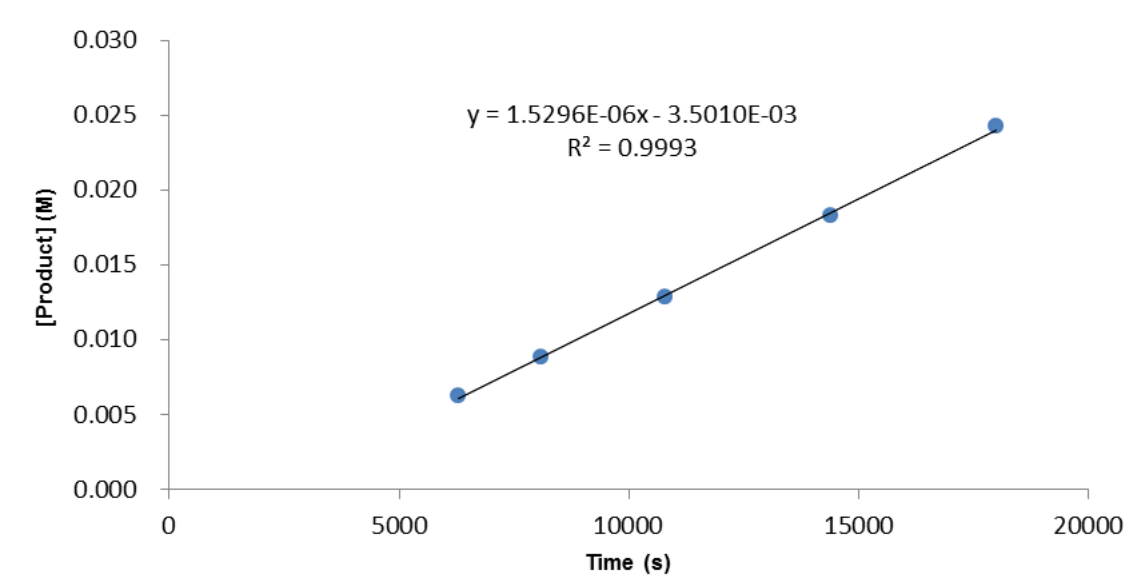

Fig S2. Initial rate of hydroalkynylation of $\beta, \gamma$-unsaturated amide

The hydroalkynylation of $\mathbf{1} \mathbf{u}$ catalyzed by Ir complex $\mathbf{6}$ has similar kinetic profile to that of catalyzed by the in-situ generated catalyst.

\section{Measurement of Kinetic Isotope Effect:}

\section{Kinetic isotopic effect of alkyne}<smiles>CCN(CC)C(=O)C=CC(=O)O[Mg]</smiles>
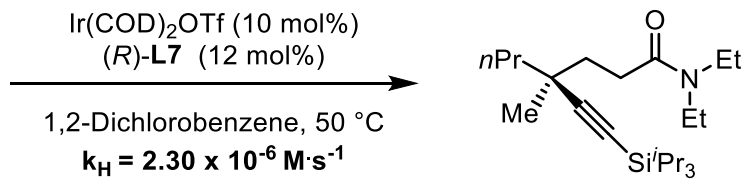

Reaction conditions: $\beta, \gamma$-unsaturated amide $=0.125 \mathrm{M}$, alkyne $=0.250 \mathrm{M}, \operatorname{Ir}(\mathrm{COD})_{2} \mathrm{OTf}=12.5$ $\mathrm{mM},(R)-\mathbf{L} 7=15 \mathrm{mM}, 50.0^{\circ} \mathrm{C}$.

The initial rate was obtained by linear regression of the concentration-time data monitored by GC.

The figure for linear regression to obtain the initial rate is shown in Fig S3.

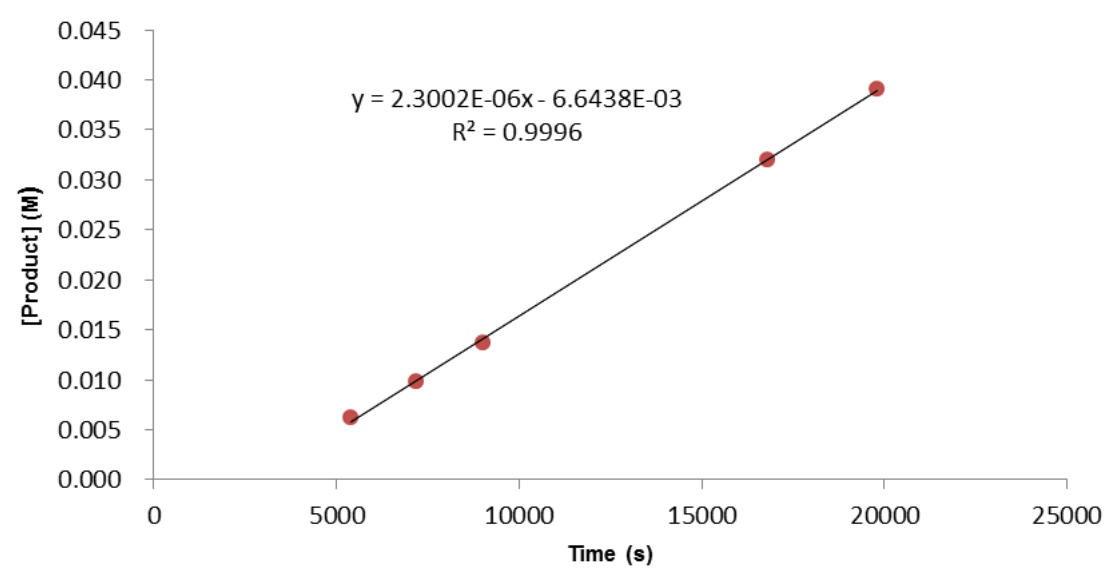


Fig S3. Initial rate of hydroalkynylation of $\beta, \gamma$-unsaturated amide

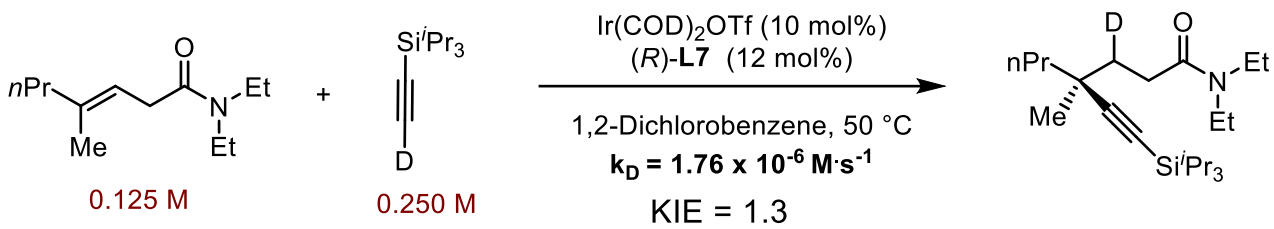

Reaction conditions: $\beta, \gamma$-unsaturated amide $=0.125 \mathrm{M}$, alkyne $=0.250 \mathrm{M}, \operatorname{Ir}(\mathrm{COD})_{2} \mathrm{OTf}=12.5$ $\mathrm{mM},(R)-\mathbf{L} 7=15 \mathrm{mM}, 50.0^{\circ} \mathrm{C}$.

The initial rate was obtained by linear regression of the concentration-time data monitored by GC.

The figure for linear regression to obtain the initial rate is shown in Fig S4.

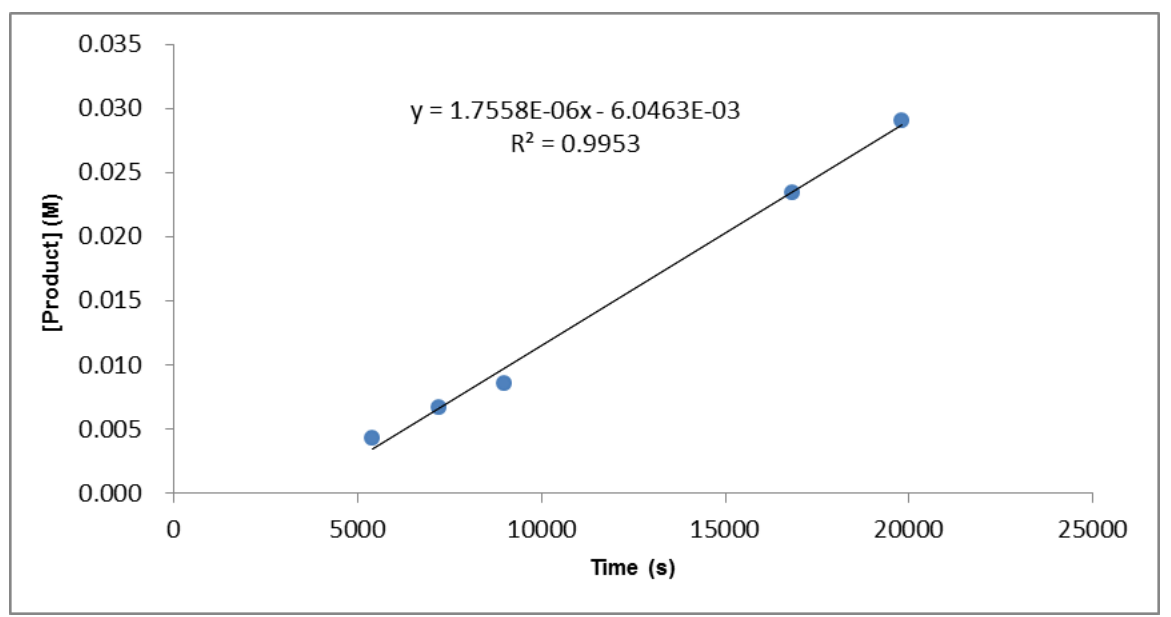

Fig S4. Initial rate of hydroalkynylation of $\beta, \gamma$-unsaturated amide

\section{Kinetic isotopic effect of alkene}<smiles>[R]N(CC)C(=O)C/C=C(\C)CCBr</smiles>

1u, $0.0587 \mathrm{M}$<smiles>[R]N(CC)C(=O)C/C(C)=C(/[2H])CCBr</smiles>

1u-d, $0.0663 \mathrm{M}$

$$
\mathrm{R}^{1}=\mathrm{CH}_{2} \mathrm{CH}_{2} \mathrm{OMe}
$$

total: $0.125 \mathrm{M}, 53 \% \mathrm{D}$

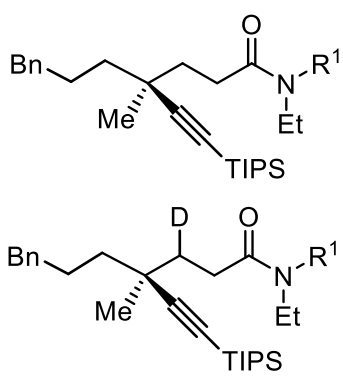

3u, $55.7 \%$ D (average of three expts)

Reaction conditions: $\beta, \gamma$-unsaturated amide (1u, $0.0587 \mathrm{M}, \mathbf{1 u}-\mathrm{d}, 0.0663 \mathrm{M}$, total of $0.125 \mathrm{M}, 53 \%$ D), alkyne $=0.250 \mathrm{M}, \operatorname{Ir}(\mathrm{COD})_{2} \mathrm{OTf}=12.5 \mathrm{mM},(R)-\mathbf{L} 7=15 \mathrm{mM}, 50.0^{\circ} \mathrm{C}$. The reaction was monitored under $30 \%$ conversion. 
The deuterated ratio of substrates and products were analyzed by ${ }^{1} \mathrm{H}$ NMR.

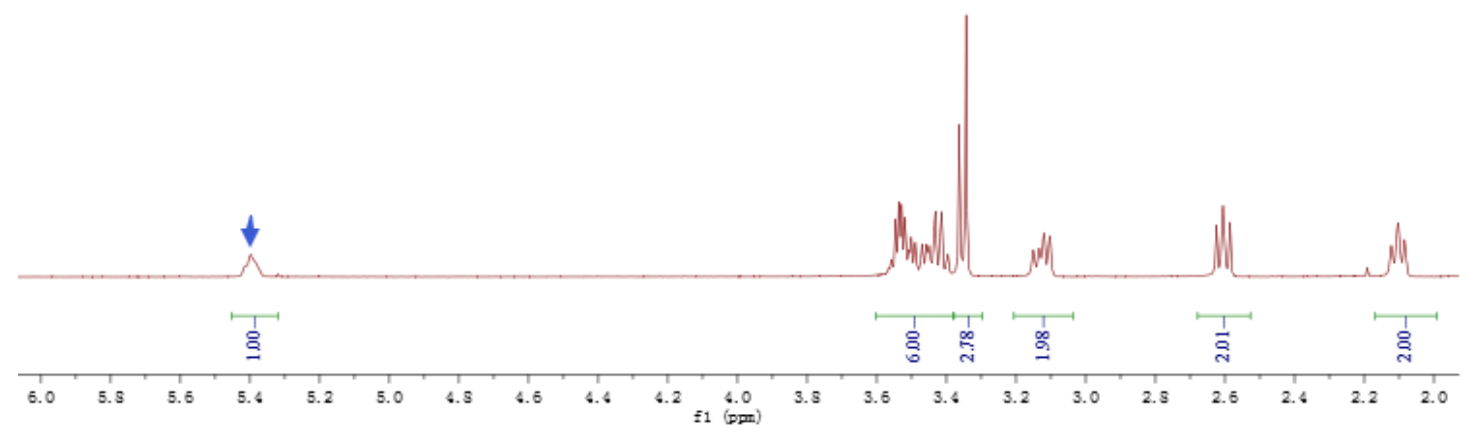

${ }^{1} \mathrm{H}$ NMR (400 MHz, $\mathrm{CDCl}_{3}$ ) of $\mathbf{1 u - H}$

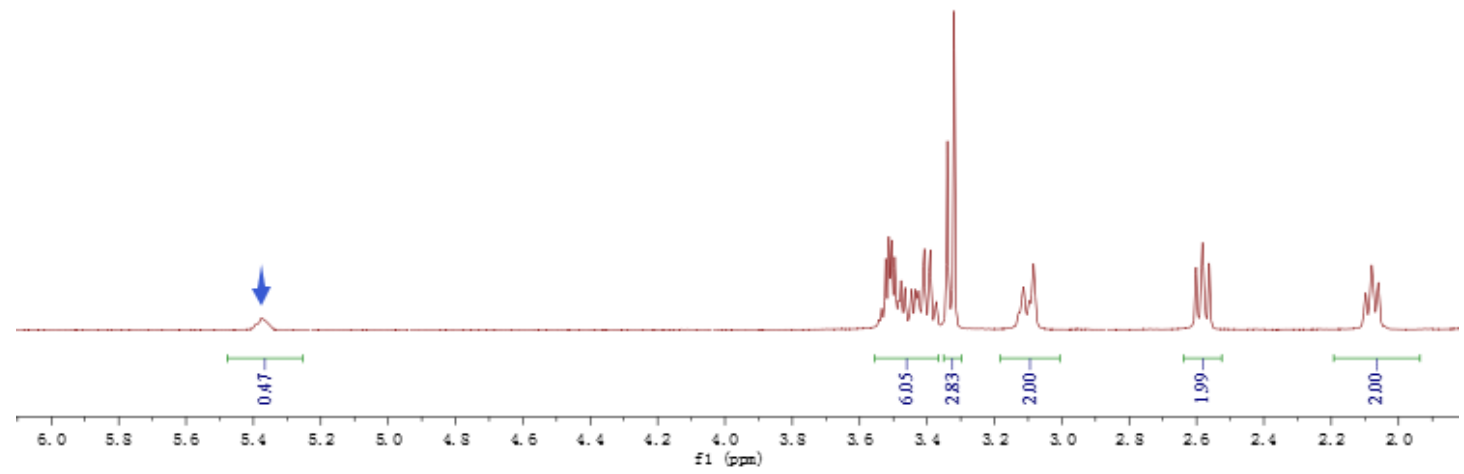

${ }^{1} \mathrm{H}$ NMR (400 MHz, $\left.\mathrm{CDCl}_{3}\right)$ of $\mathbf{1 u + 1 u - d}$

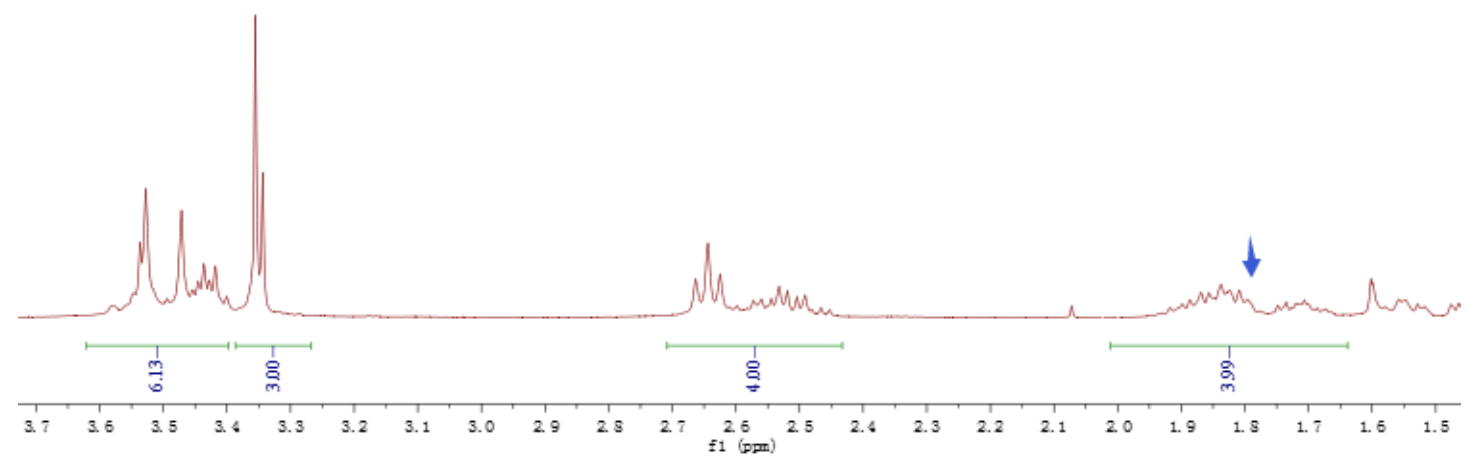

${ }^{1} \mathrm{H}$ NMR $\left(400 \mathrm{MHz}, \mathrm{CDCl}_{3}\right)$ of $\mathbf{3 u}-\mathbf{H}$ 

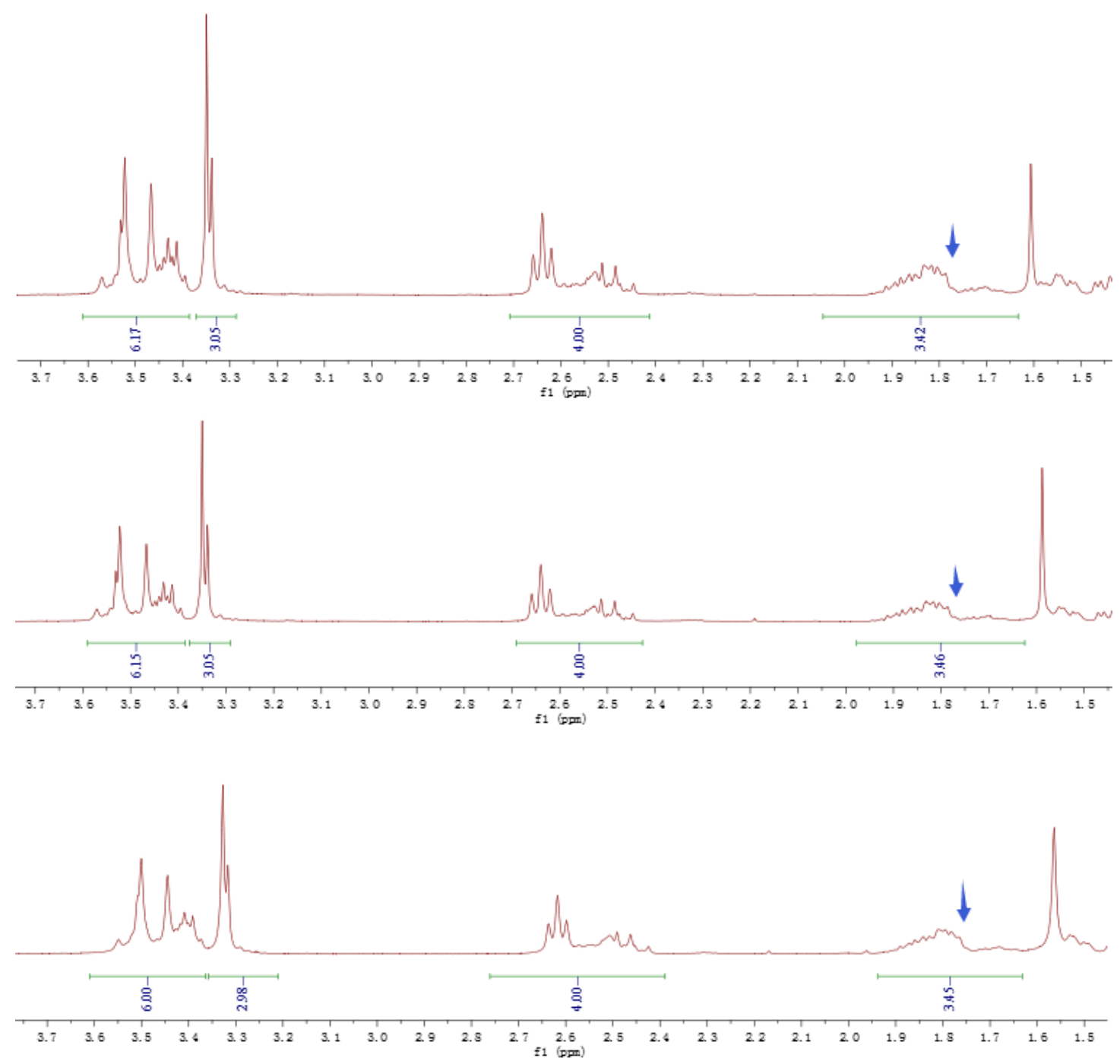

${ }^{1} \mathrm{H}$ NMR (400 MHz, $\mathrm{CDCl}_{3}$ ) of product

\section{Control experiments for $E / Z$ isomerization versus enantioselectivity:}

In an $\mathrm{N}_{2}$-filled glovebox, $0.20 \mathrm{mmol}$ of the amide was weighed into a one-dram screw-capped vial. A stir bar was added, and the substrate was dissolved in 1,2-difluorobenzene $(0.50 \mathrm{~mL})$. The resulting solution was treated with $\left[\operatorname{Ir}(\operatorname{cod})_{2}\right]$ OTf $(20.0 \mu \mathrm{mol}, 10.0 \mathrm{~mol} \%)$, CTH-P-Phos $(24.0$ $\mu \mathrm{mol}, 12.0 \mathrm{~mol} \%)$ and triisopropylsilylacetylene ( $0.40 \mathrm{mmol}, 2.0$ equiv.). Dodecane (40.0 $\mu \mathrm{L})$ was added as internal standard substance. The vial was capped with a Teflon-lined screw cap, and the resulting solution was removed from the glovebox, placed in a pre-set low temperature thermostatic stirring reaction bath at $20{ }^{\circ} \mathrm{C}$. At different time, a portion of reaction mixture was taken out through a syringe under a stream of argon for monitoring the $E / Z$ ratio of remaining alkene as well as the yield and er of product. The $E / Z$ ratio of remaining alkene and the yield were 
obtained by GC. The er of product was obtained by HPLC.

\section{General Information for Computation:}

All calculations were performed with the Gaussian 09 program. (Full citation of Gaussian 09: Frisch, M. J.; Trucks, G. W.; Schlegel, H. B.; Scuseria, G. E.; Robb, M. A.; Cheeseman, J. R.; Scalmani, G.; Barone, V.; Mennucci, B.; Petersson, G. A.; Nakatsuji, H.; Caricato, M.; Li, X.; Hratchian, H. P.; Izmaylov, A. F.; Bloino, J.; Zheng, G.; Sonnenberg, J. L.; Hada, M.; Ehara, M.; Toyota, K.; Fukuda, R.; Hasegawa, J.; Ishida, M.; Nakajima, T.; Honda, Y.; Kitao, O.; Nakai, H.; Vreven, T.; Montgomery, J. A., Jr.; Peralta, J. E.; Ogliaro, F.; Bearpark, M.; Heyd, J. J.; Brothers, E.; Kudin, K. N.; Staroverov, V. N.; Keith, T.; Kobayashi, R.; Normand, J.; Raghavachari, K.; Rendell, A.; Burant, J. C.; Iyengar, S. S.; Tomasi, J.; Cossi, M.; Rega, N.; Millam, J. M.; Klene, M.; Knox, J. E.; Cross, J. B.; Bakken, V.; Adamo, C.; Jaramillo, J.; Gomperts, R.; Stratmann, R. E.; Yazyev, O.; Austin, A. J.; Cammi, R.; Pomelli, C.; Ochterski, J. W.; Martin, R. L.; Morokuma, K.; Zakrzewski, V. G.; Voth, G. A.; Salvador, P.; Dannenberg, J. J.; Dapprich, S.; Daniels, A. D.; Farkas, O.; Foresman, J. B.; Ortiz, J. V.; Cioslowski, J.; Fox, D. J.; Gaussian 09, revision B.01; Gaussian Inc.: Wallingford, CT, 2010.) Density functional theory calculations using the M06 functional ((a) Zhao, Y.; Truhlar, D. G. Theor. Chem. Acc. 2008, 120, 215-241. (b) Zhao, Y.; Truhlar, D. G. Acc. Chem. Res. 2008, 41, 157-167.) Geometry optimizations were conducted with the Gaussian 09 software package, B3LYP functional (with gd3 dispersion correction), and LANL2DZ basis set for Rh and 6-31g(d,p) basis set for all other atoms. Single-point energy calculations were conducted with the M06 functional and SDD basis set for Rh and the $6-311++\mathrm{g}(\mathrm{d}, \mathrm{p})$ basis set for all other atoms, along with the SMD $o-\mathrm{C}_{6} \mathrm{H}_{4} \mathrm{Cl}_{2}$ solvent correction. Thermal corrections were applied to the optimized geometries to provide Gibbs free energies.

The energy profiles of the catalytic hydroalkynylation of both $E$-alkene and $Z$-alkene were computed. For each substrate, pathways that lead to both enantiomers were analyzed. Extensive conformational searches were conducted for all intermediates and transition states, and the lowest energy conformers are shown in Fig S5 and Fig S6.

In addition, many possible transition state structures were considered during the computation for the activation energies of migratory insertion without coordination of the amide group. For each 
substrate, four possible structures (from TS-2e to TS-2h for $E$-alkene and from TS-2i to TS-2l for $Z$-alkene) were computed. The structures and energies were summarized in Fig S7.

We also considered many possible transition state structures for the oxidative addition of $\mathrm{C}-\mathrm{H}$ bond. The activation free energies of transition states containing alkene coordination are significantly higher that with containing carbonyl coordination. The structures and energies were summarized in Fig S8. 


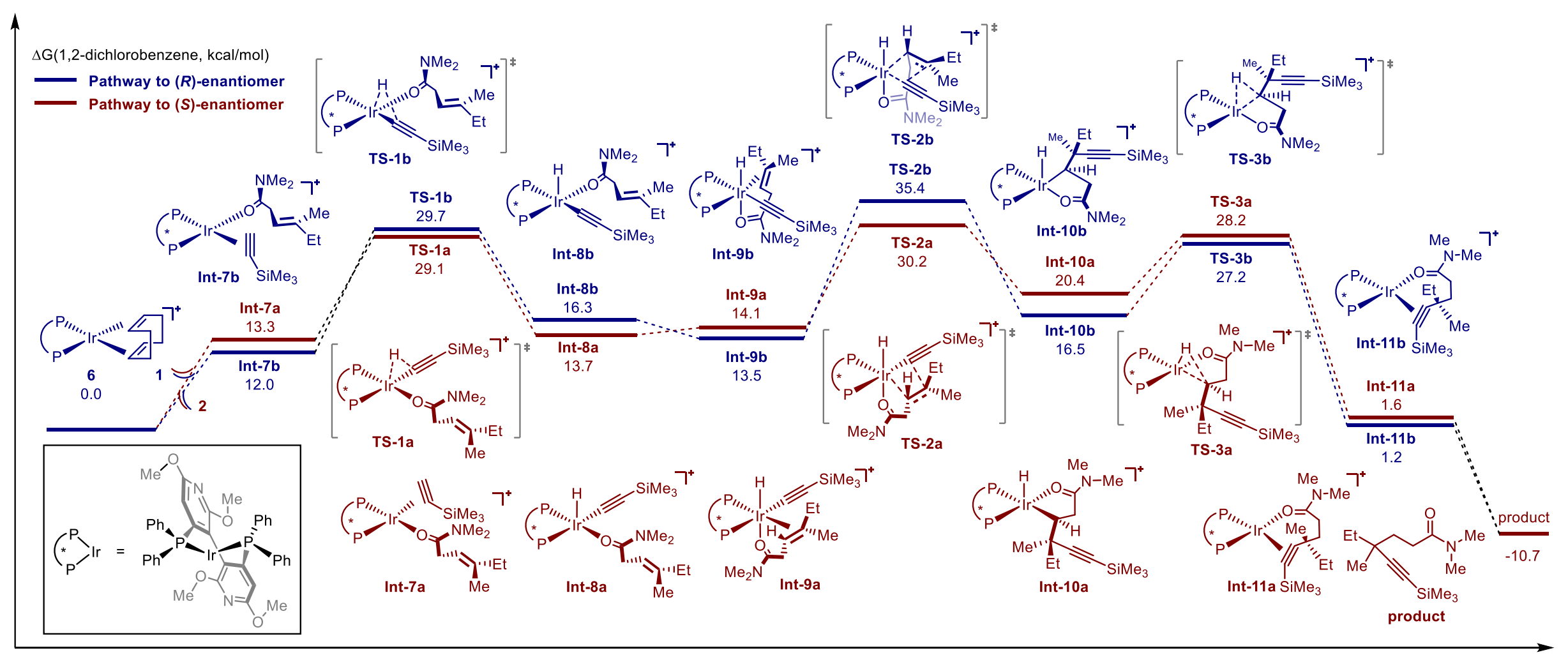

reaction coordinate

Figure S5. Energy profile for Ir-catalyzed hydroalkynylation of $E$-alkene. Gibbs free energies in $o-\mathrm{C}_{6} \mathrm{H}_{4} \mathrm{Cl}_{2}\left(\Delta G_{\text {solv298K }}\right)$ are reported. 


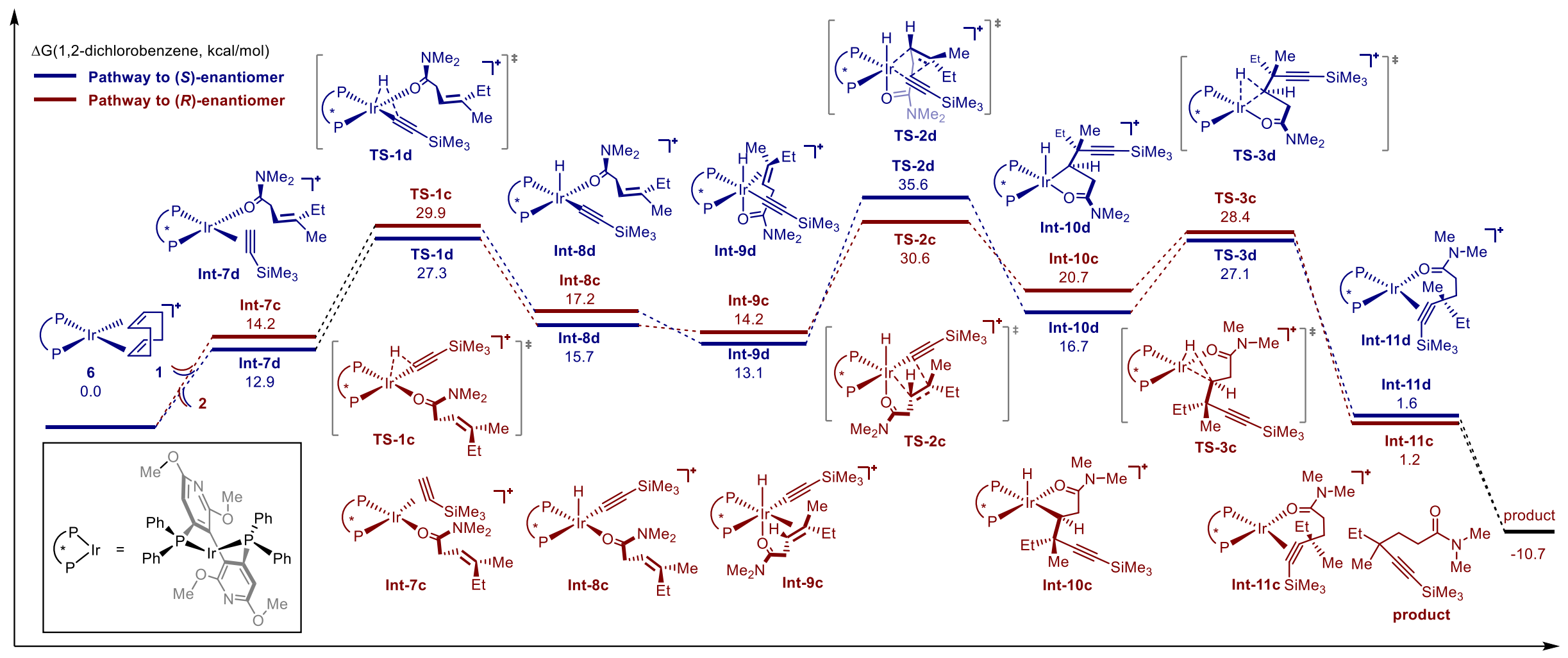

reaction coordinate

Figure S6. Energy profile for Ir-catalyzed hydroalkynylation of $Z$-alkene. Gibbs free energies in $o-\mathrm{C}_{6} \mathrm{H}_{4} \mathrm{Cl}_{2}\left(\Delta G_{\text {solv298K }}\right)$ are reported. 
From E-alkene

[]$_{\mathrm{SiMe}_{3}}$

TS-2e

$46.4 \mathrm{kcal} / \mathrm{mol}$

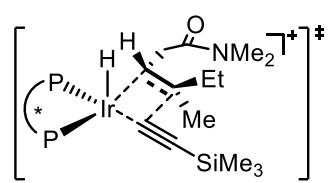

TS-2f

$42.1 \mathrm{kcal} / \mathrm{mol}$

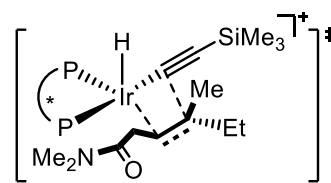

TS-2g

$38.8 \mathrm{kcal} / \mathrm{mol}$

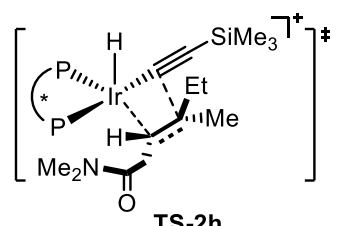

$44.4 \mathrm{kcal} / \mathrm{mol}$

From Z-alkene

[]$_{\mathrm{SiMe}_{3}}$

TS-2i

$44.4 \mathrm{kcal} / \mathrm{mol}$

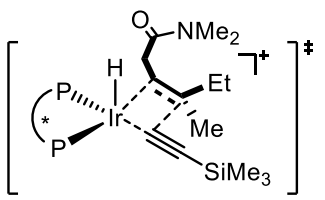

TS-2j

$46.6 \mathrm{kcal} / \mathrm{mol}$

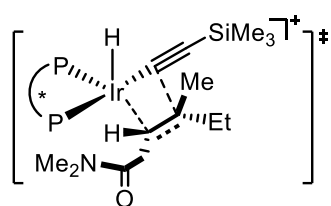

TS-2k

$44.3 \mathrm{kcal} / \mathrm{mol}$

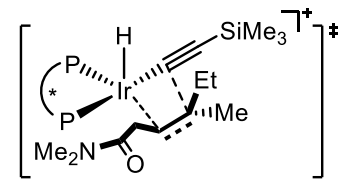

TS-2I

$41.1 \mathrm{kcal} / \mathrm{mol}$

Fig S7. Additional transition states of migratory insertion without amide coordination.

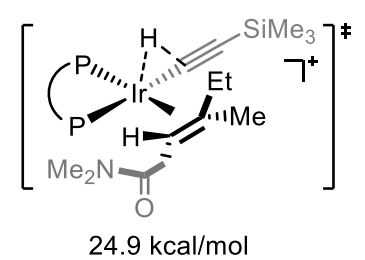

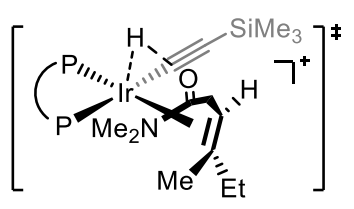

$30.0 \mathrm{kcal} / \mathrm{mol}$

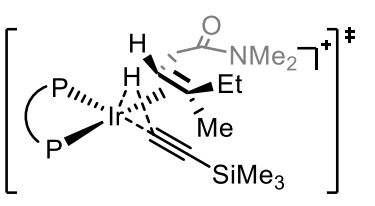

$24.6 \mathrm{kcal} / \mathrm{mol}$

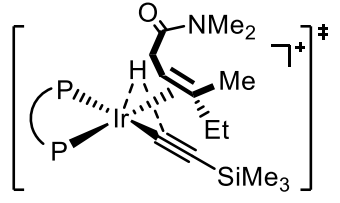

$25.3 \mathrm{kcal} / \mathrm{mol}$

Fig S8. Additional transition states of oxidative addition of C-H bond. 


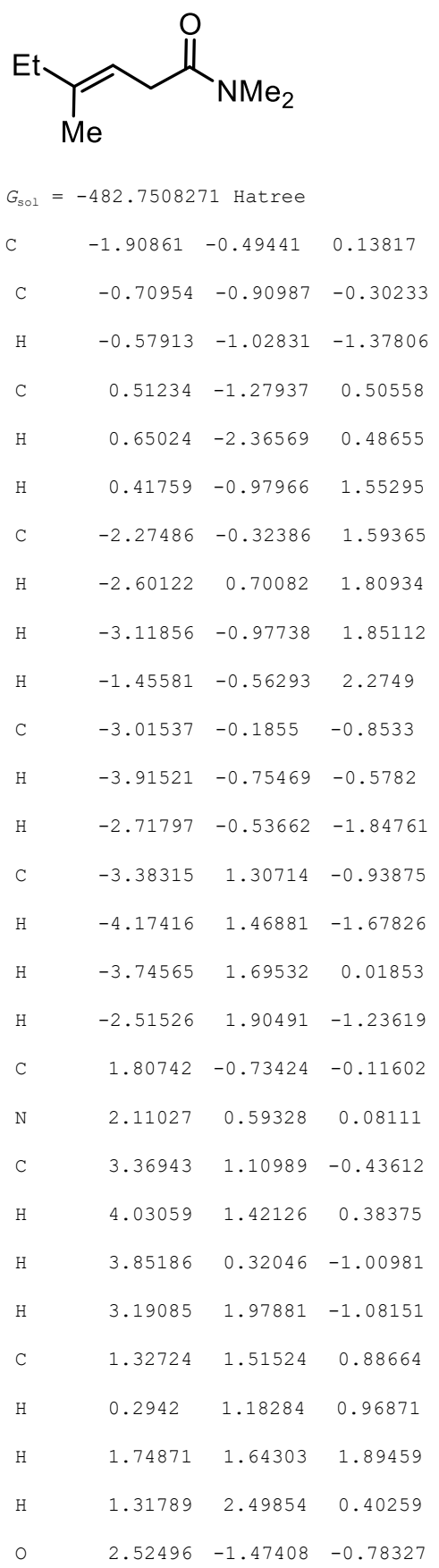

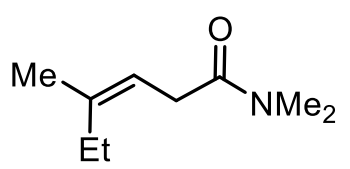

$G_{\text {sol }}=-482.7506963$ Hatree

$\begin{array}{lrrr}\text { C } & -2.24720000 & -0.49358000 & 0.19943900 \\ \text { C } & -0.95914100 & -0.77276800 & -0.04683400 \\ \text { H } & -0.67076000 & -1.78700700 & -0.30858500 \\ \text { C } & 0.18901200 & 0.20420200 & 0.00535700 \\ \text { H } & 0.15223900 & 0.80543000 & 0.92305200\end{array}$

$$
\begin{aligned}
& \begin{array}{llll}
\mathrm{H} & 0.12614000 & 0.92632600 & -0.82201900
\end{array} \\
& \begin{array}{llll}
\text { C } & -2.75460900 & 0.88811200 & 0.56213900
\end{array} \\
& \mathrm{H} \quad \begin{array}{llll}
-3.48740400 & 0.79917100 & 1.37617100
\end{array} \\
& \begin{array}{llll}
\mathrm{H} & -1.93880900 & 1.50731600 & 0.95139600
\end{array} \\
& \text { C } \quad-3.29841800 \quad-1.57467400 \quad 0.11050100 \\
& \text { H } \quad-3.80645200 \quad-1.70900800 \quad 1.07507900 \\
& \text { H } \quad-2.86493100 \quad-2.53467600 \quad-0.18180900 \\
& \text { C } \quad 1.55230200 \quad-0.50022800 \quad-0.07540700 \\
& \begin{array}{llll}
\text { N } & 2.67492500 & 0.29439100 & 0.00097300
\end{array} \\
& \begin{array}{llll}
\text { C } & 3.98746200 & -0.33361200 & -0.04997300
\end{array} \\
& \begin{array}{llll}
\mathrm{H} & 4.54127700 & -0.14955400 & 0.88006400
\end{array} \\
& \text { H } \quad 3.85107200 \quad-1.40486800 \quad-0.18531700 \\
& \text { H } \quad 4.57489400 \quad 0.07092200 \quad-0.88410100 \\
& \begin{array}{llll}
\text { C } & 2.68643700 & 1.73575200 & 0.17710900
\end{array} \\
& \begin{array}{llll}
\mathrm{H} & 1.68299100 & 2.15328200 & 0.13550800
\end{array} \\
& \mathrm{H} \quad 3.13394000 \quad 2.01101500 \quad 1.14251400 \\
& \begin{array}{llll}
\mathrm{H} & 3.28278000 & 2.20979300 & -0.61352700
\end{array} \\
& \begin{array}{llll}
0 & 1.63775400 & -1.71712600 & -0.21123300
\end{array} \\
& \mathrm{H} \quad-4.07945700 \quad-1.32219400 \quad-0.61848700 \\
& \text { C } \quad-3.41673000 \quad 1.62386500 \quad-0.61912800 \\
& \mathrm{H} \quad-4.26536600 \quad 1.05905500 \quad-1.01788300 \\
& \text { H } \quad-3.78830200 \quad 2.60672400 \quad-0.31028000 \\
& \mathrm{H} \quad-2.70506200 \quad 1.77213000 \quad-1.43794500
\end{aligned}
$$$$
\bar{\equiv} \mathrm{SiMe}_{3}
$$

$G_{\text {sol }}=-485.8121734$ Hatree

$$
\begin{array}{lrrr}
\text { C } & 2.76116700 & 0.00042400 & 0.00024400 \\
\text { H } & 3.82812700 & 0.00053400 & 0.00019600 \\
\text { C } & 1.54559200 & 0.00028900 & 0.00031000 \\
\text { Si } & -0.30017700 & -0.00010700 & -0.00006700 \\
\text { C } & -0.89989800 & -1.76420100 & -0.30589100 \\
\text { H } & -0.54550800 & -2.44484300 & 0.47474500 \\
\text { H } & -1.99503400 & -1.80516000 & -0.31480300 \\
\text { H } & -0.54251100 & -2.14425400 & -1.26822100 \\
\text { C } & -0.90058500 & 1.14679200 & -1.37453700 \\
\text { H } & -1.99573100 & 1.17172500 & -1.40702000 \\
\text { H } & -0.54650400 & 2.17101200 & -1.22010100 \\
\text { H } & -0.54376900 & 0.81388200 & -2.35437800 \\
\text { C } & -0.90109600 & 0.61701200 & 1.68009400 \\
\text { H } & -1.99623500 & 0.62792600 & 1.71952900 \\
\text { H } & -0.54295300 & -0.02530700 & 2.49090000 \\
\text { H } & -0.54848100 & 1.63409700 & 1.87877500
\end{array}
$$




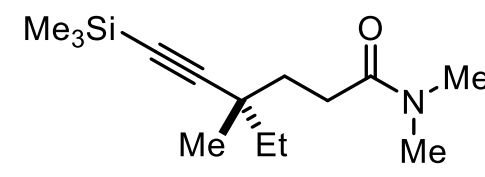

$G_{\text {sol }}=-968.5800892$ Hatree

\begin{tabular}{|c|c|c|c|}
\hline & -1.38576300 & 0.89389100 & -0.42373800 \\
\hline H & -1.23455600 & 0.57262500 & -1.45999100 \\
\hline C & 1.04299900 & 0.78791700 & 0.05991400 \\
\hline $\mathrm{H}$ & -2.23010100 & 1.59593000 & -0.4291030 \\
\hline C & -1.73597100 & -0.35386900 & 0.40819700 \\
\hline $\mathrm{H}$ & -1.94083100 & -0.09339300 & 1.45002900 \\
\hline $\mathrm{H}$ & -0.88235100 & -1.03483400 & 0.40129200 \\
\hline C & 0.11451500 & 2.82485700 & -1.00239400 \\
\hline C & -0.34460200 & 2.28201000 & 1.44903500 \\
\hline $\mathrm{H}$ & -1.20240700 & 2.96475400 & 1.43747500 \\
\hline $\mathrm{H}$ & -0.53360800 & 1.49890600 & 2.18755500 \\
\hline C & -2.88155300 & -1.13737200 & -0.23174900 \\
\hline N & -4.17746500 & -0.82699500 & 0.12312800 \\
\hline C & -5.26725400 & -1.58789200 & -0.47149600 \\
\hline $\mathrm{H}$ & -4.83933600 & -2.32713800 & -1.14613200 \\
\hline $\mathrm{H}$ & -5.85363200 & -2.09409100 & 0.30646400 \\
\hline $\mathrm{H}$ & -5.94063800 & -0.92611200 & -1.03154100 \\
\hline C & -4.58482300 & 0.19602300 & 1.06964300 \\
\hline $\mathrm{H}$ & -5.33625300 & 0.85274600 & 0.61229800 \\
\hline $\mathrm{H}$ & -5.03458200 & -0.24864700 & 1.96875500 \\
\hline $\mathrm{H}$ & -3.74515100 & 0.81593500 & 1.37657300 \\
\hline 0 & -2.63357500 & -2.00418000 & -1.06624200 \\
\hline $\mathrm{H}$ & 0.20145100 & 2.36052100 & -1.99171500 \\
\hline $\mathrm{H}$ & -0.79308700 & 3.44284500 & -1.02614300 \\
\hline C & 2.00748700 & 0.04156800 & 0.07492600 \\
\hline $\mathrm{Si}$ & 3.44649600 & -1.10489500 & 0.09361600 \\
\hline C & 4.85036400 & -0.30943700 & 1.07905300 \\
\hline $\mathrm{H}$ & 5.72490700 & -0.96944300 & 1.11082100 \\
\hline $\mathrm{H}$ & 5.16353000 & 0.63906500 & 0.63049300 \\
\hline $\mathrm{H}$ & 4.54682000 & -0.10647100 & 2.11130100 \\
\hline C & 4.00193000 & -1.42133200 & -1.68464700 \\
\hline $\mathrm{H}$ & 4.30480600 & -0.49229800 & -2.17865800 \\
\hline $\mathrm{H}$ & 4.85618400 & -2.10772500 & -1.70922900 \\
\hline H & 3.19630200 & -1.86736800 & -2.2767590 \\
\hline C & 2.92050100 & -2.72640600 & 0.90867300 \\
\hline $\mathrm{H}$ & 3.75243500 & -3.43978100 & 0.93045100 \\
\hline $\mathrm{H}$ & 2.59199800 & -2.56378000 & 1.94044600 \\
\hline $\mathrm{H}$ & 2.09317700 & -3.19271300 & 0.36393500 \\
\hline C & -0.12329400 & 1.68554200 & 0.03760500 \\
\hline $\mathrm{H}$ & 0.53254700 & 2.83977500 & $1.7845680 \mathrm{C}$ \\
\hline
\end{tabular}

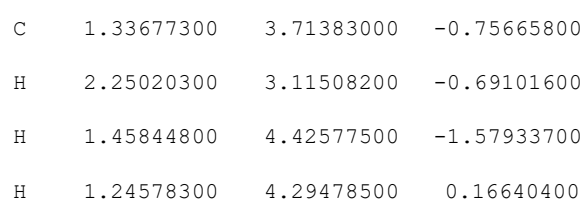

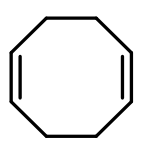

$G_{\text {sol }}=-311.7146914$ Hatree

$$
\begin{array}{lrrr}
\text { C } & -1.22081300 & 1.23015900 & -0.49560100 \\
\text { H } & -1.82957600 & 1.83240600 & -1.17129900 \\
\text { C } & 0.00009500 & 1.70379600 & -0.21974400 \\
\text { H } & 0.27913900 & 2.63400600 & -0.71442400 \\
\text { C } & -1.92237700 & -0.01882500 & -0.02235200 \\
\text { H } & -2.73933900 & 0.27307400 & 0.65601200 \\
\text { H } & -2.42472100 & -0.46485300 & -0.89259100 \\
\text { C } & -1.07638000 & -1.11175300 & 0.66491300 \\
\text { H } & -1.75961200 & -1.91671400 & 0.96021700 \\
\text { H } & -0.65887900 & -0.72747300 & 1.59814100 \\
\text { C } & -0.00000800 & -1.70379600 & -0.21969100 \\
\text { H } & -0.27899600 & -2.63389400 & -0.71461700 \\
\text { C } & 1.22087100 & -1.23007900 & -0.49558100 \\
\text { H } & 1.82939300 & -1.83210200 & -1.17170700 \\
\text { C } & 1.92247500 & 0.01893000 & -0.02233200 \\
\text { H } & 2.42495000 & 0.46500000 & -0.89246000 \\
\text { H } & 2.73929800 & -0.27320600 & 0.65611100 \\
\text { C } & 1.07626000 & 1.11160800 & 0.66502800 \\
\text { H } & 0.65839500 & 0.72690000 & 1.59787800 \\
\text { H } & 1.75920900 & 1.91661300 & 0.96090400
\end{array}
$$

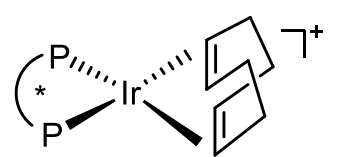

$G_{\text {sol }}=-2976.239125$ Hatree

$$
\begin{array}{lrrr}
\text { P } & -0.30442100 & 1.61503600 & -0.61975500 \\
\text { O } & 2.64787400 & 3.80743800 & 3.00800200 \\
\text { O } & 4.03758200 & -0.31239300 & 1.47616500 \\
\text { N } & 3.35409000 & 1.73566400 & 2.26170100 \\
\text { C } & 1.19820600 & 1.68563500 & 0.47178600 \\
\text { C } & 1.38996500 & 2.76731200 & 1.33331200 \\
\text { H } & 0.75041400 & 3.63555300 & 1.34420000 \\
\text { C } & 2.48844200 & 2.74295500 & 2.20260900 \\
\text { C } & 3.17028400 & 0.71625100 & 1.42873000 \\
\text { C } & 2.11686100 & 0.61632000 & 0.48510900
\end{array}
$$




\begin{tabular}{|c|c|c|c|}
\hline $\mathrm{H}$ & 3.71139300 & 4.75660500 & 4.43404000 \\
\hline $\mathrm{H}$ & 4.71357800 & 3.76184600 & 3.33143800 \\
\hline $\mathrm{H}$ & 3.73316300 & 2.97127700 & 4.58820300 \\
\hline C & 5.13826100 & -0.21396300 & 2.38818700 \\
\hline $\mathrm{H}$ & 5.71288000 & -1.12921900 & 2.24302300 \\
\hline $\mathrm{H}$ & 4.78587400 & -0.14475400 & 3.42063300 \\
\hline $\mathrm{H}$ & 5.75478000 & 0.66060100 & 2.16539500 \\
\hline C & 0.35857100 & 1.37330300 & -2.31596400 \\
\hline C & -0.30874900 & 0.52166600 & -3.20837700 \\
\hline $\mathrm{H}$ & -1.17726800 & -0.03402800 & -2.86650000 \\
\hline $\mathrm{C}$ & 0.14758300 & 0.37823500 & -4.52001300 \\
\hline $\mathrm{H}$ & -0.37350500 & -0.28543500 & -5.20343300 \\
\hline C & 1.27026900 & 1.08653700 & -4.95017000 \\
\hline $\mathrm{H}$ & 1.62277200 & 0.97854400 & -5.97154800 \\
\hline C & 1.94161000 & 1.93511300 & -4.06537200 \\
\hline $\mathrm{H}$ & 2.81547700 & 2.48751000 & -4.39756500 \\
\hline C & 1.49220200 & 2.07876100 & -2.75329100 \\
\hline $\mathrm{H}$ & 2.02580300 & 2.73307400 & -2.07142600 \\
\hline C & -0.94813400 & 3.35251300 & -0.64089800 \\
\hline C & -1.45803400 & 3.93120400 & 0.53836000 \\
\hline $\mathrm{H}$ & -1.45491300 & 3.36969800 & 1.46787000 \\
\hline C & -1.96401100 & 5.23036900 & 0.53724200 \\
\hline $\mathrm{H}$ & -2.33859100 & 5.66130700 & 1.46076000 \\
\hline C & -1.99312900 & 5.97043800 & -0.64700800 \\
\hline $\mathrm{H}$ & -2.39084800 & 6.98055300 & -0.64909900 \\
\hline C & -1.51203000 & 5.40223800 & -1.82520600 \\
\hline $\mathrm{H}$ & -1.53489200 & 5.96679300 & -2.75235000 \\
\hline C & -0.99131700 & 4.10526400 & -1.82458400 \\
\hline $\mathrm{H}$ & -0.61536400 & 3.68905700 & -2.75139800 \\
\hline C & 2.14907500 & -0.52995400 & -0.46386800 \\
\hline C & 1.27227600 & -1.63330800 & -0.46964600 \\
\hline P & -0.24609800 & -1.62574800 & 0.60257800 \\
\hline C & 0.39400700 & -1.38775900 & 2.30838500 \\
\hline C & -0.30134400 & -0.56291100 & 3.20460500 \\
\hline $\mathrm{H}$ & -1.18139700 & -0.02664400 & 2.86167600 \\
\hline C & 0.14231600 & -0.42033500 & 4.52057600 \\
\hline $\mathrm{H}$ & -0.40043200 & 0.22297400 & 5.20659900 \\
\hline C & 1.28031000 & -1.10316400 & 4.95173200 \\
\hline $\mathrm{H}$ & 1.62288500 & -0.99625600 & 5.97659800 \\
\hline $\mathrm{C}$ & 1.97994600 & -1.92456100 & 4.06329200 \\
\hline $\mathrm{H}$ & 2.86604400 & -2.45679300 & 4.39599200 \\
\hline $\mathrm{C}$ & 1.54375600 & -2.06626400 & 2.74649000 \\
\hline $\mathrm{H}$ & 2.10018600 & -2.69817800 & 2.06173000 \\
\hline C & -0.83145700 & -3.38278300 & 0.58552600 \\
\hline C & -1.31634200 & -3.94902100 & -0.61017500 \\
\hline
\end{tabular}

\begin{tabular}{|c|c|c|c|}
\hline $\mathrm{H}$ & -1.32605700 & -3.36439000 & -1.52550000 \\
\hline $\mathrm{C}$ & -1.78108000 & -5.26302600 & -0.64193600 \\
\hline $\mathrm{H}$ & -2.13741500 & -5.68426600 & -1.57708300 \\
\hline C & -1.79291400 & -6.03075100 & 0.52513100 \\
\hline $\mathrm{H}$ & -2.15901700 & -7.05249000 & 0.50169800 \\
\hline C & -1.33572600 & -5.47561000 & 1.71895600 \\
\hline $\mathrm{H}$ & -1.34573000 & -6.06216300 & 2.63257300 \\
\hline C & -0.85616700 & -4.16317000 & 1.75132300 \\
\hline $\mathrm{H}$ & -0.49822200 & -3.75634500 & 2.68943500 \\
\hline C & 1.51927500 & -2.70456600 & -1.33001700 \\
\hline H & 0.91091500 & -3.59483500 & -1.35561000 \\
\hline C & 2.63261500 & -2.63708100 & -2.17785400 \\
\hline O & 2.84690200 & -3.69234500 & -2.98266300 \\
\hline C & 3.99172100 & -3.65385800 & -3.84418600 \\
\hline H & 3.97234100 & -4.59721900 & -4.38979900 \\
\hline $\mathrm{H}$ & 4.91540300 & -3.56972600 & -3.26523900 \\
\hline $\mathrm{H}$ & 3.93139300 & -2.81181800 & -4.53914400 \\
\hline $\mathrm{N}$ & 3.46159800 & -1.59829800 & -2.21744600 \\
\hline C & 3.22327900 & -0.58799100 & -1.38725000 \\
\hline O & 4.05068800 & 0.47367700 & -1.41770600 \\
\hline C & 5.17385900 & 0.41767800 & -2.30567200 \\
\hline H & 5.70857900 & 1.35513900 & -2.15017500 \\
\hline H & 4.84723500 & 0.33298400 & -3.34539800 \\
\hline $\mathrm{H}$ & 5.81952300 & -0.43149000 & -2.06763400 \\
\hline Ir & -1.94781700 & -0.03187400 & 0.00071900 \\
\hline $\mathrm{C}$ & -3.60209700 & -1.57337000 & -0.11794700 \\
\hline H & -3.11014900 & -2.49645100 & -0.39793400 \\
\hline C & -3.44828900 & -1.17921600 & 1.21802400 \\
\hline $\mathrm{H}$ & -2.88400600 & -1.85293600 & 1.86126900 \\
\hline C & -4.73590800 & -1.16165900 & -1.04766000 \\
\hline $\mathrm{H}$ & -4.96725000 & -2.00618100 & -1.70413200 \\
\hline H & -5.64506900 & -0.97388100 & -0.47129100 \\
\hline C & -4.37618900 & 0.06254000 & -1.91370800 \\
\hline $\mathrm{H}$ & -5.28816700 & 0.55735500 & -2.27850500 \\
\hline H & -3.83568900 & -0.26914600 & -2.80686700 \\
\hline C & -4.33358100 & -0.20566300 & 1.96144300 \\
\hline $\mathrm{H}$ & -3.78649200 & 0.14178900 & 2.84455400 \\
\hline $\mathrm{H}$ & -5.22298900 & -0.72816000 & 2.34295100 \\
\hline C & -4.74717200 & 1.00821300 & 1.10448800 \\
\hline $\mathrm{H}$ & -5.66262700 & 0.79461700 & 0.54742100 \\
\hline H & -4.98891400 & 1.84504700 & 1.76702600 \\
\hline C & -3.64630200 & 1.45377400 & 0.15095900 \\
\hline H & -3.17759800 & 2.39199700 & 0.41948200 \\
\hline C & -3.50692400 & 1.06327500 & -1.18784900 \\
\hline $\mathrm{H}$ & -2.97653600 & 1.75363700 & -1.84193900 \\
\hline
\end{tabular}




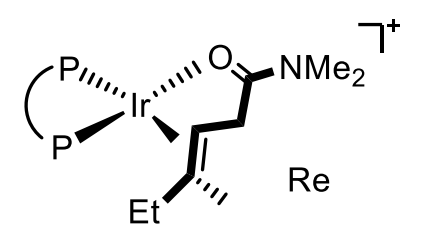

$G_{\text {sol }}=-3147.262192$ Hatree

$$
\begin{aligned}
& \text { O } \\
& \text { N } \\
& \text { C }
\end{aligned}
$$$$
0
$$

$$
\text { "н }
$$

\section{H
H
C
H}$$
\text { , }
$$ 


\begin{tabular}{|c|c|c|c|}
\hline C & -3.01014300 & 1.35094100 & -1.55663400 \\
\hline C & -4.41606700 & 1.10118500 & 0.61050300 \\
\hline $\mathrm{H}$ & -5.37841900 & 1.32855500 & 0.13394300 \\
\hline $\mathrm{H}$ & -4.43928800 & 1.58449900 & 1.59535100 \\
\hline C & -2.46613100 & 2.43787800 & -2.47245800 \\
\hline $\mathrm{H}$ & -1.83855500 & 1.99628200 & -3.25315200 \\
\hline $\mathrm{H}$ & -1.83899400 & 3.12586600 & -1.90346100 \\
\hline C & -3.79194200 & 0.28348900 & -2.30345400 \\
\hline $\mathrm{H}$ & -3.25589100 & -0.01742600 & -3.20889800 \\
\hline $\mathrm{H}$ & -3.98973800 & -0.61429000 & -1.72110000 \\
\hline C & -4.28283000 & -0.39176600 & 0.82874100 \\
\hline $\mathrm{N}$ & -5.28634400 & -1.11557500 & 1.34448400 \\
\hline C & -5.14941600 & -2.56571200 & 1.51429900 \\
\hline $\mathrm{H}$ & -5.11135900 & -2.81723200 & 2.57961200 \\
\hline $\mathrm{H}$ & -6.01600200 & -3.06254900 & 1.06750400 \\
\hline $\mathrm{H}$ & -4.23944500 & -2.90715400 & 1.02705700 \\
\hline C & -6.56534400 & -0.55512000 & 1.77787600 \\
\hline $\mathrm{H}$ & -7.37048400 & -0.87024100 & 1.10467700 \\
\hline $\mathrm{H}$ & -6.79475600 & -0.92049700 & 2.78359500 \\
\hline $\mathrm{H}$ & -6.53432000 & 0.53152500 & 1.80931700 \\
\hline 0 & -3.19331500 & -0.95247900 & 0.51839900 \\
\hline $\mathrm{H}$ & -4.75685100 & 0.69660000 & -2.62995200 \\
\hline C & -3.59098400 & 3.25430500 & -3.14274900 \\
\hline $\mathrm{H}$ & -3.15804300 & 4.06389300 & -3.73777700 \\
\hline $\mathrm{H}$ & -4.20239300 & 2.64443600 & -3.81401200 \\
\hline $\mathrm{H}$ & -4.25229500 & 3.70618000 & -2.39572600 \\
\hline
\end{tabular}

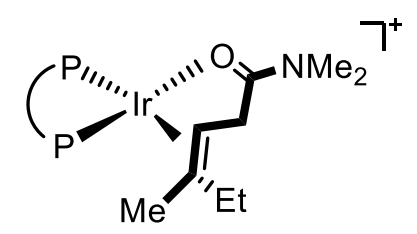

$G_{\text {sol }}=-3147.262375$ Hatree

$\begin{array}{llll}\text { P } & -0.28172300 & -1.53838100 & 0.35337800 \\ \text { O } & 2.73804300 & -3.79598900 & -3.16838800 \\ \text { O } & 4.56799400 & -0.23806900 & -0.87224900 \\ \text { N } & 3.66963100 & -2.01698300 & -2.02161700 \\ \text { C } & 1.31473700 & -1.76959500 & -0.54467900 \\ \text { C } & 1.43235500 & -2.75211800 & -1.52820900 \\ \text { H } & 0.64070600 & -3.45112500 & -1.75706100 \\ \text { C } & 2.64092600 & -2.83329300 & -2.23311900 \\ \text { C } & 3.54092400 & -1.08219700 & -1.08336700 \\ \text { C } & 2.38673300 & -0.89423300 & -0.28613700 \\ \text { C } & 3.97259900 & -3.90347300 & -3.88681900 \\ \text { H } & 3.83161900 & -4.73422600 & -4.57844000\end{array}$

$$
\begin{aligned}
& 4.80476600 \quad-4.11212200 \quad-3.20880100 \\
& 4.18813700 \quad-2.98329900 \quad-4.43686500 \\
& \begin{array}{llll}
5.75232200 & -0.42260600 & -1.65501200
\end{array} \\
& 6.44648700 \quad 0.34440400 \quad-1.31004100 \\
& \begin{array}{llll}
5.54052100 & -0.29326200 & -2.71972900
\end{array} \\
& 6.17706400-1.41731100-1.49665700 \\
& \begin{array}{lll}
0.13748000 & -1.78726200 & 2.12490000
\end{array} \\
& \begin{array}{lll}
-0.52856100 & -1.02881000 & 3.09939300
\end{array} \\
& \begin{array}{lll}
-1.24640900 & -0.27459500 & 2.79040800
\end{array} \\
& \begin{array}{lll}
-0.26114100 & -1.23011000 \quad 4.45421100
\end{array} \\
& \begin{array}{lll}
-0.77712500 & -0.63390600 & 5.20102600
\end{array} \\
& 0.67224200 \quad-2.19083600 \quad 4.84736600 \\
& 0.87955700 \quad-2.34840700 \quad 5.90165600 \\
& \begin{array}{lll}
1.34346300 & -2.94629900 & 3.88275300
\end{array} \\
& 2.07295900 \quad-3.69189600 \quad 4.18474700 \\
& \begin{array}{lll}
1.08257000 & -2.74509300 & 2.52701000
\end{array} \\
& \begin{array}{lll}
1.62178900 & -3.32446400 & 1.78440800
\end{array} \\
& \begin{array}{lll}
-1.30276600 & -3.00731200 & -0.10723800
\end{array} \\
& \begin{array}{lll}
-1.91832000 & -3.03103400 & -1.37068100
\end{array} \\
& \begin{array}{lll}
-1.78458000 & -2.19523500 & -2.05047600
\end{array} \\
& \begin{array}{lll}
-2.69907000 & -4.11850000 & -1.75971200
\end{array} \\
& \begin{array}{llll}
-3.15765300 & -4.12796700 & -2.74415900
\end{array} \\
& -2.89359800 \quad-5.19044200 \quad-0.88470700 \\
& \text { H } \quad-3.50428000 \quad-6.03626100 \quad-1.18615600 \\
& \begin{array}{llll}
\text { C } & -2.29846700 & -5.16953700 & 0.37657300
\end{array} \\
& \text { H } \quad-2.44430900 \quad-5.99899000 \quad 1.06229800 \\
& \begin{array}{llll}
\text { C } & -1.50444000 & -4.08678000 & 0.76436400
\end{array} \\
& \mathrm{H} \quad-1.04493900 \quad-4.08973500 \quad 1.74606500 \\
& \begin{array}{llll}
\text { C } & 2.40765200 & 0.13423300 & 0.78869700
\end{array} \\
& \begin{array}{llll}
\text { C } & 1.65705400 & 1.33009000 & 0.80480300
\end{array} \\
& \begin{array}{llll}
\text { P } & 0.36487500 & 1.67019900 & -0.50195600
\end{array} \\
& \text { C } \quad 1.31991700 \quad 1.48734200 \quad-2.06797600 \\
& \begin{array}{llll}
\text { C } & 0.79052400 & 0.72953400 & -3.12131200
\end{array} \\
& \text { H } \quad-0.16101700 \quad 0.22761000 \quad-2.98104100 \\
& \begin{array}{llll}
\text { C } & 1.48978300 & 0.59942400 & -4.32234800
\end{array} \\
& \text { H } \quad 1.07051200 \quad 0.00700500 \quad-5.13017500 \\
& \text { C } \quad 2.72624800 \quad 1.22672600 \quad-4.48180400 \\
& \text { H } \quad 3.26785200 \quad 1.13261800 \quad-5.41838200 \\
& \begin{array}{llll}
\text { C } & 3.27067800 & 1.96999000 & -3.43108600
\end{array} \\
& \begin{array}{llll}
\mathrm{H} & 4.23729000 & 2.45119500 & -3.54711500
\end{array} \\
& \begin{array}{llll}
\text { C } & 2.57778800 & 2.09361600 & -2.22687800
\end{array} \\
& \text { H } \quad 3.02092600 \quad 2.65455600 \quad-1.40981100 \\
& \begin{array}{llll}
\text { C } & 0.06776600 & 3.49476400 & -0.33502100
\end{array} \\
& \begin{array}{llll}
\text { C } & -0.68423400 & 3.94844600 & 0.76495200
\end{array}
\end{aligned}
$$




\begin{tabular}{|c|c|c|c|}
\hline C & -0.94880600 & 5.30629200 & 0.93986100 \\
\hline $\mathrm{H}$ & -1.51926900 & 5.63766500 & 1.80259900 \\
\hline C & -0.48311300 & 6.23626500 & 0.00693200 \\
\hline $\mathrm{H}$ & -0.69253600 & 7.29341000 & 0.13896900 \\
\hline C & 0.24851600 & 5.79780900 & -1.09578100 \\
\hline $\mathrm{H}$ & 0.60851000 & 6.51212800 & -1.83014300 \\
\hline C & 0.52575400 & 4.43852200 & -1.26595400 \\
\hline $\mathrm{H}$ & 1.09187300 & 4.12108900 & -2.13342200 \\
\hline C & 1.84341300 & 2.24072000 & 1.84626800 \\
\hline $\mathrm{H}$ & 1.32262100 & 3.18446100 & 1.90299200 \\
\hline C & 2.77085300 & 1.93017400 & 2.84914100 \\
\hline 0 & 2.93483400 & 2.83923300 & 3.82822800 \\
\hline C & 3.89617700 & 2.55069000 & 4.84991900 \\
\hline $\mathrm{H}$ & 3.86780200 & 3.40828900 & 5.52235700 \\
\hline $\mathrm{H}$ & 4.89746900 & 2.43540800 & 4.42569400 \\
\hline $\mathrm{H}$ & 3.63425600 & 1.63625000 & 5.38947900 \\
\hline $\mathrm{N}$ & 3.47590000 & 0.80334700 & 2.87337300 \\
\hline C & 3.29307300 & -0.05881100 & 1.87720500 \\
\hline 0 & 3.98692200 & -1.21377800 & 1.89547400 \\
\hline C & 4.91313900 & -1.41559500 & 2.96828600 \\
\hline $\mathrm{H}$ & 5.37710000 & -2.38212600 & 2.76863800 \\
\hline $\mathrm{H}$ & 4.39763800 & -1.43304000 & 3.93236200 \\
\hline $\mathrm{H}$ & 5.67092100 & -0.62809000 & 2.98671100 \\
\hline Ir & -1.51125100 & 0.43043500 & -0.14872800 \\
\hline C & -3.13222300 & 1.89178600 & -0.34754300 \\
\hline $\mathrm{H}$ & -2.79923600 & 2.90432400 & -0.14163500 \\
\hline C & -2.91768300 & 1.42701900 & -1.66951500 \\
\hline C & -4.30419300 & 1.46639000 & 0.53489300 \\
\hline $\mathrm{H}$ & -5.26226000 & 1.70524300 & 0.05680300 \\
\hline $\mathrm{H}$ & -4.27383500 & 2.04696700 & 1.46546000 \\
\hline C & -2.30996700 & 2.38332200 & -2.67537700 \\
\hline H & -1.71918000 & 1.86599800 & -3.43444600 \\
\hline $\mathrm{H}$ & -3.12146000 & 2.90456600 & -3.19946400 \\
\hline $\mathrm{H}$ & -1.69285100 & 3.15042000 & -2.20920500 \\
\hline C & -3.74810900 & 0.32622600 & -2.32333000 \\
\hline $\mathrm{H}$ & -3.11098000 & -0.19766300 & -3.04611800 \\
\hline $\mathrm{H}$ & -4.05353300 & -0.42543600 & -1.59572800 \\
\hline C & -4.99794800 & 0.85521400 & -3.06067700 \\
\hline $\mathrm{H}$ & -4.73643300 & 1.49878500 & -3.90423400 \\
\hline $\mathrm{H}$ & -5.65036400 & 1.43000300 & -2.39444900 \\
\hline C & -4.25703900 & -0.00145700 & 0.90565000 \\
\hline $\mathrm{N}$ & -5.28531000 & -0.60082400 & 1.52294000 \\
\hline C & -5.22620900 & -2.02835900 & 1.85331100 \\
\hline $\mathrm{H}$ & -5.17060800 & -2.16001700 & 2.93925000 \\
\hline $\mathrm{H}$ & -6.13270000 & -2.51952200 & 1.48660900 \\
\hline
\end{tabular}

$\begin{array}{llll}\text { H } & -4.35199200 & -2.47630600 & 1.38733800 \\ \text { C } & -6.51241900 & 0.08180200 & 1.92922700 \\ \text { H } & -7.36279900 & -0.27678100 & 1.33867100 \\ \text { H } & -6.71125500 & -0.13216600 & 2.98414100 \\ \text { H } & -6.42831200 & 1.15936900 & 1.80940800 \\ \text { O } & -3.21508000 & -0.66016100 & 0.62842100 \\ \text { H } & -5.58021900 & 0.01665600 & -3.45445800\end{array}$

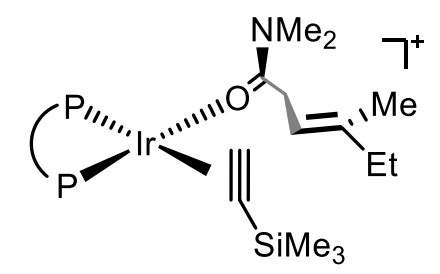

$$
\begin{aligned}
& G_{\text {sol }}=-3633.068367 \text { Hatree } \\
& \text { Ir } \quad 0.83937800 \quad 0.36597000 \quad-0.43117200 \\
& \begin{array}{llll}
\text { C } & 2.15630100 & 1.78957300 & -1.29957100
\end{array} \\
& \begin{array}{llll}
\mathrm{H} & 2.37329300 & 1.92160300 & -2.34401200
\end{array} \\
& \text { N } \quad-3.88375700 \quad-1.15025100 \quad 3.03168700 \\
& \text { C } \quad-1.79872100 \quad-1.48160400 \quad 1.20502600 \\
& \begin{array}{llll}
\text { C } & -1.73434700 & -2.07139700 & 2.46840900
\end{array} \\
& \mathrm{H} \quad-0.90002400 \quad-2.67599400 \quad 2.79326000 \\
& \begin{array}{llll}
\text { C } & -2.81350400 & -1.87706200 & 3.34048200
\end{array} \\
& \text { C } \quad-3.92838300 \quad-0.58888900 \quad 1.82625700 \\
& \begin{array}{llll}
\text { C } & -2.92095700 & -0.71476200 & 0.83920400
\end{array} \\
& \text { C } \quad-3.83790700 \quad-2.29161800 \quad 5.44587300 \\
& \mathrm{H} \quad-3.56807800 \quad-2.84719400 \quad 6.34417000 \\
& \mathrm{H} \quad-4.76204500 \quad-2.68964300 \quad 5.01772200 \\
& \mathrm{H} \quad-3.98565100 \quad-1.23441300 \quad 5.68311300 \\
& \begin{array}{llll}
\text { C } & -6.03885700 & 0.28609700 & 2.48409400
\end{array} \\
& \begin{array}{llll}
\mathrm{H} & -6.79965300 & 0.91107100 & 2.01533300
\end{array} \\
& \mathrm{H} \quad-5.66733300 \quad 0.75878400 \quad 3.39727100 \\
& \begin{array}{llll}
\text { H } & -6.45488400 & -0.69265500 & 2.73675100
\end{array} \\
& \begin{array}{llll}
\text { C } & -1.06576300 & -2.46047600 & -1.44286000
\end{array} \\
& \begin{array}{llll}
\text { C } & -0.60988000 & -2.08970900 & -2.71763400
\end{array} \\
& \text { H } \quad 0.11516000 \quad-1.28669600 \quad-2.81183900 \\
& \text { C } \quad-1.09668100 \quad-2.73691800 \quad-3.85445200 \\
& \text { H } \quad-0.74365000 \quad-2.43915100 \quad-4.83736800 \\
& \text { C } \quad-2.03991900 \quad-3.75853400 \quad-3.72816300 \\
& \mathrm{H} \quad-2.41733600 \quad-4.26275800 \quad-4.61284700 \\
& \begin{array}{llll}
\text { C } & -2.50345900 & -4.12729800 & -2.46296000
\end{array} \\
& \mathrm{H} \quad-3.24180500 \quad-4.91717300 \quad-2.36176900 \\
& \text { C } \quad-2.02503400 \quad-3.47902200-1.32354900 \\
& \mathrm{H} \quad-2.40756200 \quad-3.75695400 \quad-0.34653400 \\
& \begin{array}{llll}
\text { C } & 0.73514700 & -2.86928400 & 0.83488100
\end{array} \\
& \begin{array}{llll}
\text { C } & 1.54916800 & -2.44801400 & 1.90078100
\end{array}
\end{aligned}
$$




\begin{tabular}{|c|c|c|c|}
\hline $\mathrm{H}$ & 1.51498600 & -1.41166100 & 2.22506500 \\
\hline C & 2.39723200 & -3.34794300 & 2.54618700 \\
\hline $\mathrm{H}$ & 3.00619600 & -3.01141500 & 3.38030900 \\
\hline $\mathrm{C}$ & 2.46068200 & -4.67819400 & 2.12194300 \\
\hline $\mathrm{H}$ & 3.11971300 & -5.37994400 & 2.62451100 \\
\hline C & 1.66851400 & -5.10130500 & 1.05428100 \\
\hline $\mathrm{H}$ & 1.71020800 & -6.13402700 & 0.72057700 \\
\hline C & 0.80738100 & -4.20477400 & 0.41535900 \\
\hline $\mathrm{H}$ & 0.19337400 & -4.55275200 & -0.40767900 \\
\hline C & -3.15076000 & -0.11498800 & -0.50369700 \\
\hline C & -2.46085500 & 0.98935400 & -1.05098900 \\
\hline $\mathrm{P}$ & -0.96426200 & 1.70861900 & -0.19940700 \\
\hline C & -1.57922900 & 2.07962300 & 1.49523400 \\
\hline C & -0.77970400 & 1.77317300 & 2.60425600 \\
\hline $\mathrm{H}$ & 0.16952400 & 1.27259400 & 2.44169700 \\
\hline C & -1.20731200 & 2.09122800 & 3.89502300 \\
\hline $\mathrm{H}$ & -0.58089200 & 1.84717000 & 4.74787600 \\
\hline C & -2.43808900 & 2.72069800 & 4.08654300 \\
\hline $\mathrm{H}$ & -2.76919500 & 2.97473900 & 5.08914200 \\
\hline C & -3.24747200 & 3.01883400 & 2.98671900 \\
\hline $\mathrm{H}$ & -4.20870500 & 3.50258100 & 3.13289200 \\
\hline C & -2.82611900 & 2.69479400 & 1.69720000 \\
\hline $\mathrm{H}$ & -3.47046300 & 2.91333500 & 0.85134000 \\
\hline C & -0.75403000 & 3.37127300 & -0.99029900 \\
\hline C & -0.27536000 & 3.44118500 & -2.31142500 \\
\hline $\mathrm{H}$ & -0.04476200 & 2.52760800 & -2.84981300 \\
\hline C & -0.10142000 & 4.66977500 & -2.94547800 \\
\hline $\mathrm{H}$ & 0.25641500 & 4.70072100 & -3.97039100 \\
\hline C & -0.38341900 & 5.85629800 & -2.26366400 \\
\hline $\mathrm{H}$ & -0.24397300 & 6.81458800 & -2.75478600 \\
\hline C & -0.84302600 & 5.80096700 & -0.94932100 \\
\hline $\mathrm{H}$ & -1.06135200 & 6.71692700 & -0.40835500 \\
\hline C & -1.02939800 & 4.56843900 & -0.31609100 \\
\hline $\mathrm{H}$ & -1.38453700 & 4.55315300 & 0.70687600 \\
\hline C & -2.86149300 & 1.50193300 & -2.28593300 \\
\hline $\mathrm{H}$ & -2.39585800 & 2.36149400 & -2.74347400 \\
\hline C & -3.93436300 & 0.89182100 & -2.94864000 \\
\hline 0 & -4.30262900 & 1.42385400 & -4.12863700 \\
\hline C & -5.41086700 & 0.82651100 & -4.81137300 \\
\hline $\mathrm{H}$ & -5.53356600 & 1.40845700 & -5.72496300 \\
\hline $\mathrm{H}$ & -6.31960700 & 0.87765900 & -4.20519300 \\
\hline $\mathrm{H}$ & -5.20613300 & -0.22030000 & -5.05257500 \\
\hline N & -4.58522100 & -0.16519800 & -2.47233400 \\
\hline C & -4.19985200 & -0.64558700 & -1.29372900 \\
\hline 0 & -4.84002900 & -1.72590500 & -0.80374800 \\
\hline
\end{tabular}

\begin{tabular}{|c|c|c|c|}
\hline C & -5.92815200 & -2.25624100 & -1.56799400 \\
\hline $\mathrm{H}$ & -6.31453200 & -3.08806700 & -0.97794300 \\
\hline $\mathrm{H}$ & -5.58534300 & -2.60945300 & -2.54434000 \\
\hline $\mathrm{H}$ & -6.70695900 & -1.50404000 & -1.71787300 \\
\hline C & 5.50406200 & -0.10637700 & -0.25790200 \\
\hline $\mathrm{H}$ & 5.22158300 & 0.58432300 & -1.05093500 \\
\hline C & 6.77754100 & -0.08207700 & 0.17421600 \\
\hline C & 4.36423500 & -0.96062700 & 0.25557300 \\
\hline $\mathrm{H}$ & 3.66896600 & -0.34831700 & 0.83527500 \\
\hline $\mathrm{H}$ & 4.71569700 & -1.76180600 & 0.90997600 \\
\hline C & 7.77363000 & 0.86700800 & -0.46671600 \\
\hline C & 7.32309500 & -0.93580900 & 1.29349200 \\
\hline $\mathrm{H}$ & 7.69586600 & -0.29849700 & 2.10498500 \\
\hline $\mathrm{H}$ & 6.58655200 & -1.61745300 & 1.72407900 \\
\hline C & 3.52549100 & -1.54685000 & -0.87063000 \\
\hline $\mathrm{N}$ & 4.01440700 & -2.57953700 & -1.58698700 \\
\hline $\mathrm{C}$ & 3.21934100 & -3.20301300 & -2.64467700 \\
\hline $\mathrm{H}$ & 3.75538000 & -3.13998300 & -3.59772200 \\
\hline $\mathrm{H}$ & 3.05052000 & -4.25889400 & -2.40743400 \\
\hline $\mathrm{H}$ & 2.26227800 & -2.69656200 & -2.72990800 \\
\hline C & 5.28780100 & -3.23784800 & -1.29670200 \\
\hline $\mathrm{H}$ & 5.99372200 & -2.54313700 & -0.84754900 \\
\hline $\mathrm{H}$ & 5.14161000 & -4.10158300 & -0.63666600 \\
\hline $\mathrm{H}$ & 5.71837600 & -3.59217400 & -2.23680400 \\
\hline 0 & 2.39751200 & -1.07153900 & -1.15627900 \\
\hline $\mathrm{H}$ & 8.26256500 & 1.45732700 & 0.32056900 \\
\hline $\mathrm{H}$ & 7.23799200 & 1.57955500 & -1.10351500 \\
\hline P & -0.37353600 & -1.62339000 & 0.03803400 \\
\hline O & -4.99683800 & 0.16647200 & 1.50955200 \\
\hline $\mathrm{O}$ & -2.73653700 & -2.46806400 & 4.54714600 \\
\hline C & 2.32417800 & 2.02059900 & -0.07223300 \\
\hline Si & 3.04452900 & 2.95032900 & 1.39143200 \\
\hline C & 3.34891700 & 1.78135800 & 2.84349300 \\
\hline $\mathrm{H}$ & 3.67390700 & 2.35888400 & 3.71641100 \\
\hline $\mathrm{H}$ & 2.44716300 & 1.23187100 & 3.13056400 \\
\hline $\mathrm{H}$ & 4.13691900 & 1.05513000 & 2.62223700 \\
\hline C & 4.68029400 & 3.66974400 & 0.78052900 \\
\hline $\mathrm{H}$ & 5.37933100 & 2.87970500 & 0.49102200 \\
\hline $\mathrm{H}$ & 4.52753800 & 4.32564600 & -0.08246200 \\
\hline $\mathrm{H}$ & 5.15124700 & 4.26420100 & 1.57184100 \\
\hline C & 1.88736100 & 4.34402000 & 1.91134900 \\
\hline $\mathrm{H}$ & 1.65839600 & 5.00586700 & 1.07121600 \\
\hline $\mathrm{H}$ & 0.94305000 & 3.97219800 & 2.31765000 \\
\hline $\mathrm{H}$ & 2.37288700 & 4.94385500 & 2.69026900 \\
\hline $\mathrm{H}$ & 8.17705100 & -1.53476400 & 0.95690300 \\
\hline
\end{tabular}




$\begin{array}{llll}\text { C } & 8.85828200 & 0.16483300 & -1.30432100 \\ \text { H } & 9.53309900 & 0.90206100 & -1.74934600 \\ \text { H } & 9.46877200 & -0.51429500 & -0.70143900 \\ \text { H } & 8.41024300 & -0.41520200 & -2.11780800\end{array}$

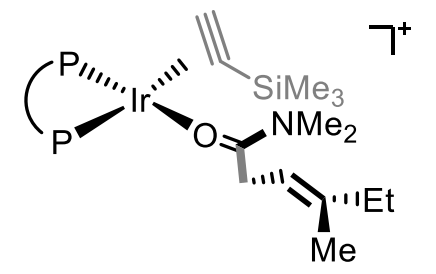

$G_{\text {sol }}=-3633.066174$ Hatree$$
\text { C }
$$ 


\begin{tabular}{|c|c|c|c|}
\hline $\mathrm{H}$ & 6.90892400 & -1.58233300 & 0.06481900 \\
\hline C & -4.99810700 & -0.73554300 & 1.04767700 \\
\hline $\mathrm{H}$ & -5.31948800 & 0.15284600 & 0.50655900 \\
\hline C & -5.71977600 & -1.10947000 & 2.11839200 \\
\hline C & -3.73483300 & -1.38627300 & 0.52362400 \\
\hline $\mathrm{H}$ & -3.67698500 & -2.44146800 & 0.80880400 \\
\hline $\mathrm{H}$ & -2.85338100 & -0.90080100 & 0.95249900 \\
\hline C & -6.93686900 & -0.30340100 & 2.52916100 \\
\hline C & -5.40012800 & -2.30149200 & 2.98719700 \\
\hline $\mathrm{H}$ & -6.29356600 & -2.91948100 & 3.13694300 \\
\hline $\mathrm{H}$ & -4.61167400 & -2.93769900 & 2.57917500 \\
\hline C & -3.55107300 & -1.27374400 & -0.98255800 \\
\hline $\mathrm{N}$ & -4.45202600 & -1.84318800 & -1.81136100 \\
\hline C & -4.26859700 & -1.75356700 & -3.26125600 \\
\hline $\mathrm{H}$ & -3.84060300 & -2.68413100 & -3.65081600 \\
\hline $\mathrm{H}$ & -5.24033400 & -1.58670800 & -3.73365500 \\
\hline $\mathrm{H}$ & -3.59916000 & -0.92844500 & -3.49125800 \\
\hline C & -5.49153600 & -2.77276100 & -1.37061800 \\
\hline $\mathrm{H}$ & -5.74970100 & -2.60437000 & -0.32784200 \\
\hline $\mathrm{H}$ & -6.39003300 & -2.61127300 & -1.97233000 \\
\hline $\mathrm{H}$ & -5.16294200 & -3.81006700 & -1.50861800 \\
\hline 0 & -2.58404700 & -0.64253900 & -1.47656000 \\
\hline $\mathrm{H}$ & -7.80687200 & -0.97357000 & 2.57580600 \\
\hline $\mathrm{H}$ & -7.15721100 & 0.44049700 & 1.75565200 \\
\hline P & 1.13515200 & 1.65044200 & -0.49426500 \\
\hline 0 & 3.88459600 & -1.36552700 & 2.72709900 \\
\hline 0 & 2.53359900 & 2.72728800 & 4.36722900 \\
\hline C & -1.96788700 & 2.09892800 & -1.83243300 \\
\hline $\mathrm{Si}$ & -3.51742900 & 3.04070700 & -1.36819900 \\
\hline C & -3.80080300 & 3.03359900 & 0.49697000 \\
\hline $\mathrm{H}$ & -4.78459900 & 3.46095700 & 0.72254100 \\
\hline $\mathrm{H}$ & -3.77363700 & 2.02297500 & 0.91486200 \\
\hline $\mathrm{H}$ & -3.05440500 & 3.63683800 & 1.01992800 \\
\hline C & -3.32777500 & 4.80265000 & -2.01427300 \\
\hline $\mathrm{H}$ & -2.46656400 & 5.30453200 & -1.56446900 \\
\hline $\mathrm{H}$ & -3.19424800 & 4.81140000 & -3.10086300 \\
\hline $\mathrm{H}$ & -4.22329800 & 5.39132000 & -1.78362600 \\
\hline C & -4.96732400 & 2.19818700 & -2.24310400 \\
\hline $\mathrm{H}$ & -4.81078700 & 2.17793900 & -3.32645500 \\
\hline $\mathrm{H}$ & -5.11578000 & 1.16765900 & -1.90667200 \\
\hline $\mathrm{H}$ & -5.89449600 & 2.75077100 & -2.05166300 \\
\hline $\mathrm{H}$ & -5.07344400 & -1.98145200 & 3.98366600 \\
\hline C & -6.78881800 & 0.40711800 & 3.88684200 \\
\hline $\mathrm{H}$ & -7.68849100 & 0.98657200 & 4.11429200 \\
\hline $\mathrm{H}$ & -6.64061300 & -0.30310500 & 4.70596900 \\
\hline
\end{tabular}

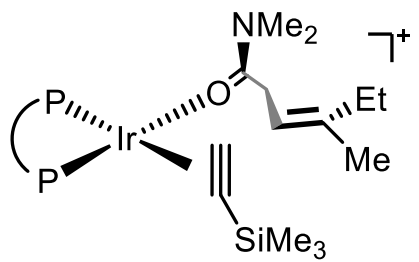

$G_{\text {sol }}=-3633.066839$ Hatree

$0.79867400 \quad 0.44455400$

1.99872000

1.93256400

2.14904600

2.08415500

$-3.65461300$

$-1.36274600$

$-1.65542000$

$-1.55094500$

3.17243700

$-1.49681900$

$-0.61926300$

$-2.16549500$

1.23250000

$-2.53681300$

$-2.73677600$

2.47570000

$-3.78853200$

$-2.83025100$

$-2.04438200$

2.74194000

$-3.43031300$

$-3.08762500$

$-0.77580100$

3.40662700

$-4.35443000$

$-0.83064400$

1.98601000

$-3.61781000$

$-5.89969200$

$-2.56120300$

0.94495500

$-6.71100600$

$-5.50228900$

$-6.25798900$

$-3.12385000$

5.54871100

$-1.02567900$

$-0.65717500$

$-2.99525400$

6.41727200

5.15700200

$-1.51889000$

5.82108700

$-0.01807500$

2.77459000

0.58319600

2. 36304500

o.

0.02394800

0.44738300

3.68056800

$-1.02105500$

3.02066500

$-1.17398600$

$-0.88918500$

$-2.45218300$

$-1.46759800$

$-2.05945800$

$-2.46007900$

$-2.43526900$

$-2.04085600$

$-2.75810400$

$-1.20329000$

$-2.87541800$

$-3.1281170$

$-2.69261300$

$-3.87891200$

$-2.36290600$

$-4.87370600$

$-3.76043200$

$-3.72090000$

$-4.26852200$

$-4.59308300$

$-4.17099800$

$-2.43959400$

$-1.92721300$

$-4.99765200$

$-2.31351000$

$-2.24232000-3.83054000$

$-3.51809500$

$-1.31568800$

$-0.32511000$

$0.91690800-2.80520500$

0.70000000

1.76248600

$-2.36160400$

1.73153800

$1.69343900-1.33366500$

2.07628800

$2.68449800 \quad-3.22913200$

2.31720700

H $\quad 3.31758500$

$-2.87677600$

3.12647300

2.79104500

$-4.54737700$

1.86496600

3.50767900

$-5.22401900$

2.32096400

$\begin{array}{lll}1.96785800 & -4.99128300 & 0.82958300\end{array}$ 


\begin{tabular}{|c|c|c|c|}
\hline $\mathrm{H}$ & 2.04299300 & -6.01496100 & 0.47455000 \\
\hline C & 1.03268500 & -4.12817400 & 0.25176000 \\
\hline $\mathrm{H}$ & 0.39475500 & -4.49217300 & -0.54568500 \\
\hline C & -3.16142900 & -0.21613400 & -0.36976200 \\
\hline C & -2.55565100 & 0.93015000 & -0.93023500 \\
\hline $\mathrm{P}$ & -1.04350000 & 1.70075100 & -0.15314900 \\
\hline C & -1.56245300 & 2.00394200 & 1.58620800 \\
\hline C & -0.67177800 & 1.72285900 & 2.63052400 \\
\hline $\mathrm{H}$ & 0.29176600 & 1.28387600 & 2.39107500 \\
\hline C & -1.02666800 & 1.98894400 & 3.95461500 \\
\hline $\mathrm{H}$ & -0.33011200 & 1.76466900 & 4.75700400 \\
\hline C & -2.27523700 & 2.54116600 & 4.24424600 \\
\hline $\mathrm{H}$ & -2.55076500 & 2.75397100 & 5.27290400 \\
\hline C & -3.17349600 & 2.81576300 & 3.20911700 \\
\hline $\mathrm{H}$ & -4.14783700 & 3.24053100 & 3.43178800 \\
\hline C & -2.82444100 & 2.54358600 & 1.88645000 \\
\hline $\mathrm{H}$ & -3.53620100 & 2.74262900 & 1.09134200 \\
\hline C & -0.96070100 & 3.38873000 & -0.91321600 \\
\hline C & -0.56174200 & 3.51227000 & -2.25652600 \\
\hline $\mathrm{H}$ & -0.31354600 & 2.62379500 & -2.82772700 \\
\hline C & -0.49028200 & 4.76162300 & -2.86950100 \\
\hline $\mathrm{H}$ & -0.19255300 & 4.83390900 & -3.91141300 \\
\hline C & -0.79762800 & 5.91610700 & -2.14504100 \\
\hline $\mathrm{H}$ & -0.73791700 & 6.89066600 & -2.62006900 \\
\hline C & -1.17925500 & 5.80820100 & -0.80907100 \\
\hline $\mathrm{H}$ & -1.41656400 & 6.69922200 & -0.23544700 \\
\hline C & -1.26274800 & 4.55432600 & -0.19642600 \\
\hline $\mathrm{H}$ & -1.55979700 & 4.49728200 & 0.84339700 \\
\hline C & -3.04660600 & 1.44570900 & -2.13067500 \\
\hline $\mathrm{H}$ & -2.64699700 & 2.33455300 & -2.59472000 \\
\hline C & -4.12343000 & 0.79680900 & -2.74825400 \\
\hline 0 & -4.57844700 & 1.33080700 & -3.89665200 \\
\hline C & -5.68924700 & 0.69028200 & -4.53459400 \\
\hline $\mathrm{H}$ & -5.88669100 & 1.28063200 & -5.42951800 \\
\hline $\mathrm{H}$ & -6.56743900 & 0.68549300 & -3.88296900 \\
\hline $\mathrm{H}$ & -5.44571400 & -0.34073100 & -4.80593900 \\
\hline $\mathrm{N}$ & -4.69699300 & -0.29878300 & -2.25983400 \\
\hline C & -4.22676200 & -0.78111300 & -1.11326900 \\
\hline 0 & -4.78952000 & -1.89839300 & -0.61164900 \\
\hline C & -5.88934800 & -2.46829100 & -1.32929000 \\
\hline $\mathrm{H}$ & -6.20403600 & -3.32794900 & -0.73653600 \\
\hline $\mathrm{H}$ & -5.58128700 & -2.78774500 & -2.32857100 \\
\hline $\mathrm{H}$ & -6.71024900 & -1.75286400 & -1.42436200 \\
\hline C & 5.49484600 & 0.12955700 & -0.56624200 \\
\hline $\mathrm{H}$ & 5.20314600 & 0.77285000 & -1.39507800 \\
\hline
\end{tabular}

\begin{tabular}{|c|c|c|c|}
\hline C & 6.75047300 & 0.24004200 & -0.09536400 \\
\hline C & 4.38249700 & -0.75734600 & -0.04511700 \\
\hline $\mathrm{H}$ & 3.68882800 & -0.17130400 & 0.56288900 \\
\hline $\mathrm{H}$ & 4.76317700 & -1.56404400 & 0.58614300 \\
\hline C & 7.70973300 & 1.21763500 & -0.73499000 \\
\hline C & 7.29736500 & -0.53585000 & 1.08692200 \\
\hline $\mathrm{H}$ & 7.59631200 & 0.18890700 & 1.85732200 \\
\hline $\mathrm{H}$ & 6.51513300 & -1.14965000 & 1.54383000 \\
\hline C & 3.52524500 & -1.34437300 & -1.15664700 \\
\hline $\mathrm{N}$ & 4.02300800 & -2.33930200 & -1.91856200 \\
\hline C & 3.20934000 & -2.96816700 & -2.95875400 \\
\hline $\mathrm{H}$ & 3.69404700 & -2.84792700 & -3.93361200 \\
\hline $\mathrm{H}$ & 3.10579900 & -4.03820400 & -2.74932000 \\
\hline $\mathrm{H}$ & 2.22504500 & -2.50957600 & -2.98130200 \\
\hline C & 5.33278300 & -2.95224900 & -1.70101900 \\
\hline $\mathrm{H}$ & 6.02309600 & -2.24612700 & -1.24539800 \\
\hline $\mathrm{H}$ & 5.24696300 & -3.84826500 & -1.07432400 \\
\hline $\mathrm{H}$ & 5.74489900 & -3.24738600 & -2.66957300 \\
\hline 0 & 2.36859100 & -0.90735400 & -1.38505400 \\
\hline $\mathrm{H}$ & 8.10976600 & 1.91743300 & 0.00938300 \\
\hline $\mathrm{H}$ & 7.22977500 & 1.79916600 & -1.52628500 \\
\hline P & -0.29261600 & -1.60378800 & -0.01284800 \\
\hline 0 & -4.90595800 & -0.06470700 & 1.74493700 \\
\hline 0 & -2.36923300 & -2.66007200 & 4.59155100 \\
\hline C & 2.23087000 & 2.15721100 & -0.23960700 \\
\hline $\mathrm{Si}$ & 2.99829600 & 3.10320700 & 1.18943800 \\
\hline C & 3.45023100 & 1.93472800 & 2.60315100 \\
\hline $\mathrm{H}$ & 3.82362000 & 2.51792500 & 3.45268600 \\
\hline $\mathrm{H}$ & 2.59147800 & 1.35515100 & 2.95606500 \\
\hline $\mathrm{H}$ & 4.24082900 & 1.23501100 & 2.31585400 \\
\hline C & 4.55571500 & 3.90726400 & 0.48661100 \\
\hline $\mathrm{H}$ & 5.26496300 & 3.15646600 & 0.12613800 \\
\hline $\mathrm{H}$ & 4.31547400 & 4.57743600 & -0.34495400 \\
\hline $\mathrm{H}$ & 5.05631500 & 4.50169900 & 1.25959200 \\
\hline C & 1.81277800 & 4.43280600 & 1.80392900 \\
\hline $\mathrm{H}$ & 1.49064700 & 5.08866500 & 0.99006000 \\
\hline $\mathrm{H}$ & 0.91974800 & 4.00988600 & 2.27156800 \\
\hline $\mathrm{H}$ & 2.32133200 & 5.05074600 & 2.55348800 \\
\hline $\mathrm{H}$ & 8.57256000 & 0.70361700 & -1.17398800 \\
\hline C & 8.51099600 & -1.42705400 & 0.76215700 \\
\hline $\mathrm{H}$ & 9.36369500 & -0.84231900 & 0.40657800 \\
\hline $\mathrm{H}$ & 8.83463400 & -1.96881100 & 1.65577900 \\
\hline $\mathrm{H}$ & 8.27044100 & -2.16733300 & -0.00839700 \\
\hline
\end{tabular}




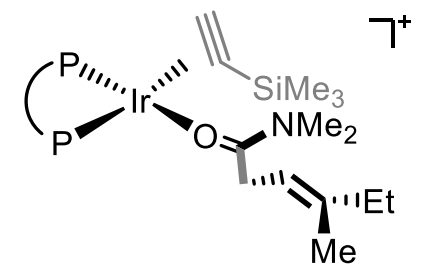

$G_{\text {sol }}=-3633.06473$ Hatree 


\begin{tabular}{|c|c|c|c|}
\hline $\mathrm{H}$ & -3.81293900 & -2.18152600 & 0.60030800 \\
\hline $\mathrm{H}$ & -2.89192500 & -0.71778900 & 0.89230600 \\
\hline & -6.77044600 & -0.05656300 & 2.80493900 \\
\hline C & -5.07047700 & -1.95177800 & 3.10773300 \\
\hline $\mathrm{H}$ & -4.02273000 & -2.17085800 & 2.87752800 \\
\hline $\mathrm{C}$ & -3.66594400 & -0.82734700 & -1.04451200 \\
\hline N & -4.63861100 & -1.21921300 & -1.89546000 \\
\hline C & -4.49367600 & -0.97257000 & -3.33146200 \\
\hline $\mathrm{H}$ & -4.14119100 & -1.87549000 & -3.84307000 \\
\hline $\mathrm{H}$ & -5.46620900 & -0.69094400 & -3.74386300 \\
\hline $\mathrm{H}$ & -3.77849900 & -0.16909500 & -3.49018400 \\
\hline C & -5.73215500 & -2.11632600 & -1.52278300 \\
\hline $\mathrm{H}$ & -5.93853200 & -2.06088400 & -0.45600500 \\
\hline $\mathrm{H}$ & -6.63640300 & -1.81695900 & -2.05945900 \\
\hline $\mathrm{H}$ & -5.49086400 & -3.15051300 & -1.79784000 \\
\hline 0 & -2.66026000 & -0.23088500 & -1.50352900 \\
\hline $\mathrm{H}$ & -7.61052500 & -0.73431200 & 2.99884100 \\
\hline $\mathrm{H}$ & -7.11708100 & 0.72308900 & 2.12165400 \\
\hline P & 1.22810000 & 1.65903900 & -0.32352900 \\
\hline 0 & 3.81560900 & -1.89037400 & 2.46348100 \\
\hline o & 2.72353000 & 2.04243800 & 4.60928000 \\
\hline C & -1.86209700 & 2.49089100 & -1.49504800 \\
\hline $\mathrm{Si}$ & -3.33561500 & 3.46202100 & -0.86791500 \\
\hline C & -3.59987200 & 3.21520100 & 0.98320200 \\
\hline $\mathrm{H}$ & -4.51672500 & 3.73060800 & 1.29212900 \\
\hline $\mathrm{H}$ & -3.70947600 & 2.15976200 & 1.25012200 \\
\hline $\mathrm{H}$ & -2.77789600 & 3.63159200 & 1.57117200 \\
\hline C & -3.03009100 & 5.27868800 & -1.27345200 \\
\hline $\mathrm{H}$ & -2.12594700 & 5.65389400 & -0.78627400 \\
\hline $\mathrm{H}$ & -2.91762000 & 5.42526100 & -2.35258000 \\
\hline $\mathrm{H}$ & -3.87605100 & 5.89107800 & -0.94027700 \\
\hline C & -4.85182700 & 2.85229600 & -1.82030700 \\
\hline $\mathrm{H}$ & -4.70846400 & 2.97018200 & -2.89922300 \\
\hline $\mathrm{H}$ & -5.07125100 & 1.79830600 & -1.62482600 \\
\hline $\mathrm{H}$ & -5.73444400 & 3.43722500 & -1.53666100 \\
\hline $\mathrm{H}$ & -5.08969400 & -1.63932600 & 4.15975100 \\
\hline $\mathrm{H}$ & -6.52468500 & 0.41491800 & 3.76481000 \\
\hline $\mathrm{C}$ & -5.90627200 & -3.23822700 & 2.96064000 \\
\hline $\mathrm{H}$ & -5.53071000 & -4.02117400 & 3.62641200 \\
\hline $\mathrm{H}$ & -6.95742600 & -3.06700700 & 3.21123100 \\
\hline & -5.86804700 & -3.62254900 & 1.93568200 \\
\hline
\end{tabular}

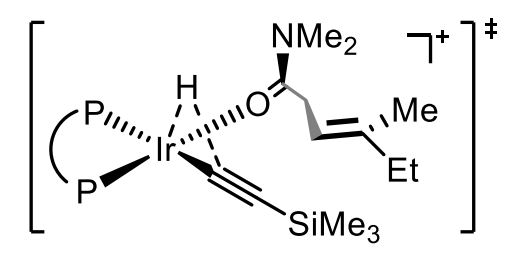

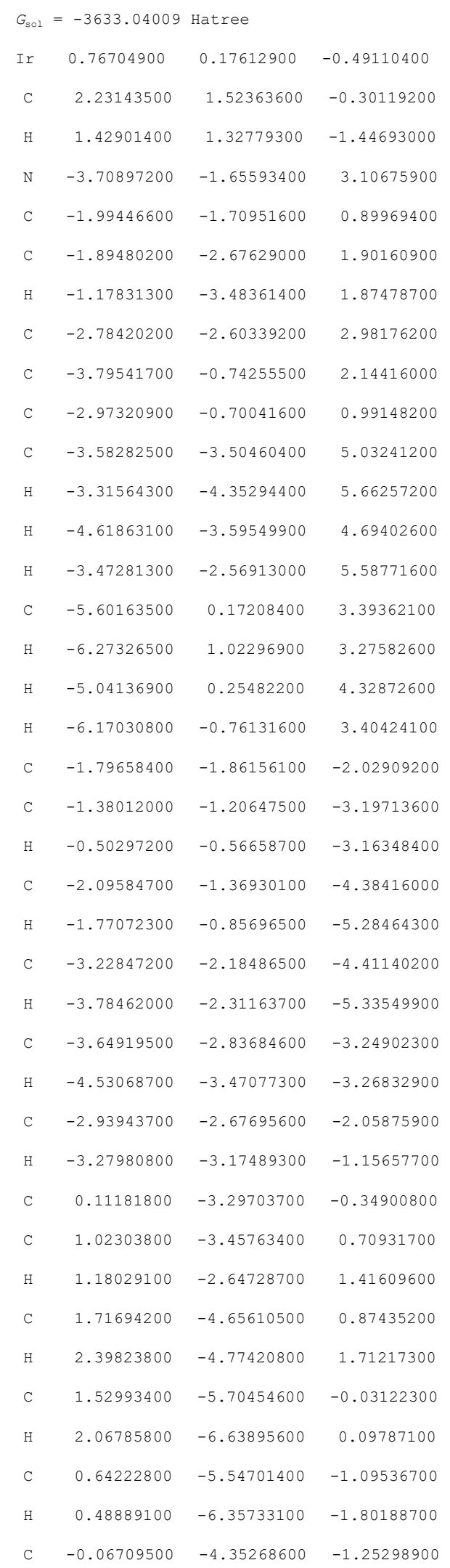




\begin{tabular}{|c|c|c|c|}
\hline $\mathrm{H}$ & -0.76077800 & -4.25161800 & -2.07970700 \\
\hline $\mathrm{C}$ & -3.27832600 & 0.30120600 & -0.06598000 \\
\hline C & -2.48982100 & 1.41732400 & -0.41951700 \\
\hline $\mathrm{P}$ & -0.78033400 & 1.68119800 & 0.26910900 \\
\hline C & -0.96164600 & 1.71782600 & 2.09359800 \\
\hline C & 0.13744200 & 1.35264700 & 2.88649600 \\
\hline $\mathrm{H}$ & 1.05176700 & 1.01484800 & 2.40852600 \\
\hline $\mathrm{C}$ & 0.05926100 & 1.44007700 & 4.27717100 \\
\hline $\mathrm{H}$ & 0.91400000 & 1.15651200 & 4.88398400 \\
\hline C & -1.11188200 & 1.89484900 & 4.88552800 \\
\hline $\mathrm{H}$ & -1.17081600 & 1.96456800 & 5.96766500 \\
\hline C & -2.20784900 & 2.26246600 & 4.10104300 \\
\hline $\mathrm{H}$ & -3.11918100 & 2.61983200 & 4.57119300 \\
\hline C & -2.13690800 & 2.17545100 & 2.71037500 \\
\hline $\mathrm{H}$ & -2.99838300 & 2.44982200 & 2.11072000 \\
\hline C & -0.39337600 & 3.42905500 & -0.19008900 \\
\hline C & -0.00490300 & 3.73148000 & -1.50645300 \\
\hline $\mathrm{H}$ & 0.08529700 & 2.93827200 & -2.24278600 \\
\hline C & 0.25629400 & 5.04693100 & -1.88603700 \\
\hline $\mathrm{H}$ & 0.54442600 & 5.26400700 & -2.91019700 \\
\hline C & 0.15219900 & 6.07982800 & -0.95060000 \\
\hline $\mathrm{H}$ & 0.35943600 & 7.10432000 & -1.24475000 \\
\hline C & -0.21947100 & 5.78829100 & 0.36148700 \\
\hline $\mathrm{H}$ & -0.30100400 & 6.58440800 & 1.09540600 \\
\hline C & -0.49570000 & 4.47212000 & 0.74067000 \\
\hline $\mathrm{H}$ & -0.78641100 & 4.26670100 & 1.76419600 \\
\hline C & -2.96319000 & 2.31524000 & -1.37838500 \\
\hline $\mathrm{H}$ & -2.41558600 & 3.19591700 & -1.67798600 \\
\hline C & -4.21222900 & 2.07359900 & -1.96427200 \\
\hline 0 & -4.64218500 & 2.96857400 & -2.87099500 \\
\hline C & -5.92611300 & 2.74958000 & -3.46756600 \\
\hline $\mathrm{H}$ & -6.07039200 & 3.58419900 & -4.15367700 \\
\hline $\mathrm{H}$ & -6.71442400 & 2.74145800 & -2.70979000 \\
\hline $\mathrm{H}$ & -5.94945600 & 1.80170800 & -4.01229900 \\
\hline $\mathrm{N}$ & -4.96892000 & 1.02090800 & -1.66790400 \\
\hline C & -4.51071600 & 0.17069800 & -0.75476800 \\
\hline 0 & -5.25856400 & -0.91005700 & -0.45678300 \\
\hline C & -6.51707700 & -1.05233800 & -1.12540000 \\
\hline $\mathrm{H}$ & -6.95482700 & -1.96541800 & -0.72058300 \\
\hline $\mathrm{H}$ & -6.37682100 & -1.14373500 & -2.20582100 \\
\hline $\mathrm{H}$ & -7.16829500 & -0.19790300 & -0.92434600 \\
\hline $\mathrm{C}$ & 5.32027400 & -0.97650200 & 0.26661800 \\
\hline $\mathrm{H}$ & 5.50339500 & -0.17801700 & -0.44959600 \\
\hline $\mathrm{C}$ & 6.32744200 & -1.34020200 & 1.08020500 \\
\hline & 3.90666100 & -1.51252200 & 0.26747500 \\
\hline
\end{tabular}

\begin{tabular}{|c|c|c|c|}
\hline $\mathrm{H}$ & 3.24056700 & -0.80255700 & 0.76884600 \\
\hline $\mathrm{H}$ & 3.83703600 & -2.45965200 & 0.81146600 \\
\hline C & 7.68249400 & -0.66792800 & 0.95779500 \\
\hline $\mathrm{C}$ & 6.22293900 & -2.39046300 & 2.15990800 \\
\hline $\mathrm{H}$ & 6.42997100 & -1.94293700 & 3.14009000 \\
\hline $\mathrm{H}$ & 5.24102200 & -2.86481200 & 2.21850400 \\
\hline $\mathrm{C}$ & 3.28468300 & -1.72480000 & -1.10616300 \\
\hline $\mathrm{N}$ & 3.88037500 & -2.51338000 & -2.02036100 \\
\hline C & 3.22980600 & -2.70619700 & -3.31984800 \\
\hline $\mathrm{H}$ & 3.99198800 & -2.97511100 & -4.05394800 \\
\hline $\mathrm{H}$ & 2.48343600 & -3.50714600 & -3.26803100 \\
\hline $\mathrm{H}$ & 2.73465400 & -1.78597300 & -3.62422900 \\
\hline $\mathrm{C}$ & 4.97974400 & -3.43154400 & -1.72478500 \\
\hline $\mathrm{H}$ & 5.50825400 & -3.12870600 & -0.82520900 \\
\hline $\mathrm{H}$ & 4.59828000 & -4.45284900 & -1.60508700 \\
\hline $\mathrm{H}$ & 5.68973800 & -3.42098800 & -2.55633200 \\
\hline 0 & 2.18844500 & -1.18625100 & -1.42345900 \\
\hline $\mathrm{H}$ & 7.97556600 & -0.27533700 & 1.94164800 \\
\hline $\mathrm{H}$ & 7.60002300 & 0.19615400 & 0.29094800 \\
\hline P & -0.78821700 & -1.69294800 & -0.50754400 \\
\hline O & -4.72431600 & 0.22586600 & 2.26242700 \\
\hline 0 & -2.67387500 & -3.55497300 & 3.92568200 \\
\hline C & 3.13658600 & 2.35367100 & -0.16127200 \\
\hline $\mathrm{Si}$ & 4.37381800 & 3.69885000 & 0.11176600 \\
\hline C & 6.09997800 & 2.95438800 & 0.28833100 \\
\hline $\mathrm{H}$ & 6.83644700 & 3.73990400 & 0.49204600 \\
\hline $\mathrm{H}$ & 6.14326600 & 2.23239300 & 1.10953400 \\
\hline $\mathrm{H}$ & 6.40694500 & 2.44338600 & -0.63046000 \\
\hline C & 4.31914700 & 4.84887000 & -1.38520600 \\
\hline $\mathrm{H}$ & 4.58515900 & 4.31849900 & -2.30541700 \\
\hline $\mathrm{H}$ & 3.32090400 & 5.27667600 & -1.51806700 \\
\hline $\mathrm{H}$ & 5.02769900 & 5.67557200 & -1.25976800 \\
\hline C & 3.89576800 & 4.62614100 & 1.68518200 \\
\hline $\mathrm{H}$ & 2.88487600 & 5.03802700 & 1.60534500 \\
\hline $\mathrm{H}$ & 3.92311700 & 3.96880200 & 2.56034400 \\
\hline $\mathrm{H}$ & 4.58609000 & 5.45709000 & 1.86959500 \\
\hline $\mathrm{H}$ & 6.96851800 & -3.18119200 & 2.01884400 \\
\hline C & 8.79942700 & -1.59617500 & 0.44541400 \\
\hline $\mathrm{H}$ & 9.74406000 & -1.04961100 & 0.36872900 \\
\hline $\mathrm{H}$ & 8.96736800 & -2.44609800 & 1.11380300 \\
\hline $\mathrm{H}$ & 8.55836800 & -1.98974900 & -0.54770400 \\
\hline
\end{tabular}




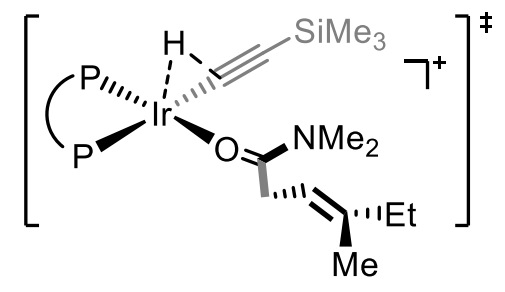

$G_{\text {sol }}=-3633.040996$ Hatree

\begin{tabular}{|c|c|c|c|}
\hline Ir & 0.78183300 & 0.22178000 & -0.54923800 \\
\hline C & 2.40290300 & -0.79879000 & -1.22476800 \\
\hline $\mathrm{H}$ & 1.39988600 & -0.39949700 & -1.97450000 \\
\hline $\mathrm{N}$ & -3.18332500 & -1.10895800 & 3.54588400 \\
\hline C & -1.56954300 & -1.48753800 & 1.29022400 \\
\hline C & -1.22845500 & -2.06093800 & 2.51658000 \\
\hline $\mathrm{H}$ & -0.35352000 & -2.67963600 & 2.65147000 \\
\hline C & -2.07639000 & -1.84294800 & 3.61061300 \\
\hline $\mathrm{C}$ & -3.49657100 & -0.56294900 & 2.37401600 \\
\hline C & -2.74249000 & -0.71052700 & 1.18383000 \\
\hline C & -2.59888400 & -2.23421900 & 5.89722300 \\
\hline $\mathrm{H}$ & -2.13640200 & -2.78732300 & 6.71485000 \\
\hline $\mathrm{H}$ & -3.59699500 & -2.62952800 & 5.68946300 \\
\hline $\mathrm{H}$ & -2.68567900 & -1.17528600 & 6.15610000 \\
\hline C & -5.40135000 & 0.33428000 & 3.47987900 \\
\hline $\mathrm{H}$ & -6.24846700 & 0.95423900 & 3.18456400 \\
\hline $\mathrm{H}$ & -4.83536200 & 0.81964800 & 4.27951300 \\
\hline $\mathrm{H}$ & -5.74958600 & -0.63959500 & 3.83349300 \\
\hline C & -1.46238900 & -2.35258800 & -1.50708300 \\
\hline C & -1.22973400 & -1.96005300 & -2.83277400 \\
\hline $\mathrm{H}$ & -0.47548800 & -1.21006700 & -3.04577000 \\
\hline C & -1.97427500 & -2.51533600 & -3.87472300 \\
\hline $\mathrm{H}$ & -1.78622400 & -2.20178400 & -4.89715300 \\
\hline C & -2.95697800 & -3.46770600 & -3.60252900 \\
\hline $\mathrm{H}$ & -3.53360900 & -3.90203200 & -4.41377600 \\
\hline C & -3.20060500 & -3.85912400 & -2.28356600 \\
\hline $\mathrm{H}$ & -3.96683100 & -4.59730600 & -2.06626100 \\
\hline C & -2.46190100 & -3.30371300 & -1.23915600 \\
\hline $\mathrm{H}$ & -2.67196300 & -3.60357300 & -0.21756700 \\
\hline C & 0.67074200 & -3.10076500 & 0.33935300 \\
\hline C & 1.71711700 & -2.86253500 & 1.24728500 \\
\hline $\mathrm{H}$ & 1.89772600 & -1.85602400 & 1.60997700 \\
\hline C & 2.53162400 & -3.90586400 & 1.68221400 \\
\hline $\mathrm{H}$ & 3.32756500 & -3.70646500 & 2.39366000 \\
\hline C & 2.32994400 & -5.20100200 & 1.19794400 \\
\hline $\mathrm{H}$ & 2.96731900 & -6.01390300 & 1.53273600 \\
\hline C & 1.30975900 & -5.44327500 & 0.27909800 \\
\hline $\mathrm{H}$ & 1.15172800 & -6.44479000 & -0.10960100 \\
\hline
\end{tabular}

$$
\begin{aligned}
& 0.48098000 \quad-4.40190900 \quad-0.14592400 \\
& \begin{array}{llll}
-0.30610500 & -4.61226400 & -0.85996400
\end{array} \\
& \begin{array}{llll}
-3.26521200 & -0.09344300 & -0.06462400
\end{array} \\
& \begin{array}{lll}
-2.68758000 & 1.01047100 & -0.72170800
\end{array} \\
& \begin{array}{lll}
-1.05988100 & 1.67726800 & -0.14136900
\end{array} \\
& \begin{array}{lll}
-1.39740000 & 2.23630900 & 1.57682500
\end{array} \\
& \begin{array}{lll}
-0.43617500 \quad 2.01815500 & 2.57418200
\end{array} \\
& \begin{array}{lll}
0.47686400 & 1.48228500 & 2.33224700
\end{array} \\
& \begin{array}{lll}
-0.65525200 & 2.46357200 & 3.87861600
\end{array} \\
& \begin{array}{lll}
0.09285800 \quad 2.28268900 & 4.64473400
\end{array} \\
& \begin{array}{lll}
-1.83772600 \quad 3.13280800 \quad 4.19770900 &
\end{array} \\
& \begin{array}{lll}
-2.00839400 \quad 3.48221200 & 5.21163900
\end{array} \\
& \begin{array}{lll}
-2.80523000 & 3.34716200 & 3.21246400
\end{array} \\
& \begin{array}{lll}
-3.72884900 \quad 3.86240400 & 3.45882600
\end{array} \\
& \begin{array}{lll}
-2.59174100 \quad 2.89701800 & 1.90957500
\end{array} \\
& \begin{array}{lll}
-3.35638900 & 3.05239700 & 1.15489200
\end{array} \\
& \begin{array}{llll}
-0.81997100 & 3.22162500 & -1.12265300
\end{array} \\
& \begin{array}{lll}
-0.44094700 & 3.09549400 & -2.47083500
\end{array} \\
& \begin{array}{lll}
-0.27520300 \quad 2.10790700 & -2.89057700
\end{array} \\
& \begin{array}{llll}
-0.27360900 \quad 4.22574700 & -3.26863100
\end{array} \\
& 0.00222400 \quad 4.11199100 \quad-4.31291600 \\
& \begin{array}{llll}
-0.45434100 \quad 5.50077500 & -2.72454800
\end{array} \\
& \begin{array}{llll}
-0.32371200 & 6.38199600 & -3.34567900
\end{array} \\
& \begin{array}{lll}
-0.80387400 & 5.63601100 & -1.38084900
\end{array} \\
& \begin{array}{llll}
-0.94265200 & 6.62311300 & -0.94978600
\end{array} \\
& \begin{array}{llll}
-0.99099900 & 4.50335700 & -0.58314100
\end{array} \\
& \begin{array}{lll}
-1.27155100 & 4.62748300 & 0.45645700
\end{array} \\
& \begin{array}{lll}
-3.31414700 & 1.56513900 & -1.83779600
\end{array} \\
& \begin{array}{lll}
-2.91951900 & 2.41735400 & -2.37167800
\end{array} \\
& \begin{array}{llll}
-4.51767500 & 0.99164100 & -2.27012900
\end{array} \\
& \begin{array}{lll}
-5.10972500 & 1.55325000 & -3.33947800
\end{array} \\
& \begin{array}{lll}
-6.34888000 & 0.99081200 & -3.78707700
\end{array} \\
& \begin{array}{lll}
-6.64407600 & 1.59277600 & -4.64660700
\end{array} \\
& \begin{array}{lll}
-7.11090500 & 1.04769900 & -3.00488800
\end{array} \\
& \begin{array}{lll}
-6.22185600 & -0.05468700 & -4.08125200
\end{array} \\
& \begin{array}{lll}
-5.08422800 & -0.05719300 & -1.68004300
\end{array} \\
& \begin{array}{lll}
-4.47483000 & -0.57701800 & -0.61784000
\end{array} \\
& \begin{array}{lll}
-5.03015100 & -1.64852500 & -0.01995700
\end{array} \\
& \begin{array}{lll}
-6.27148300 & -2.13254800 & -0.54492300
\end{array} \\
& \begin{array}{lll}
-6.54371700 & -2.97449900 & 0.09241100
\end{array} \\
& \begin{array}{lll}
-6.15660200 & -2.46060700 & -1.58148600
\end{array} \\
& \begin{array}{lll}
-7.04327200 & -1.35966100 & -0.50227100
\end{array} \\
& \begin{array}{lll}
4.74881300 & 1.20257400 & 1.21252800
\end{array} \\
& \begin{array}{llll}
5.04464800 & 0.68659600 & 0.30133000
\end{array} \\
& \begin{array}{llll}
\text { C } & 5.60716800 & 1.21054100 & 2.24743300
\end{array}
\end{aligned}
$$




\begin{tabular}{|c|c|c|c|}
\hline C & 3.35097400 & 1.77651900 & 1.18005200 \\
\hline $\mathrm{H}$ & 3.18236000 & 2.48316500 & 1.99969900 \\
\hline $\mathrm{H}$ & 2.62170300 & 0.96524600 & 1.30031000 \\
\hline C & 6.95318100 & 0.52387100 & 2.11722900 \\
\hline C & 5.33540200 & 1.85610200 & 3.58512200 \\
\hline $\mathrm{H}$ & 6.17660600 & 2.49391200 & 3.88214100 \\
\hline $\mathrm{H}$ & 4.42907700 & 2.46587700 & 3.60131700 \\
\hline C & 2.96276800 & 2.44868700 & -0.13187300 \\
\hline $\mathrm{N}$ & 3.63950500 & 3.52342800 & -0.57347200 \\
\hline C & 3.23749900 & 4.16390400 & -1.82907000 \\
\hline $\mathrm{H}$ & 2.51382000 & 4.96565400 & -1.64486200 \\
\hline $\mathrm{H}$ & 4.12543300 & 4.58613800 & -2.30539200 \\
\hline $\mathrm{H}$ & 2.77841200 & 3.42771100 & -2.48453100 \\
\hline C & 4.63078600 & 4.25311600 & 0.21593100 \\
\hline $\mathrm{H}$ & 4.99728200 & 3.64601700 & 1.03884100 \\
\hline $\mathrm{H}$ & 5.48139800 & 4.50680400 & -0.42304400 \\
\hline $\mathrm{H}$ & 4.20055200 & 5.18445400 & 0.60352600 \\
\hline 0 & 1.99389800 & 2.02339800 & -0.82071900 \\
\hline $\mathrm{H}$ & 7.74493300 & 1.24282300 & 2.37103600 \\
\hline $\mathrm{H}$ & 7.11515200 & 0.23731000 & 1.07255900 \\
\hline $\mathrm{P}$ & -0.41434400 & -1.68812700 & -0.15245300 \\
\hline 0 & -4.60486300 & 0.19789800 & 2.29787500 \\
\hline 0 & -1.72830800 & -2.42038300 & 4.77497200 \\
\hline C & 3.40490900 & -1.36555800 & -1.68075500 \\
\hline $\mathrm{Si}$ & 4.85794800 & -2.26957000 & -2.38628200 \\
\hline C & 4.82822900 & -4.04911200 & -1.76290100 \\
\hline $\mathrm{H}$ & 5.67639500 & -4.61387400 & -2.16623500 \\
\hline $\mathrm{H}$ & 4.88415200 & -4.08879500 & -0.67080300 \\
\hline $\mathrm{H}$ & 3.90818300 & -4.55935400 & -2.06440300 \\
\hline C & 4.69468300 & -2.21382000 & -4.26686200 \\
\hline $\mathrm{H}$ & 3.76657900 & -2.68899700 & -4.60013200 \\
\hline $\mathrm{H}$ & 4.70023600 & -1.18492600 & -4.64062100 \\
\hline $\mathrm{H}$ & 5.52950900 & -2.74531400 & -4.73755100 \\
\hline C & 6.44490700 & -1.40510500 & -1.83555500 \\
\hline $\mathrm{H}$ & 6.46852300 & -0.36032900 & -2.16228500 \\
\hline $\mathrm{H}$ & 6.55060800 & -1.42439800 & -0.74581600 \\
\hline $\mathrm{H}$ & 7.32107900 & -1.90693500 & -2.26134400 \\
\hline $\mathrm{H}$ & 5.22916900 & 1.09837000 & 4.37038700 \\
\hline C & 7.11043100 & -0.71826700 & 3.01332700 \\
\hline $\mathrm{H}$ & 8.09128400 & -1.17827300 & 2.86037500 \\
\hline $\mathrm{H}$ & 7.02720200 & -0.46988300 & 4.07577200 \\
\hline $\mathrm{H}$ & 6.34588700 & -1.46717800 & 2.78117100 \\
\hline
\end{tabular}

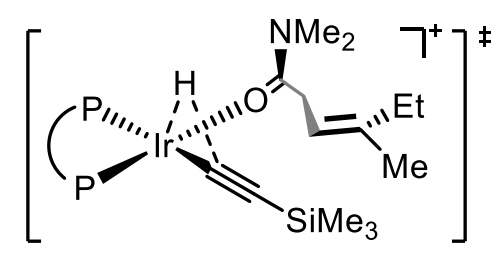

$G_{\text {sol }}=-3633.039785$ Hatree

\begin{tabular}{|c|c|c|c|}
\hline Ir & 0.77390500 & 0.23651500 & -0.54487600 \\
\hline C & 2.21480900 & 1.61269200 & -0.35287400 \\
\hline $\mathrm{H}$ & 1.38022500 & 1.45001600 & -1.46694500 \\
\hline $\mathrm{N}$ & -3.48184200 & -1.92080500 & 3.10839100 \\
\hline C & -1.88046100 & -1.79553100 & 0.82037600 \\
\hline C & -1.71718100 & -2.82577800 & 1.74725500 \\
\hline $\mathrm{H}$ & -0.99299000 & -3.61744800 & 1.62658400 \\
\hline C & -2.55141300 & -2.84151300 & 2.87302100 \\
\hline C & -3.63166100 & -0.94802300 & 2.21432600 \\
\hline C & -2.86966700 & -0.81378900 & 1.02809600 \\
\hline C & -3.23424200 & -3.89357000 & 4.89321000 \\
\hline $\mathrm{H}$ & -2.92501700 & -4.77855300 & 5.44941600 \\
\hline $\mathrm{H}$ & -4.28406900 & -3.97837000 & 4.59944000 \\
\hline $\mathrm{H}$ & -3.10986400 & -2.99672400 & 5.50624600 \\
\hline C & -5.39409100 & -0.15784700 & 3.60403000 \\
\hline $\mathrm{H}$ & -6.08662900 & 0.68387300 & 3.57395200 \\
\hline $\mathrm{H}$ & -4.79457200 & -0.12591000 & 4.51765300 \\
\hline $\mathrm{H}$ & -5.94380300 & -1.10217900 & 3.57533000 \\
\hline C & -1.83308500 & -1.73314400 & -2.11423300 \\
\hline C & -1.52276500 & -0.95512000 & -3.23878900 \\
\hline $\mathrm{H}$ & -0.67499600 & -0.27738700 & -3.19967600 \\
\hline C & -2.30644300 & -1.04492400 & -4.39038900 \\
\hline $\mathrm{H}$ & -2.06300600 & -0.43741900 & -5.25683700 \\
\hline C & -3.40118400 & -1.91016900 & -4.42569400 \\
\hline $\mathrm{H}$ & -4.00969000 & -1.98039700 & -5.32242400 \\
\hline C & -3.71604200 & -2.68568000 & -3.30641900 \\
\hline $\mathrm{H}$ & -4.56745000 & -3.35926700 & -3.33177300 \\
\hline C & -2.93846400 & -2.59884300 & -2.15183000 \\
\hline $\mathrm{H}$ & -3.19647900 & -3.19477500 & -1.28218300 \\
\hline C & 0.19033200 & -3.24714400 & -0.65504600 \\
\hline C & 1.12777600 & -3.49513200 & 0.36286800 \\
\hline $\mathrm{H}$ & 1.27913000 & -2.76285400 & 1.15105700 \\
\hline C & 1.85924000 & -4.68229800 & 0.38278400 \\
\hline $\mathrm{H}$ & 2.56093200 & -4.86983100 & 1.19058700 \\
\hline C & 1.68434000 & -5.63054700 & -0.62921500 \\
\hline $\mathrm{H}$ & 2.25173700 & -6.55626700 & -0.61333000 \\
\hline C & 0.77104400 & -5.38432500 & -1.65395900 \\
\hline $\mathrm{H}$ & 0.62658100 & -6.11618200 & -2.44315900 \\
\hline $\mathrm{C}$ & 0.02454200 & -4.20247700 & -1.66649000 \\
\hline
\end{tabular}




\begin{tabular}{|c|c|c|c|}
\hline $\mathrm{H}$ & -0.68698500 & -4.03188800 & -2.46591800 \\
\hline $\mathrm{C}$ & -3.25205800 & 0.24274700 & 0.05214700 \\
\hline C & -2.50920900 & 1.39818100 & -0.27102500 \\
\hline $\mathrm{P}$ & -0.77557400 & 1.66419000 & 0.35153900 \\
\hline C & -0.87511500 & 1.60541000 & 2.18194600 \\
\hline C & 0.26963800 & 1.24028900 & 2.90779900 \\
\hline $\mathrm{H}$ & 1.17267500 & 0.95829900 & 2.37551100 \\
\hline $\mathrm{C}$ & 0.25146300 & 1.25803400 & 4.30313200 \\
\hline $\mathrm{H}$ & 1.14130900 & 0.97469400 & 4.85731200 \\
\hline C & -0.90450600 & 1.64435200 & 4.98359500 \\
\hline $\mathrm{H}$ & -0.91684100 & 1.66038200 & 6.06938500 \\
\hline C & -2.04505700 & 2.01329400 & 4.26637000 \\
\hline $\mathrm{H}$ & -2.94465900 & 2.31837900 & 4.79259700 \\
\hline C & -2.03442700 & 1.99474400 & 2.87133100 \\
\hline $\mathrm{H}$ & -2.93023000 & 2.26934600 & 2.32468600 \\
\hline C & -0.44570300 & 3.44100500 & -0.03321500 \\
\hline C & -0.13230900 & 3.81730600 & -1.35042900 \\
\hline $\mathrm{H}$ & -0.07271300 & 3.06399300 & -2.13035200 \\
\hline C & 0.08971400 & 5.15454500 & -1.67442800 \\
\hline $\mathrm{H}$ & 0.31962700 & 5.42864700 & -2.69965100 \\
\hline C & 0.02027700 & 6.13623300 & -0.68225600 \\
\hline $\mathrm{H}$ & 0.19692000 & 7.17786100 & -0.93296200 \\
\hline C & -0.27836800 & 5.77154500 & 0.63023500 \\
\hline $\mathrm{H}$ & -0.33347700 & 6.52767800 & 1.40762500 \\
\hline C & -0.51484600 & 4.43303100 & 0.95451200 \\
\hline $\mathrm{H}$ & -0.74877300 & 4.17069600 & 1.97954800 \\
\hline C & -3.04996400 & 2.33809100 & -1.15089300 \\
\hline $\mathrm{H}$ & -2.53930900 & 3.24952700 & -1.42241500 \\
\hline C & -4.31903700 & 2.09662600 & -1.69192200 \\
\hline 0 & -4.81296500 & 3.02948600 & -2.52500100 \\
\hline $\mathrm{C}$ & -6.11755400 & 2.80937900 & -3.07447200 \\
\hline $\mathrm{H}$ & -6.31288100 & 3.67542000 & -3.70693100 \\
\hline $\mathrm{H}$ & -6.86977800 & 2.74054800 & -2.28 \\
\hline $\mathrm{H}$ & -6.14385400 & 1.89159400 & -3.66837000 \\
\hline $\mathrm{N}$ & -5.03529200 & 1.00904600 & -1.42254900 \\
\hline C & -4.51398700 & 0.12017200 & -0.58296300 \\
\hline 0 & -5.22157500 & -0.99433100 & -0.31291500 \\
\hline C & -6.50666600 & -1.13149100 & -0.92998600 \\
\hline $\mathrm{H}$ & -6.90305100 & -2.07716400 & -0.55878000 \\
\hline $\mathrm{H}$ & -6.41558600 & -1.15804100 & -2.01914800 \\
\hline $\mathrm{H}$ & -7.16751100 & -0.30705200 & -0.65030800 \\
\hline $\mathrm{C}$ & 5.33040400 & -0.83152200 & 0.18069800 \\
\hline $\mathrm{H}$ & 5.54175900 & -0.04188400 & -0.53731000 \\
\hline $\mathrm{C}$ & 6.28680300 & -1.16013900 & 1.06684200 \\
\hline & 3.93326100 & -1.40731800 & 0.10636300 \\
\hline
\end{tabular}

\begin{tabular}{|c|c|c|c|}
\hline $\mathrm{H}$ & 3.23191300 & -0.76479600 & 0.64958600 \\
\hline $\mathrm{H}$ & 3.88692600 & -2.39817400 & 0.56988300 \\
\hline C & 7.62776000 & -0.46513400 & 1.03249800 \\
\hline C & 6.12222800 & -2.20358500 & 2.15547300 \\
\hline $\mathrm{H}$ & 6.45513300 & -1.76211900 & 3.10427500 \\
\hline $\mathrm{H}$ & 5.06669900 & -2.45520600 & 2.29951400 \\
\hline C & 3.33885200 & -1.53085100 & -1.28979500 \\
\hline N & 3.99216900 & -2.19047400 & -2.26495100 \\
\hline C & 3.37744700 & -2.29871200 & -3.59113000 \\
\hline $\mathrm{H}$ & 4.17094500 & -2.36438400 & -4.33896700 \\
\hline $\mathrm{H}$ & 2.74956600 & -3.19472800 & -3.65828300 \\
\hline $\mathrm{H}$ & 2.76135300 & -1.42282800 & -3.78283400 \\
\hline C & 5.14707800 & -3.05907900 & -2.04157100 \\
\hline $\mathrm{H}$ & 5.64272100 & -2.81542800 & -1.10564300 \\
\hline $\mathrm{H}$ & 4.83345700 & -4.11017300 & -2.03245500 \\
\hline $\mathrm{H}$ & 5.86495200 & -2.91873800 & -2.85451000 \\
\hline 0 & 2.20984400 & -1.03986400 & -1.56662600 \\
\hline $\mathrm{H}$ & 7.82498300 & 0.04825400 & 1.98208000 \\
\hline $\mathrm{H}$ & 7.68516200 & 0.27287100 & 0.22913200 \\
\hline $\mathrm{P}$ & -0.74579900 & -1.65469400 & -0.63937100 \\
\hline 0 & -4.56974200 & -0.00835100 & 2.44192800 \\
\hline 0 & -2.38019500 & -3.85253900 & 3.74324400 \\
\hline C & 3.10966500 & 2.45563500 & -0.22243500 \\
\hline Si & 4.33410500 & 3.81702400 & 0.02939400 \\
\hline C & 6.07901100 & 3.10006400 & 0.10937400 \\
\hline $\mathrm{H}$ & 6.81162300 & 3.89568900 & 0.28589100 \\
\hline $\mathrm{H}$ & 6.17503600 & 2.36997900 & 0.91867800 \\
\hline H & 6.34823300 & 2.60487400 & -0.82972300 \\
\hline C & 4.18999400 & 4.99915200 & -1.43635700 \\
\hline $\mathrm{H}$ & 4.42386900 & 4.49508900 & -2.37977900 \\
\hline $\mathrm{H}$ & 3.17887300 & 5.40997600 & -1.51402800 \\
\hline $\mathrm{H}$ & 4.88785400 & 5.83668000 & -1.32353400 \\
\hline C & 3.91110200 & 4.69726900 & 1.64477900 \\
\hline $\mathrm{H}$ & 2.88989600 & 5.09029200 & 1.62092800 \\
\hline $\mathrm{H}$ & 3.99118700 & 4.02007500 & 2.50135300 \\
\hline $\mathrm{H}$ & 4.59322700 & 5.53734400 & 1.81799200 \\
\hline H & 8.44609800 & -1.18132500 & 0.89204500 \\
\hline C & 6.92120900 & -3.49851900 & 1.91109100 \\
\hline $\mathrm{H}$ & 7.99280700 & -3.30259800 & 1.81325900 \\
\hline $\mathrm{H}$ & 6.78903000 & -4.19459100 & 2.74499000 \\
\hline $\mathrm{H}$ & 6.59114900 & -4.00494300 & 0.99761000 \\
\hline
\end{tabular}




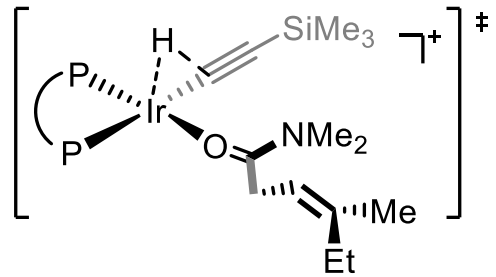

$G_{\text {sol }}=-3633.044007$ Hatree$$
\text { N }
$$$$
\mathrm{H}
$$$$
\text { C }
$$ 


\begin{tabular}{|c|c|c|c|}
\hline C & 3.47629400 & 1.76808600 & 0.94409400 \\
\hline $\mathrm{H}$ & 3.36285700 & 2.52445600 & 1.72851300 \\
\hline $\mathrm{H}$ & 2.74344000 & 0.97825100 & 1.15024700 \\
\hline C & 7.10884200 & 0.54029000 & 1.77443000 \\
\hline C & 5.57567600 & 1.97225600 & 3.23464800 \\
\hline $\mathrm{H}$ & 6.50974000 & 2.48254100 & 3.50243600 \\
\hline $\mathrm{H}$ & 4.81808100 & 2.75795200 & 3.13456300 \\
\hline C & 3.03383000 & 2.36756700 & -0.38581100 \\
\hline $\mathrm{N}$ & 3.69864000 & 3.40959000 & -0.91561100 \\
\hline C & 3.25966400 & 3.98374100 & -2.19013700 \\
\hline $\mathrm{H}$ & 2.63368800 & 4.86727000 & -2.02205900 \\
\hline $\mathrm{H}$ & 4.14149900 & 4.27678800 & -2.76571800 \\
\hline $\mathrm{H}$ & 2.68134400 & 3.24785700 & -2.74233200 \\
\hline C & 4.73357500 & 4.16901200 & -0.21503500 \\
\hline $\mathrm{H}$ & 5.13428600 & 3.60552200 & 0.62270200 \\
\hline $\mathrm{H}$ & 5.55438200 & 4.37691900 & -0.90739800 \\
\hline $\mathrm{H}$ & 4.33084300 & 5.12578000 & 0.13862500 \\
\hline 0 & 2.03352900 & 1.91120300 & -1.00668400 \\
\hline $\mathrm{H}$ & 7.93545800 & 1.26027000 & 1.82617800 \\
\hline $\mathrm{H}$ & 7.18537200 & 0.00128900 & 0.82661300 \\
\hline P & -0.42318600 & -1.70859700 & -0.05941200 \\
\hline 0 & -4.51676800 & 0.43847500 & 2.33398600 \\
\hline 0 & -1.62391900 & -2.03251100 & 4.94023800 \\
\hline C & 3.39532000 & -1.58690800 & -1.55122400 \\
\hline $\mathrm{Si}$ & 4.81423900 & -2.63484100 & -2.10980400 \\
\hline C & 4.10654600 & -4.16020600 & -2.96620800 \\
\hline $\mathrm{H}$ & 4.91187500 & -4.81519500 & -3.31745500 \\
\hline $\mathrm{H}$ & 3.47628300 & -4.73652600 & -2.28150900 \\
\hline $\mathrm{H}$ & 3.49811100 & -3.88559200 & -3.83392800 \\
\hline C & 5.87691700 & -1.62860700 & -3.30277700 \\
\hline $\mathrm{H}$ & 5.30642400 & -1.32790200 & -4.18754400 \\
\hline $\mathrm{H}$ & 6.26339600 & -0.72044200 & -2.82854700 \\
\hline $\mathrm{H}$ & 6.73556700 & -2.21731600 & -3.64478800 \\
\hline C & 5.81282000 & -3.13626500 & -0.58672200 \\
\hline $\mathrm{H}$ & 6.21891600 & -2.26453100 & -0.06370000 \\
\hline $\mathrm{H}$ & 5.19253200 & -3.69482500 & 0.12101700 \\
\hline $\mathrm{H}$ & 6.65488000 & -3.77517900 & -0.87591800 \\
\hline $\mathrm{H}$ & 7.27301600 & -0.17859900 & 2.58612200 \\
\hline C & 5.17178200 & 1.03816200 & 4.39206800 \\
\hline $\mathrm{H}$ & 5.91819900 & 0.25499800 & 4.55503900 \\
\hline $\mathrm{H}$ & 5.07303400 & 1.60054800 & 5.32551800 \\
\hline $\mathrm{H}$ & 4.21458900 & 0.54795800 & 4.18687200 \\
\hline
\end{tabular}
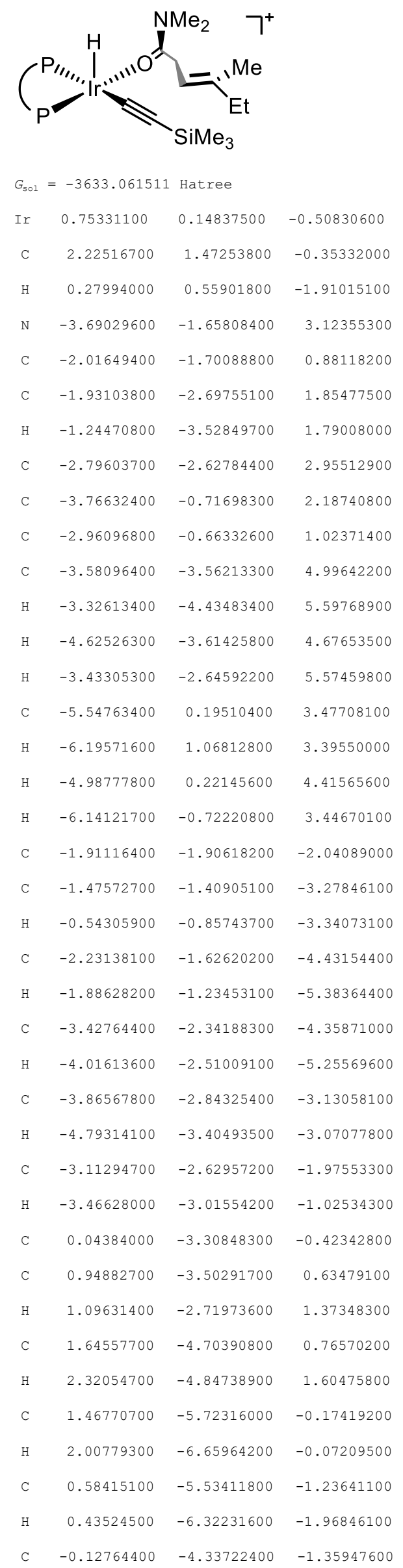


\begin{tabular}{|c|c|c|c|}
\hline $\mathrm{H}$ & -0.81884400 & -4.21333700 & -2.18508600 \\
\hline 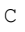 & -3.26058300 & 0.37186400 & -0.00273500 \\
\hline & -2.45163400 & 1.47510900 & -0.35158000 \\
\hline $\mathbb{P}$ & -0.72490100 & 1.71384300 & 0.29396400 \\
\hline $\mathrm{C}$ & -0.79330800 & 1.73782700 & 2.12464100 \\
\hline 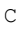 & 0.40306000 & 1.53242600 & 2.83206800 \\
\hline $\mathrm{H}$ & 1.32684200 & 1.34441100 & 2.29230400 \\
\hline C & 0.41582700 & 1.61107900 & 4.22492500 \\
\hline $\mathrm{H}$ & 1.34514000 & 1.45458700 & 4.76429200 \\
\hline C & -0.75935700 & 1.89989700 & 4.92112900 \\
\hline $\mathrm{H}$ & -0.74754500 & 1.96358200 & 6.00508000 \\
\hline $\mathrm{C}$ & -1.94886800 & 2.11218900 & 4.22138900 \\
\hline H & -2.86340100 & 2.34325500 & 4.75935100 \\
\hline C & -1.97063800 & 2.03240300 & 2.82794400 \\
\hline $\mathrm{H}$ & -2.90459100 & 2.18353300 & 2.29796500 \\
\hline C & -0.33708500 & 3.45198300 & -0.17508700 \\
\hline C & 0.20230200 & 3.73943300 & -1.43880600 \\
\hline $\mathrm{H}$ & 0.47303700 & 2.93250400 & -2.10958700 \\
\hline $\mathrm{C}$ & 0.40948900 & 5.06117400 & -1.83181100 \\
\hline $\mathrm{H}$ & 0.81988400 & 5.27045300 & -2.81503900 \\
\hline C & 0.10070100 & 6.10968000 & -0.96160200 \\
\hline $\mathrm{H}$ & 0.26900800 & 7.13802900 & -1.26694000 \\
\hline C & -0.42198000 & 5.83091100 & 0.30157000 \\
\hline H & -0.65995100 & 6.64009300 & 0.98524600 \\
\hline C & -0.64616800 & 4.50955600 & 0.69292200 \\
\hline $\mathrm{H}$ & -1.05708900 & 4.31034700 & 1.67634500 \\
\hline $\mathrm{C}$ & -2.91240200 & 2.39643500 & -1.29520700 \\
\hline $\mathrm{H}$ & -2.34081000 & 3.26143100 & -1.59663100 \\
\hline $\mathrm{C}$ & -4.17631400 & 2.19618800 & -1.86487400 \\
\hline 0 & -4.59253700 & 3.10921600 & -2.75855000 \\
\hline C & -5.88853700 & 2.92953500 & -3.34291400 \\
\hline $\mathrm{H}$ & -6.01673300 & 3.77250600 & -4.02183400 \\
\hline $\mathrm{H}$ & -6.66850000 & 2.93787200 & -2.57662500 \\
\hline $\mathrm{H}$ & -5.94310400 & 1.98645400 & -3.89367100 \\
\hline $\mathrm{N}$ & -4.95919100 & 1.16444700 & -1.56290800 \\
\hline $\mathrm{C}$ & -4.51086100 & 0.28961200 & -0.66867100 \\
\hline 0 & -5.28880200 & -0.76842600 & -0.36733500 \\
\hline C & -6.56331600 & -0.86256800 & -1.01477500 \\
\hline $\mathrm{H}$ & -7.02556200 & -1.76235100 & -0.6074580 \\
\hline $\mathrm{H}$ & -6.44465900 & -0.95203900 & -2.09787200 \\
\hline $\mathrm{H}$ & -7.18041000 & 0.01277000 & -0.79723300 \\
\hline C & 5.26406100 & -1.00636500 & 0.35352300 \\
\hline $\mathrm{H}$ & 5.45795600 & -0.17657100 & -0.32237600 \\
\hline $\mathrm{C}$ & 6.24520100 & -1.39159900 & 1.18888500 \\
\hline C & 3.85844600 & -1.55693000 & 0.28706200 \\
\hline
\end{tabular}

\begin{tabular}{|c|c|c|c|}
\hline $\mathrm{H}$ & 3.17610000 & -0.86508500 & 0.79332600 \\
\hline $\mathrm{H}$ & 3.77813400 & -2.51971000 & 0.80131600 \\
\hline C & 7.59503900 & -0.69983000 & 1.14439600 \\
\hline $\mathrm{C}$ & 6.11490000 & -2.48498300 & 2.22200100 \\
\hline $\mathrm{H}$ & 6.27903500 & -2.07365600 & 3.22585500 \\
\hline $\mathrm{H}$ & 5.13851500 & -2.97427600 & 2.22530800 \\
\hline C & 3.27756000 & -1.73279900 & -1.10990700 \\
\hline $\mathrm{N}$ & 3.92239300 & -2.45968900 & -2.04049000 \\
\hline C & 3.31581600 & -2.60135000 & -3.36768900 \\
\hline $\mathrm{H}$ & 4.10199700 & -2.84965900 & -4.08319900 \\
\hline $\mathrm{H}$ & 2.56254400 & -3.39737700 & -3.37169600 \\
\hline $\mathrm{H}$ & 2.83938700 & -1.66590000 & -3.65639900 \\
\hline C & 5.03574200 & -3.36477900 & -1.75658600 \\
\hline $\mathrm{H}$ & 5.51007800 & -3.11379600 & -0.81223900 \\
\hline $\mathrm{H}$ & 4.68028200 & -4.40192100 & -1.72840700 \\
\hline $\mathrm{H}$ & 5.78446000 & -3.27491900 & -2.54850100 \\
\hline 0 & 2.16581500 & -1.22881800 & -1.43035300 \\
\hline $\mathrm{H}$ & 7.84669700 & -0.34603900 & 2.15408800 \\
\hline $\mathrm{H}$ & 7.52602100 & 0.19089800 & 0.51249700 \\
\hline $\mathrm{P}$ & -0.84492500 & -1.69316600 & -0.55988500 \\
\hline O & -4.66753000 & 0.27311500 & 2.34886200 \\
\hline 0 & -2.69537200 & -3.60742400 & 3.87047900 \\
\hline C & 3.15006400 & 2.27960800 & -0.22308500 \\
\hline $\mathrm{Si}$ & 4.38423800 & 3.63416200 & -0.03548400 \\
\hline C & 6.09249800 & 2.91971500 & 0.34572800 \\
\hline $\mathrm{H}$ & 6.81718300 & 3.72473600 & 0.51254100 \\
\hline $\mathrm{H}$ & 6.07612700 & 2.29277600 & 1.24281900 \\
\hline $\mathrm{H}$ & 6.46111700 & 2.31142300 & -0.48731300 \\
\hline C & 4.45729100 & 4.60298300 & -1.65686100 \\
\hline $\mathrm{H}$ & 4.75950700 & 3.96148000 & -2.49114400 \\
\hline $\mathrm{H}$ & 3.48240100 & 5.03594700 & -1.90070500 \\
\hline $\mathrm{H}$ & 5.18130700 & 5.42268100 & -1.58516100 \\
\hline C & 3.83424100 & 4.75959100 & 1.37951400 \\
\hline $\mathrm{H}$ & 2.83853000 & 5.17278600 & 1.18938500 \\
\hline $\mathrm{H}$ & 3.79541000 & 4.21527900 & 2.32901500 \\
\hline $\mathrm{H}$ & 4.52967500 & 5.59746000 & 1.50448400 \\
\hline $\mathrm{H}$ & 6.87644700 & -3.25994000 & 2.07895500 \\
\hline C & 8.74154000 & -1.59330500 & 0.63544900 \\
\hline $\mathrm{H}$ & 9.68095700 & -1.03294100 & 0.61580800 \\
\hline $\mathrm{H}$ & 8.89670100 & -2.46836100 & 1.27387200 \\
\hline $\mathrm{H}$ & 8.54168900 & -1.94792400 & -0.38121300 \\
\hline
\end{tabular}




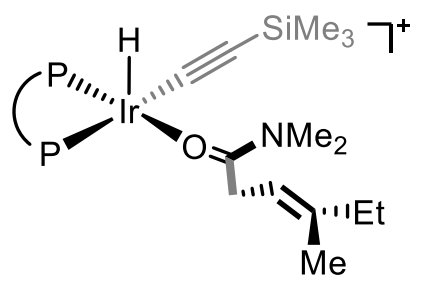

$G_{\text {sol }}=-3633.065621$ Hatree

\section{C}$$
\text { C }
$$$$
\text { C }
$$$$
\mathrm{H}
$$ 


\begin{tabular}{|c|c|c|c|}
\hline C & 3.24034900 & 1.98869500 & 1.17906500 \\
\hline $\mathrm{H}$ & 3.23279400 & 2.85559200 & 1.84793600 \\
\hline $\mathrm{H}$ & 2.33538100 & 1.41392100 & 1.42866400 \\
\hline C & 6.49349300 & 0.20799900 & 2.46457100 \\
\hline C & 5.28200600 & 2.19404900 & 3.52802500 \\
\hline $\mathrm{H}$ & 6.25580400 & 2.67966000 & 3.66478200 \\
\hline $\mathrm{H}$ & 4.53401600 & 2.97742300 & 3.38464000 \\
\hline C & 3.01346900 & 2.46760400 & -0.25046100 \\
\hline $\mathrm{N}$ & 3.87119000 & 3.31860800 & -0.83280600 \\
\hline C & 3.60865400 & 3.75511200 & -2.20889300 \\
\hline $\mathrm{H}$ & 2.90123700 & 4.59089700 & -2.22656500 \\
\hline $\mathrm{H}$ & 4.55139200 & 4.07102800 & -2.65886700 \\
\hline $\mathrm{H}$ & 3.18720500 & 2.92774500 & -2.77813200 \\
\hline C & 4.95920200 & 3.99767600 & -0.13038500 \\
\hline $\mathrm{H}$ & 5.21504700 & 3.47252000 & 0.78590900 \\
\hline $\mathrm{H}$ & 5.84491200 & 4.00765700 & -0.77105800 \\
\hline $\mathrm{H}$ & 4.68501400 & 5.03426000 & 0.09914000 \\
\hline 0 & 1.99949200 & 2.09352100 & -0.90270000 \\
\hline $\mathrm{H}$ & 7.43824700 & 0.76792200 & 2.51327700 \\
\hline $\mathrm{H}$ & 6.52410100 & -0.37864500 & 1.54075000 \\
\hline P & -0.31262800 & -1.65941100 & -0.10605700 \\
\hline 0 & -4.92079400 & -0.03787400 & 1.73580500 \\
\hline 0 & -2.39630100 & -2.77797700 & 4.45016900 \\
\hline C & 3.32369500 & -1.27690600 & -1.65792200 \\
\hline $\mathrm{Si}$ & 4.73980300 & -2.21982600 & -2.35540900 \\
\hline C & 4.22058000 & -4.01975800 & -2.60455100 \\
\hline $\mathrm{H}$ & 5.05175500 & -4.61605000 & -2.99788300 \\
\hline $\mathrm{H}$ & 3.89789400 & -4.47058800 & -1.66052400 \\
\hline $\mathrm{H}$ & 3.38904300 & -4.09930100 & -3.31258500 \\
\hline C & 5.24481100 & -1.45317200 & -4.00815000 \\
\hline $\mathrm{H}$ & 4.41675100 & -1.47066100 & -4.72432100 \\
\hline $\mathrm{H}$ & 5.55784000 & -0.41103600 & -3.88519300 \\
\hline $\mathrm{H}$ & 6.08203000 & -2.00341200 & -4.45240000 \\
\hline C & 6.19104300 & -2.13520400 & -1.14326500 \\
\hline $\mathrm{H}$ & 6.52539100 & -1.10258200 & -0.99530000 \\
\hline $\mathrm{H}$ & 5.91406000 & -2.54711700 & -0.16709900 \\
\hline $\mathrm{H}$ & 7.04589300 & -2.70951600 & -1.51787500 \\
\hline $\mathrm{H}$ & 5.04995700 & 1.69594900 & 4.47665900 \\
\hline C & 6.42752700 & -0.74842800 & 3.66892200 \\
\hline $\mathrm{H}$ & 7.26432100 & -1.45253200 & 3.64229300 \\
\hline $\mathrm{H}$ & 6.47876400 & -0.21401700 & 4.62235200 \\
\hline $\mathrm{H}$ & 5.49850900 & -1.32858500 & 3.65694000 \\
\hline
\end{tabular}

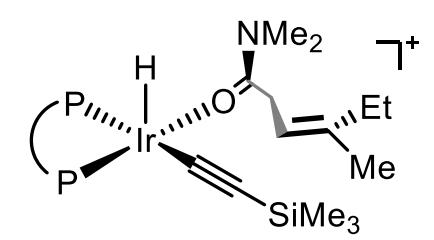

$$
{ }^{\mathrm{H}}
$$

c

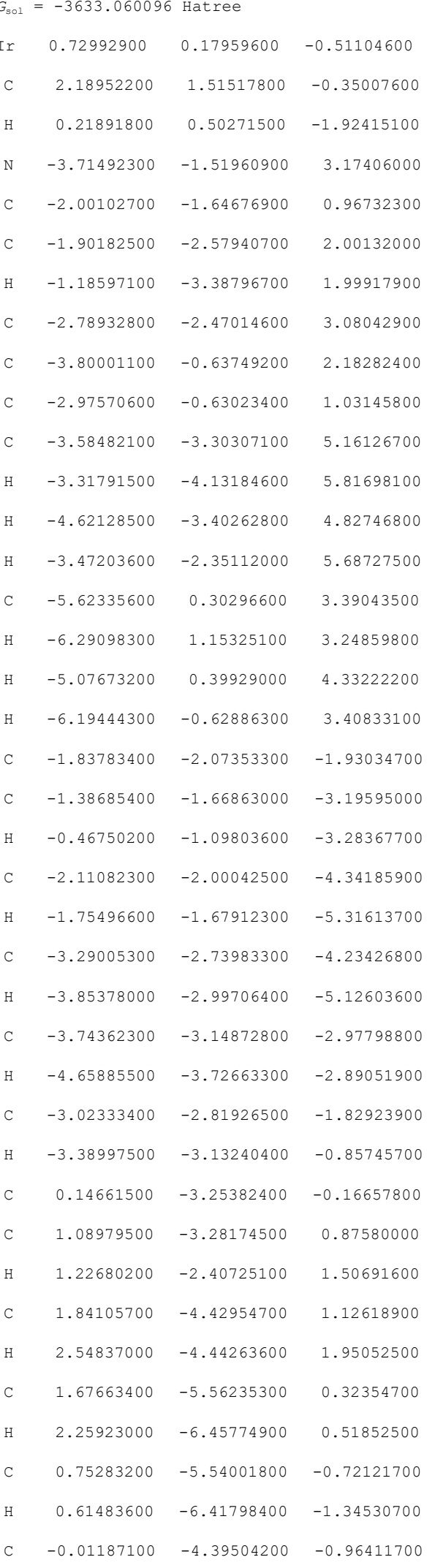




\begin{tabular}{|c|c|c|c|}
\hline $\mathrm{H}$ & -0.73368700 & -4.39820200 & -1.77295800 \\
\hline & -3.27668000 & 0.33843600 & -0.05733800 \\
\hline & -2.48465300 & 1.44054800 & -0.44707800 \\
\hline & -0.77657000 & 1.74967200 & 0.21770900 \\
\hline$C$ & -0.88029500 & 1.86314600 & 2.04302900 \\
\hline$C$ & 0.30322000 & 1.70567900 & 2.78314700 \\
\hline 11 & 1.23838700 & 1.49740200 & 2.27146200 \\
\hline $\mathrm{C}$ & 0.28709100 & 1.85582200 & 4.17009200 \\
\hline $\mathrm{H}$ & 1.20655800 & 1.73579400 & 4.73508800 \\
\hline$C$ & -0.90449600 & 2.16746000 & 4.82750000 \\
\hline H & -0.91510900 & 2.28622200 & 5.90682100 \\
\hline C & -2.08185500 & 2.33059300 & 4.09478800 \\
\hline $\mathrm{H}$ & -3.00940000 & 2.57821900 & 4.60223600 \\
\hline C & -2.07462200 & 2.17997900 & 2.70708100 \\
\hline H & -2.99928300 & 2.29348300 & 2.15182500 \\
\hline C & -0.40947700 & 3.46764800 & -0.33312800 \\
\hline C & 0.17261600 & 3.69859200 & -1.58953400 \\
\hline H & 0.48509200 & 2.86285100 & -2.20436900 \\
\hline C & 0.36777900 & 5.00156100 & -2.04610900 \\
\hline $\mathrm{H}$ & 0.81495800 & 5.16689900 & -3.02166800 \\
\hline C & 0.00012800 & 6.08778200 & -1.24856300 \\
\hline $\mathrm{H}$ & 0.15777200 & 7.10186400 & -1.60341700 \\
\hline $\mathrm{C}$ & -0.56625400 & 5.86589200 & 0.00700700 \\
\hline H & -0.84805400 & 6.70540100 & 0.63520500 \\
\hline C & -0.77515100 & 4.56343200 & 0.46296800 \\
\hline H & -1.21789200 & 4.40838000 & 1.44059200 \\
\hline C & -2.94296600 & 2.29873300 & -1.44936500 \\
\hline $\mathrm{H}$ & -2.38333000 & 3.15909600 & -1.78519100 \\
\hline C & -4.18775600 & 2.03729800 & -2.03647200 \\
\hline O & -4.60263300 & 2.89047500 & -2.98802600 \\
\hline C & -5.87860900 & 2.64736800 & -3.59310000 \\
\hline H & -6.00859700 & 3.44834800 & -4.32077700 \\
\hline $\mathrm{H}$ & -6.67810900 & 2.67882000 & -2.84782600 \\
\hline H & -5.89701100 & 1.67433700 & -4.09166700 \\
\hline $\mathrm{N}$ & -4.95383100 & 1.00442500 & -1.69741000 \\
\hline C & -4.50788300 & 0.19016400 & -0.74629200 \\
\hline 0 & -5.26811400 & -0.86893000 & -0.40590400 \\
\hline C & -6.52300300 & -1.03019700 & -1.07789400 \\
\hline H & -6.97257600 & -1.91952500 & -0.63500600 \\
\hline H & -6.37495300 & -1.17257500 & -2.15167200 \\
\hline $\mathrm{H}$ & -7.16732700 & -0.16146100 & -0.92129700 \\
\hline C & 5.41672300 & -0.75211800 & -0.13603700 \\
\hline $\mathrm{F}$ & 5.48666900 & -0.07122300 & -0.98188200 \\
\hline 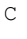 & 6.50332500 & -0.91749900 & 0.63896100 \\
\hline & 4.04811300 & -1.36540700 & 0.07006000 \\
\hline
\end{tabular}

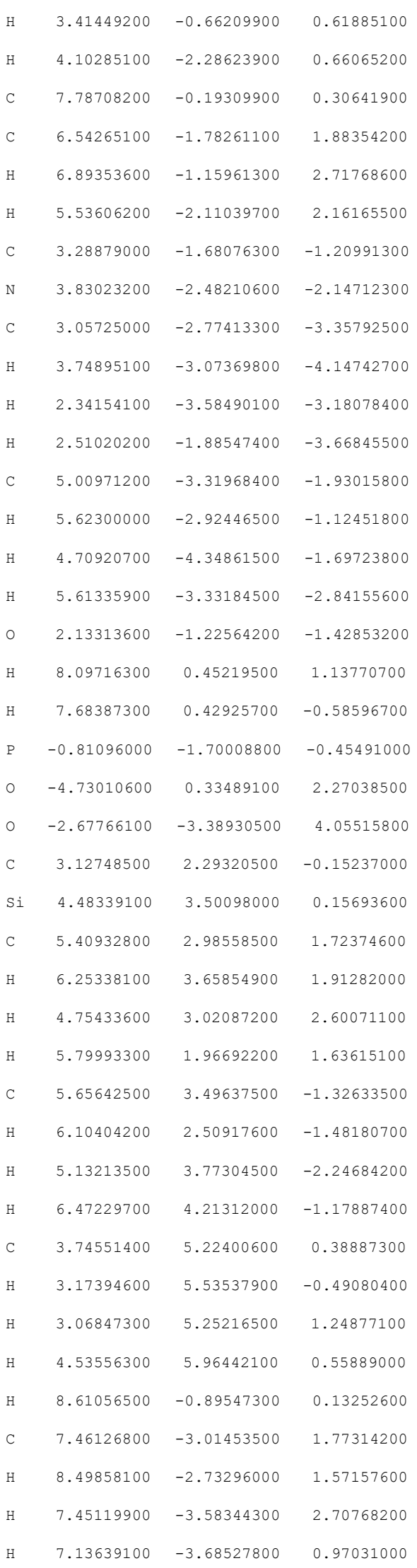




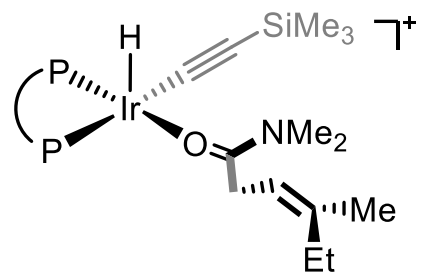

$G_{\text {sol }}=-3633.062357$ Hatree

C $2.27419400-1.10539800$

H $\quad 0.12961700 \quad-0.06718000$

$\mathrm{N} \quad-3.74273100 \quad-0.63283400$

C $\quad-1.80382700-1.26600000$

C $\quad-1.68577300 \quad-1.72198800$

$\mathrm{H} \quad-0.86377900 \quad-2.33659900$

C $\quad-2.68890000-1.38006900$

C $\quad-3.84022100 \quad-0.19463000$

C $\quad-2.91041600$

C $\quad-3.57776200$

H $\quad-3.26877400$

H $\quad-4.54660700$

$\mathrm{H} \quad-3.66094000$

C $\quad-5.86747900$

H $\quad-6.62875100$

$\mathrm{H} \quad-5.42487300$

$\mathrm{H} \quad-6.30678900$

C $\quad-1.20555500$

$-0.51779900$

0.43323800

C $\quad-1.04421400$

$\mathrm{H} \quad-0.50668700$

C $\quad-2.25312300$

$\mathrm{H} \quad-2.65994100$

C $\quad-2.93568600$

H $\quad-3.87331900$

C -2.41638800

H $\quad-2.96349800$

C $\quad 0.61418600$

C $\quad 1.59719700$

H $\quad 1.74693200$

C $\quad 2.39342700$

$\mathrm{H} \quad 3.14859600$

C 2.22493000

$\mathrm{H} \quad 2.84885200$

C $\quad 1.25324000$

H 1.11835100
0.44747000$$
\text { - }
$$

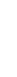$$
-2
$$$$
\text { C }
$$

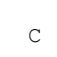$$
\text { c }
$$$$
\text { H }
$$$$
\text { C }
$$ 


\begin{tabular}{|c|c|c|c|}
\hline C & 3.37904400 & 1.83728100 & 0.87667300 \\
\hline $\mathrm{H}$ & 3.43493600 & 2.79525000 & 1.40333200 \\
\hline $\mathrm{H}$ & 2.43633000 & 1.37190500 & 1.20375100 \\
\hline C & 6.47819600 & 0.02267600 & 2.44958500 \\
\hline C & 5.36741500 & 2.18009600 & 3.26807100 \\
\hline $\mathrm{H}$ & 4.50388300 & 2.83357500 & 3.10892200 \\
\hline C & 3.18151100 & 2.10658500 & -0.61116400 \\
\hline $\mathrm{N}$ & 4.09782900 & 2.79158200 & -1.31201500 \\
\hline C & 3.86544000 & 3.03199900 & -2.74048900 \\
\hline $\mathrm{H}$ & 3.26205700 & 3.93324800 & -2.89323600 \\
\hline $\mathrm{H}$ & 4.83125400 & 3.15718200 & -3.23347600 \\
\hline $\mathrm{H}$ & 3.33948700 & 2.18097100 & -3.16986200 \\
\hline C & 5.23055600 & 3.49438300 & -0.71080700 \\
\hline $\mathrm{H}$ & 5.47163000 & 3.07569100 & 0.26280100 \\
\hline $\mathrm{H}$ & 6.10516400 & 3.37409100 & -1.35520400 \\
\hline $\mathrm{H}$ & 5.01669800 & 4.56552200 & -0.61315500 \\
\hline 0 & 2.13852700 & 1.71347500 & -1.20362700 \\
\hline $\mathrm{H}$ & 7.47993300 & 0.45608200 & 2.34858800 \\
\hline $\mathrm{H}$ & 6.39299400 & -0.79630600 & 1.73174000 \\
\hline P & -0.44170900 & -1.68107300 & 0.16956700 \\
\hline 0 & -4.89030400 & 0.58321000 & 1.70180200 \\
\hline 0 & -2.55231000 & -1.84513100 & 4.84609100 \\
\hline C & 3.19795400 & -1.83847500 & -1.40202200 \\
\hline $\mathrm{Si}$ & 4.51945600 & -3.00691100 & -1.92115100 \\
\hline C & 3.80394600 & -4.75502200 & -1.98468300 \\
\hline $\mathrm{H}$ & 4.57262600 & -5.48176700 & -2.27117300 \\
\hline $\mathrm{H}$ & 3.40440100 & -5.05239600 & -1.00971400 \\
\hline $\mathrm{H}$ & 2.99001000 & -4.82560700 & -2.71377200 \\
\hline C & 5.16366100 & -2.50515200 & -3.62635600 \\
\hline $\mathrm{H}$ & 4.36156800 & -2.51035500 & -4.37170900 \\
\hline $\mathrm{H}$ & 5.59684100 & -1.49954900 & -3.60920000 \\
\hline $\mathrm{H}$ & 5.94107000 & -3.19787200 & -3.96820400 \\
\hline C & 5.92944300 & -2.94036100 & -0.66016800 \\
\hline $\mathrm{H}$ & 6.38178300 & -1.94341100 & -0.62201700 \\
\hline $\mathrm{H}$ & 5.57271000 & -3.19209100 & 0.34414600 \\
\hline $\mathrm{H}$ & 6.72002600 & -3.65247000 & -0.92230000 \\
\hline $\mathrm{H}$ & 5.21717200 & 1.72455100 & 4.25710000 \\
\hline $\mathrm{H}$ & 6.42300200 & -0.39887400 & 3.46123700 \\
\hline C & 6.64458300 & 3.03991800 & 3.31967900 \\
\hline $\mathrm{H}$ & 6.54064600 & 3.82952500 & 4.06959900 \\
\hline $\mathrm{H}$ & 7.52422100 & 2.44772400 & 3.58579600 \\
\hline $\mathrm{H}$ & 6.84584900 & 3.51984500 & 2.35585800 \\
\hline
\end{tabular}

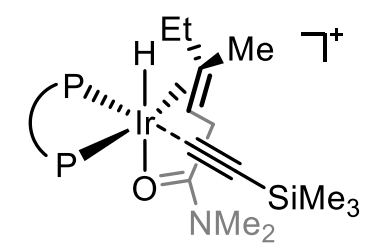

Ir $\quad-1.04419000 \quad 0.46180100 \quad-0.64559400$

C $\quad-2.86805600 \quad-0.33444400 \quad-0.43963400$

$\begin{array}{llll}\text { C } & -2.25498100 & 2.00428800 & -2.51099400\end{array}$

C $\quad-2.08978900 \quad 2.53843800 \quad-1.24850900$

C $\quad 1.44963000 \quad-2.00884200 \quad-0.29293300$

C $\quad 1.62354700 \quad-3.03334800 \quad-1.22703100$

H $\quad 0.80199900 \quad-3.61041500 \quad-1.62370700$

$\begin{array}{llll}\text { C } & 2.92272700 & -3.33524600 & -1.65338200\end{array}$

$\begin{array}{llll}\text { C } \quad 3.82819100 & -1.71547200 & -0.33665100\end{array}$

$\begin{array}{llll}\text { C } & 2.57425600 & -1.30463700 & 0.18471200\end{array}$

$\begin{array}{llll}\text { C } & 4.37711100 & -4.66309800 & -2.98564400\end{array}$

$\mathrm{H} \quad 4.24773800 \quad-5.47789400 \quad-3.69807700$

H $\quad 4.99790400 \quad-4.98904300 \quad-2.14650700$

$\mathrm{H} \quad 4.85701600 \quad-3.80774800 \quad-3.46912600$

$\begin{array}{llll}\text { C } & 6.19259600 & -1.46781500 & -0.38735300\end{array}$

$\begin{array}{llll}\mathrm{H} & 6.91423700 & -0.81336100 & 0.10264100\end{array}$

H $\quad 6.25732100 \quad-1.36140700 \quad-1.47331100$

H $\quad 6.38475500 \quad-2.51059300 \quad-0.12178300$

C $\quad-0.37671100 \quad-1.77286900 \quad 2.02981200$

$\begin{array}{llll}\text { C } & -1.48888500 & -1.25053400 & 2.70954300\end{array}$

H $\quad-2.23869000 \quad-0.69147400 \quad 2.16284100$

C $\quad-1.63763000 \quad-1.47342400 \quad 4.07858800$

$\mathrm{H} \quad-2.50455800 \quad-1.07390700 \quad 4.59694400$

C $\quad-0.68516500 \quad-2.21507600 \quad 4.78045700$

$\mathrm{H} \quad-0.80586700 \quad-2.38835300 \quad 5.84577600$

$\begin{array}{llll}\text { C } & 0.41871900 & -2.73987400 \quad 4.10706200\end{array}$

H $\quad 1.15932600 \quad-3.32430000 \quad 4.64475300$

$\begin{array}{llll}\text { C } & 0.57415600 & -2.52488500 & 2.73642800\end{array}$

H $\quad 1.43926000 \quad-2.93491900 \quad 2.22874300$

$\begin{array}{llll}\text { C } & -1.25573400 & -3.02406400 & -0.42925600\end{array}$

C $\quad-1.73581100 \quad-3.04108000 \quad-1.74806800$

$\mathrm{H} \quad-1.60881200 \quad-2.17020900 \quad-2.37973400$

C $\quad-2.39158000 \quad-4.16496900 \quad-2.24818600$

H $\quad-2.75347300 \quad-4.16362200 \quad-3.27198700$

C $\quad-2.59281400 \quad-5.28217400 \quad-1.43389200$

H $\quad-3.10908300 \quad-6.15477600 \quad-1.82266400$

C $\quad-2.12857300 \quad-5.26995100 \quad-0.11876000$

H $\quad-2.28176400 \quad-6.13225400 \quad 0.52310700$

$\begin{array}{llll}\text { C } & -1.45935400 & -4.15065700 & 0.38125100\end{array}$ 


\begin{tabular}{|c|c|c|c|}
\hline $\mathrm{H}$ & -1.10256000 & -4.16297600 & 1.40453200 \\
\hline C & 2.56863500 & -0.20922200 & 1.19088400 \\
\hline 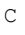 & 2.10798400 & 1.10365100 & 0.95316400 \\
\hline$P$ & 1.20496800 & 1.49061000 & -0.63321100 \\
\hline $\mathrm{C}$ & 2.42045900 & 1.02471800 & -1.93785800 \\
\hline C & 1.99860900 & 0.36682200 & -3.10107300 \\
\hline H & 0.96015400 & 0.07241600 & -3.20379000 \\
\hline C & 2.90857400 & 0.07761700 & -4.12036200 \\
\hline $\mathrm{H}$ & 2.56901900 & -0.43501300 & -5.01539800 \\
\hline C & 4.24753900 & 0.44536000 & -3.98784200 \\
\hline $\mathrm{H}$ & 4.95400600 & 0.22560800 & -4.78279300 \\
\hline C & 4.67863900 & 1.09724500 & -2.82882300 \\
\hline $\mathrm{H}$ & 5.72012600 & 1.38523200 & -2.72050800 \\
\hline C & 3.77391100 & 1.38286800 & -1.80752800 \\
\hline $\mathrm{H}$ & 4.12097400 & 1.88169100 & -0.90826300 \\
\hline $\mathrm{C}$ & 1.22060300 & 3.34820700 & -0.71161900 \\
\hline C & 0.50959900 & 4.09709400 & 0.24699500 \\
\hline $\mathrm{H}$ & -0.02158900 & 3.58506100 & 1.04147200 \\
\hline C & 0.48030200 & 5.49032200 & 0.18901800 \\
\hline $\mathrm{H}$ & -0.05809400 & 6.04849800 & 0.94950600 \\
\hline C & 1.13911800 & 6.16568200 & -0.84086500 \\
\hline $\mathrm{H}$ & 1.11467300 & 7.25012800 & -0.88701300 \\
\hline $\mathrm{C}$ & 1.82570300 & 5.43589400 & -1.80957300 \\
\hline $\mathrm{H}$ & 2.33757800 & 5.94900700 & -2.61818800 \\
\hline C & 1.86933500 & 4.04035000 & -1.74644900 \\
\hline $\mathrm{H}$ & 2.41727600 & 3.49804400 & -2.50719000 \\
\hline C & 2.29835400 & 2.08324100 & 1.92817100 \\
\hline H & 2.00578000 & 3.11299900 & 1.79690400 \\
\hline C & 2.93666600 & 1.72309500 & 3.12164500 \\
\hline 0 & 3.10683700 & 2.69516300 & 4.03732700 \\
\hline C & 3.78779000 & 2.35379100 & 5.25074200 \\
\hline H & 3.81690500 & 3.27473200 & 5.83333400 \\
\hline $\mathrm{H}$ & 4.80324100 & 2.00365000 & 5.04593600 \\
\hline $\mathrm{H}$ & 3.24934500 & 1.57493800 & 5.79775400 \\
\hline $\mathrm{N}$ & 3.37335800 & 0.49413800 & 3.37895300 \\
\hline C & 3.19935700 & -0.43011500 & 2.43953200 \\
\hline 0 & 3.64912200 & -1.67810900 & 2.68232300 \\
\hline C & 4.34381900 & -1.90613000 & 3.91414900 \\
\hline $\mathrm{H}$ & 4.63844000 & -2.95569200 & 3.88698700 \\
\hline H & 3.69401200 & -1.71423200 & 4.77215100 \\
\hline $\mathrm{H}$ & 5.22674800 & -1.26632200 & 3.99001300 \\
\hline $\mathrm{N}$ & 4.00351900 & -2.69417700 & -1.21850900 \\
\hline H & -1.24617500 & 3.20819300 & -1.12817700 \\
\hline 0 & 4.91241200 & -1.04293200 & 0.09376000 \\
\hline C & -3.15990500 & 2.71848600 & -0.19161500 \\
\hline
\end{tabular}

\begin{tabular}{|c|c|c|}
\hline-4.02533200 & 2.07684900 & -0.37757600 \\
\hline-3.50259800 & 3.75968700 & -0.21012900 \\
\hline-1.22252300 & 2.30040300 & -3.57986000 \\
\hline-3.52357300 & 1.36672400 & -3.00761400 \\
\hline-4.14161200 & 2.14321500 & -3.47715500 \\
\hline-3.30735900 & 0.61844200 & -3.77542600 \\
\hline-2.60516900 & 2.36274900 & 1.18345900 \\
\hline-3.20956500 & 2.82795200 & 2.29156700 \\
\hline-2.65342800 & 2.53928900 & 3.61569600 \\
\hline-1.77432300 & 1.90878900 & 3.50811100 \\
\hline-2.37806500 & 3.47436600 & 4.11581800 \\
\hline-3.40205700 & 2.02531600 & 4.22719400 \\
\hline-4.40867500 & 3.66568800 & 2.27915900 \\
\hline-5.04669100 & 3.37301200 & 3.11702500 \\
\hline-4.15702000 & 4.72692700 & 2.39376300 \\
\hline-4.97999700 & 3.52770300 & 1.36328900 \\
\hline-1.57956800 & 1.63740400 & 1.27051300 \\
\hline-1.00809000 & 1.38381300 & -4.14027700 \\
\hline-0.28897900 & 2.63022000 & -3.11882100 \\
\hline-0.28858100 & -1.58301800 & 0.20670400 \\
\hline 3.05419100 & -4.33039200 & -2.54802100 \\
\hline-3.98852200 & -0.78878500 & -0.20189400 \\
\hline-5.62071100 & -1.55315700 & 0.15334000 \\
\hline-5.43725700 & -2.76261100 & 1.59516000 \\
\hline-6.39593500 & -3.23843800 & 1.83104600 \\
\hline-4.71990500 & -3.55260400 & 1.35102600 \\
\hline-5.08207200 & -2.25939500 & 2.50070300 \\
\hline-6.23552100 & -2.47219600 & -1.37946500 \\
\hline-5.52675500 & -3.25267200 & -1.67426700 \\
\hline-7.20231400 & -2.95064300 & -1.18597200 \\
\hline-6.36287900 & -1.79623500 & -2.23162400 \\
\hline-6.85573100 & -0.19308100 & 0.61946300 \\
\hline-7.84007400 & -0.62040800 & 0.84147900 \\
\hline-6.52791000 & 0.35748800 & 1.50825200 \\
\hline-6.98651000 & 0.52726000 & -0.19560400 \\
\hline-0.79224000 & -0.25696300 & -2.00768400 \\
\hline-4.09779200 & 0.88940500 & -2.21680900 \\
\hline-1.70709600 & 3.38701700 & -4.56196500 \\
\hline-2.59736400 & 3.07386100 & -5.11396800 \\
\hline-1.94016300 & 4.31882500 & -4.03668900 \\
\hline-0.92217900 & 3.60100400 & -5.29290400 \\
\hline
\end{tabular}




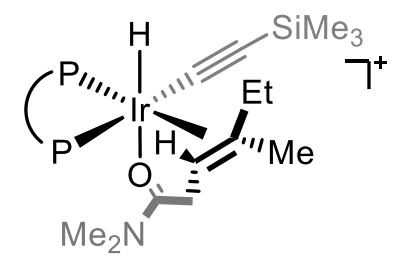

$G_{\text {sol }}=-3633.064933$ Hatree$$
\text { C }
$$$$
\text { H }
$$

\section{C}




\begin{tabular}{|c|c|c|c|}
\hline $\mathrm{H}$ & 1.25176100 & 3.86230900 & 0.96322300 \\
\hline $\mathrm{H}$ & 3.00345100 & 3.93326500 & 1.02648000 \\
\hline $\mathrm{C}$ & 3.68403000 & 1.87873900 & -2.33045100 \\
\hline C & 4.65714600 & 1.97138100 & 0.04009300 \\
\hline $\mathrm{H}$ & 4.48921000 & 1.64731100 & 1.06806900 \\
\hline C & 2.09401300 & 2.31113900 & 2.13680200 \\
\hline $\mathrm{N}$ & 2.46113200 & 2.74518000 & 3.35831900 \\
\hline C & 2.45667300 & 1.83593600 & 4.50595200 \\
\hline $\mathrm{H}$ & 2.16498100 & 0.84106200 & 4.17951100 \\
\hline $\mathrm{H}$ & 3.45653900 & 1.79764500 & 4.95124600 \\
\hline $\mathrm{H}$ & 1.75068700 & 2.19157300 & 5.26385500 \\
\hline $\mathrm{C}$ & 2.91788600 & 4.10689200 & 3.63875600 \\
\hline $\mathrm{H}$ & 2.55777900 & 4.39754200 & 4.62897600 \\
\hline $\mathrm{H}$ & 4.01271300 & 4.17024100 & 3.63982900 \\
\hline $\mathrm{H}$ & 2.51732200 & 4.81745100 & 2.91803900 \\
\hline 0 & 1.66591800 & 1.13837000 & 1.97535300 \\
\hline $\mathrm{H}$ & 2.90260368 & 2.47523650 & -2.80925717 \\
\hline$P$ & 0.19723700 & -1.58165700 & 0.10840000 \\
\hline $\mathrm{N}$ & -3.68293300 & -1.62347500 & 2.67578200 \\
\hline C & 3.89086600 & -0.86892400 & -0.73727100 \\
\hline $\mathrm{Si}$ & 5.29081200 & -2.05362000 & -1.02408800 \\
\hline C & 6.25529400 & -2.27270700 & 0.58209000 \\
\hline $\mathrm{H}$ & 7.08016000 & -2.98056100 & 0.44266600 \\
\hline $\mathrm{H}$ & 6.68518500 & -1.32686100 & 0.92814800 \\
\hline $\mathrm{H}$ & 5.60837100 & -2.66088000 & 1.37473700 \\
\hline $\mathrm{C}$ & 6.40297800 & -1.32246500 & -2.36774600 \\
\hline $\mathrm{H}$ & 6.84053500 & -0.36454600 & -2.06753000 \\
\hline $\mathrm{H}$ & 7.23226000 & -2.00801800 & -2.57647700 \\
\hline $\mathrm{H}$ & 5.85786000 & -1.16845500 & -3.30455900 \\
\hline C & 4.55439200 & -3.69239200 & -1.59799900 \\
\hline $\mathrm{H}$ & 5.35615600 & -4.40591000 & -1.82008600 \\
\hline $\mathrm{H}$ & 3.91575700 & -4.13157900 & -0.82613300 \\
\hline $\mathrm{H}$ & 3.95251100 & -3.56874600 & -2.50359800 \\
\hline $\mathrm{H}$ & 0.76541900 & 0.44201000 & -1.72375300 \\
\hline $\mathrm{H}$ & 5.48825200 & 1.39341200 & -0.36725100 \\
\hline $\mathrm{H}$ & 3.61840008 & 0.86981083 & -2.74284397 \\
\hline $\mathrm{H}$ & 4.94615300 & 3.03103800 & 0.06108300 \\
\hline C & 5.06617974 & 2.47770355 & -2.65644074 \\
\hline $\mathrm{H}$ & 5.16922072 & 3.50107666 & -2.28340380 \\
\hline $\mathrm{H}$ & 5.88013602 & 1.87813722 & -2.23800817 \\
\hline $\mathrm{H}$ & 5.20309886 & 2.50315671 & -3.74152109 \\
\hline
\end{tabular}

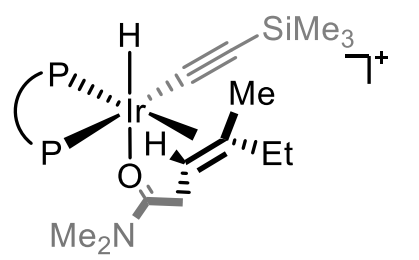

$G_{\text {sol }}=-3633.064734$ Hatree

Ir $\quad 1.08909800 \quad 0.56707100 \quad-0.39358900$

$\begin{array}{llll}\text { C } & 1.90658100 & 2.85917400 & -0.71848800\end{array}$

$\begin{array}{llll}\text { C } \quad 3.05404500 & 2.35299000 & -1.28921000\end{array}$

$\begin{array}{llll}\text { C } & 2.81474400 & -0.42385500 & -0.64092000\end{array}$

$\begin{array}{llll}\text { C } & -1.21787800 & -1.35108900 & 1.39284000\end{array}$

$\begin{array}{llll}\text { C } & -1.05234600 & -1.53778000 & 2.76803400\end{array}$

H $\quad-0.11017900 \quad-1.82776100 \quad 3.20890500$

C $\quad-2.16529300 \quad-1.36908900 \quad 3.60075500$

$\begin{array}{llll}\text { C } & -3.51733000 & -0.84502000 & 1.84543800\end{array}$

$\begin{array}{llll}\text { C } & -2.48254300 & -0.98773900 & 0.88577000\end{array}$

C $\quad-3.11383700 \quad-1.43843100 \quad 5.77998100$

$\mathrm{H} \quad-2.74094400 \quad-1.64901800 \quad 6.78248100$

H $\quad-3.89441600 \quad-2.15442400 \quad 5.50860300$

H $\quad-3.53055400 \quad-0.42840400 \quad 5.73344600$

$\begin{array}{llll}\text { C } & -5.79538300 & -0.36230200 & 2.32732800\end{array}$

$\mathrm{H} \quad-6.67076700 \quad-0.08931600 \quad 1.73721900$

$\mathrm{H} \quad-5.58015000 \quad 0.41173300 \quad 3.06863100$

H $\quad-5.96674600 \quad-1.31043200 \quad 2.84355100$

$\begin{array}{llll}\text { C } & -0.13539900 & -2.69978300 & -1.02225100\end{array}$

$\begin{array}{llll}\text { C } & 0.62137300 & -2.70559200 & -2.20435600\end{array}$

H $\quad 1.40766400 \quad-1.97316800 \quad-2.34525200$

$\begin{array}{llll}\text { C } & 0.38419000 & -3.67213700 & -3.18226500\end{array}$

$\mathrm{H} \quad 0.97532900 \quad-3.66837400 \quad-4.09314800$

C $\quad-0.60250900 \quad-4.64082100 \quad-2.99085200$

$\mathrm{H} \quad-0.78421800 \quad-5.39185100 \quad-3.75391700$

$\begin{array}{llll}\text { C } & -1.35224700 & -4.64397500 & -1.81348600\end{array}$

H $\quad-2.11788700 \quad-5.39760900-1.65522000$

C $\quad-1.12112200 \quad-3.68087400 \quad-0.83011000$

H $\quad-1.72065200 \quad-3.69001500 \quad 0.07306100$

$\begin{array}{llll}\text { C } & 1.46373800 & -2.44480100 & 1.38498300\end{array}$

$\begin{array}{llll}\text { C } & 2.32100300 & -1.76157000 & 2.26144800\end{array}$

$\begin{array}{llll}\mathrm{H} & 2.34138300 & -0.67938800 & 2.25923200\end{array}$

$\begin{array}{llll}\text { C } & 3.15843100 & -2.47182900 & 3.12131500\end{array}$

H $\quad 3.81802200 \quad-1.93256900 \quad 3.79512000$

$\begin{array}{llll}\text { C } \quad 3.16241500 & -3.86857000 & 3.10970600\end{array}$

H $\quad 3.81992600 \quad-4.41853700 \quad 3.77635800$

$\begin{array}{llll}\text { C } & 2.31990400 & -4.55299400 & 2.23411600\end{array}$

$\mathrm{H} \quad 2.31868900 \quad-5.63858900 \quad 2.21249800$

C $\quad 1.47267100 \quad-3.84732100 \quad 1.37695500$ 


\begin{tabular}{|c|c|c|c|}
\hline $\mathrm{H}$ & 0.83008600 & -4.39845700 & 0.70079200 \\
\hline$C$ & -2.81861400 & -0.77432300 & -0.54576800 \\
\hline & -2.38221300 & 0.32236200 & -1.31612900 \\
\hline & -1.17428900 & 1.53692300 & -0.57977600 \\
\hline $\mathrm{C}$ & -2.11335900 & 2.17808900 & 0.87332300 \\
\hline C & -1.50022200 & 2.24516500 & 2.13224700 \\
\hline $\mathrm{H}$ & -0.49532500 & 1.85990000 & 2.25726800 \\
\hline $\mathrm{C}$ & -2.19659700 & 2.75631500 & 3.23022200 \\
\hline $\mathrm{H}$ & -1.71453100 & 2.79631600 & 4.20271200 \\
\hline C & -3.50941000 & 3.20425400 & 3.08174200 \\
\hline H & -4.04809700 & 3.60729400 & 3.93427400 \\
\hline C & -4.13446500 & 3.12283700 & 1.83403900 \\
\hline H & -5.16024600 & 3.45877600 & 1.71483200 \\
\hline C & -3.44545400 & 2.60660900 & 0.73739400 \\
\hline H & -3.94505200 & 2.53769400 & -0.22388800 \\
\hline $\mathrm{C}$ & -1.20334300 & 2.94700100 & -1.78474700 \\
\hline C & -0.67199600 & 2.75002300 & -3.07505500 \\
\hline H & -0.28488500 & 1.77573300 & -3.35742900 \\
\hline C & -0.63444900 & 3.79242400 & -3.99993700 \\
\hline H & -0.23655400 & 3.61382100 & -4.99440500 \\
\hline C & -1.10175600 & 5.06138800 & -3.64714100 \\
\hline H & -1.06884000 & 5.87447900 & -4.36578900 \\
\hline $\mathrm{C}$ & -1.60632800 & 5.27573000 & -2.36599600 \\
\hline $\mathrm{H}$ & -1.96784700 & 6.25892600 & -2.07972300 \\
\hline C & -1.66004200 & 4.22806200 & -1.44123300 \\
\hline H & -2.06726000 & 4.41797500 & -0.45515700 \\
\hline C & -2.86172100 & 0.48302300 & -2.61632200 \\
\hline $\mathrm{H}$ & -2.59297200 & 1.31509500 & -3.24848500 \\
\hline C & -3.76569400 & -0.46490300 & -3.1144970 \\
\hline o & -4.21477900 & -0.27457500 & -4.36773400 \\
\hline C & -5.14947400 & -1.22521100 & -4.89283500 \\
\hline H & -5.37281700 & -0.88087900 & -5.90265300 \\
\hline $\mathrm{H}$ & -6.06212100 & -1.25219100 & -4.29110900 \\
\hline $\mathrm{H}$ & -4.71438800 & -2.22799400 & -4.9211270 \\
\hline $\mathrm{N}$ & -4.19195900 & -1.51223500 & -2.4143960 \\
\hline C & -3.73282200 & -1.65333200 & -1.1750480 \\
\hline 0 & -4.15278000 & -2.71183600 & -0.45384000 \\
\hline C & -5.12071100 & -3.58191800 & -1.05234300 \\
\hline H & -5.32618300 & -4.34304300 & -0.2989950 \\
\hline H & -4.72538700 & -4.04180400 & -1.96187600 \\
\hline $\mathrm{H}$ & -6.03558200 & -3.03773500 & -1.30087600 \\
\hline 0 & -1.97637900 & -1.56665900 & 4.91871800 \\
\hline $\mathrm{H}$ & 1.13600800 & 3.19001700 & -1.40134700 \\
\hline 0 & -4.72601200 & -0.47763400 & 1.3822030 \\
\hline & 1.87619400 & 3.52376800 & 0.6513300 \\
\hline
\end{tabular}

\begin{tabular}{|c|c|c|}
\hline 0.90717100 & 4.02580900 & 0.77290600 \\
\hline 2.63543000 & 4.30937800 & 0.70128300 \\
\hline 3.13176500 & 2.06614800 & -2.76283500 \\
\hline 4.35995800 & 2.25868200 & -0.53727100 \\
\hline 4.18398400 & 2.14188500 & 0.53476800 \\
\hline 2.01574800 & 2.57085400 & 1.82848200 \\
\hline 2.41530500 & 3.05284600 & 3.02256500 \\
\hline 2.58976300 & 2.14951300 & 4.16371700 \\
\hline 2.18361500 & 1.17197800 & 3.91692400 \\
\hline 3.65258300 & 2.04975500 & 4.41078400 \\
\hline 2.06401900 & 2.55289000 & 5.03407300 \\
\hline 2.80934300 & 4.44781800 & 3.23887800 \\
\hline 2.71524900 & 4.66898200 & 4.30340100 \\
\hline 3.84968700 & 4.62728700 & 2.94314500 \\
\hline 2.15721700 & 5.13549000 & 2.69981600 \\
\hline 1.70530900 & 1.36244300 & 1.69577900 \\
\hline 2.17607300 & 2.21488200 & -3.26644400 \\
\hline 0.28416300 & -1.50937900 & 0.31120100 \\
\hline-3.37176500 & -1.03273100 & 3.15369600 \\
\hline 3.81575800 & -1.10356800 & -0.87161000 \\
\hline 5.27396600 & -2.18275700 & -1.15736800 \\
\hline 6.41175300 & -2.11196800 & 0.35265600 \\
\hline 7.27927100 & -2.76864300 & 0.22102600 \\
\hline 6.78647100 & -1.09719800 & 0.52474700 \\
\hline 5.88184400 & -2.43116400 & 1.25588300 \\
\hline 6.21413400 & -1.58016900 & -2.68686000 \\
\hline 6.57506600 & -0.55337800 & -2.56206800 \\
\hline 7.08652000 & -2.21476500 & -2.88013000 \\
\hline 5.58169000 & -1.60575400 & -3.58075400 \\
\hline 4.68710800 & -3.95840600 & -1.43642600 \\
\hline 5.53455500 & -4.62477000 & -1.63393900 \\
\hline 4.15586900 & -4.33920600 & -0.55825300 \\
\hline 4.00521400 & -4.02237000 & -2.29087500 \\
\hline 0.77605100 & 0.11905500 & -1.85089200 \\
\hline 4.88673300 & 1.35714000 & -0.85734500 \\
\hline 3.47201200 & 1.03930500 & -2.92244500 \\
\hline 3.87298000 & 2.73195300 & -3.22132200 \\
\hline 5.24615000 & 3.50018500 & -0.78483100 \\
\hline 6.17789600 & 3.40400900 & -0.22026700 \\
\hline 5.51107000 & 3.60578100 & -1.84034200 \\
\hline 4.75787700 & 4.42909200 & -0.47145700 \\
\hline
\end{tabular}




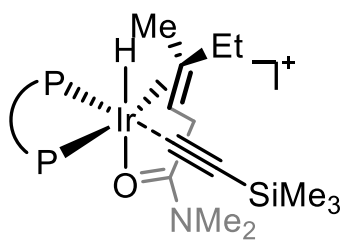

$G_{\text {sol }}=-3633.066637$ Hatree$$
\text { C }
$$ 


\begin{tabular}{|c|c|c|c|}
\hline $\mathrm{H}$ & -4.06560800 & 1.93131200 & -0.40843900 \\
\hline $\mathrm{H}$ & -3.59672800 & 3.63774900 & -0.34644600 \\
\hline $\mathrm{C}$ & -1.36162600 & 2.05339900 & -3.69188500 \\
\hline C & -3.62318200 & 1.10762000 & -3.01232300 \\
\hline $\mathrm{H}$ & -3.37405100 & 0.32665900 & -3.73929000 \\
\hline C & -2.61843500 & 2.35189300 & 1.09577800 \\
\hline $\mathrm{N}$ & -3.21447900 & 2.85569800 & 2.19133100 \\
\hline $\mathrm{C}$ & -2.61965800 & 2.65969100 & 3.51553600 \\
\hline $\mathrm{H}$ & -1.70468000 & 2.08070400 & 3.41823700 \\
\hline $\mathrm{H}$ & -2.39499500 & 3.63113300 & 3.96928800 \\
\hline $\mathrm{H}$ & -3.32423300 & 2.12768700 & 4.16315400 \\
\hline C & -4.44680300 & 3.64346500 & 2.16339500 \\
\hline $\mathrm{H}$ & -5.05098800 & 3.37567800 & 3.03400800 \\
\hline $\mathrm{H}$ & -4.23572300 & 4.71861100 & 2.20967000 \\
\hline $\mathrm{H}$ & -5.03532200 & 3.42974400 & 1.27318100 \\
\hline 0 & -1.56393700 & 1.67144000 & 1.19626700 \\
\hline $\mathrm{H}$ & -1.07623700 & 1.12176100 & -4.18794000 \\
\hline $\mathrm{H}$ & -0.46454600 & 2.57147000 & -3.35077900 \\
\hline P & -0.18230100 & -1.56498000 & 0.27482700 \\
\hline 0 & 3.19824900 & -4.34870000 & -2.40195300 \\
\hline C & -3.91421500 & -0.91809700 & -0.04977500 \\
\hline $\mathrm{Si}$ & -5.51197100 & -1.70206900 & 0.40343000 \\
\hline C & -5.24295300 & -2.85580000 & 1.87665800 \\
\hline $\mathrm{H}$ & -6.18353500 & -3.33025000 & 2.17881500 \\
\hline $\mathrm{H}$ & -4.53124300 & -3.64836000 & 1.62456500 \\
\hline $\mathrm{H}$ & -4.84666100 & -2.31671800 & 2.74365900 \\
\hline $\mathrm{C}$ & -6.17392100 & -2.68591300 & -1.06850100 \\
\hline $\mathrm{H}$ & -5.46490500 & -3.46617600 & -1.36334100 \\
\hline $\mathrm{H}$ & -7.12403400 & -3.17152400 & -0.81852900 \\
\hline $\mathrm{H}$ & -6.34663200 & -2.04434800 & -1.93907000 \\
\hline C & -6.75257300 & -0.34897600 & 0.87660700 \\
\hline $\mathrm{H}$ & -7.71758700 & -0.78535500 & 1.15800900 \\
\hline $\mathrm{H}$ & -6.39560000 & 0.23860500 & 1.72977800 \\
\hline $\mathrm{H}$ & -6.93380700 & 0.33943600 & 0.04374500 \\
\hline $\mathrm{H}$ & -0.77852500 & -0.38412400 & -1.98324200 \\
\hline $\mathrm{H}$ & -4.09213700 & 0.60643600 & -2.16593100 \\
\hline $\mathrm{H}$ & -1.84208100 & 2.68028400 & -4.45411300 \\
\hline C & -4.61531900 & 2.09999500 & -3.65727700 \\
\hline $\mathrm{H}$ & -4.20091300 & 2.58310000 & -4.54712000 \\
\hline $\mathrm{H}$ & -5.52104900 & 1.56810400 & -3.96191700 \\
\hline H & 6500 & 2.88679800 & -2.95 \\
\hline
\end{tabular}

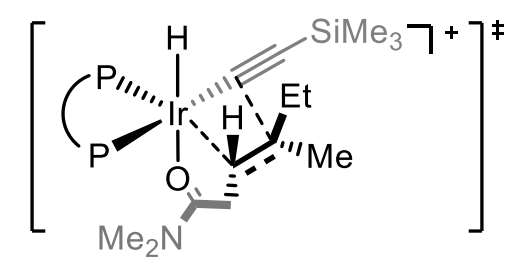

$G_{\text {sol }}=-3633.039678$ Hatree

$\begin{array}{llll}\text { Ir } & 1.04781900 \quad 0.64000800 & -0.20086700\end{array}$

$\begin{array}{llll}\text { C } & 2.17592500 & 2.48136100 & -0.39075300\end{array}$

$\begin{array}{llll}\text { C } & 3.41947900 & 1.83111500 & -0.82615900\end{array}$

$\begin{array}{llll}\text { C } & 2.95696400 & -0.06857900 & -0.55392200\end{array}$

$\begin{array}{llll}\text { C } & -1.38234000 & -1.64641600 & 1.08039300\end{array}$

$\begin{array}{llll}\text { C } & -1.35749800 & -2.15938100 & 2.37947600\end{array}$

$\begin{array}{llll}\mathrm{H} & -0.47073700 & -2.59307700 & 2.81861500\end{array}$

$\begin{array}{llll}\text { C } & -2.53833400 & -2.12478200 & 3.13095200\end{array}$

C $\quad-3.69465800 \quad-1.13079400 \quad 1.44049200$

$\begin{array}{llll}\text { C } & -2.57857800 & -1.10262000 & 0.56780800\end{array}$

$\begin{array}{llll}\text { C } & -3.69378300 & -2.63505200 & 5.14524500\end{array}$

$\mathrm{H} \quad-3.42901600 \quad-3.09463700 \quad 6.09778800$

$\mathrm{H} \quad-4.47644900 \quad-3.21613600 \quad 4.64963100$

H $\quad-4.05912100 \quad-1.61644200 \quad 5.30366600$

$\begin{array}{llll}\text { C } & -5.99129200 & -0.64844500 & 1.82643500\end{array}$

$\mathrm{H} \quad-6.79871100 \quad-0.20317000 \quad 1.24400400$

$\begin{array}{llll}\mathrm{H} & -5.82104900 & -0.07398200 & 2.74091700\end{array}$

$\mathrm{H} \quad-6.24196100 \quad-1.67763700 \quad 2.09638000$

$\begin{array}{llll}\text { C } & -0.13837900 & -2.55826100 & -1.41655600\end{array}$

C $\quad 0.64612900 \quad-2.33643600 \quad-2.55918500$

H $\quad 1.39911900 \quad-1.55644200 \quad-2.55072700$

$\begin{array}{llll}\text { C } & 0.46615200 & -3.11889900 & -3.70055300\end{array}$

$\mathrm{H} \quad 1.07730600 \quad-2.93623400 \quad-4.57963400$

C $\quad-0.49620300 \quad-4.13030300 \quad-3.71479600$

$\mathrm{H} \quad-0.63530500 \quad-4.73782000 \quad-4.60417000$

$\begin{array}{llll}\text { C } & -1.27933400 & -4.35778100 & -2.58193500\end{array}$

\begin{tabular}{l}
$c-1.27933400-4.35778100-2.58193500$ \\
\hline
\end{tabular}

H $\quad-2.02944700 \quad-5.14314100 \quad-2.58569400$

C $\quad-1.10300800 \quad-3.57819200 \quad-1.43725800$

$\mathrm{H} \quad-1.72992000 \quad-3.75448400 \quad-0.57004000$

$\begin{array}{llll}\text { C } & 1.32416100 & -2.68104500 & 1.07870500\end{array}$

$\begin{array}{llll}\text { C } & 2.10739200 & -2.11956100 & 2.10003900\end{array}$

H $\quad 2.08640100 \quad-1.04823500 \quad 2.26361000$

$\begin{array}{llll}\text { C } & 2.91113500 & -2.93201400 & 2.90197400\end{array}$

$\begin{array}{llll}\text { H } & 3.50552500 & -2.48662300 & 3.69479200\end{array}$

$\begin{array}{llll}\text { C } & 2.95614500 & -4.31099300 & 2.68596300\end{array}$

H $\quad 3.58196600 \quad-4.94188200 \quad 3.31027800$

$\begin{array}{llll}\text { C } & 2.19265100 & -4.87488100 & 1.66237100\end{array}$

H $\quad 2.22339700 \quad-5.94588900 \quad 1.48527000$ 


\begin{tabular}{|c|c|c|c|}
\hline $\mathrm{H}$ & 0.79245100 & -4.52246900 & 0.07528000 \\
\hline$C$ & -2.78019100 & -0.56090400 & -0.80503400 \\
\hline & -2.29173100 & 0.67624700 & -1.27667600 \\
\hline$P$ & -1.09981800 & 1.65462200 & -0.23526600 \\
\hline $\mathrm{C}$ & -2.06883800 & 1.95649100 & 1.30440600 \\
\hline $\mathrm{C}$ & -1.49541100 & 1.74005600 & 2.56516100 \\
\hline $\mathrm{H}$ & -0.48941900 & 1.34338200 & 2.63636600 \\
\hline $\mathrm{C}$ & -2.22850900 & 2.00278800 & 3.72502900 \\
\hline $\mathrm{H}$ & -1.77943500 & 1.82365800 & 4.69771200 \\
\hline C & -3.53454500 & 2.48516000 & 3.63714500 \\
\hline H & -4.10060000 & 2.69470500 & 4.54006200 \\
\hline $\mathrm{C}$ & -4.11712800 & 2.69100300 & 2.38336300 \\
\hline H & -5.13639000 & 3.05833900 & 2.30882900 \\
\hline C & -3.39305800 & 2.42222400 & 1.22286200 \\
\hline H & -3.85819400 & 2.57204300 & 0.25347400 \\
\hline C & -1.02704300 & 3.32414900 & -1.03980300 \\
\hline C & -0.43607000 & 3.44568900 & -2.31273700 \\
\hline H & -0.06152000 & 2.56212500 & -2.82023900 \\
\hline C & -0.32565900 & 4.68839400 & -2.93559900 \\
\hline H & 0.11627600 & 4.75556500 & -3.92533100 \\
\hline C & -0.77748900 & 5.84099100 & -2.28787000 \\
\hline H & -0.68850700 & 6.80936900 & -2.77078800 \\
\hline C & -1.34079900 & 5.73824400 & -1.01712300 \\
\hline H & -1.69245200 & 6.62793400 & -0.50304600 \\
\hline C & -1.46870000 & 4.49123900 & -0.39763200 \\
\hline H & -1.92286400 & 4.43612800 & 0.58469100 \\
\hline C & -2.67350800 & 1.13423900 & -2.53798500 \\
\hline $\mathrm{H}$ & -2.35776900 & 2.08450900 & -2.94139500 \\
\hline C & -3.53038100 & 0.33423900 & -3.30480500 \\
\hline o & -3.88733100 & 0.80835000 & -4.51282700 \\
\hline C & -4.77530100 & 0.01043100 & -5.30426900 \\
\hline H & -4.92856600 & 0.57614000 & -6.22345100 \\
\hline $\mathrm{H}$ & -5.72836400 & -0.14333700 & -4.79060000 \\
\hline $\mathrm{H}$ & -4.33327100 & -0.96449500 & -5.52775400 \\
\hline $\mathrm{N}$ & -3.99638700 & -0.84230200 & -2.89649700 \\
\hline C & -3.63390400 & -1.26488300 & -1.68893500 \\
\hline 0 & -4.10433500 & -2.45332000 & -1.26046200 \\
\hline C & -5.00678800 & -3.15905400 & -2.11925500 \\
\hline H & -5.27392700 & -4.06417400 & -1.57265500 \\
\hline H & -4.52685300 & -3.41440700 & -3.0677520 \\
\hline H & -5.90014400 & -2.56343500 & -2.32442800 \\
\hline 0 & -2.48612600 & -2.63568300 & 4.37606200 \\
\hline $\mathrm{H}$ & 1.72554400 & 3.07149300 & -1.18542100 \\
\hline 0 & -4.84340900 & -0.60495100 & 0.97280500 \\
\hline & 2.15798700 & 3.24236100 & 0.93800200 \\
\hline
\end{tabular}

\begin{tabular}{|c|c|c|}
\hline 1.25176100 & 3.86230900 & 0.96322300 \\
\hline 3.00345100 & 3.93326500 & 1.02648000 \\
\hline 3.68403000 & 1.87873900 & -2.33045100 \\
\hline 4.65714600 & 1.97138100 & 0.04009300 \\
\hline 4.48921000 & 1.64731100 & 1.06806900 \\
\hline 2.09401300 & 2.31113900 & 2.13680200 \\
\hline 2.46113200 & 2.74518000 & 3.35831900 \\
\hline 2.45667300 & 1.83593600 & 4.50595200 \\
\hline 2.16498100 & 0.84106200 & 4.17951100 \\
\hline 3.45653900 & 1.79764500 & 4.95124600 \\
\hline 1.75068700 & 2.19157300 & 5.26385500 \\
\hline 2.91788600 & 4.10689200 & 3.63875600 \\
\hline 2.55777900 & 4.39754200 & 4.62897600 \\
\hline 4.01271300 & 4.17024100 & 3.63982900 \\
\hline 2.51732200 & 4.81745100 & 2.91803900 \\
\hline 1.66591800 & 1.13837000 & 1.97535300 \\
\hline 2.76923100 & 1.59954300 & -2.86043600 \\
\hline 0.19723700 & -1.58165700 & 0.10840000 \\
\hline-3.68293300 & -1.62347500 & 2.67578200 \\
\hline 3.89086600 & -0.86892400 & -0.73727100 \\
\hline 5.29081200 & -2.05362000 & -1.02408800 \\
\hline 6.25529400 & -2.27270700 & 0.58209000 \\
\hline 7.08016000 & -2.98056100 & 0.44266600 \\
\hline 6.68518500 & -1.32686100 & 0.92814800 \\
\hline 5.60837100 & -2.66088000 & 1.37473700 \\
\hline 6.40297800 & -1.32246500 & -2.36774600 \\
\hline 6.84053500 & -0.36454600 & -2.06753000 \\
\hline 7.23226000 & -2.00801800 & -2.57647700 \\
\hline 5.85786000 & -1.16845500 & -3.30455900 \\
\hline 4.55439200 & -3.69239200 & -1.59799900 \\
\hline 5.35615600 & -4.40591000 & -1.82008600 \\
\hline 3.91575700 & -4.13157900 & -0.82613300 \\
\hline 3.95251100 & -3.56874600 & -2.50359800 \\
\hline 0.76541900 & 0.44201000 & -1.72375300 \\
\hline 5.48825200 & 1.39341200 & -0.36725100 \\
\hline 4.44525000 & 1.13977500 & -2.58888400 \\
\hline 4.94615300 & 3.03103800 & 0.06108300 \\
\hline 4.14553300 & 3.27456000 & -2.79312200 \\
\hline 3.40080100 & 4.04643600 & -2.57714000 \\
\hline 5.08883100 & 3.56927600 & -2.32363100 \\
\hline 4.30846100 & 3.26205600 & -3.87483000 \\
\hline
\end{tabular}




$$
\begin{aligned}
& {[\underbrace{\mathrm{MiMe}_{3}}_{\mathrm{NMe}_{2}}]^{+}} \\
& G_{\text {sol }}=-3633.031058 \text { Hatree } \\
& \text { Ir } \quad-1.06412500 \quad 0.37250000 \quad-0.45331300 \\
& \text { C } \quad-2.98292000 \quad-0.35276600 \quad-0.36364000 \\
& \text { C } \quad-3.42547300 \quad 0.99321900 \quad-1.75021800 \\
& \text { C } \quad-2.25104100 \quad 1.85543600 \quad-1.53511500 \\
& \text { C } \quad 1.41397100 \quad-2.08939900 \quad-0.27287500 \\
& \text { C } \quad 1.34089600 \quad-3.21163400 \quad-1.10047800 \\
& \text { H } \quad 0.46731400 \quad-3.84286100 \quad-1.15731100 \\
& \text { C } \quad 2.45611400 \quad-3.54418200 \quad-1.87822200 \\
& \text { C } \quad 3.65170300 \quad-1.78335000 \quad-1.08007700 \\
& \begin{array}{llll}
\text { C } & 2.60403300 & -1.33497600 & -0.23766400
\end{array} \\
& \begin{array}{llll}
\text { C } & 3.48979000 & -5.00318600 & -3.44561300
\end{array} \\
& \text { H } \quad 3.18879700 \quad-5.90039500 \quad-3.98689500 \\
& \mathrm{H} \quad 4.35459400 \quad-5.21653600 \quad-2.81111700 \\
& \mathrm{H} \quad 3.75541300 \quad-4.20775700 \quad-4.14759100 \\
& \text { C } \quad 5.87211000 \quad-1.51393100 \quad-1.88860700 \\
& \mathrm{H} \quad 6.68919700 \quad-0.81769400 \quad-1.69594600 \\
& \text { H } \quad 5.59383900 \quad-1.48963500 \quad-2.94566300 \\
& \text { H } \quad 6.17148900 \quad-2.53159000 \quad-1.62490300 \\
& \begin{array}{llll}
\text { C } & 0.42245900 & -1.36860400 & 2.40294100
\end{array} \\
& \text { C } \quad \begin{array}{llll}
-0.15554800 & -0.36181500 & 3.18988200
\end{array} \\
& \begin{array}{llll}
\mathrm{H} & -0.84529100 & 0.34326300 & 2.73742100
\end{array} \\
& \begin{array}{llll}
\text { C } & 0.16880400 & -0.25816600 & 4.54454100
\end{array} \\
& \begin{array}{llll}
\mathrm{H} & -0.28124200 & 0.52454500 & 5.14845500
\end{array} \\
& \text { C } \quad 1.06896800 \quad-1.15413100 \quad 5.12216300 \\
& \text { H } \quad 1.31790000 \quad-1.07321300 \quad 6.17622200 \\
& \text { C } \quad 1.65364000 \quad-2.15437100 \quad 4.34091800 \\
& \mathrm{H} \quad 2.35715700 \quad-2.85203900 \quad 4.78575400 \\
& \begin{array}{llll}
\text { C } & 1.33549100 & -2.26248300 & 2.98729000
\end{array} \\
& \begin{array}{llll}
\mathrm{H} & 1.80677800 & -3.03232100 & 2.38508300
\end{array} \\
& \text { C } \quad-1.16272300 \quad-3.04399500 \quad 0.63717200 \\
& \text { C } \quad-1.84226200 \quad-3.39362600 \quad-0.54325200 \\
& \mathrm{H} \quad-1.80805400 \quad-2.73042100 \quad-1.39983800 \\
& \text { C } \quad-2.55231400 \quad-4.59135500 \quad-0.62960500 \\
& \text { H } \quad-3.04809400 \quad-4.85547400 \quad-1.55918800 \\
& \text { C } \quad-2.62330700 \quad-5.44707700 \quad 0.47230800 \\
& \mathrm{H} \quad-3.17449200 \quad-6.38032600 \quad 0.40489000 \\
& \text { C } \quad-1.98420100 \quad-5.09270300 \quad 1.66048900 \\
& \mathrm{H} \quad-2.04015100 \quad-5.74569600 \quad 2.52640500 \\
& \begin{array}{llll}
\text { C } & -1.25806800 & -3.90176900 & 1.74312000
\end{array}
\end{aligned}
$$




\begin{tabular}{|c|c|c|c|}
\hline $\mathrm{H}$ & -3.40673000 & 3.61415000 & -0.92279800 \\
\hline $\mathrm{H}$ & -1.67486000 & 3.83720800 & -0.92758600 \\
\hline $\mathrm{C}$ & -3.43291000 & 0.16925600 & -3.03561500 \\
\hline C & -4.79575800 & 1.51237900 & -1.35569600 \\
\hline $\mathrm{H}$ & -5.02830300 & 2.38154200 & -1.98614700 \\
\hline $\mathrm{H}$ & -5.56175400 & 0.75392600 & -1.52420200 \\
\hline $\mathrm{C}$ & -2.36711200 & 2.83225000 & 0.80289500 \\
\hline $\mathrm{N}$ & -2.93423600 & 3.68563000 & 1.67904200 \\
\hline $\mathrm{C}$ & -2.93405100 & 3.38113300 & 3.11089900 \\
\hline $\mathrm{H}$ & -2.71585600 & 2.32528800 & 3.25489700 \\
\hline $\mathrm{H}$ & -2.18197500 & 3.97944900 & 3.63817000 \\
\hline $\mathrm{H}$ & -3.91892000 & 3.61127800 & 3.52727700 \\
\hline $\mathrm{C}$ & -3.54227600 & 4.95880500 & 1.28769400 \\
\hline $\mathrm{H}$ & -4.61334500 & 4.85117400 & 1.07849000 \\
\hline $\mathrm{H}$ & -3.42561200 & 5.66511700 & 2.11284400 \\
\hline $\mathrm{H}$ & -3.04426800 & 5.38390100 & 0.41670600 \\
\hline 0 & -1.75049800 & 1.81721600 & 1.21584700 \\
\hline $\mathrm{H}$ & -4.12068400 & -0.67232400 & -2.91959700 \\
\hline $\mathrm{H}$ & -2.43747700 & -0.24777400 & -3.20054400 \\
\hline P & -0.10255300 & -1.52533400 & 0.64913500 \\
\hline 0 & 2.35135100 & -4.63659500 & -2.65847300 \\
\hline C & -4.00137700 & -0.95176600 & 0.02908800 \\
\hline $\mathrm{Si}$ & -5.53089500 & -1.78424100 & 0.68021800 \\
\hline C & -6.19169200 & -2.98862000 & -0.61184000 \\
\hline $\mathrm{H}$ & -7.09320600 & -3.48915400 & -0.24092000 \\
\hline $\mathrm{H}$ & -6.45811700 & -2.47963300 & -1.54416200 \\
\hline $\mathrm{H}$ & -5.45202400 & -3.75960100 & -0.84632200 \\
\hline C & -6.83129900 & -0.46541700 & 1.06604200 \\
\hline $\mathrm{H}$ & -7.18239700 & 0.05820800 & 0.17173300 \\
\hline $\mathrm{H}$ & -7.70408900 & -0.93993300 & 1.52926900 \\
\hline $\mathrm{H}$ & -6.44930900 & 0.28117600 & 1.76988200 \\
\hline C & -5.04527900 & -2.66983200 & 2.27221000 \\
\hline $\mathrm{H}$ & -5.92518300 & -3.16158800 & 2.70281500 \\
\hline $\mathrm{H}$ & -4.28155300 & -3.43150300 & 2.09656500 \\
\hline $\mathrm{H}$ & -4.65835600 & -1.96467600 & 3.01451000 \\
\hline $\mathrm{H}$ & -0.73684500 & -0.49257100 & -1.70998600 \\
\hline $\mathrm{H}$ & -4.84580500 & 1.82884000 & -0.31414600 \\
\hline C & -3.85047100 & 1.00568400 & -4.26171600 \\
\hline $\mathrm{H}$ & -4.87862200 & 1.37010100 & -4.18252900 \\
\hline $\mathrm{H}$ & -3.19387700 & 1.86867000 & -4.40945300 \\
\hline H & 074700 & 0.38507100 & 46600 \\
\hline
\end{tabular}

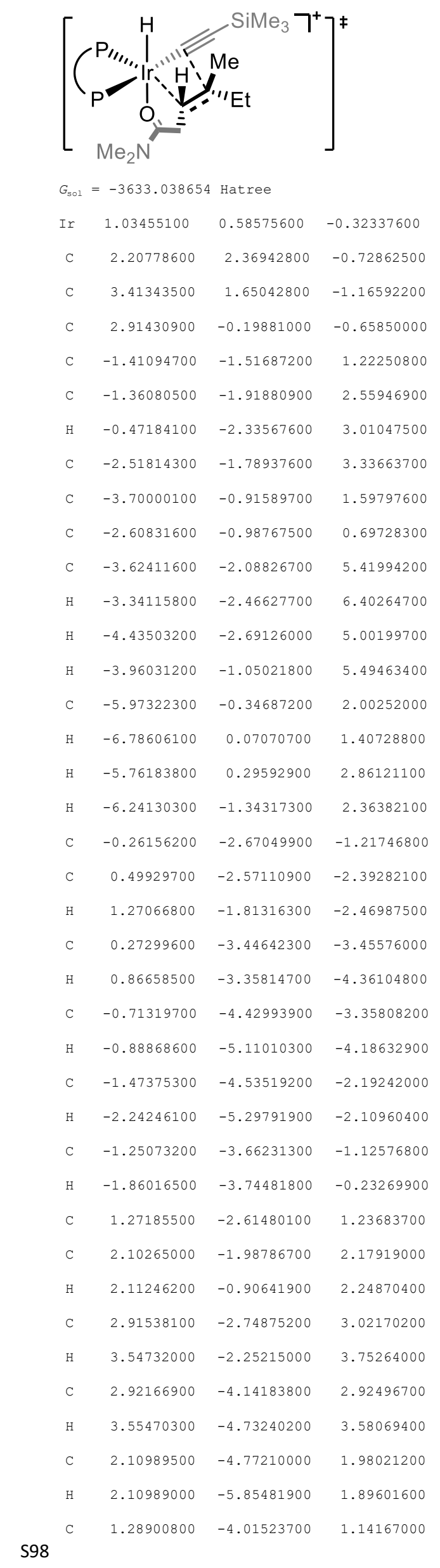




\begin{tabular}{|c|c|c|c|}
\hline $\mathrm{H}$ & 0.66293600 & -4.52144000 & 0.41575000 \\
\hline$C$ & -2.83649600 & -0.55247100 & -0.70916600 \\
\hline & -2.33715700 & 0.63358800 & -1.28888800 \\
\hline & -1.08669400 & 1.65695900 & -0.36484100 \\
\hline $\mathrm{C}$ & -1.99695800 & 2.09731600 & 1.17835900 \\
\hline C & -1.39060900 & 1.95757800 & 2.43465900 \\
\hline $\mathrm{H}$ & -0.39282600 & 1.54118300 & 2.50731900 \\
\hline $\mathrm{C}$ & -2.08176700 & 2.32177000 & 3.59290500 \\
\hline $\mathrm{H}$ & -1.60778000 & 2.20044600 & 4.56269900 \\
\hline C & -3.37785400 & 2.83068200 & 3.50793300 \\
\hline H & -3.91087100 & 3.11906300 & 4.40921200 \\
\hline $\mathrm{C}$ & -3.99320000 & 2.96110400 & 2.25974600 \\
\hline H & -5.00516500 & 3.34860000 & 2.18793100 \\
\hline C & -3.31191800 & 2.58981200 & 1.10157600 \\
\hline H & -3.80387100 & 2.67974200 & 0.13802900 \\
\hline $\mathrm{C}$ & -1.00062500 & 3.25919400 & -1.29498000 \\
\hline C & -0.46030900 & 3.26883400 & -2.59583400 \\
\hline H & -0.13152300 & 2.33952600 & -3.05055600 \\
\hline C & -0.34156600 & 4.45862200 & -3.31329700 \\
\hline H & 0.06045200 & 4.43947800 & -4.32195500 \\
\hline C & -0.73324700 & 5.66904300 & -2.73578300 \\
\hline H & -0.63777200 & 6.59610800 & -3.29282100 \\
\hline $\mathrm{C}$ & -1.24453000 & 5.67732200 & -1.43919300 \\
\hline H & -1.54870900 & 6.61270900 & -0.97874100 \\
\hline C & -1.38085100 & 4.48355300 & -0.72411100 \\
\hline H & -1.79240000 & 4.51510500 & 0.27776700 \\
\hline C & -2.75086000 & 1.00469300 & -2.56849200 \\
\hline $\mathrm{H}$ & -2.43016900 & 1.91494700 & -3.05199600 \\
\hline C & -3.64864500 & 0.16941600 & -3.24562100 \\
\hline o & -4.03553800 & 0.56072300 & -4.47391500 \\
\hline C & -4.96471500 & -0.27302300 & -5.17604500 \\
\hline H & -5.13693600 & 0.22731900 & -6.12915600 \\
\hline $\mathrm{H}$ & -5.90331500 & -0.36770000 & -4.62295900 \\
\hline $\mathrm{H}$ & -4.54965500 & -1.27117500 & -5.34106100 \\
\hline $\mathrm{N}$ & -4.12372100 & -0.96296900 & -2.73601900 \\
\hline C & -3.73189400 & -1.30132200 & -1.51109500 \\
\hline 0 & -4.21370700 & -2.44262500 & -0.97946300 \\
\hline C & -5.15784400 & -3.18920300 & -1.75489700 \\
\hline H & -5.42802300 & -4.04340500 & -1.1330190 \\
\hline H & -4.71356600 & -3.52746700 & -2.69478400 \\
\hline $\mathrm{H}$ & -6.04355200 & -2.58900700 & -1.97892000 \\
\hline O & -2.44093300 & -2.19036400 & 4.62012200 \\
\hline $\mathrm{F}$ & 1.73086200 & 2.89817000 & -1.55112600 \\
\hline 0 & -4.85017200 & -0.40510600 & 1.11730000 \\
\hline & 2.24764100 & 3.23649900 & 0.53545700 \\
\hline
\end{tabular}

\begin{tabular}{|c|c|c|}
\hline 1.34486500 & 3.86246400 & 0.53435700 \\
\hline 3.09610000 & 3.92650900 & 0.54364100 \\
\hline 3.56196200 & 1.51382800 & -2.67134500 \\
\hline 4.73216800 & 1.86254800 & -0.42268700 \\
\hline 4.56953800 & 1.82875900 & 0.65848800 \\
\hline 2.20884000 & 2.41566100 & 1.81287700 \\
\hline 2.64321400 & 2.94176600 & 2.97494200 \\
\hline 2.66428300 & 2.13573400 & 4.19720200 \\
\hline 2.36322500 & 1.11726300 & 3.96592900 \\
\hline 3.67497300 & 2.13296900 & 4.61854600 \\
\hline 1.97790000 & 2.55769800 & 4.93914100 \\
\hline 3.13830500 & 4.31176800 & 3.11804400 \\
\hline 2.84876500 & 4.68308700 & 4.10424600 \\
\hline 4.23143500 & 4.35447900 & 3.04258800 \\
\hline 2.69994300 & 4.97359100 & 2.37307700 \\
\hline 1.73641300 & 1.24995700 & 1.77507800 \\
\hline 2.63510700 & 1.17114800 & -3.13223000 \\
\hline 0.14041900 & -1.57428400 & 0.20740600 \\
\hline-3.66394300 & -1.30115700 & 2.87042200 \\
\hline 3.82271700 & -1.03749700 & -0.79333700 \\
\hline 5.18481100 & -2.27719300 & -1.02616000 \\
\hline 6.19172000 & -2.40056500 & 0.56382600 \\
\hline 6.99985900 & -3.13233800 & 0.45291800 \\
\hline 6.64736100 & -1.44092500 & 0.82937400 \\
\hline 5.56117400 & -2.71848100 & 1.39981600 \\
\hline 6.26888200 & -1.67304600 & -2.45360700 \\
\hline 6.73055700 & -0.70292700 & -2.24162800 \\
\hline 7.08032100 & -2.38742000 & -2.63373200 \\
\hline 5.69661200 & -1.58194900 & -3.38247400 \\
\hline 4.39152600 & -3.93387300 & -1.45338400 \\
\hline 5.16770100 & -4.68517500 & -1.63869100 \\
\hline 3.76227700 & -4.29310300 & -0.63389000 \\
\hline 3.76882800 & -3.86351200 & -2.35052500 \\
\hline 0.68425500 & 0.25888400 & -1.80985200 \\
\hline 5.40865100 & 1.03873600 & -0.65659700 \\
\hline 4.36677600 & 0.82832100 & -2.94005300 \\
\hline 3.79512700 & 2.50464300 & -3.08151500 \\
\hline 5.41699800 & 3.18894600 & -0.81647700 \\
\hline 4.77183300 & 4.06273700 & -0.68829100 \\
\hline 6.30559300 & 3.33665300 & -0.19551600 \\
\hline 5.74446000 & 3.17248000 & -1.85935000 \\
\hline
\end{tabular}




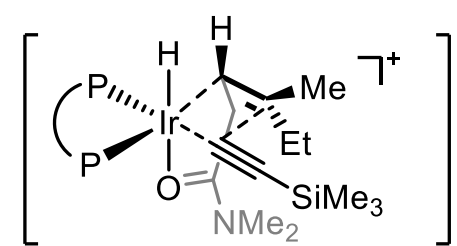

$G_{\text {sol }}=-3633.030626$ Hatree

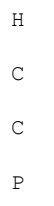



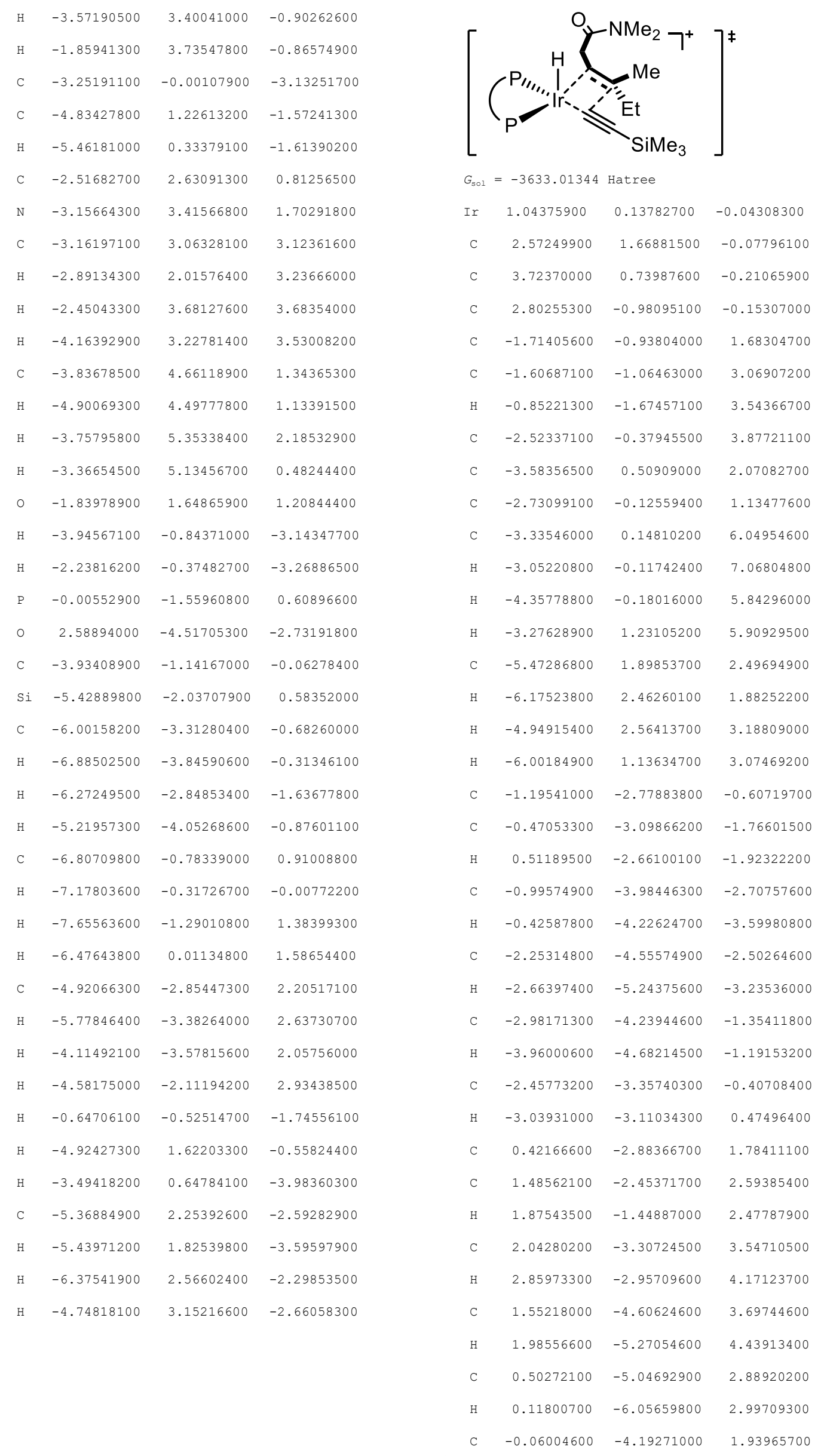


\begin{tabular}{|c|c|c|c|}
\hline $\mathrm{H}$ & -0.87551300 & -4.55201500 & 1.32207400 \\
\hline C & -3.05574200 & 0.00097500 & -0.31310900 \\
\hline C & -2.30722700 & 0.69164900 & -1.28858000 \\
\hline P & -0.65785400 & 1.48399200 & -0.93880600 \\
\hline C & -1.07379900 & 3.15884900 & -0.29444700 \\
\hline C & -0.10389500 & 4.17835800 & -0.32698100 \\
\hline $\mathrm{H}$ & 0.86922800 & 3.99650300 & -0.76925000 \\
\hline C & -0.39186000 & 5.44683700 & 0.17569500 \\
\hline $\mathrm{H}$ & 0.36282300 & 6.22617300 & 0.12691100 \\
\hline C & -1.64745500 & 5.71521500 & 0.72571400 \\
\hline $\mathrm{H}$ & -1.87211100 & 6.70362500 & 1.11506000 \\
\hline C & -2.61388600 & 4.71068600 & 0.75971900 \\
\hline $\mathrm{H}$ & -3.59658700 & 4.91381600 & 1.17457200 \\
\hline C & -2.33499900 & 3.44011000 & 0.25052600 \\
\hline $\mathrm{H}$ & -3.10909900 & 2.68292300 & 0.27742900 \\
\hline C & 0.01484200 & 1.76681200 & -2.63734000 \\
\hline C & 0.65267500 & 0.67809200 & -3.26080000 \\
\hline $\mathrm{H}$ & 0.68670000 & -0.29049400 & -2.76459400 \\
\hline C & 1.20696600 & 0.80957400 & -4.53364900 \\
\hline $\mathrm{H}$ & 1.69160400 & -0.04132600 & -5.00279300 \\
\hline C & 1.12543200 & 2.03137800 & -5.20613500 \\
\hline $\mathrm{H}$ & 1.55381200 & 2.13657700 & -6.19811400 \\
\hline C & 0.48150700 & 3.11233400 & -4.60331000 \\
\hline $\mathrm{H}$ & 0.40441000 & 4.06054100 & -5.12656000 \\
\hline C & -0.07300700 & 2.98381900 & -3.32713100 \\
\hline $\mathrm{H}$ & -0.57694000 & 3.83212400 & -2.87852600 \\
\hline C & -2.80033900 & 0.79573300 & -2.59260600 \\
\hline $\mathrm{H}$ & -2.27237600 & 1.31392400 & -3.37928700 \\
\hline C & -4.03768700 & 0.21215800 & -2.88913700 \\
\hline 0 & -4.49176600 & 0.35037400 & -4.14638500 \\
\hline C & -5.75763300 & -0.23916900 & -4.46831900 \\
\hline H & -5.92371900 & -0.00609900 & -5.52002300 \\
\hline $\mathrm{H}$ & -6.55636300 & 0.18703700 & -3.85512000 \\
\hline $\mathrm{H}$ & -5.73515000 & -1.32210000 & -4.31847000 \\
\hline $\mathrm{N}$ & -4.75915600 & -0.46110400 & -1.99695300 \\
\hline C & -4.27798100 & -0.56362300 & -0.76332500 \\
\hline 0 & -4.98981800 & -1.26615700 & 0.14124400 \\
\hline C & -6.25936500 & -1.78771500 & -0.27033200 \\
\hline $\mathrm{H}$ & -6.65847000 & -2.29564400 & 0.60807000 \\
\hline $\mathrm{H}$ & -6.14598600 & -2.49117400 & -1.09933200 \\
\hline $\mathrm{H}$ & -6.93064100 & -0.98298600 & -0.58134400 \\
\hline 0 & -2.39686100 & -0.53158300 & 5.20697600 \\
\hline $\mathrm{H}$ & 2.47162400 & 2.25839600 & -0.99026400 \\
\hline 0 & -4.56100300 & 1.29650600 & 1.56929500 \\
\hline C & 2.53664400 & 2.57787600 & 1.15256000 \\
\hline
\end{tabular}

\begin{tabular}{|c|c|c|}
\hline 2.71553500 & 2.01083700 & 2.07236900 \\
\hline 1.53846300 & 3.00724500 & 1.26390500 \\
\hline 4.38146200 & 0.81198400 & -1.58571600 \\
\hline 4.67354300 & 0.57043500 & 0.95825500 \\
\hline 5.25046900 & 1.49956400 & 1.03789700 \\
\hline 4.15007200 & 0.40368700 & 1.90063000 \\
\hline 3.57039900 & 3.71465700 & 1.04460700 \\
\hline 3.56089000 & 4.68399300 & 2.00994600 \\
\hline 4.50353400 & 5.79446500 & 1.91368300 \\
\hline 5.12978900 & 5.64346600 & 1.03684600 \\
\hline 3.96645500 & 6.74645600 & 1.82118300 \\
\hline 5.13127300 & 5.83926000 & 2.81134800 \\
\hline 2.64225000 & 4.74227300 & 3.13733400 \\
\hline 3.20432200 & 4.90162800 & 4.06489900 \\
\hline 1.93180800 & 5.57240500 & 3.02702000 \\
\hline 2.07679600 & 3.81875100 & 3.24173800 \\
\hline 4.37419800 & 3.73499100 & 0.10860300 \\
\hline 3.60619100 & 0.73959800 & -2.35533600 \\
\hline 4.73227500 & 1.85415400 & -1.60747000 \\
\hline-0.38154900 & -1.68645700 & 0.62561500 \\
\hline-3.48826600 & 0.39705000 & 3.39199700 \\
\hline 3.39349800 & -2.05294400 & -0.38471100 \\
\hline 4.22977400 & -3.70010500 & -0.66321400 \\
\hline 5.83884400 & -3.70483100 & 0.32159600 \\
\hline 6.34172100 & -4.67248300 & 0.21363600 \\
\hline 6.53257000 & -2.93188000 & -0.02373100 \\
\hline 5.65321900 & -3.54366100 & 1.38815200 \\
\hline 4.55704800 & -3.93201500 & -2.50834700 \\
\hline 5.25477300 & -3.19269800 & -2.91184300 \\
\hline 4.99292300 & -4.92328300 & -2.67814700 \\
\hline 3.63094800 & -3.87629900 & -3.08984200 \\
\hline 3.05758300 & -5.04427600 & -0.05633400 \\
\hline 3.50775100 & -6.02731400 & -0.23733500 \\
\hline 2.85654000 & -4.95502300 & 1.01413400 \\
\hline 2.09911700 & -5.01372500 & -0.58318100 \\
\hline 0.70094300 & 0.65372800 & 1.36966000 \\
\hline 5.35939200 & -0.26048100 & 0.79862600 \\
\hline 5.54856700 & -0.11307900 & -1.90748900 \\
\hline 5.25416400 & -1.16421100 & -1.85997100 \\
\hline 5.90158600 & 0.08509000 & -2.92402400 \\
\hline 6.39649600 & 0.03939800 & -1.23400800 \\
\hline
\end{tabular}




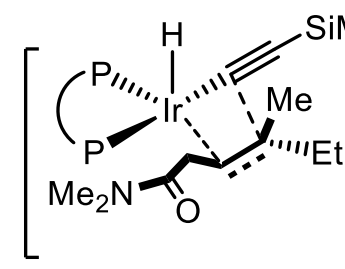

$G_{\text {sol }}=-3633.025648$ Hatree 


\begin{tabular}{|c|c|c|c|}
\hline $\mathrm{H}$ & -2.64672800 & -2.78357000 & -0.66591200 \\
\hline & -3.87896800 & -1.61573600 & -1.06447800 \\
\hline C & -3.14366500 & 0.62131000 & 2.49725900 \\
\hline C & -4.99535500 & 0.14229800 & 0.80849100 \\
\hline $\mathrm{H}$ & -5.49956400 & -0.63743300 & 1.38917300 \\
\hline H & -5.39238200 & 1.11986700 & 1.09198100 \\
\hline C & -4.42985600 & -3.02771000 & 0.50447500 \\
\hline N & -5.13597800 & -3.93227800 & -0.24045800 \\
\hline C & -6.12718300 & -4.77770700 & 0.41699900 \\
\hline $\mathrm{H}$ & -6.15391600 & -4.52440400 & 1.47479100 \\
\hline $\mathrm{H}$ & -7.11777100 & -4.61766300 & -0.02571900 \\
\hline H & -5.86484000 & -5.83617200 & 0.30057400 \\
\hline C & -5.00960300 & -4.12788600 & -1.67611000 \\
\hline $\mathrm{H}$ & -4.82780700 & -5.18732200 & -1.89382500 \\
\hline $\mathrm{H}$ & -5.93146600 & -3.83245900 & -2.19468900 \\
\hline $\mathrm{H}$ & -4.18017900 & -3.55687000 & -2.08693200 \\
\hline 0 & -4.59047700 & -2.92497300 & 1.72329700 \\
\hline H & -3.42051100 & 1.67872500 & 2.54100900 \\
\hline H & -2.05057300 & 0.58205600 & 2.62289700 \\
\hline P & 0.61540300 & 1.76475100 & -0.36016600 \\
\hline 0 & 2.99646500 & 2.80403900 & 4.08235400 \\
\hline E & -3.36112400 & 2.24373900 & -0.50708200 \\
\hline $\mathrm{Si}$ & -4.42488100 & 3.59380200 & -1.22045500 \\
\hline C & -4.97187800 & 4.74104000 & 0.17261300 \\
\hline H & -5.60041800 & 5.54563500 & -0.22541700 \\
\hline H & -5.55825000 & 4.21061600 & 0.93001300 \\
\hline $\mathrm{H}$ & -4.11109300 & 5.20051800 & 0.66733500 \\
\hline $\mathrm{C}$ & -5.91825600 & 2.79855300 & -2.05740500 \\
\hline H & -6.54273500 & 2.24973700 & -1.34546300 \\
\hline $\mathrm{H}$ & -6.54553000 & 3.56999600 & -2.51838500 \\
\hline $\mathrm{H}$ & -5.61163100 & 2.10415000 & -2.84620500 \\
\hline c & -3.35633900 & 4.50271400 & -2.48219200 \\
\hline $\mathrm{H}$ & -3.93279100 & 5.31571900 & -2.93817200 \\
\hline $\mathrm{H}$ & -2.46716300 & 4.93663000 & -2.01619200 \\
\hline $\mathrm{H}$ & -3.03044200 & 3.83367700 & -3.28467600 \\
\hline $\mathrm{H}$ & -1.19531600 & -0.20497800 & -1.45821100 \\
\hline $\mathrm{H}$ & -5.20643600 & -0.02871200 & -0.24647400 \\
\hline C & -3.81262200 & -0.14362700 & 3.65577300 \\
\hline $\mathrm{H}$ & -4.89170800 & 0.03098500 & 3.67089800 \\
\hline $\mathrm{H}$ & -3.65636700 & -1.22170900 & 3.57532900 \\
\hline & -3.40642400 & 0.20 & 4.60877600 \\
\hline
\end{tabular}

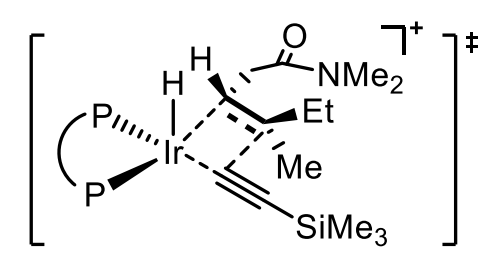

\begin{tabular}{|c|c|c|c|}
\hline$G_{\text {sol }}$ & $=-3633.020289$ & Hatree & \\
\hline Ir & -1.03435000 & 0.06719700 & 0.16195800 \\
\hline $\mathrm{C}$ & -2.62300900 & 1.37203700 & 0.00109700 \\
\hline $\mathrm{C}$ & -3.59330200 & 0.14285700 & 1.10154900 \\
\hline $\mathrm{C}$ & -2.78361600 & -1.07950700 & 0.86094400 \\
\hline C & 2.03171600 & 1.43343100 & 0.99375500 \\
\hline C & 2.10408100 & 2.18437100 & 2.16878800 \\
\hline $\mathrm{H}$ & 1.47503800 & 3.04158700 & 2.35783800 \\
\hline C & 3.04546800 & 1.81610400 & 3.13914200 \\
\hline C & 3.79759100 & 0.07474400 & 1.88211700 \\
\hline C & 2.90417500 & 0.33919500 & 0.81491300 \\
\hline C & 4.06151600 & 2.21837700 & 5.25161200 \\
\hline $\mathrm{H}$ & 3.92729100 & 2.94908500 & 6.04922400 \\
\hline H & 5.07747300 & 2.27656600 & 4.85150900 \\
\hline $\mathrm{H}$ & 3.88748200 & 1.20716800 & 5.62960600 \\
\hline C & 5.55599500 & -1.24769400 & 2.78589800 \\
\hline $\mathrm{H}$ & 6.12975500 & -2.11081500 & 2.44693300 \\
\hline $\mathrm{H}$ & 5.03491800 & -1.48058100 & 3.71820600 \\
\hline $\mathrm{H}$ & 6.21816300 & -0.39446600 & 2.95404000 \\
\hline C & 1.36783100 & 1.96415000 & -1.86924100 \\
\hline C & 0.54854700 & 1.68826300 & -2.97441200 \\
\hline $\mathrm{H}$ & -0.46349600 & 1.32359500 & -2.81760600 \\
\hline C & 1.01857400 & 1.89223900 & -4.27260800 \\
\hline $\mathrm{H}$ & 0.37571700 & 1.67740600 & -5.12102800 \\
\hline C & 2.31347600 & 2.37138900 & -4.47752800 \\
\hline $\mathrm{H}$ & 2.68125000 & 2.52969800 & -5.48684100 \\
\hline C & 3.13616900 & 2.64559600 & -3.38232400 \\
\hline $\mathrm{H}$ & 4.14412100 & 3.01815500 & -3.53920300 \\
\hline C & 2.66831500 & 2.44683700 & -2.08260600 \\
\hline $\mathrm{H}$ & 3.32064900 & 2.64859800 & -1.23909900 \\
\hline C & 0.11344900 & 3.48593200 & 0.24238800 \\
\hline C & -0.72658500 & 3.68721300 & 1.35042900 \\
\hline $\mathrm{H}$ & -1.10342100 & 2.83377200 & 1.90237000 \\
\hline C & -1.07926600 & 4.97546100 & 1.75265100 \\
\hline $\mathrm{H}$ & -1.71559400 & 5.11197100 & 2.62203000 \\
\hline C & -0.61884500 & 6.08398100 & 1.03795900 \\
\hline $\mathrm{H}$ & -0.89529300 & 7.08703500 & 1.34873300 \\
\hline C & 0.19896800 & 5.89510500 & -0.07659300 \\
\hline $\mathrm{H}$ & 0.56075500 & 6.75036400 & -0.63945200 \\
\hline C & 0.56700900 & 4.60645900 & -0.47063100 \\
\hline
\end{tabular}




\begin{tabular}{|c|c|c|c|}
\hline $\mathrm{H}$ & 1.21090200 & 4.48275500 & -1.33353900 \\
\hline C & 3.03627300 & -0.46589100 & -0.42740100 \\
\hline C & 2.11265100 & -1.42007900 & -0.89489400 \\
\hline P & 0.51378900 & -1.73046900 & 0.01385300 \\
\hline C & 1.07097800 & -2.54262300 & 1.56726600 \\
\hline C & 0.33432000 & -2.38337700 & 2.75028000 \\
\hline $\mathrm{H}$ & -0.54051500 & -1.74409900 & 2.75930800 \\
\hline C & 0.72199700 & -3.03973600 & 3.91924900 \\
\hline $\mathrm{H}$ & 0.14206400 & -2.90787300 & 4.82755700 \\
\hline C & 1.85085900 & -3.86002900 & 3.91876900 \\
\hline $\mathrm{H}$ & 2.15207300 & -4.37166500 & 4.82798000 \\
\hline C & 2.59404700 & -4.01997100 & 2.74689200 \\
\hline $\mathrm{H}$ & 3.47504800 & -4.65473900 & 2.74209000 \\
\hline C & 2.21081000 & -3.36466000 & 1.57689400 \\
\hline $\mathrm{H}$ & 2.80177400 & -3.49002500 & 0.67603200 \\
\hline C & -0.23025000 & -3.07743700 & -1.00499500 \\
\hline C & -0.76457900 & -2.73710800 & -2.26155300 \\
\hline $\mathrm{H}$ & -0.72222600 & -1.70736300 & -2.60863800 \\
\hline C & -1.32205400 & -3.71491200 & -3.08360400 \\
\hline $\mathrm{H}$ & -1.71260700 & -3.44206100 & -4.05954800 \\
\hline C & -1.36966100 & -5.04523700 & -2.65381800 \\
\hline $\mathrm{H}$ & -1.80025400 & -5.80870300 & -3.29468600 \\
\hline C & -0.85838800 & -5.38811500 & -1.40221200 \\
\hline $\mathrm{H}$ & -0.89383200 & -6.41880700 & -1.06255500 \\
\hline C & -0.28685800 & -4.41132400 & -0.58109400 \\
\hline $\mathrm{H}$ & 0.11646000 & -4.69462400 & 0.38473100 \\
\hline C & 2.41310900 & -2.16585200 & -2.03839200 \\
\hline $\mathrm{H}$ & 1.75791900 & -2.92524300 & -2.43635600 \\
\hline C & 3.63445900 & -1.93491900 & -2.68440500 \\
\hline 0 & 3.89780700 & -2.68108000 & -3.77199200 \\
\hline C & 5.14876900 & -2.47022900 & -4.43805100 \\
\hline $\mathrm{H}$ & 5.15230900 & -3.17209200 & -5.27202800 \\
\hline $\mathrm{H}$ & 5.98918800 & -2.67299800 & -3.76857200 \\
\hline $\mathrm{H}$ & 5.22914900 & -1.44345500 & -4.80552600 \\
\hline $\mathrm{N}$ & 4.52132900 & -1.03301000 & -2.27377200 \\
\hline C & 4.22422000 & -0.32683500 & -1.18786800 \\
\hline 0 & 5.10357000 & 0.60718900 & -0.77306000 \\
\hline C & 6.34514000 & 0.71258800 & -1.48019200 \\
\hline $\mathrm{H}$ & 6.90036500 & 1.49944400 & -0.96891500 \\
\hline $\mathrm{H}$ & 6.17999100 & 0.98040100 & -2.52717600 \\
\hline $\mathrm{H}$ & 6.89857700 & -0.22927200 & -1.44099200 \\
\hline $\mathrm{N}$ & 3.86850600 & 0.77939800 & 3.00774400 \\
\hline $\mathrm{H}$ & -2.46786000 & -1.51396000 & 1.80961100 \\
\hline 0 & 4.62597300 & -0.97643500 & 1.73099900 \\
\hline C & -3.38008500 & -2.16283500 & -0.03110100 \\
\hline
\end{tabular}

\begin{tabular}{|c|c|c|}
\hline-2.59890400 & -2.86801400 & -0.31975500 \\
\hline-3.76291900 & -1.74400200 & -0.96790200 \\
\hline-3.46223400 & 0.77409600 & 2.49200800 \\
\hline-5.00561000 & 0.21242000 & 0.54278400 \\
\hline-5.60345700 & -0.53682200 & 1.07187100 \\
\hline-5.43784500 & 1.20065500 & 0.71508000 \\
\hline-4.48084600 & -2.96714700 & 0.69044100 \\
\hline-5.17102800 & -3.90114500 & -0.03361300 \\
\hline-6.22700500 & -4.66870600 & 0.61942600 \\
\hline-6.29495000 & -4.35371800 & 1.65858000 \\
\hline-7.18795100 & -4.49759500 & 0.11903200 \\
\hline-6.00185100 & -5.74098300 & 0.57566000 \\
\hline-4.97523900 & -4.19494400 & -1.44440500 \\
\hline-4.84427800 & -5.27486500 & -1.58348500 \\
\hline-5.84750100 & -3.88489700 & -2.03578100 \\
\hline-4.09056800 & -3.70143100 & -1.83992000 \\
\hline-4.70823500 & -2.77882700 & 1.88722200 \\
\hline-3.69569700 & 1.84126900 & 2.42900400 \\
\hline-2.42246600 & 0.69306600 & 2.82229400 \\
\hline 0.64247900 & 1.76886200 & -0.19449100 \\
\hline 3.09503400 & 2.56758400 & 4.25298500 \\
\hline-3.21063500 & 2.31043700 & -0.56873100 \\
\hline-4.10492700 & 3.68796200 & -1.44832000 \\
\hline-4.69976400 & 4.93533700 & -0.16695700 \\
\hline-5.21545100 & 5.76589000 & -0.66200000 \\
\hline-5.40220100 & 4.48749500 & 0.54334000 \\
\hline-3.85946400 & 5.34923300 & 0.39836100 \\
\hline-5.55724700 & 2.93528300 & -2.39054500 \\
\hline-6.26658300 & 2.43997600 & -1.72007600 \\
\hline-6.10357300 & 3.71888400 & -2.92772500 \\
\hline-5.21860400 & 2.20080300 & -3.12843100 \\
\hline-2.86372900 & 4.46552400 & -2.63711700 \\
\hline-3.34636200 & 5.27022500 & -3.20340900 \\
\hline-2.01171200 & 4.89275400 & -2.10061000 \\
\hline-2.48226600 & 3.73409800 & -3.35640300 \\
\hline-0.57987100 & 0.21395100 & 1.62320100 \\
\hline-5.04847400 & -0.00340700 & -0.52447200 \\
\hline-4.38326900 & 0.10569500 & 3.53156200 \\
\hline-5.43633000 & 0.32897700 & 3.33955600 \\
\hline-4.26929600 & -0.98015400 & 3.53228400 \\
\hline-4.14035600 & 0.49246800 & 4.52596900 \\
\hline
\end{tabular}




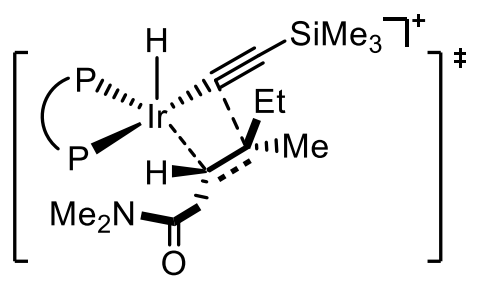

$G_{\text {sol }}=-3633.016709$ Hatree

\begin{tabular}{|c|c|c|c|}
\hline Ir & -1.03750700 & -0.12899500 & -0.30523400 \\
\hline C & -2.57317600 & -1.62618400 & -0.25576400 \\
\hline C & -3.68520600 & -0.76464600 & -0.72471600 \\
\hline C & -2.81995700 & 0.91396100 & -0.49702300 \\
\hline $\mathrm{C}$ & 1.57588900 & 1.18108400 & 1.56595000 \\
\hline $\mathrm{C}$ & 1.41889100 & 1.47705600 & 2.92165500 \\
\hline $\mathrm{H}$ & 0.60912400 & 2.08446200 & 3.29807100 \\
\hline C & 2.37217600 & 0.99144300 & 3.82592400 \\
\hline $\mathrm{C}$ & 3.56273800 & -0.01258000 & 2.16550400 \\
\hline C & 2.67061000 & 0.39437000 & 1.14235100 \\
\hline C & 3.17846200 & 0.83880100 & 6.05819400 \\
\hline $\mathrm{H}$ & 2.84397800 & 1.20319200 & 7.02948000 \\
\hline $\mathrm{H}$ & 4.16553300 & 1.24477600 & 5.82067100 \\
\hline $\mathrm{H}$ & 3.23642800 & -0.25321000 & 6.06177900 \\
\hline C & 5.59289200 & -1.09338500 & 2.79198700 \\
\hline $\mathrm{H}$ & 6.36628800 & -1.65375800 & 2.26604000 \\
\hline $\mathrm{H}$ & 5.14117900 & -1.70764900 & 3.57527800 \\
\hline $\mathrm{H}$ & 6.01870700 & -0.19674000 & 3.24952300 \\
\hline C & 1.15911300 & 2.79308400 & -0.87556700 \\
\hline C & 0.60976300 & 2.92431200 & -2.16003900 \\
\hline $\mathrm{H}$ & -0.28283300 & 2.36675300 & -2.42628500 \\
\hline C & 1.19704300 & 3.77620500 & -3.09648800 \\
\hline $\mathrm{H}$ & 0.76337000 & 3.86823800 & -4.08771700 \\
\hline C & 2.33797300 & 4.50596500 & -2.75923500 \\
\hline $\mathrm{H}$ & 2.79484800 & 5.16915900 & -3.48763100 \\
\hline C & 2.89078400 & 4.38135500 & -1.48274700 \\
\hline $\mathrm{H}$ & 3.77676700 & 4.94942300 & -1.21492300 \\
\hline C & 2.30650700 & 3.53102600 & -0.54292700 \\
\hline $\mathrm{H}$ & 2.75225600 & 3.43406400 & 0.44110200 \\
\hline C & -0.69263300 & 2.97814700 & 1.34283500 \\
\hline C & -1.67938500 & 2.51307300 & 2.22875000 \\
\hline $\mathrm{H}$ & -1.88954700 & 1.45003300 & 2.29421200 \\
\hline C & -2.39279800 & 3.40025100 & 3.03412300 \\
\hline $\mathrm{H}$ & -3.14112500 & 3.02050900 & 3.72344400 \\
\hline C & -2.14658900 & 4.77312900 & 2.95107000 \\
\hline $\mathrm{H}$ & -2.70211900 & 5.46568800 & 3.57622300 \\
\hline C & -1.18230400 & 5.24873600 & 2.06190300 \\
\hline $\mathrm{H}$ & -0.98536300 & 6.31417200 & 1.98959800 \\
\hline
\end{tabular}

\begin{tabular}{|c|c|c|c|}
\hline C & -0.45721500 & 4.35898900 & 1.26531100 \\
\hline $\mathrm{H}$ & 0.29316200 & 4.74805000 & 0.58708800 \\
\hline C & 3.02707900 & 0.09629000 & -0.27490800 \\
\hline C & 2.36470900 & -0.79741500 & -1.14933500 \\
\hline P & 0.76238800 & -1.64999900 & -0.72050500 \\
\hline C & 1.18309800 & -2.96625200 & 0.50697600 \\
\hline C & 0.20975000 & -3.94118500 & 0.79768900 \\
\hline $\mathrm{H}$ & -0.74753000 & -3.93390700 & 0.28742300 \\
\hline C & 0.48178700 & -4.96748500 & 1.70091000 \\
\hline $\mathrm{H}$ & -0.27701400 & -5.71750400 & 1.90311400 \\
\hline $\mathrm{C}$ & 1.72988700 & -5.04121300 & 2.32455200 \\
\hline $\mathrm{H}$ & 1.94305200 & -5.84266400 & 3.02550000 \\
\hline C & 2.70685500 & -4.09409800 & 2.02228100 \\
\hline $\mathrm{H}$ & 3.68902100 & -4.15865200 & 2.48105100 \\
\hline C & 2.44088000 & -3.06402600 & 1.11539200 \\
\hline $\mathrm{H}$ & 3.22712800 & -2.35908800 & 0.87835400 \\
\hline C & 0.41107500 & -2.66693100 & -2.22917400 \\
\hline C & -0.39324500 & -2.15665700 & -3.26013600 \\
\hline $\mathrm{H}$ & -0.84086400 & -1.17475600 & -3.15814000 \\
\hline C & -0.62182500 & -2.89626100 & -4.42095100 \\
\hline $\mathrm{H}$ & -1.24750400 & -2.48416300 & -5.20694200 \\
\hline C & -0.05113000 & -4.16114500 & -4.56860700 \\
\hline $\mathrm{H}$ & -0.23301000 & -4.74028000 & -5.46880600 \\
\hline C & 0.75732000 & -4.67678200 & -3.55447500 \\
\hline $\mathrm{H}$ & 1.21048500 & -5.65741200 & -3.66355600 \\
\hline C & 0.99119300 & -3.93617900 & -2.39546800 \\
\hline $\mathrm{H}$ & 1.62895900 & -4.35018700 & -1.62278900 \\
\hline C & 2.87876000 & -1.00974600 & -2.43075900 \\
\hline $\mathrm{H}$ & 2.41765500 & -1.67740600 & -3.14269700 \\
\hline $\mathrm{C}$ & 4.04886700 & -0.33822900 & -2.80696300 \\
\hline 0 & 4.51899600 & -0.58034500 & -4.04234200 \\
\hline C & 5.71925800 & 0.09150300 & -4.44380000 \\
\hline $\mathrm{H}$ & 5.90951100 & -0.24481900 & -5.46297700 \\
\hline $\mathrm{H}$ & 6.55601300 & -0.17812200 & -3.79351200 \\
\hline $\mathrm{H}$ & 5.58769700 & 1.17677700 & -4.42017800 \\
\hline $\mathrm{N}$ & 4.69413900 & 0.50702000 & -2.00940700 \\
\hline C & 4.19430900 & 0.71296400 & -0.79528100 \\
\hline 0 & 4.83214400 & 1.58367400 & 0.01299500 \\
\hline $\mathrm{C}$ & 6.03598500 & 2.18543400 & -0.47733600 \\
\hline $\mathrm{H}$ & 6.38860400 & 2.82263200 & 0.33435100 \\
\hline $\mathrm{H}$ & 5.83941400 & 2.78129300 & -1.37252100 \\
\hline $\mathrm{H}$ & 6.78480500 & 1.42596500 & -0.71638600 \\
\hline 0 & 2.19493600 & 1.29866700 & 5.12288800 \\
\hline $\mathrm{H}$ & -2.38343100 & -2.45381500 & -0.93639300 \\
\hline 0 & 4.63157200 & -0.74627100 & 1.78711700 \\
\hline
\end{tabular}



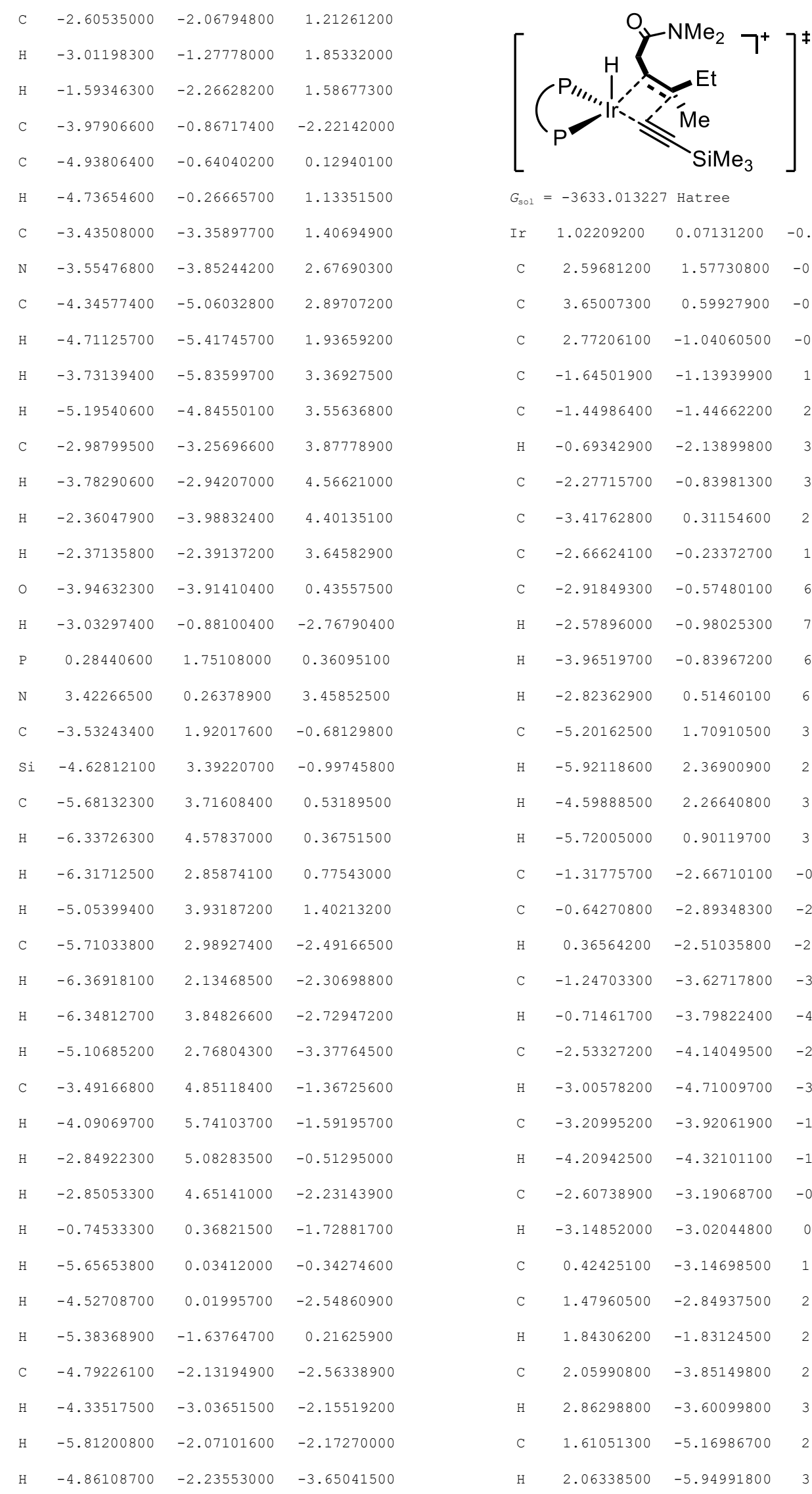

\begin{tabular}{|c|c|c|c|}
\hline$G_{\text {sol }}=$ & $=-3633.013227$ & Hatree & \\
\hline Ir & 1.02209200 & 0.07131200 & -0.16363300 \\
\hline C & 2.59681200 & 1.57730800 & -0.23722600 \\
\hline C & 3.65007300 & 0.59927900 & -0.62309700 \\
\hline $\mathrm{C}$ & 2.77206100 & -1.04060500 & -0.31852000 \\
\hline C & -1.64501900 & -1.13939900 & 1.63411600 \\
\hline C & -1.44986400 & -1.44662200 & 2.98219500 \\
\hline $\mathrm{H}$ & -0.69342900 & -2.13899800 & 3.32071900 \\
\hline C & -2.27715700 & -0.83981300 & 3.93578300 \\
\hline C & -3.41762800 & 0.31154600 & 2.33997900 \\
\hline C & -2.66624100 & -0.23372700 & 1.26946400 \\
\hline C & -2.91849300 & -0.57480100 & 6.21040800 \\
\hline $\mathrm{H}$ & -2.57896000 & -0.98025300 & 7.16351600 \\
\hline $\mathrm{H}$ & -3.96519700 & -0.83967200 & 6.03748900 \\
\hline H & -2.82362900 & 0.51460100 & 6.20575400 \\
\hline C & -5.20162500 & 1.70910500 & 3.07717300 \\
\hline $\mathrm{H}$ & -5.92118600 & 2.36900900 & 2.59162100 \\
\hline $\mathrm{H}$ & -4.59888500 & 2.26640800 & 3.79898200 \\
\hline $\mathrm{H}$ & -5.72005000 & 0.90119700 & 3.59964100 \\
\hline C & -1.31775700 & -2.66710100 & -0.91954500 \\
\hline C & -0.64270800 & -2.89348300 & -2.13020800 \\
\hline $\mathrm{H}$ & 0.36564200 & -2.51035800 & -2.26686500 \\
\hline C & -1.24703300 & -3.62717800 & -3.15161000 \\
\hline $\mathrm{H}$ & -0.71461700 & -3.79822400 & -4.08251100 \\
\hline C & -2.53327200 & -4.14049500 & -2.97484400 \\
\hline $\mathrm{H}$ & -3.00578200 & -4.71009700 & -3.76933800 \\
\hline C & -3.20995200 & -3.92061900 & -1.77345300 \\
\hline $\mathrm{H}$ & -4.20942500 & -4.32101100 & -1.63148100 \\
\hline C & -2.60738900 & -3.19068700 & -0.74677500 \\
\hline $\mathrm{H}$ & -3.14852000 & -3.02044800 & 0.17803400 \\
\hline C & 0.42425100 & -3.14698500 & 1.32417400 \\
\hline $\mathrm{C}$ & 1.47960500 & -2.84937500 & 2.20262500 \\
\hline $\mathrm{H}$ & 1.84306200 & -1.83124500 & 2.28115400 \\
\hline C & 2.05990800 & -3.85149800 & 2.98168300 \\
\hline $\mathrm{H}$ & 2.86298800 & -3.60099800 & 3.66858300 \\
\hline $\mathrm{C}$ & 1.61051300 & -5.16986700 & 2.87787700 \\
\hline $\mathrm{H}$ & 2.06338500 & -5.94991800 & 3.48252300 \\
\hline C & 0.57459000 & -5.47932100 & 1.99512100 \\
\hline $\mathrm{H}$ & 0.21858300 & -6.50151700 & 1.90836800 \\
\hline C & -0.01813600 & -4.47569200 & 1.22603100 \\
\hline
\end{tabular}




\begin{tabular}{|c|c|c|c|}
\hline $\mathrm{H}$ & -0.82927700 & -4.73332000 & 0.55457500 \\
\hline 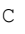 & -3.10424500 & 0.08504000 & -0.11781900 \\
\hline & -2.41828700 & 0.87918000 & -1.05853600 \\
\hline & -0.70989000 & 1.56082400 & -0.76969100 \\
\hline$C$ & -0.99453200 & 3.17044800 & 0.07283100 \\
\hline$C$ & 0.02966800 & 4.13498600 & 0.11091100 \\
\hline $\mathrm{H}$ & 0.98280400 & 3.96634500 & -0.37723300 \\
\hline $\mathrm{C}$ & -0.17274100 & 5.35116400 & 0.76344900 \\
\hline $\mathrm{H}$ & 0.62485300 & 6.08782100 & 0.76853800 \\
\hline C & -1.38851400 & 5.61765900 & 1.39614800 \\
\hline H & -1.54398900 & 6.56530000 & 1.90314500 \\
\hline C & -2.40483600 & 4.66297400 & 1.36665800 \\
\hline $\mathrm{H}$ & -3.35610800 & 4.86415900 & 1.85022300 \\
\hline C & -2.21573900 & 3.44682000 & 0.70705200 \\
\hline H & -3.02424700 & 2.72583200 & 0.69283500 \\
\hline C & -0.16256000 & 1.94570300 & -2.49153800 \\
\hline C & 0.18429900 & 0.84335000 & -3.29741200 \\
\hline H & 0.04596200 & -0.16906000 & -2.92266200 \\
\hline C & 0.65054100 & 1.03220700 & -4.59822600 \\
\hline $\mathrm{H}$ & 0.90416000 & 0.17269000 & -5.21156800 \\
\hline C & 0.77594400 & 2.32491700 & -5.11333100 \\
\hline $\mathrm{H}$ & 1.13686500 & 2.47524000 & -6.12611800 \\
\hline C & 0.42249400 & 3.42105700 & -4.32615200 \\
\hline H & 0.50585700 & 4.42722600 & -4.72543400 \\
\hline C & -0.04634000 & 3.23658200 & -3.02229800 \\
\hline H & -0.32628800 & 4.10049100 & -2.43116000 \\
\hline C & -3.02679900 & 1.18638800 & -2.27940900 \\
\hline $\mathrm{H}$ & -2.55024000 & 1.79694200 & -3.03227400 \\
\hline C & -4.31105500 & 0.69117600 & -2.53106500 \\
\hline o & -4.87593700 & 1.02371800 & -3.70436500 \\
\hline C & -6.19372900 & 0.53037200 & -3.97481700 \\
\hline H & -6.44796300 & 0.91645300 & -4.96187600 \\
\hline $\mathrm{H}$ & -6.90854300 & 0.89263900 & -3.23082900 \\
\hline H & -6.20989100 & -0.56300100 & -3.97887500 \\
\hline $\mathrm{N}$ & -4.97407700 & -0.08323600 & -1.67555900 \\
\hline C & -4.38630900 & -0.37344900 & -0.52101100 \\
\hline 0 & -5.04164900 & -1.17606300 & 0.34328800 \\
\hline C & -6.36782500 & -1.59009300 & -0.00798500 \\
\hline H & -6.70749300 & -2.20215000 & 0.82797200 \\
\hline H & -6.36665900 & -2.17376900 & -0.9323230 \\
\hline $\mathrm{H}$ & -7.02521200 & -0.72660900 & -0.13876300 \\
\hline 0 & -2.06822000 & -1.16968800 & 5.22233000 \\
\hline $\mathrm{H}$ & 2.39879400 & 2.26457000 & -1.05943900 \\
\hline 0 & -4.38895100 & 1.19274400 & 2.01533700 \\
\hline & 2.72799300 & 2.33954100 & 1.08684000 \\
\hline
\end{tabular}

\begin{tabular}{|c|c|c|c|}
\hline $\mathrm{H}$ & 3.25945800 & 1.74273600 & 1.83052900 \\
\hline $\mathrm{H}$ & 1.73575100 & 2.52562000 & 1.51253300 \\
\hline C & 3.91872000 & 0.56307400 & -2.12237500 \\
\hline C & 4.92513300 & 0.45163800 & 0.21853900 \\
\hline $\mathrm{H}$ & 4.67235500 & 0.36431700 & 1.27838800 \\
\hline C & 3.37720000 & 3.72358800 & 0.90941400 \\
\hline $\mathrm{N}$ & 3.82169400 & 4.37580700 & 2.02676900 \\
\hline $\mathrm{C}$ & 4.46515800 & 5.67855100 & 1.88153100 \\
\hline $\mathrm{H}$ & 4.51030200 & 5.92895000 & 0.82364200 \\
\hline $\mathrm{H}$ & 3.89711300 & 6.44883600 & 2.41695300 \\
\hline $\mathrm{H}$ & 5.47957400 & 5.64852000 & 2.29669200 \\
\hline C & 3.73648500 & 3.87481600 & 3.39147100 \\
\hline $\mathrm{H}$ & 4.72017400 & 3.56263000 & 3.76714400 \\
\hline $\mathrm{H}$ & 3.36432300 & 4.66964600 & 4.04748000 \\
\hline $\mathrm{H}$ & 3.04889800 & 3.03505000 & 3.46928400 \\
\hline O & 3.44712900 & 4.23456800 & -0.21218400 \\
\hline $\mathrm{H}$ & 2.99575700 & 0.49163400 & -2.70103500 \\
\hline $\mathrm{H}$ & 4.40153300 & 1.50783200 & -2.39791500 \\
\hline P & -0.40610900 & -1.77531000 & 0.40245600 \\
\hline $\mathrm{N}$ & -3.23442200 & 0.02993000 & 3.62600200 \\
\hline C & 3.38877200 & -2.11107500 & -0.48702500 \\
\hline $\mathrm{Si}$ & 4.35557700 & -3.67158700 & -0.81396000 \\
\hline C & 5.35873500 & -4.10925000 & 0.71954600 \\
\hline $\mathrm{H}$ & 5.95710900 & -5.00878400 & 0.53612300 \\
\hline $\mathrm{H}$ & 6.04673300 & -3.30453200 & 0.99788900 \\
\hline $\mathrm{H}$ & 4.70507200 & -4.30929100 & 1.57354400 \\
\hline C & 5.48930100 & -3.32183000 & -2.28429300 \\
\hline $\mathrm{H}$ & 6.21107000 & -2.52713200 & -2.07024900 \\
\hline $\mathrm{H}$ & 6.05973200 & -4.22436600 & -2.53178600 \\
\hline $\mathrm{H}$ & 4.91997700 & -3.03491600 & -3.17407000 \\
\hline C & 3.11701500 & -5.02859800 & -1.23750100 \\
\hline $\mathrm{H}$ & 3.65000400 & -5.95874900 & -1.46563800 \\
\hline $\mathrm{H}$ & 2.43590300 & -5.22577300 & -0.40488600 \\
\hline $\mathrm{H}$ & 2.51632700 & -4.76442700 & -2.11349500 \\
\hline $\mathrm{H}$ & 0.88002600 & 0.38981300 & 1.33405800 \\
\hline $\mathrm{H}$ & 5.40579700 & -0.49033700 & -0.05688200 \\
\hline $\mathrm{H}$ & 4.57477000 & -0.26492300 & -2.39802600 \\
\hline $\mathrm{C}$ & 5.92466100 & 1.60401600 & -0.00225200 \\
\hline $\mathrm{H}$ & 6.36868400 & 1.55326600 & -1.00030000 \\
\hline $\mathrm{H}$ & 5.47209300 & 2.59094000 & 0.10272000 \\
\hline $\mathrm{H}$ & 6.73867700 & 1.52035000 & 0.72417200 \\
\hline
\end{tabular}




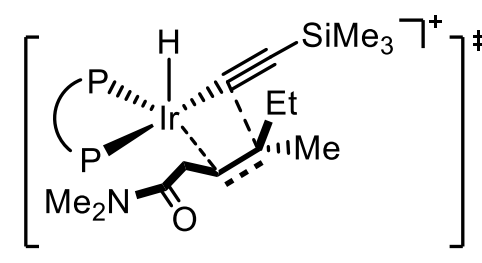

$G_{\text {sol }}=-3633.021933$ Hatree$$
\text { C }
$$

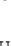




\begin{tabular}{|c|c|c|c|}
\hline$H$ & -2.74983100 & -2.38518500 & -0.89749500 \\
\hline$H$ & -4.08287100 & -1.26580500 & -0.80867500 \\
\hline & -2.67281000 & 0.60082300 & 2.68284900 \\
\hline c & -4.84443300 & 0.30018100 & 1.39116500 \\
\hline H & -5.04358200 & 1.36907200 & 1.50371100 \\
\hline H & -5.25229600 & -0.00073600 & 0.42464000 \\
\hline C & -4.27936600 & -3.06378800 & 0.42499800 \\
\hline $\mathrm{N}$ & -5.12713900 & -3.76540400 & -0.38925300 \\
\hline $\mathrm{c}$ & -5.88232200 & -4.88236700 & 0.16926400 \\
\hline H & -5.74665900 & -4.88888300 & 1.24874000 \\
\hline $\mathrm{H}$ & -6.94588200 & -4.77401200 & -0.07176400 \\
\hline $\mathrm{H}$ & -5.53030100 & -5.83548300 & -0.24563700 \\
\hline C & -5.24119400 & -3.60326200 & -1.83020800 \\
\hline $\mathrm{H}$ & -4.83589700 & -4.47941100 & -2.35390200 \\
\hline $\mathrm{H}$ & -6.29502000 & -3.49874600 & -2.11449800 \\
\hline $\mathrm{H}$ & -4.70575500 & -2.72277700 & -2.17847300 \\
\hline 0 & -4.15884300 & -3.33393100 & 1.62232900 \\
\hline $\mathrm{H}$ & -1.57451500 & 0.59412900 & 2.63313300 \\
\hline $\mathrm{H}$ & -2.95669800 & -0.05554300 & 3.51369100 \\
\hline$P$ & 0.64073800 & 1.78037300 & -0.36774500 \\
\hline D & 3.20035900 & 2.94055500 & 3.94032000 \\
\hline C & -3.38148700 & 2.22353100 & -0.37612800 \\
\hline $\mathrm{Si}$ & -4.49825500 & 3.52957800 & -1.08580800 \\
\hline C & -4.99290400 & 4.71738700 & 0.29324400 \\
\hline $\mathrm{H}$ & -5.64777900 & 5.50315000 & -0.09983900 \\
\hline H & -5.53673800 & 4.20752500 & 1.09515900 \\
\hline $\mathrm{H}$ & -4.11383100 & 5.20009200 & 0.73071200 \\
\hline C & -6.01780100 & 2.68779800 & -1.82391000 \\
\hline $\mathrm{H}$ & -6.59280900 & 2.14532500 & -1.06662400 \\
\hline $\mathrm{H}$ & -6.68392700 & 3.43480400 & -2.27023500 \\
\hline $\mathrm{H}$ & -5.73891100 & 1.97893000 & -2.61019200 \\
\hline C & -3.50351000 & 4.41105000 & -2.42524700 \\
\hline $\mathrm{H}$ & -4.11450900 & 5.19449800 & -2.88796100 \\
\hline $\mathrm{H}$ & -2.60554100 & 4.87997000 & -2.01271100 \\
\hline $\mathrm{H}$ & -3.19487600 & 3.71868600 & -3.21475100 \\
\hline $\mathrm{H}$ & -1.17078000 & -0.20058300 & -1.35549600 \\
\hline C & -5.55170000 & -0.47335600 & 2.52473000 \\
\hline $\mathrm{H}$ & -5.31627600 & -0.05078000 & 3.50597700 \\
\hline $\mathrm{H}$ & -6.63402800 & -0.38852000 & 2.38648600 \\
\hline $\mathrm{H}$ & -5.28563000 & -1.53207800 & 2.52815900 \\
\hline & -2.99006900 & 1.61896600 & 2.91842100 \\
\hline
\end{tabular}

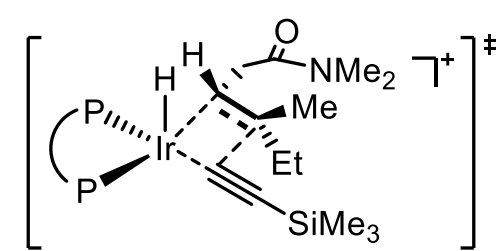

$G_{\text {sol }}=-3633.016625$ Hatree

$\begin{array}{llll}\text { Ir } & -1.04333200 & 0.16554300 & 0.16847900\end{array}$

$\begin{array}{llll}\text { C } & -2.49875600 & 1.62808400 & -0.02527800\end{array}$

$\begin{array}{llll}\text { C } & -3.77679500 & 0.28245200 & 0.45002500\end{array}$

$\begin{array}{llll}\text { C } & -2.88313600 & -0.86381300 & 0.76793100\end{array}$

$\begin{array}{llll}\text { C } & 2.10793100 & 1.05957100 & 1.27939700\end{array}$

$\begin{array}{llll}\text { C } & 2.22504800 \quad 1.55617800 & 2.57853400\end{array}$

H $\quad 1.66342400 \quad 2.40743000 \quad 2.93471300$

$\begin{array}{llll}\text { C } & 3.11982100 & 0.92582800 & 3.45464300\end{array}$

$\begin{array}{llll}\text { C } & 3.73988200 & -0.59379000 & 1.87743700\end{array}$

$\begin{array}{llll}\text { C } & 2.88820300 & -0.04881900 & 0.88681300\end{array}$

$\begin{array}{llll}\text { C } & 4.13419200 & 0.81526800 & 5.60293100\end{array}$

$\begin{array}{llll}\mathrm{H} & 4.05110400 & 1.38427800 & 6.52890500\end{array}$

$\begin{array}{llll}\mathrm{H} & 5.15752000 & 0.86106200 & 5.22038700\end{array}$

$\begin{array}{llll}\mathrm{H} & 3.86712500 & -0.23105400 & 5.77489900\end{array}$

$\begin{array}{llll}\text { C } & 5.36398100 & -2.21719500 & 2.49862700\end{array}$

$\begin{array}{llll}\mathrm{H} & 5.87626900 & -3.03529900 & 1.99119700\end{array}$

H $\quad 4.80902300 \quad-2.59401600 \quad 3.36187100$

$\begin{array}{llll}\mathrm{H} & 6.08641700 & -1.47150600 & 2.83998700\end{array}$

$\begin{array}{llll}\text { C } & 1.60061800 & 2.24138000 & -1.40019000\end{array}$

$\begin{array}{llll}\text { C } & 0.83807600 & 2.22695900 & -2.57748700\end{array}$

H $\quad-0.19046200 \quad 1.87774500 \quad-2.54829700$

$\begin{array}{llll}\text { C } & 1.38869300 & 2.66638700 & -3.78245400\end{array}$

H $\quad 0.79003400 \quad 2.65083600 \quad-4.68833600$

$\begin{array}{llll}\text { C } & 2.70732200 & 3.12212400 & -3.82136400\end{array}$

$\mathrm{H} \quad 3.13687100 \quad 3.46450100 \quad-4.75806100$

$\begin{array}{llll}\text { C } & 3.47505900 & 3.13479900 & -2.65423100\end{array}$

H $\quad 4.50174400 \quad 3.48771300 \quad-2.68214800$

$\begin{array}{llll}\text { C } & 2.92756800 & 2.69786200 & -1.44730500\end{array}$

H $\quad 3.53690600 \quad 2.69579700 \quad-0.54924700$

$\begin{array}{llll}\text { C } & 0.35448900 & 3.35522200 & 0.96933000\end{array}$

$\begin{array}{llll}\text { C } & -0.53383300 & 3.36152500 & 2.05829400\end{array}$

$\begin{array}{llll}\mathrm{H} & -1.00335200 & 2.43680500 & 2.37533400\end{array}$

$\begin{array}{llll}\text { C } & -0.81673100 \quad 4.54505200 & 2.74009500\end{array}$

$\mathrm{H} \quad-1.49425100 \quad 4.52797000 \quad 3.58856000$

$\begin{array}{llll}\text { C } & -0.23489900 & 5.74698600 & 2.32942500\end{array}$

$\begin{array}{llll}\mathrm{H} & -0.45643700 & 6.66917400 & 2.85825700\end{array}$

$\begin{array}{llll}\text { C } & 0.63307700 \quad 5.75589900 & 1.23704100\end{array}$

$\mathrm{H} \quad 1.08852600 \quad 6.68570300 \quad 0.90954800$

$\begin{array}{llll}\text { C } & 0.93053400 \quad 4.56890700 & 0.56324700\end{array}$ 


\begin{tabular}{|c|c|c|c|}
\hline $\mathrm{H}$ & 1.61487400 & 4.59651900 & -0.27663300 \\
\hline C & 2.95558500 & -0.60157400 & -0.49075500 \\
\hline C & 1.94539600 & -1.35059200 & -1.12285700 \\
\hline P & 0.32884200 & -1.69222400 & -0.26778800 \\
\hline C & 0.77555200 & -2.85061600 & 1.08558300 \\
\hline C & -0.00239800 & -2.88426700 & 2.25305600 \\
\hline $\mathrm{H}$ & -0.83114400 & -2.19467300 & 2.37067600 \\
\hline C & 0.28921200 & -3.79503400 & 3.26919200 \\
\hline $\mathrm{H}$ & -0.31879000 & -3.81007400 & 4.16861800 \\
\hline C & 1.36144800 & -4.67791200 & 3.13040000 \\
\hline $\mathrm{H}$ & 1.58892200 & -5.38587500 & 3.92174000 \\
\hline C & 2.14551300 & -4.64546600 & 1.97488900 \\
\hline $\mathrm{H}$ & 2.98422500 & -5.32644900 & 1.86558200 \\
\hline C & 1.85929500 & -3.73546100 & 0.95676700 \\
\hline $\mathrm{H}$ & 2.48377300 & -3.70826200 & 0.07018800 \\
\hline C & -0.54406200 & -2.71136500 & -1.53766500 \\
\hline C & -1.22550700 & -2.03426600 & -2.56365900 \\
\hline $\mathrm{H}$ & -1.23205400 & -0.94608200 & -2.58392700 \\
\hline C & -1.87707700 & -2.74099600 & -3.57379100 \\
\hline $\mathrm{H}$ & -2.39199900 & -2.20421000 & -4.36488400 \\
\hline C & -1.85766000 & -4.13872100 & -3.56996600 \\
\hline $\mathrm{H}$ & -2.35986700 & -4.69193800 & -4.35773300 \\
\hline C & -1.18439000 & -4.82003800 & -2.55462600 \\
\hline $\mathrm{H}$ & -1.16162100 & -5.90575600 & -2.55017800 \\
\hline C & -0.52980000 & -4.11222500 & -1.54223700 \\
\hline $\mathrm{H}$ & -0.00775100 & -4.65530400 & -0.76162500 \\
\hline C & 2.15887300 & -1.85370400 & -2.40925200 \\
\hline $\mathrm{H}$ & 1.42550100 & -2.43824700 & -2.94365000 \\
\hline C & 3.39276300 & -1.60383200 & -3.02408800 \\
\hline 0 & 3.57393800 & -2.11641600 & -4.25418700 \\
\hline C & 4.83397700 & -1.88291500 & -4.89528100 \\
\hline H & 4.76200400 & -2.38301000 & -5.86114600 \\
\hline $\mathrm{H}$ & 5.65620300 & -2.30454600 & -4.31064900 \\
\hline $\mathrm{H}$ & 5.01112500 & -0.81285100 & -5.03411600 \\
\hline $\mathrm{N}$ & 4.36725000 & -0.90221100 & -2.45244500 \\
\hline C & 4.14743800 & -0.41658800 & -1.23444200 \\
\hline 0 & 5.11577000 & 0.31903200 & -0.65454300 \\
\hline C & 6.35238700 & 0.46759500 & -1.36245000 \\
\hline $\mathrm{H}$ & 6.98281300 & 1.07585500 & -0.71329000 \\
\hline $\mathrm{H}$ & 6.19808100 & 0.96723600 & -2.32247000 \\
\hline $\mathrm{H}$ & 6.81930700 & -0.50436200 & -1.54220100 \\
\hline $\mathrm{N}$ & 3.85448200 & -0.13137200 & 3.11934100 \\
\hline $\mathrm{H}$ & -2.75285600 & -0.90789600 & 1.85196700 \\
\hline 0 & 4.47659200 & -1.66382400 & 1.52002900 \\
\hline C & -3.26164900 & -2.26325400 & 0.29032600 \\
\hline
\end{tabular}

\begin{tabular}{|c|c|c|}
\hline-2.39769400 & -2.92708700 & 0.38599300 \\
\hline-3.53096600 & -2.28423600 & -0.76916900 \\
\hline-4.43589300 & 0.94035000 & 1.64943500 \\
\hline-4.65203100 & 0.22465600 & -0.81011100 \\
\hline-4.92116000 & 1.24774700 & -1.07610700 \\
\hline-4.39639000 & -2.85423800 & 1.15526200 \\
\hline-4.88329800 & -4.08905600 & 0.81617500 \\
\hline-5.92790800 & -4.68919600 & 1.64105800 \\
\hline-6.26371900 & -3.95329800 & 2.36856100 \\
\hline-6.76951100 & -5.00124700 & 1.01228900 \\
\hline-5.54478000 & -5.57222800 & 2.16792000 \\
\hline-4.35911100 & -4.94725400 & -0.23490900 \\
\hline-3.89105200 & -5.84379500 & 0.19377000 \\
\hline-5.17362900 & -5.27687700 & -0.89116900 \\
\hline-3.61780800 & -4.43483600 & -0.84311500 \\
\hline-4.83878700 & -2.22739000 & 2.11919400 \\
\hline-4.98795300 & 1.84000600 & 1.36759800 \\
\hline-3.70345300 & 1.19987300 & 2.41654400 \\
\hline 0.78360800 & 1.74477800 & 0.16820600 \\
\hline 3.21749700 & 1.43718900 & 4.69410200 \\
\hline-2.88379100 & 2.74112100 & -0.43023100 \\
\hline-3.50531400 & 4.38407300 & -1.05598300 \\
\hline-4.02460300 & 5.43647900 & 0.41719500 \\
\hline-4.39584800 & 6.40870200 & 0.07384200 \\
\hline-4.82412800 & 4.96118300 & 0.99449000 \\
\hline-3.18040300 & 5.61550300 & 1.08944200 \\
\hline-4.97191400 & 4.04016800 & -2.19588300 \\
\hline-5.80809300 & 3.57418300 & -1.66489900 \\
\hline-5.33792100 & 4.98293700 & -2.61831300 \\
\hline-4.69236900 & 3.38906800 & -3.03019200 \\
\hline-2.09618400 & 5.19305300 & -2.01365800 \\
\hline-2.42823900 & 6.15576900 & -2.41879700 \\
\hline-1.23144800 & 5.37829000 & -1.36995900 \\
\hline-1.76795500 & 4.57225100 & -2.85304700 \\
\hline-0.64613300 & 0.02172600 & 1.64887600 \\
\hline-4.06547500 & -0.16847400 & -1.64778700 \\
\hline-5.11122600 & 0.19502200 & 2.08309300 \\
\hline-5.95939000 & -0.58050000 & -0.66586600 \\
\hline-5.80219800 & -1.65361300 & -0.55748100 \\
\hline-6.55257400 & -0.24295300 & 0.18738500 \\
\hline-6.55852400 & -0.42490500 & -1.56860100 \\
\hline
\end{tabular}




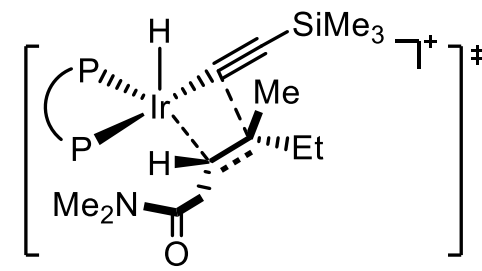

$G_{\text {sol }}=-3633.016900$ Hatree

\begin{tabular}{|c|c|c|c|}
\hline Ir & 1.03948300 & 0.19735400 & -0.37852500 \\
\hline C & 2.58843100 & 1.67255500 & -0.52955300 \\
\hline C & 3.63576700 & 0.79436900 & -1.09362000 \\
\hline C & 2.79117200 & -0.87966700 & -0.61117200 \\
\hline $\mathrm{C}$ & -1.60219300 & -1.13891000 & 1.58070100 \\
\hline C & -1.51121100 & -1.37283000 & 2.95455500 \\
\hline $\mathrm{H}$ & -0.67743800 & -1.88974600 & 3.40666300 \\
\hline C & -2.56257000 & -0.94230000 & 3.77401100 \\
\hline C & -3.71975300 & -0.10506000 & 2.00032300 \\
\hline C & -2.72667200 & -0.46562600 & 1.05568100 \\
\hline C & -3.52519000 & -0.77303700 & 5.94219600 \\
\hline H & -3.22715500 & -1.07003400 & 6.94772700 \\
\hline $\mathrm{H}$ & -4.45890100 & -1.26829700 & 5.66203400 \\
\hline $\mathrm{H}$ & -3.67060500 & 0.30973700 & 5.89373000 \\
\hline C & -5.86550000 & 0.82812100 & 2.45134500 \\
\hline H & -6.64126500 & 1.31018500 & 1.85588400 \\
\hline $\mathrm{H}$ & -5.51306900 & 1.50105200 & 3.23738100 \\
\hline $\mathrm{H}$ & -6.25370800 & -0.08228900 & 2.91522300 \\
\hline C & -0.93587500 & -2.88604300 & -0.69099400 \\
\hline C & -0.30556400 & -3.09893600 & -1.92720700 \\
\hline $\mathrm{H}$ & 0.56514800 & -2.51050100 & -2.19811800 \\
\hline C & -0.78419600 & -4.07225800 & -2.80504000 \\
\hline $\mathrm{H}$ & -0.29095100 & -4.22560300 & -3.76027400 \\
\hline C & -1.89400500 & -4.84429700 & -2.45694700 \\
\hline $\mathrm{H}$ & -2.26624000 & -5.60166100 & -3.14034300 \\
\hline C & -2.52463900 & -4.64001400 & -1.22804100 \\
\hline $\mathrm{H}$ & -3.38698600 & -5.23954300 & -0.95181000 \\
\hline C & -2.05049500 & -3.66698300 & -0.34663300 \\
\hline $\mathrm{H}$ & -2.55700300 & -3.50905000 & 0.59918900 \\
\hline C & 0.84059200 & -2.69573400 & 1.59390100 \\
\hline C & 1.76312000 & -2.05557200 & 2.43741100 \\
\hline $\mathrm{H}$ & 1.87148900 & -0.97564300 & 2.39729400 \\
\hline C & 2.53926200 & -2.78760600 & 3.33499300 \\
\hline $\mathrm{H}$ & 3.23887300 & -2.27483600 & 3.98844200 \\
\hline C & 2.41800000 & -4.17855900 & 3.39049500 \\
\hline $\mathrm{H}$ & 3.02205800 & -4.75100700 & 4.08799200 \\
\hline C & 1.51467000 & -4.82743600 & 2.54799300 \\
\hline $\mathrm{H}$ & 1.41376900 & -5.90798800 & 2.58557900 \\
\hline
\end{tabular}

$$
\begin{aligned}
& 0.72772300 \quad-4.09229100 \quad 1.65740400 \\
& \begin{array}{lll}
0.02465400 & -4.61224400 & 1.01667500
\end{array} \\
& \begin{array}{llll}
-2.99636000 & -0.23009900 & -0.39177700
\end{array} \\
& \begin{array}{llll}
-2.33988800 & 0.69913900 & -1.23394200
\end{array} \\
& \begin{array}{lll}
-0.82835300 & 1.66105500 & -0.71784000
\end{array} \\
& \begin{array}{lll}
-1.36447700 & 2.84345600 & 0.59135400
\end{array} \\
& \begin{array}{lll}
-0.41673600 & 3.76627600 & 1.06812600
\end{array} \\
& \begin{array}{llll}
0.58319800 & 3.79409200 & 0.65003600
\end{array} \\
& \begin{array}{lll}
-0.77363100 \quad 4.71418100 \quad 2.02583600
\end{array} \\
& \begin{array}{lll}
-0.03265000 & 5.42734400 & 2.37420500
\end{array} \\
& \begin{array}{lll}
-2.08068600 \quad 4.75876000 & 2.51666900
\end{array} \\
& \begin{array}{lll}
-2.35885400 \quad 5.49788100 & 3.26199800
\end{array} \\
& \begin{array}{lll}
-3.03233400 \quad 3.86641200 & 2.02428100
\end{array} \\
& \begin{array}{lll}
-4.05799200 & 3.91348300 & 2.37803000
\end{array} \\
& \begin{array}{lll}
-2.68172800 & 2.91567300 & 1.06187300
\end{array} \\
& \begin{array}{lll}
-3.44366000 & 2.24897400 & 0.67885200
\end{array} \\
& \begin{array}{lll}
-0.53767600 & 2.80092800 & -2.14590000
\end{array} \\
& \begin{array}{llll}
0.25569200 & 2.38015200 & -3.22453900
\end{array} \\
& \begin{array}{llll}
0.71020700 & 1.39647400 & -3.19920200
\end{array} \\
& \begin{array}{llll}
0.45579700 & 3.20689900 & -4.32956200
\end{array} \\
& \begin{array}{lll}
1.07133000 & 2.86360300 & -5.15573300
\end{array} \\
& \begin{array}{llll}
-0.13014400 \quad 4.47337500 & -4.36931900
\end{array} \\
& \begin{array}{llll}
0.03109200 & 5.12212500 & -5.22488300
\end{array} \\
& \begin{array}{llll}
-0.92529800 & 4.90088700 & -3.30544900
\end{array} \\
& \begin{array}{llll}
-1.38780200 & 5.88287500 & -3.33075300
\end{array} \\
& \begin{array}{llll}
-1.13307400 \quad 4.07130800 & -2.20258400
\end{array} \\
& \begin{array}{llll}
-1.75820600 & 4.41957000 & -1.38841500
\end{array} \\
& \begin{array}{llll}
-2.77218700 & 0.85305000 & -2.55299300
\end{array} \\
& \begin{array}{lll}
-2.31360800 & 1.54729500 & -3.24113500
\end{array} \\
& \begin{array}{lll}
-3.85333800 & 0.08329400 & -3.00107300
\end{array} \\
& \begin{array}{llll}
-4.24510600 & 0.26744100 & -4.27341900
\end{array} \\
& \begin{array}{llll}
-5.35481100 & -0.50413700 & -4.74892600
\end{array} \\
& \begin{array}{llll}
-5.49204900 & -0.20052300 & -5.78674300
\end{array} \\
& \begin{array}{lll}
-6.25754000 & -0.29143100 & -4.16963600
\end{array} \\
& \begin{array}{lll}
-5.14117900 & -1.57503000 & -4.69143600
\end{array} \\
& \begin{array}{lll}
-4.48921500 & -0.79845800 & -2.23631100
\end{array} \\
& \begin{array}{lll}
-4.07030100 & -0.94357200 & -0.98309900
\end{array} \\
& \begin{array}{llll}
-4.70256300 & -1.84411100 & -0.20393800
\end{array} \\
& \begin{array}{lll}
-5.81862300 & -2.54286700 & -0.76770900
\end{array} \\
& \begin{array}{llll}
-6.18959500 & -3.18346500 & 0.03314600
\end{array} \\
& \begin{array}{llll}
-5.51072300 & -3.14563600 & -1.62619900
\end{array} \\
& \begin{array}{lll}
-6.59742300 & -1.84556100 & -1.08680900
\end{array} \\
& \begin{array}{lll}
-2.44842800 & -1.18570600 \quad 5.09158000
\end{array} \\
& 2.34681200 \quad 2.52374000 \quad-1.16440700 \\
& \begin{array}{llll}
0 & -4.81644700 & 0.52176200 & 1.52431200
\end{array}
\end{aligned}
$$




\begin{tabular}{|c|c|c|c|}
\hline C & 2.70877300 & 2.04821900 & 0.95650500 \\
\hline $\mathrm{H}$ & 3.42622500 & 1.40651000 & 1.47132900 \\
\hline $\mathrm{H}$ & 1.74972800 & 1.89632400 & 1.48956500 \\
\hline C & 3.70063800 & 0.76672900 & -2.61134900 \\
\hline C & 5.02311800 & 0.71708800 & -0.44910100 \\
\hline $\mathrm{H}$ & 5.56038900 & -0.12379100 & -0.89720800 \\
\hline C & 3.07878300 & 3.53209400 & 1.17214500 \\
\hline $\mathrm{N}$ & 3.38126900 & 3.92756200 & 2.44556700 \\
\hline C & 3.81579700 & 5.30441900 & 2.67429900 \\
\hline $\mathrm{H}$ & 3.75776500 & 5.84885800 & 1.73410100 \\
\hline $\mathrm{H}$ & 3.17238300 & 5.78534500 & 3.41980600 \\
\hline $\mathrm{H}$ & 4.84772800 & 5.32350500 & 3.04490900 \\
\hline C & 3.42949000 & 3.05370000 & 3.61009300 \\
\hline $\mathrm{H}$ & 4.45924300 & 2.76175000 & 3.85744800 \\
\hline $\mathrm{H}$ & 3.01491300 & 3.58407100 & 4.47332000 \\
\hline $\mathrm{H}$ & 2.83449400 & 2.15376200 & 3.46241800 \\
\hline 0 & 3.07083300 & 4.31523000 & 0.22351400 \\
\hline $\mathrm{H}$ & 2.71927400 & 0.62561700 & -3.06110800 \\
\hline $\mathrm{P}$ & -0.20953500 & -1.66343800 & 0.47452200 \\
\hline N & -3.64578900 & -0.32657600 & 3.30919700 \\
\hline C & 3.51848100 & -1.88213400 & -0.74994400 \\
\hline $\mathrm{Si}$ & 4.61313700 & -3.37097300 & -0.97601300 \\
\hline C & 5.67069500 & -3.59722600 & 0.56783200 \\
\hline $\mathrm{H}$ & 6.30583800 & -4.48470000 & 0.46876800 \\
\hline $\mathrm{H}$ & 6.32735200 & -2.73801400 & 0.73852200 \\
\hline $\mathrm{H}$ & 5.04531000 & -3.72774600 & 1.45637100 \\
\hline C & 5.69108400 & -3.06808800 & -2.49730200 \\
\hline $\mathrm{H}$ & 6.35034400 & -2.20305300 & -2.37186900 \\
\hline $\mathrm{H}$ & 6.32898200 & -3.94080200 & -2.67786900 \\
\hline $\mathrm{H}$ & 5.08610600 & -2.90822600 & -3.39544700 \\
\hline C & 3.47494100 & -4.85082600 & -1.24466100 \\
\hline $\mathrm{H}$ & 4.07127400 & -5.75589700 & -1.40680800 \\
\hline $\mathrm{H}$ & 2.83415100 & -5.01976500 & -0.37449400 \\
\hline $\mathrm{H}$ & 2.83099400 & -4.71129600 & -2.11826700 \\
\hline $\mathrm{H}$ & 0.64514600 & -0.34759000 & -1.76172700 \\
\hline $\mathrm{H}$ & 4.36830900 & -0.01852100 & -2.96984300 \\
\hline $\mathrm{H}$ & 4.94885200 & 0.49439500 & 0.61715800 \\
\hline $\mathrm{H}$ & 4.08934800 & 1.73353400 & -2.95296500 \\
\hline C & 5.82454200 & 2.01735100 & -0.66146200 \\
\hline $\mathrm{H}$ & 6.76267600 & 1.96208700 & -0.10100900 \\
\hline $\mathrm{H}$ & 6.07982700 & 2.16157000 & -1.71490200 \\
\hline $\mathrm{H}$ & 5.27891100 & 2.90422600 & -0.33030200 \\
\hline
\end{tabular}

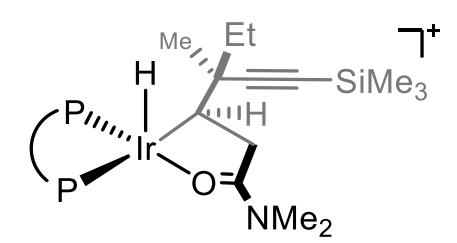

$$
\begin{aligned}
& G_{\text {sol }}=-3633.061205 \text { Hatree } \\
& \text { Ir }-0.67224800 \quad 0.48992400 \quad-0.11992100 \\
& \begin{array}{llll}
\text { C } & -5.00414500 & -0.29484300 & 0.83496800
\end{array} \\
& \begin{array}{llll}
\text { C } & -3.60631300 & 0.04847400 & 1.16934000
\end{array} \\
& \begin{array}{llll}
\text { C } & -2.75040200 & -0.07166400 & -0.15238300
\end{array} \\
& \begin{array}{llll}
\text { C } & 2.80881700 & 0.92302500 & 0.85776300
\end{array} \\
& \begin{array}{llll}
\text { C } & 3.27497500 & 1.58649500 & 1.99329100
\end{array} \\
& \text { H } \quad 2.93039600 \quad 2.57052600 \quad 2.27900100 \\
& \begin{array}{llll}
\text { C } & 4.24126800 & 0.94523600 & 2.78073200
\end{array} \\
& \begin{array}{llll}
\text { C } & 4.26884100 & -0.88644500 & 1.42349900
\end{array} \\
& \begin{array}{llll}
\text { C } & 3.30136700 & -0.35783100 & 0.53558100
\end{array} \\
& \begin{array}{llll}
\text { C } & 5.68621700 & 0.97673400 & 4.66919400
\end{array} \\
& \mathrm{H} \quad 5.89626300 \quad 1.68273800 \quad 5.47271400 \\
& \begin{array}{llll}
\mathrm{H} & 6.59252000 & 0.78217500 & 4.08917200
\end{array} \\
& \begin{array}{llll}
\mathrm{H} & 5.32006600 & 0.03172200 & 5.07993200
\end{array} \\
& \begin{array}{llll}
\text { C } & 5.73205300 & -2.66306300 & 2.02155600
\end{array} \\
& \mathrm{H} \quad 5.97977600 \quad-3.63844200 \quad 1.60147100 \\
& \text { H } \quad 5.33645100 \quad-2.77395800 \quad 3.03464900 \\
& \begin{array}{llll}
\mathrm{H} & 6.62119800 & -2.02826400 & 2.05621900
\end{array} \\
& \begin{array}{llll}
\text { C } & 2.20093700 & 1.96080000 & -1.81194900
\end{array} \\
& \begin{array}{llll}
\text { C } & 1.34416200 & 2.07283200 & -2.91871300
\end{array} \\
& \begin{array}{llll}
\mathrm{H} & 0.27156500 & 1.96823800 & -2.78304700
\end{array} \\
& \begin{array}{llll}
\text { C } & 1.85824400 & 2.34263700 & -4.18728600
\end{array} \\
& \text { H } \quad 1.18641500 \quad 2.42650000 \quad-5.03644900 \\
& \begin{array}{llll}
\text { C } & 3.23368000 & 2.50575700 & -4.36329500
\end{array} \\
& \mathrm{H} \quad 3.63471300 \quad 2.71693800 \quad-5.35007800 \\
& \begin{array}{llll}
\text { C } \quad 4.09235600 & 2.39797600 & -3.26747400
\end{array} \\
& \text { H } \quad 5.16242900 \quad 2.52786800 \quad-3.39956800 \\
& \begin{array}{llll}
\text { C } & 3.58218100 & 2.12584800 & -1.99681000
\end{array} \\
& \begin{array}{llll}
\mathrm{H} & 4.25976100 & 2.02988800 & -1.15533800
\end{array} \\
& \begin{array}{llll}
\text { C } & 1.23915100 & 3.36624000 & 0.53930500
\end{array} \\
& \begin{array}{llll}
\text { C } & 0.39993000 & 3.54449900 & 1.65237100
\end{array} \\
& \begin{array}{llll}
\mathrm{H} & -0.11710500 & 2.69254100 & 2.08191400
\end{array} \\
& \begin{array}{llll}
\text { C } & 0.22345400 \quad 4.80873300 & 2.21410100
\end{array} \\
& \text { H } \quad-0.42234000 \quad 4.92846700 \quad 3.07894700 \\
& \begin{array}{llll}
\text { C } & 0.87198200 & 5.91634900 & 1.66320700
\end{array} \\
& \begin{array}{llll}
\mathrm{H} & 0.73256700 & 6.90143100 & 2.09834900
\end{array} \\
& \begin{array}{llll}
\text { C } & 1.69982000 & 5.75177300 & 0.55207400
\end{array} \\
& \text { H } \quad 2.20707200 \quad 6.60842100 \quad 0.11834000 \\
& \begin{array}{llll}
\text { C } & 1.88528000 \quad 4.48557400 & -0.00679700
\end{array}
\end{aligned}
$$




\begin{tabular}{|c|c|c|c|}
\hline $\mathrm{H}$ & 2.53415300 & 4.37442500 & -0.86850500 \\
\hline $\mathrm{C}$ & 2.95176100 & -1.13211700 & -0.68829500 \\
\hline $\mathrm{C}$ & 1.72425500 & -1.78547100 & -0.94121600 \\
\hline P & 0.26947200 & -1.58103000 & 0.20890700 \\
\hline $\mathrm{C}$ & 0.94931700 & -2.06493300 & 1.84843500 \\
\hline C & 0.67780400 & -1.32864700 & 3.00905500 \\
\hline $\mathrm{H}$ & 0.09830700 & -0.41489700 & 2.94811000 \\
\hline $\mathrm{C}$ & 1.15985800 & -1.75357100 & 4.24818400 \\
\hline $\mathrm{H}$ & 0.94218800 & -1.17097300 & 5.13805200 \\
\hline C & 1.91709300 & -2.92147400 & 4.34101100 \\
\hline $\mathrm{H}$ & 2.28564600 & -3.25615900 & 5.30604000 \\
\hline C & 2.20240600 & -3.65812900 & 3.18856600 \\
\hline $\mathrm{H}$ & 2.79519200 & -4.56557300 & 3.25332300 \\
\hline C & 1.72854800 & -3.23151400 & 1.94882300 \\
\hline $\mathrm{H}$ & 1.96709900 & -3.80623300 & 1.05957700 \\
\hline C & -0.87322600 & -2.96476700 & -0.24130200 \\
\hline C & -1.54966600 & -2.91550900 & -1.47495400 \\
\hline $\mathrm{H}$ & -1.38384000 & -2.08209100 & -2.15203400 \\
\hline C & -2.43604100 & -3.92451700 & -1.84653200 \\
\hline $\mathrm{H}$ & -2.93779400 & -3.87347200 & -2.80808600 \\
\hline C & -2.68354300 & -4.99193400 & -0.97997400 \\
\hline $\mathrm{H}$ & -3.37845900 & -5.77574400 & -1.26514300 \\
\hline C & -2.03953400 & -5.03964400 & 0.25498600 \\
\hline $\mathrm{H}$ & -2.23313300 & -5.85963500 & 0.93981300 \\
\hline C & -1.13939800 & -4.03588600 & 0.62355200 \\
\hline $\mathrm{H}$ & -0.65276800 & -4.09602500 & 1.58934800 \\
\hline C & 1.57994900 & -2.55373000 & -2.09874900 \\
\hline $\mathrm{H}$ & 0.68225100 & -3.10526000 & -2.33224700 \\
\hline C & 2.66456600 & -2.64610300 & -2.98077200 \\
\hline 0 & 2.49052600 & -3.40025100 & -4.08065500 \\
\hline C & 3.59405600 & -3.52248700 & -4.98666300 \\
\hline $\mathrm{H}$ & 3.23759000 & -4.16970900 & -5.78801800 \\
\hline $\mathrm{H}$ & 4.45928600 & -3.97264500 & -4.49241400 \\
\hline $\mathrm{H}$ & 3.88304900 & -2.54664800 & -5.38666400 \\
\hline $\mathrm{N}$ & 3.82731500 & -2.03555400 & -2.77718700 \\
\hline C & 3.95916600 & -1.31143700 & -1.66935300 \\
\hline 0 & 5.13390100 & -0.68913200 & -1.45173400 \\
\hline C & 6.18324900 & -0.89490300 & -2.40480800 \\
\hline $\mathrm{H}$ & 7.03438600 & -0.33278300 & -2.01918300 \\
\hline $\mathrm{H}$ & 5.89551100 & -0.52185100 & -3.39134900 \\
\hline $\mathrm{H}$ & 6.43569200 & -1.95510600 & -2.48740400 \\
\hline $\mathrm{N}$ & 4.72617200 & -0.26283100 & 2.50614100 \\
\hline $\mathrm{H}$ & -2.86102000 & -1.11075000 & -0.46329000 \\
\hline 0 & 4.74196000 & -2.11576900 & 1.14369400 \\
\hline C & -3.35676300 & 0.78906100 & -1.30065900 \\
\hline
\end{tabular}

\begin{tabular}{|c|c|c|}
\hline-4.44820900 & 0.81582100 & -1.23765300 \\
\hline-3.11087700 & 0.32653400 & -2.26797100 \\
\hline-3.57586600 & 1.48539800 & 1.77355000 \\
\hline-3.11572400 & -0.99002800 & 2.20577700 \\
\hline-2.12003000 & -0.72868200 & 2.57166700 \\
\hline-3.79290000 & -1.04471300 & 3.06109800 \\
\hline-2.74398800 & 2.16402900 & -1.32095800 \\
\hline-3.36676800 & 3.24337200 & -1.80154700 \\
\hline-2.72650500 & 4.56269000 & -1.74939300 \\
\hline-1.83979200 & 4.51542900 & -1.12238900 \\
\hline-2.44563900 & 4.88425400 & -2.75791600 \\
\hline-3.43568700 & 5.28541000 & -1.33548500 \\
\hline-4.69610400 & 3.22249500 & -2.41450300 \\
\hline-5.42558200 & 3.70970200 & -1.75884800 \\
\hline-4.65875500 & 3.77050200 & -3.36077100 \\
\hline-5.02213600 & 2.20567900 & -2.61710200 \\
\hline-1.55011800 & 2.28078000 & -0.88108200 \\
\hline-3.93284900 & 2.19328300 & 1.01498300 \\
\hline-2.52825100 & 1.74319100 & 1.96654900 \\
\hline 1.45848600 & 1.67006700 & -0.15451800 \\
\hline 4.68509300 & 1.60911100 & 3.86276300 \\
\hline-6.15770800 & -0.57654300 & 0.55063600 \\
\hline-7.90577800 & -1.03736100 & 0.18159500 \\
\hline-8.94644300 & -0.75669300 & 1.73086400 \\
\hline-9.99444900 & -1.01862100 & 1.54639700 \\
\hline-8.59137000 & -1.36951200 & 2.56561700 \\
\hline-8.91557000 & 0.29065800 & 2.04852000 \\
\hline-7.95834900 & -2.85348300 & -0.33240700 \\
\hline-7.57773500 & -3.50199400 & 0.46322200 \\
\hline-8.98607800 & -3.16315900 & -0.55304000 \\
\hline-7.35557900 & -3.03357000 & -1.22859700 \\
\hline-8.53345900 & 0.05491300 & -1.22925600 \\
\hline-9.57401800 & -0.18860300 & -1.47159400 \\
\hline-8.49677600 & 1.11580100 & -0.95987800 \\
\hline-7.94135600 & -0.08468600 & -2.14018300 \\
\hline-0.72690700 & 0.58018500 & 1.40674000 \\
\hline-3.06594800 & -1.98554500 & 1.75754700 \\
\hline-4.40289100 & 1.69159200 & 3.04720000 \\
\hline-5.44724900 & 1.40594500 & 2.89108400 \\
\hline-4.01597800 & 1.10641400 & 3.88660300 \\
\hline-4.38271500 & 2.74440700 & 3.34650600 \\
\hline
\end{tabular}

$\begin{array}{lll}-3.35676300 & 0.78906100 & -1.30065900\end{array}$ 


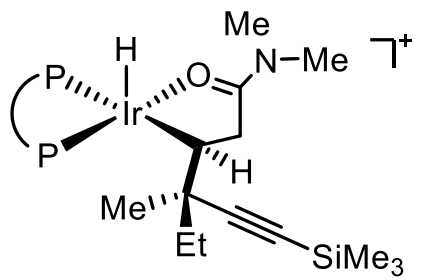

$$
\begin{aligned}
& G_{\text {sol }}=-3633.054969 \text { Hatree } \\
& \text { Ir } \quad-0.69157900 \quad 0.22583800 \quad-0.59077400 \\
& \text { C } \quad-2.76977600 \quad-0.28145400 \quad-0.43884000 \\
& \text { H } \quad-0.03132900 \quad-0.05302100 \quad-1.95397100 \\
& \begin{array}{llll}
\text { C } & -4.95333000 & -1.03684200 & 0.40362300
\end{array} \\
& \text { C } \quad 2.26988800 \quad-1.54697800 \quad-0.65119700 \\
& \text { C } \quad 2.63031300 \quad-2.30795700 \quad-1.76516200 \\
& \text { H } \quad 1.96060500 \quad-3.00425800 \quad-2.24560500 \\
& \begin{array}{llll}
\text { C } & 3.93149900 & -2.18509200 & -2.27003900
\end{array} \\
& \begin{array}{llll}
\text { C } & 4.49626600 & -0.65068800 & -0.69070300
\end{array} \\
& \begin{array}{llll}
\text { C } & 3.22285300 & -0.68384700 & -0.06853800
\end{array} \\
& \text { C } \quad 5.57428800 \quad-2.84620800 \quad-3.85690000 \\
& \text { H } \quad 5.60457000 \quad-3.53695000 \quad-4.69958800 \\
& \mathrm{H} \quad 6.31229300 \quad-3.13501400 \quad-3.10349800 \\
& \text { H } \quad 5.79106500 \quad-1.82818200 \quad-4.19211600 \\
& \begin{array}{llll}
\text { C } & 6.70617700 & 0.22555300 & -0.76220100
\end{array} \\
& \text { H } \quad 7.27541900 \quad 0.94333000 \quad-0.17067700 \\
& \text { H } \quad 6.64499900 \quad 0.55319200 \quad-1.80339400 \\
& \mathrm{H} \quad 7.18279800 \\
& \text { C } \quad 0.75138000 \quad-2.02467200 \\
& \begin{array}{llll}
\text { C } & -0.00263900 & -1.34192100 & 2.74839600
\end{array} \\
& \mathrm{H} \quad-0.71116700 \quad-0.58117900 \\
& \text { C } \quad 0.15087400 \quad-1.63008400 \\
& \mathrm{H} \quad-0.43997100 \quad-1.09315500 \\
& \text { C } \quad 1.06021200 \quad-2.60758400 \\
& \mathrm{H} \quad 1.17484400 \quad-2.83863400 \\
& \text { C } \quad 1.82736500 \quad-3.28373500 \\
& \text { H } \quad 2.54293200 \quad-4.03843000 \\
& \text { C } \quad 1.68346000 \quad-2.98928700 \\
& \mathrm{H} \quad 2.30553800 \quad-3.50228700 \\
& \text { C } \quad-0.08949600 \quad-3.21358800 \\
& \text { C } \quad-0.44278500 \quad-3.17857300 \\
& \mathrm{H} \quad-0.41852900-2.23655600 \\
& \text { C } \quad-0.83195100 \quad-4.34047100 \quad-2.80939100 \\
& \text { H } \quad-1.08823200 \quad-4.29670200 \quad-3.86364800 \\
& \text { C } \quad-0.90678300 \quad-5.55165500 \quad-2.11750900 \\
& \text { H } \quad-1.21770000 \quad-6.45550500 \quad-2.63236600 \\
& \text { C } \quad-0.58768500 \quad-5.59164600 \quad-0.76071700 \\
& \text { H } \quad-0.65472600 \quad-6.52607300 \quad-0.21183600
\end{aligned}
$$

\begin{tabular}{|c|c|c|c|}
\hline C & -0.17352900 & -4.43347800 & -0.09773800 \\
\hline $\mathrm{H}$ & 0.07338200 & -4.48953300 & 0.95575700 \\
\hline C & 3.02707200 & 0.14178400 & 1.15178900 \\
\hline C & 2.23159000 & 1.30170900 & 1.21071500 \\
\hline $\mathrm{P}$ & 1.17193600 & 1.74277400 & -0.24015800 \\
\hline C & 2.34987600 & 2.15645800 & -1.58770700 \\
\hline C & 1.94938100 & 1.99241900 & -2.92236600 \\
\hline $\mathrm{H}$ & 0.97076800 & 1.57919400 & -3.14561700 \\
\hline C & 2.79911300 & 2.36499500 & -3.96476500 \\
\hline $\mathrm{H}$ & 2.48003200 & 2.23219400 & -4.99417600 \\
\hline C & 4.05574100 & 2.90410100 & -3.68376600 \\
\hline $\mathrm{H}$ & 4.71704100 & 3.19367100 & -4.49498400 \\
\hline $\mathrm{C}$ & 4.46113000 & 3.07120800 & -2.35747900 \\
\hline $\mathrm{H}$ & 5.43676500 & 3.49293300 & -2.13479400 \\
\hline C & 3.61469600 & 2.70059900 & -1.31206500 \\
\hline $\mathrm{H}$ & 3.94206800 & 2.82602800 & -0.28545100 \\
\hline C & 0.42628500 & 3.36625100 & 0.22765000 \\
\hline C & -0.58239600 & 3.39437600 & 1.20725700 \\
\hline $\mathrm{H}$ & -0.89199500 & 2.47601100 & 1.69934600 \\
\hline C & -1.18681800 & 4.59545400 & 1.57376700 \\
\hline $\mathrm{H}$ & -1.95467700 & 4.60015800 & 2.34168900 \\
\hline C & -0.80412500 & 5.78858700 & 0.95465300 \\
\hline $\mathrm{H}$ & -1.27441800 & 6.72513200 & 1.23891500 \\
\hline C & 0.18796500 & 5.77073800 & -0.02551200 \\
\hline $\mathrm{H}$ & 0.49366400 & 6.69396700 & -0.50883200 \\
\hline C & 0.80242700 & 4.56818100 & -0.38711200 \\
\hline $\mathrm{H}$ & 1.57538100 & 4.57367300 & -1.14718700 \\
\hline C & 2.19899500 & 2.06645700 & 2.37770500 \\
\hline $\mathrm{H}$ & 1.61846900 & 2.97270500 & 2.47173100 \\
\hline C & 2.97685500 & 1.64262700 & 3.46418900 \\
\hline 0 & 2.93868100 & 2.40357100 & 4.57267300 \\
\hline C & 3.74525500 & 2.00127300 & 5.68634600 \\
\hline $\mathrm{H}$ & 3.57016500 & 2.75423300 & 6.45487000 \\
\hline $\mathrm{H}$ & 4.80416800 & 1.97557100 & 5.41508400 \\
\hline $\mathrm{H}$ & 3.44939200 & 1.01288100 & 6.04877400 \\
\hline $\mathrm{N}$ & 3.73291500 & 0.54817400 & 3.44333000 \\
\hline C & 3.75456300 & -0.17148700 & 2.32444500 \\
\hline 0 & 4.50872900 & -1.28620400 & 2.29108300 \\
\hline C & 5.28666200 & -1.59733000 & 3.45254700 \\
\hline $\mathrm{H}$ & 5.83317000 & -2.50566600 & 3.19690200 \\
\hline $\mathrm{H}$ & 4.64341900 & -1.77088100 & 4.31930200 \\
\hline $\mathrm{H}$ & 5.98381500 & -0.78839500 & 3.68570400 \\
\hline 0 & 4.24299200 & -2.94351800 & -3.33618800 \\
\hline $\mathrm{H}$ & -2.84155400 & 0.40116000 & 0.44590100 \\
\hline 0 & 5.40687400 & 0.18900500 & -0.16068700 \\
\hline
\end{tabular}




\begin{tabular}{|c|c|c|c|}
\hline C & -3.49198300 & 0.45316200 & -1.59800100 \\
\hline $\mathrm{H}$ & -4.56472500 & 0.54306200 & -1.39816200 \\
\hline $\mathrm{H}$ & -3.38479900 & -0.12005000 & -2.52825100 \\
\hline C & -3.79319100 & -2.55272000 & -1.16301300 \\
\hline C & -3.00422400 & -2.20893300 & 1.24570100 \\
\hline $\mathrm{H}$ & -2.05557100 & -2.68731600 & 1.00275700 \\
\hline $\mathrm{H}$ & -2.83981000 & -1.47487200 & 2.03861800 \\
\hline C & -2.85892500 & 1.79340600 & -1.81644400 \\
\hline $\mathrm{N}$ & -3.49860600 & 2.80802900 & -2.40823300 \\
\hline C & -2.86502500 & 4.12438900 & -2.53011400 \\
\hline $\mathrm{H}$ & -1.97373000 & 4.16317400 & -1.90862300 \\
\hline $\mathrm{H}$ & -2.59315100 & 4.31797600 & -3.57353800 \\
\hline $\mathrm{H}$ & -3.57306900 & 4.89193800 & -2.20407500 \\
\hline C & -4.84319400 & 2.70000900 & -2.97564700 \\
\hline $\mathrm{H}$ & -5.58278400 & 3.17219000 & -2.31939100 \\
\hline $\mathrm{H}$ & -4.85883800 & 3.21253000 & -3.94164800 \\
\hline $\mathrm{H}$ & -5.12122200 & 1.66088000 & -3.13715300 \\
\hline 0 & -1.66676200 & 1.95457400 & -1.40921300 \\
\hline $\mathrm{H}$ & -4.30119900 & -2.04875200 & -1.99267100 \\
\hline $\mathrm{H}$ & -2.79767200 & -2.82351200 & -1.51875600 \\
\hline $\mathrm{P}$ & 0.51851100 & -1.65312200 & -0.00010400 \\
\hline $\mathrm{N}$ & 4.84646000 & -1.37241500 & -1.75071400 \\
\hline C & -6.05188400 & -0.62681300 & 0.74409800 \\
\hline Si & -7.72616600 & -0.06922400 & 1.28224800 \\
\hline C & -8.85537900 & -1.57663400 & 1.39924700 \\
\hline $\mathrm{H}$ & -9.86187500 & -1.28430300 & 1.71940500 \\
\hline $\mathrm{H}$ & -8.94629500 & -2.08176800 & 0.43216000 \\
\hline $\mathrm{H}$ & -8.47377300 & -2.30490400 & 2.12208700 \\
\hline C & -8.39663600 & 1.14817100 & -0.00082600 \\
\hline $\mathrm{H}$ & -8.47325000 & 0.68394700 & -0.98969300 \\
\hline $\mathrm{H}$ & -9.39753600 & 1.49355100 & 0.28137400 \\
\hline $\mathrm{H}$ & -7.75523300 & 2.03191600 & -0.08952000 \\
\hline $\mathrm{C}$ & -7.57565200 & 0.77997600 & 2.96218200 \\
\hline $\mathrm{H}$ & -8.55749200 & 1.11685500 & 3.31339200 \\
\hline $\mathrm{H}$ & -7.16732800 & 0.09969600 & 3.71650400 \\
\hline $\mathrm{H}$ & -6.92089600 & 1.65628300 & 2.91179000 \\
\hline C & -3.61678200 & -1.53304200 & 0.00187900 \\
\hline $\mathrm{H}$ & -3.67739300 & -2.97095800 & 1.64407400 \\
\hline C & -4.58331300 & -3.81957100 & -0.81728800 \\
\hline $\mathrm{H}$ & -5.56807700 & -3.57464900 & -0.40760000 \\
\hline $\mathrm{H}$ & -4.73533000 & -4.42413100 & -1.71691500 \\
\hline $\mathrm{H}$ & -4.05778500 & -4.44562500 & -0.09059700 \\
\hline
\end{tabular}
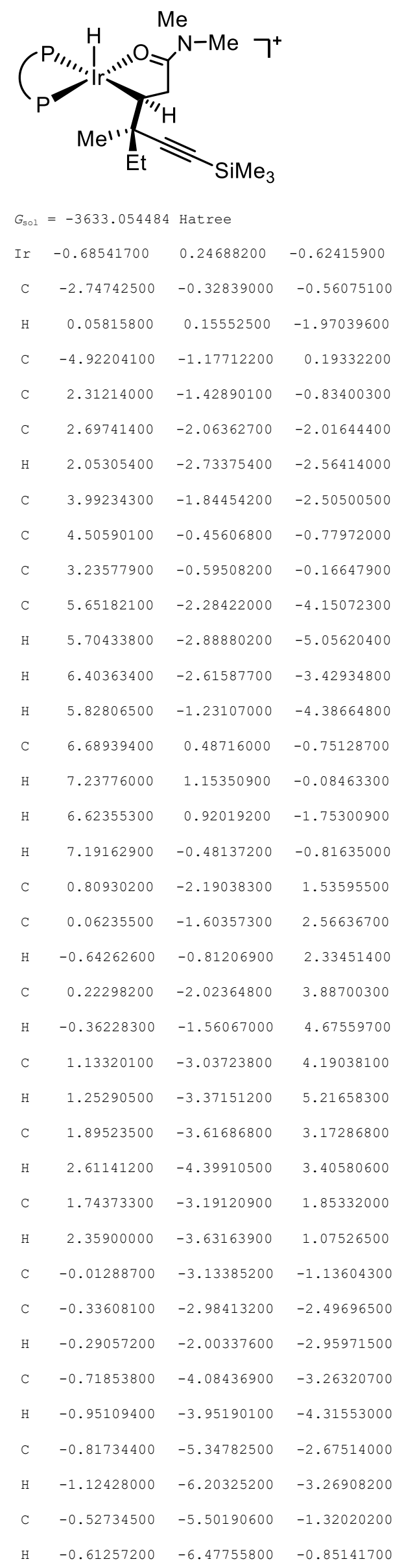


\begin{tabular}{|c|c|c|c|}
\hline C & -0.11918400 & -4.40572300 & -0.55588300 \\
\hline $\mathrm{H}$ & 0.10510300 & -4.55017100 & 0.49428700 \\
\hline C & 3.01812000 & 0.08144700 & 1.13960400 \\
\hline $\mathrm{C}$ & 2.18347300 & 1.19782800 & 1.33645000 \\
\hline P & 1.09995500 & 1.77599600 & -0.04520200 \\
\hline C & 2.23929500 & 2.43338000 & -1.32618700 \\
\hline $\mathrm{C}$ & 1.79639300 & 2.49278800 & -2.65689700 \\
\hline $\mathrm{H}$ & 0.81228900 & 2.11386500 & -2.91531600 \\
\hline C & 2.60998700 & 3.04548500 & -3.64627400 \\
\hline $\mathrm{H}$ & 2.25935700 & 3.08506900 & -4.67327000 \\
\hline C & 3.87218900 & 3.54282200 & -3.31639500 \\
\hline $\mathrm{H}$ & 4.50597600 & 3.97189600 & -4.08675500 \\
\hline C & 4.31889100 & 3.48773700 & -1.99427500 \\
\hline $\mathrm{H}$ & 5.29909500 & 3.87587400 & -1.73380900 \\
\hline C & 3.50850300 & 2.93685400 & -1.00042300 \\
\hline $\mathrm{H}$ & 3.86820700 & 2.88949400 & 0.02171300 \\
\hline C & 0.26392000 & 3.27249200 & 0.64013000 \\
\hline C & -0.77270300 & 3.10090900 & 1.57422200 \\
\hline $\mathrm{H}$ & -1.05043000 & 2.10244600 & 1.90171500 \\
\hline C & -1.44366100 & 4.20154100 & 2.10409800 \\
\hline $\mathrm{H}$ & -2.23461000 & 4.05163400 & 2.83296200 \\
\hline C & -1.09699000 & 5.49306900 & 1.69802700 \\
\hline H & -1.61889800 & 6.35177000 & 2.10951300 \\
\hline C & -0.07408800 & 5.67419200 & 0.76715700 \\
\hline $\mathrm{H}$ & 0.20425100 & 6.67514500 & 0.45092900 \\
\hline C & 0.60550700 & 4.57177900 & 0.24117200 \\
\hline $\mathrm{H}$ & 1.40211700 & 4.72986900 & -0.47698400 \\
\hline C & 2.12241500 & 1.81188200 & 2.58851900 \\
\hline $\mathrm{H}$ & 1.50677000 & 2.67656100 & 2.78979800 \\
\hline C & 2.91337400 & 1.28486100 & 3.61886300 \\
\hline 0 & 2.84684300 & 1.90037100 & 4.81295900 \\
\hline C & 3.66372800 & 1.38893600 & 5.87310700 \\
\hline $\mathrm{H}$ & 3.45354400 & 2.02712100 & 6.73144000 \\
\hline $\mathrm{H}$ & 4.72426300 & 1.44240600 & 5.61206300 \\
\hline $\mathrm{H}$ & 3.40777500 & 0.35029100 & 6.09970100 \\
\hline $\mathrm{N}$ & 3.70691900 & 0.22791200 & 3.46748000 \\
\hline C & 3.75368000 & -0.34915300 & 2.26959500 \\
\hline 0 & 4.54375400 & -1.42652300 & 2.10473900 \\
\hline C & 5.31892600 & -1.86122900 & 3.22762900 \\
\hline $\mathrm{H}$ & 5.90526200 & -2.70481500 & 2.86196900 \\
\hline $\mathrm{H}$ & 4.67125900 & -2.17636600 & 4.05018600 \\
\hline $\mathrm{H}$ & 5.97891900 & -1.06511700 & 3.58131200 \\
\hline 0 & 4.32755100 & -2.48209100 & -3.64066500 \\
\hline $\mathrm{H}$ & -2.84208200 & 0.28518900 & 0.37208100 \\
\hline 0 & 5.38956300 & 0.35405900 & -0.16454400 \\
\hline
\end{tabular}

\begin{tabular}{|c|c|c|c|}
\hline C & -3.47114200 & 0.47715700 & -1.66999600 \\
\hline $\mathrm{H}$ & -4.54799000 & 0.52992400 & -1.47961700 \\
\hline $\mathrm{H}$ & -3.34104500 & -0.02049900 & -2.64025800 \\
\hline $\mathrm{C}$ & -3.72090400 & -2.57322800 & -1.43141400 \\
\hline C & -2.93700000 & -2.36698200 & 0.98974200 \\
\hline $\mathrm{H}$ & -1.93378100 & -2.68083800 & 0.69657900 \\
\hline $\mathrm{H}$ & -2.82252100 & -1.64070100 & 1.80323600 \\
\hline C & -2.86989700 & 1.84649900 & -1.77540400 \\
\hline $\mathrm{N}$ & -3.52566600 & 2.88070400 & -2.31407000 \\
\hline C & -2.93397300 & 4.22172500 & -2.32519100 \\
\hline $\mathrm{H}$ & -2.02957300 & 4.22934800 & -1.72175100 \\
\hline $\mathrm{H}$ & -2.69326100 & 4.51814600 & -3.35178300 \\
\hline $\mathrm{H}$ & -3.65442700 & 4.93527400 & -1.91392300 \\
\hline C & -4.86014600 & 2.77432900 & -2.90578500 \\
\hline $\mathrm{H}$ & -5.62510800 & 3.14985800 & -2.21686200 \\
\hline $\mathrm{H}$ & -4.88757000 & 3.37680800 & -3.81786900 \\
\hline $\mathrm{H}$ & -5.09313600 & 1.74520500 & -3.17089500 \\
\hline 0 & -1.69461400 & 2.01264400 & -1.32404300 \\
\hline $\mathrm{H}$ & -4.18273800 & -2.06654400 & -2.28294400 \\
\hline $\mathrm{H}$ & -2.74821000 & -2.95550400 & -1.73979500 \\
\hline P & 0.56456700 & -1.64738000 & -0.20247100 \\
\hline $\mathrm{N}$ & 4.87853600 & -1.05450500 & -1.90700700 \\
\hline C & -6.02654500 & -0.79131200 & 0.54292900 \\
\hline $\mathrm{Si}$ & -7.70409700 & -0.24190400 & 1.07923200 \\
\hline C & -8.95343500 & -1.59280600 & 0.66220000 \\
\hline $\mathrm{H}$ & -9.96257300 & -1.29521600 & 0.96878500 \\
\hline $\mathrm{H}$ & -8.97760400 & -1.79704500 & -0.41312700 \\
\hline $\mathrm{H}$ & -8.71191200 & -2.52962200 & 1.17450900 \\
\hline C & -8.13179700 & 1.35023300 & 0.15005500 \\
\hline $\mathrm{H}$ & -8.14424100 & 1.18793400 & -0.93309700 \\
\hline $\mathrm{H}$ & -9.12362700 & 1.71412600 & 0.44087900 \\
\hline $\mathrm{H}$ & -7.41057000 & 2.14555600 & 0.36795100 \\
\hline C & -7.67137600 & 0.08447100 & 2.93862400 \\
\hline $\mathrm{H}$ & -8.65351200 & 0.41986800 & 3.29041500 \\
\hline $\mathrm{H}$ & -7.40921500 & -0.82037600 & 3.49636100 \\
\hline $\mathrm{H}$ & -6.94204700 & 0.85949500 & 3.19641400 \\
\hline C & -3.57221500 & -1.62516800 & -0.21873300 \\
\hline $\mathrm{H}$ & -4.36471100 & -3.41864300 & -1.17917800 \\
\hline C & -3.70489400 & -3.58324000 & 1.51761600 \\
\hline $\mathrm{H}$ & -4.73202600 & -3.32134600 & 1.78693200 \\
\hline $\mathrm{H}$ & -3.74202500 & -4.39455200 & 0.78443100 \\
\hline $\mathrm{H}$ & -3.21186400 & -3.97575800 & 2.41270700 \\
\hline
\end{tabular}




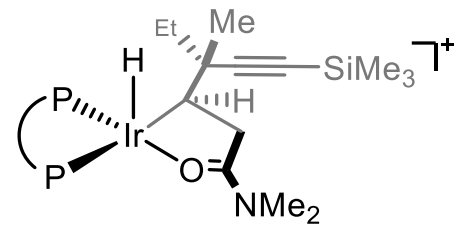

$G_{\text {sol }}=-3633.060887$ Hatree

\section{C}

3.60985800

C $\quad 2.75216100$

C -2.79107000

C $\quad-3.22076400$

H $\quad-2.88160000$

C -4.14265900

C -4.19402500

C -3.27386200

C $\quad-5.51061500$

H $\quad-5.70089200$

H $\quad-6.43562200$

H -5.11189000

C $\quad-5.60328300$

H $\quad-5.84699300$

H -5.16976800

H $\quad-6.50286200$

C -2.24229500

C $\quad-1.38794800$

H $\quad-0.31303700$

C -1.90683300

H -1.23662400

C -3.28589500

H $\quad-3.69137800$

C $\quad-4.14270200$

2.86460900

$-5.21570800$

$-3.62708700$

2.56797700

2.16092600

H -4.30286800

2.01647500

C $\quad-1.31659200$

3.38471500

C -0.39573800

3.57983700

H $\quad 0.21799400$

C $\quad-0.25920400$

2.75146400

H $\quad 0.45228200$

4.83080800

4.96441200

C $\quad-1.03297300$

5.90716400

H $\quad-0.92541700$

6.88186500

$-1.94418800$

5.72583200

H $\quad-2.54739000$

6.55907500

C $\quad-2.08755400$
0.09750100

$-0.80908300$

$-1.17010300$

0.15555600

$-0.92896000$

$-2.11717600$

$-2.44038300$

$-2.90685900$

$-1.45490300$

$-0.55807000$

$-4.84955500$

$-5.69713200$

$-4.29546900$

$-5.19473300$

$-2.01337800$

$-1.55456700$

$-3.00537000$

$-2.11014600$

1.72173100

2. 82246400

2.68790100

4. 08222200

4.92685400

4.25469900

5.23445600

3.16494100

3.29507600

1.90308200

1.06699400

$-0.66616200$

$-1.70865800$

$-2.04618900$

$-2.31096800$

$-3.12053600$

$-1.87135700$

$-2.33785200$

$-0.82985200$

$-0.48192700$

$-0.23026300$

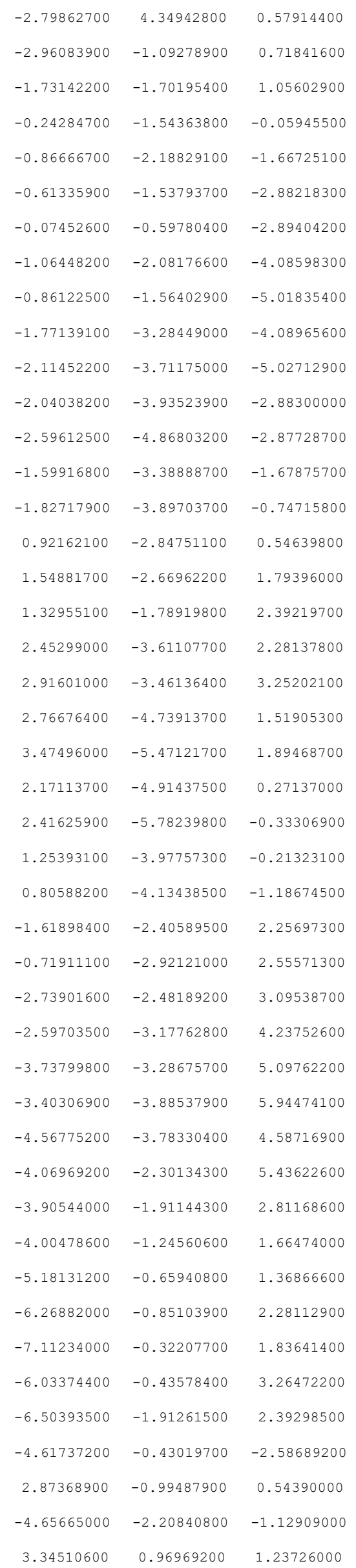$$
\text { P }
$$

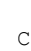




\begin{tabular}{|c|c|c|c|}
\hline $\mathrm{H}$ & 4.43778800 & 0.98080400 & 1.19606400 \\
\hline H & 3.07214700 & 0.59843800 & 2.23612600 \\
\hline C & 3.57136300 & 1.43273600 & -1.87626300 \\
\hline C & 3.11786000 & -1.06293100 & -2.13681000 \\
\hline $\mathrm{H}$ & 2.08548200 & -0.82815800 & -2.41154900 \\
\hline C & 2.74493800 & 2.34472000 & 1.11127600 \\
\hline N & 3.37513200 & 3.46418800 & 1.47616600 \\
\hline C & 2.74930700 & 4.77525600 & 1.26759800 \\
\hline H & 1.90619600 & 4.67582300 & 0.58825200 \\
\hline $\mathrm{H}$ & 2.40284700 & 5.18582900 & 2.22208100 \\
\hline $\mathrm{H}$ & 3.49140300 & 5.45582100 & 0.84092400 \\
\hline C & 4.69202000 & 3.49648900 & 2.11529400 \\
\hline $\mathrm{H}$ & 5.44395700 & 3.88841700 & 1.42227800 \\
\hline $\mathrm{H}$ & 4.64598000 & 4.15468700 & 2.98805700 \\
\hline $\mathrm{H}$ & 4.99432000 & 2.50629200 & 2.44678900 \\
\hline 0 & 1.55661200 & 2.42417600 & 0.64977500 \\
\hline $\mathrm{H}$ & 3.92860600 & 2.23287000 & -1.22127700 \\
\hline $\mathrm{H}$ & 2.55113800 & 1.67559700 & -2.18789700 \\
\hline P & -1.48334900 & 1.70396000 & 0.07878500 \\
\hline 0 & -4.55370400 & 1.36750300 & -4.03977700 \\
\hline C & 6.17004700 & -0.47145100 & -0.50034700 \\
\hline $\mathrm{Si}$ & 7.93051700 & -0.85725700 & -0.10389000 \\
\hline C & 8.76657600 & 0.71529900 & 0.53037500 \\
\hline $\mathrm{H}$ & 9.81925400 & 0.52351500 & 0.76668500 \\
\hline $\mathrm{H}$ & 8.73480100 & 1.51344700 & -0.21850900 \\
\hline H & 8.28653000 & 1.08758200 & 1.44181000 \\
\hline C & 8.78254700 & -1.46115000 & -1.67582000 \\
\hline $\mathrm{H}$ & 8.75386800 & -0.70025600 & -2.46242600 \\
\hline $\mathrm{H}$ & 9.83396100 & -1.70020100 & -1.48019300 \\
\hline $\mathrm{H}$ & 8.30221300 & -2.36422700 & -2.06612300 \\
\hline C & 7.97489600 & -2.19826800 & 1.22598600 \\
\hline $\mathrm{H}$ & 9.00914000 & -2.45558300 & 1.48097500 \\
\hline $\mathrm{H}$ & 7.47731600 & -1.87094300 & 2.14492000 \\
\hline $\mathrm{H}$ & 7.47884300 & -3.11223400 & 0.88359100 \\
\hline $\mathrm{H}$ & 0.66888400 & 0.55313100 & -1.43197100 \\
\hline $\mathrm{H}$ & 3.08455100 & -2.00239300 & -1.57394600 \\
\hline $\mathrm{H}$ & 4.21545700 & 1.43632000 & -2.75840200 \\
\hline C & 3.94520900 & -1.27369800 & -3.40938300 \\
\hline $\mathrm{H}$ & 3.53859200 & -2.11197900 & -3.98412400 \\
\hline $\mathrm{H}$ & 3.93293800 & -0.39620800 & -4.06371400 \\
\hline $\mathrm{H}$ & 4.98815700 & -1.50286600 & -3.17359100 \\
\hline
\end{tabular}

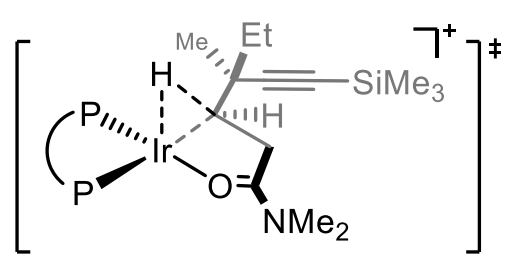




\begin{tabular}{|c|c|c|c|}
\hline $\mathrm{H}$ & 2.50949200 & 4.22313500 & -1.48765600 \\
\hline C & 3.04774100 & -1.18520900 & -0.53733700 \\
\hline C & 1.83509600 & -1.86750900 & -0.77440200 \\
\hline P & 0.33441400 & -1.51807200 & 0.27143900 \\
\hline C & 0.91001100 & -1.88108300 & 1.98065200 \\
\hline C & 0.57976800 & -1.01156400 & 3.02966000 \\
\hline $\mathrm{H}$ & 0.03427500 & -0.09963500 & 2.80926200 \\
\hline C & 0.97235800 & -1.30116200 & 4.33779400 \\
\hline $\mathrm{H}$ & 0.71274000 & -0.61930700 & 5.14206000 \\
\hline C & 1.69968600 & -2.46075000 & 4.60894600 \\
\hline $\mathrm{H}$ & 2.00019900 & -2.68929100 & 5.62718200 \\
\hline C & 2.04703000 & -3.32503400 & 3.56692900 \\
\hline $\mathrm{H}$ & 2.61959400 & -4.22462700 & 3.77250400 \\
\hline C & 1.66161500 & -3.03570900 & 2.25814600 \\
\hline $\mathrm{H}$ & 1.95006600 & -3.70627500 & 1.45454900 \\
\hline C & -0.82809700 & -2.89713400 & -0.14029600 \\
\hline C & -1.46582800 & -2.88261000 & -1.39547700 \\
\hline $\mathrm{H}$ & -1.25153000 & -2.08225300 & -2.09852200 \\
\hline C & -2.36846100 & -3.88417500 & -1.74970500 \\
\hline $\mathrm{H}$ & -2.84054300 & -3.86329200 & -2.72745600 \\
\hline C & -2.66708500 & -4.90831100 & -0.84719500 \\
\hline $\mathrm{H}$ & -3.37359500 & -5.68628700 & -1.11981200 \\
\hline C & -2.05636100 & -4.92251300 & 0.40576500 \\
\hline $\mathrm{H}$ & -2.28745400 & -5.71073300 & 1.11596500 \\
\hline C & -1.14097500 & -3.92639100 & 0.75844300 \\
\hline $\mathrm{H}$ & -0.67778000 & -3.95853200 & 1.73743100 \\
\hline C & 1.74896700 & -2.76999800 & -1.83573300 \\
\hline $\mathrm{H}$ & 0.85882100 & -3.34133600 & -2.05266200 \\
\hline C & 2.88100600 & -2.96478900 & -2.63844100 \\
\hline 0 & 2.76922700 & -3.85464300 & -3.64138500 \\
\hline C & 3.92148300 & -4.08235300 & -4.46154900 \\
\hline $\mathrm{H}$ & 3.61147900 & -4.82987600 & -5.19189800 \\
\hline $\mathrm{H}$ & 4.75860400 & -4.45840200 & -3.86693400 \\
\hline $\mathrm{H}$ & 4.23176700 & -3.16351600 & -4.96684400 \\
\hline $\mathrm{N}$ & 4.03033700 & -2.32245600 & -2.45382900 \\
\hline C & 4.10237500 & -1.46390100 & -1.44049600 \\
\hline 0 & 5.25624000 & -0.79529700 & -1.24954800 \\
\hline C & 6.35055000 & -1.09359100 & -2.12359800 \\
\hline $\mathrm{H}$ & 7.17497100 & -0.47169700 & -1.77318400 \\
\hline $\mathrm{H}$ & 6.10250000 & -0.84856500 & -3.15988000 \\
\hline $\mathrm{H}$ & 6.62247600 & -2.15070200 & -2.06585300 \\
\hline $\mathrm{N}$ & 4.62961200 & 0.05496800 & 2.63532300 \\
\hline $\mathrm{H}$ & -2.83478900 & -1.08125300 & -0.30937300 \\
\hline 0 & 4.75794800 & -1.92692800 & 1.47581800 \\
\hline C & -3.35667500 & 0.82269800 & -1.18135000 \\
\hline
\end{tabular}

\begin{tabular}{|c|c|c|}
\hline-4.44928900 & 0.84810500 & -1.15502800 \\
\hline-3.06934500 & 0.33803700 & -2.12317900 \\
\hline-3.79598300 & 1.49496200 & 1.85894700 \\
\hline-3.31724900 & -0.97268400 & 2.33026500 \\
\hline-2.34735100 & -0.69145500 & 2.75371200 \\
\hline-4.03897000 & -1.03327100 & 3.14707900 \\
\hline-2.74675800 & 2.20342800 & -1.21052800 \\
\hline-3.39270200 & 3.26867600 & -1.70264500 \\
\hline-2.75933300 & 4.59192500 & -1.68135800 \\
\hline-1.88023100 & 4.56837100 & -1.04210800 \\
\hline-2.46583200 & 4.88617500 & -2.69483000 \\
\hline-3.47702900 & 5.32283500 & -1.29748700 \\
\hline-4.72527100 & 3.22743800 & -2.30623400 \\
\hline-5.46731100 & 3.67610600 & -1.63679000 \\
\hline-4.70759000 & 3.80049800 & -3.23795600 \\
\hline-5.02572400 & 2.20888200 & -2.53934700 \\
\hline-1.56272100 & 2.33451600 & -0.77703000 \\
\hline-4.12031500 & 2.19185100 & 1.07668200 \\
\hline-2.76231800 & 1.76714900 & 2.10841700 \\
\hline 1.47435400 & 1.62882200 & -0.37493500 \\
\hline 4.47169600 & 2.05365500 & 3.78792000 \\
\hline-6.25887600 & -0.63399200 & 0.47387900 \\
\hline-7.96865000 & -1.14409400 & -0.01160400 \\
\hline-9.01570600 & -1.28512900 & 1.55108500 \\
\hline-10.03975800 & -1.58660700 & 1.30345700 \\
\hline-8.60586800 & -2.03061600 & 2.24000800 \\
\hline-9.06883600 & -0.33020200 & 2.08383400 \\
\hline-7.86927900 & -2.80661800 & -0.90051600 \\
\hline-7.43913800 & -3.57812600 & -0.25389400 \\
\hline-8.86828100 & -3.14185000 & -1.20115400 \\
\hline-7.25330100 & -2.74336000 & -1.80357600 \\
\hline-8.68297200 & 0.17156300 & -1.16521700 \\
\hline-9.70146000 & -0.09655700 & -1.46770300 \\
\hline-8.72998100 & 1.15029800 & -0.67652700 \\
\hline-8.08546400 & 0.27608800 & -2.07728700 \\
\hline-3.77560500 & 0.05335900 & 1.26502700 \\
\hline-3.22448400 & -1.96904500 & 1.89080400 \\
\hline-4.69372100 & 1.69267200 & 3.08445700 \\
\hline-5.72388600 & 1.39212000 & 2.87182300 \\
\hline-4.34438800 & 1.11689400 & 3.94623600 \\
\hline-4.70422400 & 2.74667700 & 3.37876700 \\
\hline
\end{tabular}




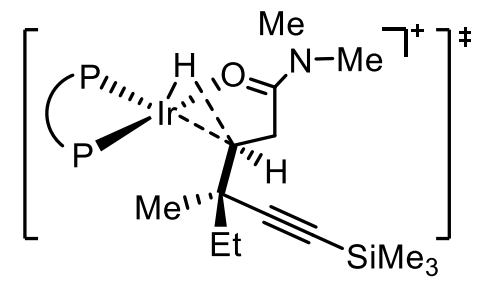

$$
\begin{aligned}
& G_{\text {sol }}=-3633.042196 \text { Hatree } \\
& \text { Ir } \quad-0.68250900 \quad 0.17358600 \quad-0.60649800 \\
& \text { C } \quad-2.88745300 \quad-0.22637100 \quad-0.42255000 \\
& \text { H } \quad-1.65025800 \quad-0.88802400 \quad-1.33112200 \\
& \text { C } \quad-5.03228500 \quad-1.02344800 \quad 0.39264500 \\
& \begin{array}{llll}
\text { C } & 2.31331800 & -1.53822400 & -0.61068400
\end{array} \\
& \text { C } \quad 2.67136600 \quad-2.32427500 \quad-1.70789800 \\
& \mathrm{H} \quad 2.00441100 \quad-3.04116500 \quad-2.16079800 \\
& \begin{array}{llll}
\text { C } & 3.96374700 & -2.19972600 & -2.23245000
\end{array} \\
& \begin{array}{llll}
\text { C } & 4.52583300 & -0.61065100 & -0.70834400
\end{array} \\
& \text { C } \quad 3.26437900 \quad-0.64965200 \quad-0.06338200 \\
& \text { C } \quad 5.59770200 \quad-2.88923400 \quad-3.81619100 \\
& \mathrm{H} \quad 5.62841000 \quad-3.60491000 \quad-4.63790800 \\
& \text { H } \quad 6.34749700 \quad-3.14577400 \quad-3.06262400 \\
& \mathrm{H} \quad 5.79854300 \quad-1.87935100 \quad-4.18443500 \\
& \begin{array}{llll}
\text { C } & 6.71776000 & 0.30219000 & -0.84145900
\end{array} \\
& \text { H } \quad 7.28304900 \quad 1.04871800 \quad-0.28251200 \\
& \text { H } \quad 6.63732400 \quad 0.59337900 \quad-1.89223600 \\
& \mathrm{H} \quad 7.21169800 \quad-0.67101500 \quad-0.78113900 \\
& \begin{array}{llll}
\text { C } & 0.80503800 & -1.94963400 & 1.84819400
\end{array} \\
& \begin{array}{llll}
\text { C } & 0.00099900 & -1.27729400 & 2.77874500
\end{array} \\
& \mathrm{H} \quad-0.72912100 \quad-0.55815200 \quad 2.42179300 \\
& \text { C } \quad 0.14348700 \quad-1.52205900 \quad 4.14542300 \\
& \mathrm{H} \quad-0.48396200 \quad-0.99376000 \quad 4.85704600 \\
& \text { C } \quad 1.09054200 \quad-2.44356000 \quad 4.59448000 \\
& \text { H } \quad 1.19832900 \quad-2.63913400 \quad 5.65725100 \\
& \begin{array}{llll}
\text { C } & 1.90417800 & -3.11065900 & 3.67494900
\end{array} \\
& \text { H } \quad 2.64697400 \quad-3.82336800 \quad 4.02078900 \\
& \begin{array}{llll}
\text { C } & 1.76943500 & -2.86190500 & 2.30922400
\end{array} \\
& \text { H } \quad 2.42293100 \quad-3.36897000 \quad 1.60653500 \\
& \text { C } \quad-0.02742200 \quad-3.24900800 \quad-0.64811400 \\
& \text { C } \quad-0.37699800 \quad-3.29569200 \quad-2.01016700 \\
& \text { H } \quad-0.32052300 \quad-2.39113400 \quad-2.60875100 \\
& \text { C } \quad-0.78556800 \quad-4.49063500 \quad-2.60180100 \\
& \mathrm{H} \quad-1.03671500 \quad-4.51001300 \quad-3.65815200 \\
& \text { C } \quad-0.87806600 \quad-5.65537200 \quad-1.83581000 \\
& \text { H } \quad-1.20248400 \quad-6.58500000 \quad-2.29341300 \\
& \text { C } \quad-0.55415800 \quad-5.61612300 \quad-0.48025200 \\
& \text { H } \quad-0.63068800 \quad-6.51446800 \quad 0.12492400
\end{aligned}
$$

\begin{tabular}{|c|c|c|c|}
\hline & -0.12447400 & -4.42428100 & 0.11009600 \\
\hline $\mathrm{H}$ & 0.12440200 & -4.41844000 & 1.16461200 \\
\hline & 3.07893300 & 0.20159600 & 1.14117600 \\
\hline & 2.25711700 & 1.34208100 & 1.19524100 \\
\hline $\mathrm{P}$ & 1.14000900 & 1.72128000 & -0.22520600 \\
\hline$C$ & 2.23316500 & 2.03228200 & -1.66119400 \\
\hline$c$ & 1.78718100 & 1.67544600 & -2.94280000 \\
\hline $\mathrm{H}$ & 0.82798500 & 1.17801500 & -3.05358900 \\
\hline C & 2.57661400 & 1.94952700 & -4.06028400 \\
\hline $\mathrm{H}$ & 2.22788200 & 1.66697600 & -5.04912200 \\
\hline $\mathrm{C}$ & 3.81343000 & 2.57854700 & -3.90651800 \\
\hline $\mathrm{H}$ & 4.42703800 & 2.79110700 & -4.77695900 \\
\hline C & 4.26468100 & 2.93058600 & -2.63182800 \\
\hline $\mathrm{H}$ & 5.22786100 & 3.41699100 & -2.50957600 \\
\hline $\mathrm{C}$ & 3.48122600 & 2.65801200 & -1.51005000 \\
\hline $\mathrm{H}$ & 3.84623800 & 2.91871900 & -0.52177300 \\
\hline C & 0.40159400 & 3.35669500 & 0.20451400 \\
\hline C & -0.62570200 & 3.40252900 & 1.16329800 \\
\hline $\mathrm{H}$ & -0.96832600 & 2.48533200 & 1.63433000 \\
\hline C & -1.20719300 & 4.61756100 & 1.52157500 \\
\hline $\mathrm{H}$ & -1.99118200 & 4.63890800 & 2.27280400 \\
\hline $\mathrm{C}$ & -0.78330100 & 5.80312800 & 0.91472400 \\
\hline $\mathrm{H}$ & -1.23694900 & 6.74977000 & 1.19263100 \\
\hline $\mathrm{C}$ & 0.22645700 & 5.76536400 & -0.04672300 \\
\hline $\mathrm{H}$ & 0.56230100 & 6.68254500 & -0.52145400 \\
\hline C & 0.81936000 & 4.54971500 & -0.40047700 \\
\hline $\mathrm{H}$ & 1.60644200 & 4.53849100 & -1.14607200 \\
\hline C & 2.23322300 & 2.13561300 & 2.34295300 \\
\hline $\mathrm{H}$ & 1.62748200 & 3.02590000 & 2.43117300 \\
\hline C & 3.04923000 & 1.75848500 & 3.41813800 \\
\hline 0 & 3.02145600 & 2.54585200 & 4.50869100 \\
\hline C & 3.86279600 & 2.18723500 & 5.61130500 \\
\hline $\mathrm{H}$ & 3.68839000 & 2.95326400 & 6.36701900 \\
\hline $\mathrm{H}$ & 4.91551900 & 2.17922100 & 5.31576300 \\
\hline $\mathrm{H}$ & 3.59840700 & 1.20088500 & 6.00243500 \\
\hline $\mathrm{N}$ & 3.83112600 & 0.68232500 & 3.40372800 \\
\hline C & 3.84091900 & -0.06570300 & 2.30360400 \\
\hline 0 & 4.61731500 & -1.16512800 & 2.28044800 \\
\hline C & 5.42940600 & -1.42998900 & 3.42973300 \\
\hline $\mathrm{H}$ & 5.98483000 & -2.33614700 & 3.18580100 \\
\hline $\mathrm{H}$ & 4.81177100 & -1.58906400 & 4.31765600 \\
\hline $\mathrm{H}$ & 6.11840400 & -0.60337600 & 3.62190900 \\
\hline o & 4.27374000 & -2.98731100 & -3.27866300 \\
\hline $\mathrm{H}$ & -2.86296900 & 0.40734800 & 0.47306200 \\
\hline & 5.42797600 & 0.26430500 & -0.22088700 \\
\hline
\end{tabular}




\begin{tabular}{|c|c|c|c|}
\hline & -3.57218300 & 0.55303400 & -1.56234900 \\
\hline 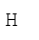 & -4.61286800 & 0.73972800 & -1.28477200 \\
\hline $\mathrm{H}$ & -3.58723400 & -0.03779100 & -2.48664000 \\
\hline 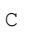 & -3.85912800 & -2.50919700 & -1.20411200 \\
\hline & -3.05852800 & -2.19018700 & 1.20690500 \\
\hline I & -2.10160000 & -2.64196100 & 0.94641700 \\
\hline H & -2.90233000 & -1.46661600 & 2.01025200 \\
\hline & -2.82795200 & 1.82704800 & -1.84695500 \\
\hline & -3.40821800 & 2.87164000 & -2.45345000 \\
\hline$c$ & -2.65909300 & 4.11197400 & -2.67432100 \\
\hline t & -1.76210800 & 4.11488200 & -2.05988600 \\
\hline & -2.37988200 & 4.20489900 & -3.72980200 \\
\hline $\mathrm{H}$ & -3.29262900 & 4.96118500 & -2.40273700 \\
\hline$c$ & -4.76888700 & 2.86035500 & -2.98831000 \\
\hline & -5.40957300 & 3.54316600 & -2.41949800 \\
\hline $\mathrm{H}$ & -4.74777500 & 3.19434000 & -4.03056700 \\
\hline H & -5.20017900 & 1.86253300 & -2.95994100 \\
\hline & -1.61324200 & 1.89300400 & -1.50104300 \\
\hline & -4.54160137 & -2.07373299 & -1.94235935 \\
\hline $\mathrm{H}$ & -2.88497399 & -2.61475484 & -1.69099261 \\
\hline 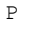 & 0.56295500 & -1.63706800 & 0.05053800 \\
\hline & 4.87306300 & -1.35709300 & -1.75187400 \\
\hline C & -6.13106100 & -0.62662100 & 0.74581300 \\
\hline $\mathrm{Si}$ & -7.80748800 & -0.08712200 & 1.30937800 \\
\hline 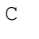 & -8.90024000 & -1.61606900 & 1.47212600 \\
\hline $\mathrm{H}$ & -9.90657800 & -1.33852100 & 1.80554400 \\
\hline $\mathrm{H}$ & -8.99845200 & -2.13979800 & 0.51575800 \\
\hline H & -8.49080300 & -2.32310800 & 2.20071200 \\
\hline 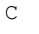 & -8.51932900 & 1.09470700 & 0.01717200 \\
\hline H & -8.60922500 & 0.61030700 & -0.96076900 \\
\hline $\mathrm{H}$ & -9.51945900 & 1.43025500 & 0.31349100 \\
\hline I & -7.89423400 & 1.986350 & -0.1017820 \\
\hline C & -7.63101800 & 0.79007000 & 2.97124600 \\
\hline $\mathrm{H}$ & -8.60973700 & 1.12018900 & 3.33714500 \\
\hline H & -7.1990900 & 0.1267960 & 3.72748900 \\
\hline $\mathrm{H}$ & -6.98903100 & 1.67377500 & 2.89405300 \\
\hline C & -3.69758200 & -1.50793100 & -0.02158700 \\
\hline $\mathrm{H}$ & -3.71439400 & -2.97340900 & 1.59175000 \\
\hline$c$ & -4.38848669 & -3.89507795 & -0.82010218 \\
\hline $\mathrm{H}$ & -5.33507638 & -3.82098668 & -0.27626747 \\
\hline & -4.56422673 & -4.49097560 & -1.72077716 \\
\hline & -3.67816534 & -4.44680048 & -0.19833683 \\
\hline
\end{tabular}

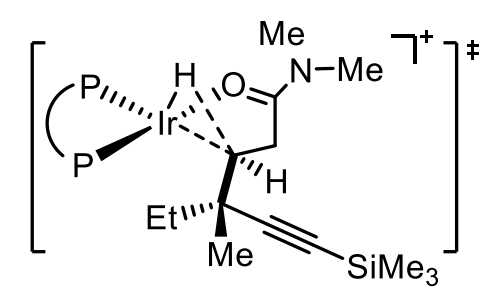

$$
\begin{aligned}
& G_{\text {sol }}=-3633.042137 \text { Hatree } \\
& \text { Ir }-0.67843800 \quad 0.23834100 \quad-0.64564100 \\
& \text { C } \quad-2.88186000 \quad-0.19002400 \quad-0.52432200 \\
& \text { H } \quad-1.63603500 \quad-0.74763600 \quad-1.48409100 \\
& \begin{array}{lrrr}
\text { C } \quad-5.04081200 & -1.09275400 & 0.13585800
\end{array} \\
& \begin{array}{llll}
\text { C } & 2.32634000 & -1.44454300 & -0.77606000
\end{array} \\
& \begin{array}{llll}
\text { C } & 2.71303200 & -2.09244200 & -1.95113600
\end{array} \\
& \mathrm{H} \quad 2.06351200 \quad-2.75719000 \quad-2.49935500 \\
& \begin{array}{llll}
\text { C } & 4.01378200 & -1.89504700 & -2.43128400
\end{array} \\
& \begin{array}{llll}
\text { C } & 4.53103600 & -0.49420200 & -0.71809900
\end{array} \\
& \begin{array}{llll}
\text { C } & 3.25765900 & -0.61878600 & -0.10889000
\end{array} \\
& \begin{array}{llll}
\text { C } & 5.68192800 & -2.37466200 & -4.05679500
\end{array} \\
& \text { H } \quad 5.73570600 \quad-2.99029900 \quad-4.95483400 \\
& \text { H } \quad 6.42267700 \quad-2.70752500 \quad-3.32450900 \\
& \text { H } \quad 5.87496700 \quad-1.32676200 \quad-4.30300500 \\
& \begin{array}{llll}
\text { C } & 6.71475800 & 0.44689400 & -0.69786100
\end{array} \\
& \begin{array}{llll}
\mathrm{H} & 7.26180500 & 1.12503100 & -0.04200500
\end{array} \\
& \begin{array}{llll}
\mathrm{H} & 6.65048400 & 0.86242200 & -1.70714700
\end{array} \\
& \text { H } \quad 7.21781300 \quad-0.52232200 \quad-0.74560300 \\
& \begin{array}{llll}
\text { C } & 0.77841300 & -2.13786600 & 1.59089900
\end{array} \\
& \begin{array}{llll}
\text { C } & -0.03168300 & -1.56200700 & 2.57937900
\end{array} \\
& \begin{array}{llll}
\mathrm{H} & -0.74811100 & -0.79760500 & 2.29582900
\end{array} \\
& \begin{array}{llll}
\text { C } & 0.08853300 & -1.95762900 & 3.91250200
\end{array} \\
& \mathrm{H} \quad-0.54296700 \quad-1.50269900 \quad 4.66977800 \\
& \begin{array}{llll}
\text { C } & 1.01831600 & -2.93519500 & 4.26962500
\end{array} \\
& \begin{array}{llll}
\mathrm{H} & 1.10765300 & -3.24900800 & 5.30544200
\end{array} \\
& \begin{array}{llll}
\text { C } & 1.83941800 & -3.50544900 & 3.29319500
\end{array} \\
& \begin{array}{llll}
\text { H } & 2.56996500 & -4.26046800 & 3.56809600
\end{array} \\
& \begin{array}{llll}
\text { C } & 1.72821800 & -3.10511600 & 1.96172600
\end{array} \\
& \text { H } \quad 2.38797700 \quad-3.53783700 \quad 1.21632100 \\
& \text { C } \quad-0.00209000 \quad-3.15374100 \quad-1.04986700 \\
& \text { C } \quad-0.32109100 \quad-3.05431600 \quad-2.41685500 \\
& \mathrm{H} \quad-0.25720300 \quad-2.09124200 \quad-2.91504600 \\
& \text { C } \quad-0.71412200 \quad-4.17920000 \quad-3.14168000 \\
& \mathrm{H} \quad-0.94204800 \quad-4.08501000 \quad-4.19927300 \\
& \text { C } \quad-0.82273300 \quad-5.41914100 \quad-2.50710900 \\
& \mathrm{H} \quad-1.13582100 \quad-6.29397700 \quad-3.06879700 \\
& \text { C } \quad-0.53111000 \quad-5.52469800 \quad-1.14791200 \\
& \text { H } \quad-0.62115400 \quad-6.48203100 \quad-0.64342500
\end{aligned}
$$




\begin{tabular}{|c|c|c|c|}
\hline C & -0.11605800 & -4.40329200 & -0.42445300 \\
\hline $\mathrm{H}$ & 0.10804600 & -4.51044500 & 0.63018100 \\
\hline C & 3.03729300 & 0.08666400 & 1.18153400 \\
\hline $\mathrm{C}$ & 2.20536200 & 1.20928200 & 1.34541800 \\
\hline P & 1.12574900 & 1.74301100 & -0.05449400 \\
\hline C & 2.26387200 & 2.21790500 & -1.40802200 \\
\hline $\mathrm{C}$ & 1.88689600 & 1.96593500 & -2.73563600 \\
\hline $\mathrm{H}$ & 0.95320700 & 1.44781700 & -2.93429700 \\
\hline C & 2.71425800 & 2.36573900 & -3.78594000 \\
\hline $\mathrm{H}$ & 2.41942800 & 2.16413100 & -4.81143000 \\
\hline C & 3.91953200 & 3.01742800 & -3.51827100 \\
\hline $\mathrm{H}$ & 4.56170400 & 3.32976800 & -4.33647400 \\
\hline C & 4.30328000 & 3.26352600 & -2.19733200 \\
\hline $\mathrm{H}$ & 5.24281200 & 3.76590700 & -1.98723600 \\
\hline C & 3.48292500 & 2.86253100 & -1.14268800 \\
\hline $\mathrm{H}$ & 3.79531100 & 3.03927300 & -0.11840400 \\
\hline C & 0.35925000 & 3.31332800 & 0.53768200 \\
\hline C & -0.69692700 & 3.23684400 & 1.46221400 \\
\hline $\mathrm{H}$ & -1.04127200 & 2.26614300 & 1.80794500 \\
\hline C & -1.30441600 & 4.39600000 & 1.94174600 \\
\hline $\mathrm{H}$ & -2.11116300 & 4.32234400 & 2.66508100 \\
\hline C & -0.87690100 & 5.64825900 & 1.49132700 \\
\hline H & -1.35044200 & 6.55172600 & 1.86373300 \\
\hline C & 0.16144800 & 5.73268400 & 0.56382100 \\
\hline $\mathrm{H}$ & 0.49942200 & 6.70224200 & 0.21011100 \\
\hline C & 0.78007200 & 4.57232200 & 0.08914300 \\
\hline $\mathrm{H}$ & 1.58871500 & 4.65485700 & -0.62827000 \\
\hline C & 2.14080600 & 1.86155200 & 2.57731500 \\
\hline $\mathrm{H}$ & 1.52448400 & 2.73174200 & 2.75155500 \\
\hline C & 2.92856100 & 1.36346200 & 3.62415000 \\
\hline 0 & 2.86208200 & 2.01487200 & 4.79946900 \\
\hline C & 3.67241000 & 1.52944200 & 5.87648400 \\
\hline $\mathrm{H}$ & 3.46339100 & 2.19329600 & 6.71547800 \\
\hline $\mathrm{H}$ & 4.73412000 & 1.56892400 & 5.61765200 \\
\hline $\mathrm{H}$ & 3.40949400 & 0.49926900 & 6.13207400 \\
\hline $\mathrm{N}$ & 3.71930200 & 0.30029700 & 3.50583100 \\
\hline C & 3.76710700 & -0.31318900 & 2.32606300 \\
\hline 0 & 4.55300400 & -1.39831800 & 2.19634800 \\
\hline C & 5.32232600 & -1.80185500 & 3.33459600 \\
\hline $\mathrm{H}$ & 5.90290600 & -2.66149700 & 2.99829700 \\
\hline $\mathrm{H}$ & 4.67065800 & -2.08441500 & 4.16579200 \\
\hline $\mathrm{H}$ & 5.98782000 & -0.99959500 & 3.66340400 \\
\hline 0 & 4.35073300 & -2.54930900 & -3.55790400 \\
\hline $\mathrm{H}$ & -2.87297500 & 0.33264100 & 0.44072400 \\
\hline 0 & 5.41422500 & 0.32360200 & -0.11179700 \\
\hline
\end{tabular}

\begin{tabular}{|c|c|c|c|}
\hline C & -3.56099300 & 0.71128100 & -1.57414200 \\
\hline $\mathrm{H}$ & -4.60194100 & 0.87016600 & -1.28107600 \\
\hline $\mathrm{H}$ & -3.57581100 & 0.22140800 & -2.55576800 \\
\hline C & -3.81395900 & -2.38029000 & -1.56633200 \\
\hline C & -3.05282600 & -2.32436600 & 0.87492600 \\
\hline $\mathrm{H}$ & -2.03657500 & -2.58870600 & 0.57861500 \\
\hline $\mathrm{H}$ & -2.97076100 & -1.65492500 & 1.73836800 \\
\hline C & -2.81501000 & 2.00721800 & -1.72282900 \\
\hline $\mathrm{N}$ & -3.39379600 & 3.10736300 & -2.22314700 \\
\hline C & -2.64845900 & 4.36623600 & -2.31129700 \\
\hline $\mathrm{H}$ & -1.74258700 & 4.30075000 & -1.71374400 \\
\hline $\mathrm{H}$ & -2.38512700 & 4.57890300 & -3.35335300 \\
\hline $\mathrm{H}$ & -3.27737400 & 5.17838800 & -1.93521800 \\
\hline C & -4.75703500 & 3.14948800 & -2.75046700 \\
\hline $\mathrm{H}$ & -5.40808200 & 3.73270200 & -2.08973700 \\
\hline $\mathrm{H}$ & -4.74420200 & 3.62904800 & -3.73408300 \\
\hline $\mathrm{H}$ & -5.17040900 & 2.15031000 & -2.86550900 \\
\hline 0 & -1.60197500 & 2.03765100 & -1.36654500 \\
\hline $\mathrm{H}$ & -4.27915100 & -1.82884700 & -2.38772900 \\
\hline $\mathrm{H}$ & -2.83233200 & -2.73259700 & -1.88992000 \\
\hline P & 0.56517800 & -1.62774400 & -0.16546800 \\
\hline N & 4.90535000 & -1.10854500 & -1.83602200 \\
\hline C & -6.15315000 & -0.74843000 & 0.50136800 \\
\hline $\mathrm{Si}$ & -7.85239800 & -0.28963500 & 1.06829400 \\
\hline C & -8.98266600 & -1.78097100 & 0.83300900 \\
\hline $\mathrm{H}$ & -10.00252800 & -1.54695400 & 1.15838800 \\
\hline $\mathrm{H}$ & -9.02905600 & -2.08553700 & -0.21748600 \\
\hline $\mathrm{H}$ & -8.63587200 & -2.64048800 & 1.41552200 \\
\hline C & -8.45658700 & 1.16408000 & 0.02107100 \\
\hline $\mathrm{H}$ & -8.49071700 & 0.90389700 & -1.04209600 \\
\hline $\mathrm{H}$ & -9.46808300 & 1.45943900 & 0.32169400 \\
\hline $\mathrm{H}$ & -7.80968800 & 2.04048600 & 0.13652400 \\
\hline C & -7.76557200 & 0.20756300 & 2.88694200 \\
\hline $\mathrm{H}$ & -8.75836500 & 0.48499500 & 3.25868100 \\
\hline $\mathrm{H}$ & -7.39576100 & -0.61496900 & 3.50752100 \\
\hline $\mathrm{H}$ & -7.10192100 & 1.06537400 & 3.03761300 \\
\hline C & -3.68974500 & -1.51526500 & -0.29124200 \\
\hline $\mathrm{H}$ & -4.44363100 & -3.25099400 & -1.37409100 \\
\hline $\mathrm{C}$ & -3.80199100 & -3.59265000 & 1.29370500 \\
\hline $\mathrm{H}$ & -3.32424500 & -4.03052200 & 2.17558900 \\
\hline $\mathrm{H}$ & -4.84362500 & -3.37709300 & 1.54846300 \\
\hline $\mathrm{H}$ & -3.79238200 & -4.35357400 & 0.50794600 \\
\hline
\end{tabular}




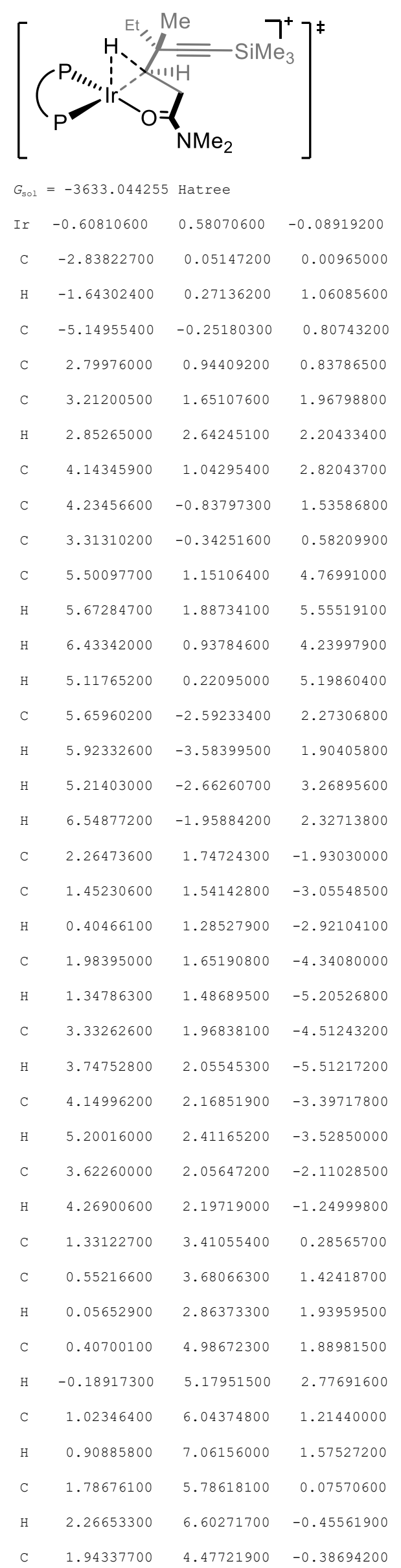

\begin{tabular}{|c|c|c|c|}
\hline & 2.54105500 & 4.29580600 & -1.27270800 \\
\hline & 3.01914200 & -1.15984300 & -0.62637600 \\
\hline & 1.79316800 & -1.79793000 & -0.91184500 \\
\hline & 0.29890400 & -1.50315100 & 0.15945100 \\
\hline & 0.86365200 & -2.01178800 & 1.83476700 \\
\hline & 0.53432600 & -1.22960500 & 2.95077000 \\
\hline & -0.00933200 & -0.30190300 & 2.80511100 \\
\hline & 0.92247900 & -1.62671600 & 4.23166800 \\
\hline & 0.66432300 & -1.01147900 & 5.08839800 \\
\hline 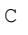 & 1.64349900 & -2.80822500 & 4.40853600 \\
\hline $\mathrm{r}$ & 1.94129300 & -3.12021400 & 5.40520800 \\
\hline 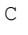 & 1.98732800 & -3.58740800 & 3.30040300 \\
\hline $\mathrm{H}$ & 2.55392200 & -4.50425700 & 3.43338500 \\
\hline 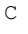 & 1.60669300 & -3.19045800 & 2.01873600 \\
\hline F & 1.89236300 & -3.79568700 & 1.16396500 \\
\hline$C$ & -0.88913300 & -2.82383700 & -0.36181300 \\
\hline e & -1.52595300 & -2.69924000 & -1.61137400 \\
\hline $\mathrm{H}$ & -1.29684100 & -1.85154300 & -2.25141900 \\
\hline$C$ & -2.44741500 & -3.65257700 & -2.04173300 \\
\hline $\mathrm{H}$ & -2.91860200 & -3.54626600 & -3.01435900 \\
\hline$C$ & -2.76542000 & -4.73862800 & -1.22208000 \\
\hline $\mathrm{H}$ & -3.48625700 & -5.47960300 & -1.55392000 \\
\hline C & -2.15509700 & -4.86278900 & 0.02500000 \\
\hline $\mathrm{H}$ & -2.40100000 & -5.69995000 & 0.67136300 \\
\hline C & -1.22176800 & -3.91439500 & 0.45375600 \\
\hline $\mathrm{H}$ & -0.76060800 & -4.03060800 & 1.42731400 \\
\hline$C$ & 1.68781400 & -2.61225900 & -2.04078400 \\
\hline $\mathrm{H}$ & 0.78646000 & -3.14791100 & -2.29805600 \\
\hline C & 2.81305600 & -2.76282200 & -2.86201200 \\
\hline 0 & 2.68116200 & -3.56660200 & -3.93300400 \\
\hline C & 3.82721100 & -3.75131100 & -4.77244200 \\
\hline $\mathrm{H}$ & 3.50115900 & -4.43244800 & -5.55859700 \\
\hline H & 4.65771200 & -4.18950200 & -4.21210400 \\
\hline $\mathrm{H}$ & 4.15487700 & -2.80169500 & -5.20451900 \\
\hline N & 3.97474300 & -2.15860600 & -2.63203300 \\
\hline C & 4.06625900 & -1.38670800 & -1.55277300 \\
\hline 0 & 5.23325200 & -0.75792200 & -1.31342 \\
\hline $\mathrm{C}$ & 6.31817500 & -1.00253400 & -2.21536400 \\
\hline H & 7.15500500 & -0.42565600 & -1.82022600 \\
\hline $\mathrm{H}$ & 6.06966000 & -0.66906800 & -3.226540 \\
\hline $\mathrm{H}$ & 6.57165300 & -2.06533100 & -2.24596900 \\
\hline $\mathrm{N}$ & 4.64082800 & -0.17341000 & 2.61464400 \\
\hline $\mathrm{H}$ & -2.85323100 & -0.96002100 & -0.38834600 \\
\hline O & 4.71693600 & -2.07710200 & 1.32632200 \\
\hline 0 & -3.34211800 & 1.02468800 & -1.08553300 \\
\hline
\end{tabular}




\begin{tabular}{|c|c|c|c|}
\hline $\mathrm{H}$ & -4.43431500 & 1.05782600 & -1.06739200 \\
\hline $\mathrm{H}$ & -3.04814600 & 0.63044700 & -2.06651200 \\
\hline C & -3.78853400 & 1.41807600 & 1.99859300 \\
\hline C & -3.33738900 & -1.08422800 & 2.25457400 \\
\hline $\mathrm{H}$ & -2.31932100 & -0.85638200 & 2.59367100 \\
\hline C & -2.71706700 & 2.39396700 & -0.97309400 \\
\hline $\mathrm{N}$ & -3.35142300 & 3.51406300 & -1.34372200 \\
\hline C & -2.69924700 & 4.81889200 & -1.18538500 \\
\hline $\mathrm{H}$ & -1.82483800 & 4.71818300 & -0.54712700 \\
\hline $\mathrm{H}$ & -2.39492400 & 5.21021200 & -2.16220500 \\
\hline $\mathrm{H}$ & -3.40961700 & 5.51704500 & -0.73310500 \\
\hline C & -4.68251800 & 3.55656300 & -1.95018700 \\
\hline $\mathrm{H}$ & -5.41285900 & 3.97104500 & -1.24673700 \\
\hline $\mathrm{H}$ & -4.64824200 & 4.20118900 & -2.83370800 \\
\hline $\mathrm{H}$ & -5.01047900 & 2.56831300 & -2.26293400 \\
\hline 0 & -1.52992800 & 2.46368900 & -0.53389400 \\
\hline $\mathrm{H}$ & -4.10559200 & 2.22316200 & 1.32963600 \\
\hline $\mathrm{H}$ & -2.78696600 & 1.65647800 & 2.37240000 \\
\hline $\mathrm{P}$ & 1.50064500 & 1.65844000 & -0.26320900 \\
\hline 0 & 4.53740200 & 1.74965900 & 3.89509000 \\
\hline C & -6.27999600 & -0.49425100 & 0.41685400 \\
\hline $\mathrm{Si}$ & -8.00123600 & -0.88116000 & -0.13657200 \\
\hline C & -9.20247800 & 0.20655600 & 0.83030200 \\
\hline $\mathrm{H}$ & -10.23610300 & -0.00255800 & 0.53240200 \\
\hline $\mathrm{H}$ & -9.12397400 & 0.02880600 & 1.90757700 \\
\hline $\mathrm{H}$ & -9.01342200 & 1.27025300 & 0.65214400 \\
\hline C & -8.34057600 & -2.70831100 & 0.18941200 \\
\hline $\mathrm{H}$ & -8.25404000 & -2.94604700 & 1.25449300 \\
\hline $\mathrm{H}$ & -9.35378100 & -2.97599600 & -0.13080600 \\
\hline $\mathrm{H}$ & -7.63799000 & -3.34772600 & -0.35475400 \\
\hline C & -8.12645400 & -0.50984500 & -1.98576500 \\
\hline $\mathrm{H}$ & -9.13341600 & -0.73416800 & -2.35509500 \\
\hline $\mathrm{H}$ & -7.92597200 & 0.54587200 & -2.19792700 \\
\hline $\mathrm{H}$ & -7.41902600 & -1.11186900 & -2.56549900 \\
\hline C & -3.78445500 & 0.04740600 & 1.27873900 \\
\hline $\mathrm{H}$ & -3.27189300 & -2.01576100 & 1.68164800 \\
\hline $\mathrm{H}$ & -4.48172300 & 1.41092100 & 2.84193300 \\
\hline C & -4.23357800 & -1.30758200 & 3.47665400 \\
\hline $\mathrm{H}$ & -5.26261800 & -1.52812000 & 3.17944500 \\
\hline $\mathrm{H}$ & -3.86267000 & -2.15609000 & 4.05967200 \\
\hline $\mathrm{H}$ & -4.25151600 & -0.43933500 & 4.14250200 \\
\hline
\end{tabular}

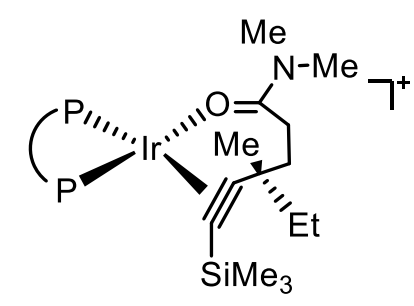

$\begin{array}{llll}\text { Ir } & 1.20672800 & 0.08743000 & 0.00833600\end{array}$

$\begin{array}{llll}\text { C } & 5.23219300 & 0.59465000 & 0.55357400\end{array}$

$\begin{array}{llll}\text { H } & 5.95702100 & 0.17494200 & -0.15158200\end{array}$

$\begin{array}{llll}\text { C } & 3.20887200 & -0.81176500 & 0.16095600\end{array}$

$\begin{array}{llll}\text { C } & -1.66385300 & -1.34010000 & 1.22485600\end{array}$

$\begin{array}{llll}\text { C } & -1.66669100 & -1.94191700 & 2.48426500\end{array}$

$\begin{array}{llll}\mathrm{H} & -0.91951800 & -2.65326200 & 2.79917200\end{array}$

$\begin{array}{llll}\text { C } \quad-2.70665700 & -1.63953400 & 3.37244300\end{array}$

C $\quad-3.68519300 \quad-0.22510000 \quad 1.88703500$

$\begin{array}{llll}\text { C } & -2.71373800 & -0.45757900 & 0.88294900\end{array}$

C $\quad-3.74491500 \quad-1.97117300 \quad 5.48554100$

H $\quad-3.52878700 \quad-2.56812000 \quad 6.37177300$

$\mathrm{H} \quad-4.71245100 \quad-2.25686100 \quad 5.06349700$

$\mathrm{H} \quad-3.77164600 \quad-0.90814700 \quad 5.74104400$

$\begin{array}{llll}\text { C } & -5.67774300 & 0.87453100 & 2.57734300\end{array}$

$\mathrm{H} \quad-6.37128400 \quad 1.58232700 \quad 2.12186600$

$\mathrm{H} \quad-5.24787900 \quad 1.29694800 \quad 3.48961500$

H $\quad-6.19553400 \quad-0.05487200 \quad 2.82823100$

C $\quad-1.13783300 \quad-2.12500700 \quad-1.49814800$

$\begin{array}{llll}\text { C } & -0.80081600 & -1.52875900 & -2.71908100\end{array}$

$\mathrm{H} \quad-0.02833300 \quad-0.76809600 \quad-2.73281800$

C $\quad-1.46258500 \quad-1.89156000 \quad-3.89410900$

H $\quad-1.19383200 \quad-1.41803700 \quad-4.83373000$

$\begin{array}{llll}\text { C } & -2.46592900 & -2.86075400 & -3.85837000\end{array}$

H $\quad-2.97616000 \quad-3.15059300 \quad-4.77217600$

C $\quad-2.81758000 \quad-3.45379500 \quad-2.64258300$

H $\quad-3.60313600 \quad-4.20280600 \quad-2.60826400$

C $\quad-2.16644100 \quad-3.08251700 \quad-1.46665300$

H $\quad-2.46357800 \quad-3.53550900 \quad-0.52602500$

$\begin{array}{llll}\text { C } & 0.48878900 & -3.23599300 & 0.65549400\end{array}$

$\begin{array}{llll}\text { C } & 1.15953300 & -3.23893800 & 1.89060900\end{array}$

$\begin{array}{llll}\mathrm{H} & 1.29842700 & -2.30678800 & 2.42841700\end{array}$

$\begin{array}{llll}\text { C } & 1.63653900 & -4.42781800 & 2.44155900\end{array}$

H $2.12834000 \quad-4.41232100 \quad 3.40939600$

$\begin{array}{llll}\text { C } & 1.49675900 & -5.62999800 & 1.74526900\end{array}$

H $\quad 1.87268400 \quad-6.55512000 \quad 2.17155700$

$\begin{array}{lllll}\text { C } & 0.87988500 & -5.63149700 & 0.49508600\end{array}$

$\begin{array}{llll}\mathrm{H} & 0.77989200 & -6.55743600 & -0.06343100\end{array}$ 


\begin{tabular}{|c|c|c|c|}
\hline C & 0.37225200 & -4.44648800 & -0.04372500 \\
\hline $\mathrm{H}$ & -0.11616700 & -4.47768700 & -1.00925000 \\
\hline C & -2.90842300 & 0.19458200 & -0.43914200 \\
\hline C & -2.08101100 & 1.20227600 & -0.96638600 \\
\hline P & -0.54103200 & 1.68014300 & -0.06879400 \\
\hline $\mathrm{C}$ & -1.14255000 & 2.31623100 & 1.55005900 \\
\hline C & -0.37407900 & 2.09176500 & 2.70294200 \\
\hline $\mathrm{H}$ & 0.54413800 & 1.51824700 & 2.62703200 \\
\hline C & -0.79200200 & 2.59229900 & 3.93708400 \\
\hline $\mathrm{H}$ & -0.19377200 & 2.40756100 & 4.82459500 \\
\hline C & -1.97939300 & 3.32063600 & 4.03183100 \\
\hline $\mathrm{H}$ & -2.30368800 & 3.71050800 & 4.99215700 \\
\hline C & -2.75394700 & 3.54044000 & 2.89055500 \\
\hline $\mathrm{H}$ & -3.68204200 & 4.09990700 & 2.96098200 \\
\hline C & -2.34342900 & 3.03688800 & 1.65540300 \\
\hline $\mathrm{H}$ & -2.96631500 & 3.19109900 & 0.78017100 \\
\hline C & 0.05582400 & 3.16685600 & -0.98996700 \\
\hline C & 0.88572900 & 2.96276600 & -2.10511500 \\
\hline $\mathrm{H}$ & 1.17817600 & 1.95108400 & -2.37221400 \\
\hline C & 1.34082600 & 4.04459200 & -2.85860900 \\
\hline $\mathrm{H}$ & 1.97250000 & 3.87114200 & -3.72507100 \\
\hline C & 0.98521400 & 5.34724200 & -2.49913800 \\
\hline $\mathrm{H}$ & 1.33993400 & 6.19037600 & -3.08449400 \\
\hline C & 0.17045400 & 5.55985100 & -1.38657200 \\
\hline $\mathrm{H}$ & -0.11036700 & 6.56957500 & -1.10166100 \\
\hline C & -0.29530900 & 4.47688800 & -0.63658300 \\
\hline $\mathrm{H}$ & -0.92878300 & 4.66036200 & 0.22409100 \\
\hline C & -2.38970200 & 1.79753900 & -2.19043400 \\
\hline $\mathrm{H}$ & -1.78777200 & 2.57853600 & -2.63287600 \\
\hline C & -3.54415600 & 1.36502000 & -2.85609700 \\
\hline 0 & -3.83453800 & 1.96415500 & -4.02590400 \\
\hline C & -5.02223900 & 1.54833400 & -4.70992400 \\
\hline $\mathrm{H}$ & -5.06052300 & 2.15512200 & -5.61479100 \\
\hline $\mathrm{H}$ & -5.91090300 & 1.72523100 & -4.09765900 \\
\hline $\mathrm{H}$ & -4.97675300 & 0.48627300 & -4.96631400 \\
\hline $\mathrm{N}$ & -4.34540300 & 0.41098300 & -2.38998900 \\
\hline C & -4.03131000 & -0.15510800 & -1.22746200 \\
\hline 0 & -4.81838300 & -1.14146100 & -0.75988900 \\
\hline C & -5.97421700 & -1.49149000 & -1.52835100 \\
\hline $\mathrm{H}$ & -6.46283800 & -2.28710800 & -0.96499500 \\
\hline $\mathrm{H}$ & -5.69009300 & -1.84778500 & -2.52205300 \\
\hline $\mathrm{H}$ & -6.64667400 & -0.63659400 & -1.63812000 \\
\hline 0 & -2.68048500 & -2.25385200 & 4.57013600 \\
\hline $\mathrm{H}$ & 5.81440700 & 0.99503200 & 1.39263700 \\
\hline 0 & -4.66607700 & 0.65030700 & 1.58996800 \\
\hline
\end{tabular}

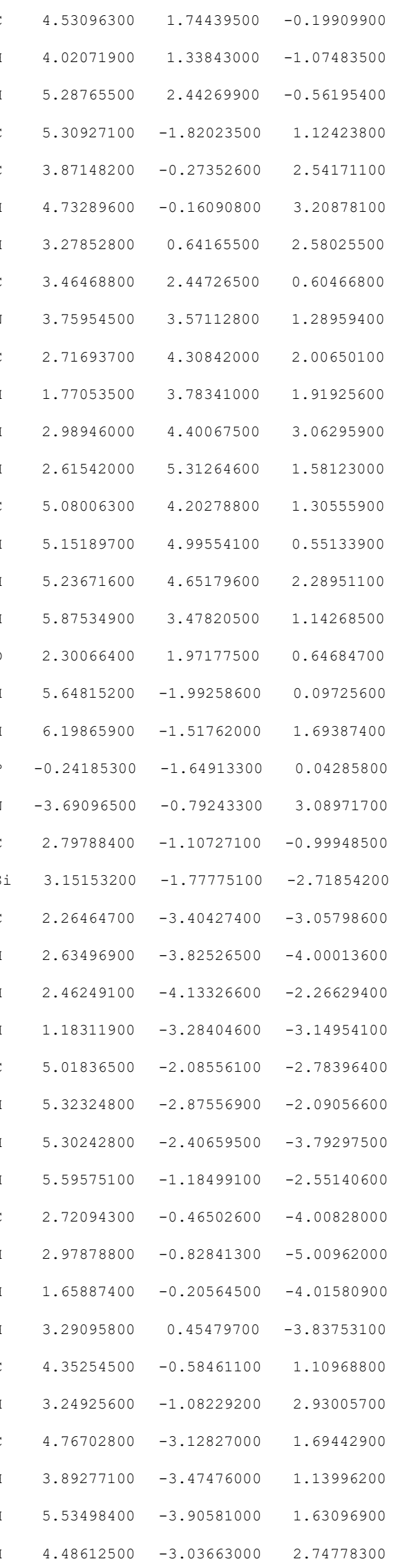




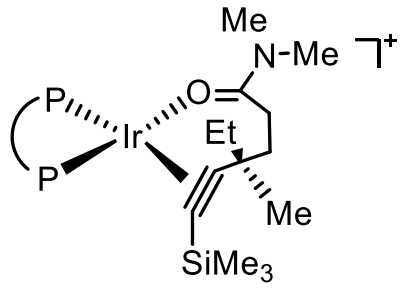

$G_{\text {sol }}=-3633.085469$ Hatree

Ir $1.18087700 \quad 0.03034400$

C $\quad 5.23043600 \quad 0.10221500$$$
\text { C } \quad 3.06301600 \quad-0.99648800
$$$$
\text { C } \quad-1.59827100 \quad-1.37928800
$$$$
\text { C } \quad-1.49593400 \quad-2.03725600
$$$$
\mathrm{H}
$$$$
\therefore
$$ \\ C}$$
\mathrm{H}
$$$$
\text { (1) }
$$$$
\text { н }
$$

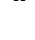




\begin{tabular}{|c|c|c|c|}
\hline C & 4.76567700 & 1.25819400 & -0.31538100 \\
\hline $\mathrm{H}$ & 4.32047900 & 0.83430100 & -1.21644300 \\
\hline $\mathrm{H}$ & 5.64038300 & 1.83209000 & -0.62836500 \\
\hline C & 4.79363800 & -2.25379700 & 1.36247000 \\
\hline C & 3.55420700 & -0.34416200 & 2.51298200 \\
\hline $\mathrm{H}$ & 3.18020800 & 0.67286000 & 2.36401300 \\
\hline $\mathrm{C}$ & 3.69888700 & 2.16407500 & 0.26311200 \\
\hline $\mathrm{N}$ & 4.05216200 & 3.32577200 & 0.85201200 \\
\hline C & 3.03258200 & 4.27288500 & 1.30919800 \\
\hline $\mathrm{H}$ & 2.06384500 & 3.99616200 & 0.90361500 \\
\hline $\mathrm{H}$ & 2.98139100 & 4.28222700 & 2.40368000 \\
\hline $\mathrm{H}$ & 3.29752300 & 5.27627300 & 0.96236900 \\
\hline C & 5.43298300 & 3.76326400 & 1.06134500 \\
\hline $\mathrm{H}$ & 5.74433400 & 4.47816800 & 0.29043400 \\
\hline $\mathrm{H}$ & 5.49613200 & 4.25925700 & 2.03363500 \\
\hline $\mathrm{H}$ & 6.12461800 & 2.92376900 & 1.07357500 \\
\hline 0 & 2.48176500 & 1.85402600 & 0.18182400 \\
\hline $\mathrm{H}$ & 5.10174400 & -2.67178900 & 0.40089000 \\
\hline $\mathrm{H}$ & 5.68267300 & -2.16764500 & 1.99432500 \\
\hline P & -0.30134300 & -1.67350500 & -0.06115900 \\
\hline $\mathrm{N}$ & -3.39847000 & -0.84159400 & 3.33569700 \\
\hline C & 2.74239400 & -1.24941300 & -1.08231800 \\
\hline $\mathrm{Si}$ & 3.11794500 & -1.86475400 & -2.81161300 \\
\hline C & 2.15382500 & -3.42084100 & -3.25234700 \\
\hline $\mathrm{H}$ & 2.49767100 & -3.79461500 & -4.22400400 \\
\hline $\mathrm{H}$ & 2.31474000 & -4.21060900 & -2.51284700 \\
\hline $\mathrm{H}$ & 1.07884800 & -3.23922700 & -3.32773000 \\
\hline C & 4.97010000 & -2.25816100 & -2.82338800 \\
\hline $\mathrm{H}$ & 5.21654100 & -3.05489800 & -2.11400900 \\
\hline $\mathrm{H}$ & 5.27511200 & -2.59919700 & -3.81942100 \\
\hline $\mathrm{H}$ & 5.57858800 & -1.38219900 & -2.57539000 \\
\hline C & 2.76968100 & -0.48058000 & -4.05072900 \\
\hline $\mathrm{H}$ & 3.05297000 & -0.79868100 & -5.06047700 \\
\hline $\mathrm{H}$ & 1.71148600 & -0.20394400 & -4.07717000 \\
\hline $\mathrm{H}$ & 3.34613900 & 0.42052500 & -3.81534000 \\
\hline C & 4.13243000 & -0.86765800 & 1.16456800 \\
\hline $\mathrm{H}$ & 2.67204300 & -0.94417600 & 2.75931100 \\
\hline $\mathrm{H}$ & 4.10418100 & -2.95944400 & 1.83063600 \\
\hline C & 4.50844800 & -0.36178000 & 3.71416100 \\
\hline $\mathrm{H}$ & 4.01112500 & 0.07315000 & 4.58669700 \\
\hline $\mathrm{H}$ & 4.81109600 & -1.37640100 & 3.98683300 \\
\hline & 5.42002900 & 0.2204250 & 3.54189000 \\
\hline
\end{tabular}




\section{References}

1 Tsuchikama, K.; Kasagawa, M.; Endo, K.; Shibata, T. Org. Lett. 2009, 11, 1821.

2 Sawano, T.; Ou, K.; Nishimura, T.; Hayashi, T. Chem. Commun. 2012, 48, 6106.

3 Ganesh, T.; Jiang, J. X.; Yang, M.-S.; Dingledine, R. J. Med. Chem. 2014, 57, 4173.

4 Kim, J. G.; Waltz, K. M.; Garcia, I. F.; Kwiatkowski, D.; Walsh, P. J. J. Am. Chem. Soc. 2004, $126,12580$.

5 Tietze, L. F.; Schiemann, K.; Wegner, C.; Wulff, C. Chem. Eur. J. 1998, 4, 1862. 


\section{Copies of NMR Spectra}<smiles>CCN(CC)C(=O)C/C=C(\C)C(C)C</smiles>

${ }^{1} \mathrm{HNMR}\left(400 \mathrm{MHz}, \mathrm{CDCl}_{3}\right)$

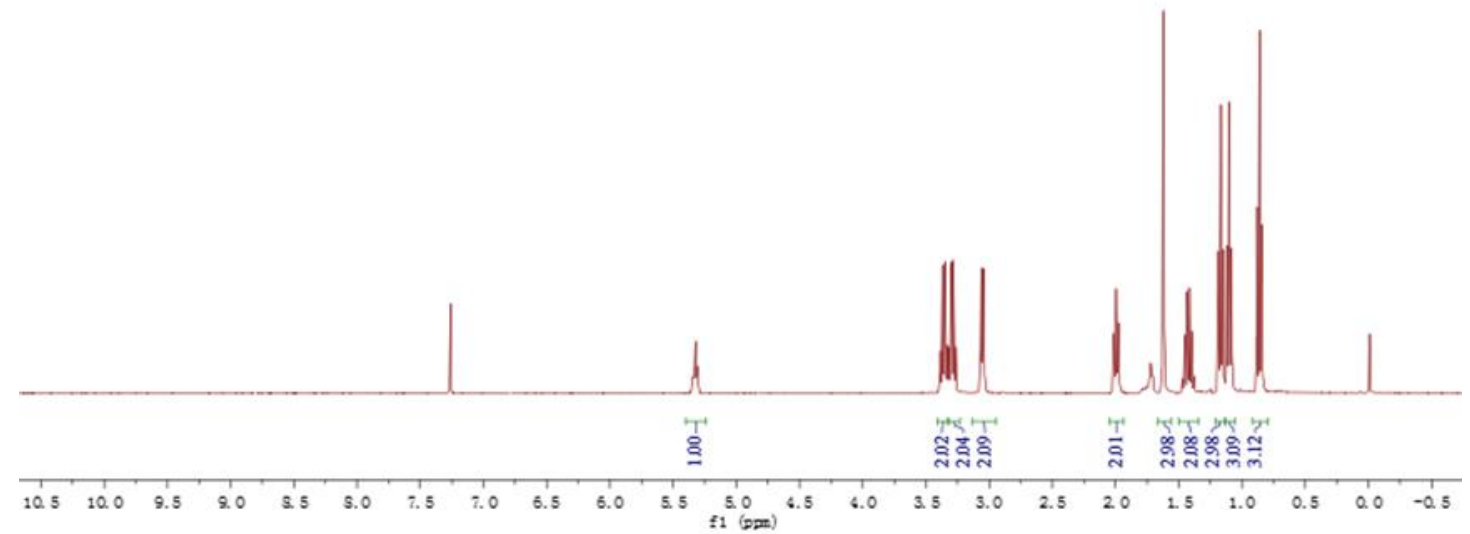

${ }^{13} \mathrm{CNMR}\left(101 \mathrm{MHz}, \mathrm{CDCl}_{3}\right)$

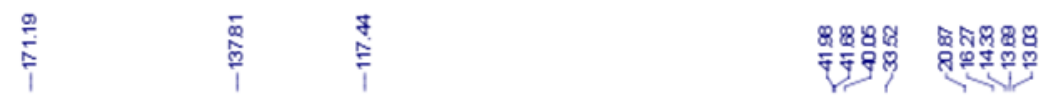

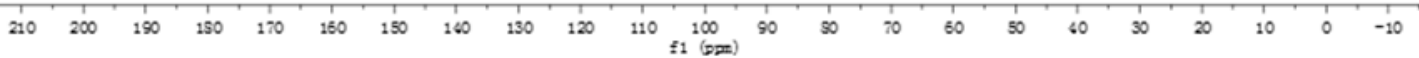


<smiles>CCCCN(CCCC)C(=O)C/C=C(\C)C(C)C</smiles>

${ }^{1} \mathrm{H} \mathrm{NMR}\left(400 \mathrm{MHz}, \mathrm{CDCl}_{3}\right)$
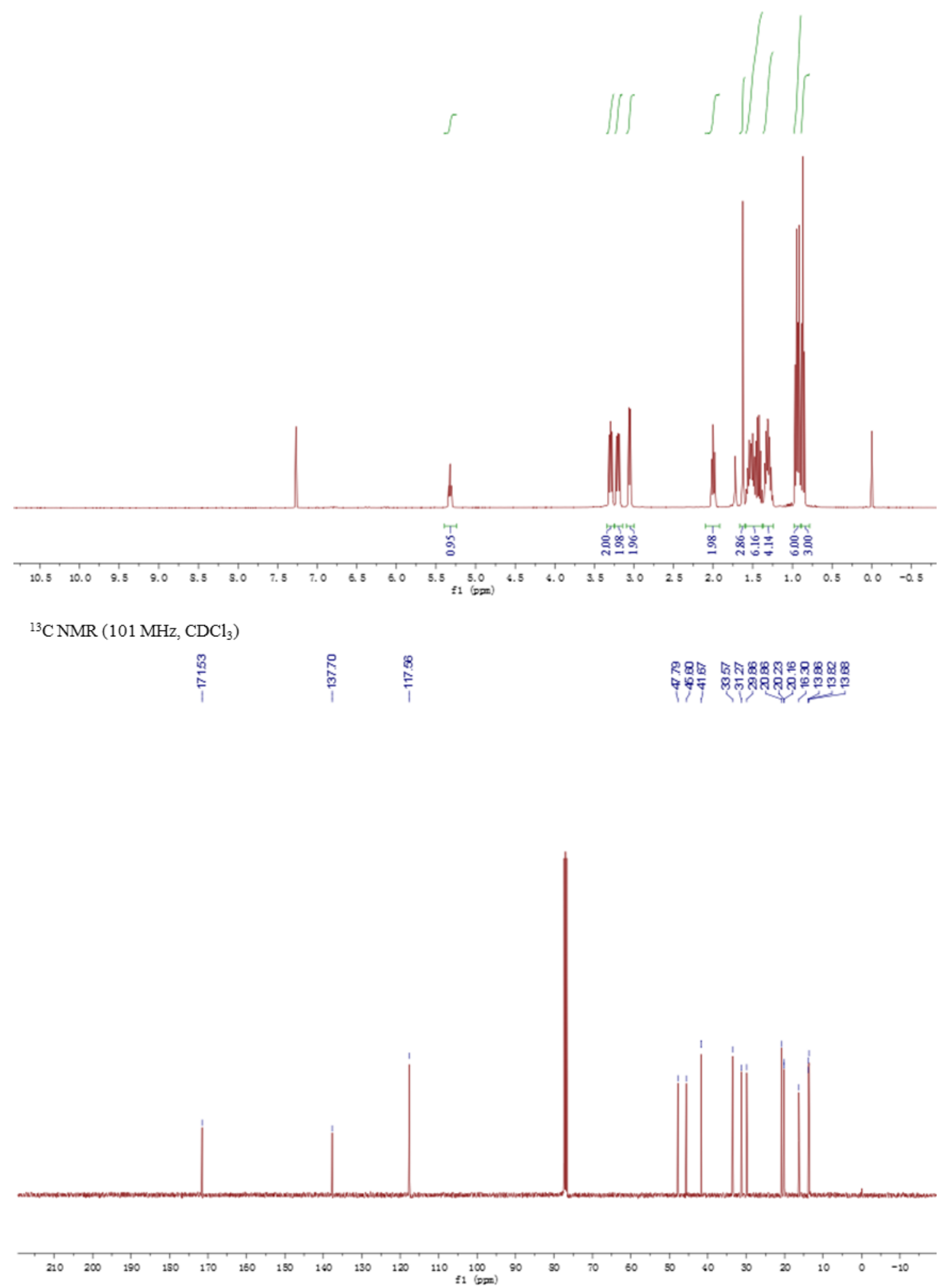
<smiles>CCCCCCCN(CC)C(=O)C/C=C(\C)C(C)C</smiles>

${ }^{1} \mathrm{H} \mathrm{NMR}\left(400 \mathrm{MHz}, \mathrm{CDCl}_{3}\right)$

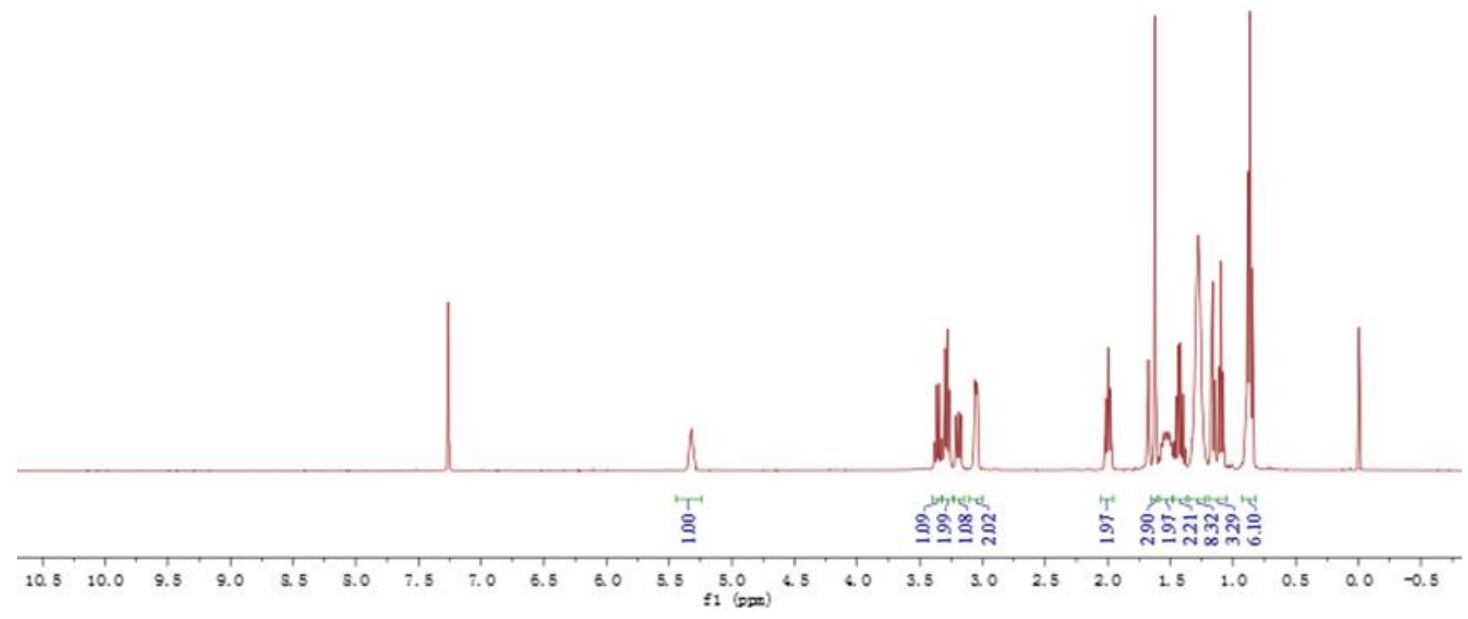

${ }^{13} \mathrm{CNMR}\left(101 \mathrm{MHz}, \mathrm{CDCl}_{3}\right)$

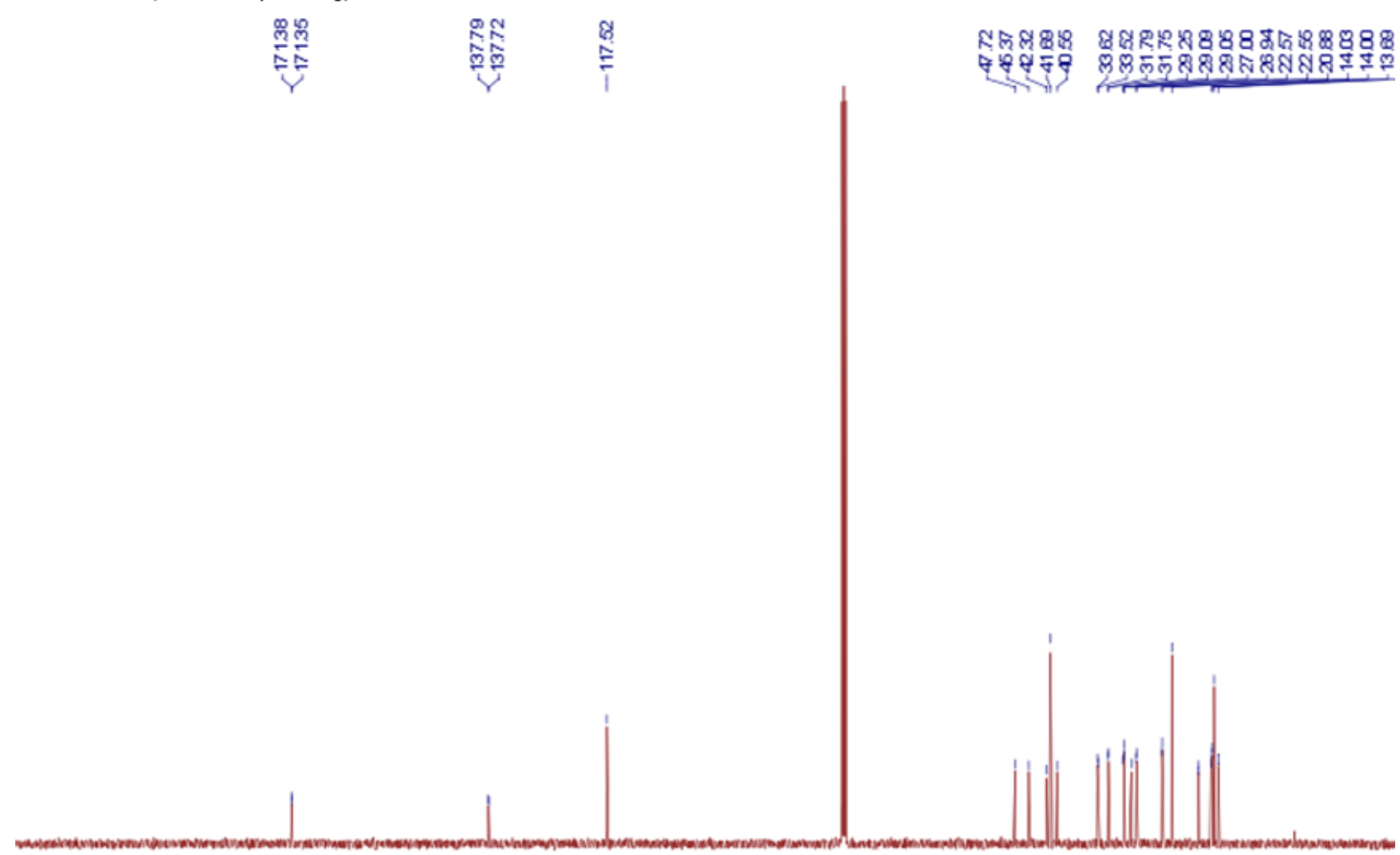

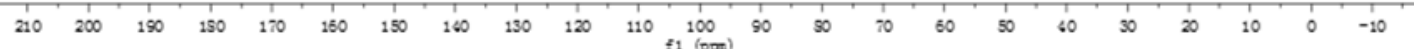


<smiles>CCN(CCc1ccccc1)C(=O)C/C=C(\C)C(C)C</smiles>

${ }^{1} \mathrm{H} \mathrm{NMR}\left(400 \mathrm{MHz}, \mathrm{CDCl}_{3}\right)$
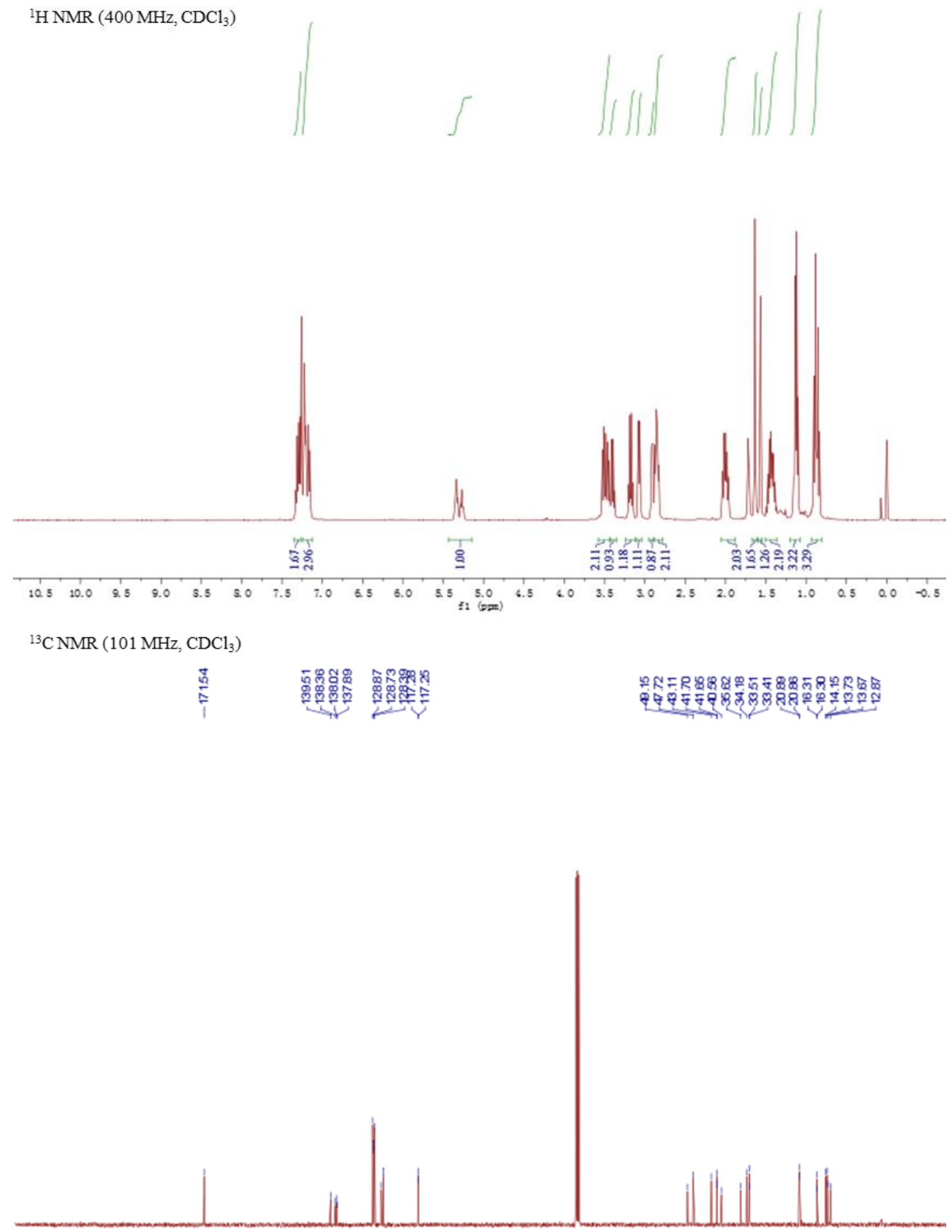

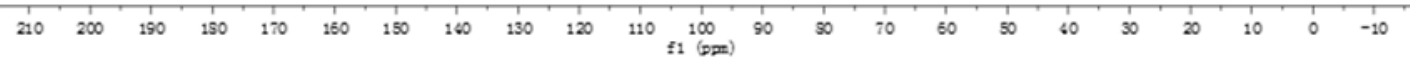


<smiles>CCN(CCc1cccs1)C(=O)C/C=C(\C)C(C)C</smiles>

${ }^{1} \mathrm{H} \mathrm{NMR}\left(400 \mathrm{MHz}, \mathrm{CDCl}_{3}\right)$

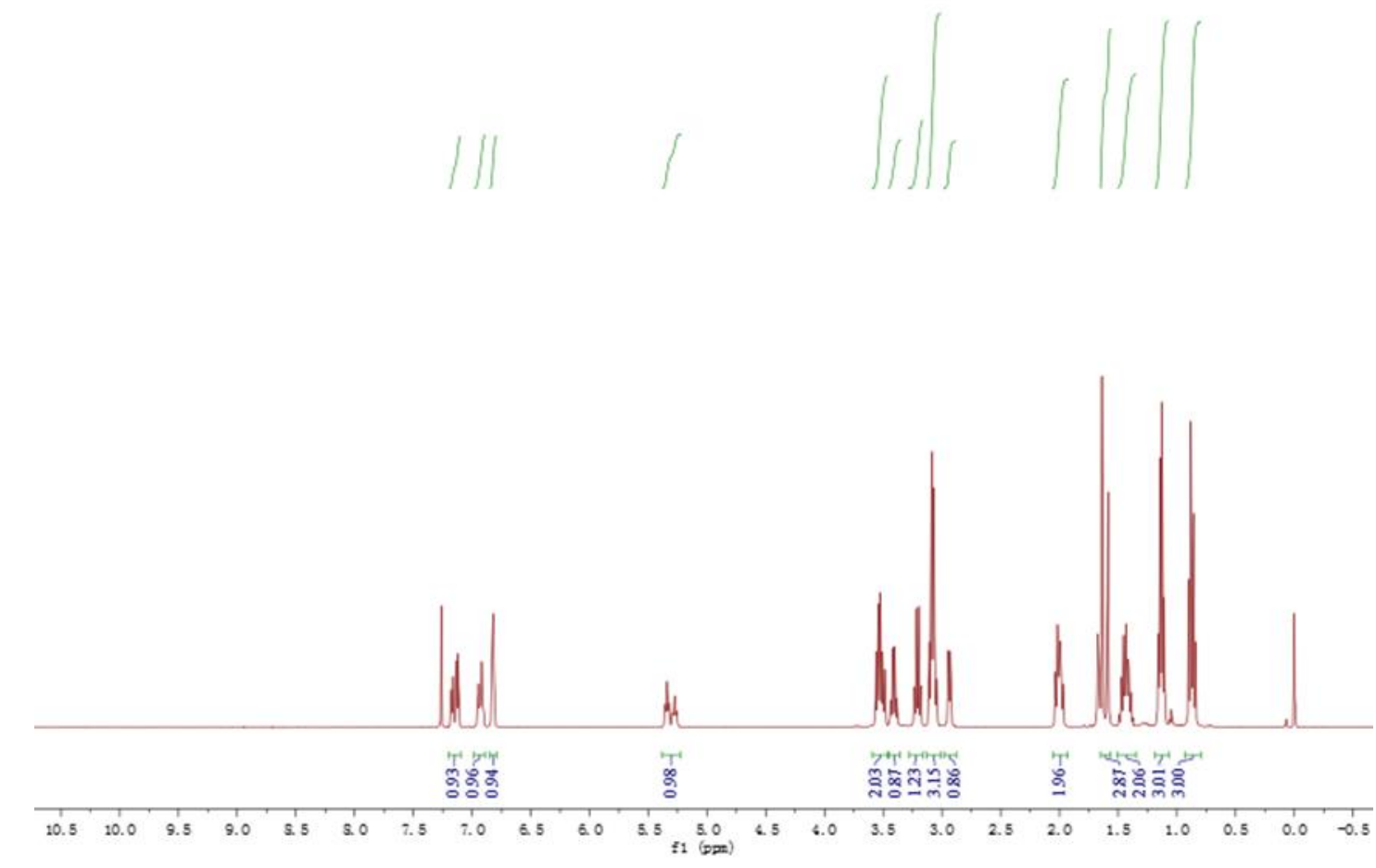

${ }^{13} \mathrm{CNMR}\left(101 \mathrm{MHz}, \mathrm{CDCl}_{3}\right)$

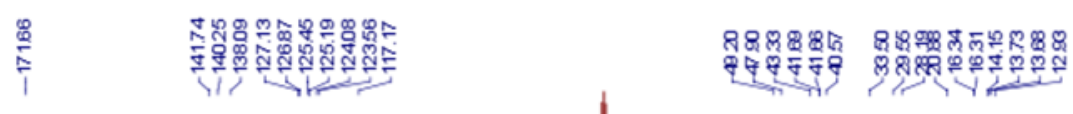

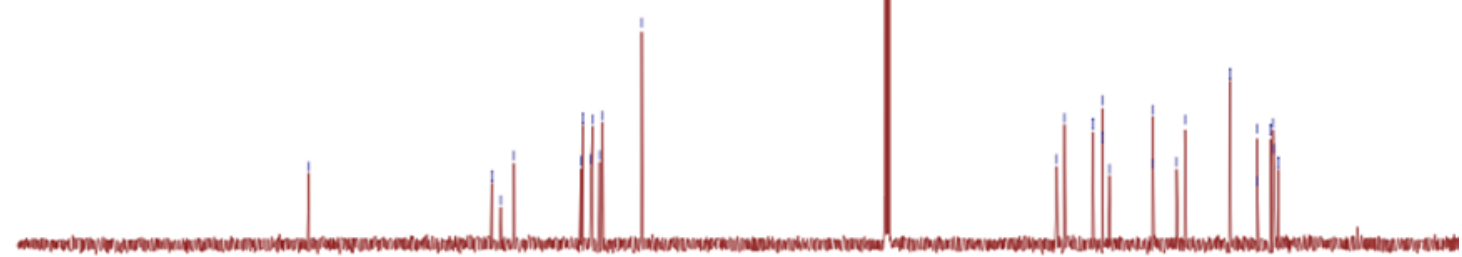

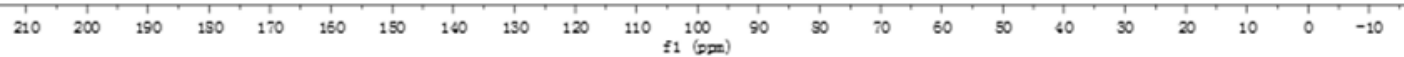


<smiles>CCN(CCc1ccco1)C(=O)C/C=C(\C)C(C)C</smiles>

${ }^{1} \mathrm{H}$ NMR $\left(400 \mathrm{MHz}, \mathrm{CDCl}_{3}\right)$

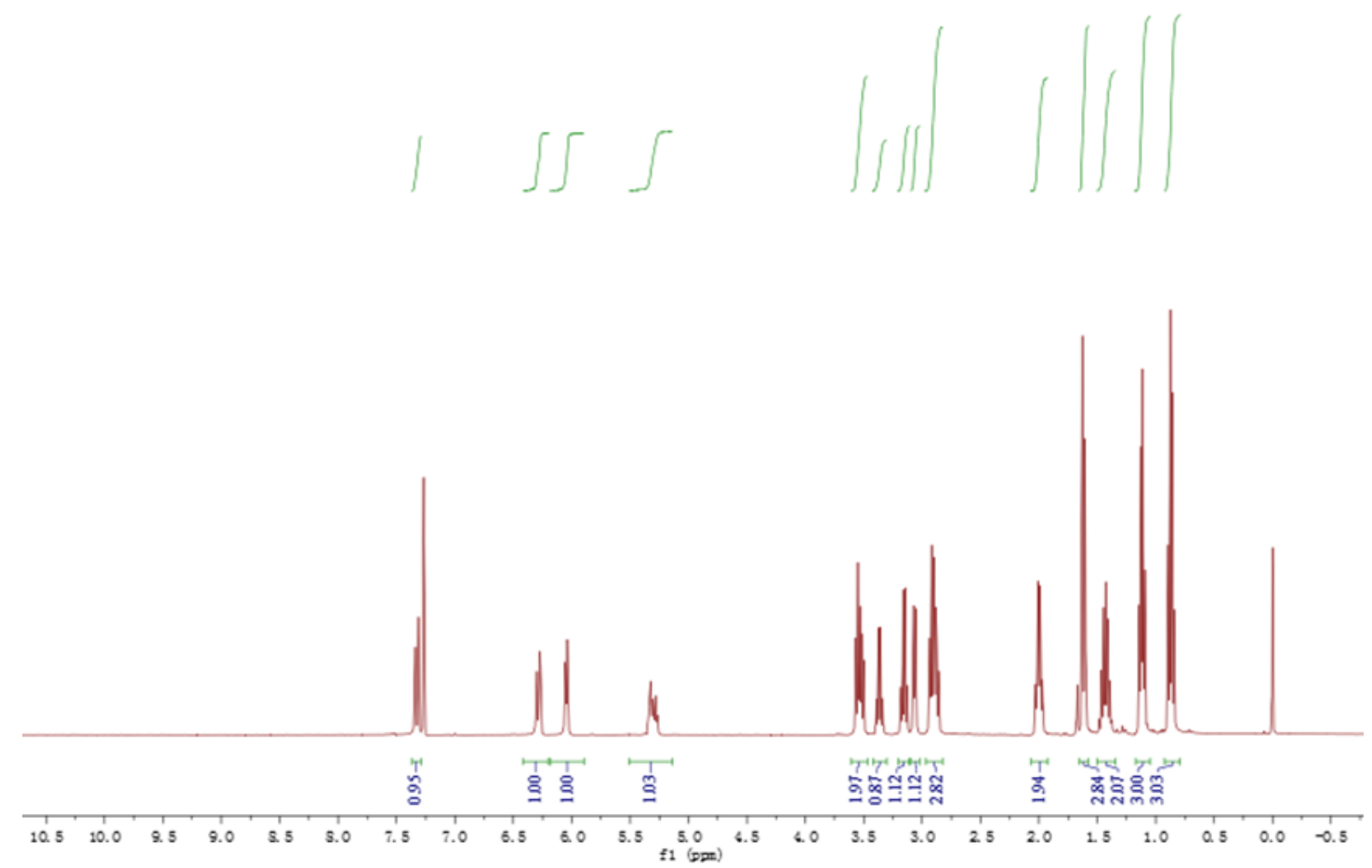

${ }^{13} \mathrm{CNMR}\left(101 \mathrm{MHz}, \mathrm{CDCl}_{3}\right)$

Nक्ष

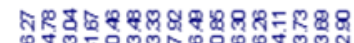

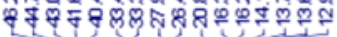

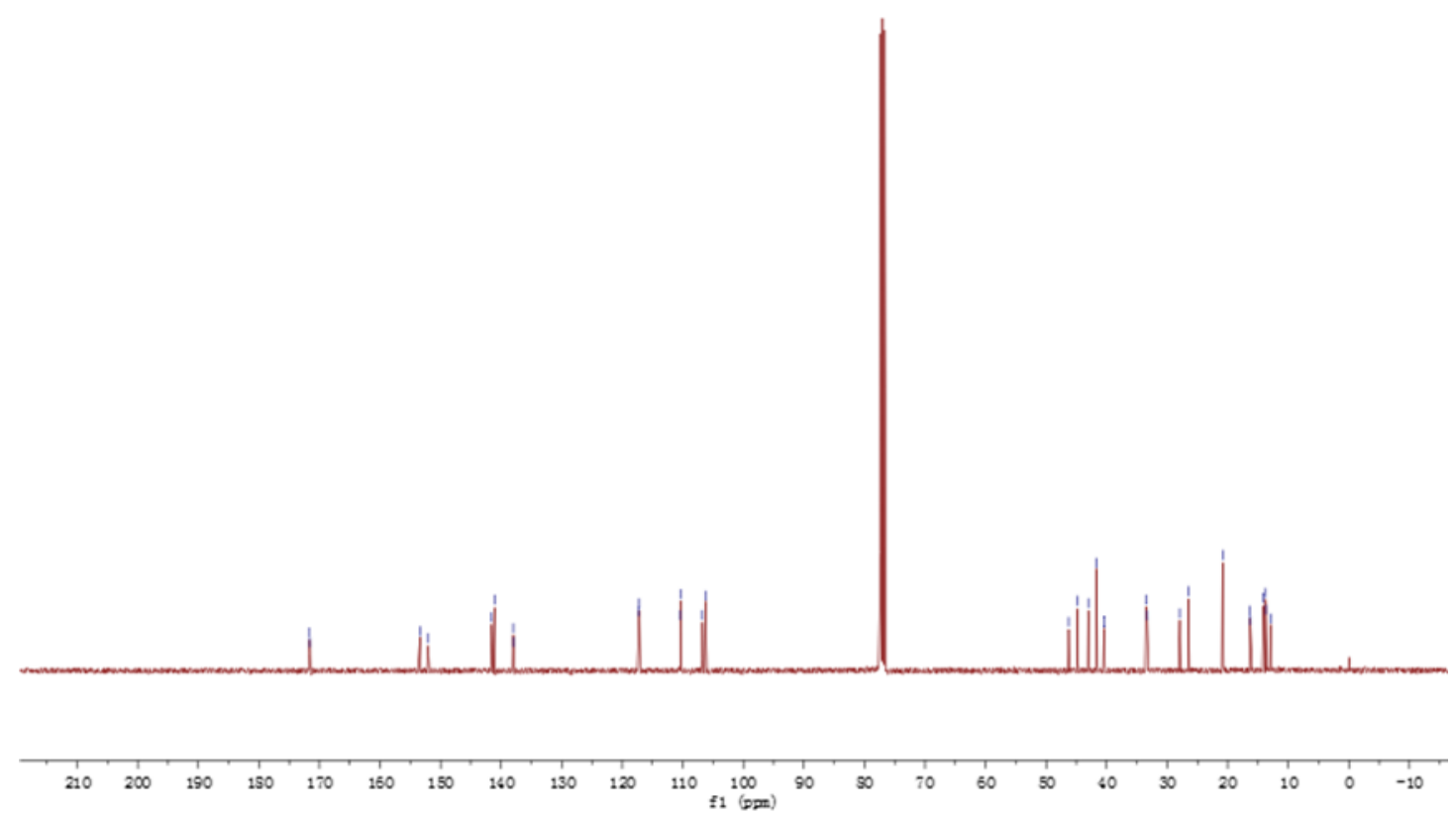


<smiles>CCN(CCOC)C(=O)C/C=C(\C)C(C)C</smiles>

${ }^{1} \mathrm{H} \mathrm{NMR}\left(400 \mathrm{MHz}, \mathrm{CDCl}_{3}\right)$

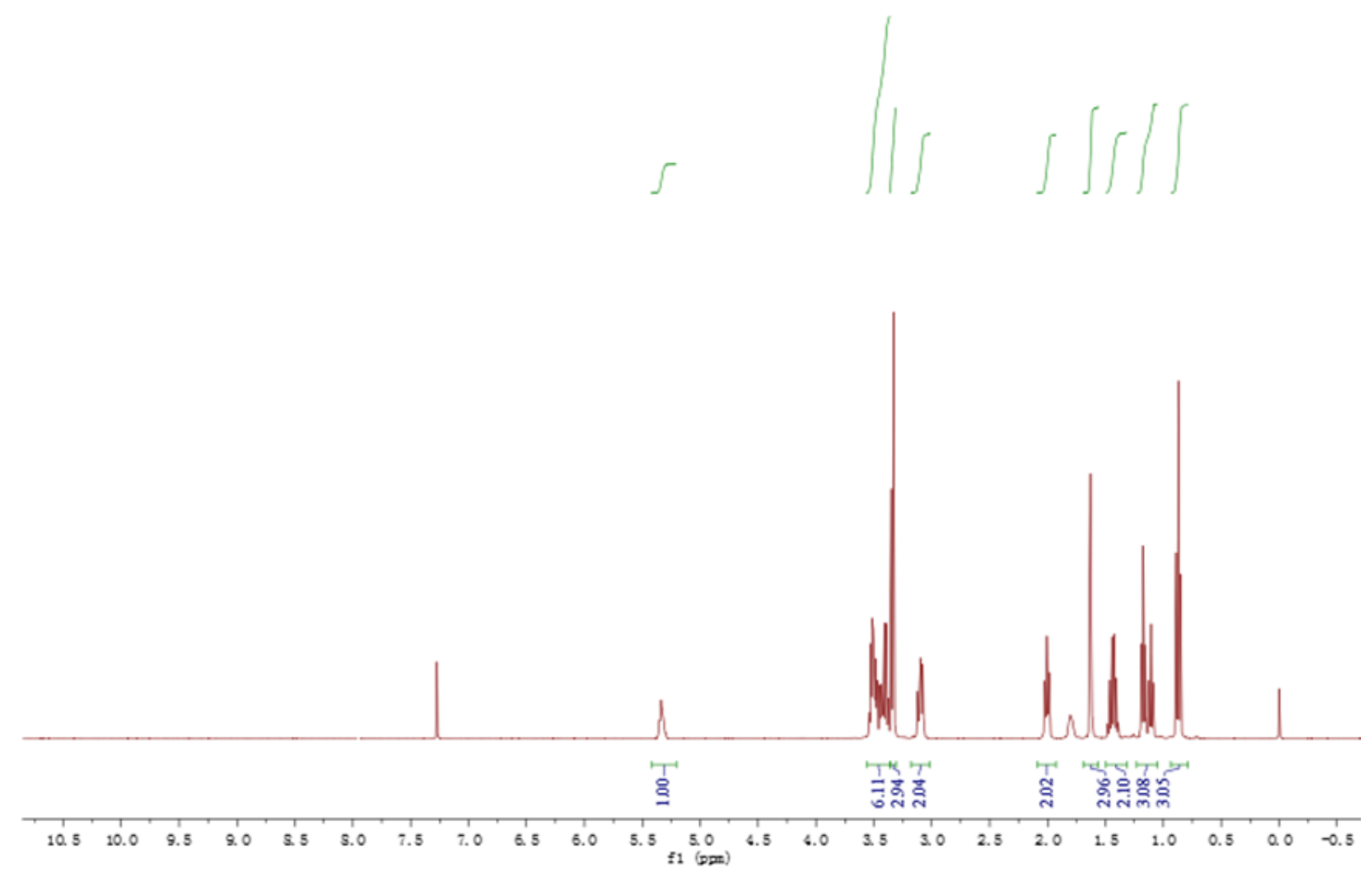

${ }^{13} \mathrm{CNMR}\left(101 \mathrm{MHz}, \mathrm{CDCl}_{3}\right)$

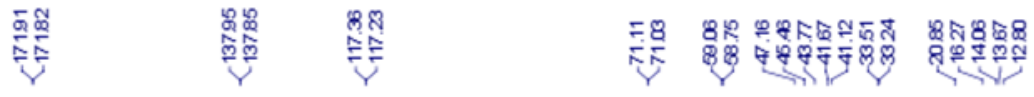

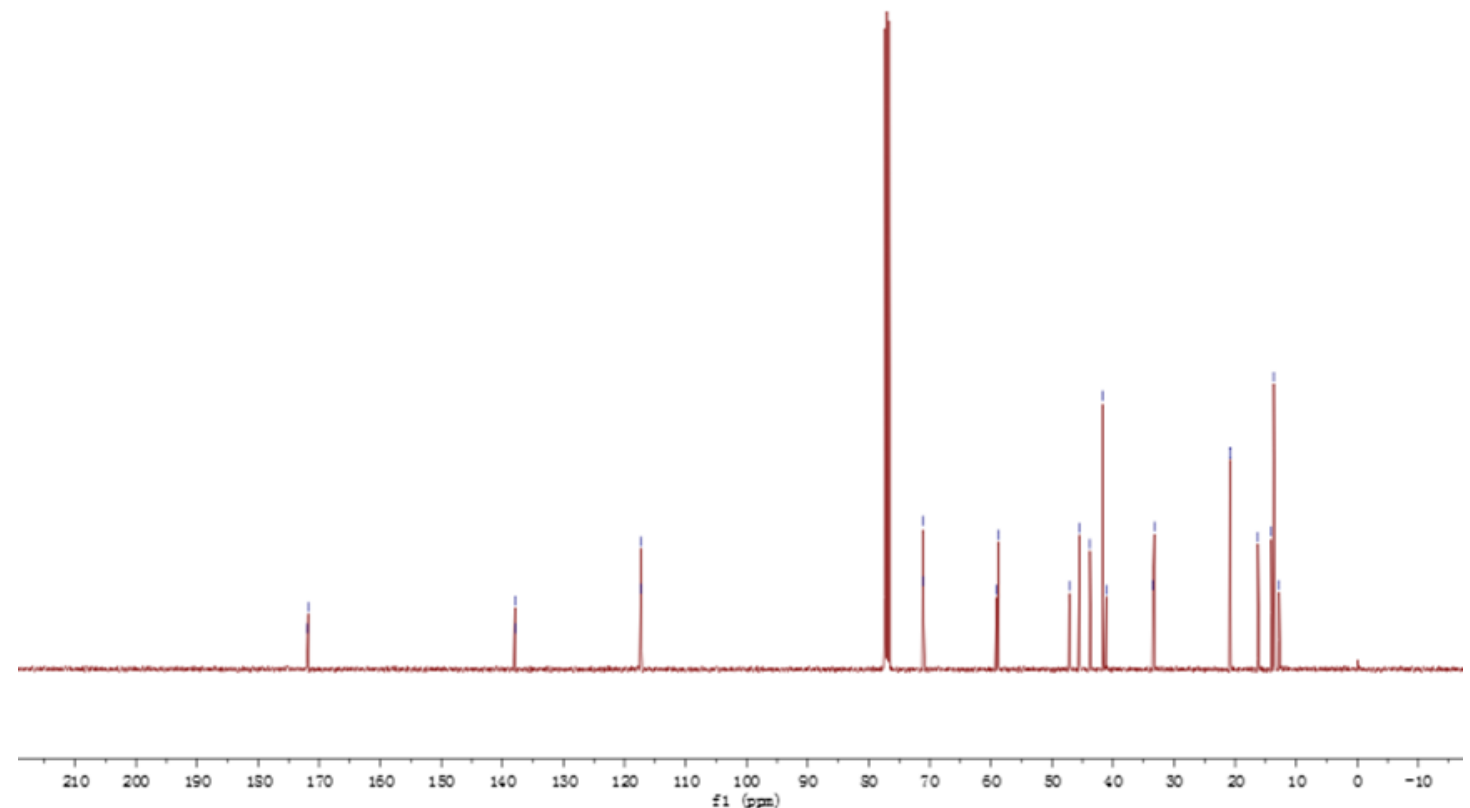


<smiles>CCN(CCOC)C(=O)C/C=C(/C)C(C)C</smiles>

${ }^{1} \mathrm{H} \mathrm{NMR}\left(400 \mathrm{MHz}, \mathrm{CDCl}_{3}\right)$

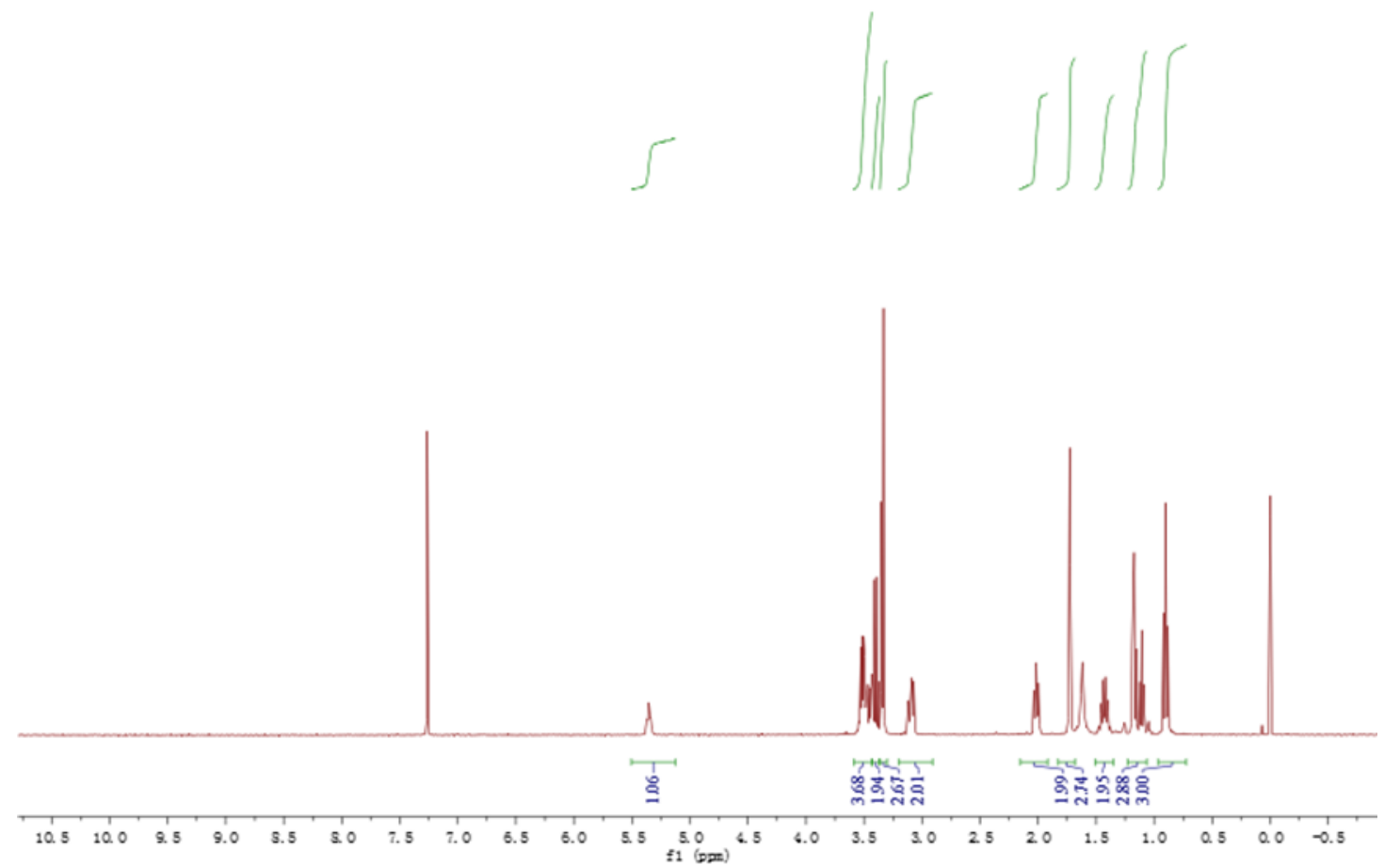

${ }^{13} \mathrm{CNMR}\left(101 \mathrm{MHz}, \mathrm{CDCl}_{3}\right)$

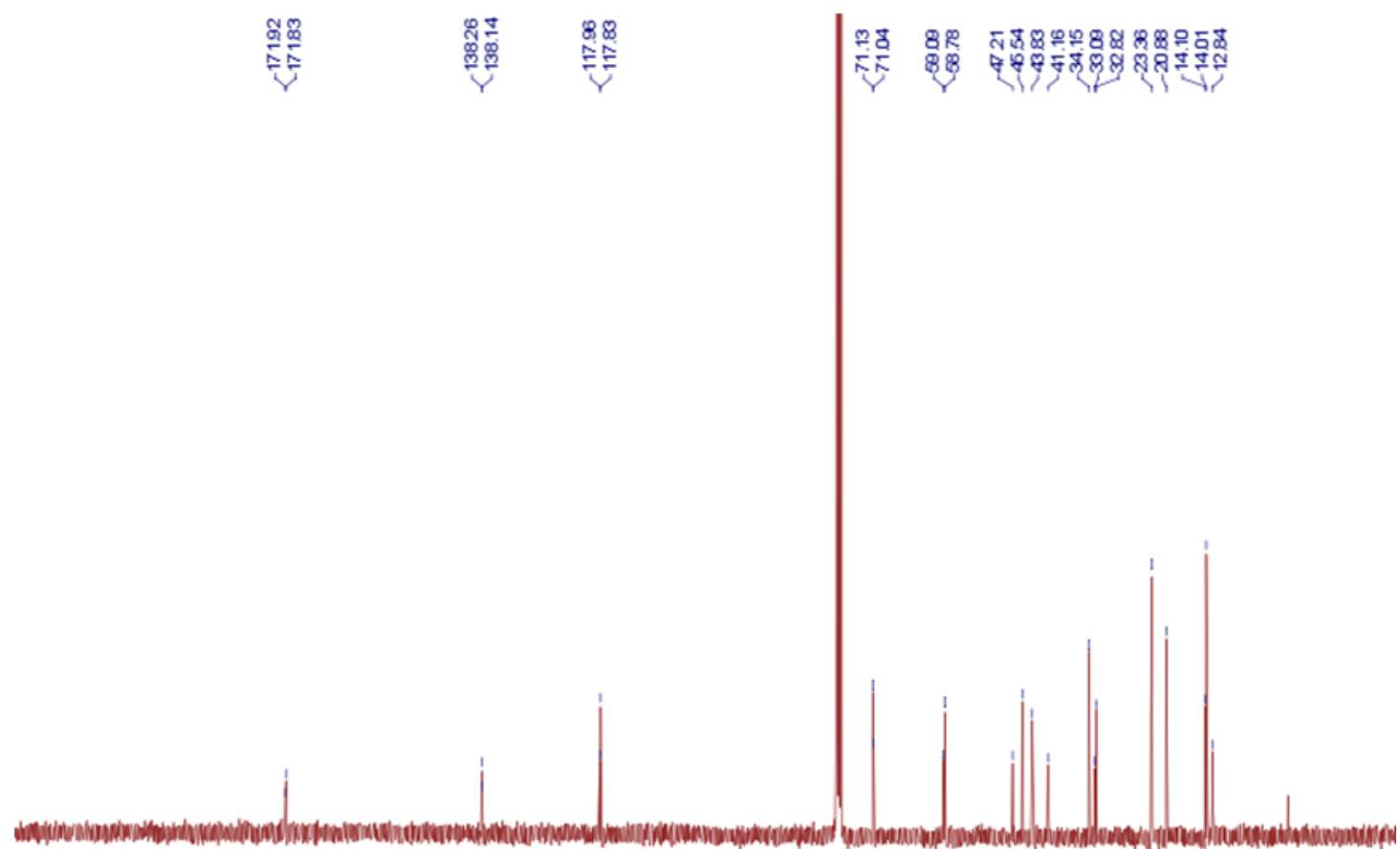

$\begin{array}{llllllllllllllllllllllll}210 & 200 & 190 & 150 & 170 & 160 & 150 & 160 & 130 & 120 & 110 & 100 & 90 & 50 & 70 & 60 & 50 & 40 & 30 & 20 & 10 & 0 & -10\end{array}$ 
<smiles>CCN(CCOC)C(=O)C/C=C(\C)C(C)C</smiles>

${ }^{1} \mathrm{H}$ NMR $\left(400 \mathrm{MHz}, \mathrm{CDCl}_{3}\right)$

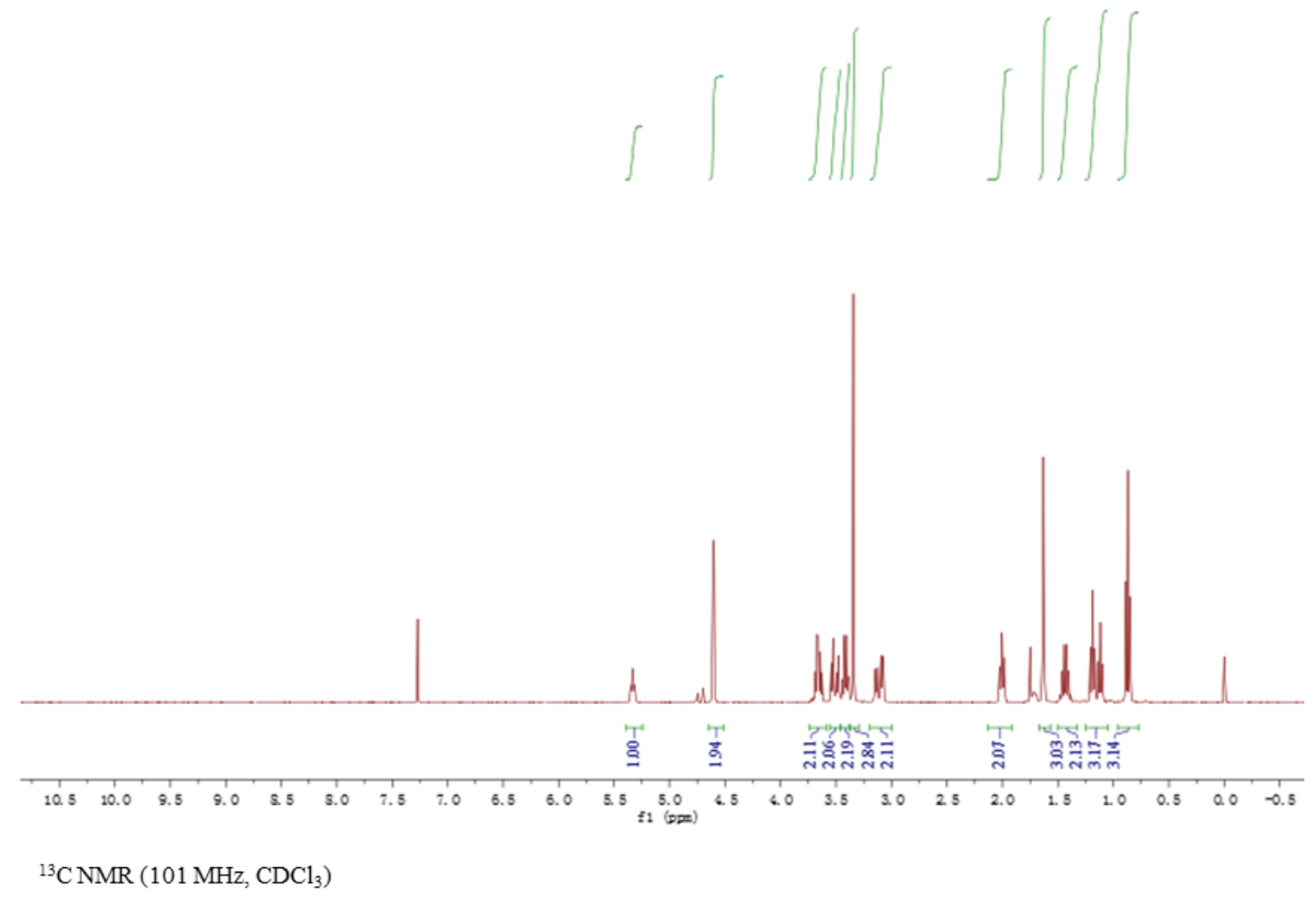

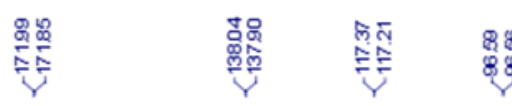

8 \&

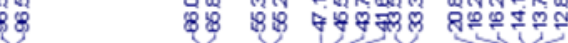

$\begin{array}{lllllllllllllllllllllllll}210 & 200 & 190 & 150 & 170 & 160 & 150 & 160 & 130 & 120 & 110 & 100 & 90 & 50 & 70 & 60 & 50 & 40 & 30 & 20 & 10 & 0 & -10\end{array}$ 


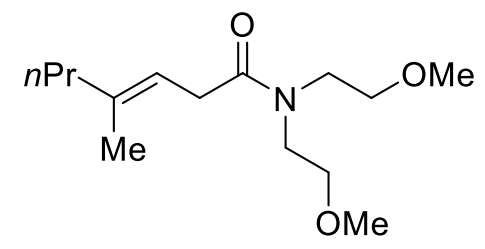

${ }^{1} \mathrm{H} \mathrm{NMR}\left(400 \mathrm{MHz}, \mathrm{CDCl}_{3}\right)$

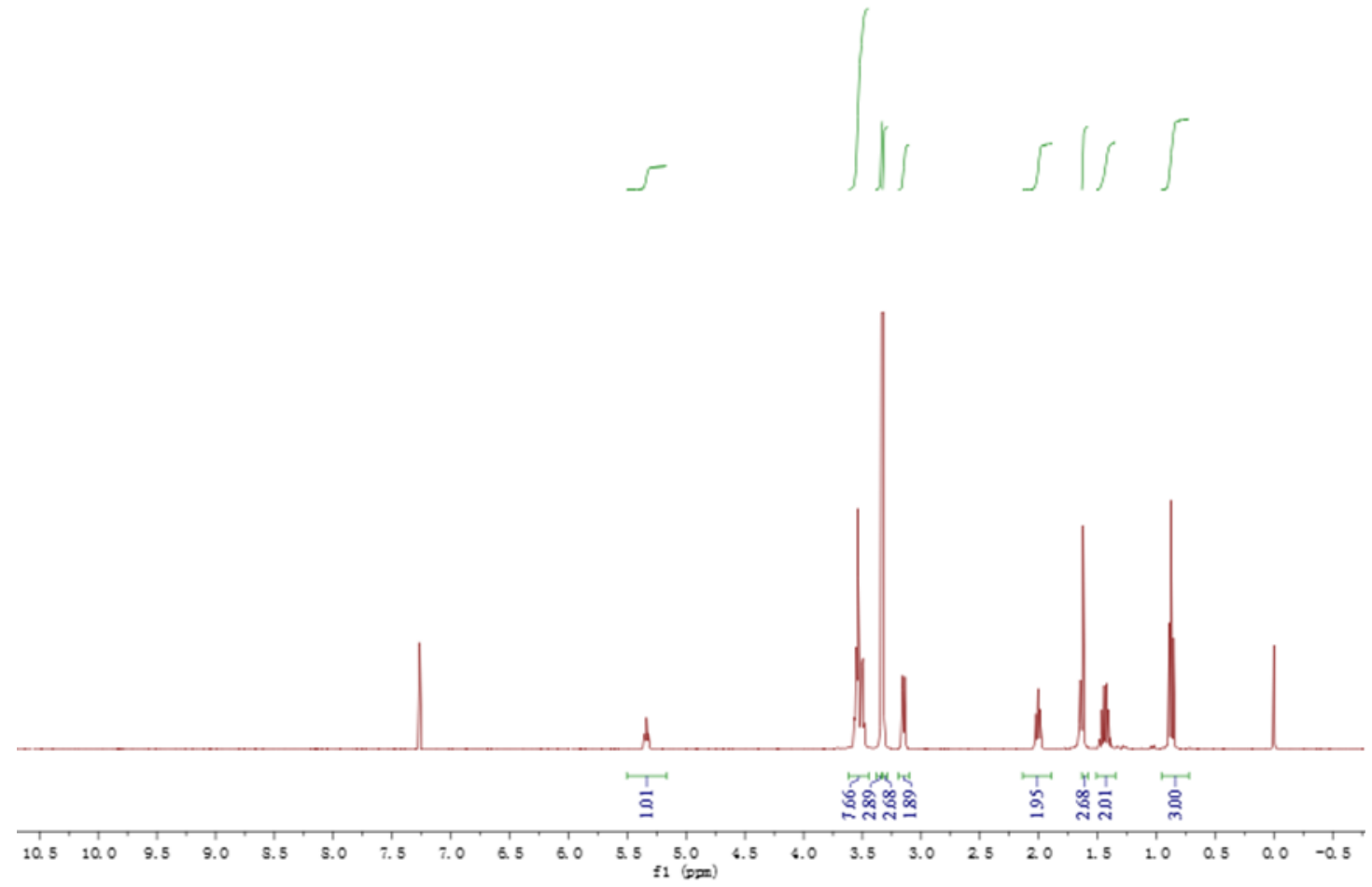

${ }^{13} \mathrm{CNMR}\left(101 \mathrm{MHz}, \mathrm{CDCl}_{3}\right)$

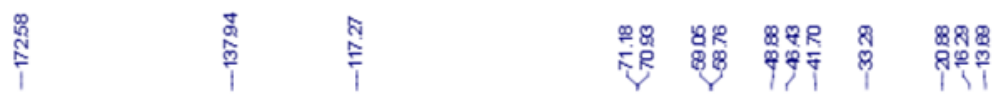

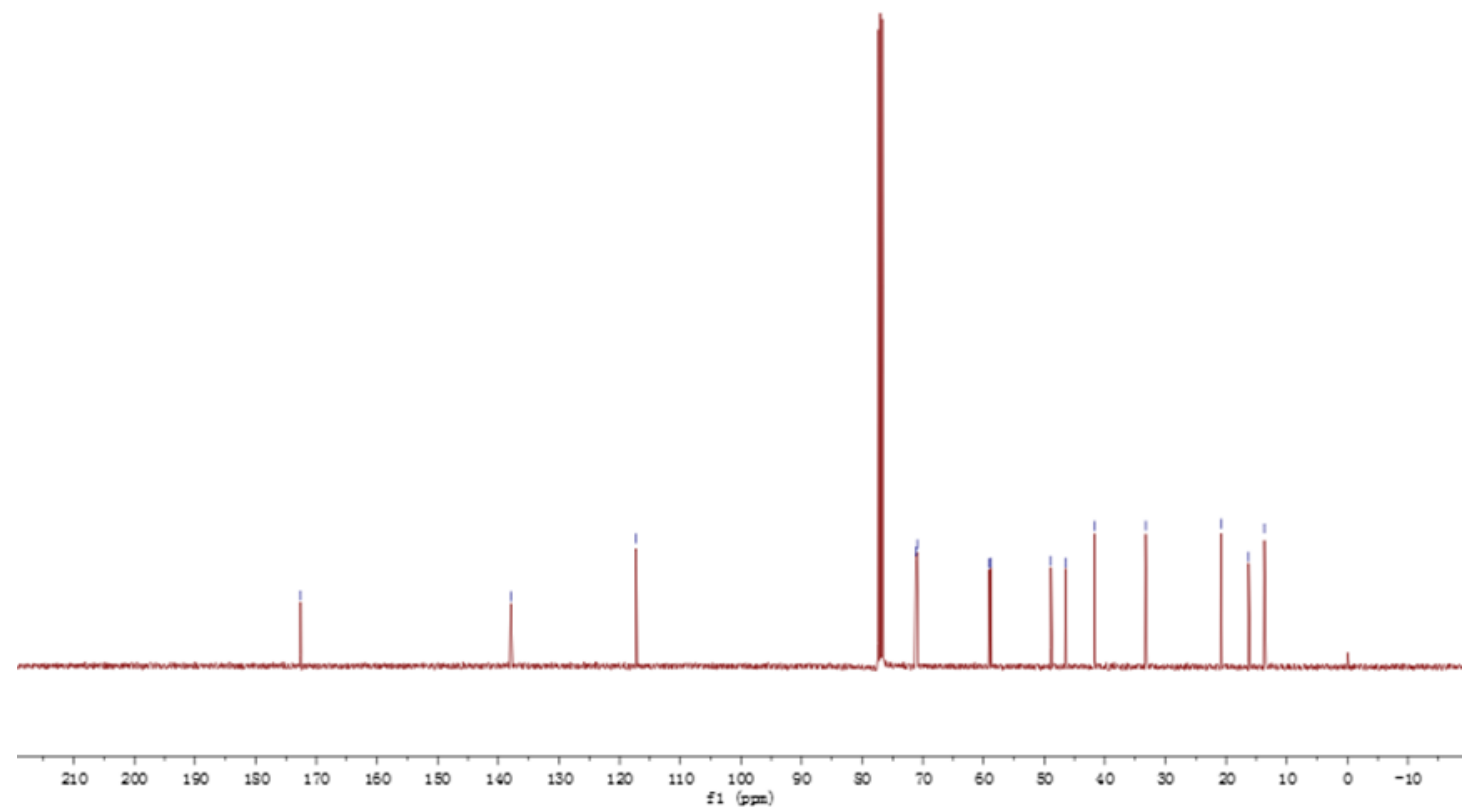


<smiles>CCN(CCC1=CCCCC1)C(=O)C/C=C(\C)C(C)C</smiles>

${ }^{1} \mathrm{H}$ NMR $\left(400 \mathrm{MHz}, \mathrm{CDCl}_{3}\right)$

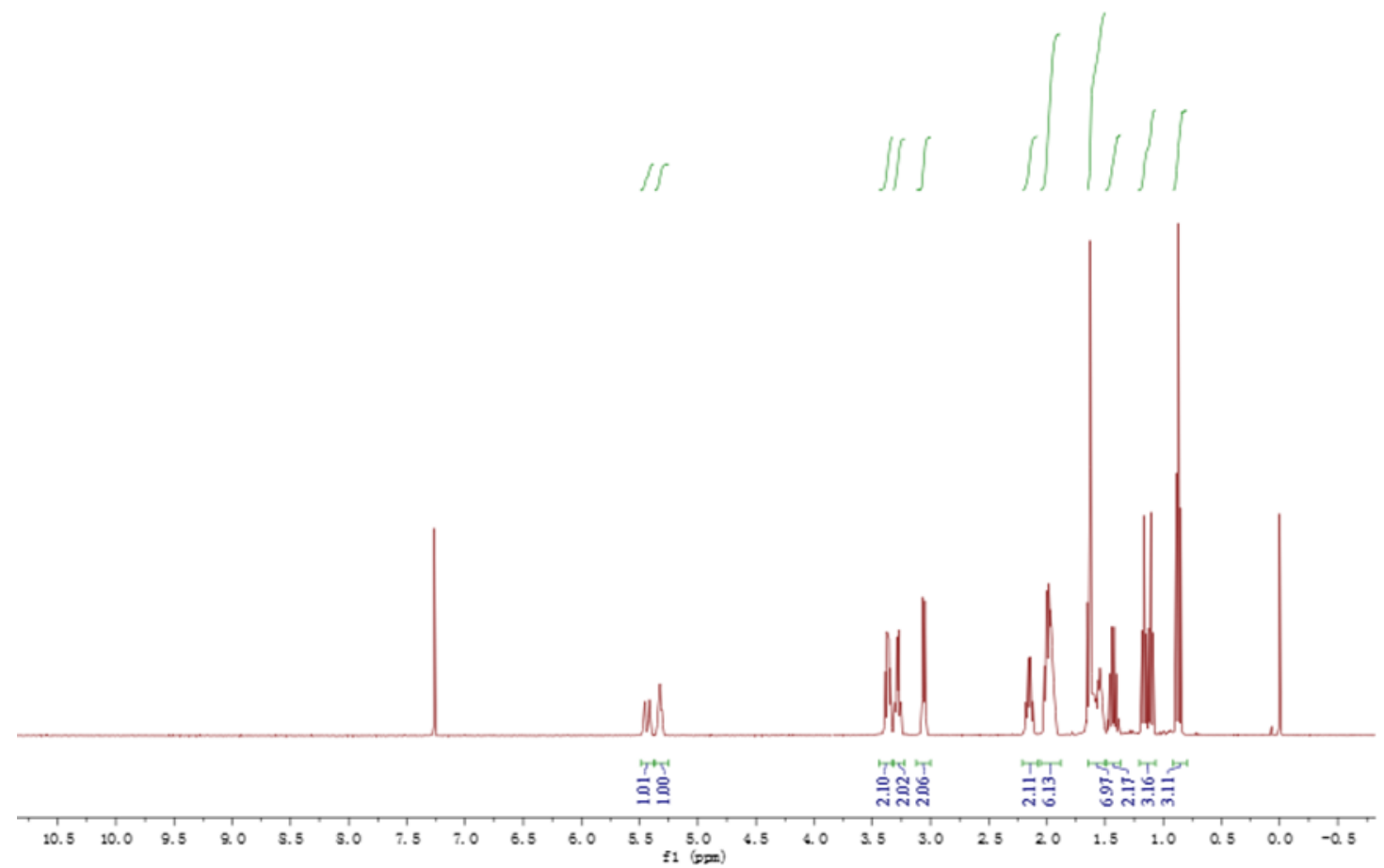

${ }^{13} \mathrm{CNMR}\left(101 \mathrm{MHz}, \mathrm{CDCl}_{3}\right)$

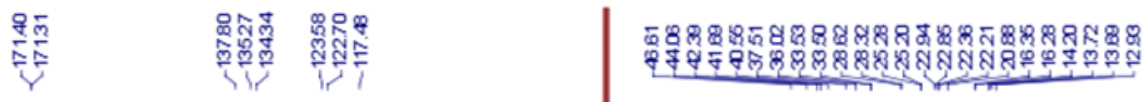

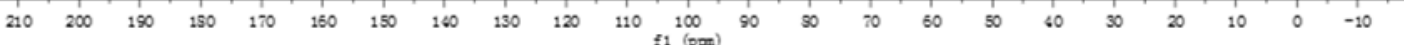


<smiles>CC(=CCC(=O)N1CCOCC1)C(C)C</smiles>

${ }^{1} \mathrm{H} \mathrm{NMR}\left(400 \mathrm{MHz}, \mathrm{CDCl}_{3}\right)$

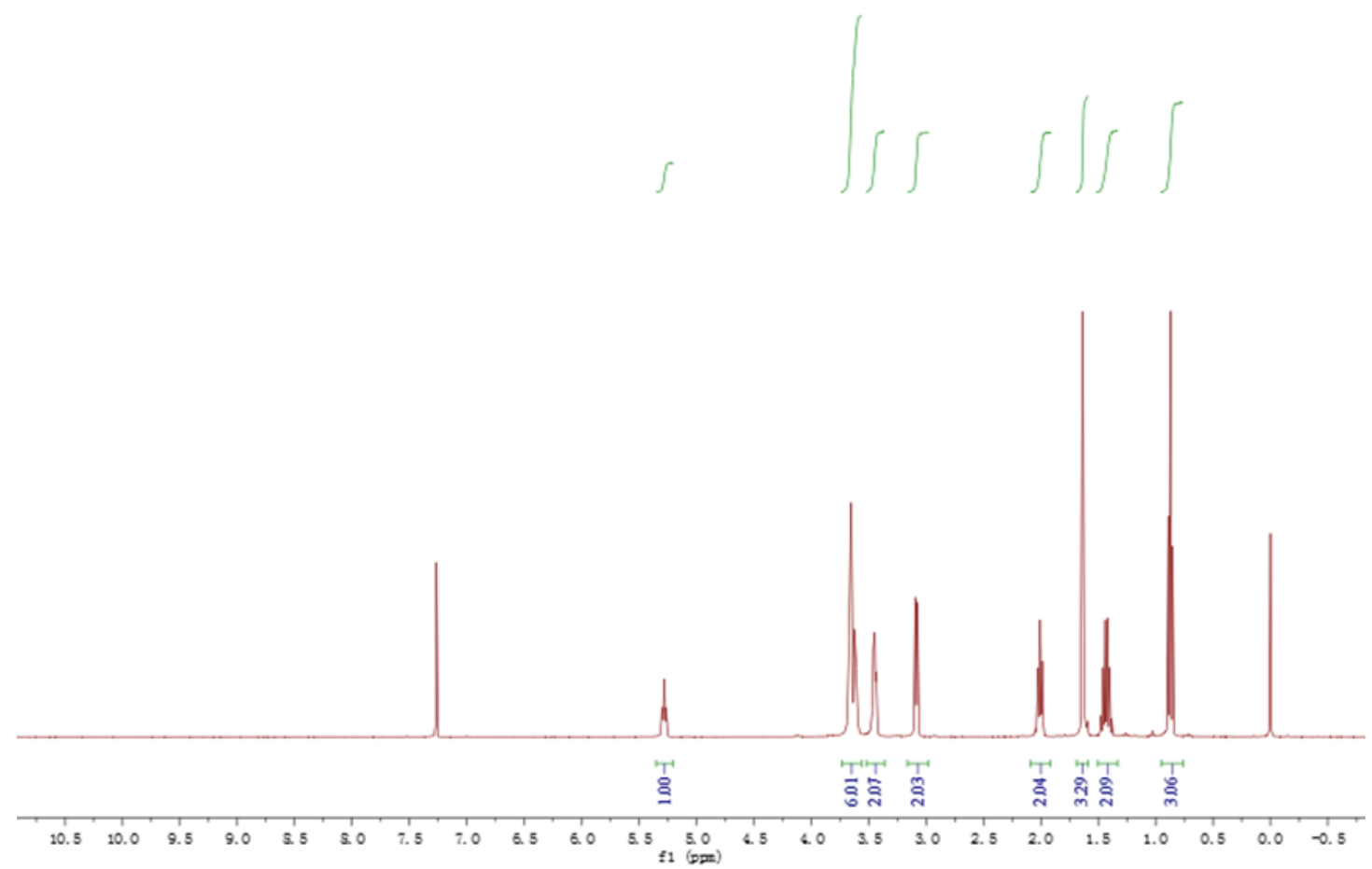

${ }^{13} \mathrm{CNMR}\left(101 \mathrm{MHz}, \mathrm{CDCl}_{3}\right)$

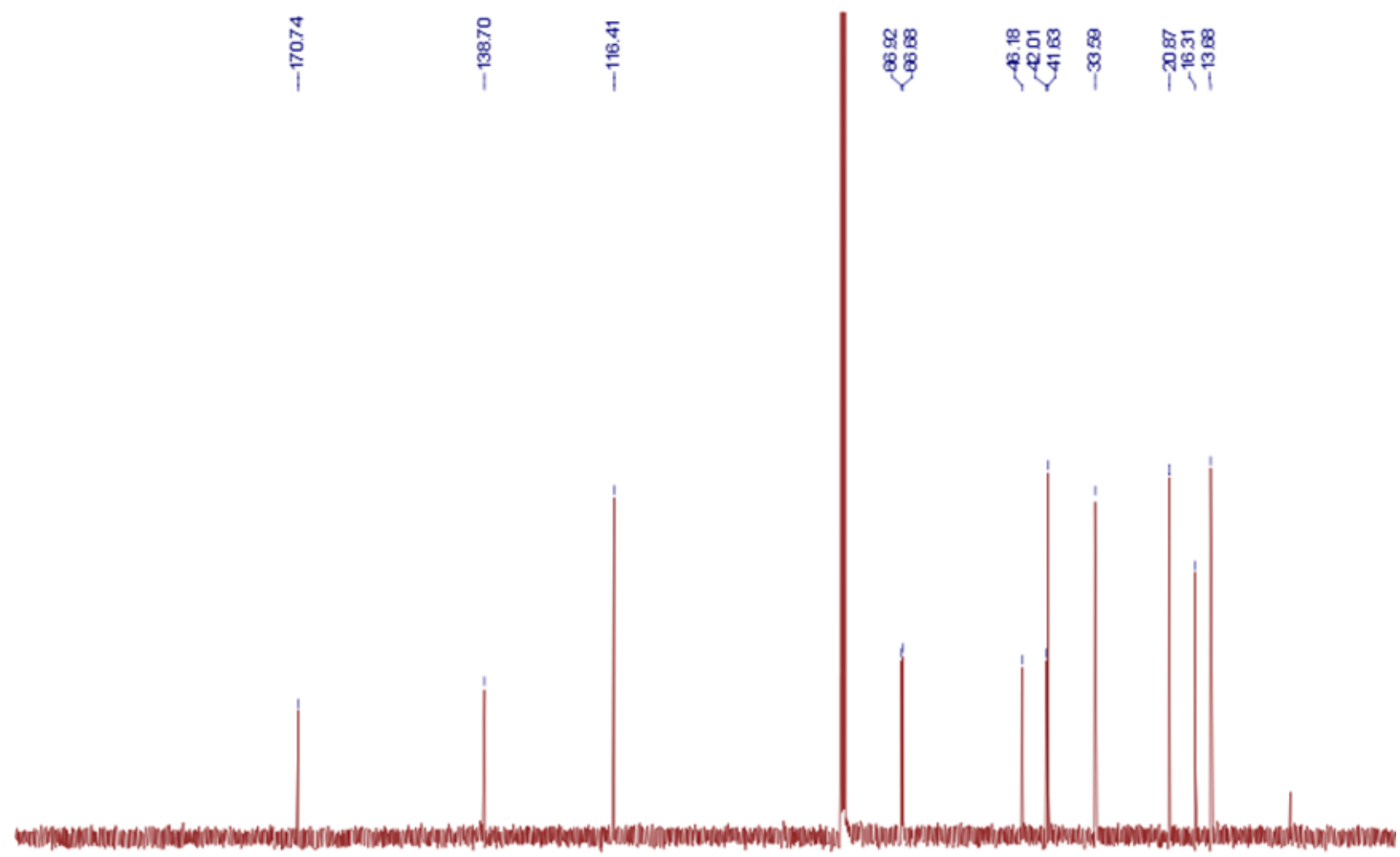

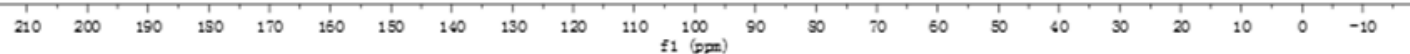


<smiles>CCN(CCOC)C(=O)C/C=C(\C)c1ccccc1</smiles>

${ }^{1} \mathrm{H}$ NMR $\left(400 \mathrm{MHz}, \mathrm{CDCl}_{3}\right)$

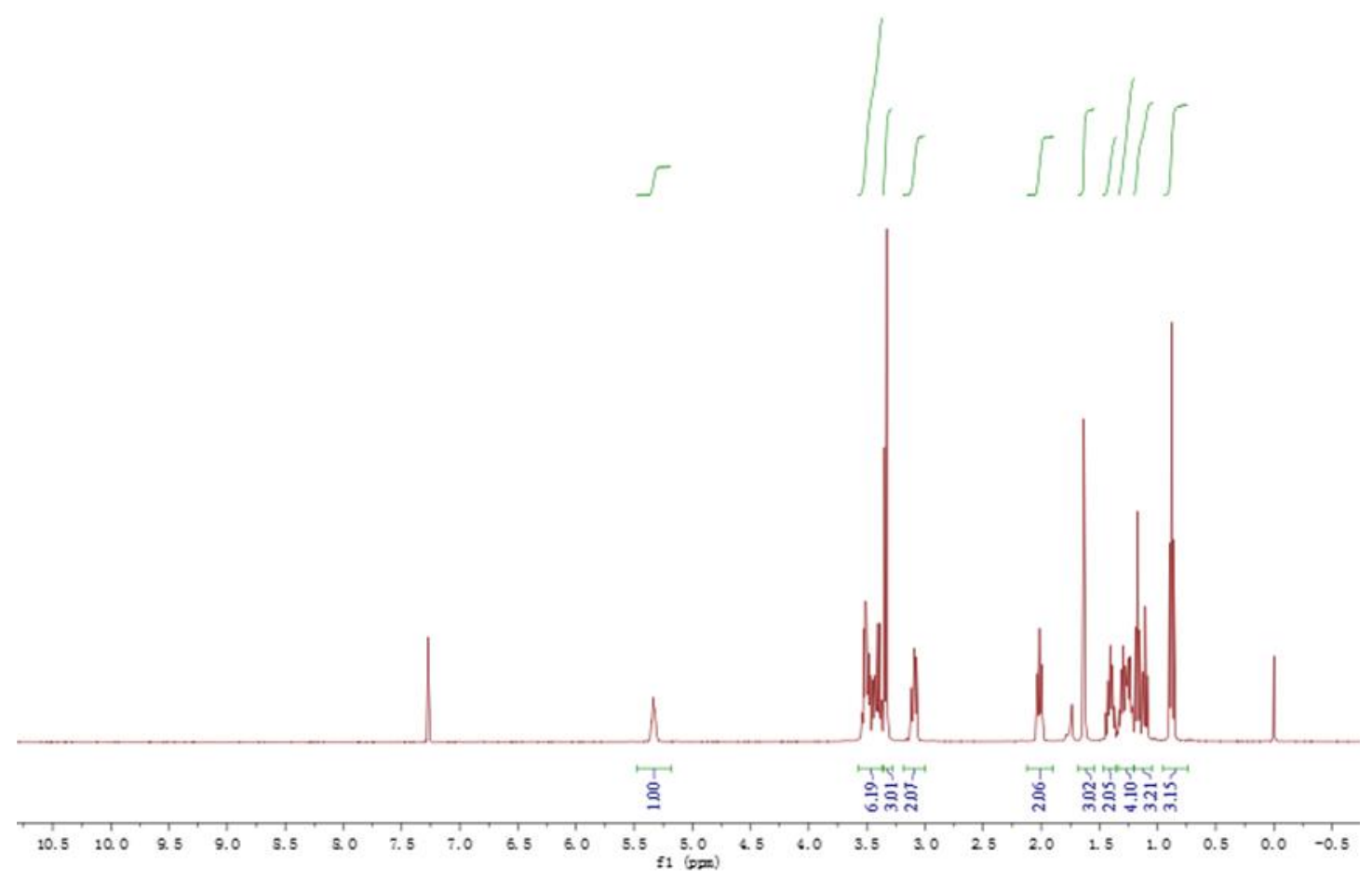

${ }^{13} \mathrm{CNMR}\left(101 \mathrm{MHz}, \mathrm{CDCl}_{3}\right)$

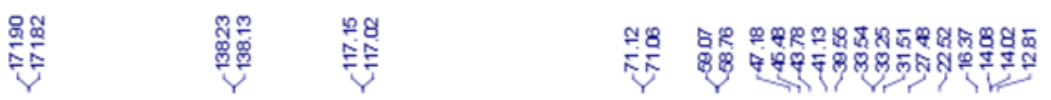

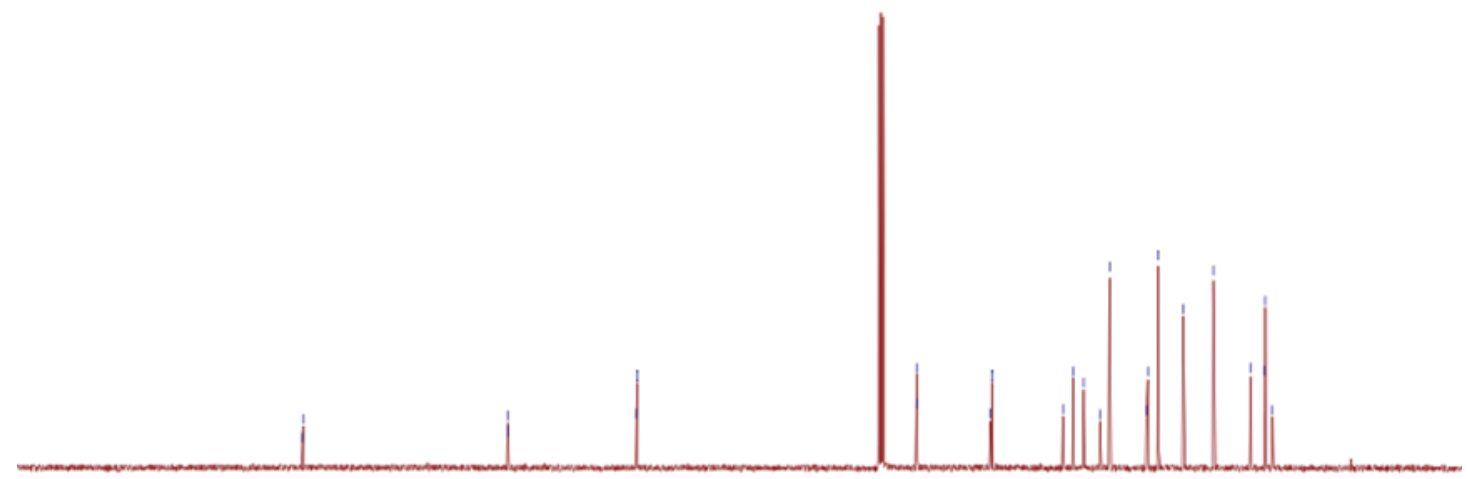

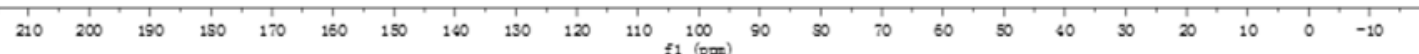


<smiles>CCN(CCOC)C(=O)C/C=C(\C)[In]c1ccccc1</smiles>

${ }^{1} \mathrm{H} \mathrm{NMR}\left(400 \mathrm{MHz}, \mathrm{CDCl}_{3}\right)$

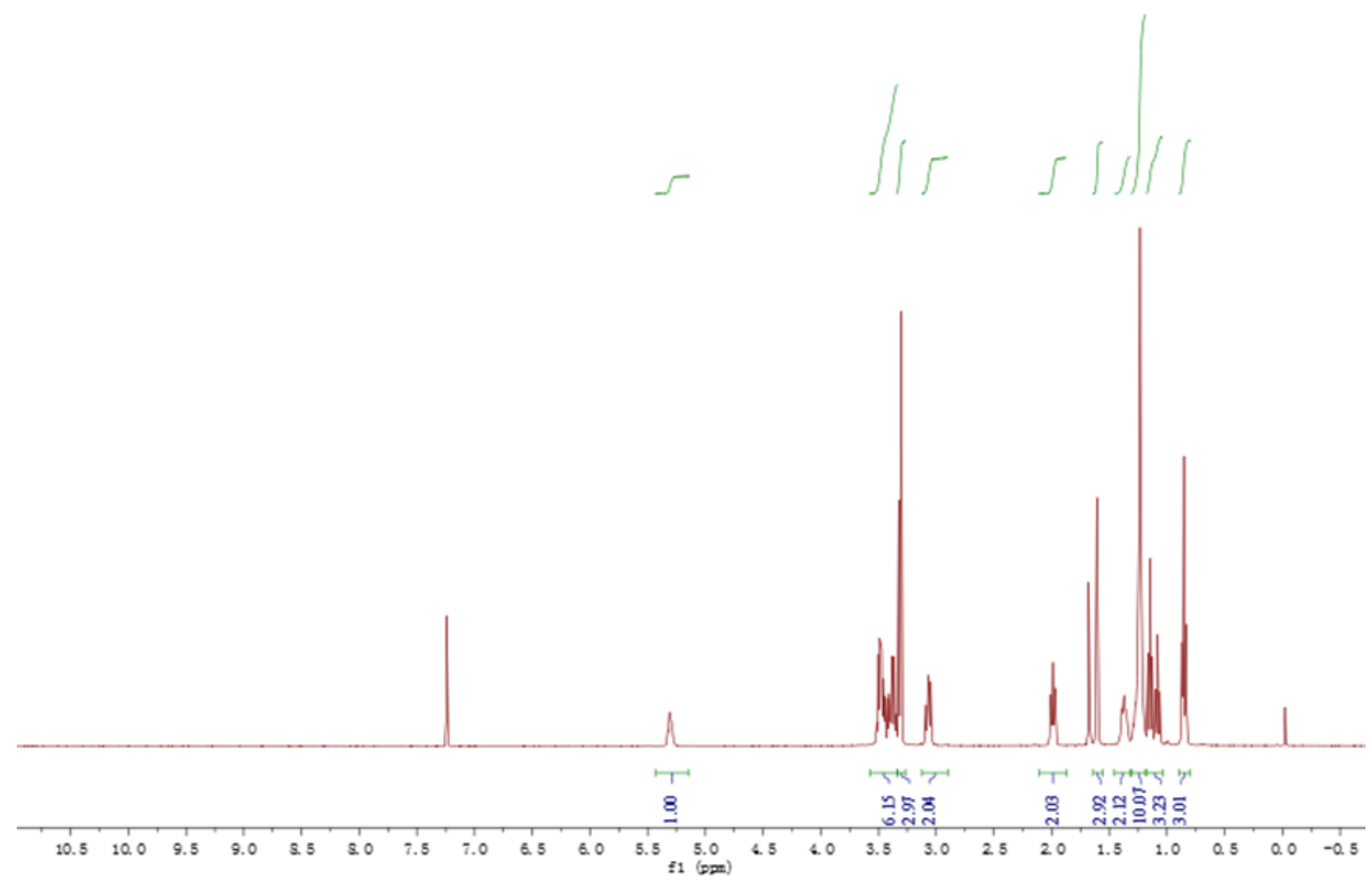
${ }^{13} \mathrm{CNMR}\left(101 \mathrm{MHz}, \mathrm{CDCl}_{3}\right)$

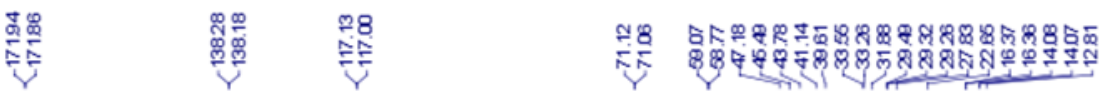

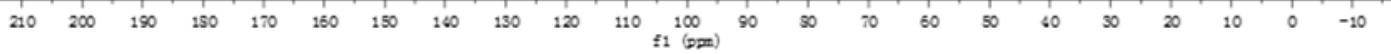


<smiles>CCN(CCOC)C(=O)C/C=C(/C)CCC(C)C</smiles>

${ }^{1} \mathrm{H} \mathrm{NMR}\left(400 \mathrm{MHz}, \mathrm{CDCl}_{3}\right)$

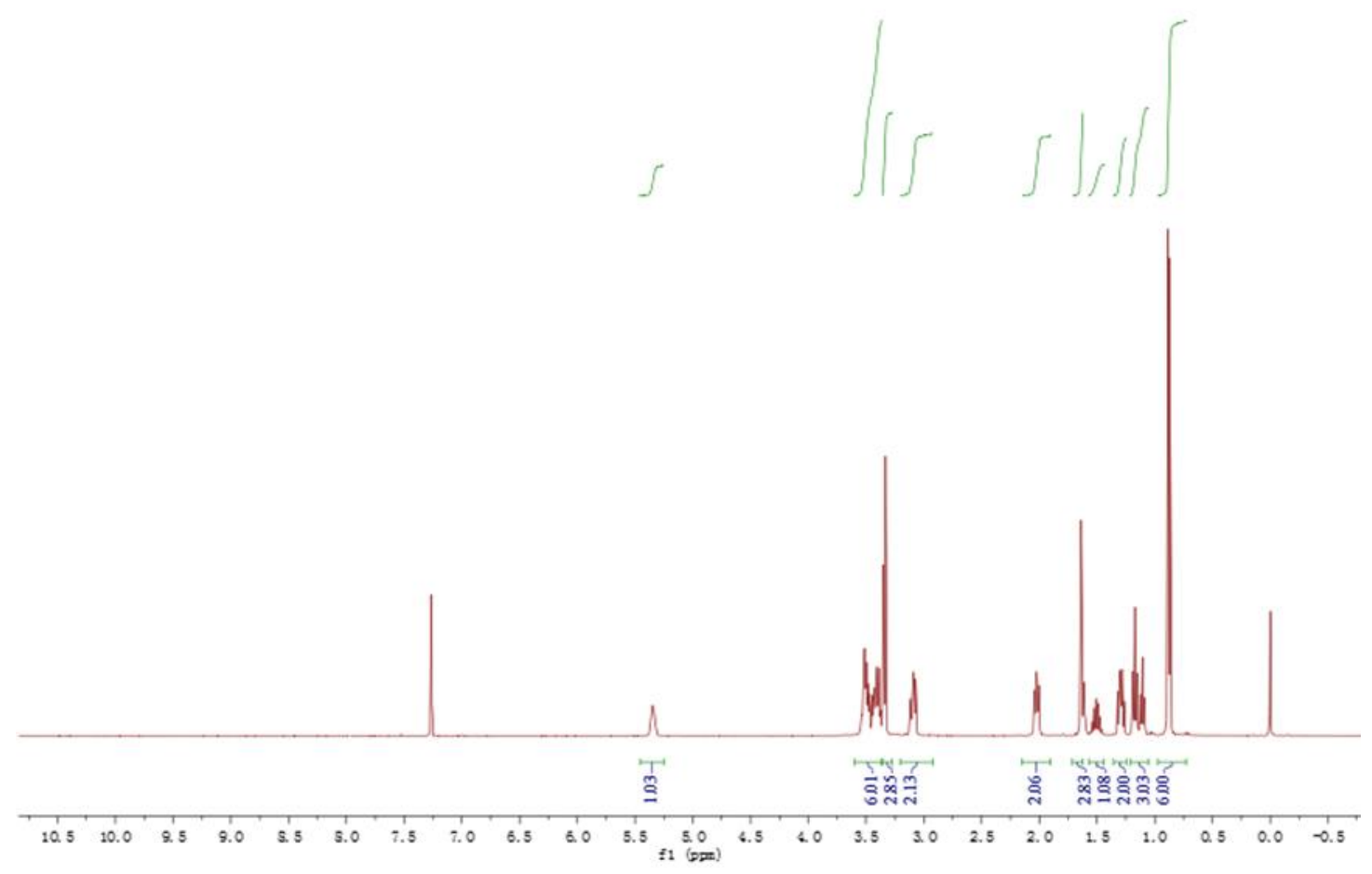

${ }^{13} \mathrm{CNMR}\left(101 \mathrm{MHz}, \mathrm{CDCl}_{3}\right)$

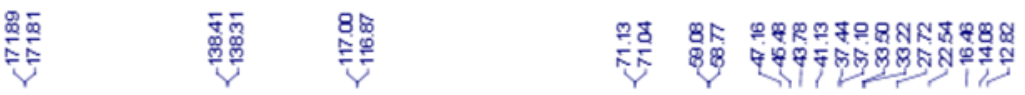

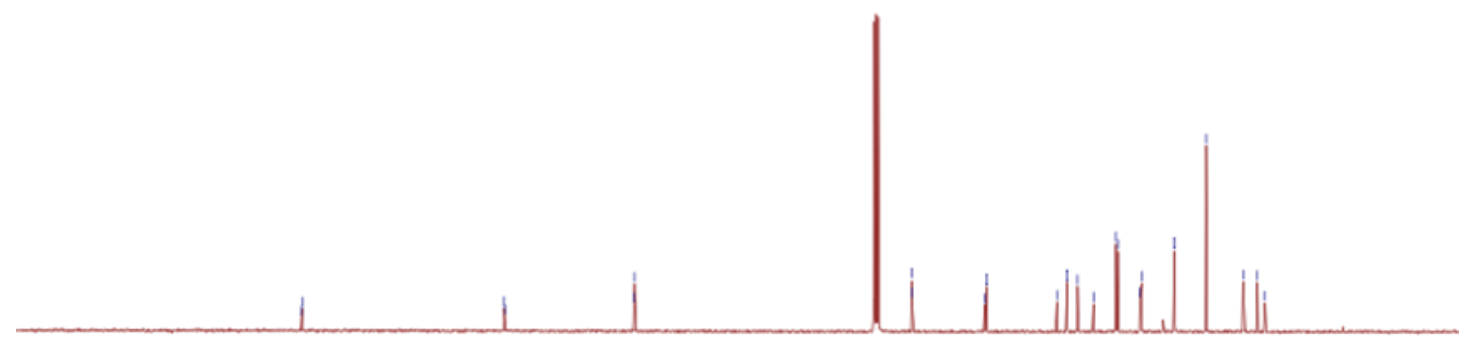

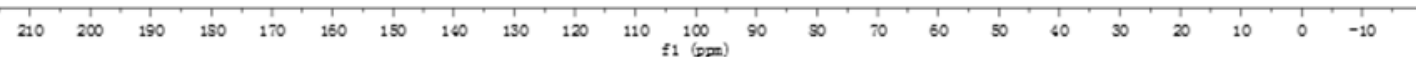


<smiles>CCN(CCOC)C(=O)C/C=C(\C)CC(C)C</smiles>

${ }^{1} \mathrm{H} \mathrm{NMR}\left(400 \mathrm{MHz}, \mathrm{CDCl}_{3}\right)$

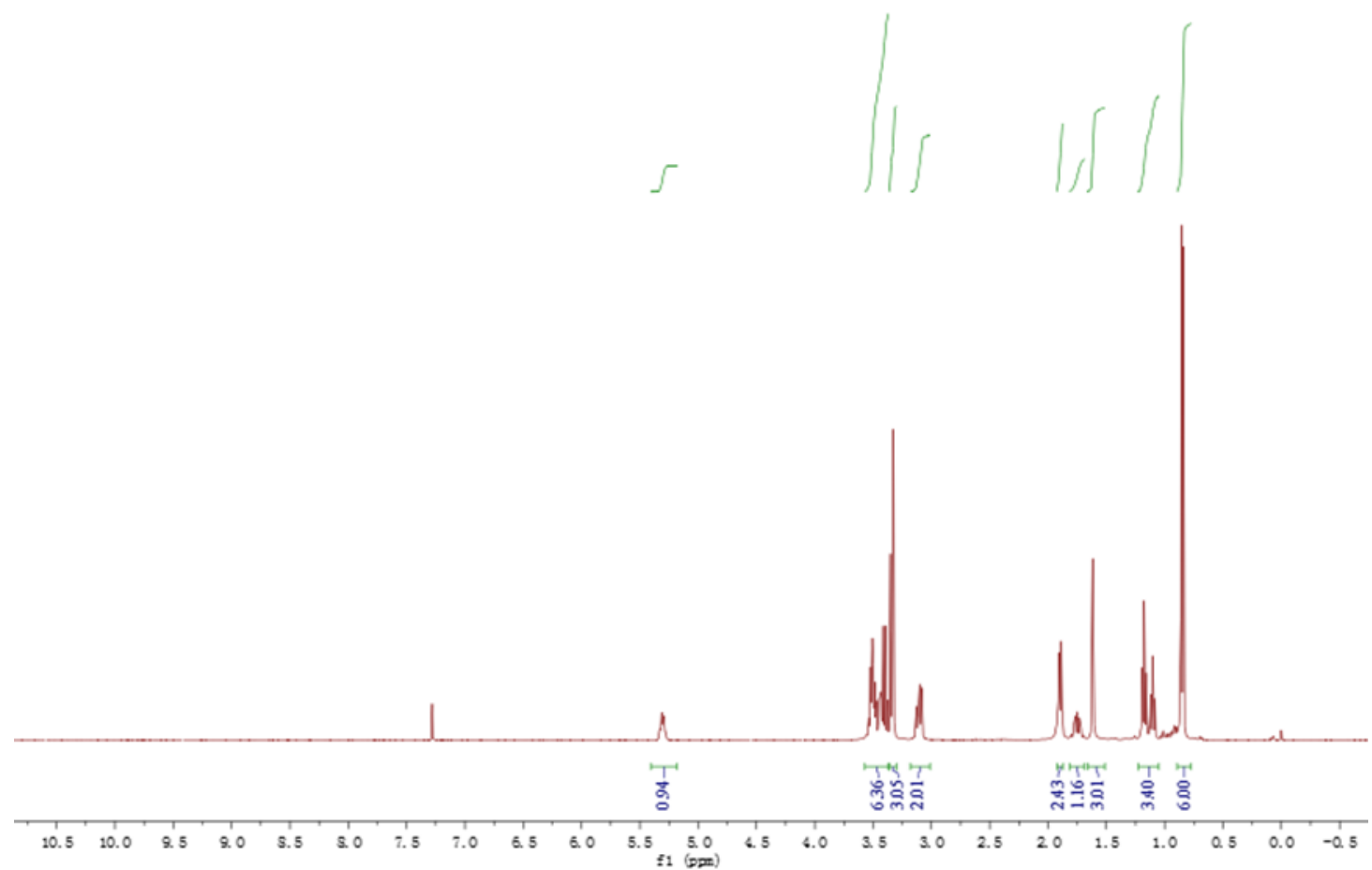

${ }^{13} \mathrm{CNMR}\left(101 \mathrm{MHz}, \mathrm{CDCl}_{3}\right)$

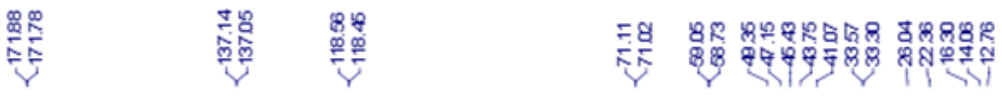

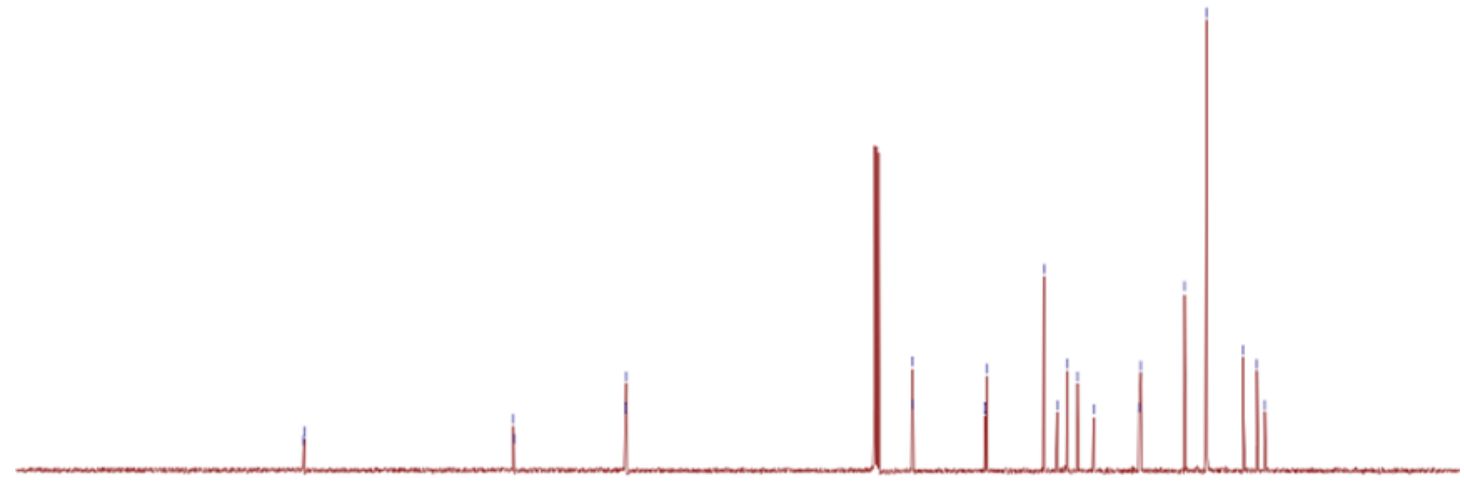

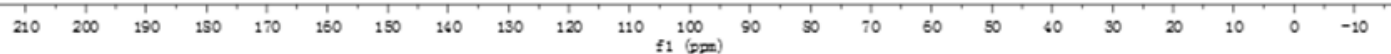


<smiles>CCN(CCOC)C(=O)C/C=C(\C)CCCCl</smiles>

${ }^{1} \mathrm{H}$ NMR $\left(400 \mathrm{MHz}, \mathrm{CDCl}_{3}\right)$

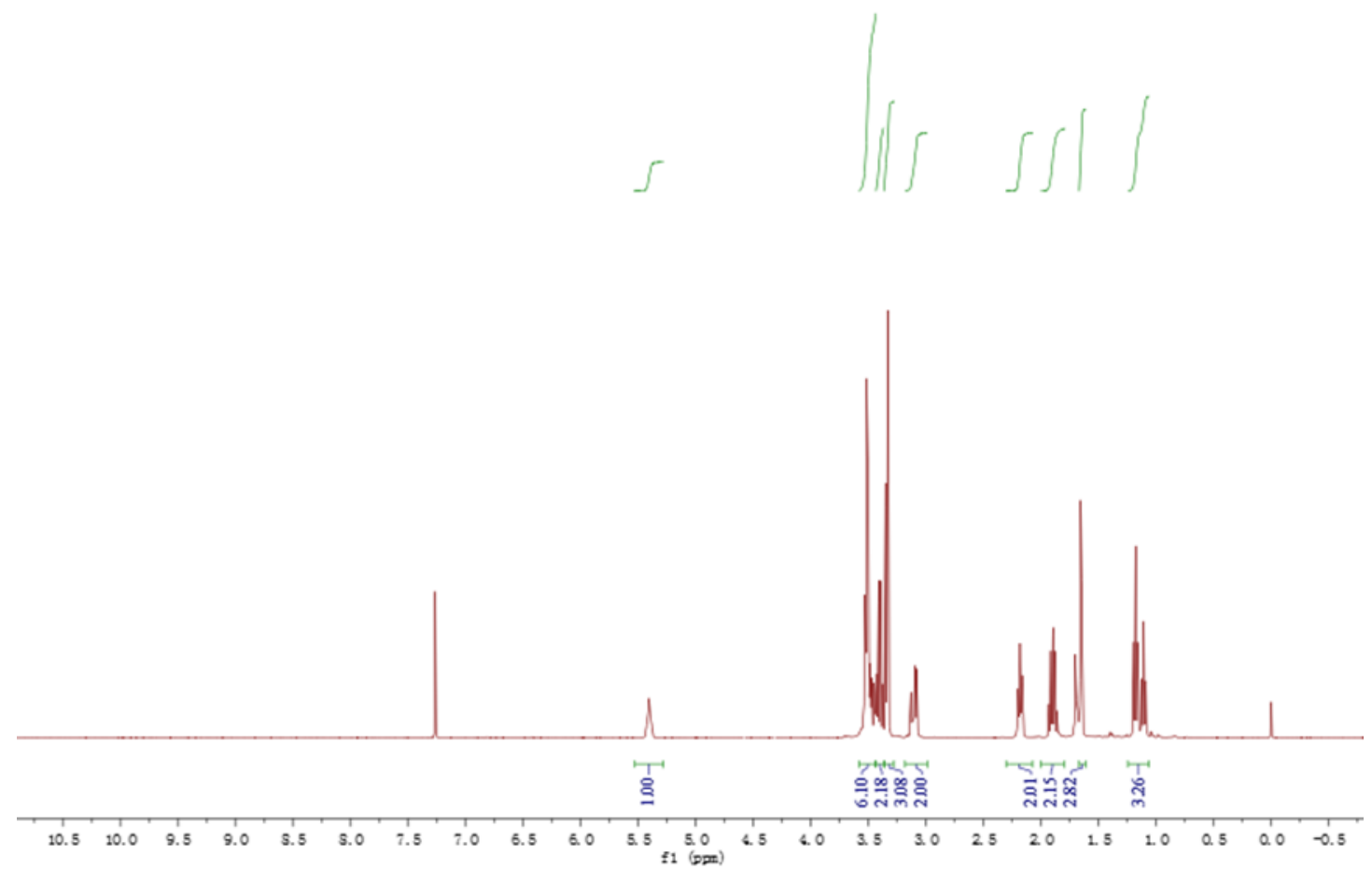

${ }^{13} \mathrm{CNMR}\left(101 \mathrm{MHz}, \mathrm{CDCl}_{3}\right)$

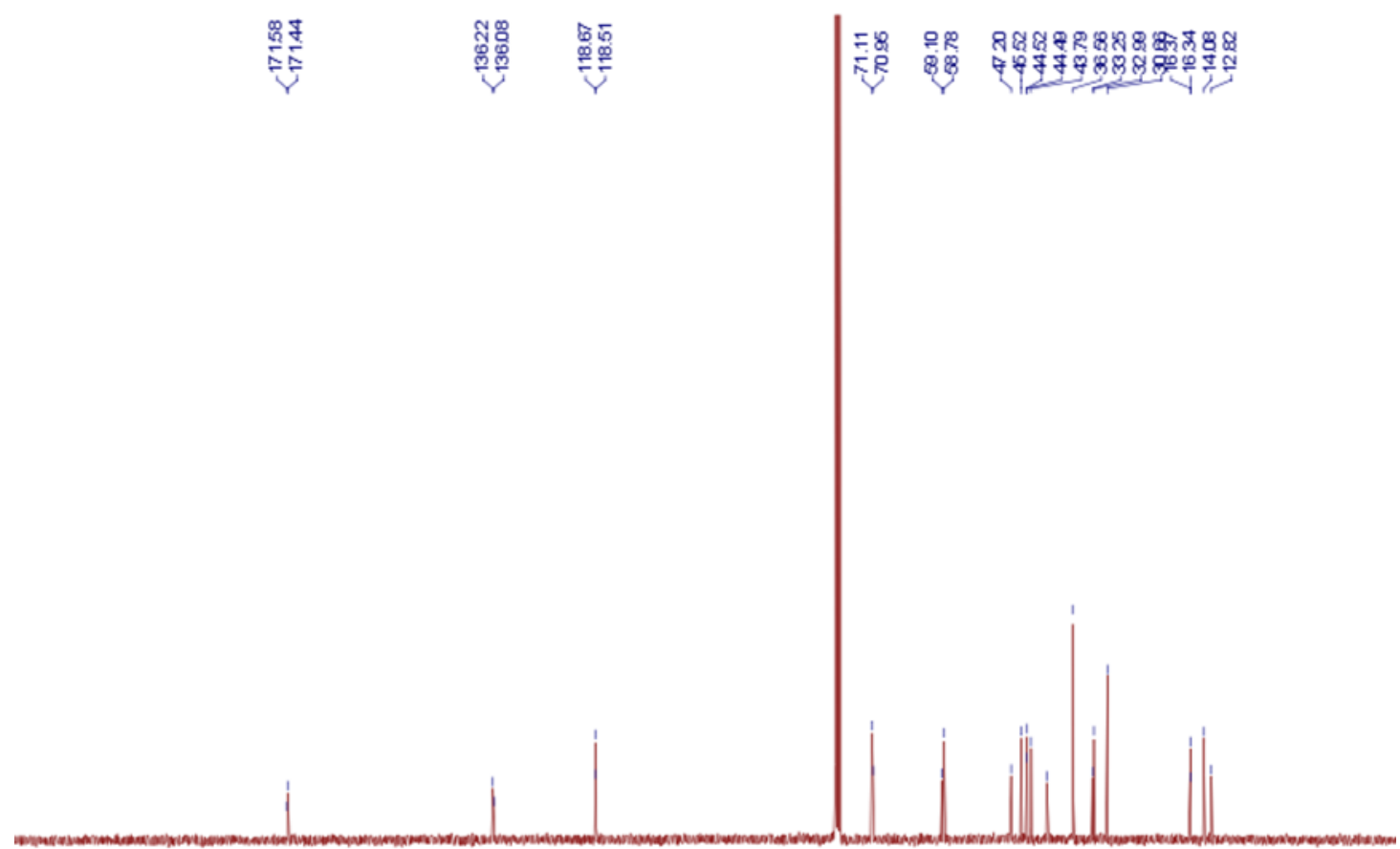

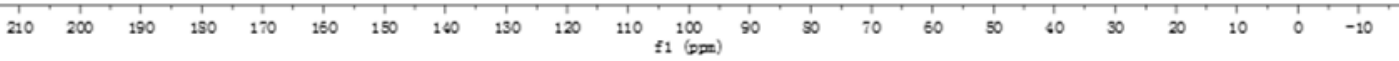


<smiles>CCN(CCOC)C(=O)C/C=C(\C)CCCOC(C)=O</smiles>

${ }^{1} \mathrm{H}$ NMR $\left(400 \mathrm{MHz}, \mathrm{CDCl}_{3}\right)$

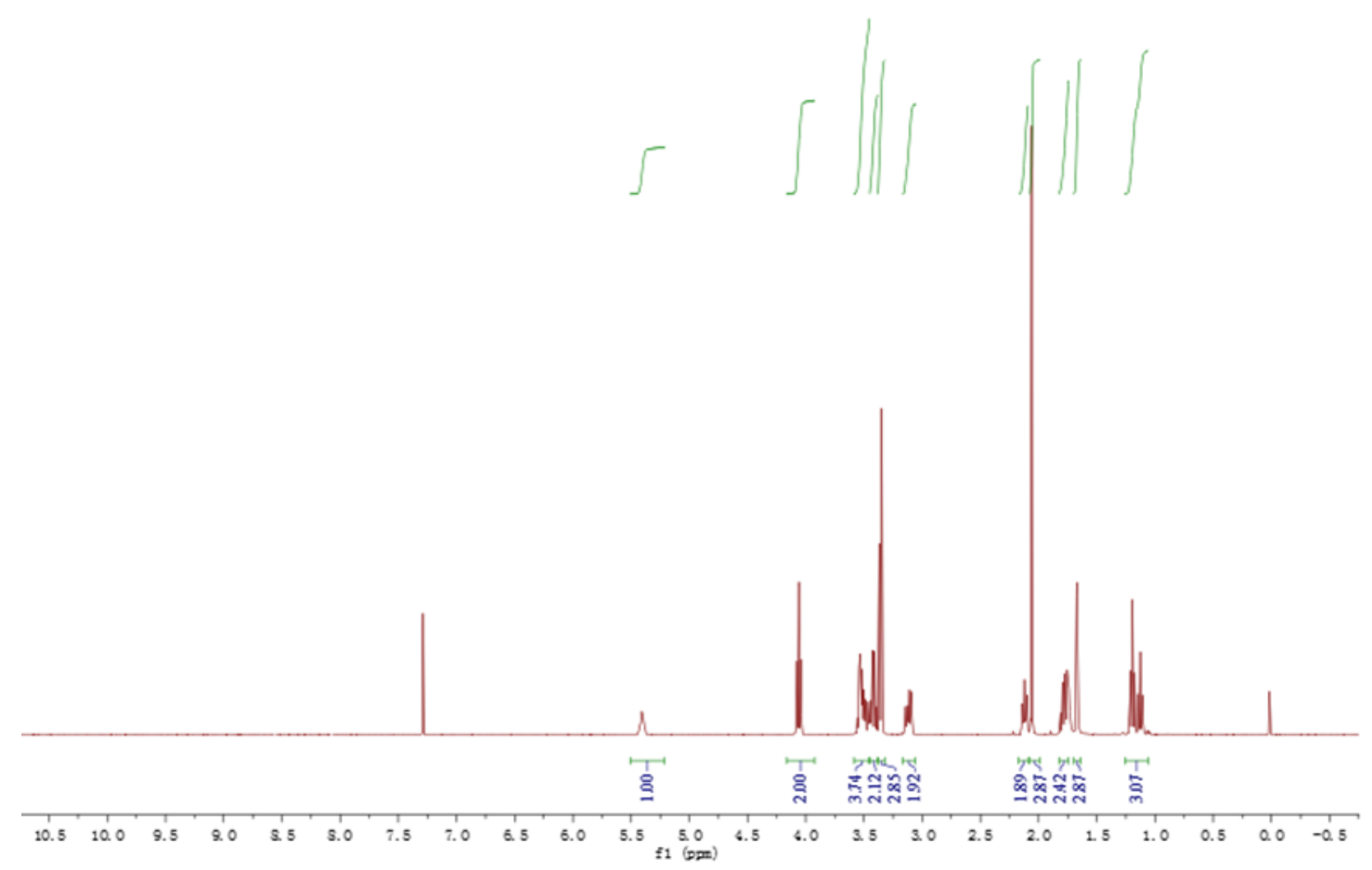

${ }^{13} \mathrm{CNMR}\left(101 \mathrm{MHz}, \mathrm{CDCl}_{3}\right)$

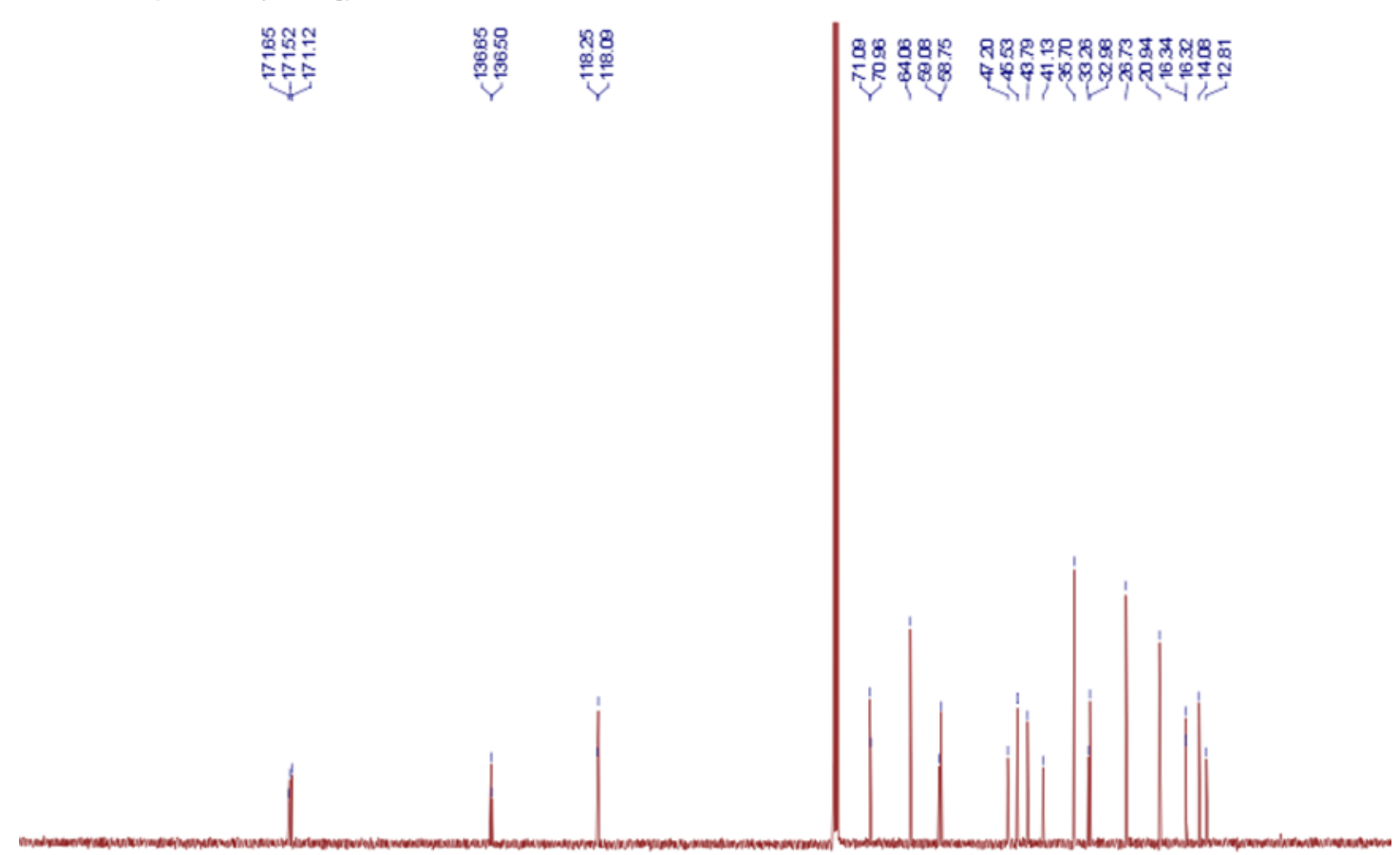

$\begin{array}{llllllllllllllllllllllll}210 & 200 & 190 & 150 & 170 & 160 & 150 & 160 & 130 & 120 & 110 & 100 & 90 & 50 & 70 & 60 & 50 & 40 & 30 & 20 & 10 & 0 & -10\end{array}$ 
<smiles>CCN(CCOC)C(=O)C/C=C(\C)CCCOCc1ccccc1</smiles>

${ }^{1} \mathrm{H} \mathrm{NMR}\left(400 \mathrm{MHz}, \mathrm{CDCl}_{3}\right)$

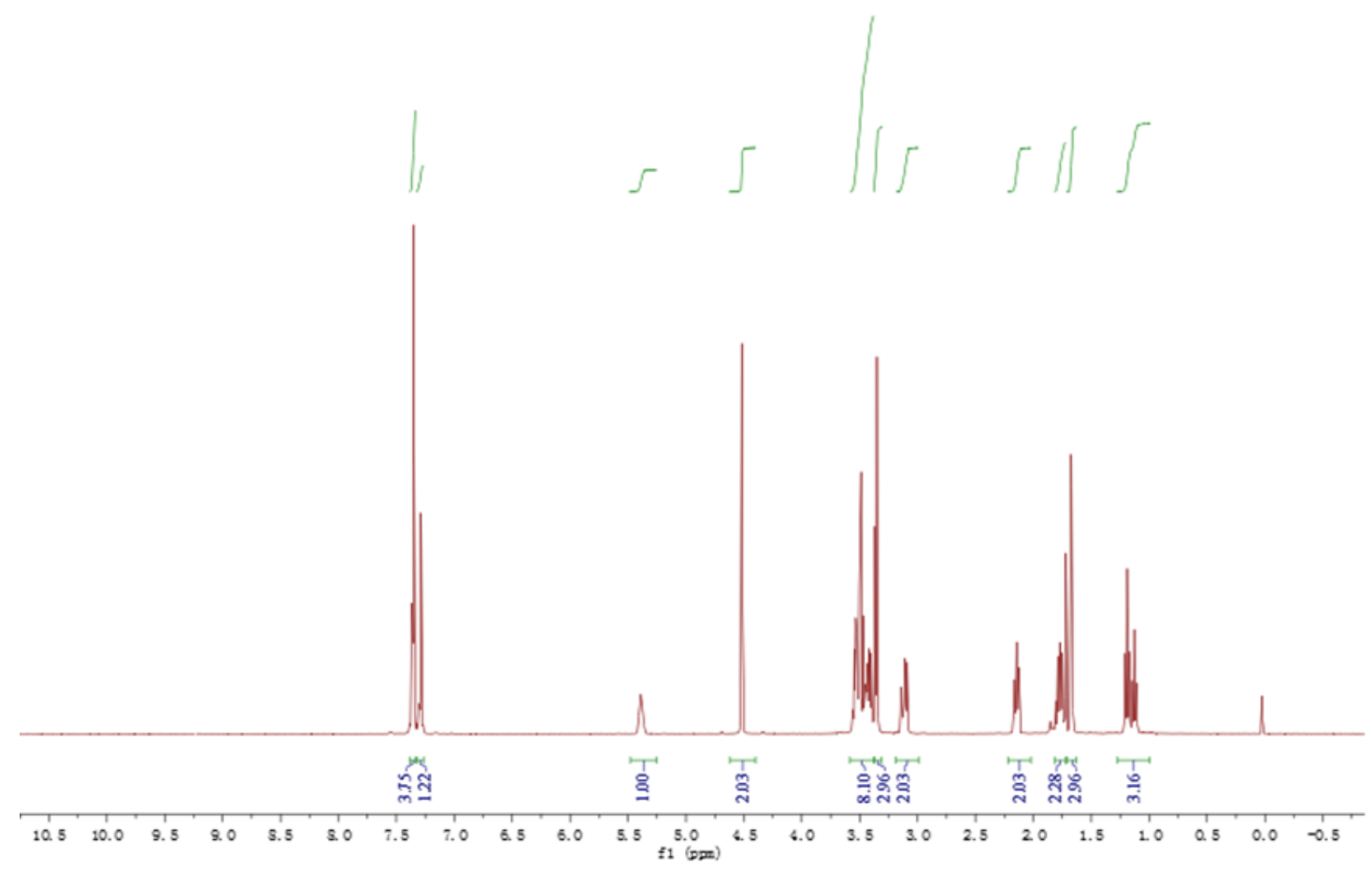

${ }^{13} \mathrm{CNMR}\left(101 \mathrm{MHz}, \mathrm{CDCl}_{3}\right)$

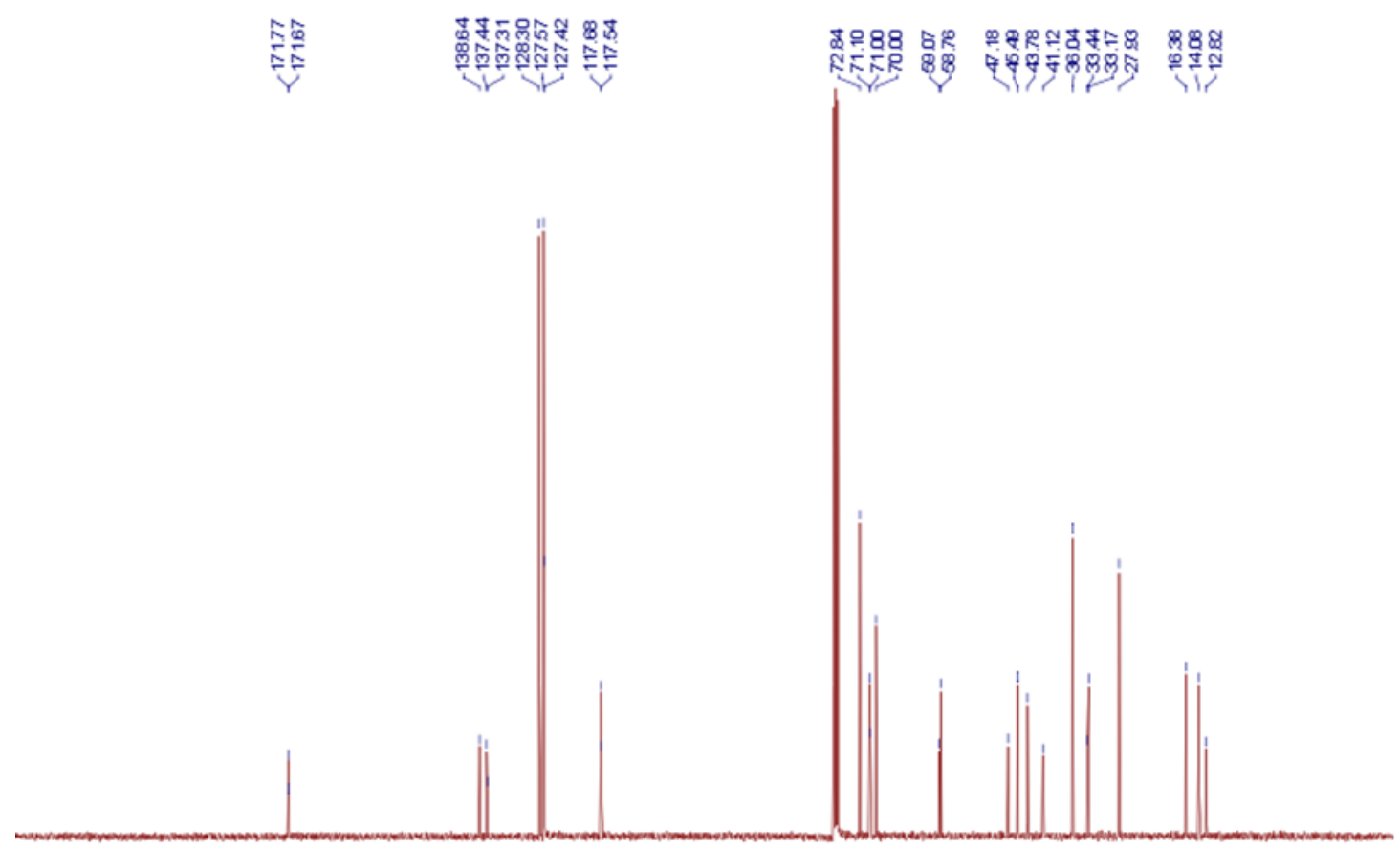

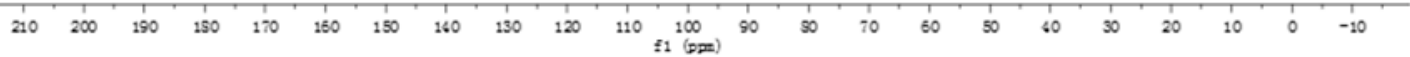


<smiles>CCN(CCOC)C(=O)C/C=C(\C)CCCc1ccccc1</smiles>

${ }^{1} \mathrm{H}$ NMR $\left(400 \mathrm{MHz}, \mathrm{CDCl}_{3}\right)$

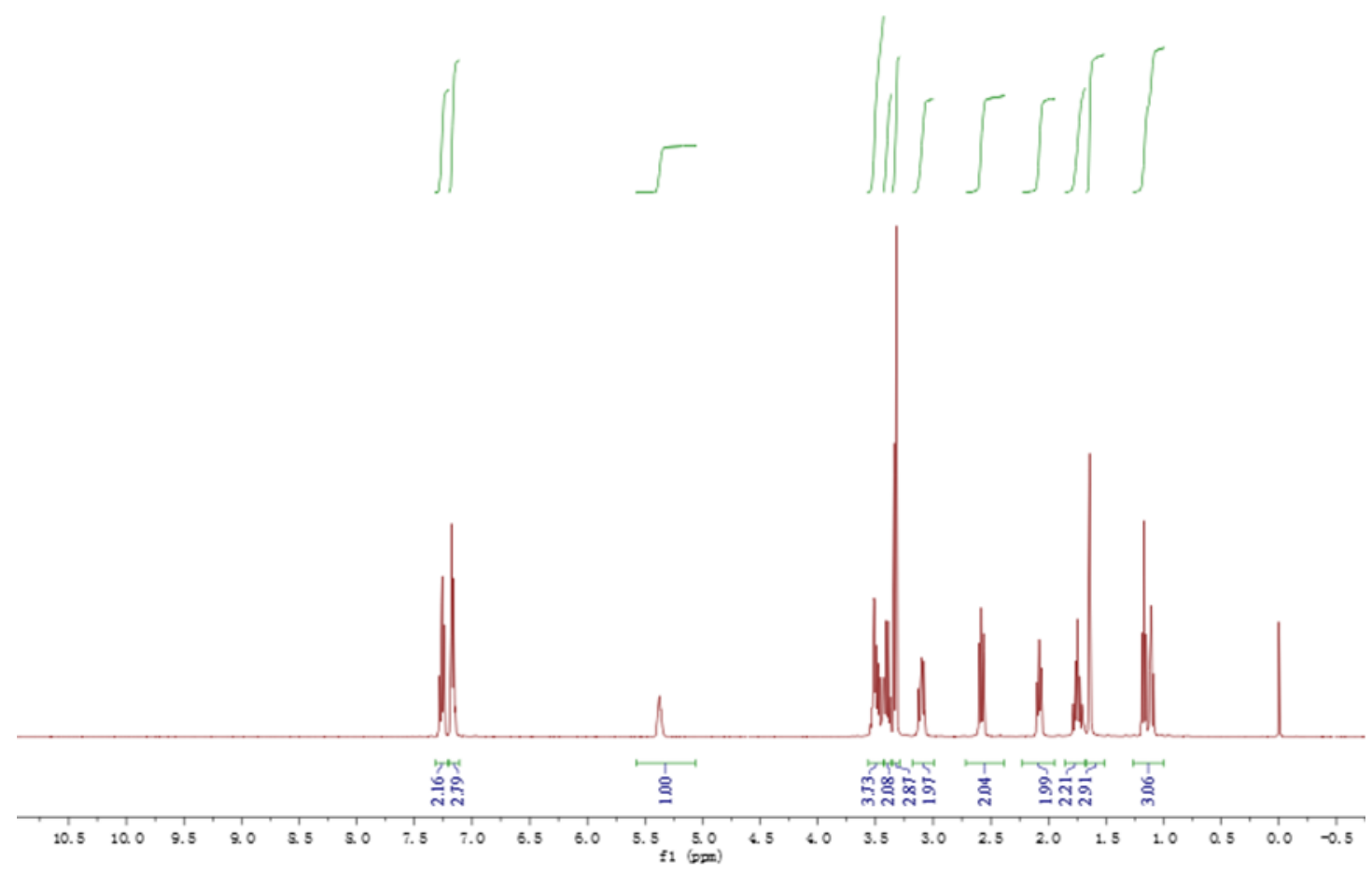

${ }^{13} \mathrm{CNMR}\left(101 \mathrm{MHz}, \mathrm{CDCl}_{3}\right)$

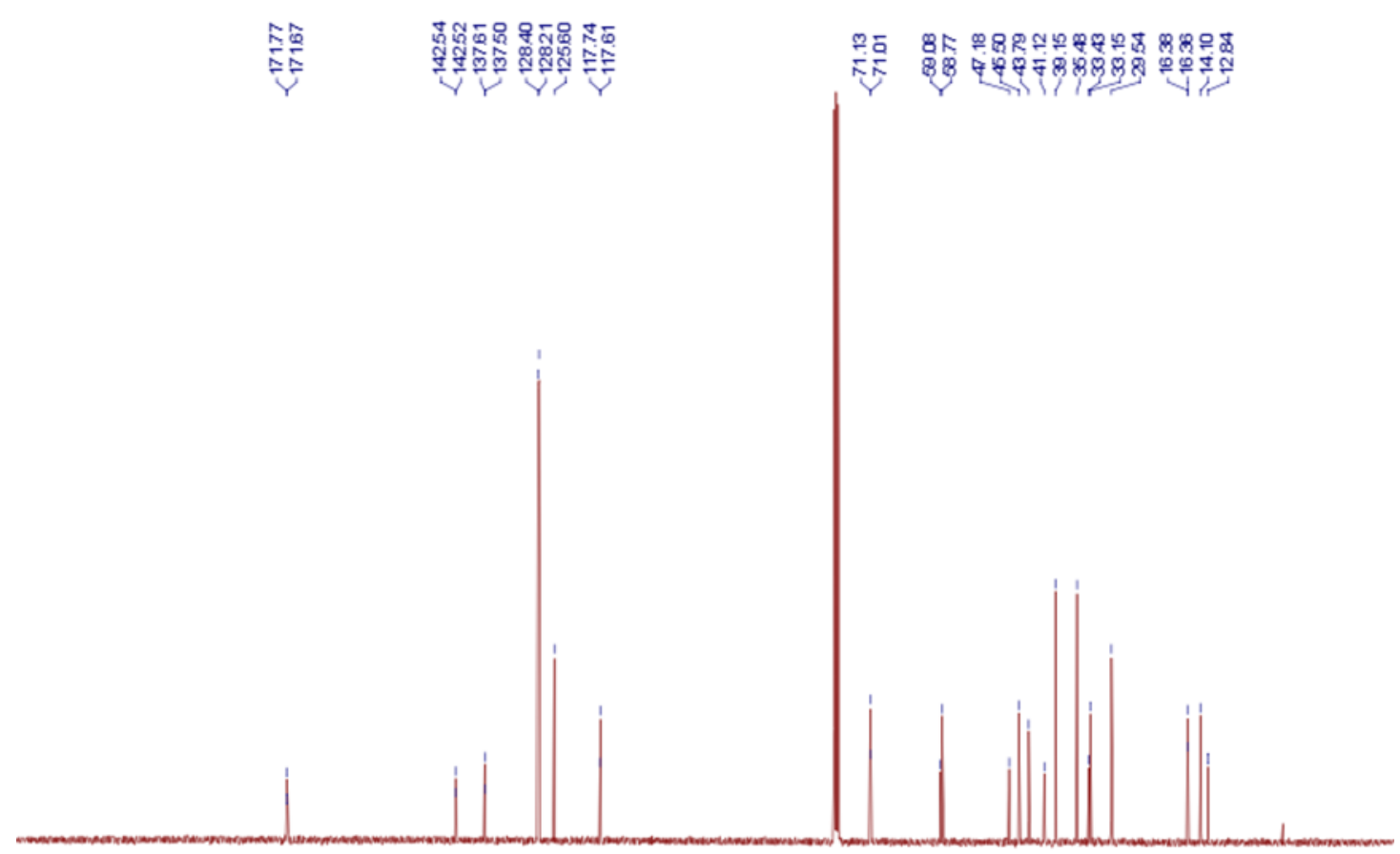

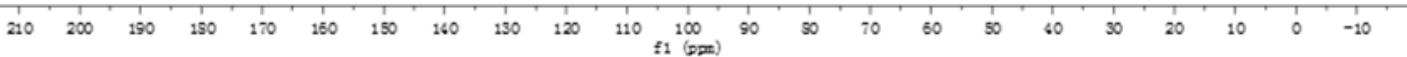


<smiles>CCN(CCOC)C(=O)C/C=C(/C)CCCc1ccccc1</smiles>

${ }^{1} \mathrm{H} \mathrm{NMR}\left(400 \mathrm{MHz}, \mathrm{CDCl}_{3}\right)$

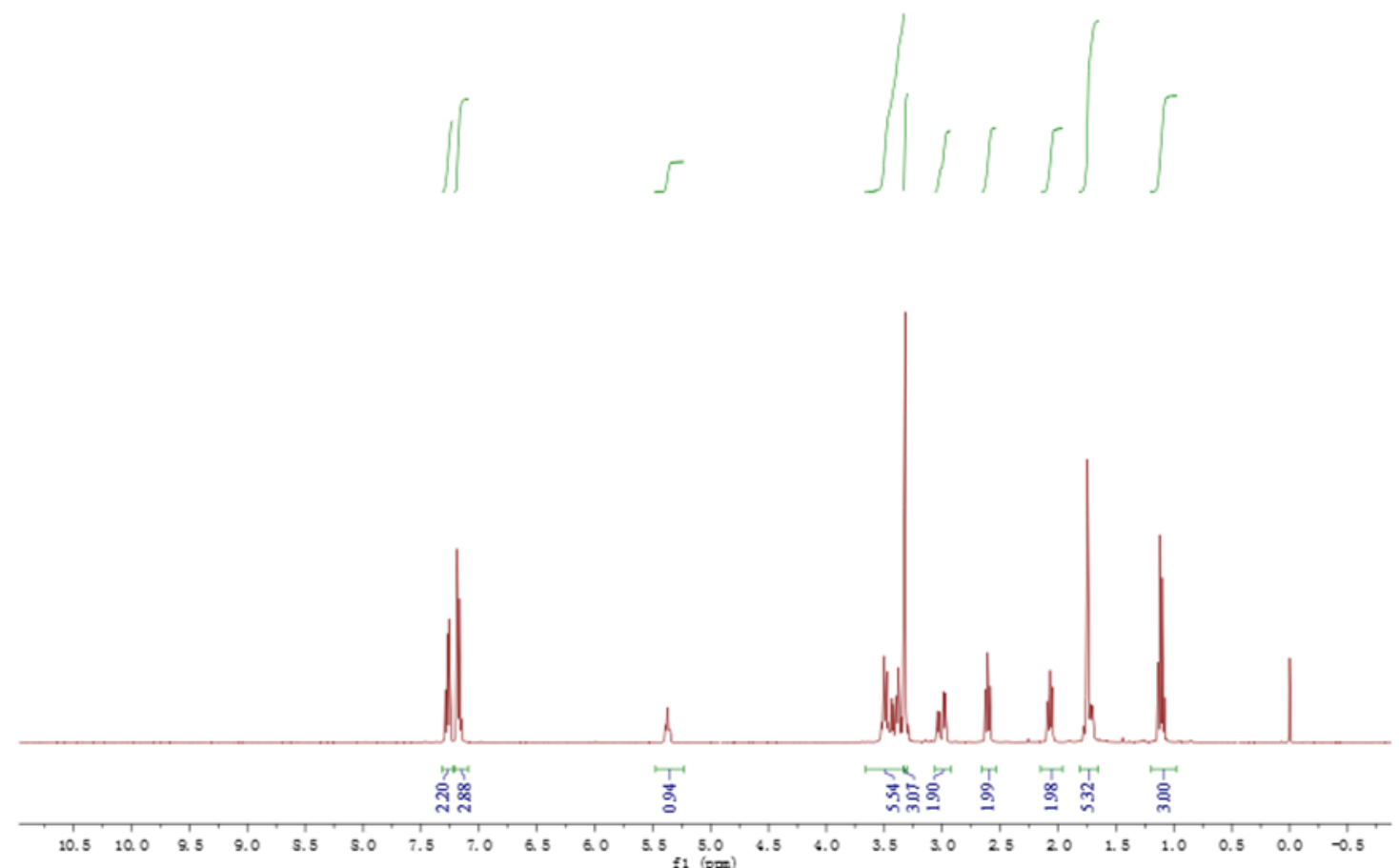

${ }^{13} \mathrm{CNMR}\left(101 \mathrm{MHz}, \mathrm{CDCl}_{3}\right)$

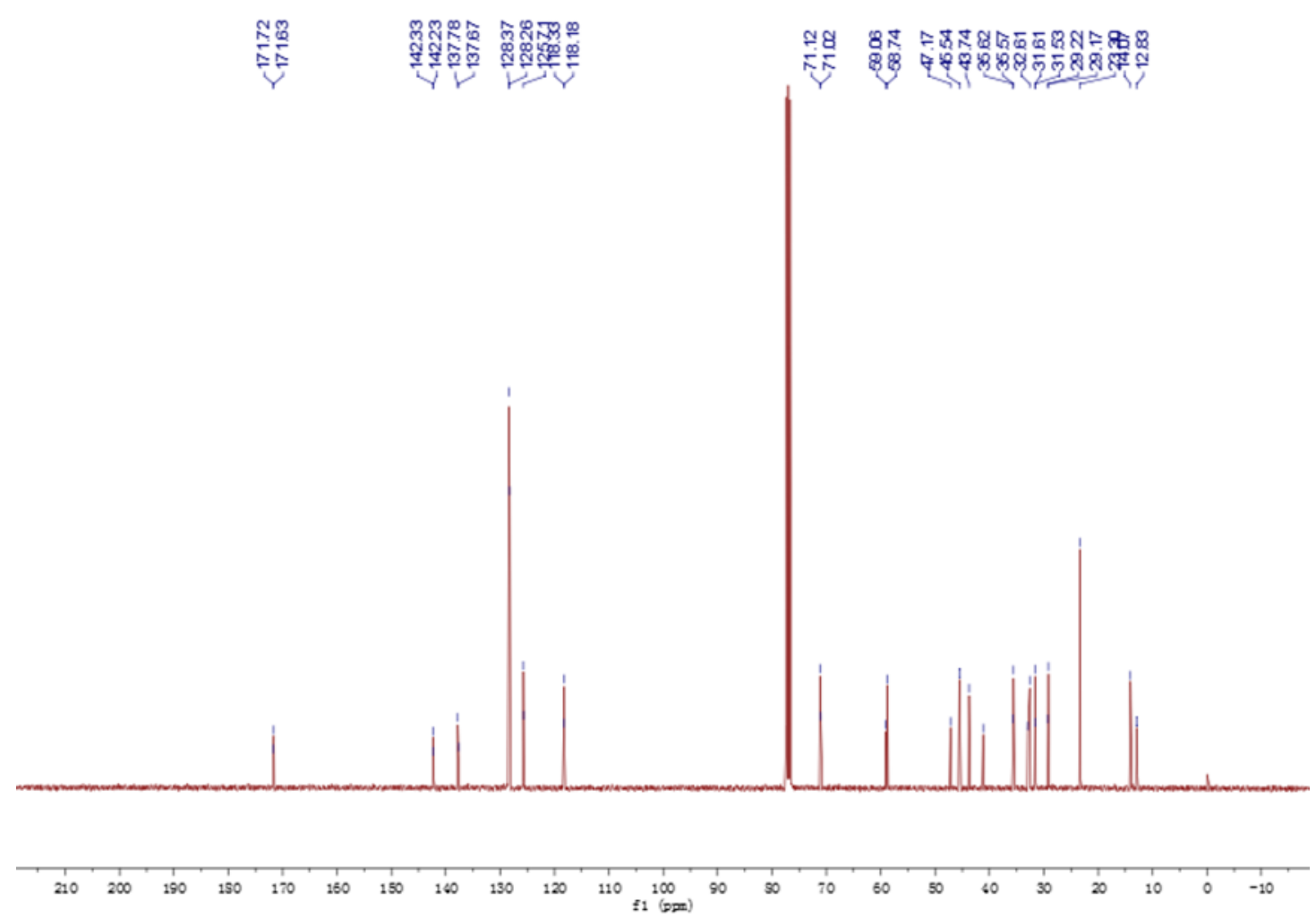


<smiles>CCN(CCOC)C(=O)C/C=C(\C)CCc1ccccc1</smiles>

${ }^{1} \mathrm{H} \mathrm{NMR}\left(400 \mathrm{MHz}, \mathrm{CDCl}_{3}\right)$

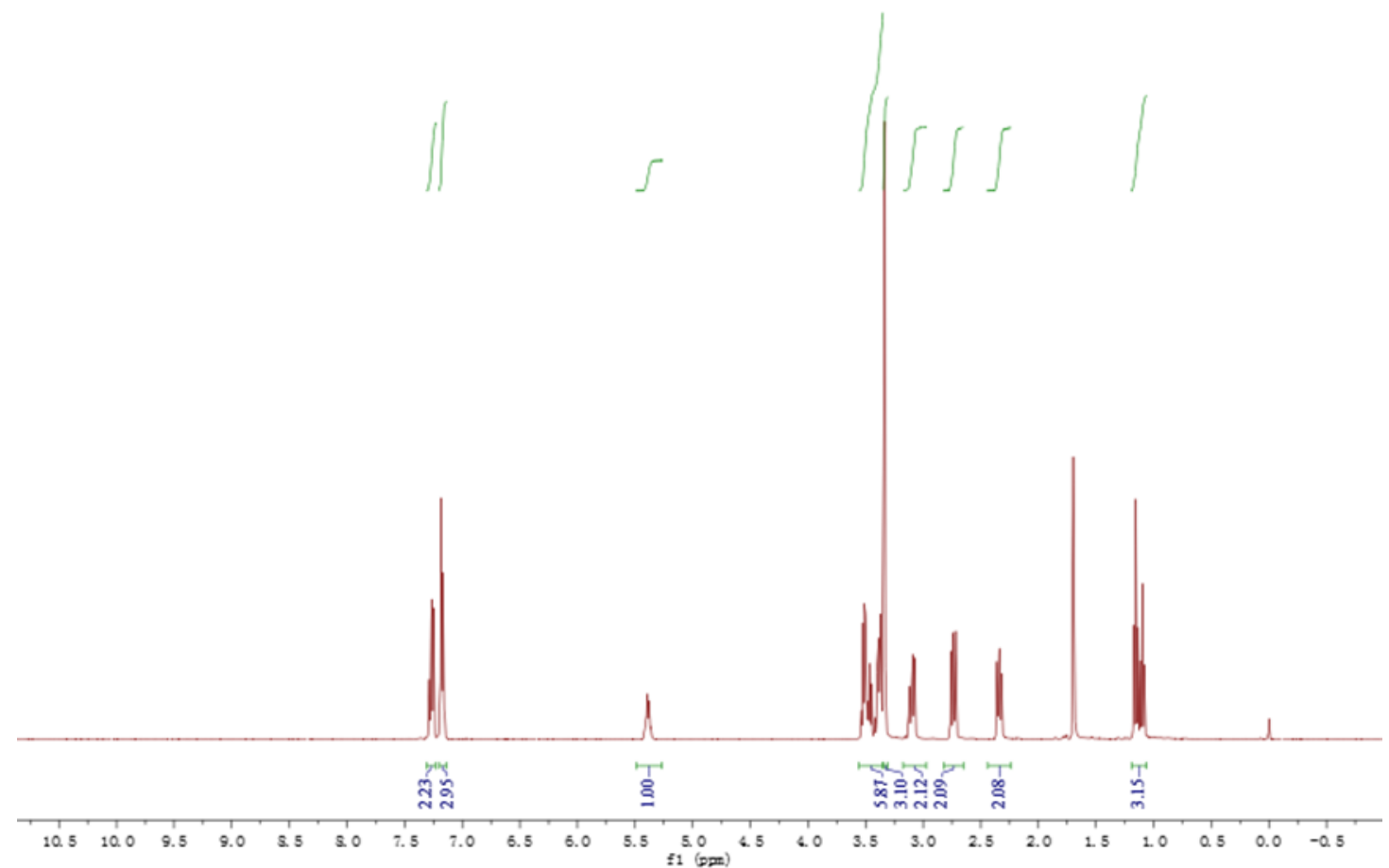

${ }^{13} \mathrm{CNMR}\left(101 \mathrm{MHz}, \mathrm{CDCl}_{3}\right)$

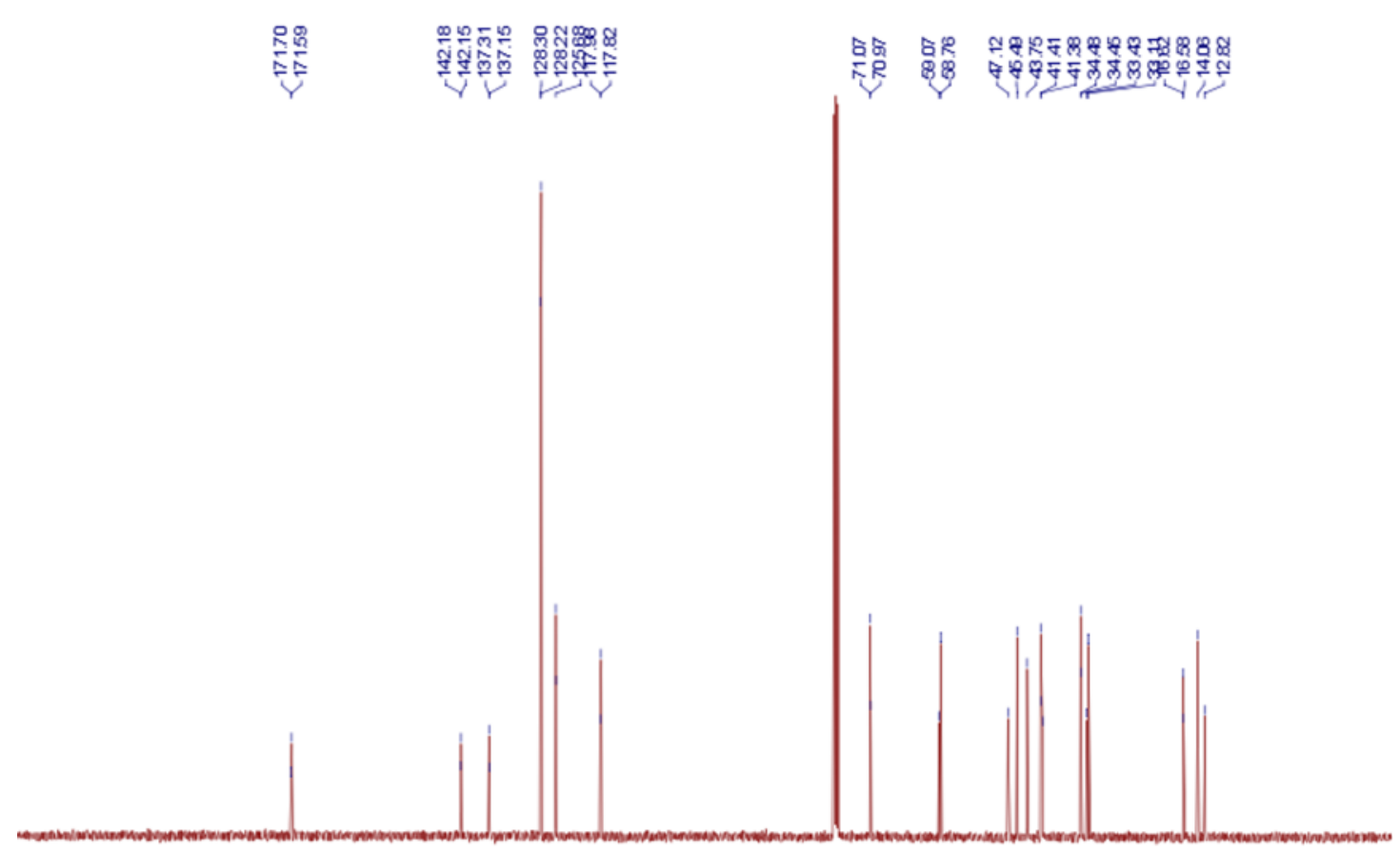

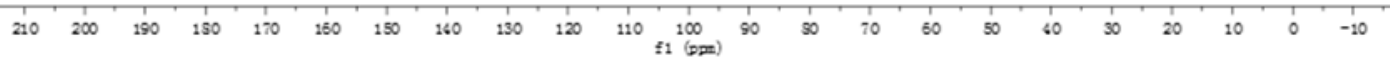


<smiles>CC/C(=C\CC(=O)N(CC)CCOC)[PH2+]c1ccccc1</smiles>

${ }^{1} \mathrm{H} \mathrm{NMR}\left(400 \mathrm{MHz}, \mathrm{CDCl}_{3}\right)$

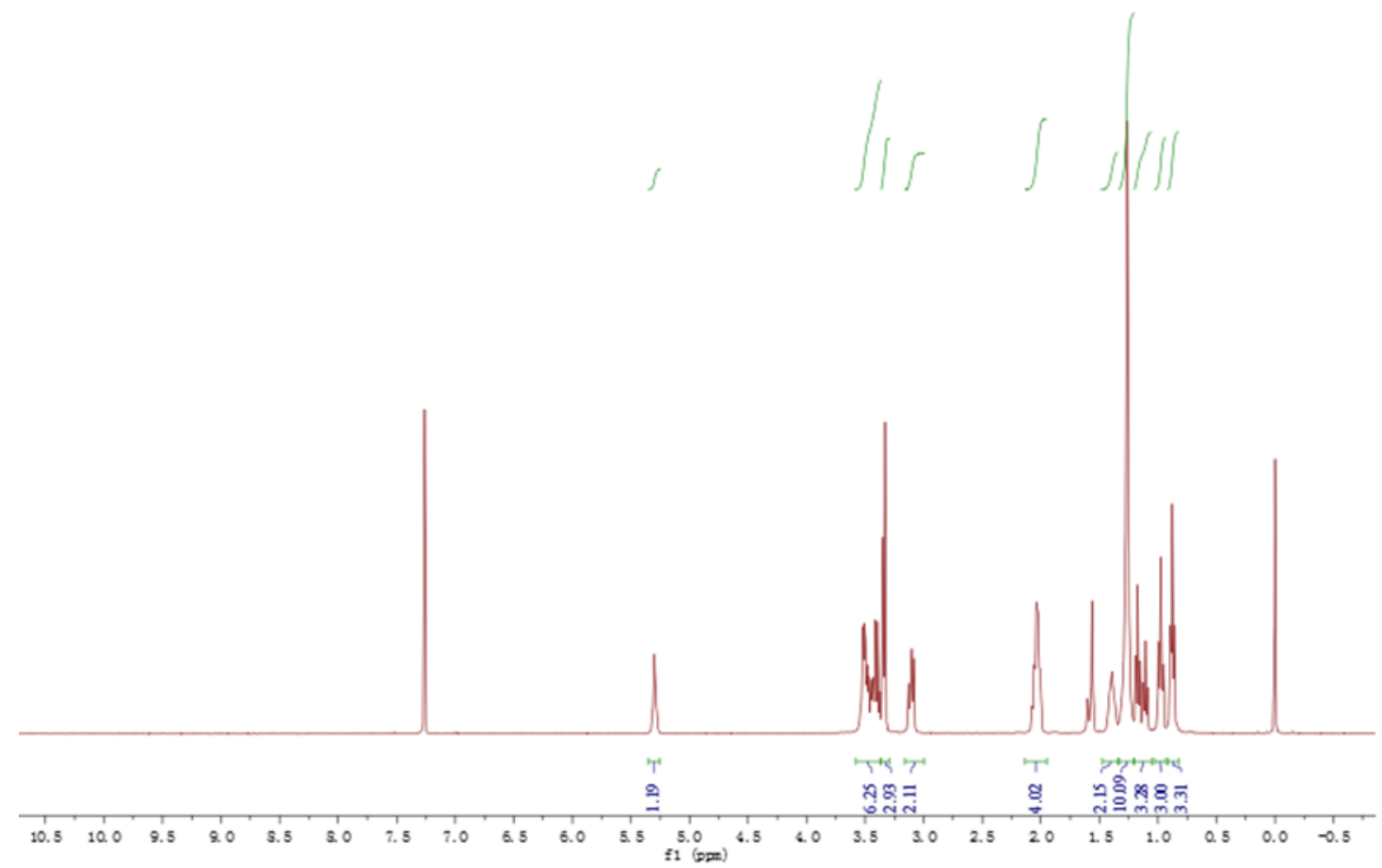

${ }^{13} \mathrm{CNMR}\left(101 \mathrm{MHz}, \mathrm{CDCl}_{3}\right)$

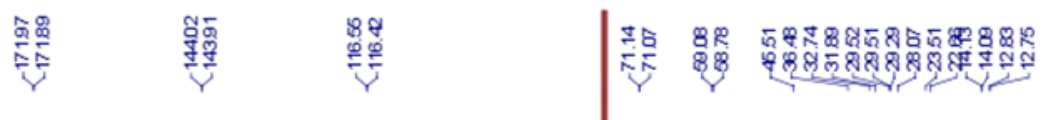

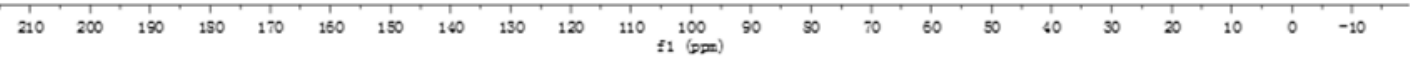


<smiles>CCN(CC)C(=O)CC[C@](C)(C#C[Se-])C(C)C</smiles>

${ }^{1} \mathrm{H} \mathrm{NMR}\left(400 \mathrm{MHz}, \mathrm{CDCl}_{3}\right)$

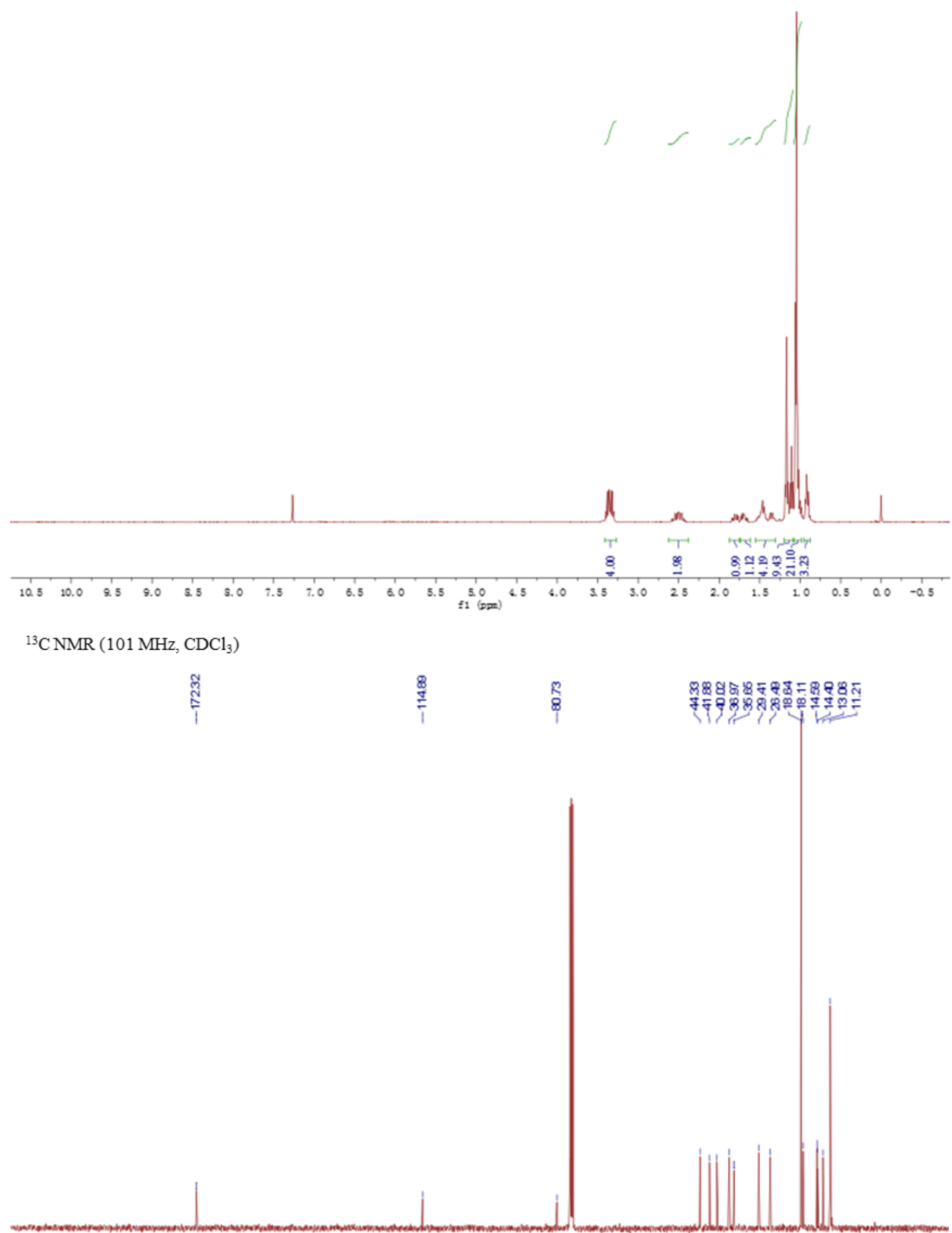

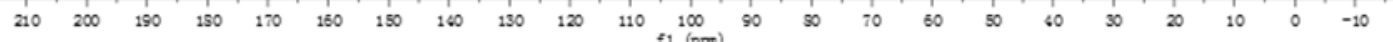


<smiles>CCN(CC)C(=O)CC[C@](C)(C#Cc1ccccc1)C(C)C</smiles>

${ }^{1} \mathrm{H} \mathrm{NMR}\left(400 \mathrm{MHz}, \mathrm{CDCl}_{3}\right)$

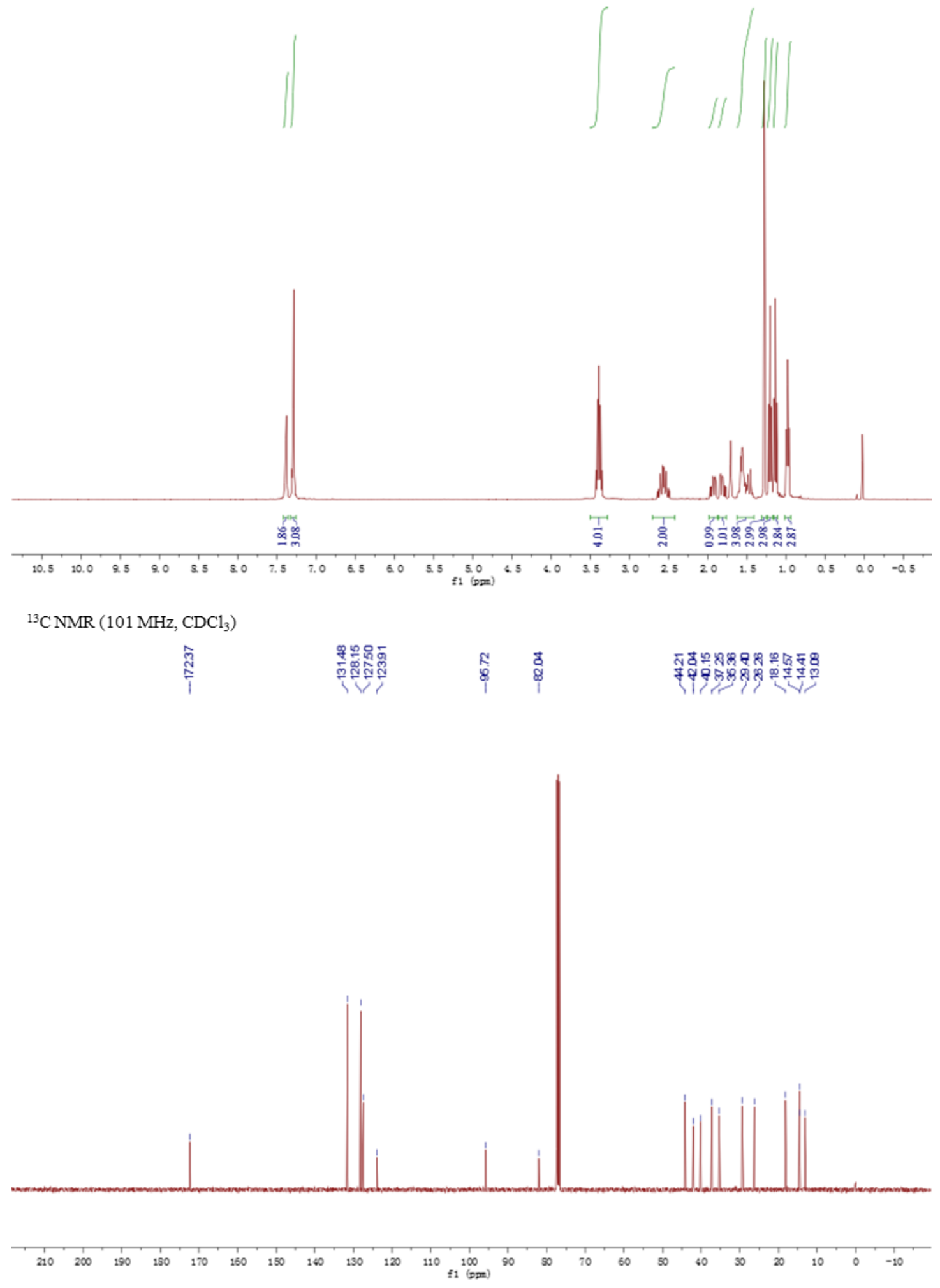




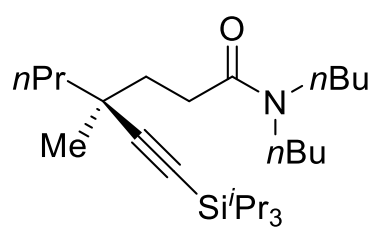

${ }^{1} \mathrm{H} \mathrm{NMR}\left(400 \mathrm{MHz}, \mathrm{CDCl}_{3}\right)$

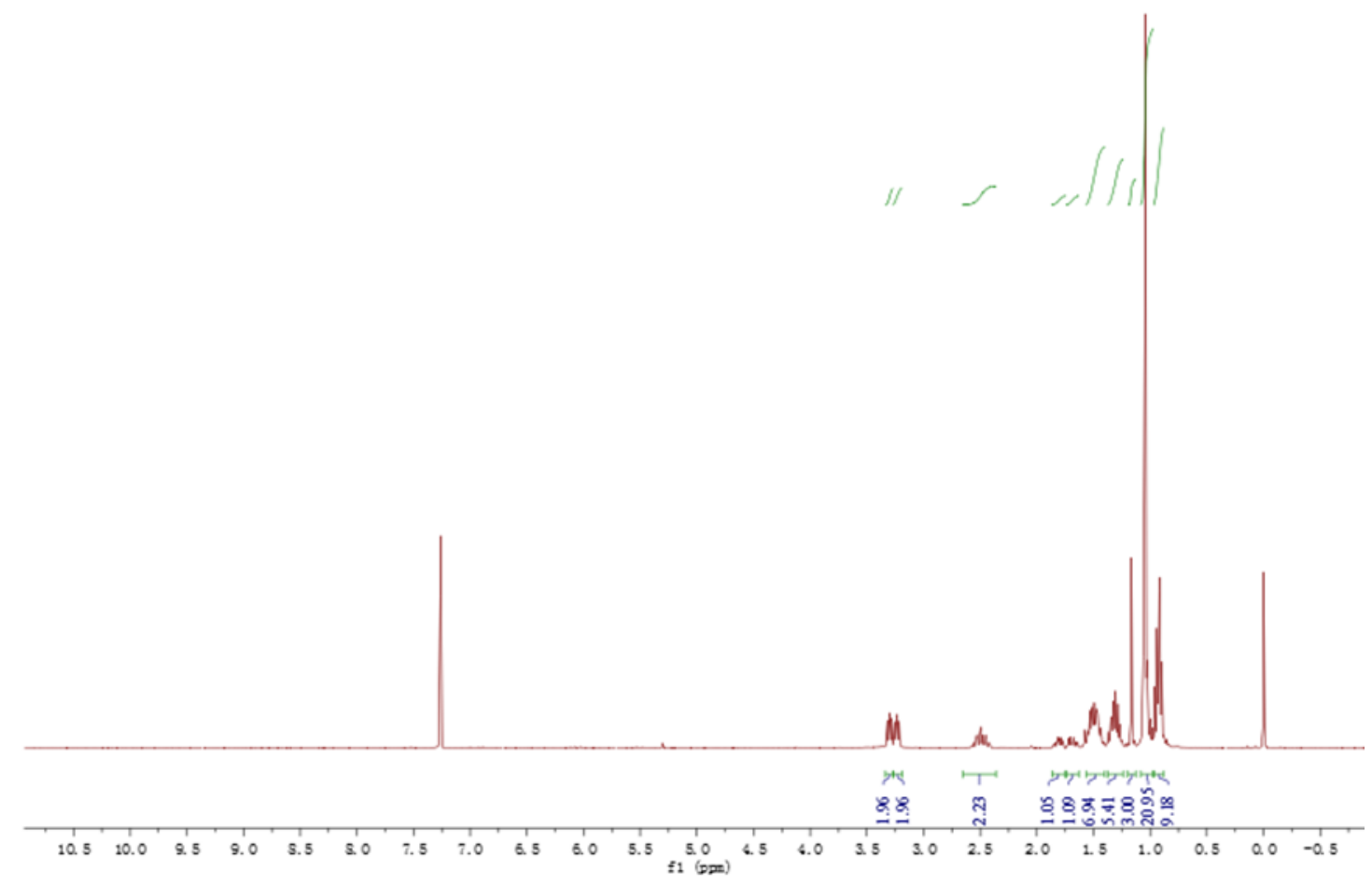

${ }^{13} \mathrm{C} \mathrm{NMR}\left(101 \mathrm{MHz}, \mathrm{CDCl}_{3}\right)$

\begin{tabular}{|c|c|c|}
\hline 导 & $\stackrel{8}{\frac{8}{\square}}$ & $\stackrel{8}{8}$ \\
\hline
\end{tabular}

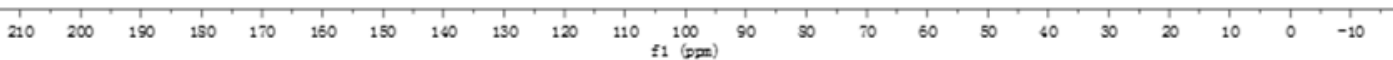




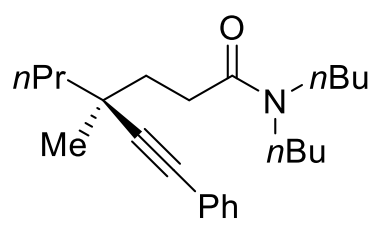

${ }^{1} \mathrm{H} \mathrm{NMR}\left(400 \mathrm{MHz}, \mathrm{CDCl}_{3}\right)$

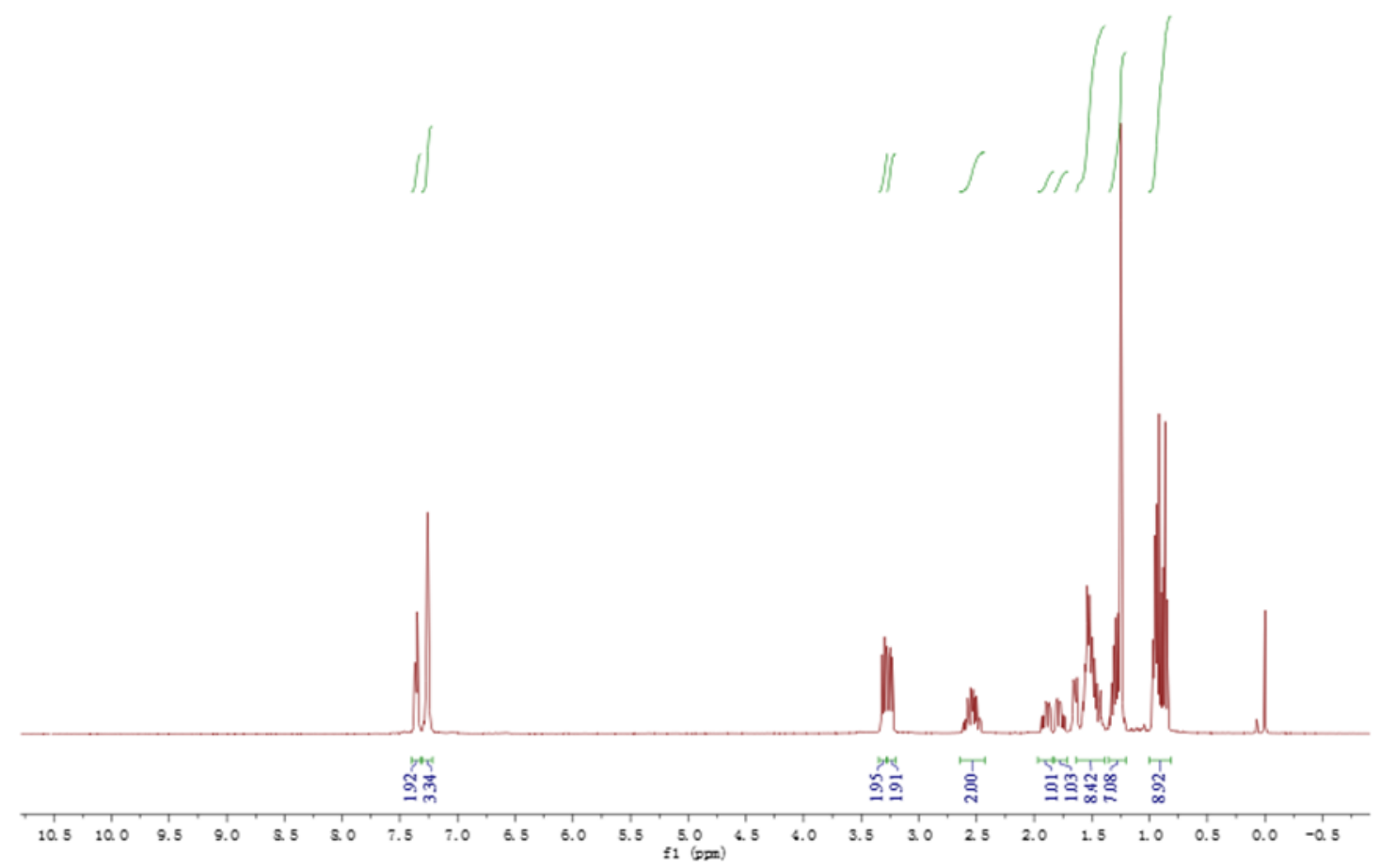

${ }^{13} \mathrm{CNMR}\left(101 \mathrm{MHz}, \mathrm{CDCl}_{3}\right)$

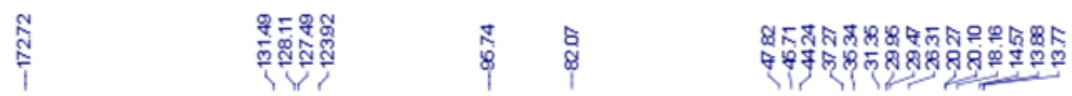

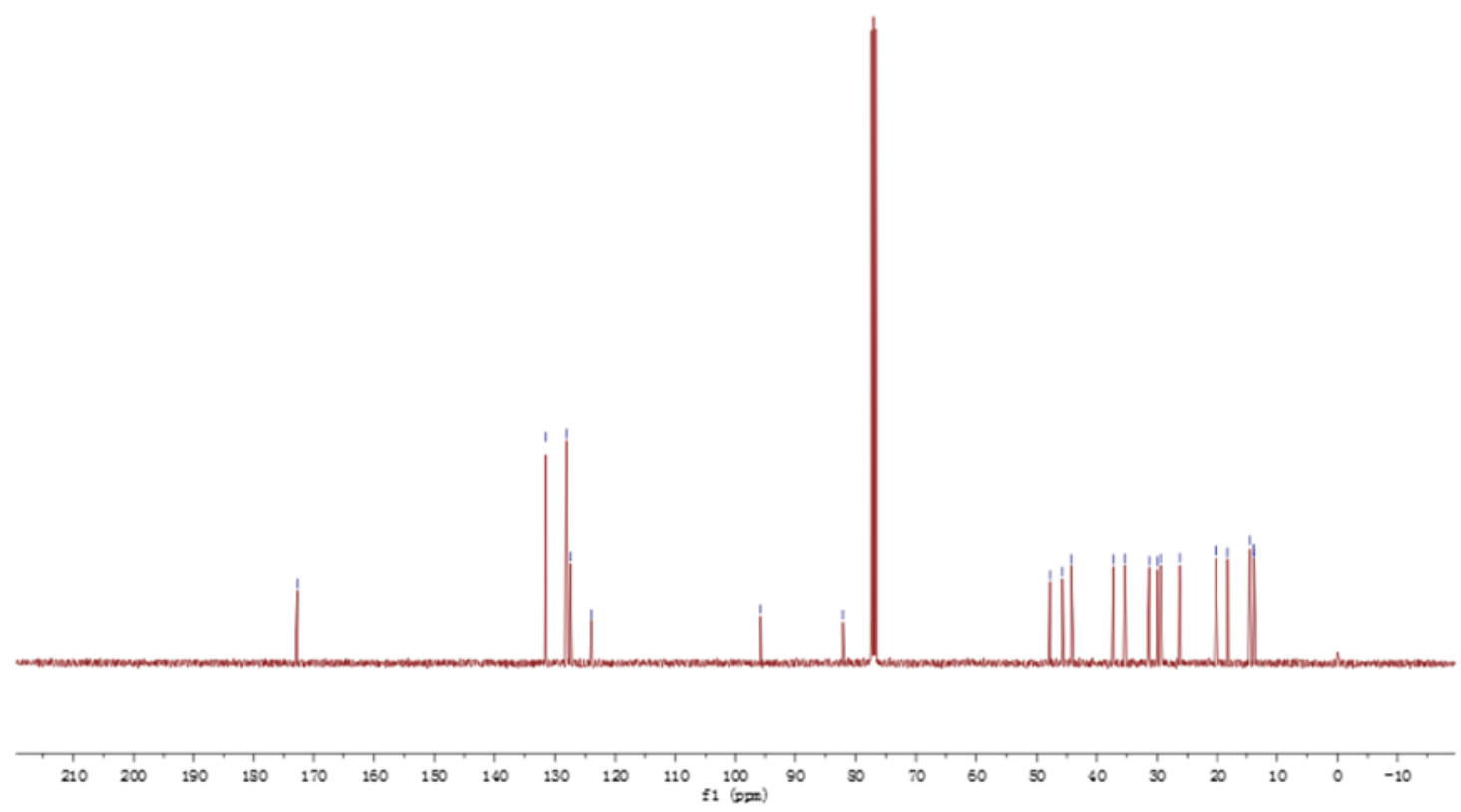




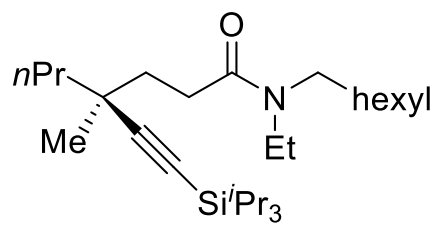

${ }^{1} \mathrm{H} \mathrm{NMR}\left(400 \mathrm{MHz}, \mathrm{CDCl}_{3}\right)$

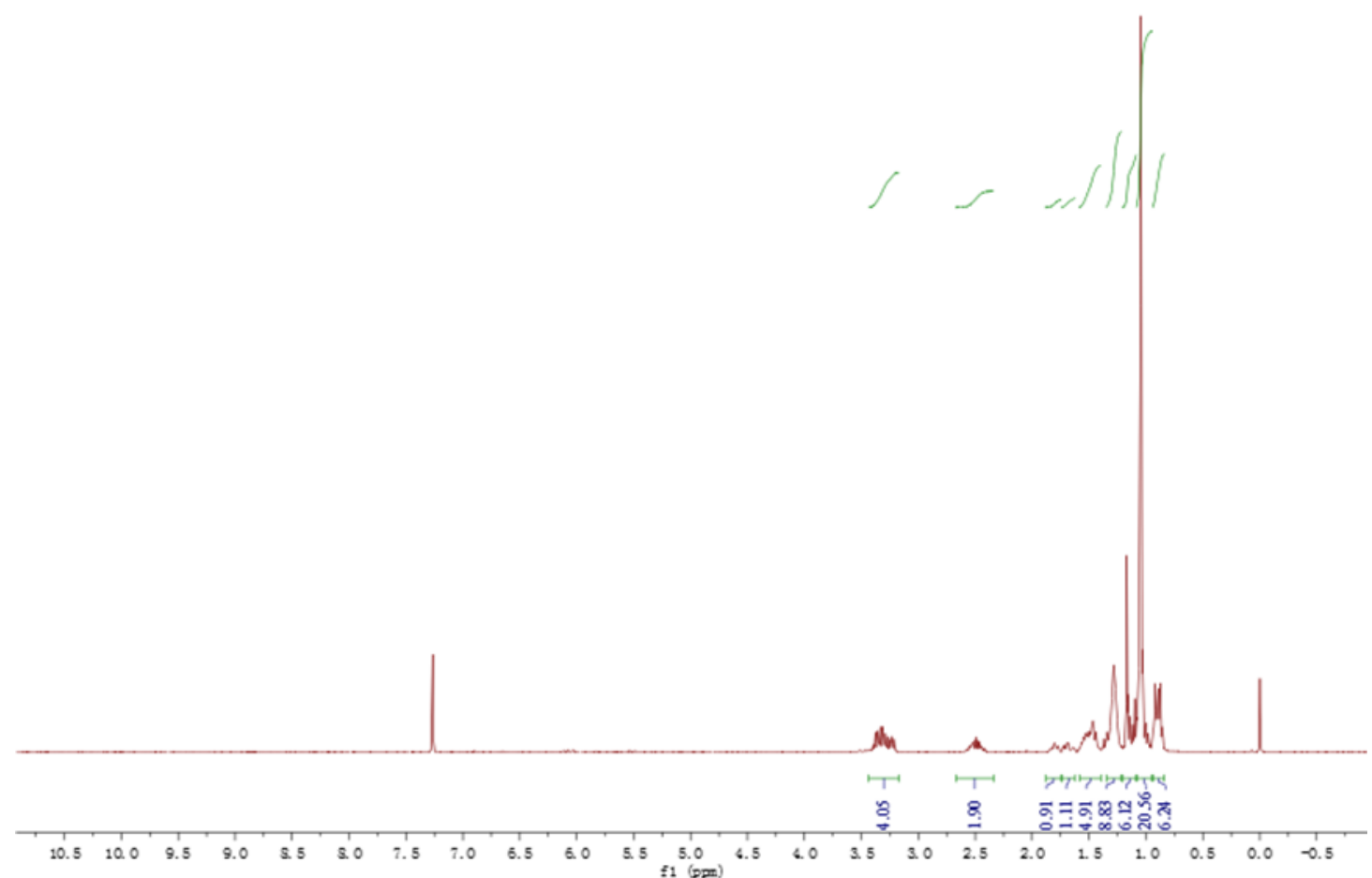

${ }^{13} \mathrm{C} \mathrm{NMR}\left(101 \mathrm{MHz}, \mathrm{CDCl}_{3}\right)$

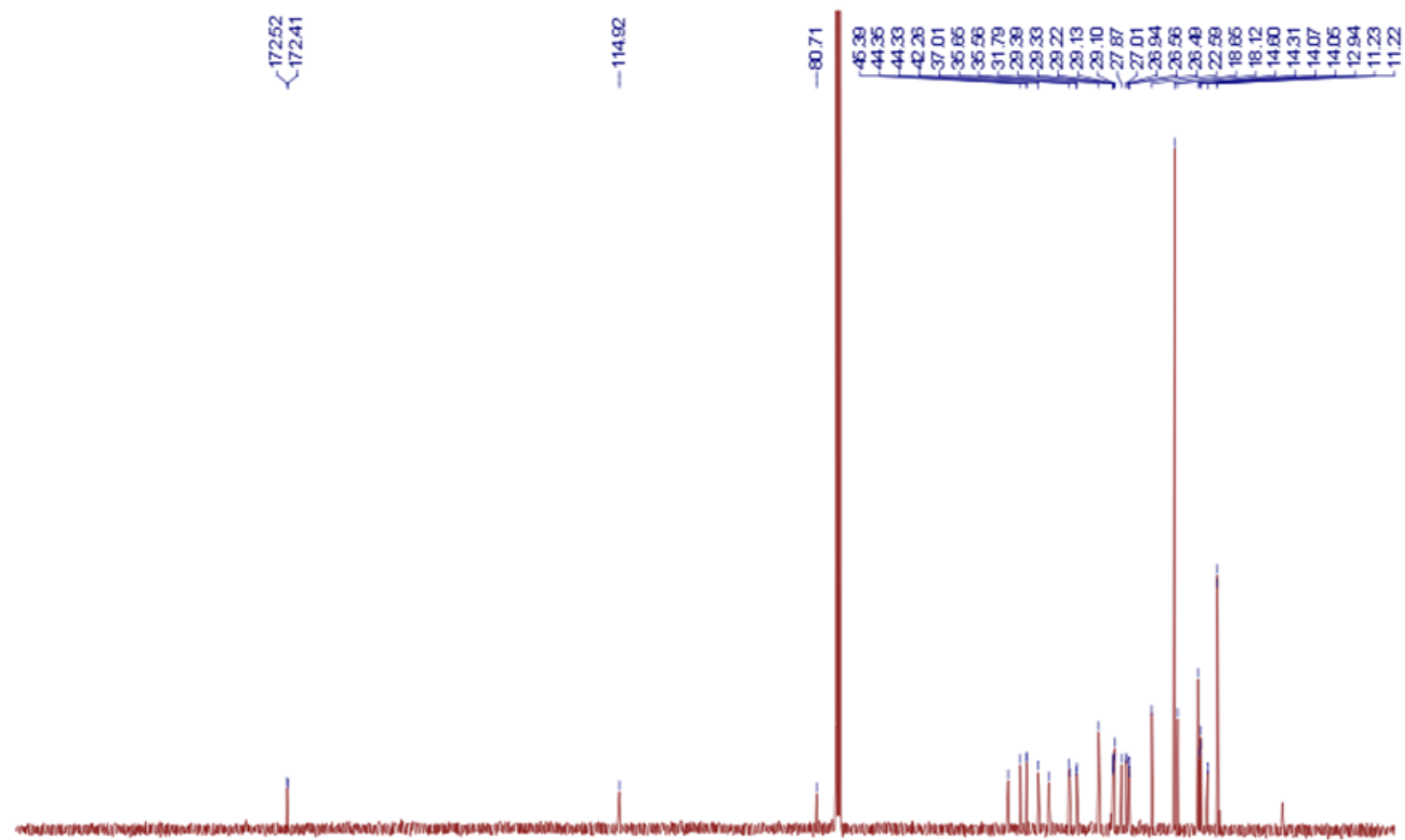

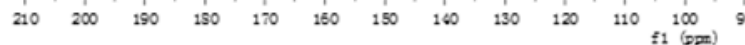


<smiles>CCN(C[14CH3])C(=O)CC[C@](C)(C#Cc1ccccc1)C(=O)c1ccccc1</smiles>

${ }^{1} \mathrm{H} \mathrm{NMR}\left(400 \mathrm{MHz}, \mathrm{CDCl}_{3}\right)$

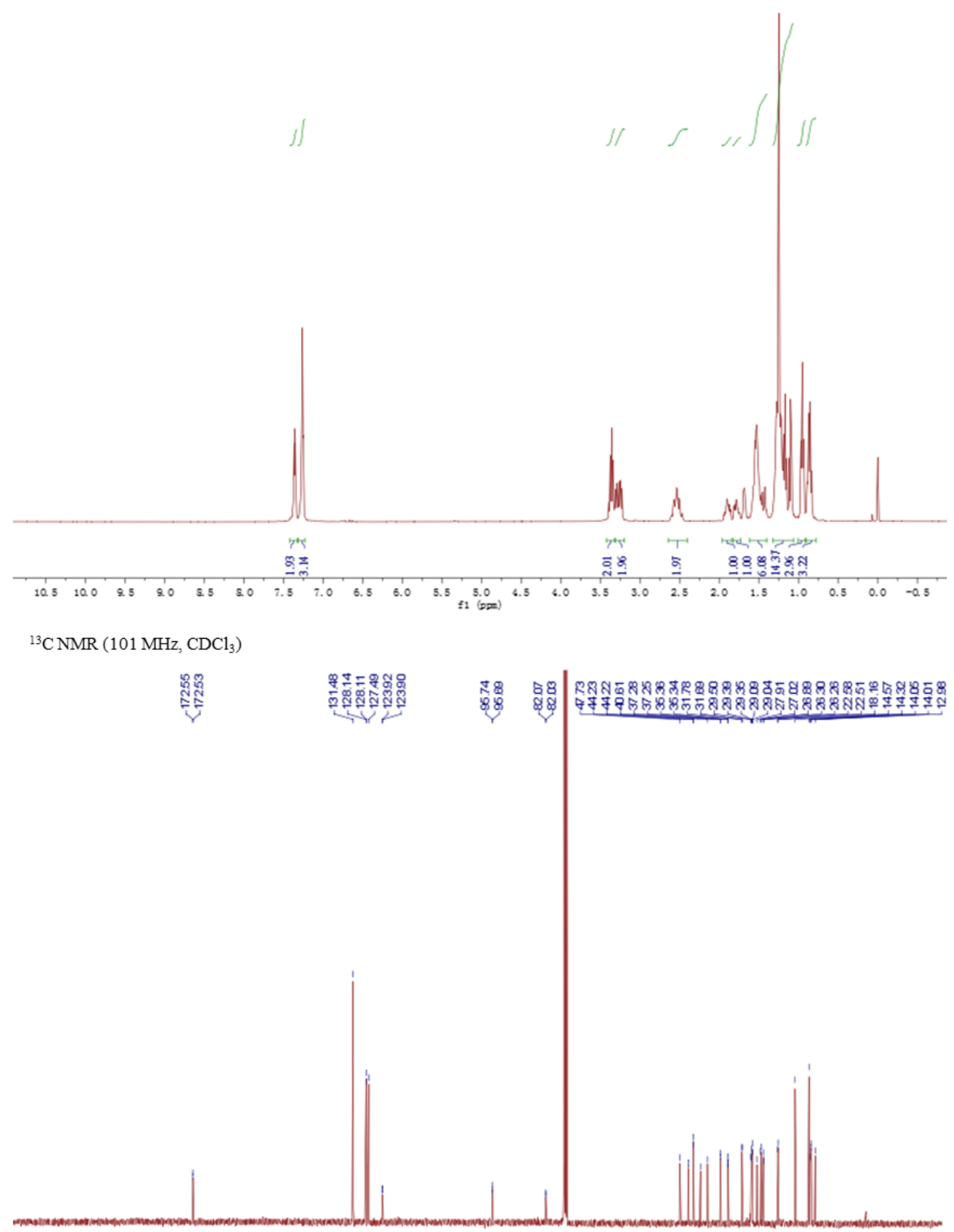

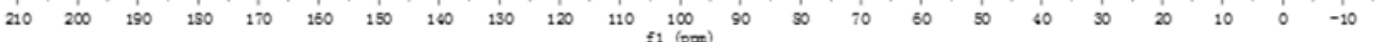


<smiles>CCN(Cc1ccccc1)C(=O)CC[C@](C)(C#C[Se-])C(C)C</smiles>

${ }^{1} \mathrm{H} \mathrm{NMR}\left(400 \mathrm{MHz}, \mathrm{CDCl}_{3}\right)$

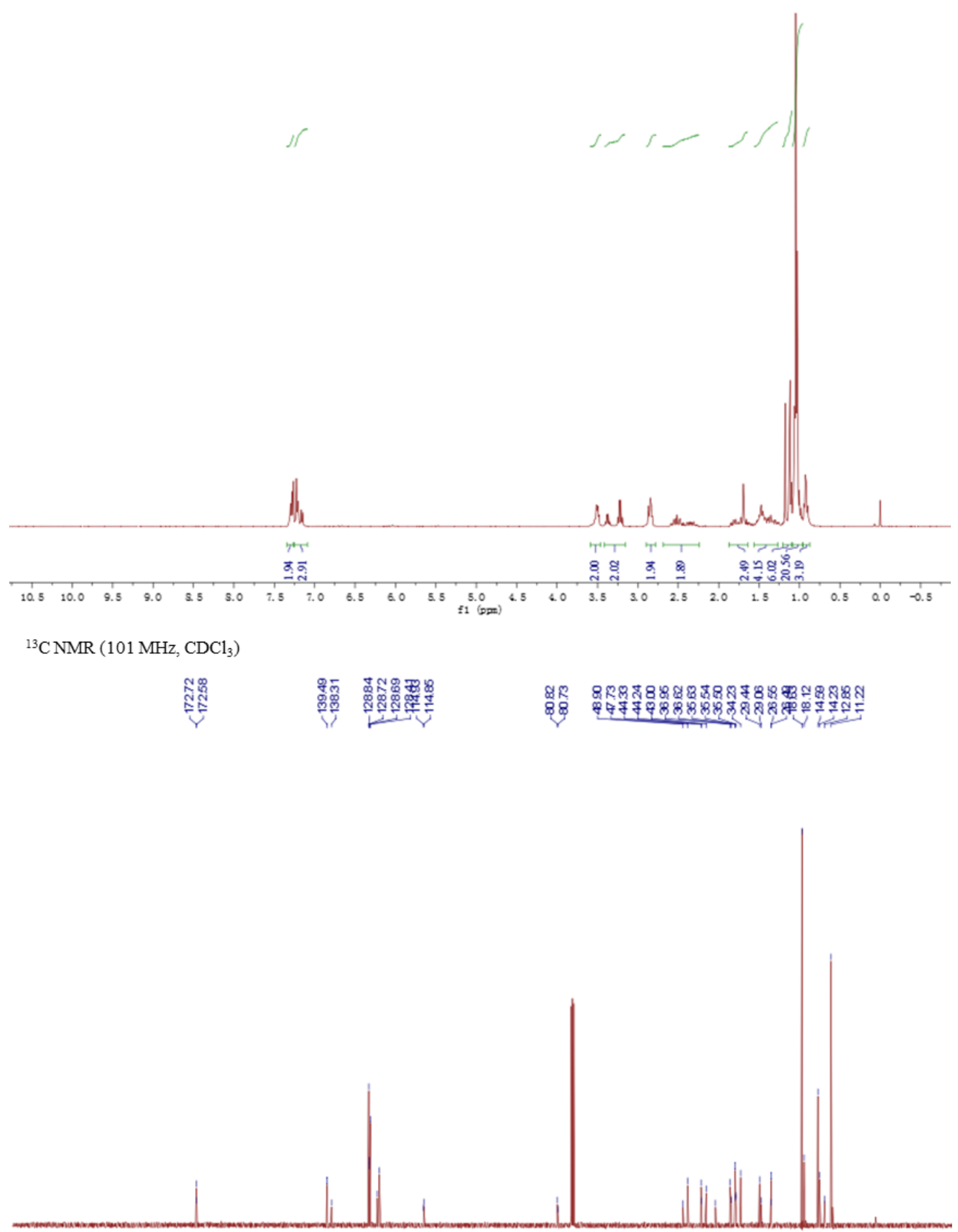

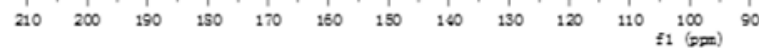


<smiles>CCN(CCc1cccs1)C(=O)CC[C@](C)(C#C[Se][SnH3])C(C)C</smiles>

${ }^{1} \mathrm{H} \mathrm{NMR}\left(400 \mathrm{MHz}, \mathrm{CDCl}_{3}\right)$

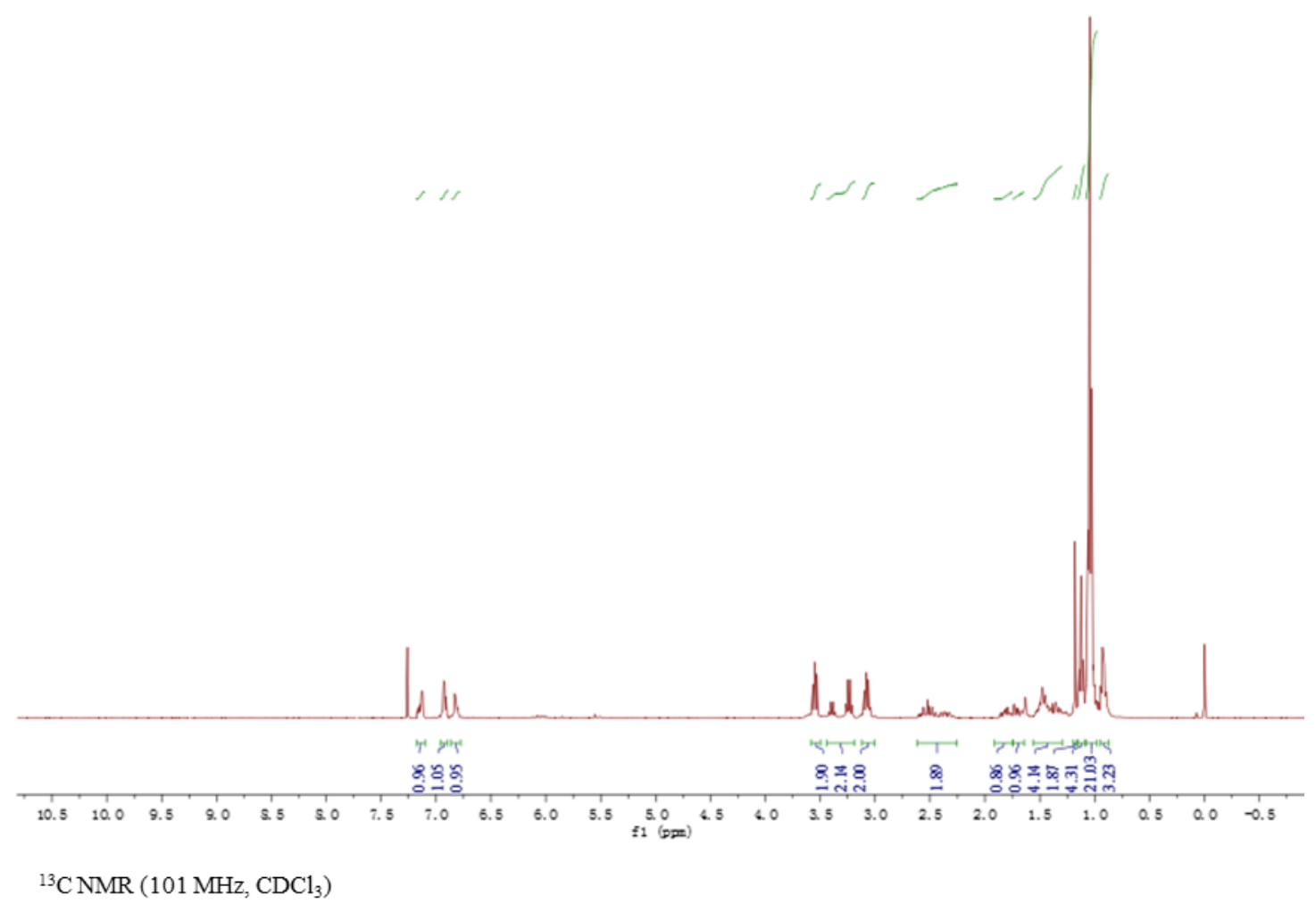

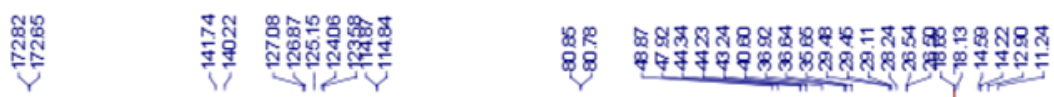

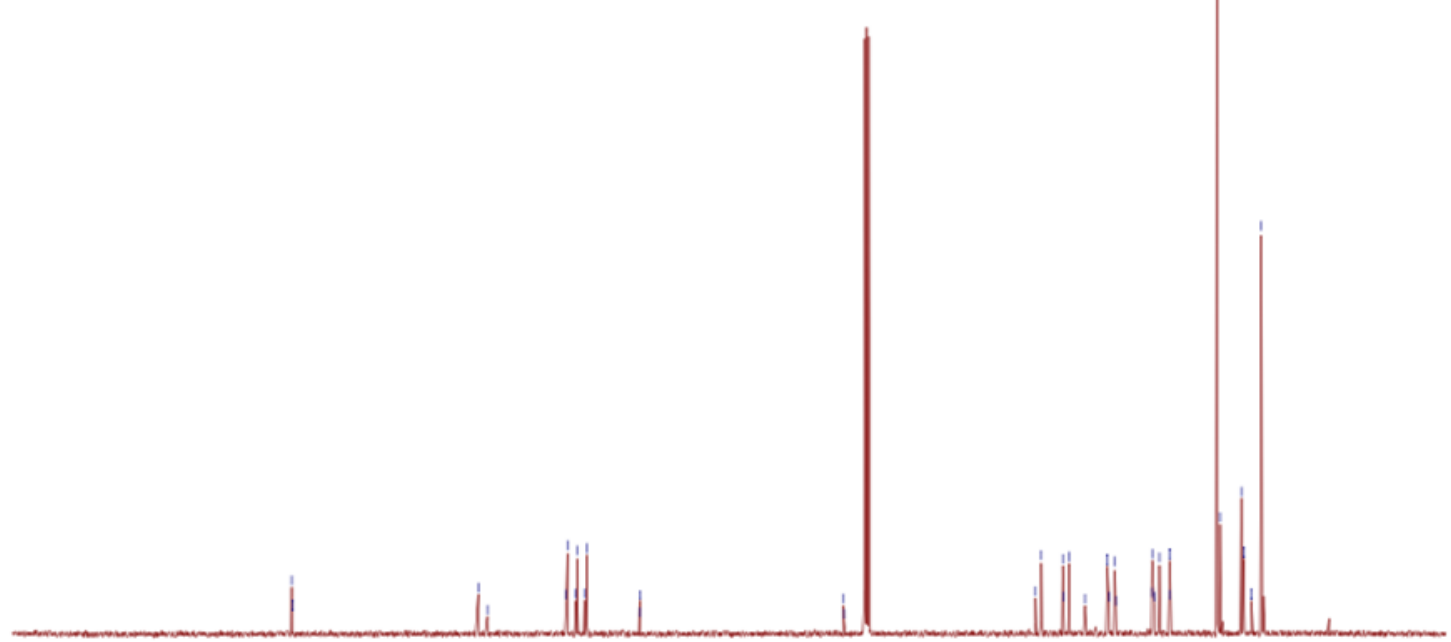

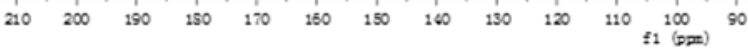


<smiles>CCN(CCc1ccco1)C(=O)CC[C@](C)(C#C[Se][SnH3])C(C)C</smiles>

${ }^{1} \mathrm{H} \mathrm{NMR}\left(400 \mathrm{MHz}, \mathrm{CDCl}_{3}\right)$

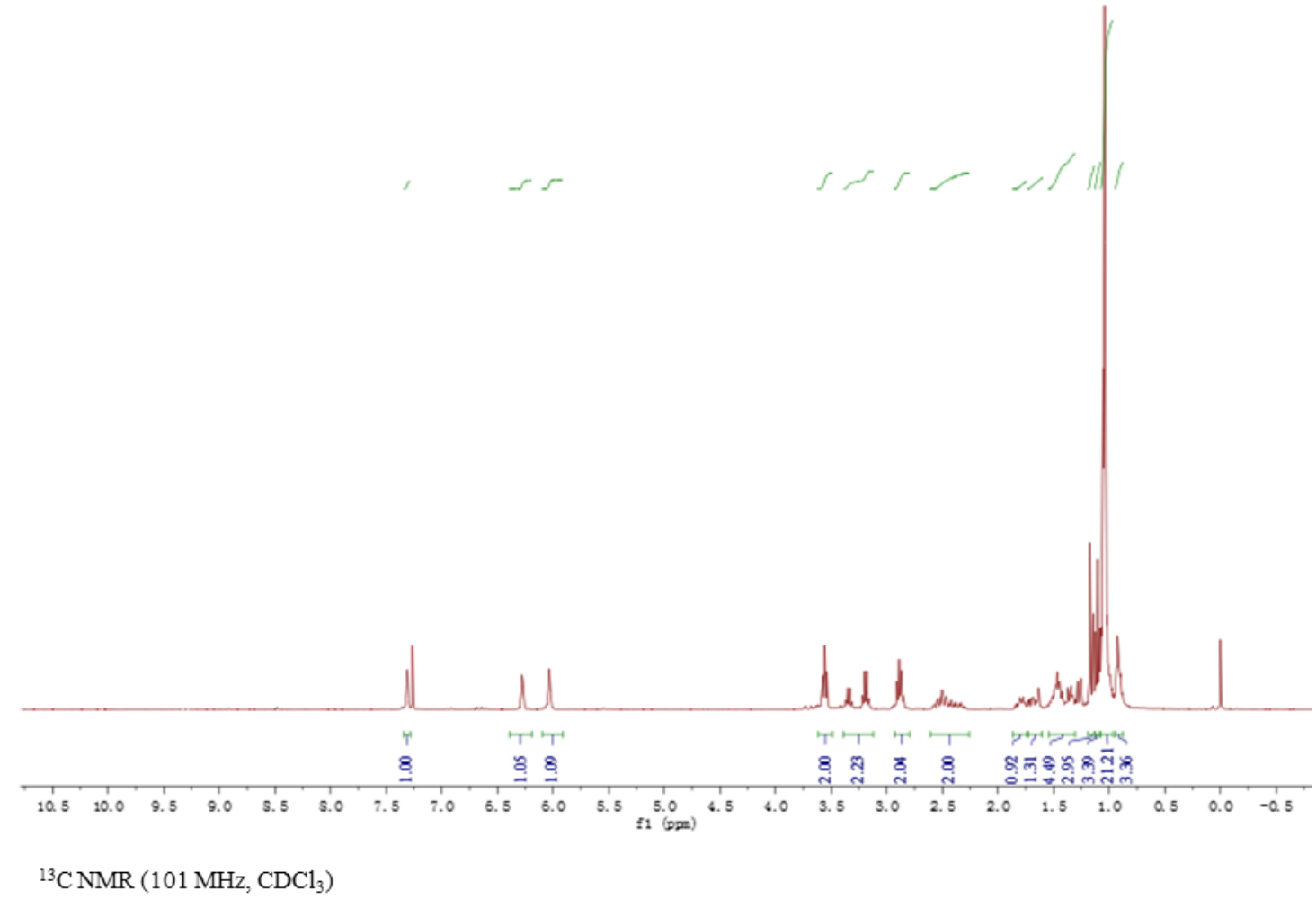

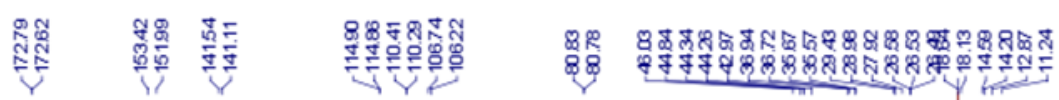

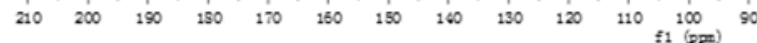


<smiles>CCN(CCOC)C(=O)CC[C@](C)(C#C[Se-][SnH3])C(C)C</smiles>

${ }^{1} \mathrm{H} \mathrm{NMR}\left(400 \mathrm{MHz}, \mathrm{CDCl}_{3}\right)$

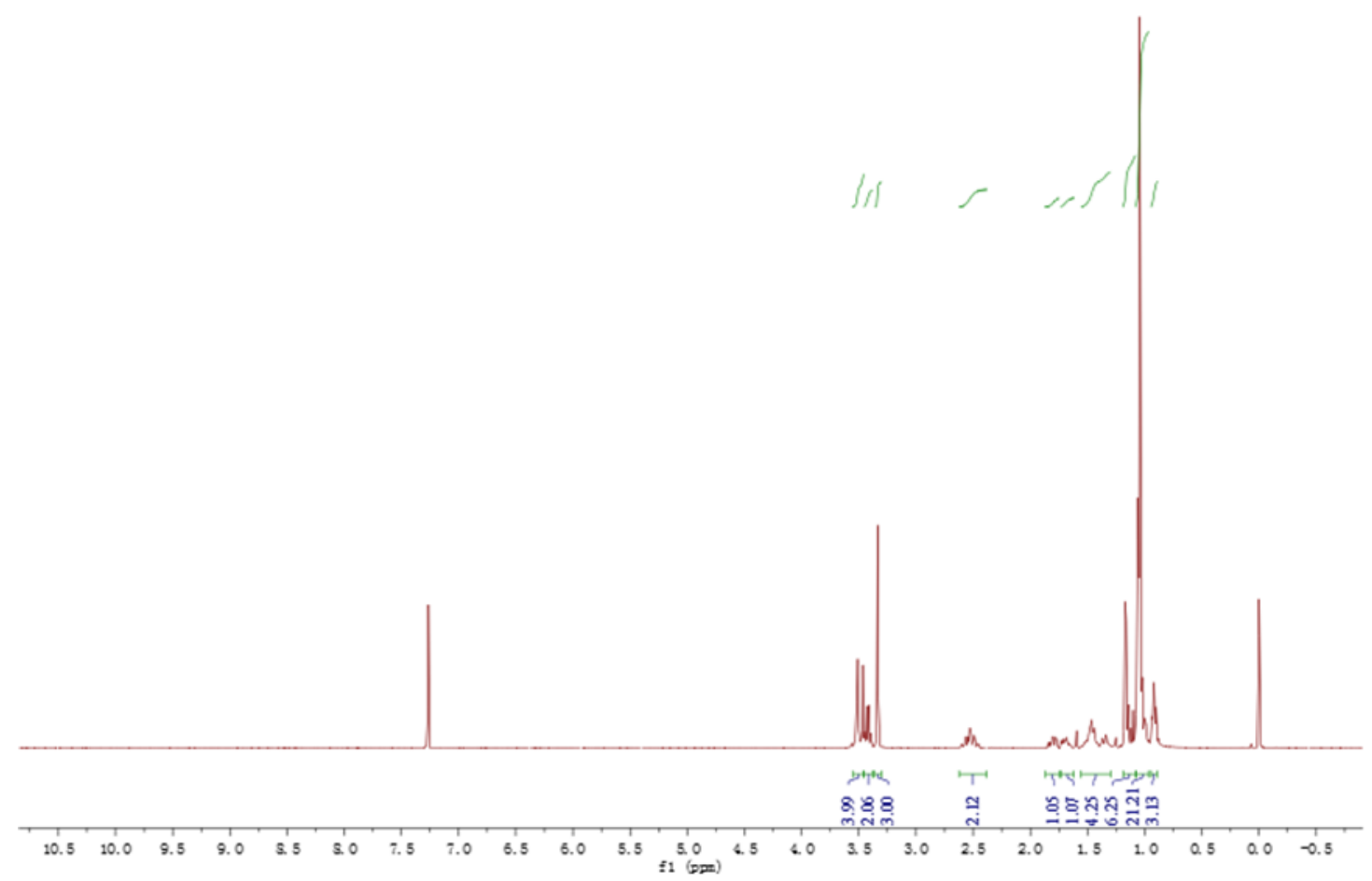
${ }^{13} \mathrm{CNMR}\left(101 \mathrm{MHz}, \mathrm{CDCl}_{3}\right)$

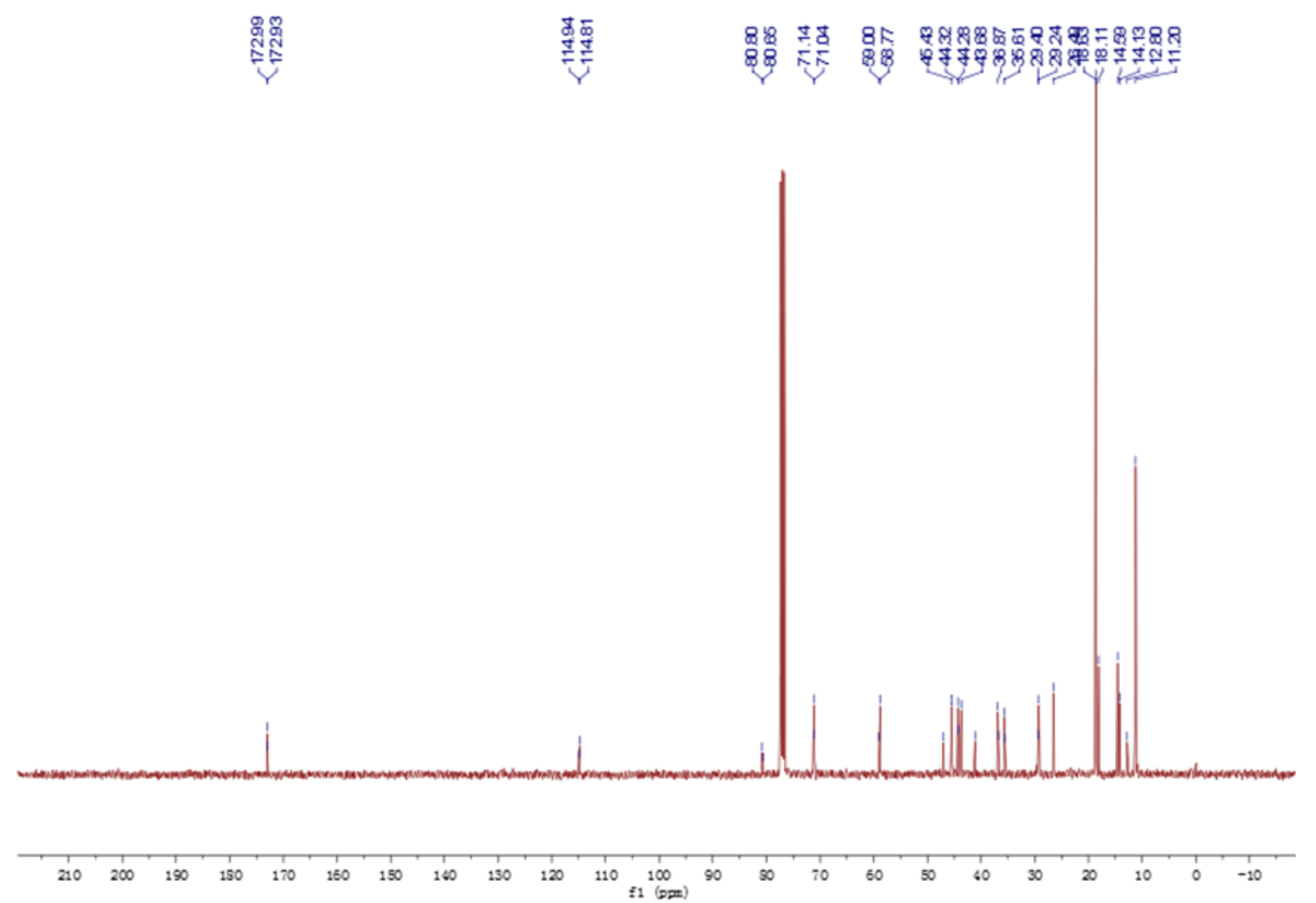


<smiles>C#CC#C[C@](C)(CCC(=O)N(CC)CCOC)C(C)C</smiles>

${ }^{1} \mathrm{H}$ NMR $\left(400 \mathrm{MHz}, \mathrm{CDCl}_{3}\right)$

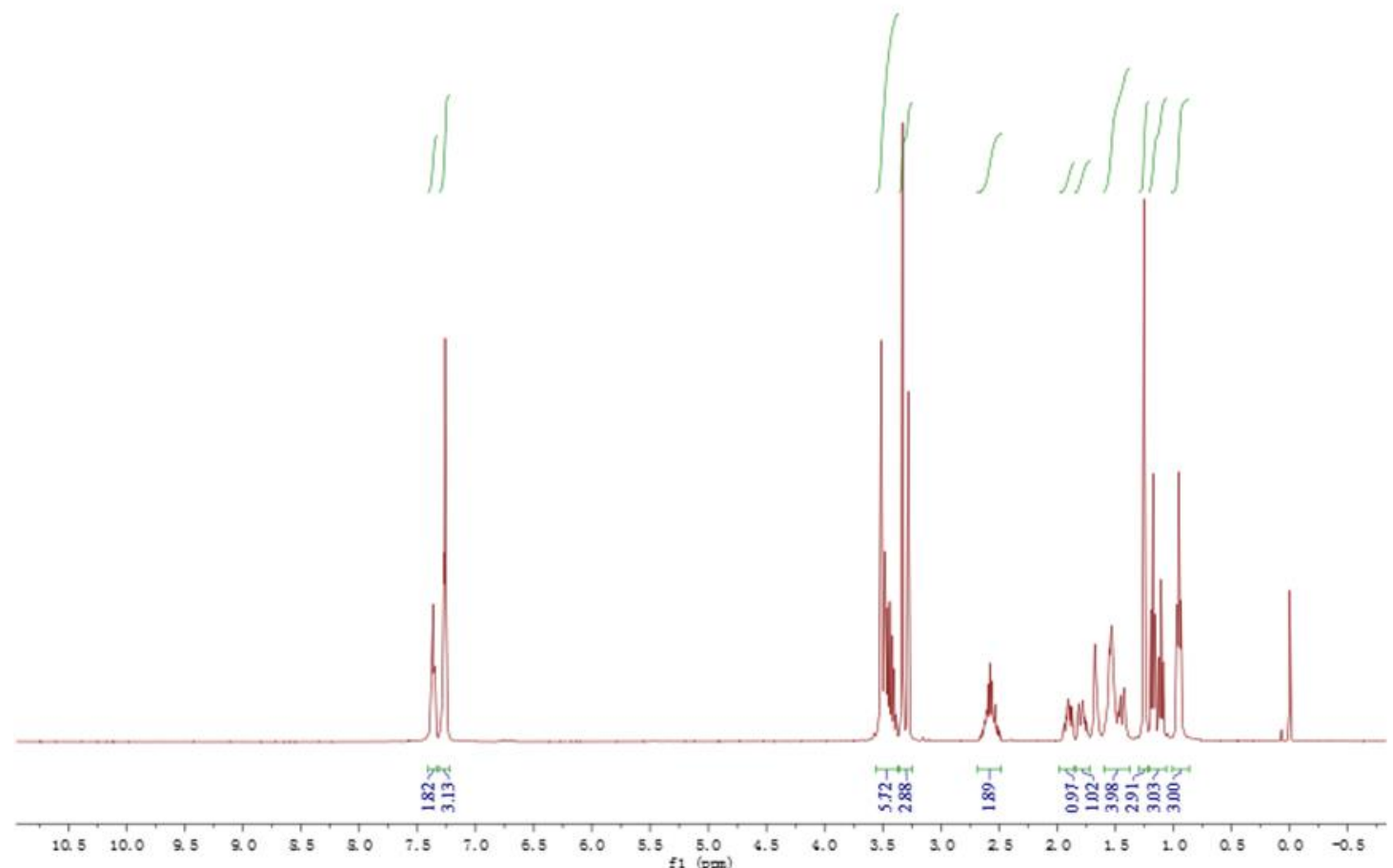

${ }^{13} \mathrm{C} \mathrm{NMR}\left(101 \mathrm{MHz}, \mathrm{CDCl}_{3}\right)$

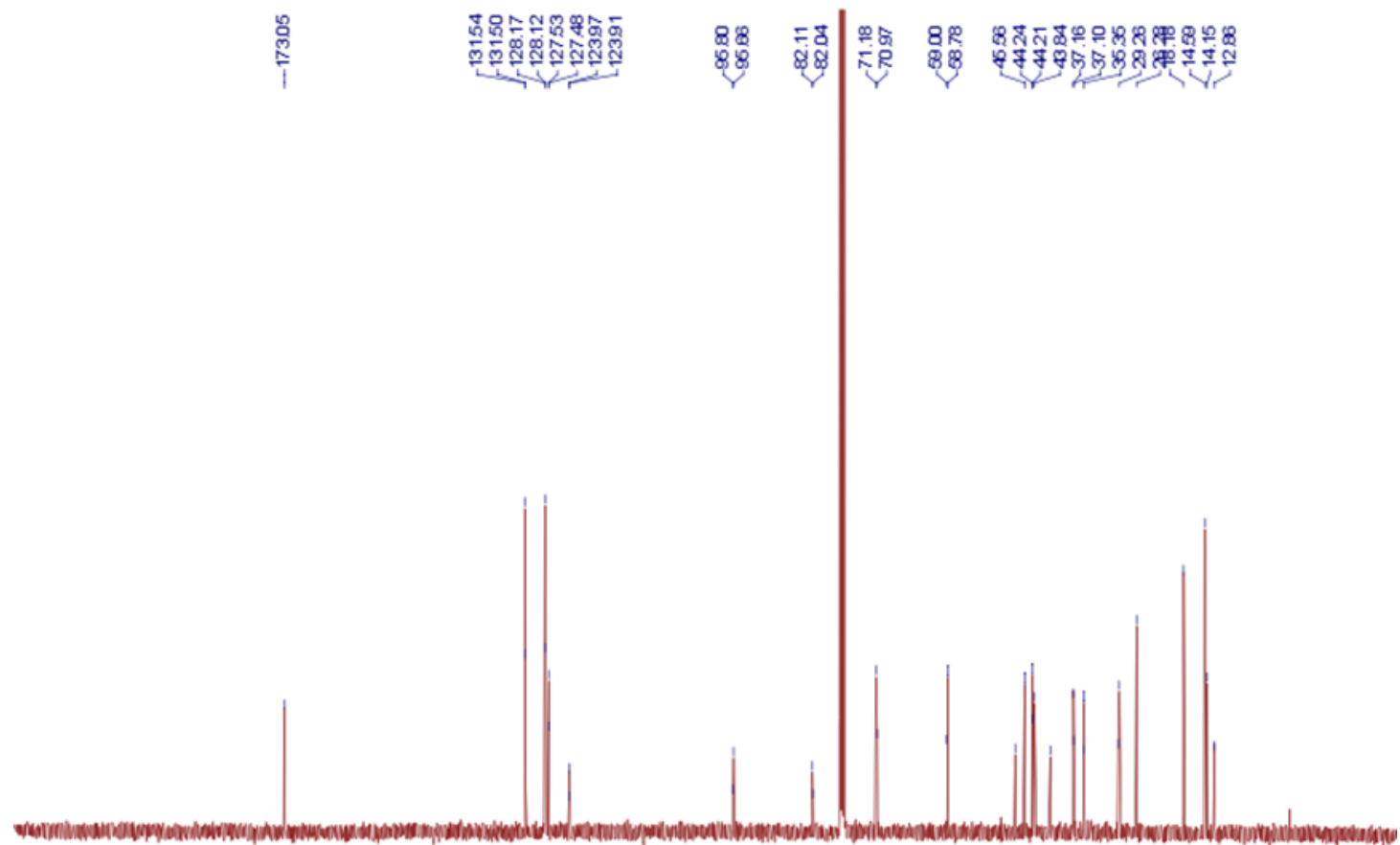

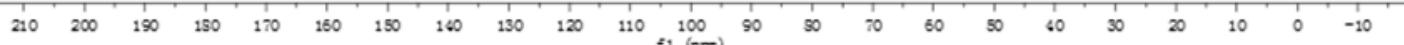




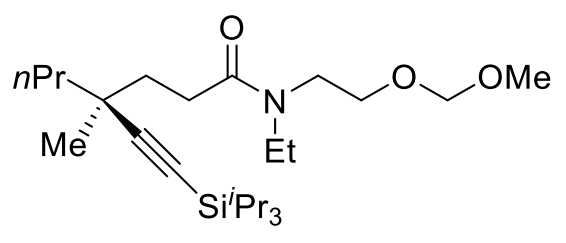

${ }^{1} \mathrm{H} \mathrm{NMR}\left(400 \mathrm{MHz}, \mathrm{CDCl}_{3}\right)$
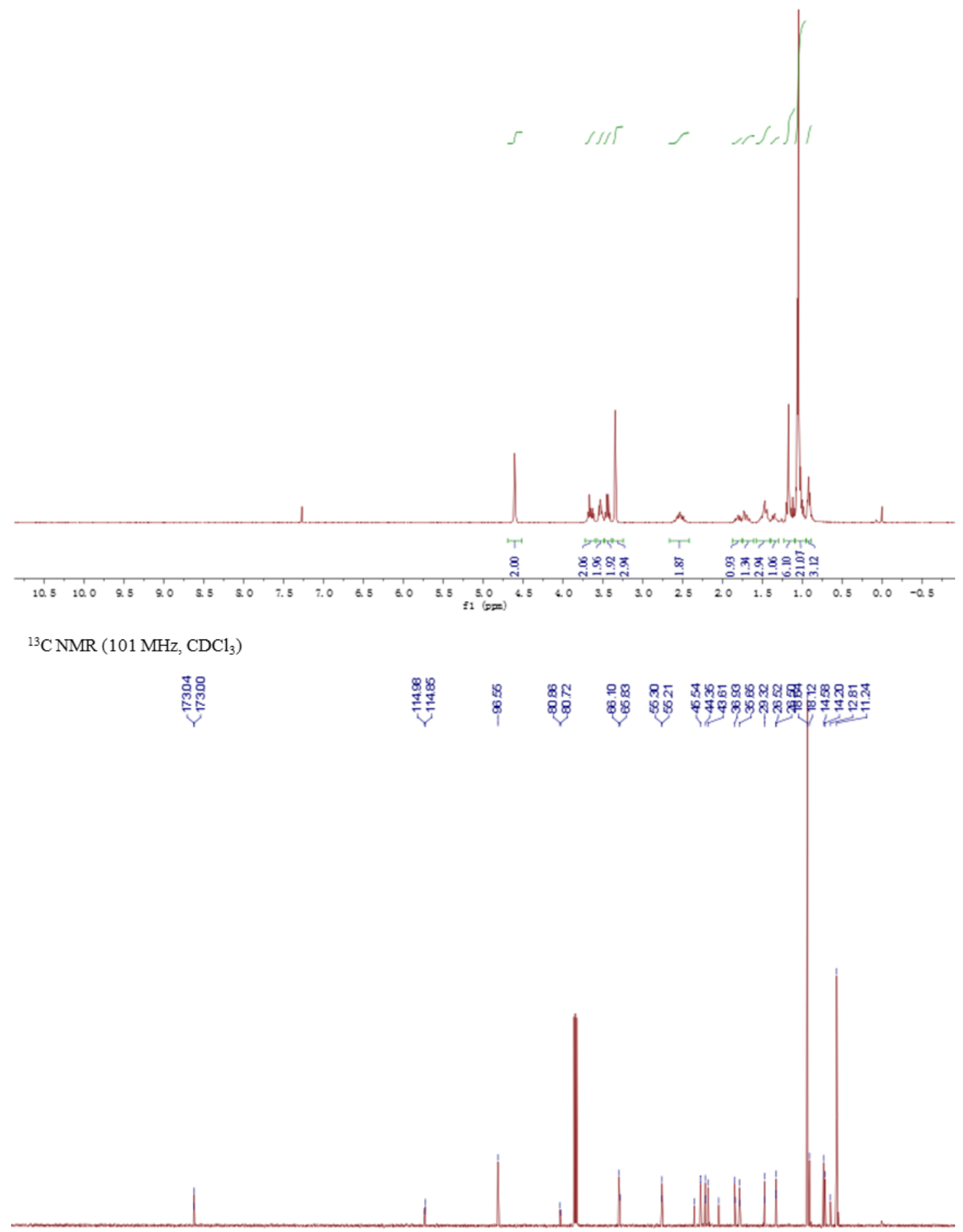

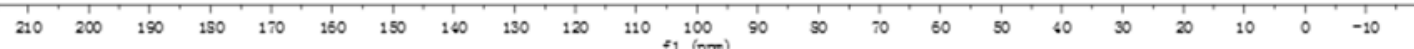


<smiles>CCN(CCOCOC)C(=O)CC[C@](C)(C#Cc1ccccc1)C(C)C</smiles>

${ }^{1} \mathrm{H} \mathrm{NMR}\left(400 \mathrm{MHz}, \mathrm{CDCl}_{3}\right)$

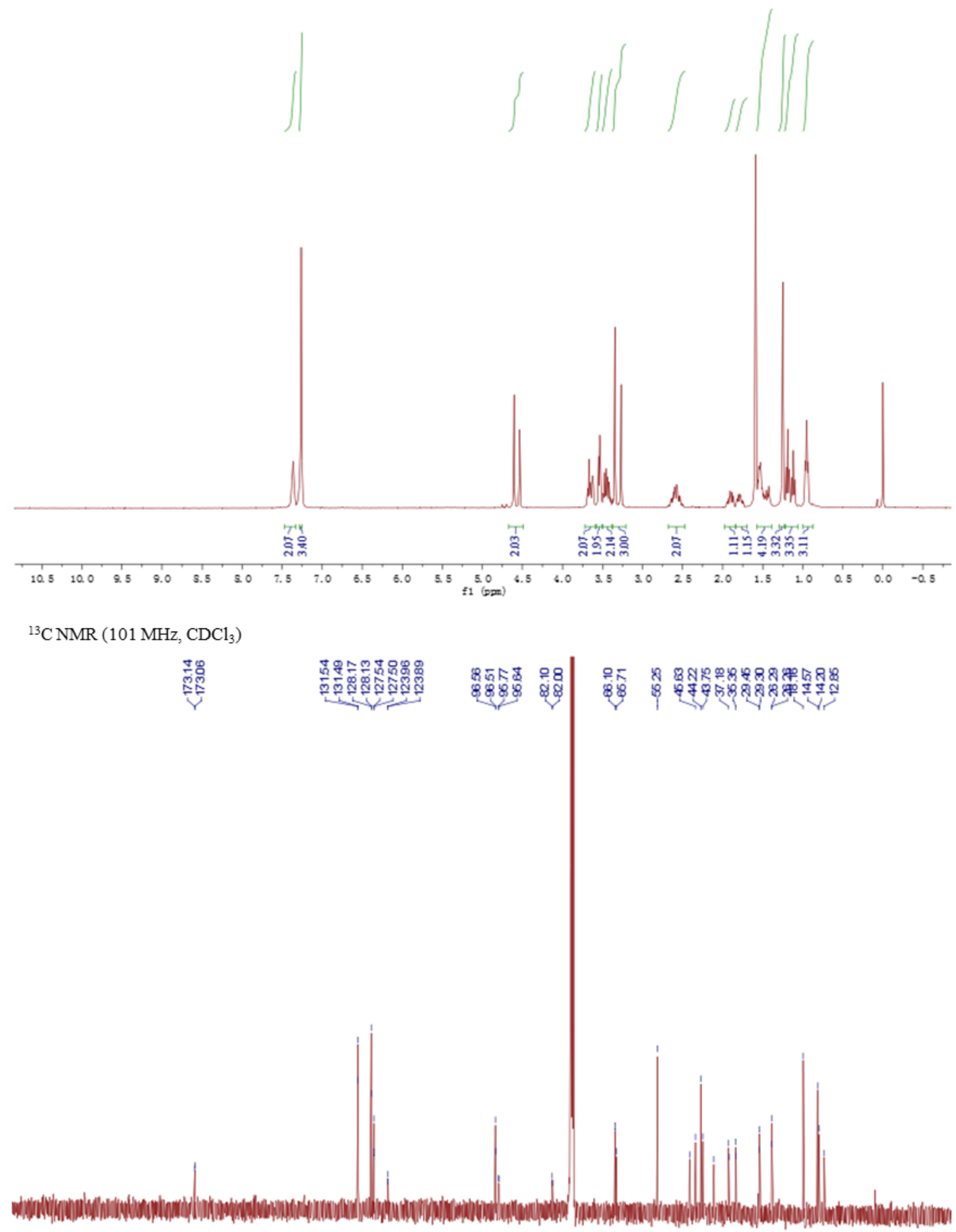

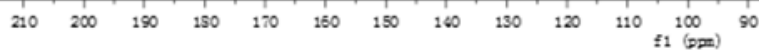




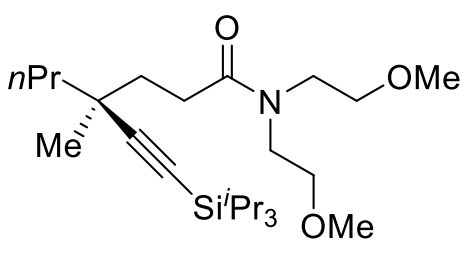

${ }^{1} \mathrm{H} \mathrm{NMR}\left(400 \mathrm{MHz}, \mathrm{CDCl}_{3}\right)$

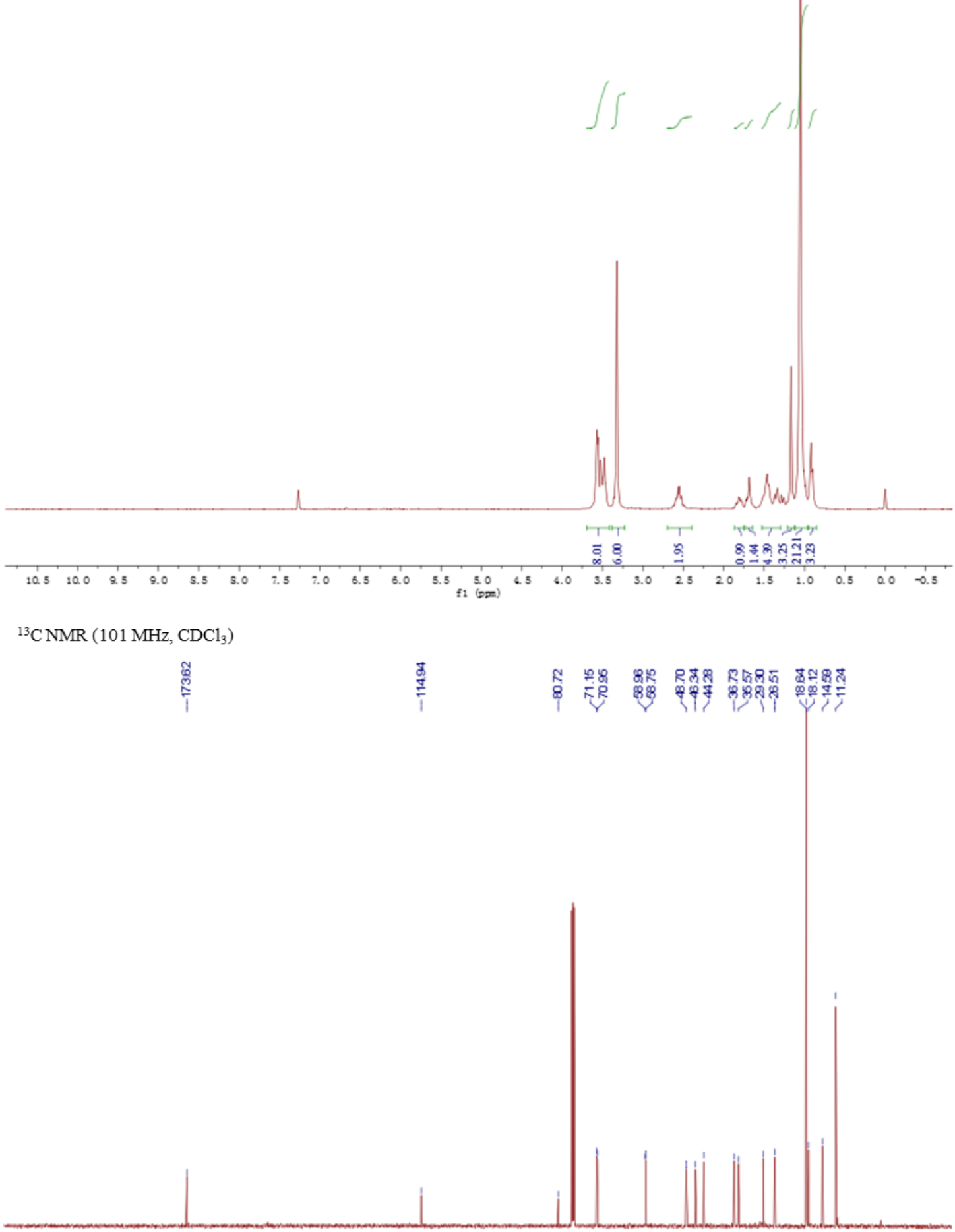

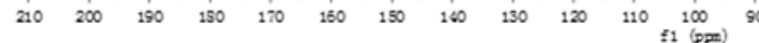




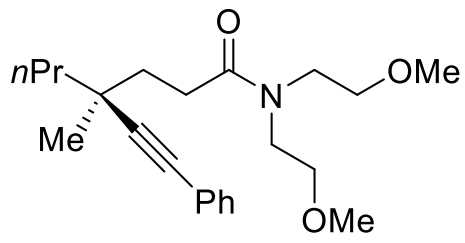

${ }^{1} \mathrm{H} \mathrm{NMR}\left(400 \mathrm{MHz}, \mathrm{CDCl}_{3}\right)$

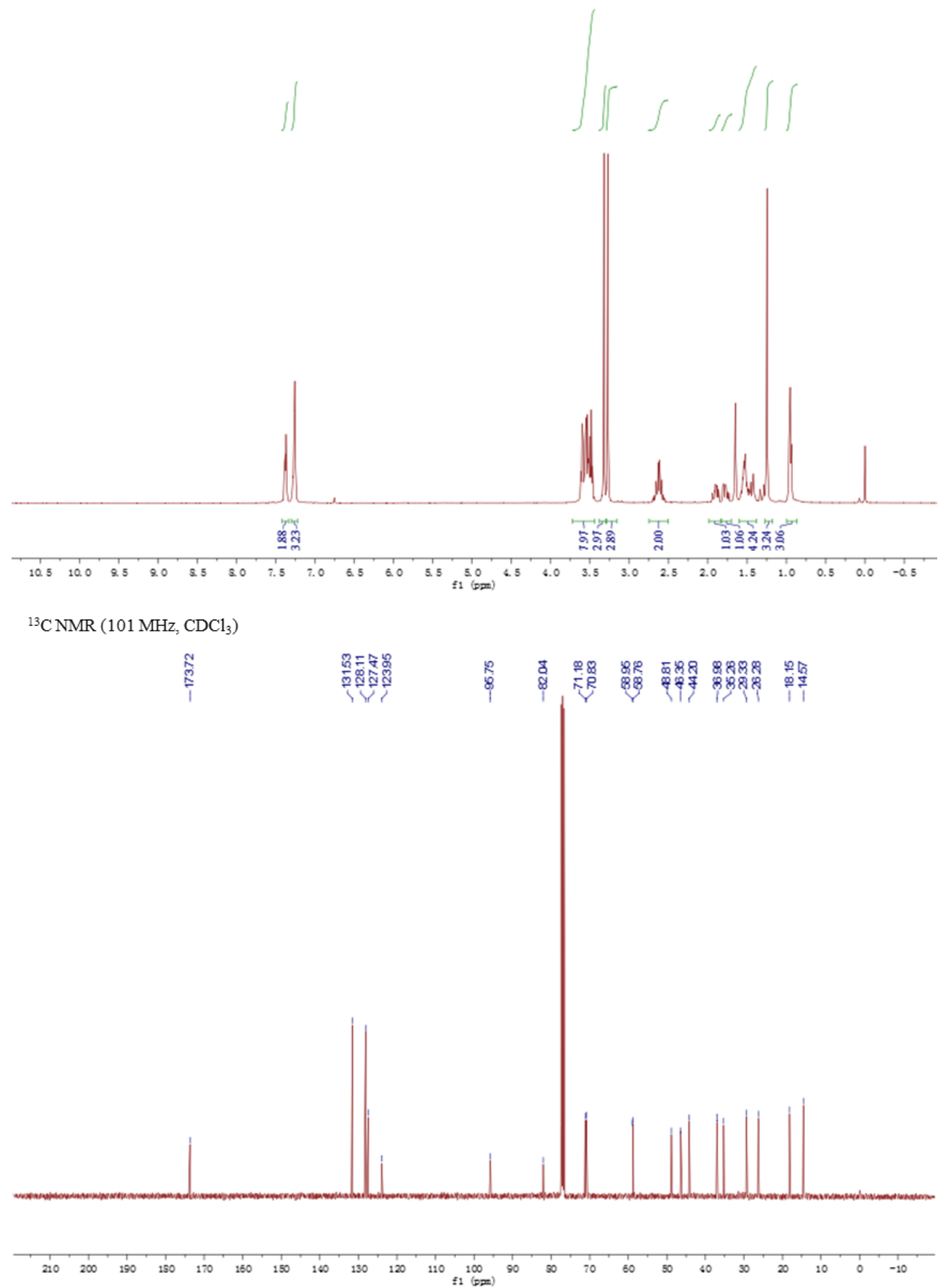


<smiles>CCN(CCC1=CCCCC1)C(=O)CC[C@](C)(C#C[Se]C(C)C)C(C)C</smiles>

$\left.{ }^{1} \mathrm{H} \mathrm{NMR} \mathrm{(400} \mathrm{MHz,} \mathrm{CDCl}_{3}\right)$
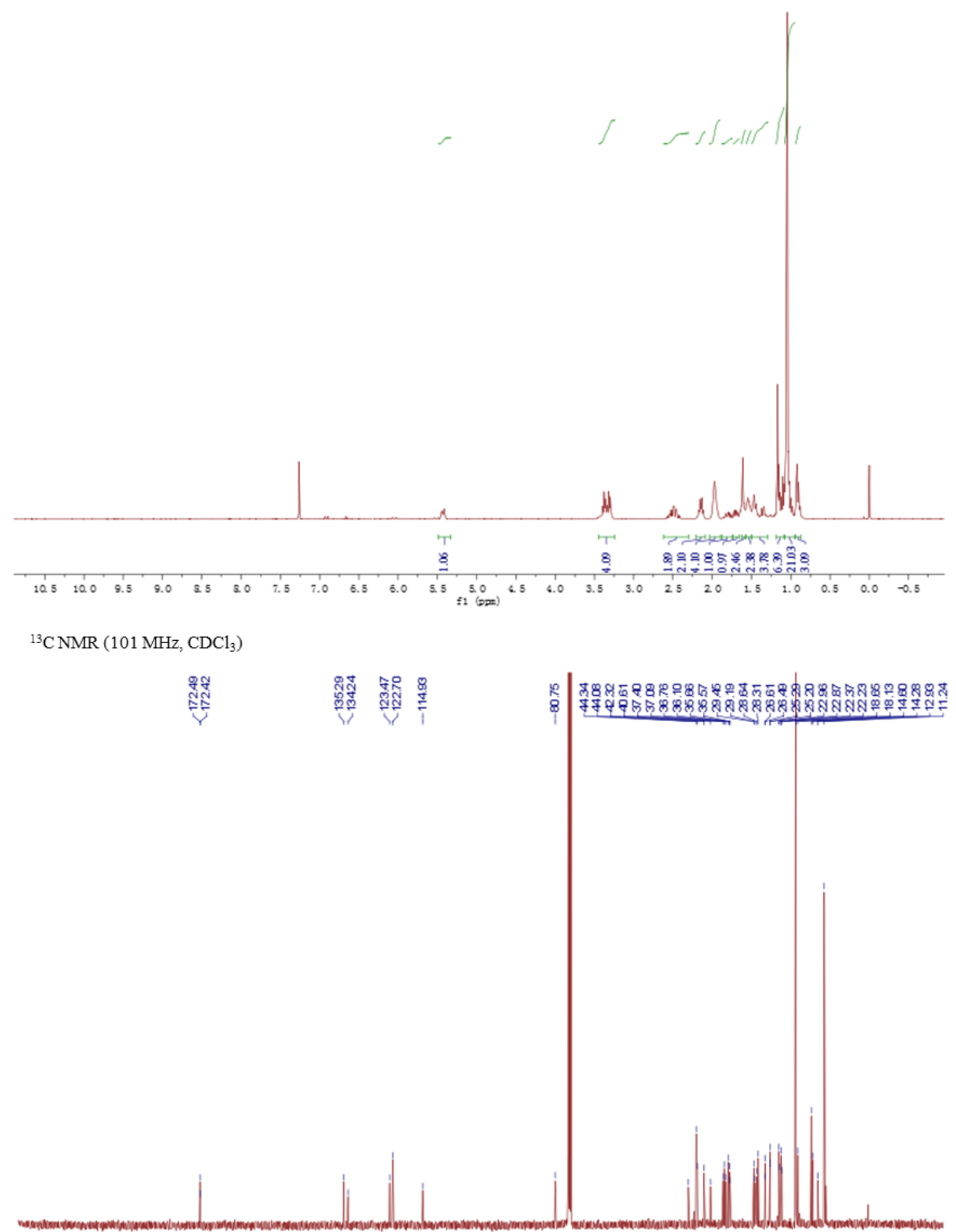

$\begin{array}{llllllllllll}210 & 200 & 190 & 150 & 170 & 160 & 150 & 160 & 130 & 120 & 110 & 100 \\ \mathrm{f1}(\mathrm{fm}) & 90\end{array}$ 
<smiles>CCN(CCC1=CCCCC1)C(=O)CC[C@](C)(C#CPc1ccccc1)C(C)C</smiles>

${ }^{1} \mathrm{H} \mathrm{NMR}\left(400 \mathrm{MHz}, \mathrm{CDCl}_{3}\right)$
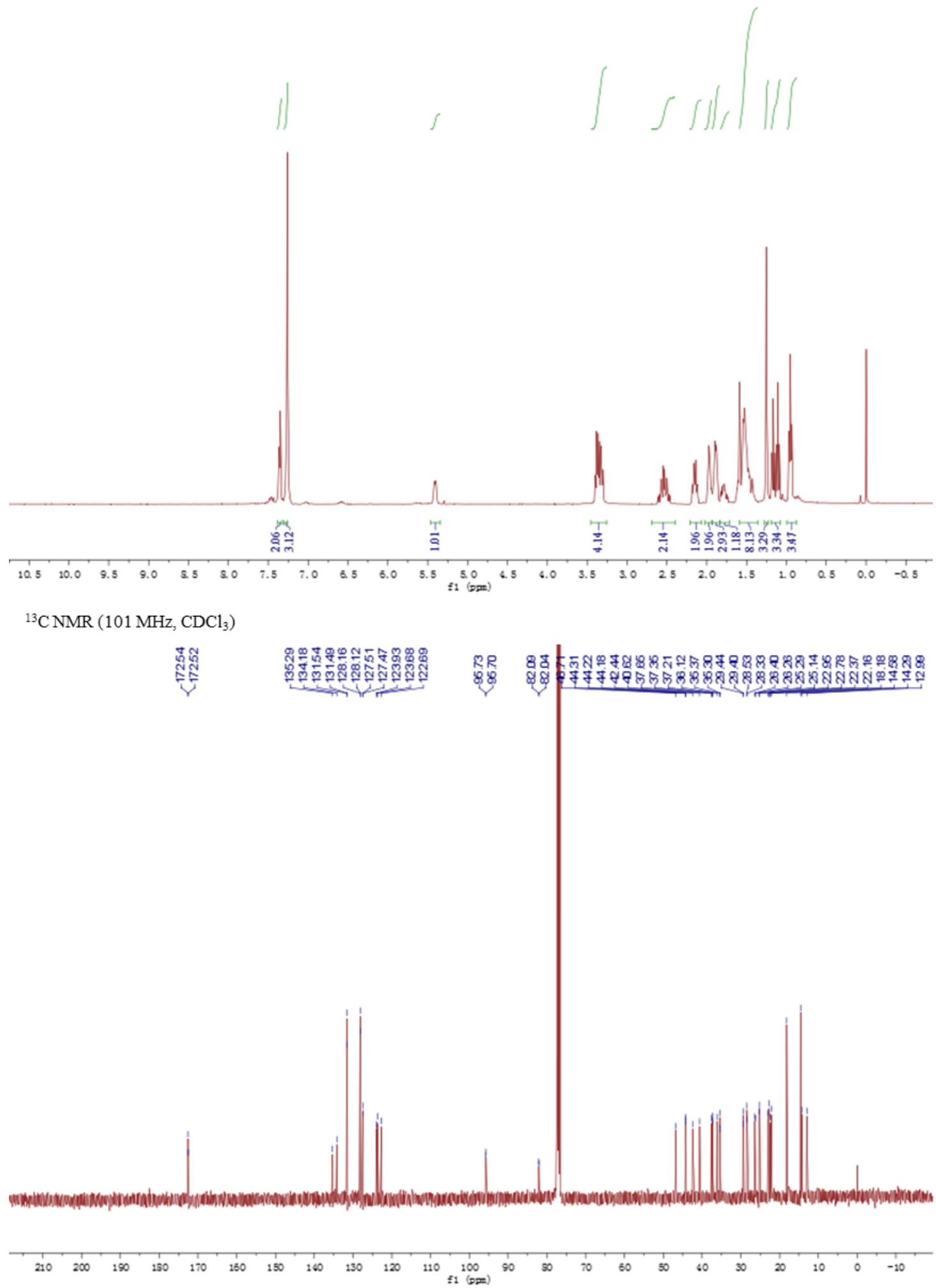


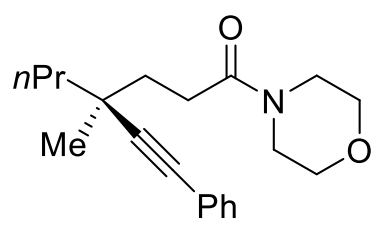

${ }^{1} \mathrm{H} \mathrm{NMR}\left(400 \mathrm{MHz}, \mathrm{CDCl}_{3}\right.$ )

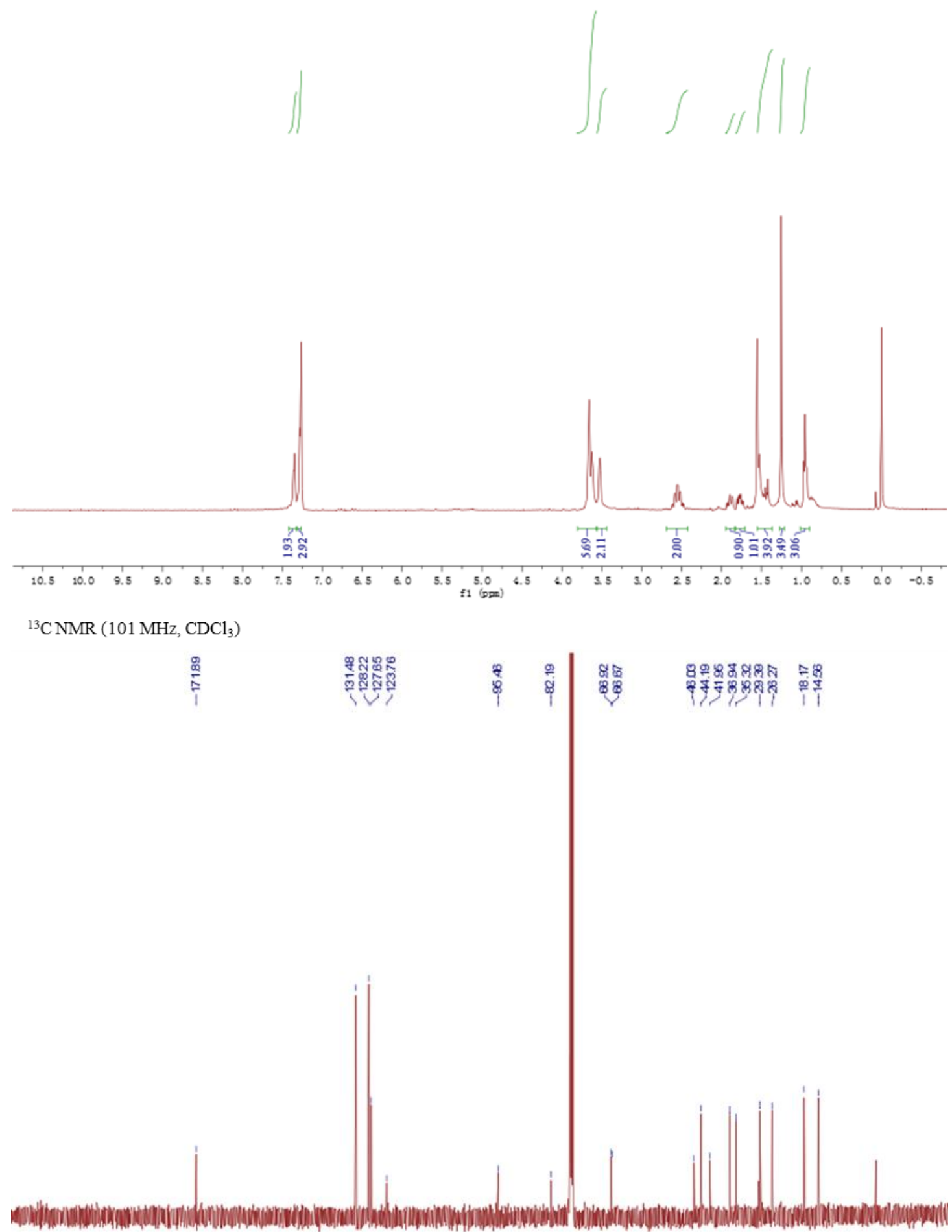

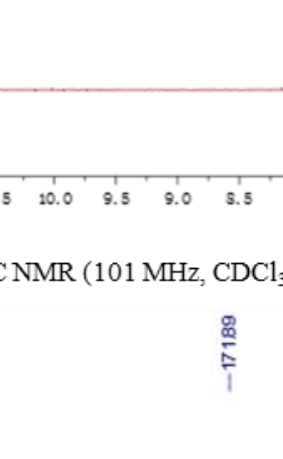




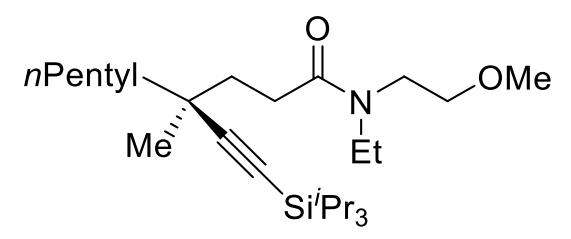

${ }^{1} \mathrm{H} \mathrm{NMR}\left(400 \mathrm{MHz}, \mathrm{CDCl}_{3}\right)$

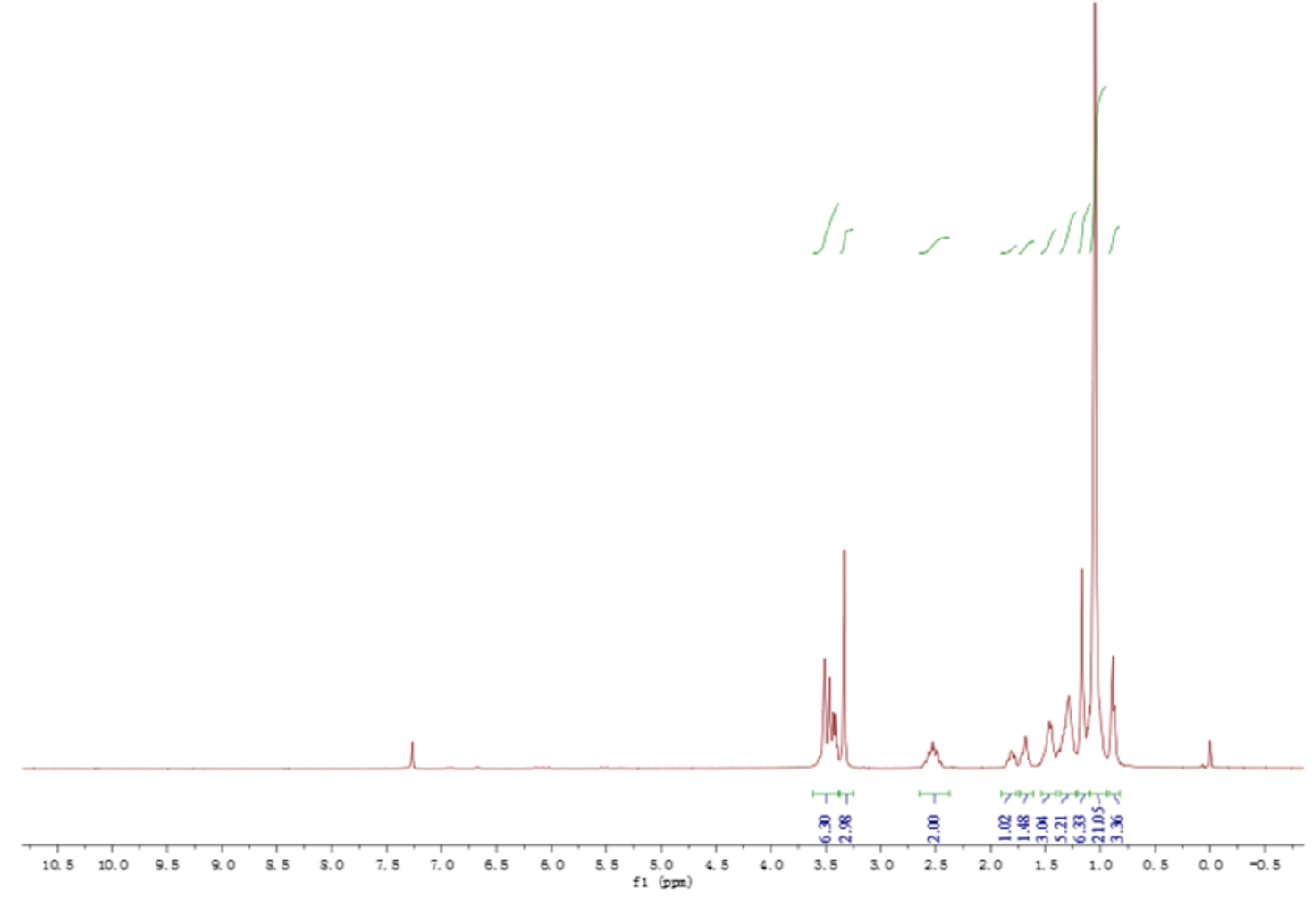

${ }^{13} \mathrm{CNMR}\left(101 \mathrm{MHz}, \mathrm{CDCl}_{3}\right)$

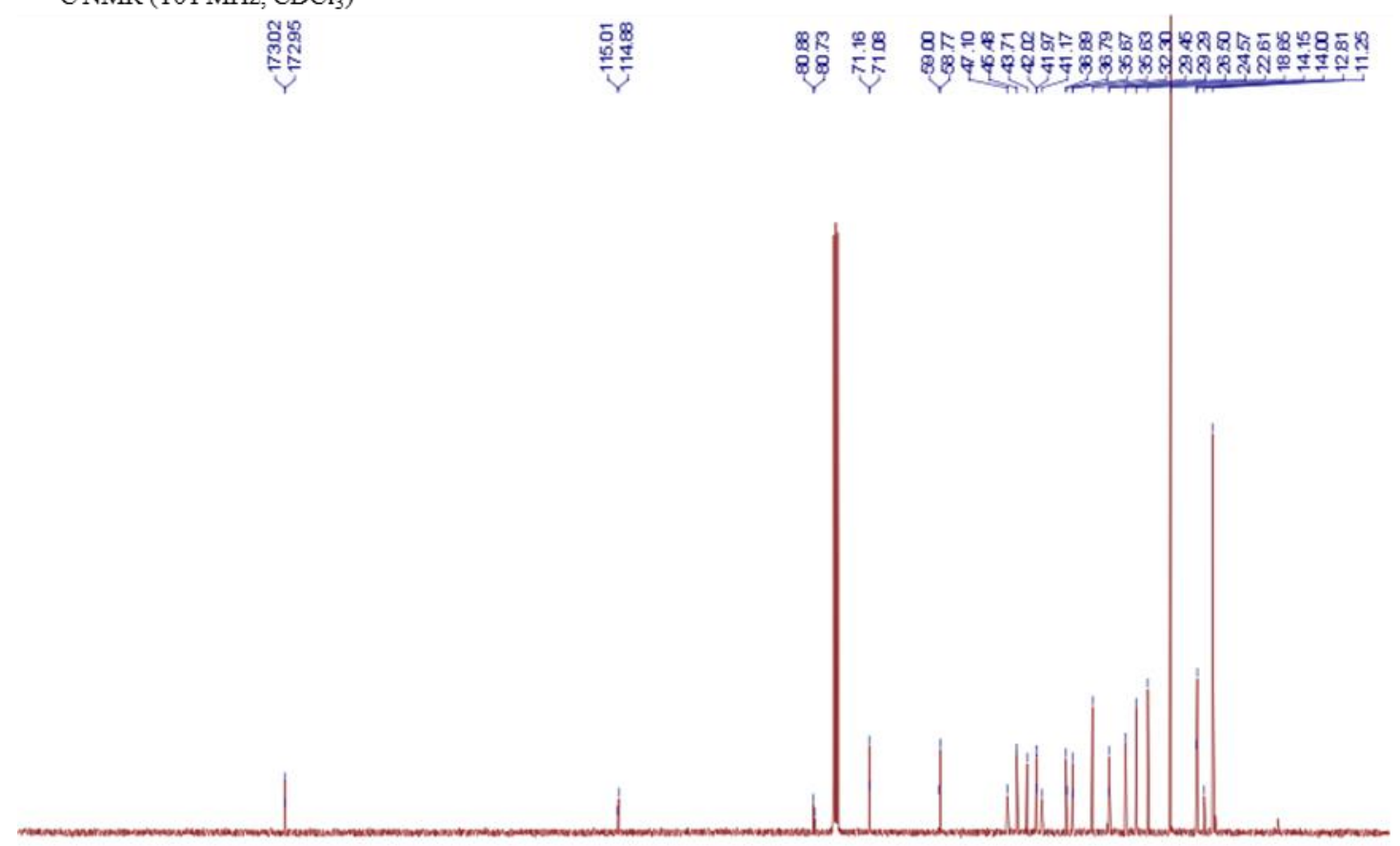

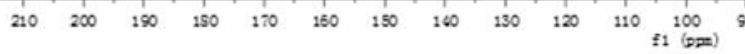




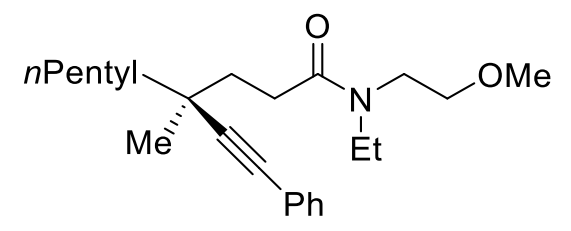

${ }^{1} \mathrm{H} \mathrm{NMR}\left(400 \mathrm{MHz}, \mathrm{CDCl}_{3}\right)$

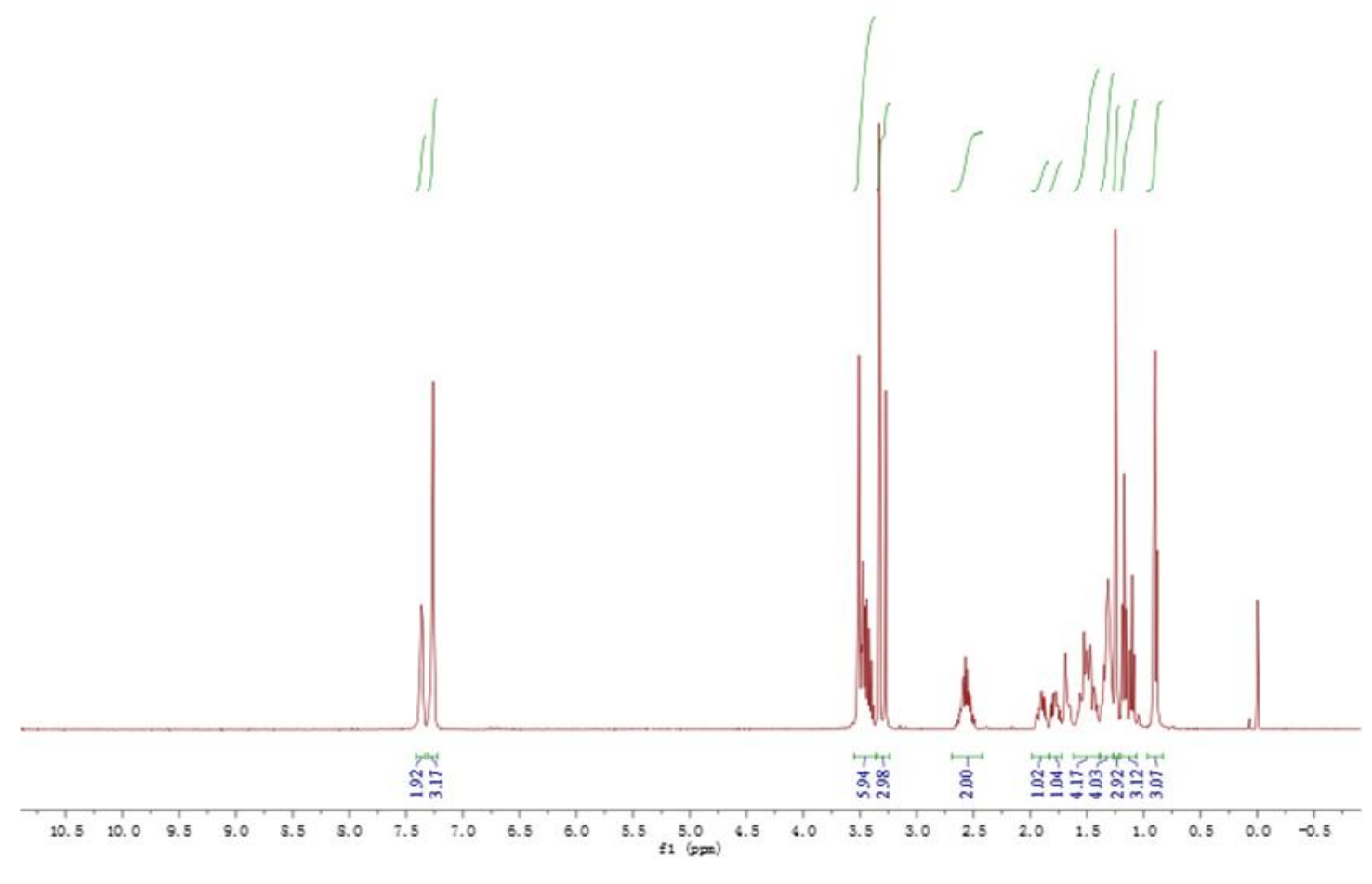

${ }^{13} \mathrm{CNMR}\left(101 \mathrm{MHz}, \mathrm{CDCl}_{3}\right)$

\begin{tabular}{|c|c|c|c|c|c|}
\hline on & \multicolumn{2}{|c|}{ 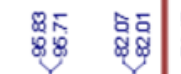 } & \multicolumn{2}{|c|}{ 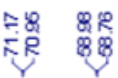 } & \\
\hline
\end{tabular}

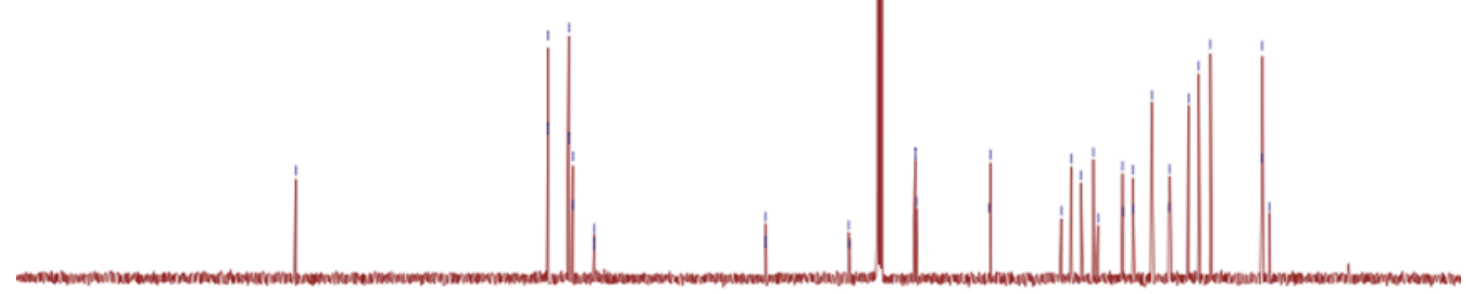

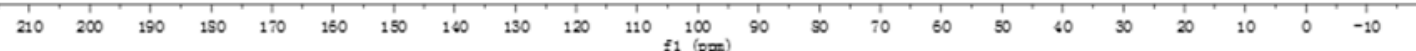


<smiles>CCN(CCOC)C(=O)CC[C@](C)(C#C[SeH])C(=O)O</smiles>

${ }^{1} \mathrm{H} \mathrm{NMR}\left(400 \mathrm{MHz}, \mathrm{CDCl}_{3}\right)$

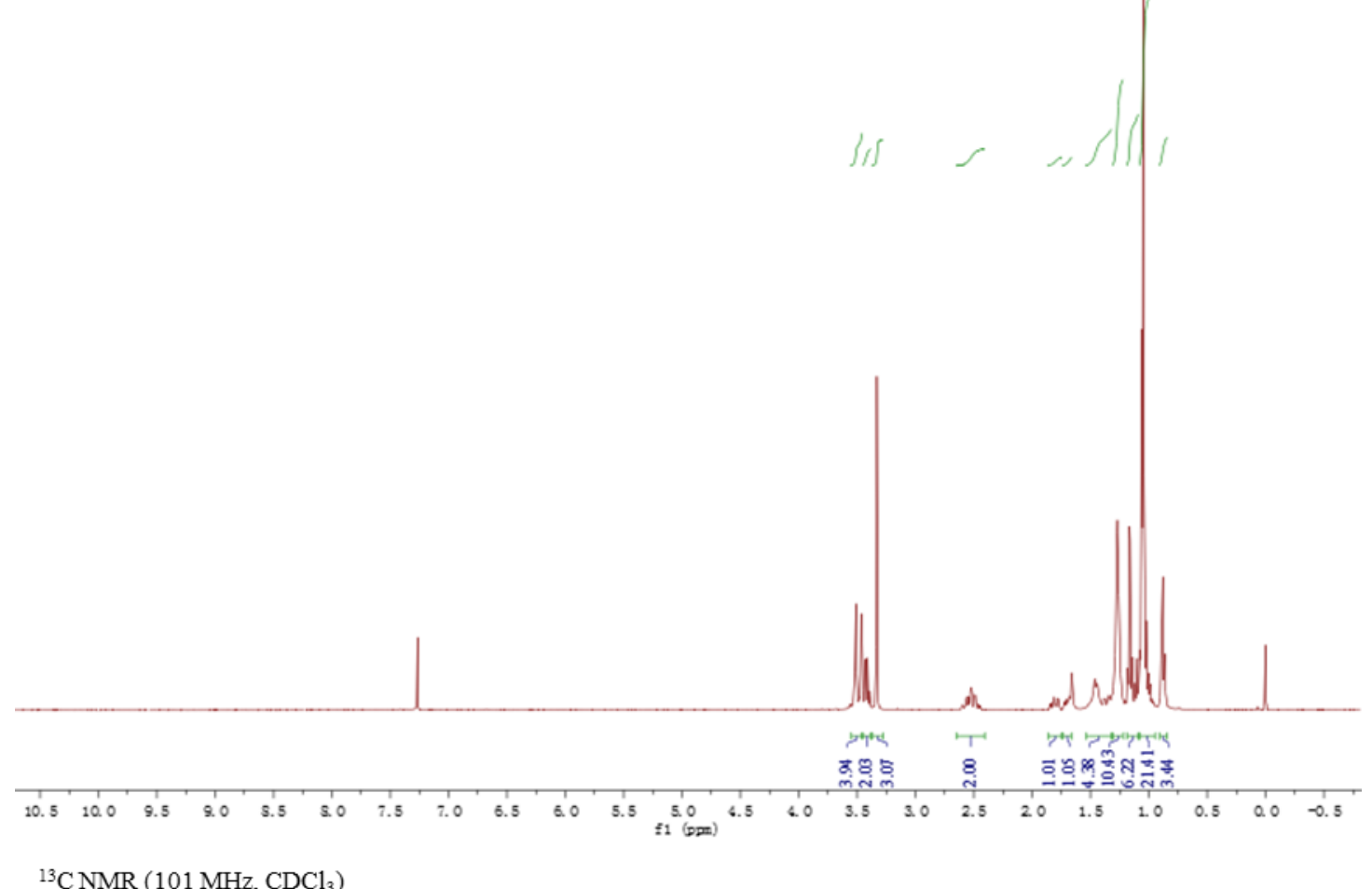

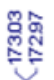

要要

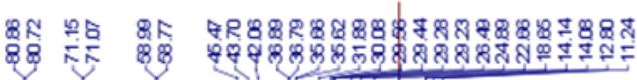

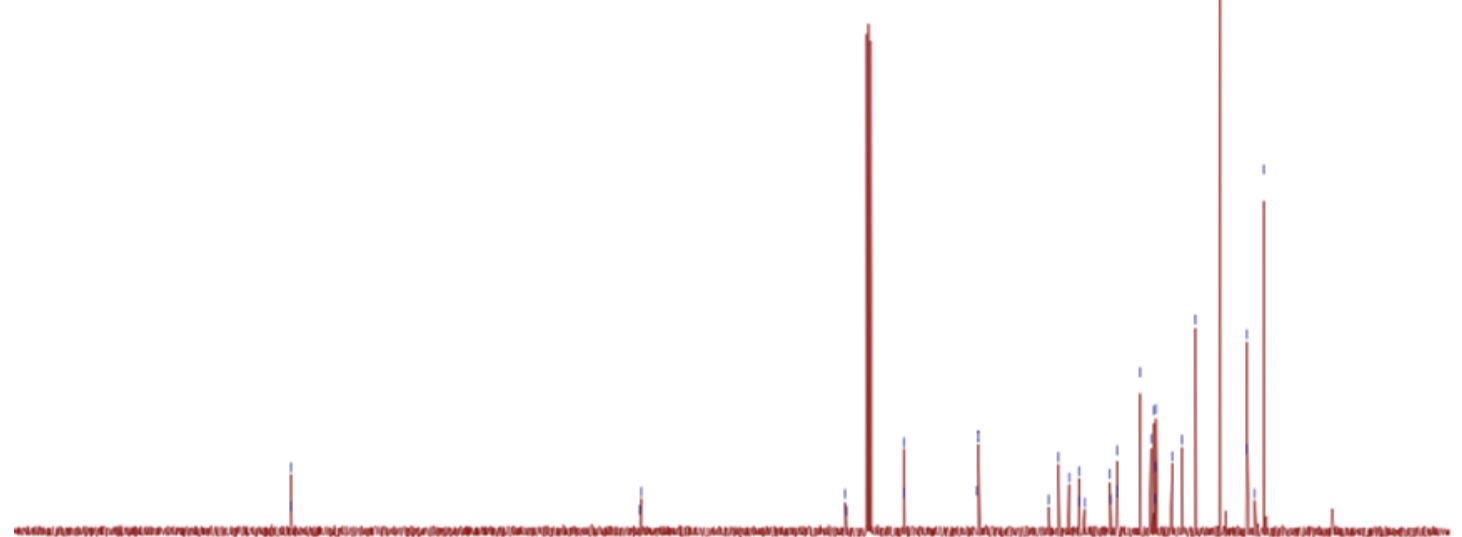

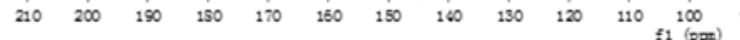


<smiles>CCN(CCOC)C(=O)CC[C@](C)(C#CPc1ccccc1)C(=O)O</smiles>

${ }^{1} \mathrm{H} \mathrm{NMR}\left(400 \mathrm{MHz}, \mathrm{CDCl}_{3}\right)$

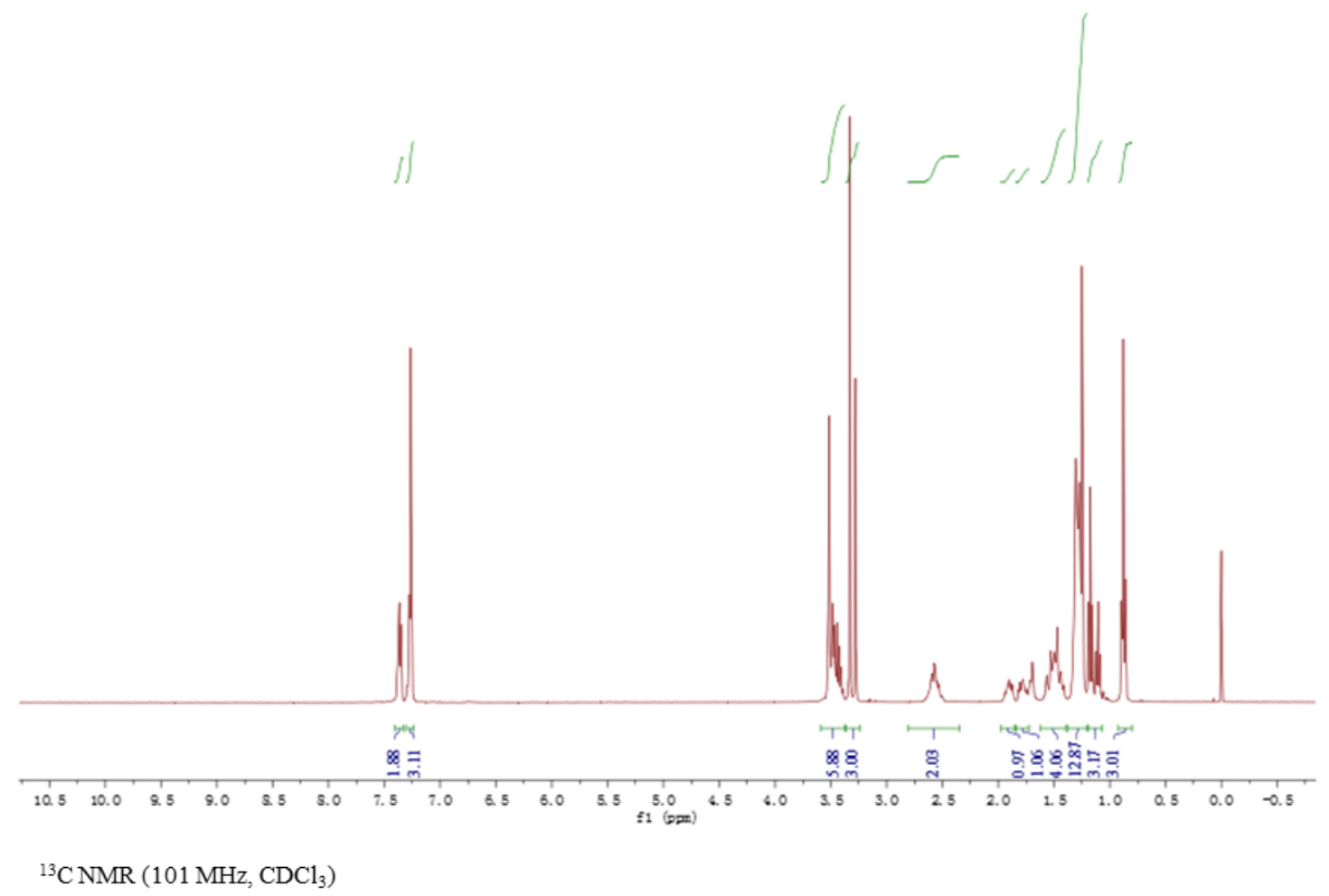

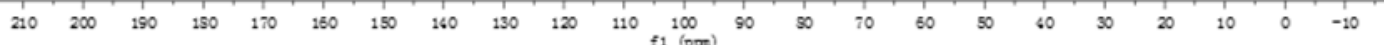


<smiles></smiles>

${ }^{1} \mathrm{H} \mathrm{NMR}\left(400 \mathrm{MHz}, \mathrm{CDCl}_{3}\right)$

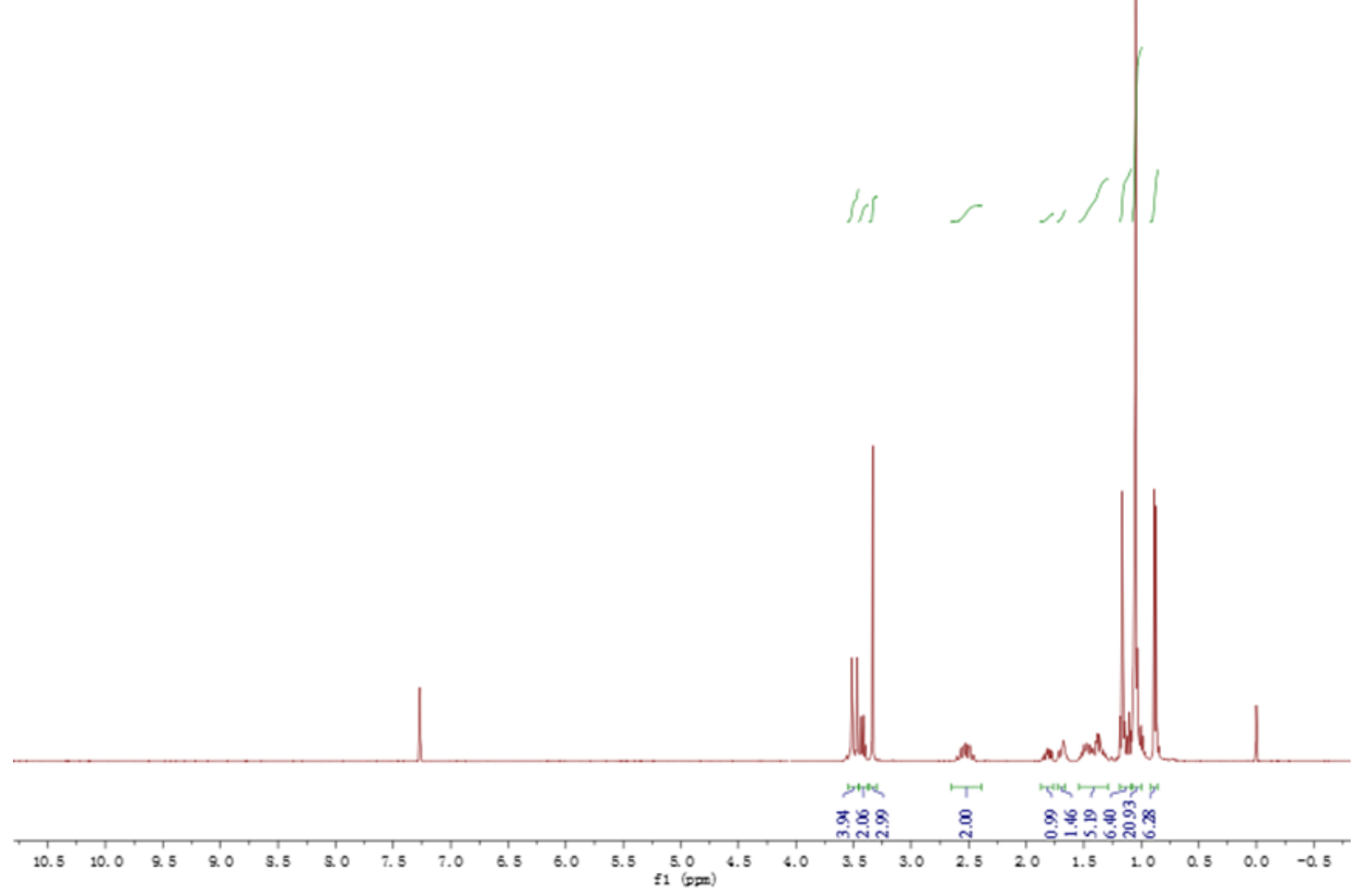

${ }^{13} \mathrm{CNMR}\left(101 \mathrm{MHz}, \mathrm{CDCl}_{3}\right)$

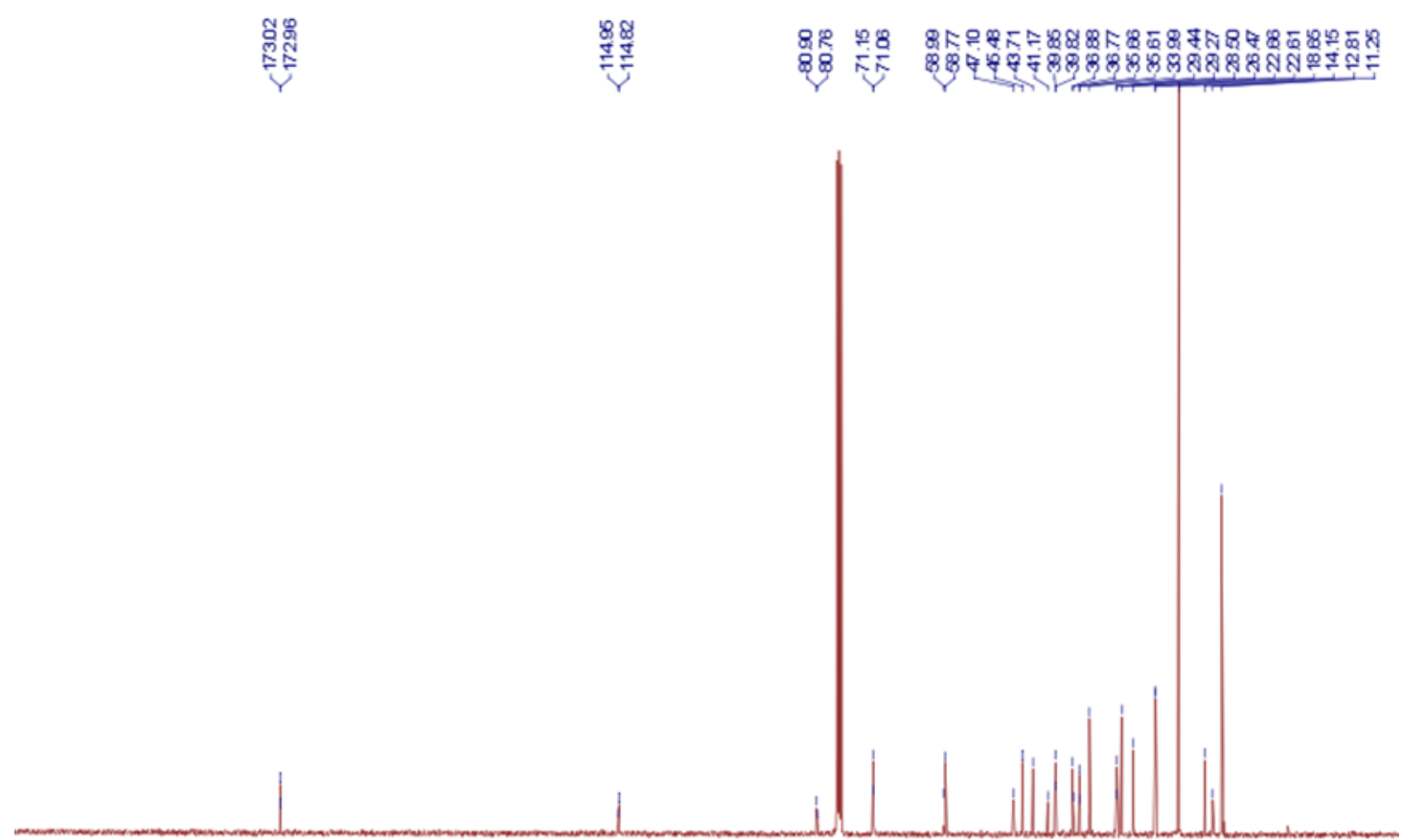

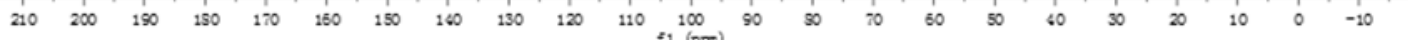


<smiles>CCN(CCOC)C(=O)CC[C@](C)(C#CCC(C)C)CCC(C)C</smiles>

${ }^{1} \mathrm{H} \mathrm{NMR}\left(400 \mathrm{MHz}, \mathrm{CDCl}_{3}\right)$

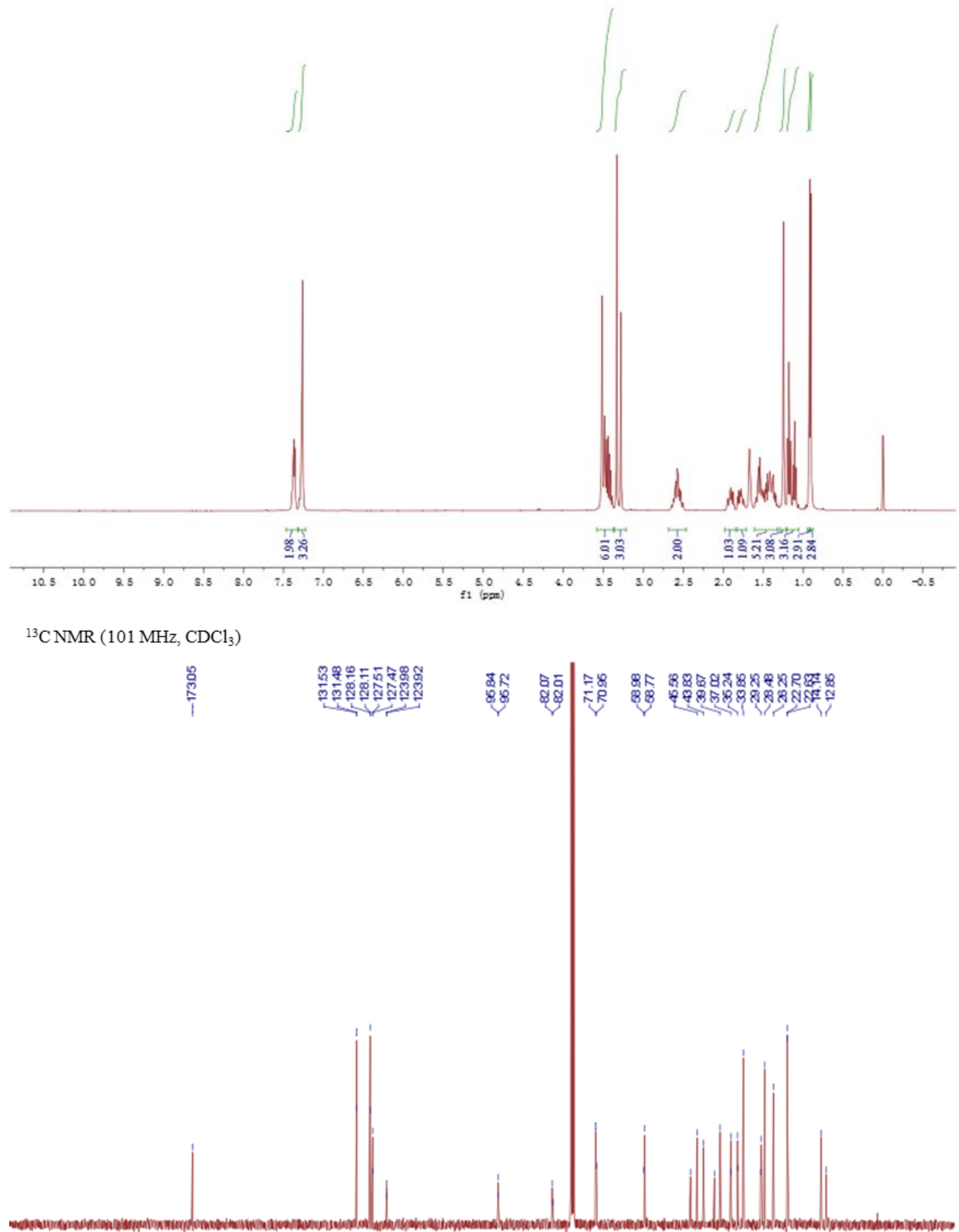

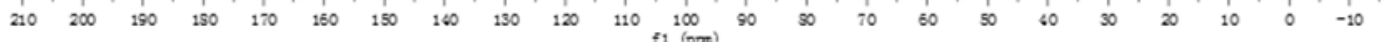


<smiles>CCN(CCOC)C(=O)CC[C@@](C)(C#C[SnH2])CC(C)C</smiles>

${ }^{1} \mathrm{H} \mathrm{NMR}\left(400 \mathrm{MHz}, \mathrm{CDCl}_{3}\right)$

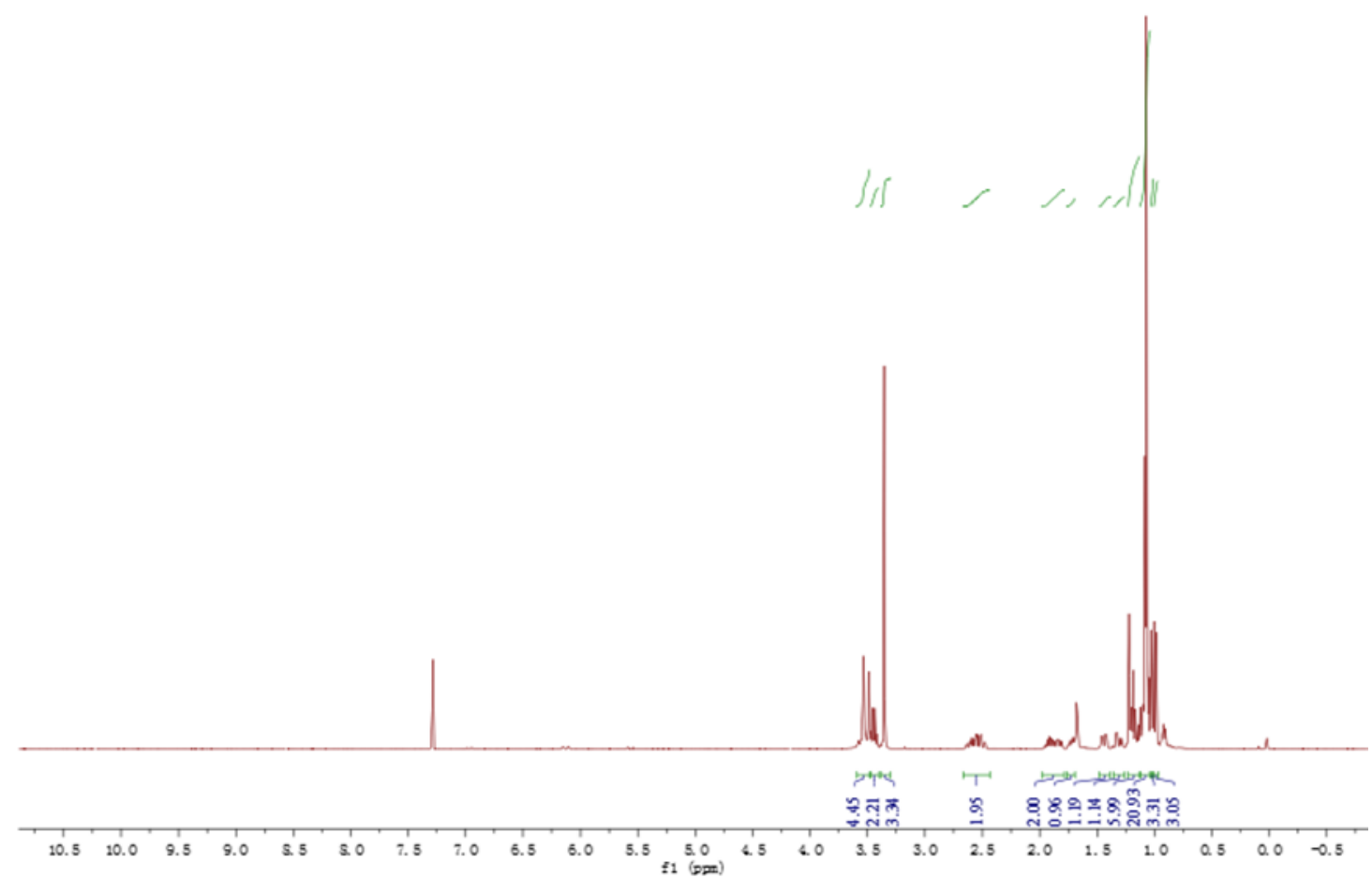
${ }^{13} \mathrm{CNMR}\left(101 \mathrm{MHz}, \mathrm{CDCl}_{3}\right)$

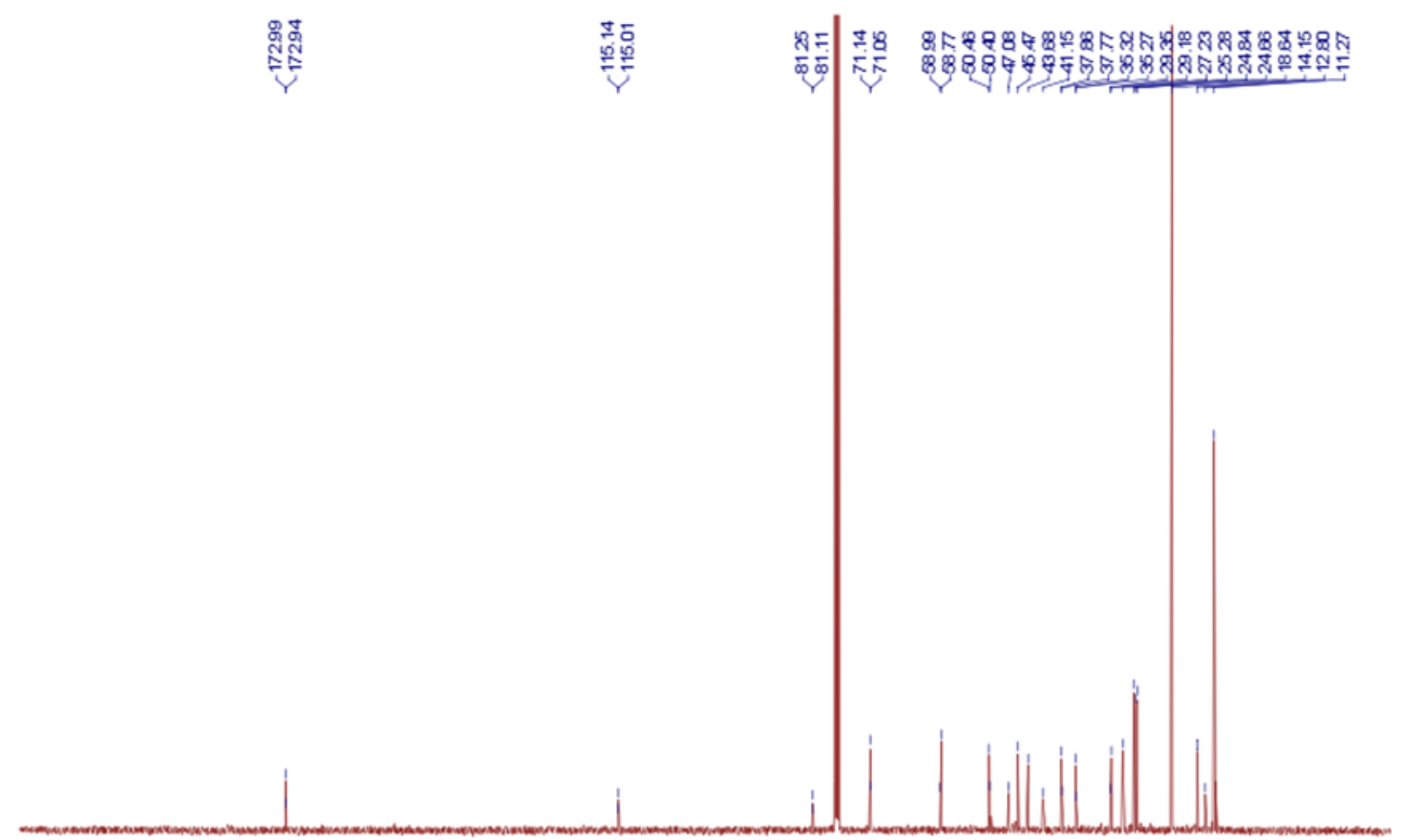

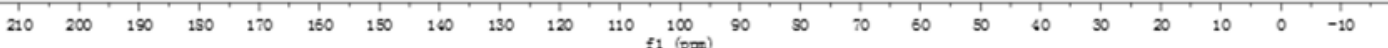


<smiles>CCN(CCOC)C(=O)CC[C@](C)(C#CC(C)C)CC(C)C</smiles>

${ }^{1} \mathrm{H} \mathrm{NMR}\left(400 \mathrm{MHz}, \mathrm{CDCl}_{3}\right)$

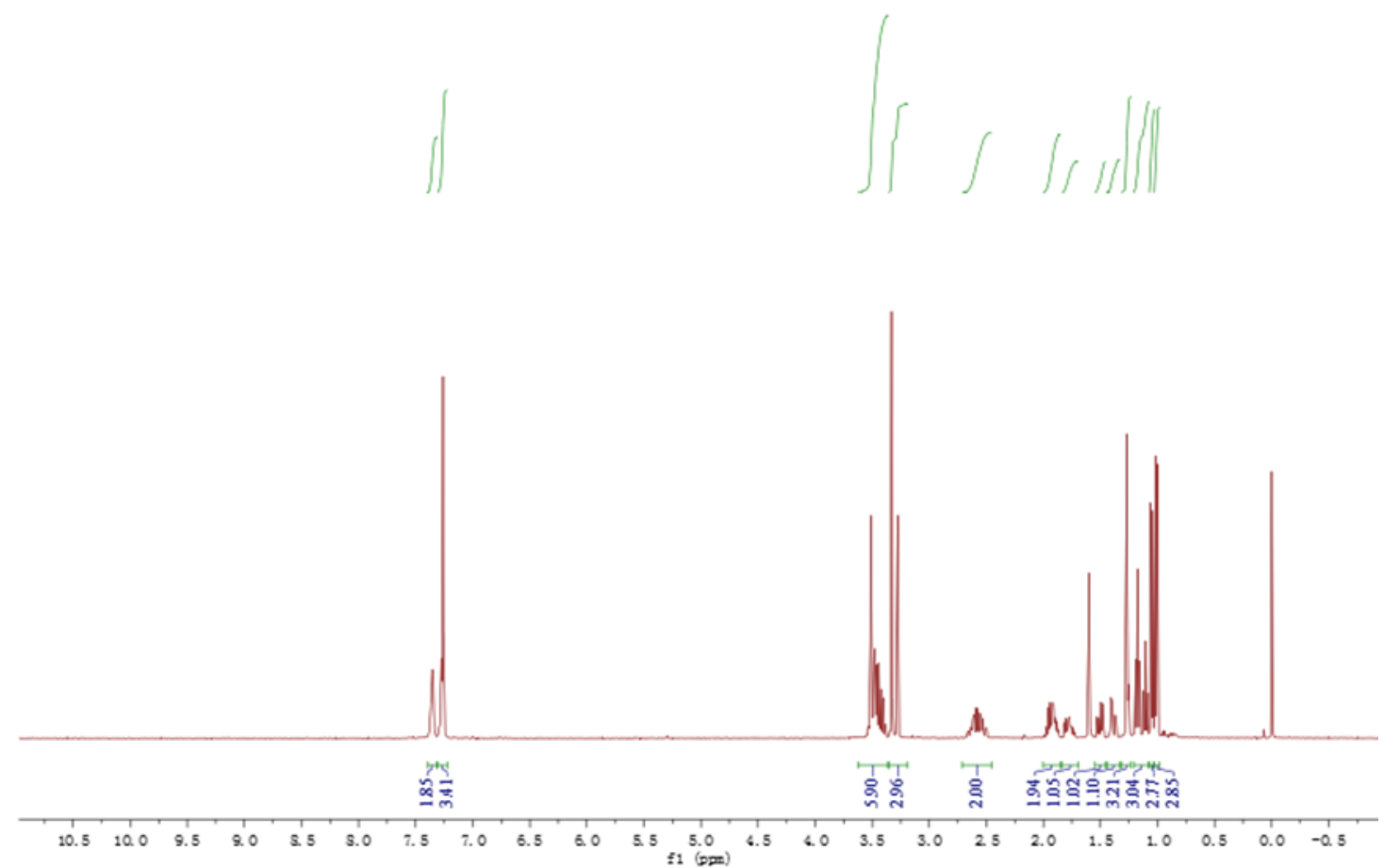

${ }^{13} \mathrm{C} \mathrm{NMR}\left(101 \mathrm{MHz}, \mathrm{CDCl}_{3}\right)$

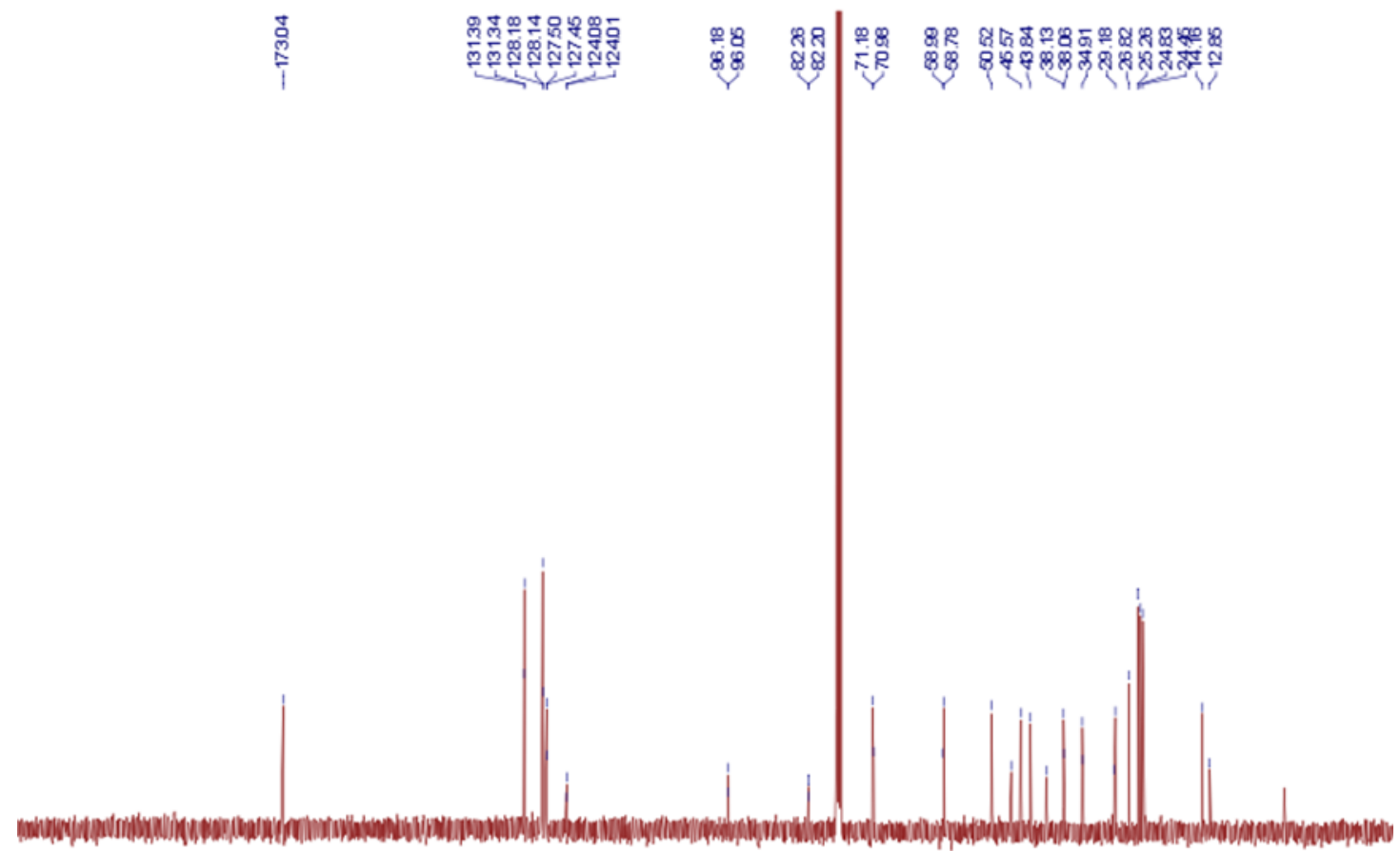

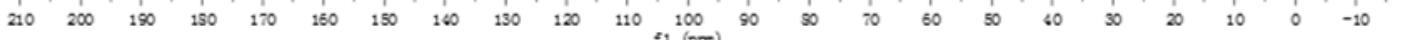




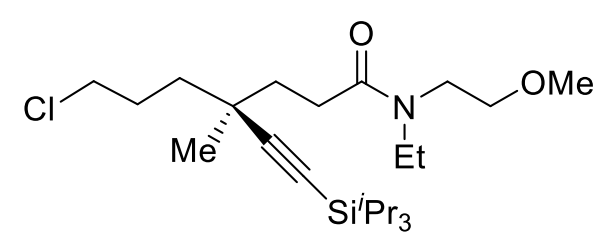

${ }^{1} \mathrm{H} \mathrm{NMR}\left(400 \mathrm{MHz}, \mathrm{CDCl}_{3}\right)$

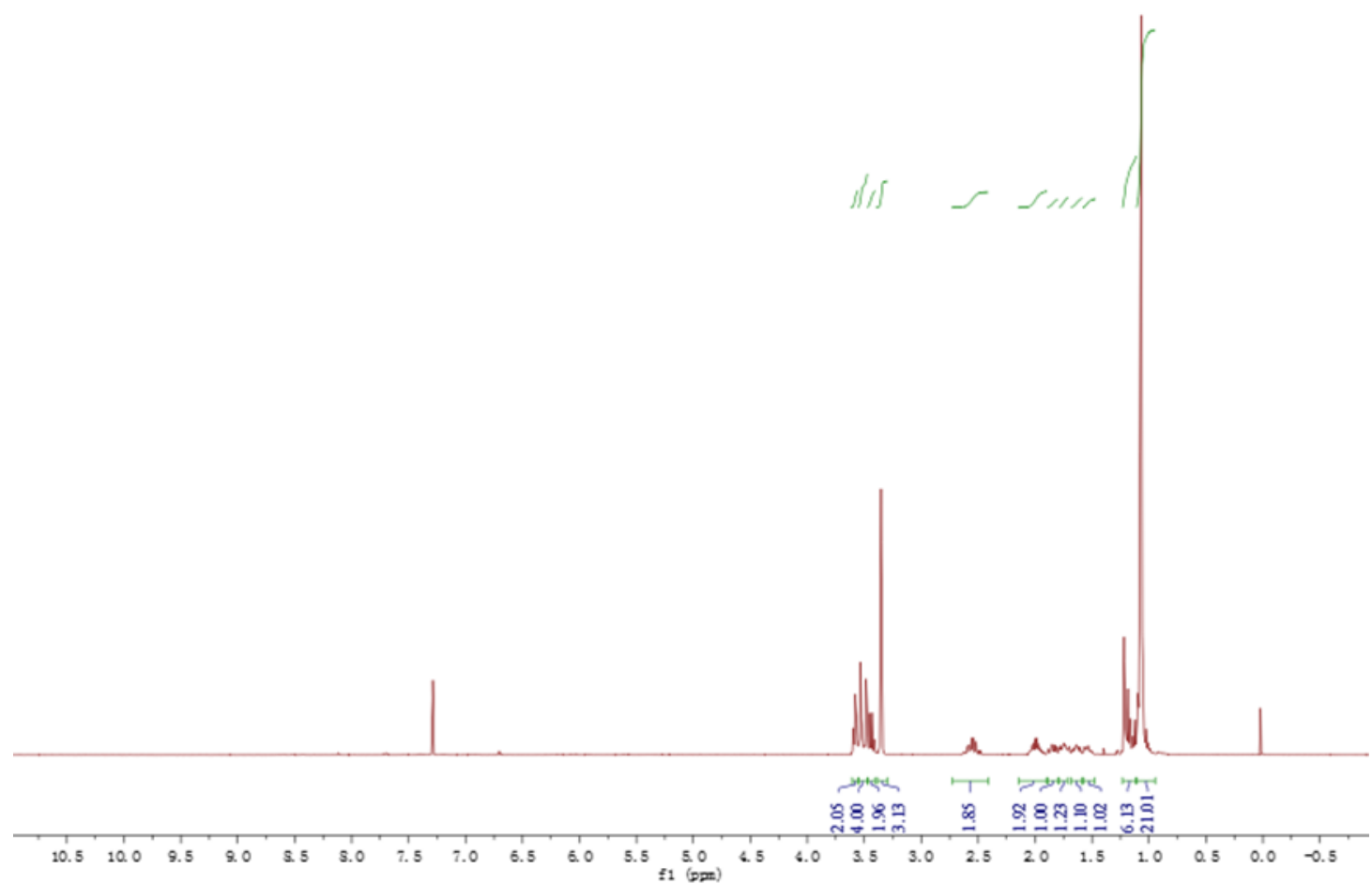

${ }^{13} \mathrm{C} \mathrm{NMR}\left(101 \mathrm{MHz}, \mathrm{CDCl}_{3}\right)$

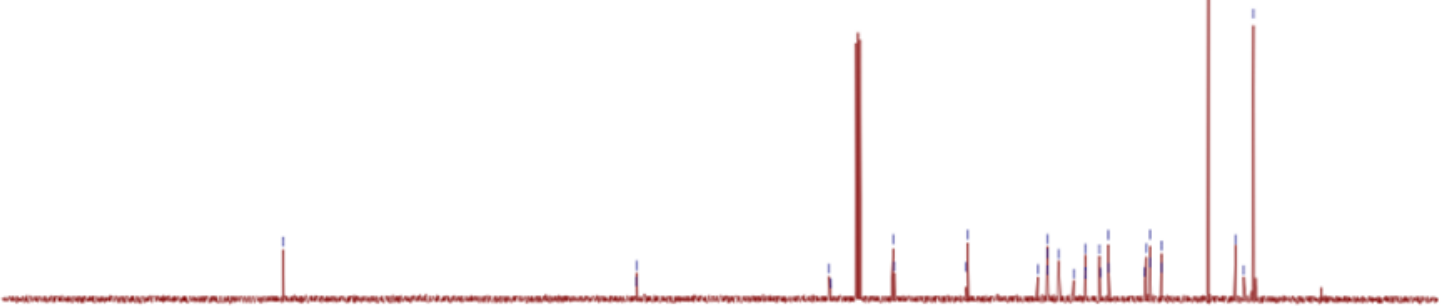


<smiles>CCN(CCOC)C(=O)CC[C@](C)(C#CCCCl)CCCCl</smiles>

$\left.{ }^{1} \mathrm{H} \mathrm{NMR} \mathrm{(400} \mathrm{MHz,} \mathrm{CDCl}_{3}\right)$
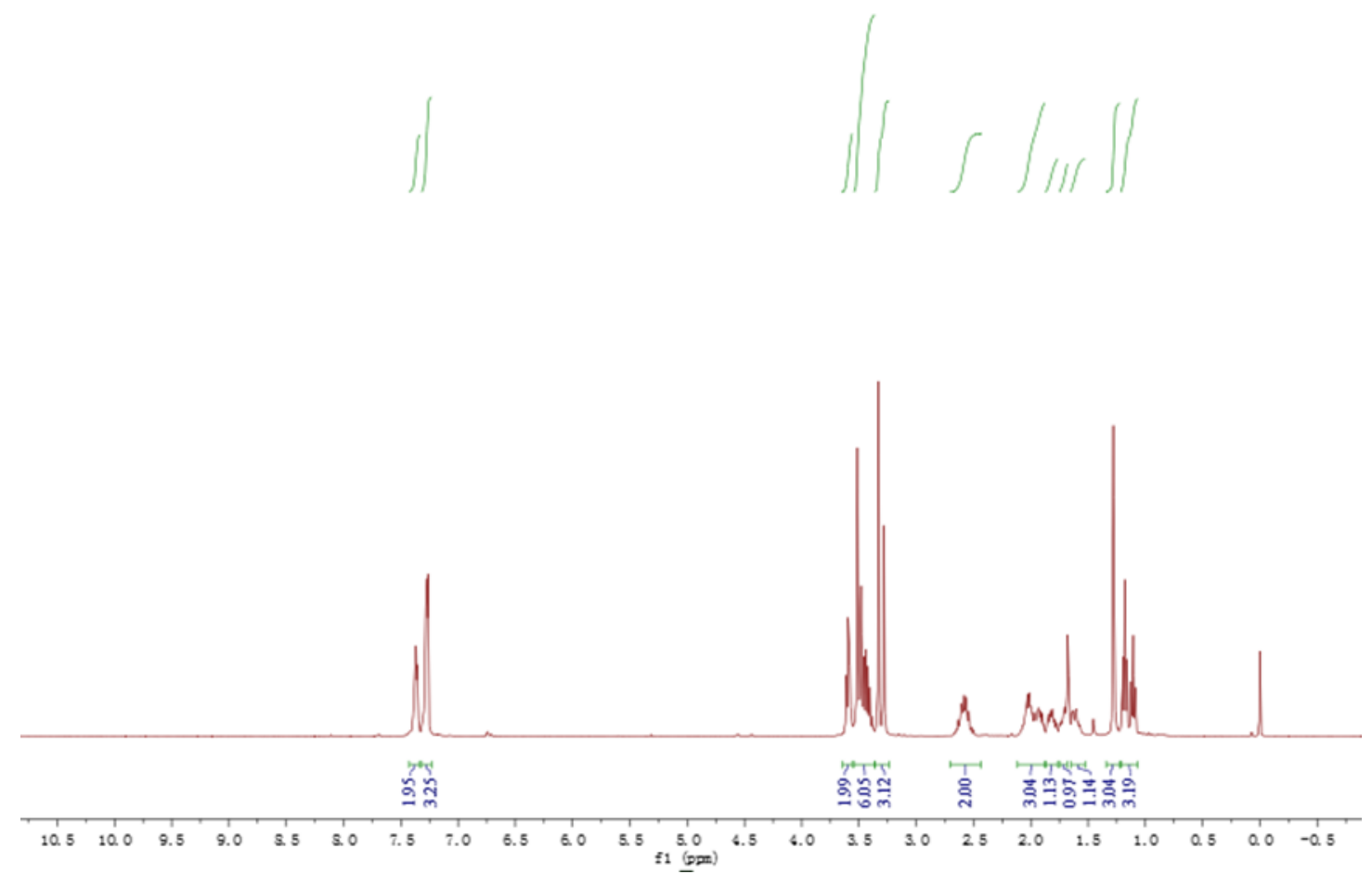

${ }^{13} \mathrm{CNMR}\left(101 \mathrm{MHz}, \mathrm{CDCl}_{3}\right)$

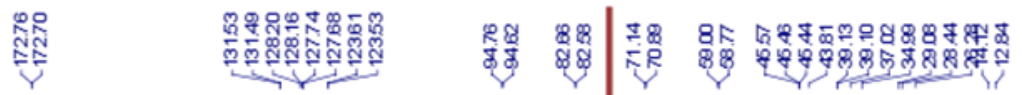

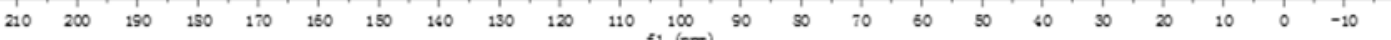


<smiles>CCN(CCOC)C(=O)CC[C@](C)(C#C[SnH2])CCCOC(C)=O</smiles>

${ }^{1} \mathrm{H} \mathrm{NMR}\left(400 \mathrm{MHz}, \mathrm{CDCl}_{3}\right)$

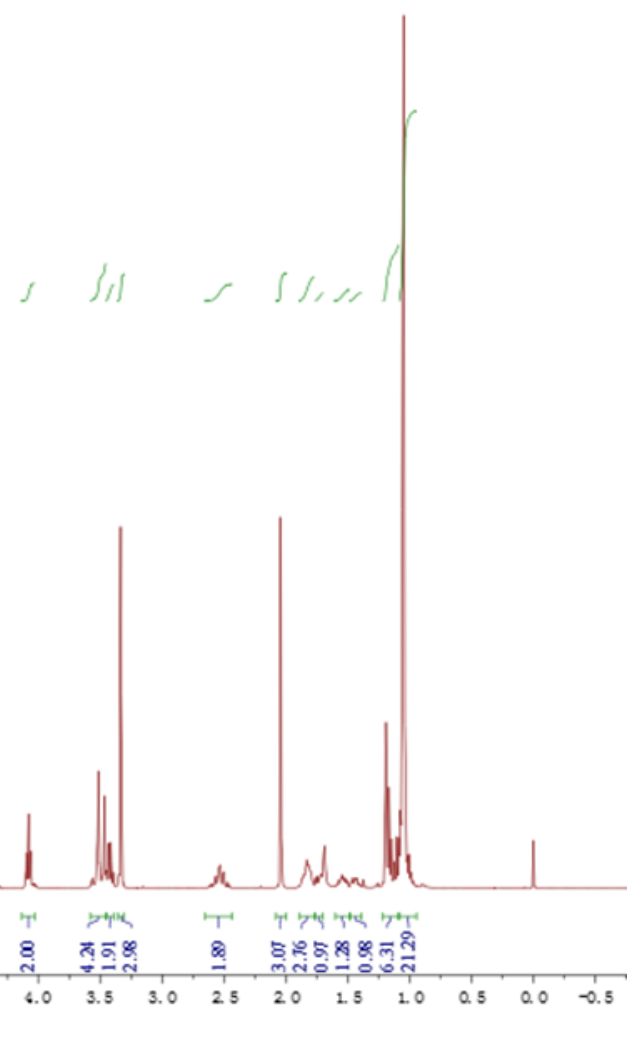

${ }^{13} \mathrm{CNMR}\left(101 \mathrm{MHz}, \mathrm{CDCl}_{3}\right)$

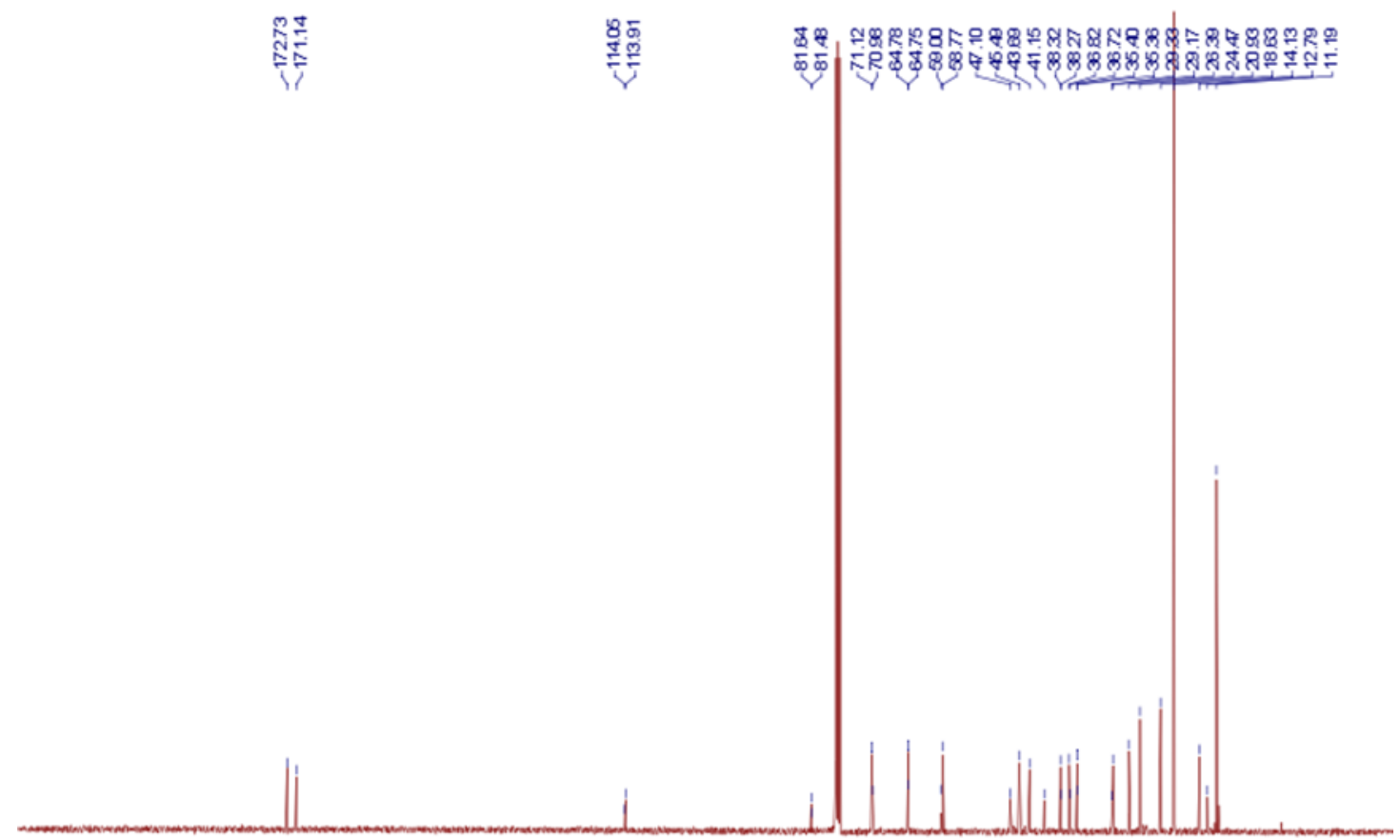

$\begin{array}{lllllllllllllllllllllll}210 & 200 & 190 & 150 & 170 & 160 & 150 & 160 & 130 & 120 & 110 & 100 & 90 & 50 & 70 & 60 & 50 & 40 & 30 & 20 & 10 & 0 & -10\end{array}$ 
<smiles>CCN(CCOC)C(=O)CC[C@](C)(C#CCCOC(C)C)CCCOC</smiles>

${ }^{1} \mathrm{H} \mathrm{NMR}\left(400 \mathrm{MHz}, \mathrm{CDCl}_{3}\right)$

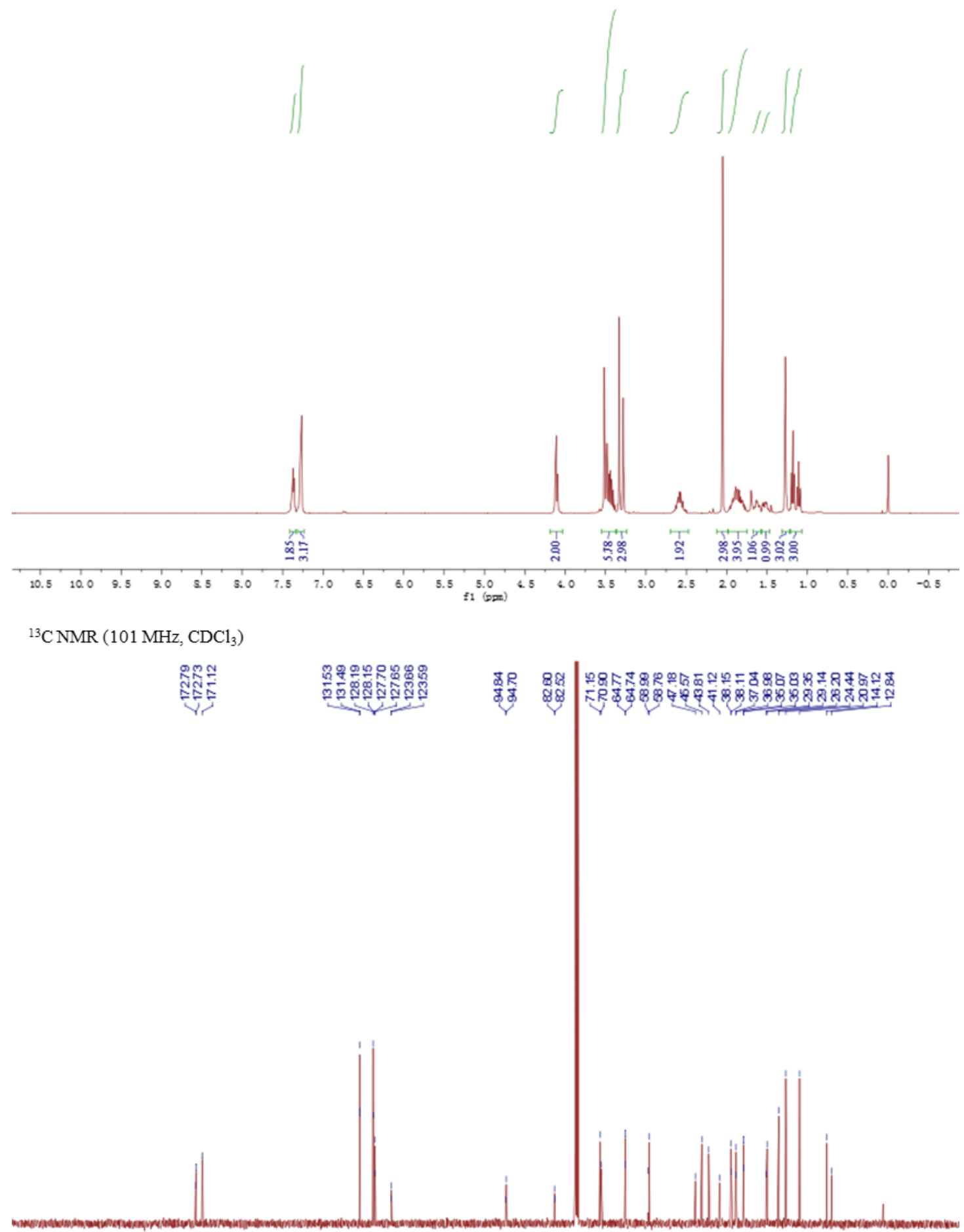

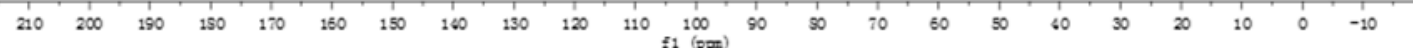


<smiles>CCN(CCOC)C(=O)CC[C@](C)(C#C[AsH2-])CCCCOc1ccccc1</smiles>

${ }^{1} \mathrm{H} \mathrm{NMR}\left(400 \mathrm{MHz}, \mathrm{CDCl}_{3}\right)$
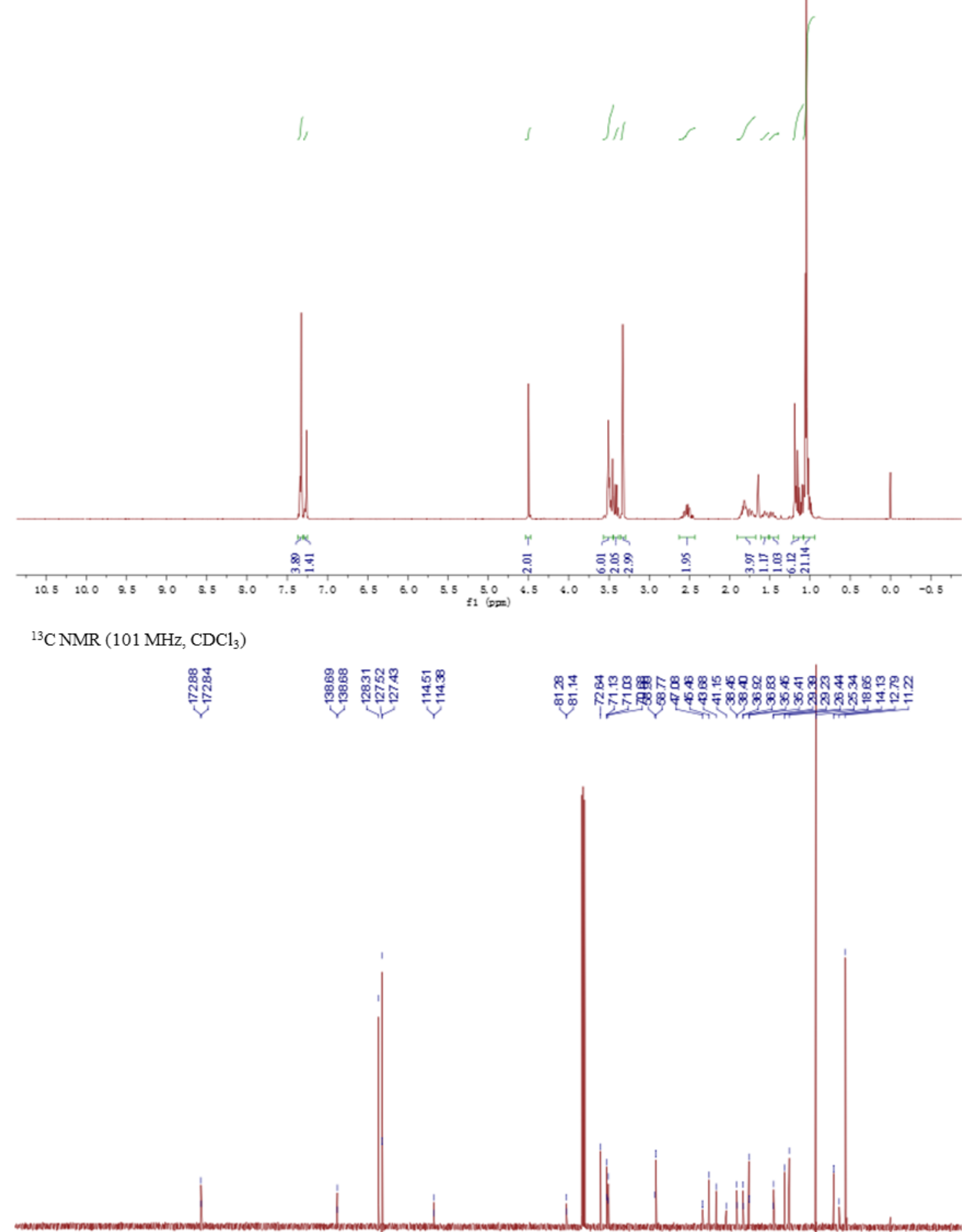

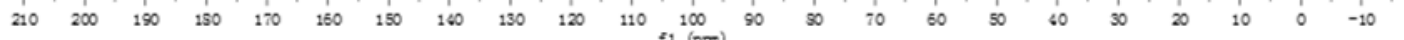


<smiles>CCN(CCOC)C(=O)CC[C@](C)(C#C[Se-])CCCc1ccccc1</smiles>

${ }^{1} \mathrm{H} \mathrm{NMR}\left(400 \mathrm{MHz}, \mathrm{CDCl}_{3}\right)$

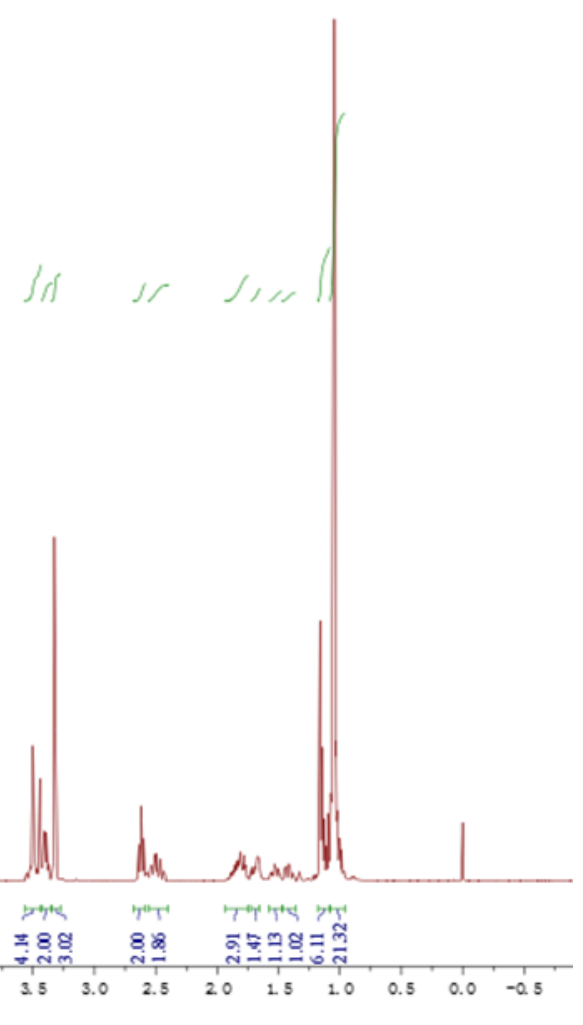

${ }^{13} \mathrm{CNMR}\left(101 \mathrm{MHz}, \mathrm{CDCl}_{3}\right)$

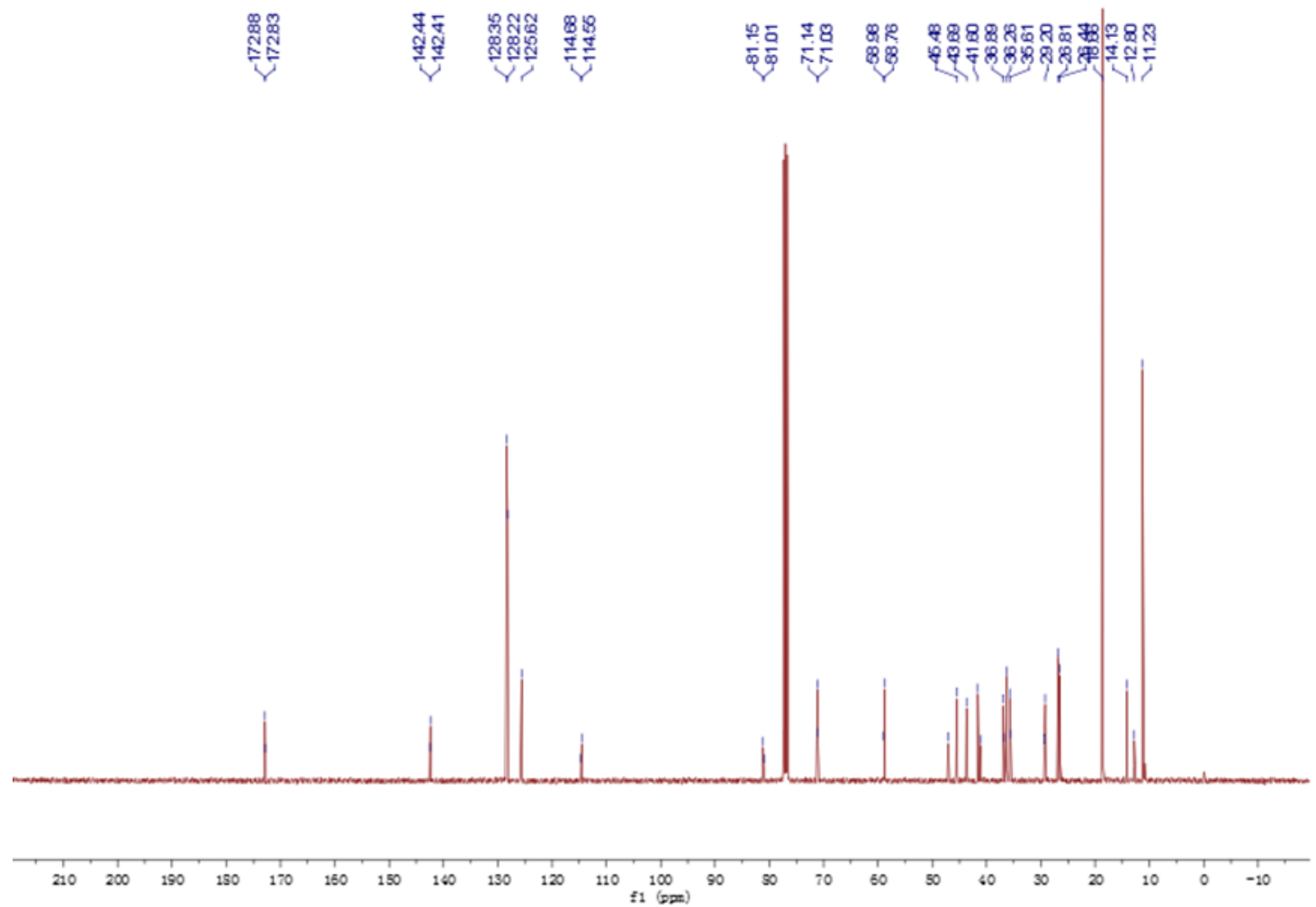


<smiles>CCN(CCOC)C(=O)CC[C@](C)(C#C[SiH2])CCc1ccccc1</smiles>

${ }^{1} \mathrm{H} \mathrm{NMR}\left(400 \mathrm{MHz}, \mathrm{CDCl}_{3}\right)$

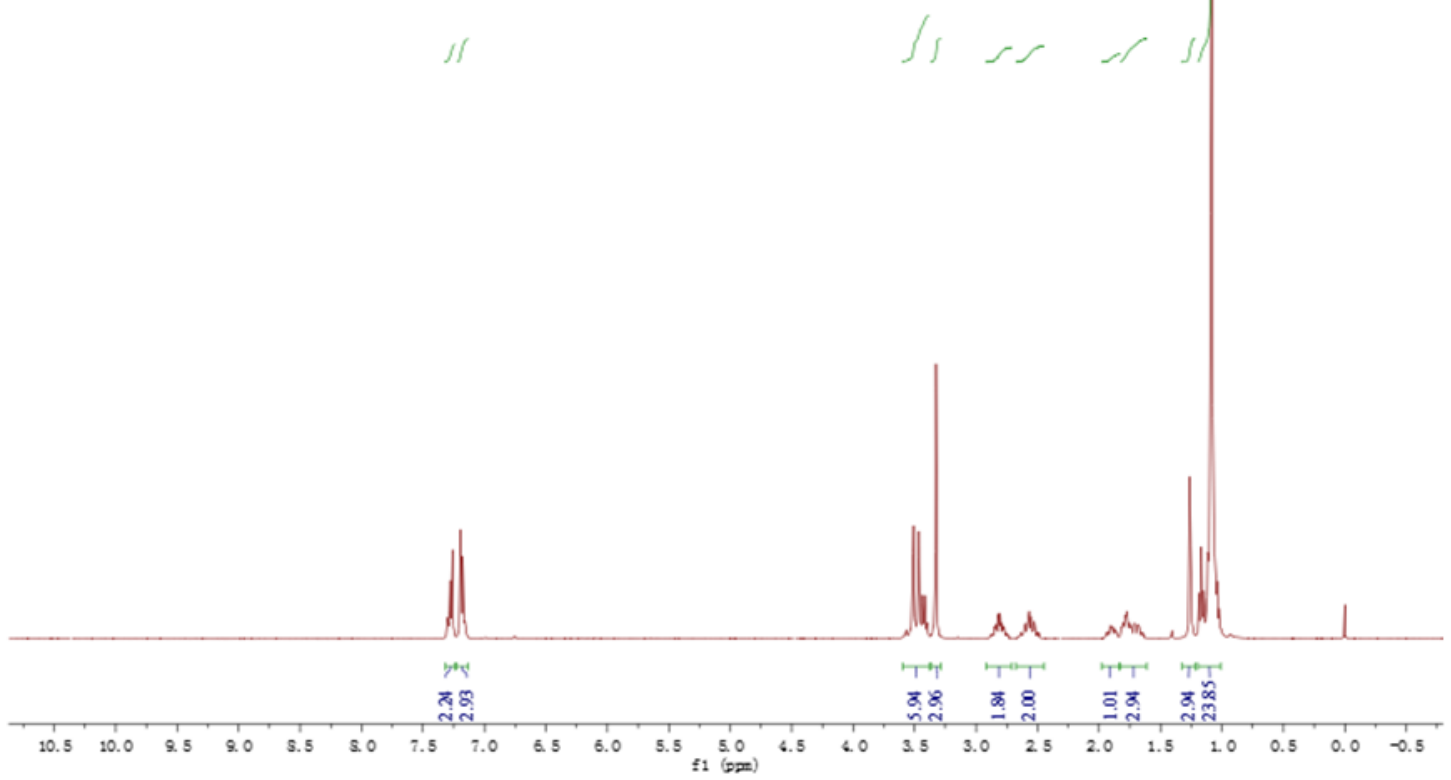

${ }^{13} \mathrm{C} \mathrm{NMR}\left(101 \mathrm{MHz}, \mathrm{CDCl}_{3}\right)$
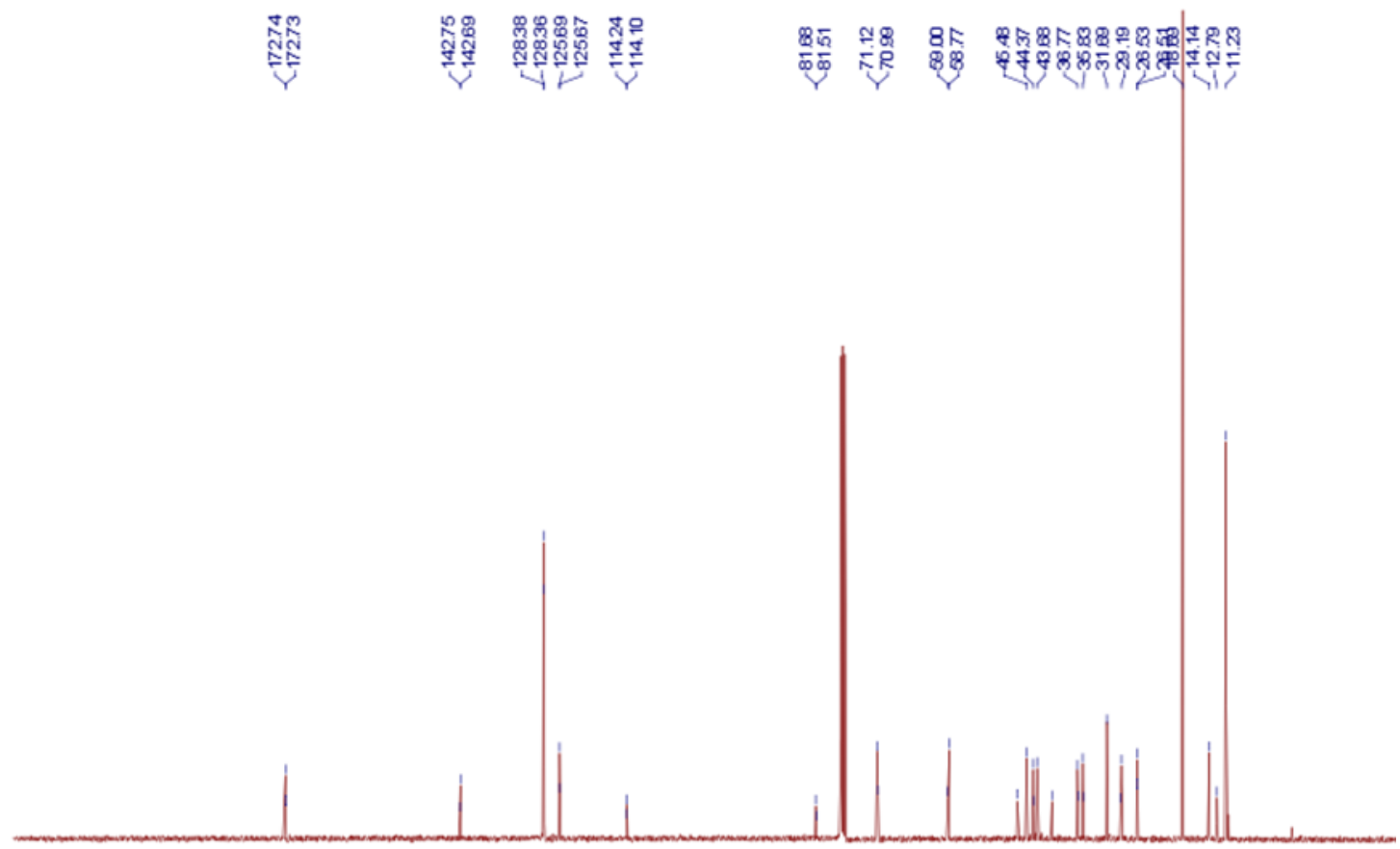

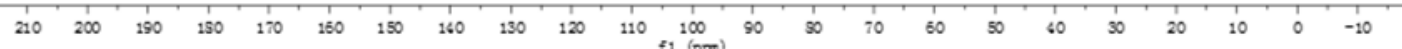


<smiles>CCN(CCOC)C(=O)CCC(C#CPc1ccccc1)(CC)[N+](=O)[O-]</smiles>

${ }^{1} \mathrm{H} \mathrm{NMR}\left(400 \mathrm{MHz}, \mathrm{CDCl}_{3}\right)$

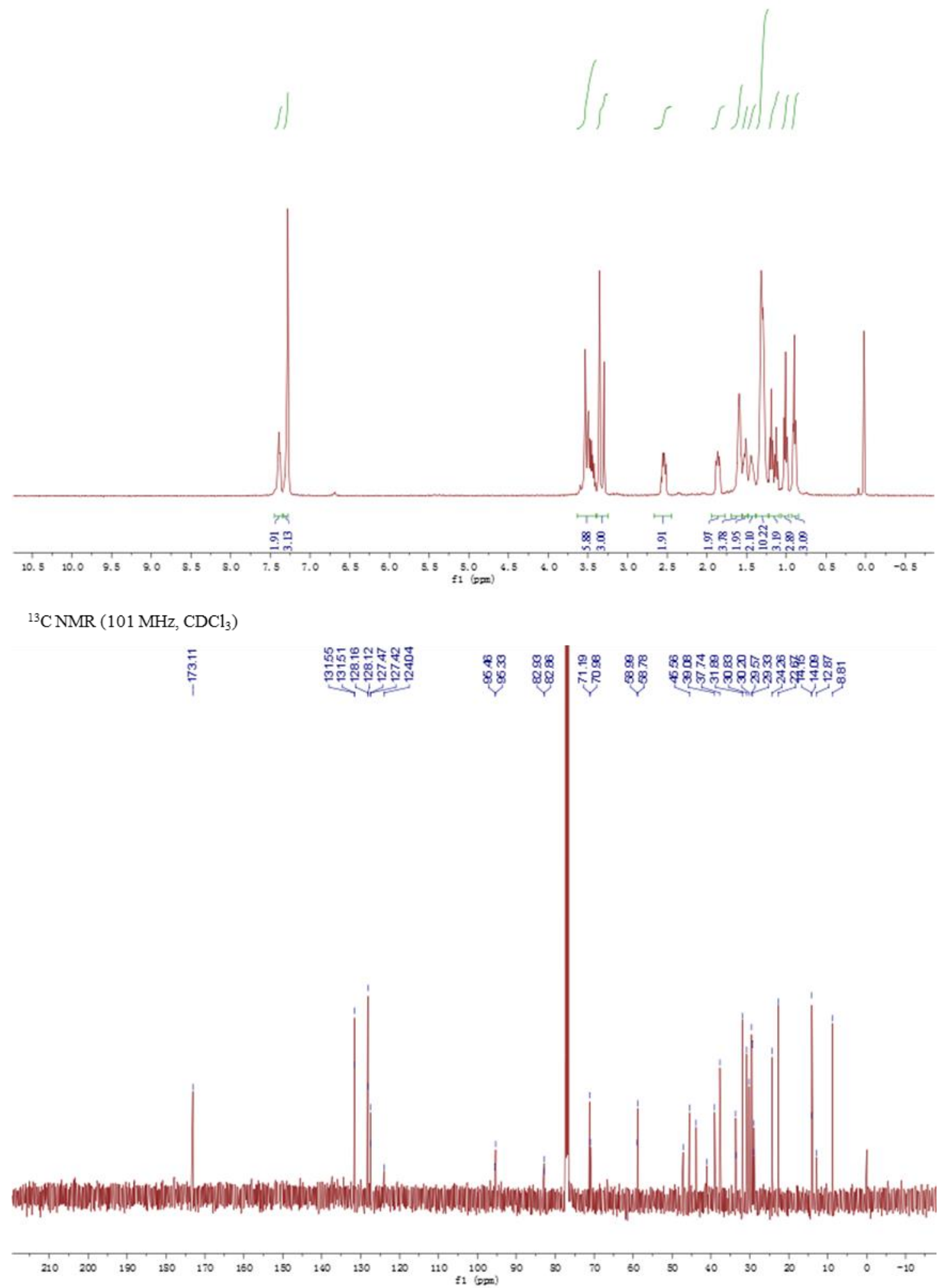




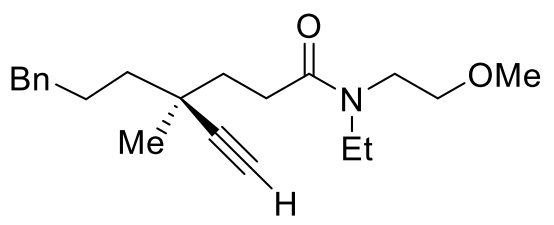

${ }^{1} \mathrm{H} \mathrm{NMR}\left(400 \mathrm{MHz}, \mathrm{CDCl}_{3}\right)$

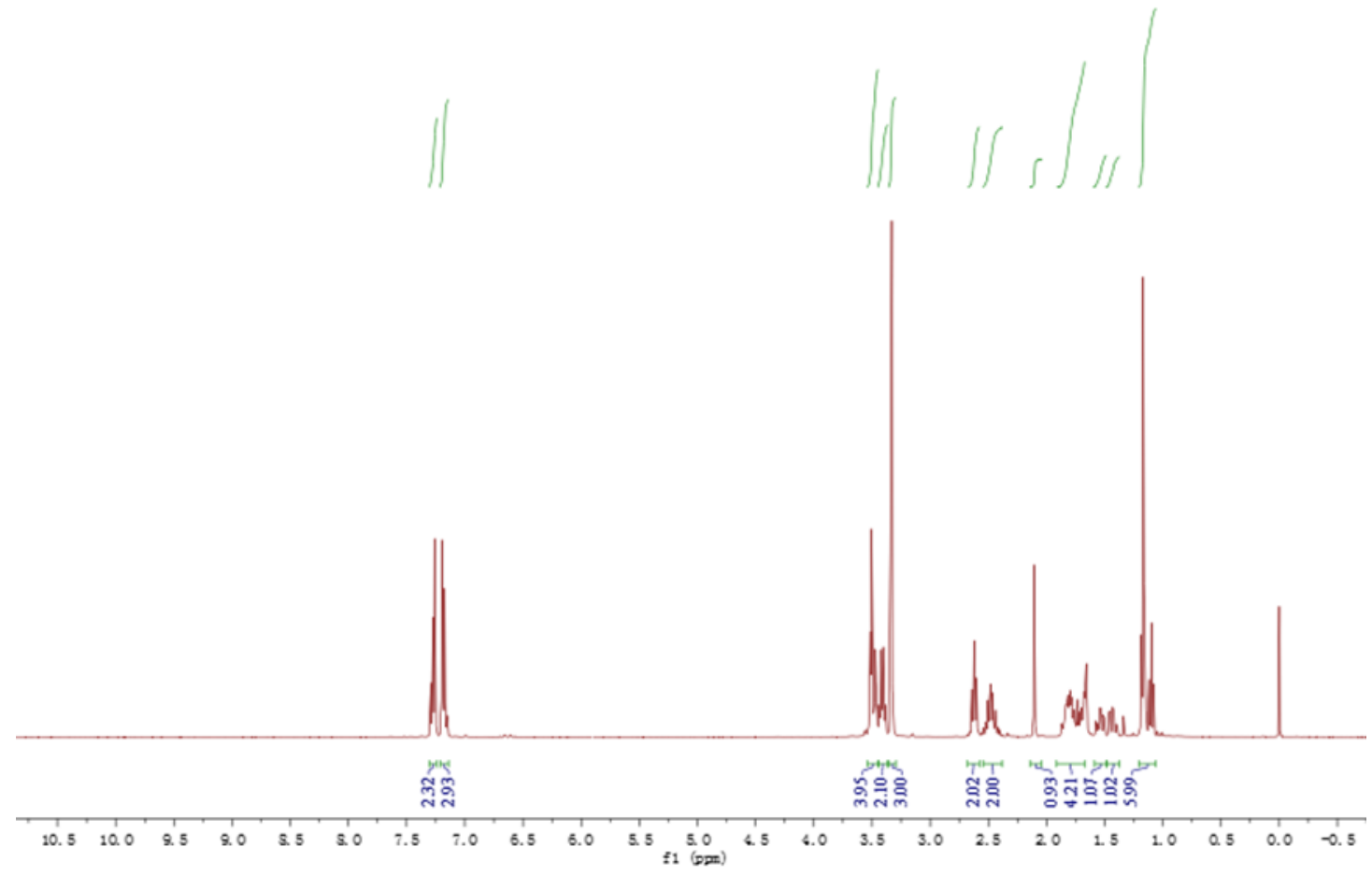

${ }^{13} \mathrm{C} \mathrm{NMR}\left(101 \mathrm{MHz}, \mathrm{CDCl}_{3}\right)$ 
<smiles>C=C[C@](C)(CCC(=O)N(CC)CCOC)CCc1ccccc1</smiles>

${ }^{1} \mathrm{H}$ NMR $\left(400 \mathrm{MHz}, \mathrm{CDCl}_{3}\right)$

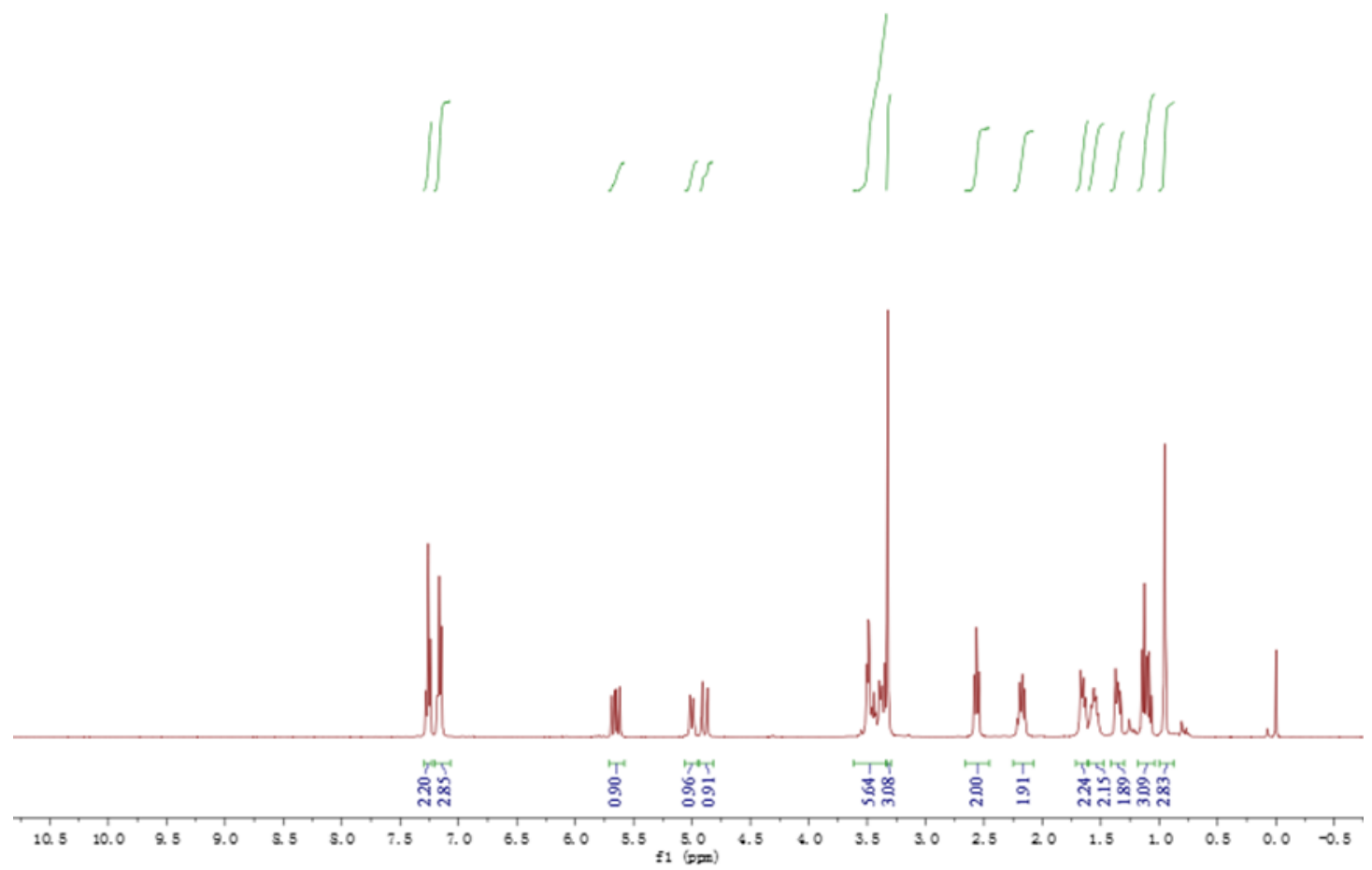

${ }^{13} \mathrm{CNMR}\left(101 \mathrm{MHz}, \mathrm{CDCl}_{3}\right)$

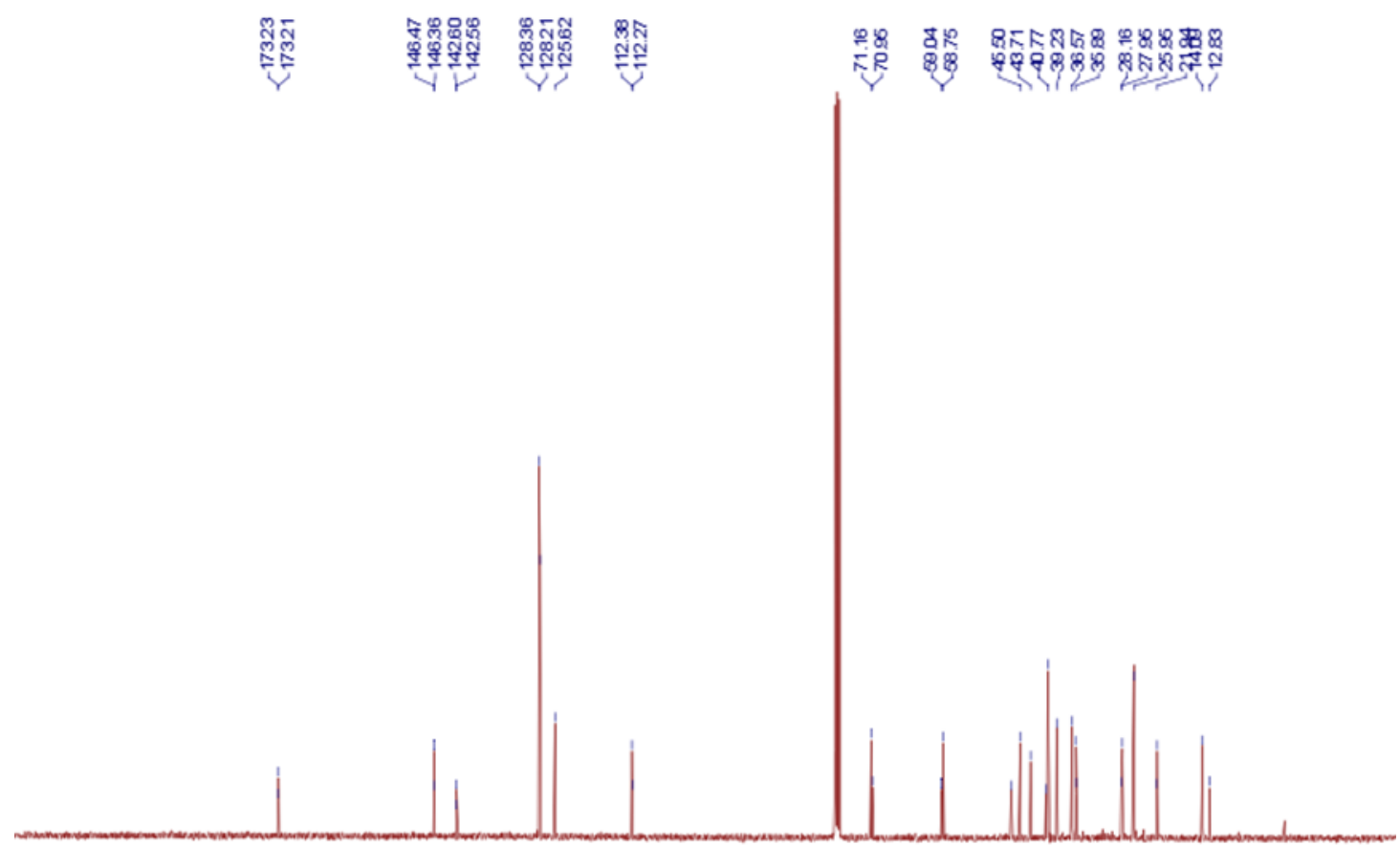

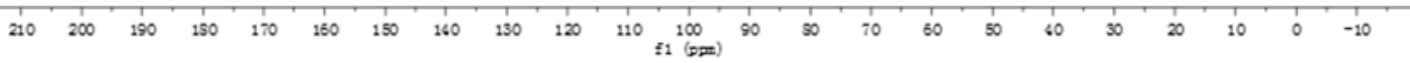


<smiles>CCN(CCOC)C(=O)CC[C@](C)(CC)CCc1ccccc1</smiles>

${ }^{1} \mathrm{H}$ NMR $\left(400 \mathrm{MHz}, \mathrm{CDCl}_{3}\right)$

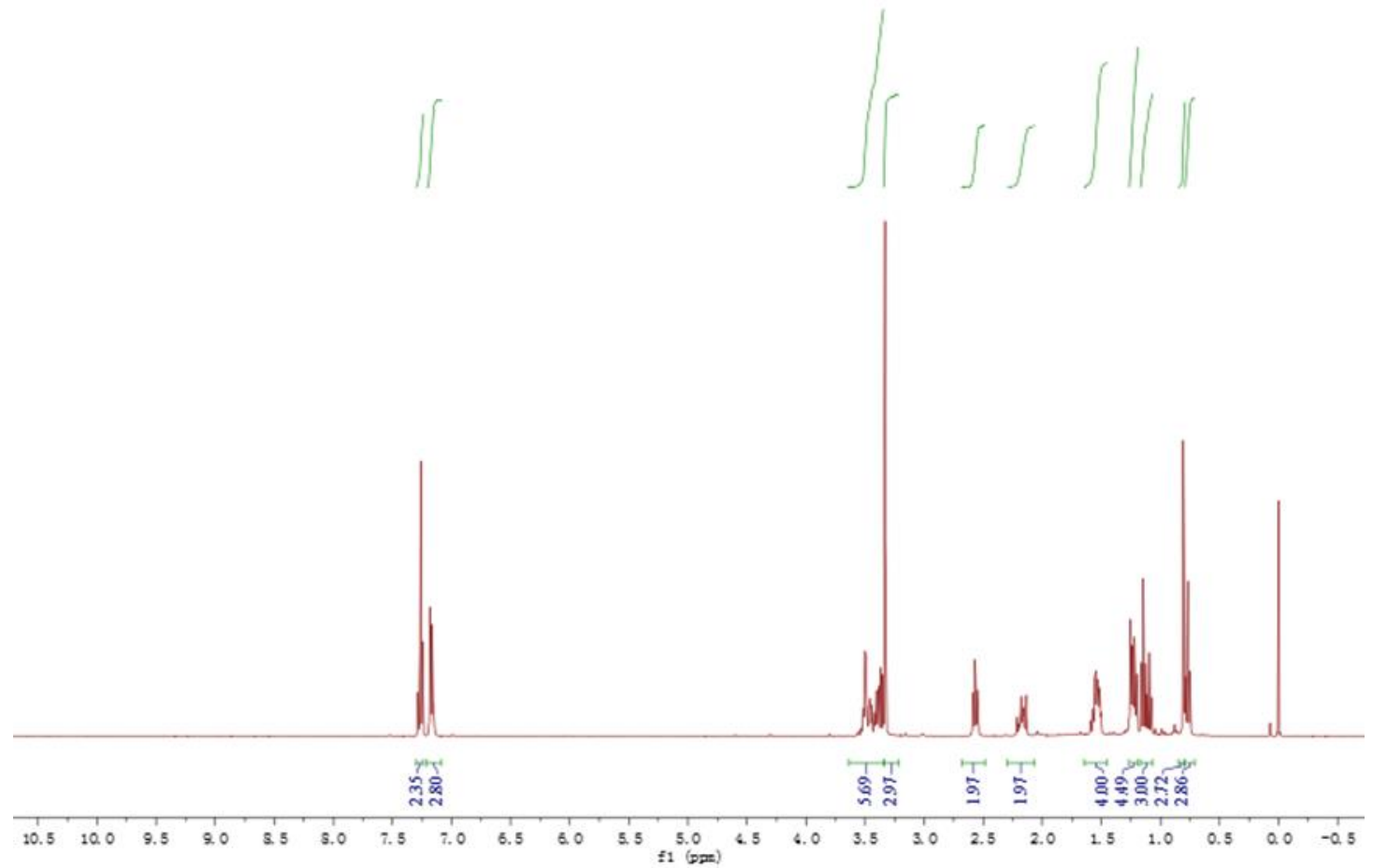

${ }^{13} \mathrm{CNMR}\left(101 \mathrm{MHz}, \mathrm{CDCl}_{3}\right)$

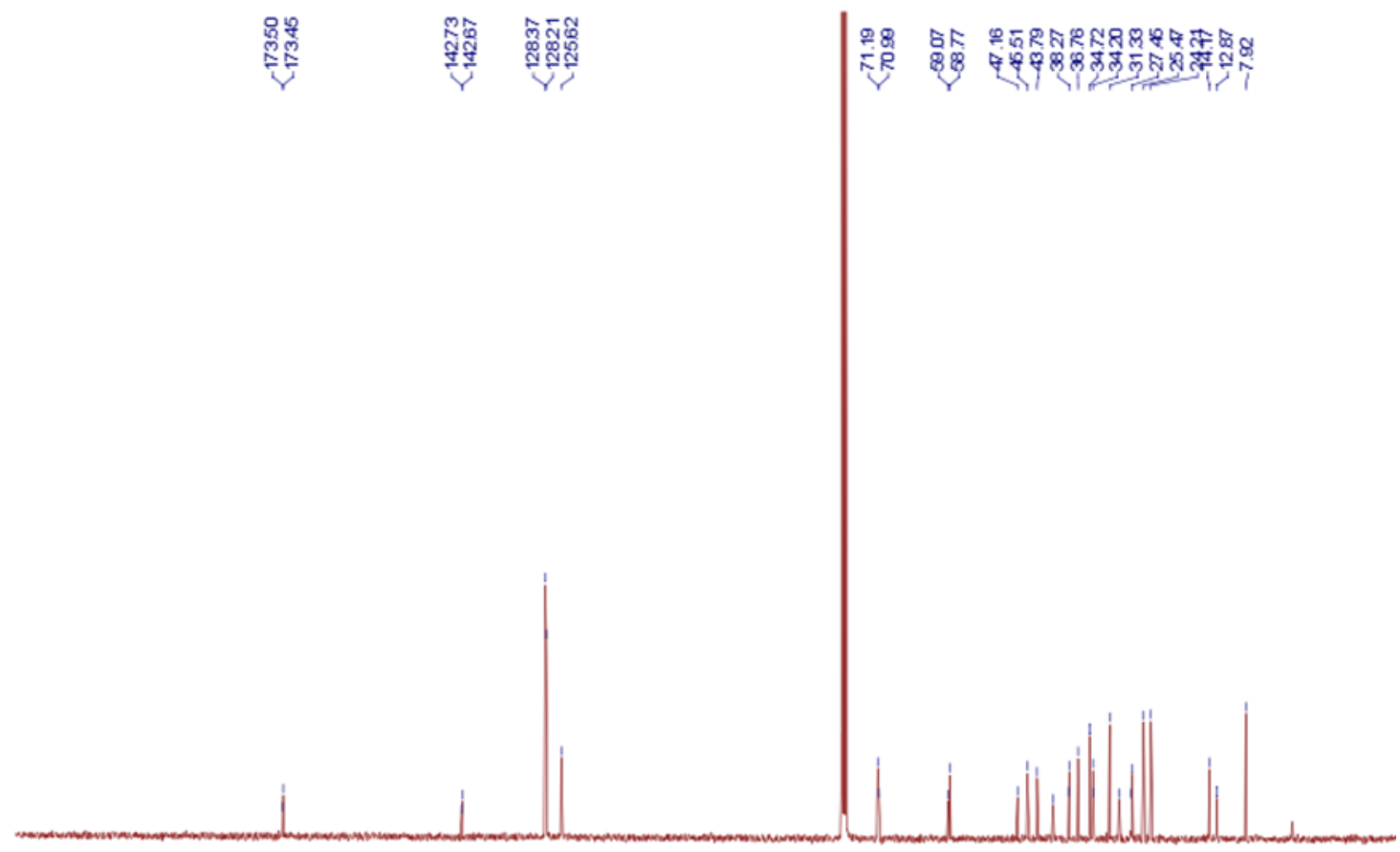

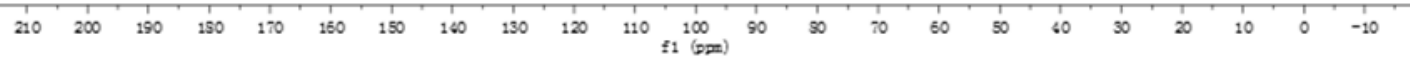




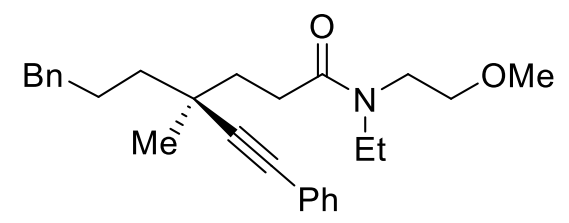

${ }^{1} \mathrm{H} \mathrm{NMR}\left(400 \mathrm{MHz}, \mathrm{CDCl}_{3}\right)$

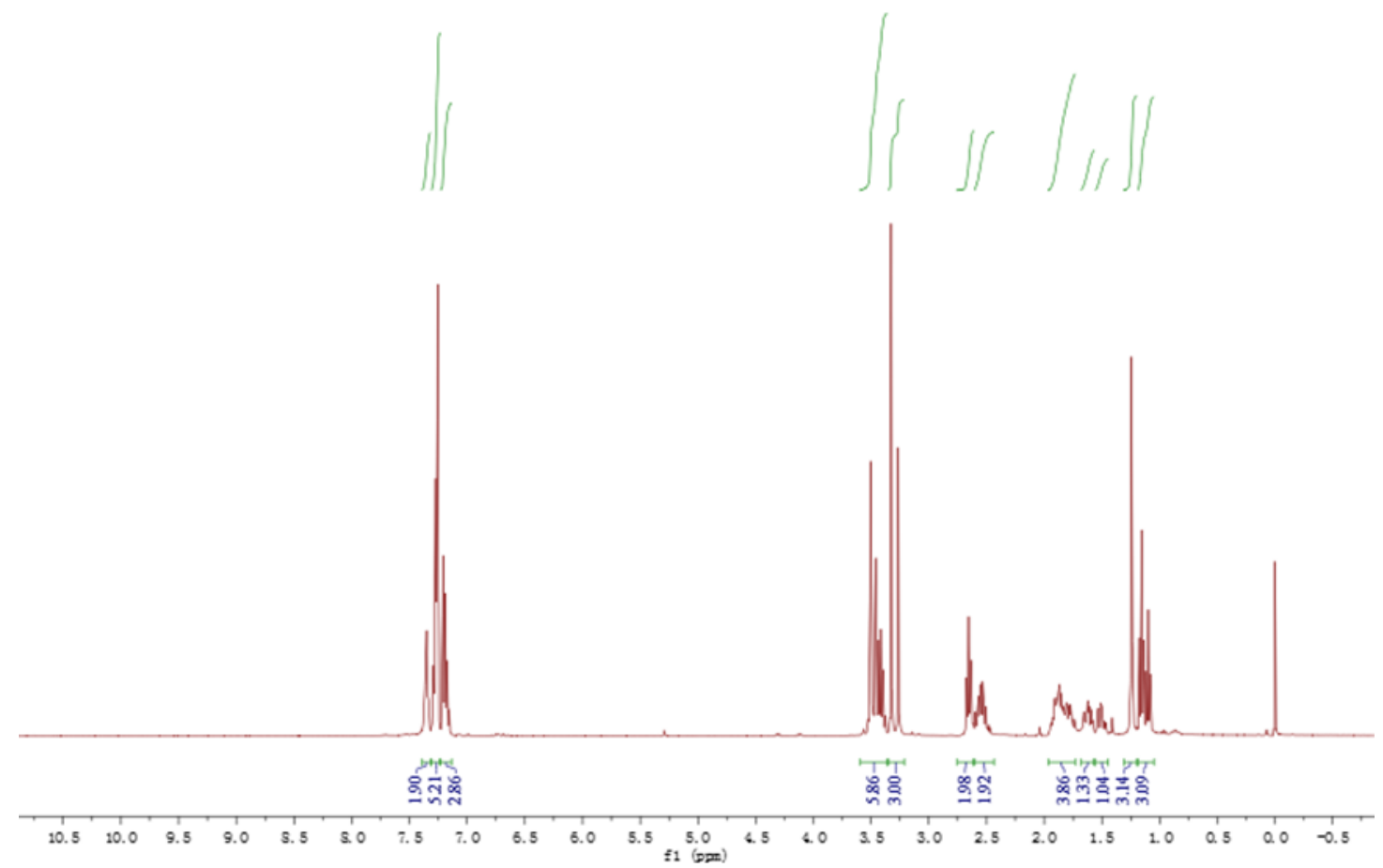

${ }^{13} \mathrm{CNMR}\left(101 \mathrm{MHz}, \mathrm{CDCl}_{3}\right)$

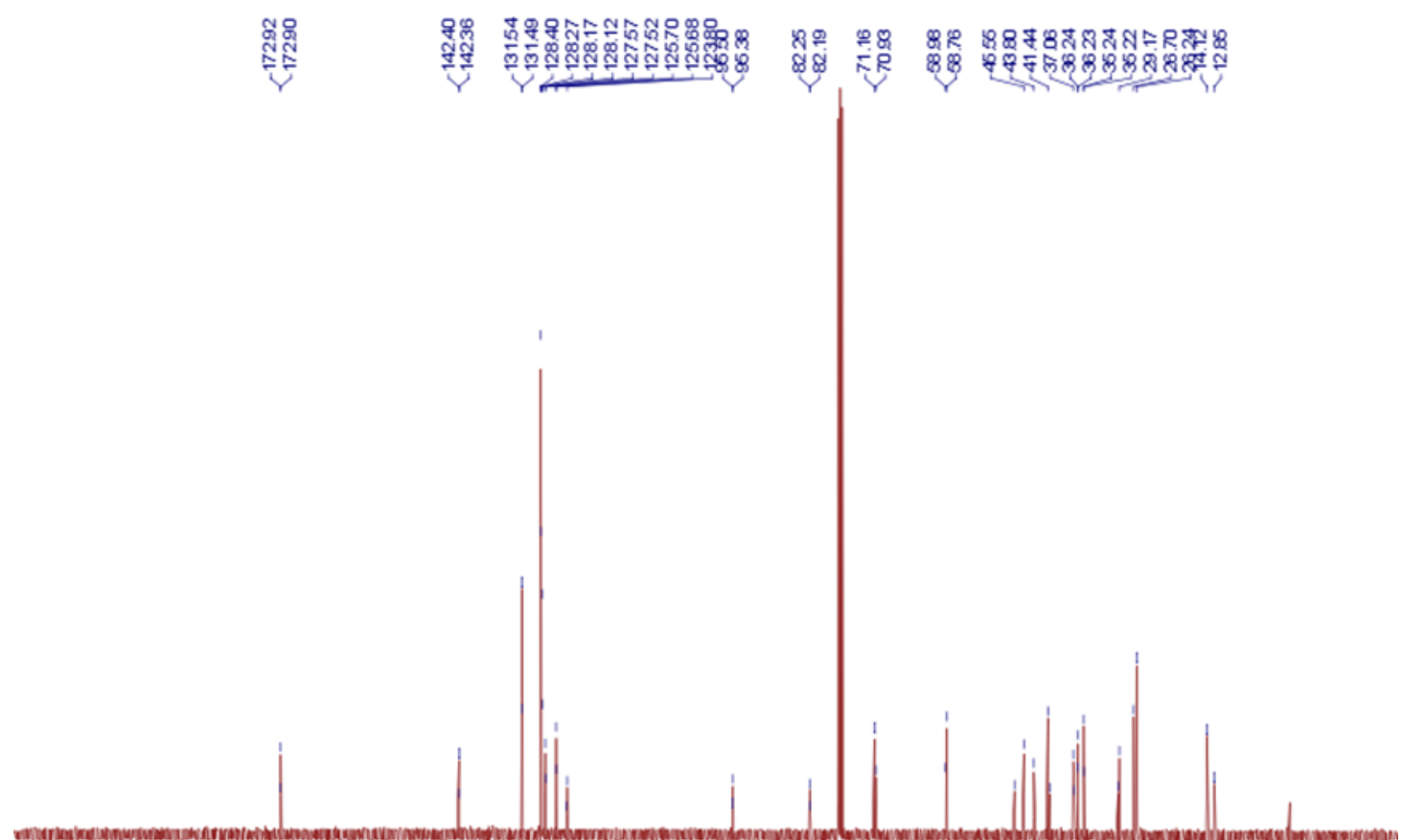

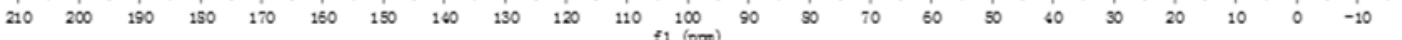


<smiles>CCN(CCOC)C(=O)CC[C@](C)(CCc1ccccc1)c1cn(C)nn1</smiles>

${ }^{1} \mathrm{H} \mathrm{NMR}\left(400 \mathrm{MHz}, \mathrm{CDCl}_{3}\right)$
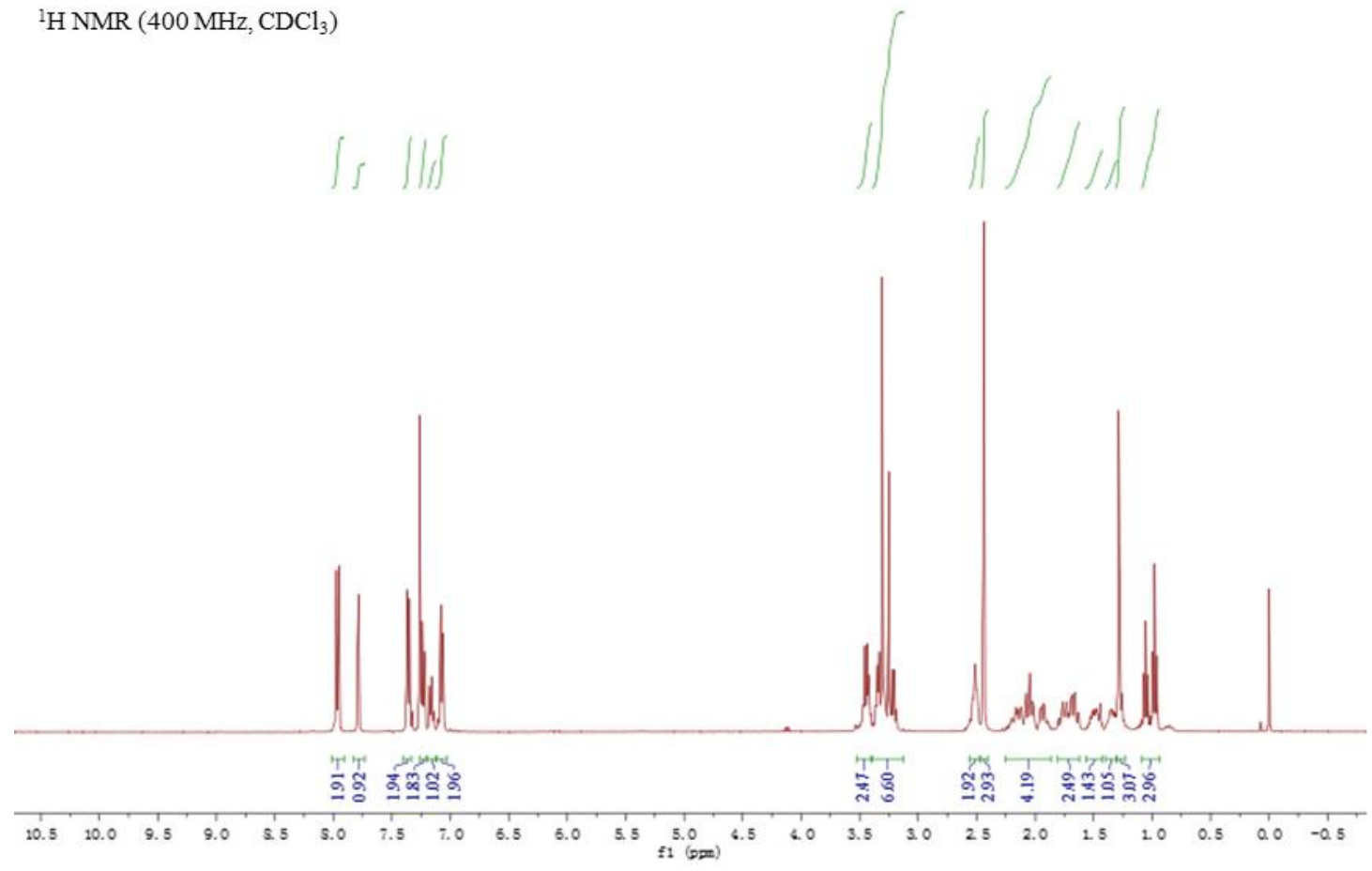

${ }^{13} \mathrm{CNMR}\left(101 \mathrm{MHz}, \mathrm{CDCl}_{3}\right)$

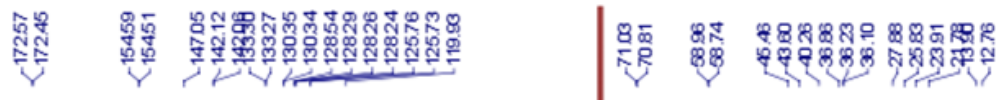

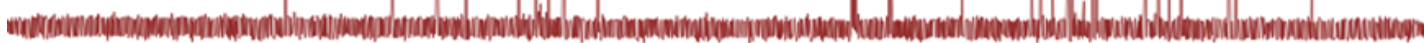

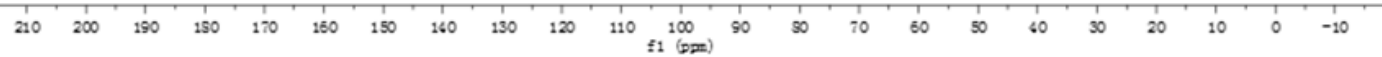


<smiles>[3H]n1c([C@@](C)(CCBr)CCC(=O)N(CC)CCOC)cc2ccccc21</smiles>

${ }^{1} \mathrm{H}$ NMR $\left(400 \mathrm{MHz}, \mathrm{CDCl}_{3}\right)$
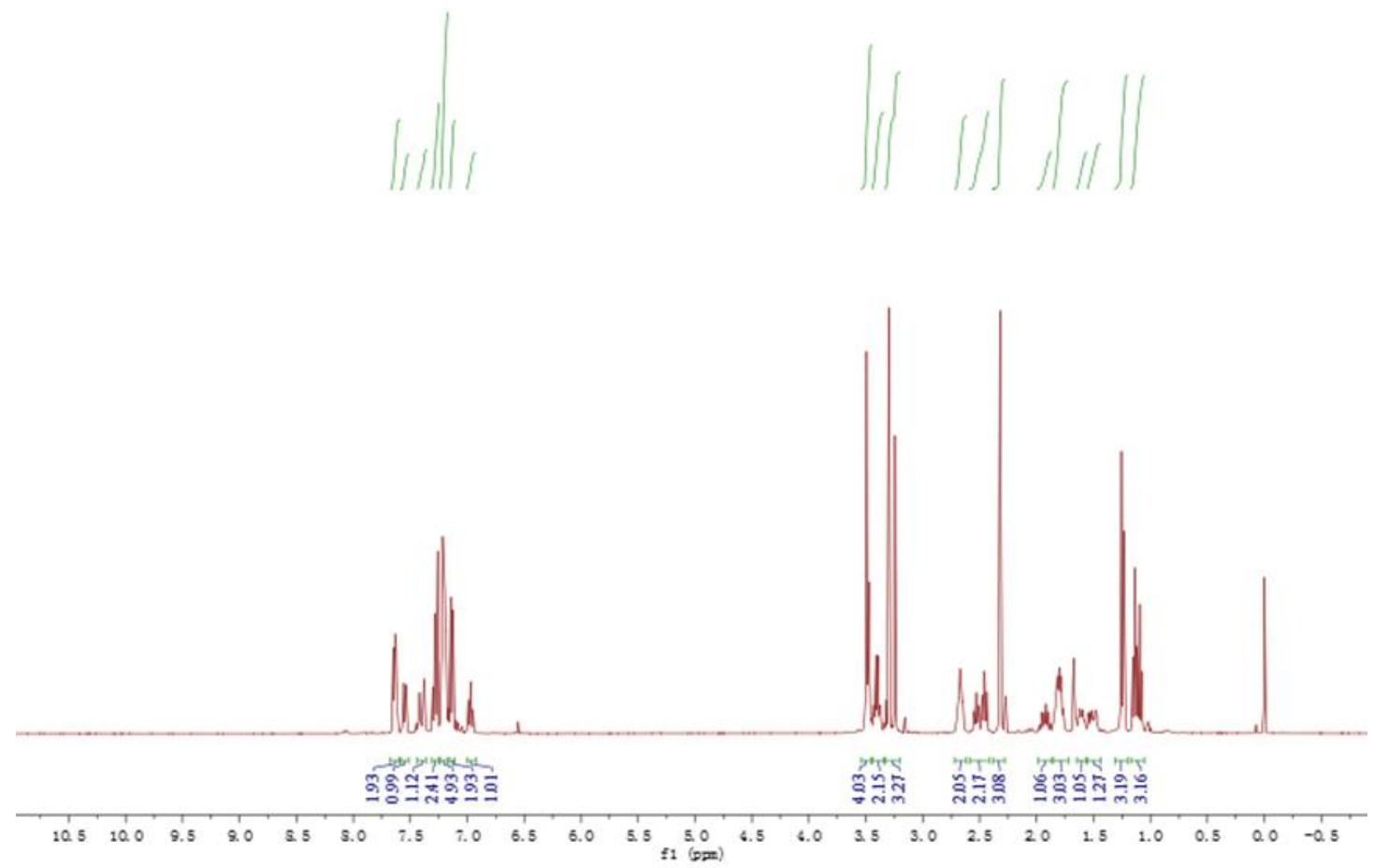

${ }^{13} \mathrm{C} \mathrm{NMR}\left(101 \mathrm{MHz}, \mathrm{CDCl}_{3}\right)$

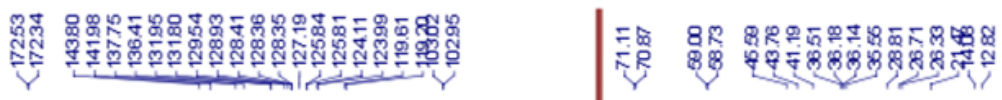

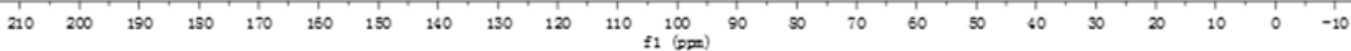


<smiles>CCC[C@](C)(CCC(=O)N(CC)CC)C(=O)O</smiles>

${ }^{1} \mathrm{H}$ NMR $\left(400 \mathrm{MHz}, \mathrm{CDCl}_{3}\right)$

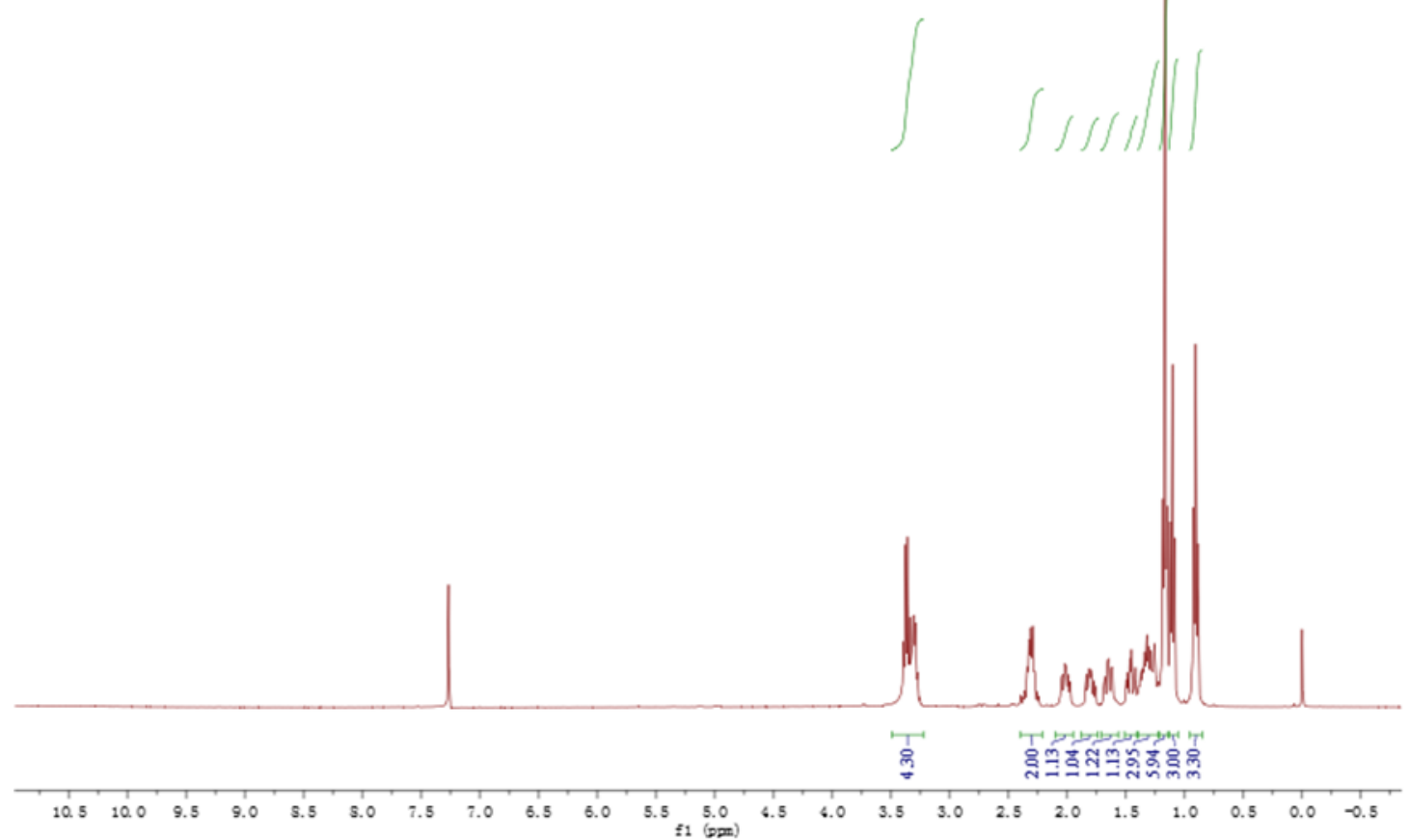

${ }^{13} \mathrm{C} \mathrm{NMR}\left(101 \mathrm{MHz}, \mathrm{CDCl}_{3}\right)$

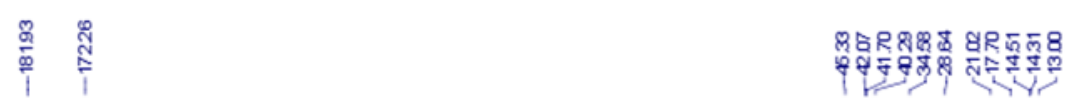

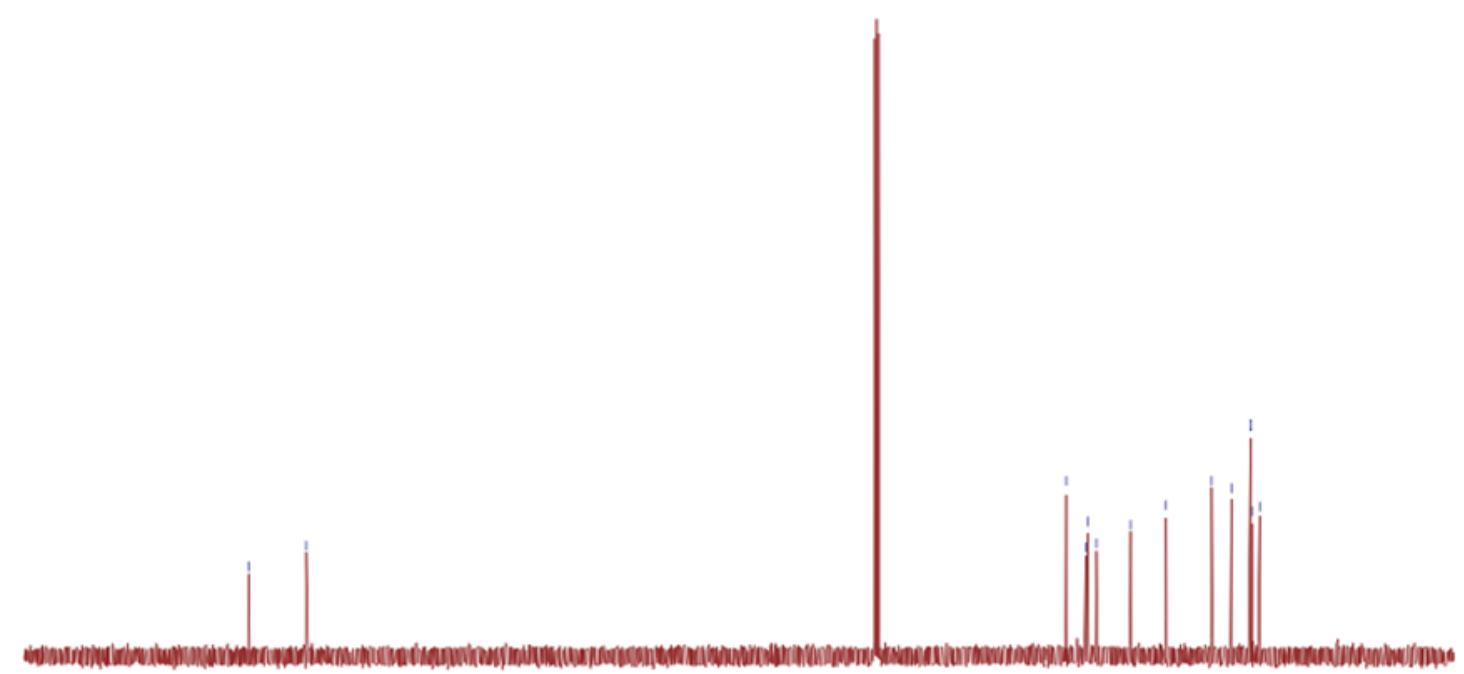

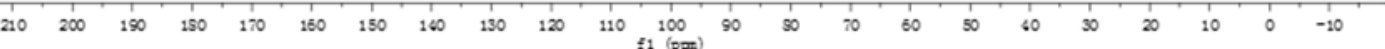




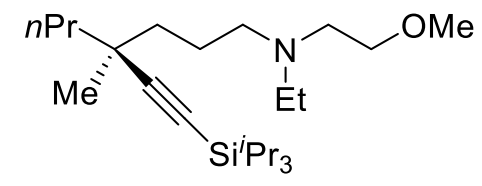

${ }^{1} \mathrm{H} \mathrm{NMR}\left(400 \mathrm{MHz}, \mathrm{CDCl}_{3}\right)$
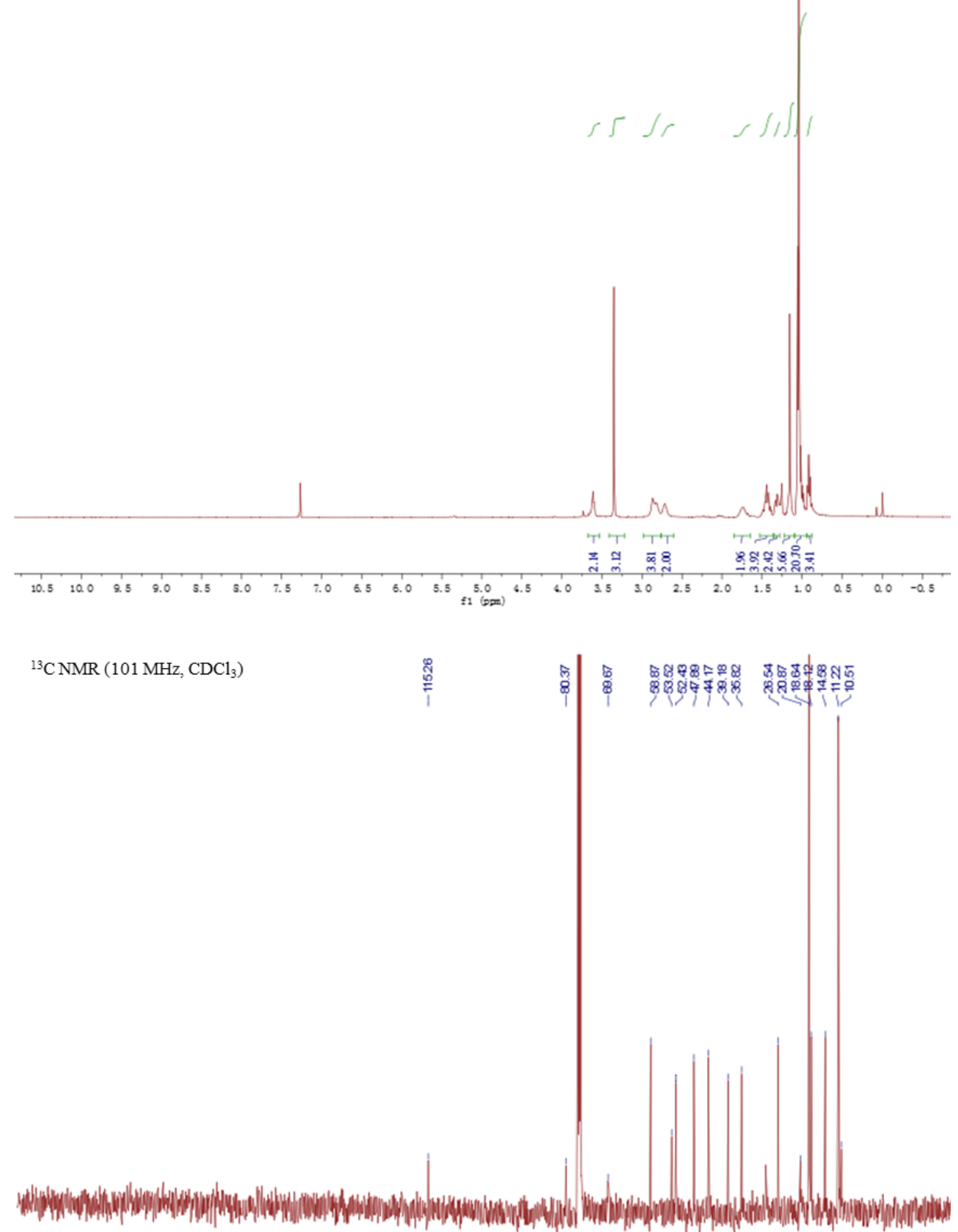

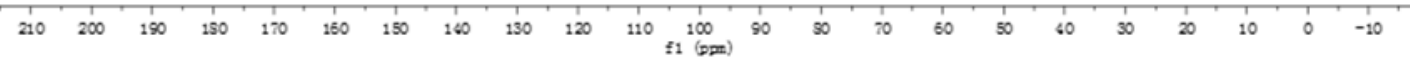


<smiles>CCN(CCOC)C(=O)CC[C@](C)(O)CCc1ccccc1</smiles>

${ }^{1} \mathrm{H}$ NMR $\left(400 \mathrm{MHz}, \mathrm{CDCl}_{3}\right)$

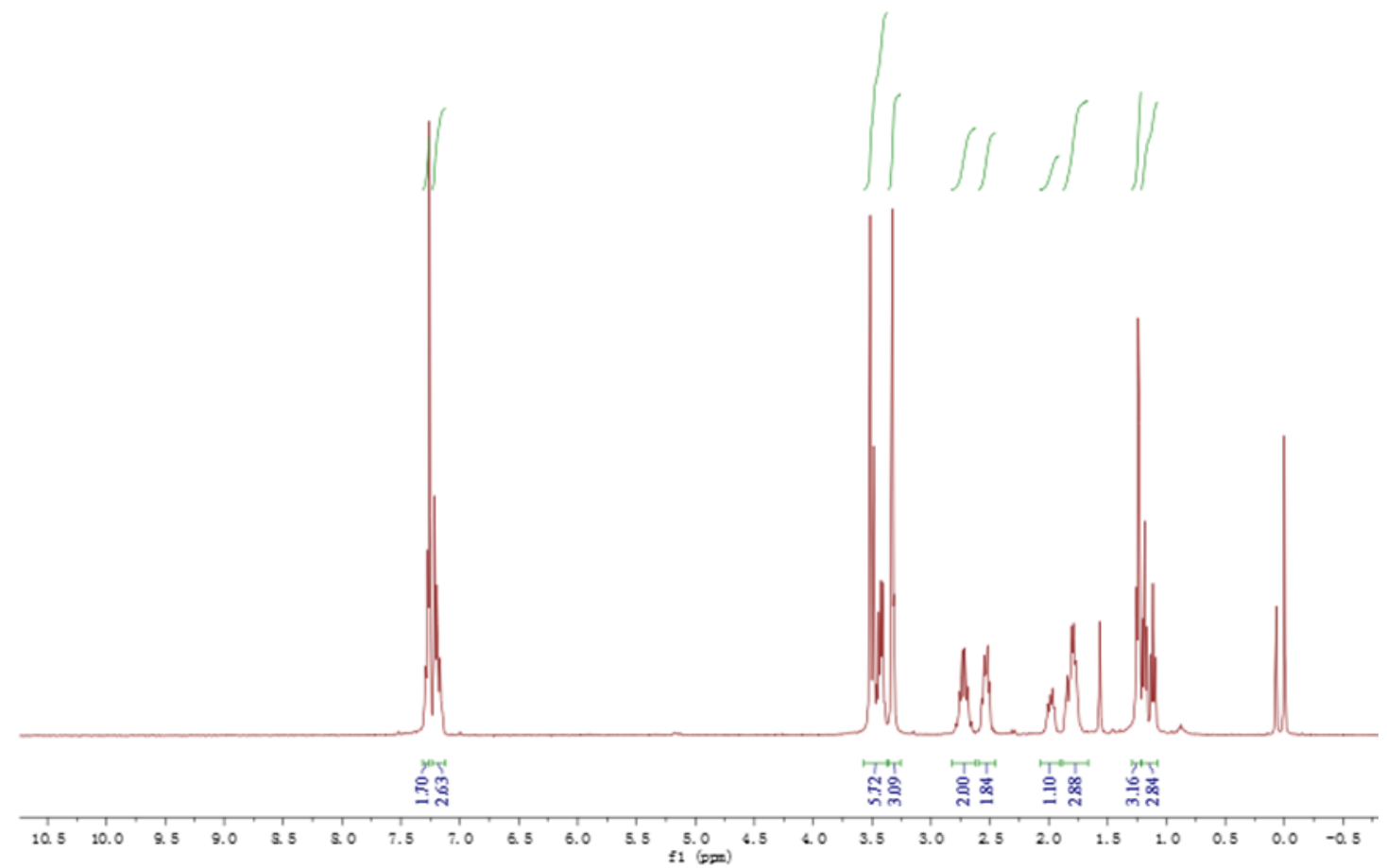

${ }^{13} \mathrm{C} \mathrm{NMR}\left(101 \mathrm{MHz}, \mathrm{CDCl}_{3}\right)$

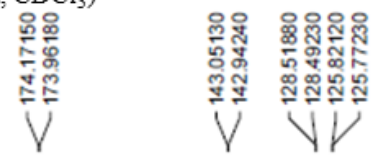

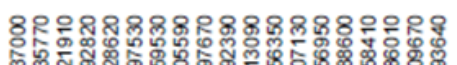

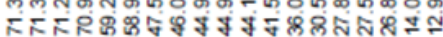

रह5,

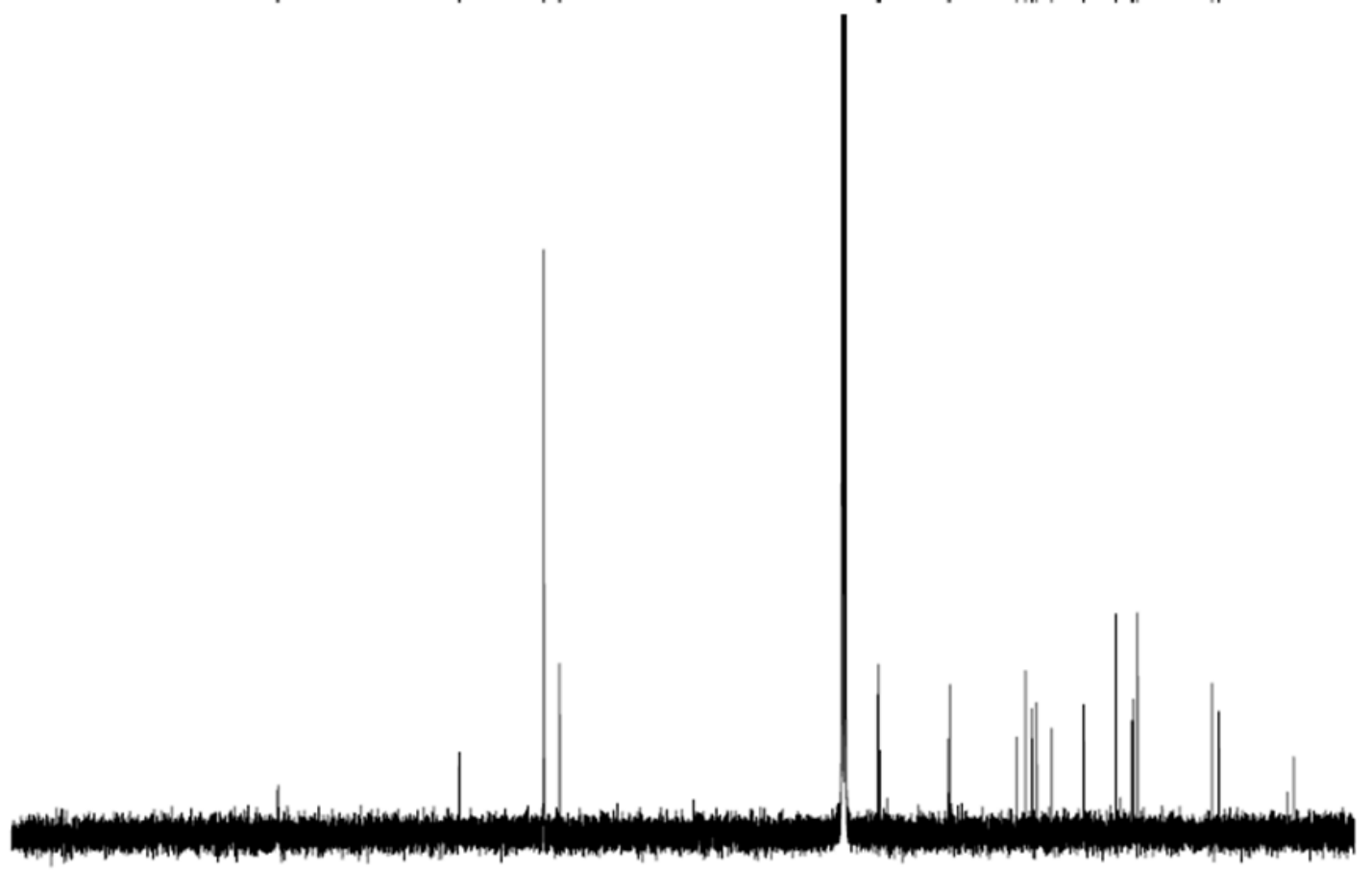

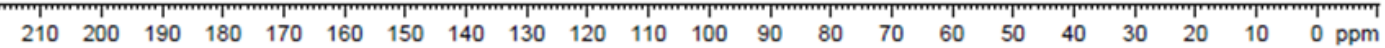


$\operatorname{Ir}(\mathrm{COD})(\mathrm{CTH}-(R)-\mathrm{P}-\mathrm{Phos}) \mathrm{OTf}$ (complex 6)

${ }^{31} \mathrm{PNMR}\left(162 \mathrm{MHz}, \mathrm{CDCl}_{3}\right)$

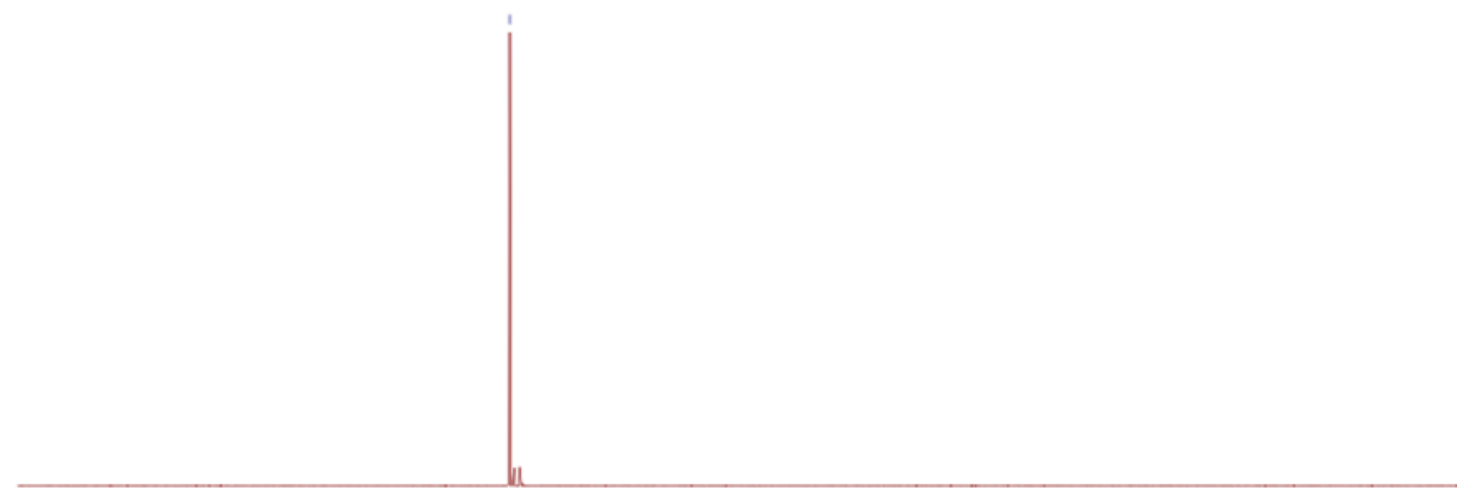

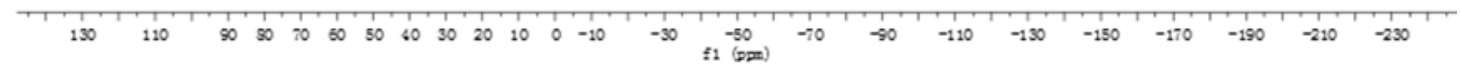

${ }^{1} \mathrm{H} \mathrm{NMR}\left(400 \mathrm{MHz}, \mathrm{CDCl}_{3}\right)$

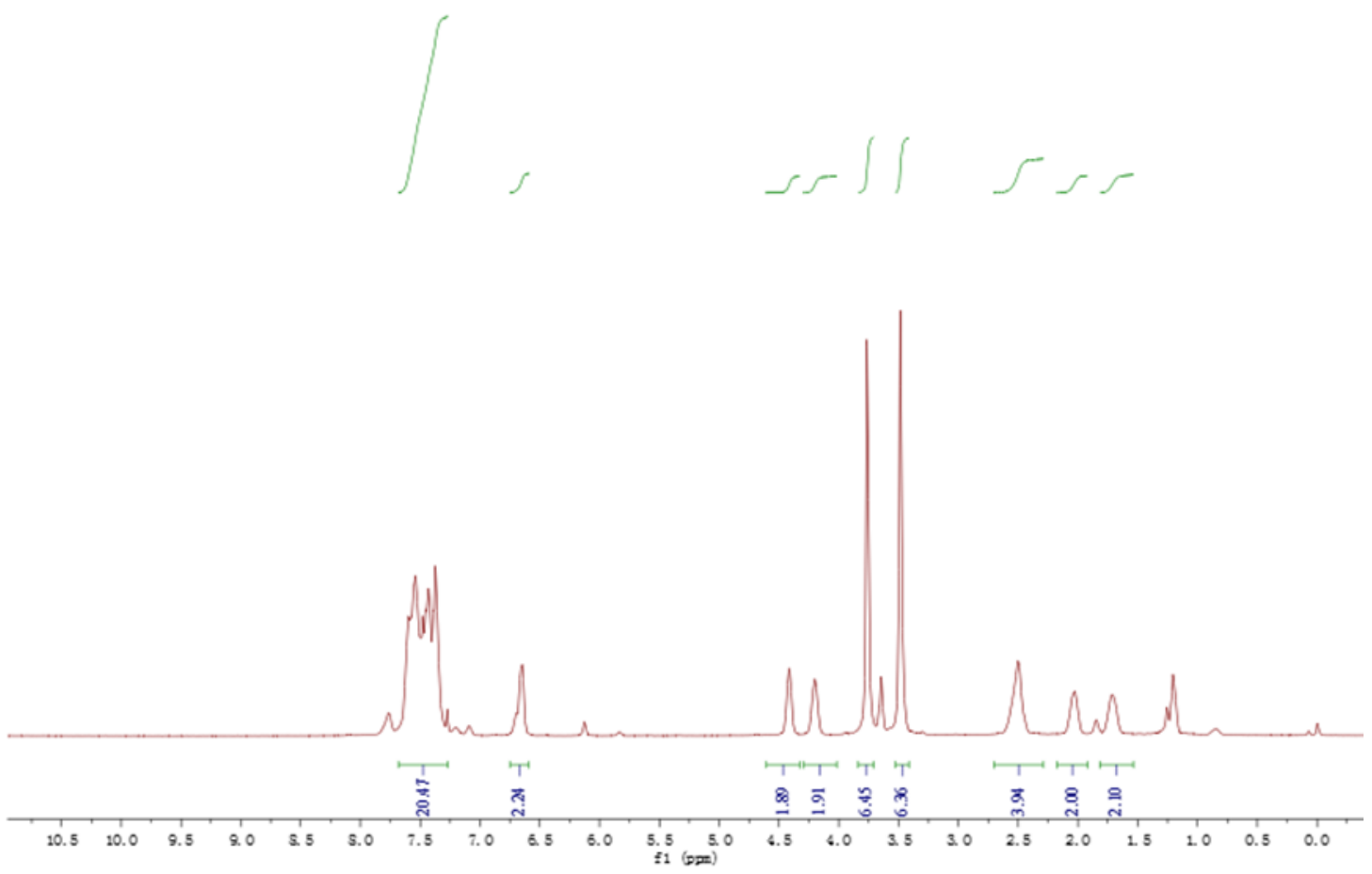


${ }^{19} \mathrm{~F} \mathrm{NMR}\left(376 \mathrm{MHz}, \mathrm{CDCl}_{3}\right)$

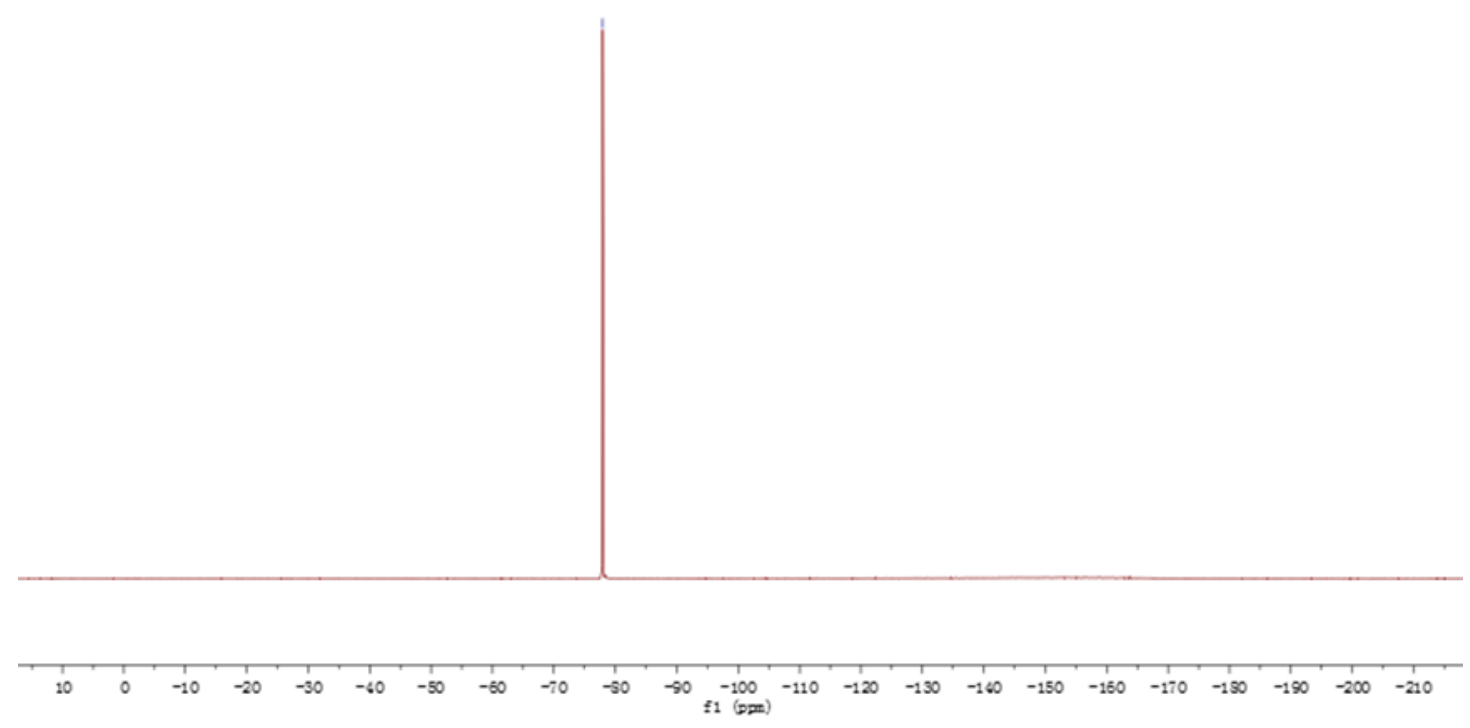

